Supporting Information For

\title{
Enantioselective Hydrogenation of Racemic $\alpha$-Arylamino Lactones to Chiral Amino Diols with Site-Specifically Modified Chiral Spiro Iridium Catalysts
}

Xue-Song Gu, Na Yu, Xiao-Hui Yang, An-Te Zhu, Jian-Hua Xie*, Qi-Lin Zhou

State Key Laboratory and Institute of Elemento-organic Chemistry, College of Chemistry, Nankai University, Tianjin 300071, China

\section{Contents}

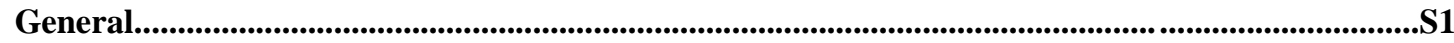

(A) Preparation of New SpiroPAP ligands and Their Iridium Catalysts.......................................S2

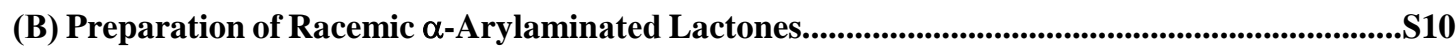

(C) Asymmetric Hydrogenation of Racemic $\alpha$-Arylaminated Lactones.........................S17

(D) Gram-Scale Asymmetric Hydrogenation and Its Applications..............................S28

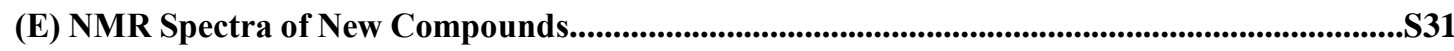

(F) HPLC Charts for Hydrogenation Products.................................................................................S134

General: All reactions and manipulations which are sensitive to moisture or air were performed under inert atmosphere of nitrogen or argon. All chemicals were purchased from J \& K, Acros and Aldrich and were used as received. 3-Bromotetrahydro-2H-pyran-2-one was synthesized according to the literature method. ${ }^{1}$ Hydrogen gas (99.999\%) was purchased from Boc Gas Inc., Tianjin. Anhydrous THF was distilled from sodium benzophenone ketyl. Anhydrous $\mathrm{CH}_{2} \mathrm{Cl}_{2}, n \mathrm{PrOH}$ and $\mathrm{Et}_{3} \mathrm{~N}$ were freshly distilled from calcium hydride. Anhydrous $\mathrm{EtOH}$ was freshly distilled from magnesium. Melting points were measured on a RY-I apparatus and uncorrected. ${ }^{1} \mathrm{H}$ NMR spectra were recorded at $400 \mathrm{MHz}$ on Bruker AV 400 spectrometer. ${ }^{13} \mathrm{C}$ NMR spectra were recorded at $100 \mathrm{MHz}$ on Bruker AV 400 spectrometer. NMR spectra were recorded in deuterated chloroform $\left(\mathrm{CDCl}_{3}\right)$ as a solvent, with residual chloroform $(\delta$ $7.26 \mathrm{ppm}$. for ${ }^{1} \mathrm{H}$ NMR and $\delta 77.00 \mathrm{ppm}$. for ${ }^{13} \mathrm{C} \mathrm{NMR}$ ) or tetramethylsilane (TMS, $\delta 0.00 \mathrm{ppm}$. for ${ }^{1} \mathrm{H}$ NMR) taken as the inert standard, and were reported in ppm. Abbreviations for signal coupling are as follows: s, singlet; $d$, doublet; t, triplet; q, quartet; $m$, multiplet. Coupling constants were taken from the spectra directly and are uncorrected. Optical rotations were determined using a Perkin Elmer 341 polarimeter. HRMS were recorded on APEXII and ZAB-HS spectrometer. HPLC analyses were performed using Hewlett Packard Model HP1100 instruments with Chiralcel OJ-3, OD-3, AD-3, IC-3, IF-3, IG-3, IH-3 column. 


\section{(A) Preparation of New SpiroPAP ligands and Their Iridium Catalysts}

The new SpiroPAP ligands and their iridium catalysts were synthesized from 4-substituted picolinaldehydes with DTB-SpiroAP ligand in the presence of $\mathrm{NaBH}_{3} \mathrm{CN}$ and the corresponding chiral spiro iridium catalysts were prepared by in situ reaction of the SpiroPAP ligands with $[\operatorname{Ir}(\operatorname{cod}) \mathrm{Cl}]_{2}$ under $\mathrm{H}_{2}$ atmosphere according to our previously reported method. ${ }^{2}$

\section{Synthesis of 4-substituted picolinaldehydes}

The 4-substituted picolinaldehydes were prepared from methyl 2-methylisonicotinate via the reaction with Grignard reagents and followed by oxidation with selenium dioxide.<smiles>CC(=O)c1ccnc([N+](=O)[O-])c1</smiles>

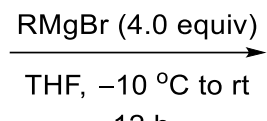
$12 \mathrm{~h}$<smiles>[R]C([R])(O)c1ccnc([N+](=O)[O-])c1</smiles>

s1

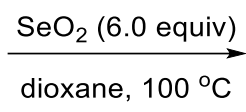

$12 \mathrm{~h}$<smiles>[R]C([R])(O)c1ccnc(C=O)c1</smiles>

S2

\section{Synthesis of 4-substituted 2-methylpyridines S1}

General procedure: To a solution of methyl 2-methylisonicotinate $(1.0 \mathrm{~g}, 6.6 \mathrm{mmol})$ in anhydrous THF $(10 \mathrm{~mL})$ was added $\mathrm{RMgBr}\left(26.4 \mathrm{mmol}\right.$, in THF) dropwise at $-10{ }^{\circ} \mathrm{C}$ under $\mathrm{N}_{2}$ atmosphere and the resulting reaction mixture was then allowed to warm to room temperature and stirred for further $10 \mathrm{~h}$. The reaction mixture was quenched with saturated $\mathrm{NH}_{4} \mathrm{Cl}(10 \mathrm{~mL})$ and extracted with EtOAc $(10 \mathrm{~mL} \times$ 3). The combined organic layer was washed with brine, dried over anhydrous $\mathrm{MgSO}_{4}$ and concentrated in vacuo. The residue was chromatographed on silica gel column with ethyl acetate/petroleum ether (from 1:5 to 1:3) as an eluent to offer the corresponding products as white/yellow solids or red oils.

\section{4-(2-Hydroxypropan-2-yl)-2-methylpyridine (S1a)}<smiles>Cc1cc(C(C)(C)O)ccn1</smiles>
White solid, mp $98-99^{\circ} \mathrm{C}, 0.8 \mathrm{~g}, 80 \%$ yield. ${ }^{1} \mathrm{H}$ NMR $\left(400 \mathrm{MHz}, \mathrm{CDCl}_{3}\right) \delta: 8.43(\mathrm{~d}, J=$ $5.2 \mathrm{~Hz}, 1 \mathrm{H}), 7.27$ (s, 1H), 7.17 (dd, $J=5.2,1.8 \mathrm{~Hz}, 1 \mathrm{H}), 2.56(\mathrm{~s}, 3 \mathrm{H}), 2.08$ (br, $1 \mathrm{H}), 1.56$ (s, 6H). ${ }^{13} \mathrm{C}$ NMR (101 MHz, $\left.\mathrm{CDCl}_{3}\right) \delta: 159.4,157.9,148.3,119.5,117.2,71.2,31.3$, 24.1. HRMS(ESI) Calcd for $\mathrm{C}_{9} \mathrm{H}_{14} \mathrm{NO}\left([\mathrm{M}+\mathrm{H}]^{+}\right)$: 152.1070; Found: 152.1073 .

\section{4-(Diethylhydroxymethyl)-2-methylpyridine (S1b)}<smiles>CCOC(O)(CC)c1ccnc(C)c1</smiles>

Yellow solid, mp $102-103{ }^{\circ} \mathrm{C}, 0.79 \mathrm{~g}, 60 \%$ yield. ${ }^{1} \mathrm{H}$ NMR (400 MHz, $\left.\mathrm{CDCl}_{3}\right) \delta: 8.42$ (d, $J=5.2 \mathrm{~Hz}, 1 \mathrm{H}), 7.18(\mathrm{~s}, 1 \mathrm{H}), 7.07(\mathrm{dd}, J=5.2,1.8 \mathrm{~Hz}, 1 \mathrm{H}), 2.56(\mathrm{~s}, 3 \mathrm{H}), 2.02-1.92$ (m, 1H), 1.87-1.77 (m, 4H), $0.76(\mathrm{t}, J=7.2 \mathrm{~Hz}, 6 \mathrm{H}) .{ }^{13} \mathrm{C} \mathrm{NMR}\left(101 \mathrm{MHz}, \mathrm{CDCl}_{3}\right) \delta$ : 158.2, 155.2, 148.8, 120.3, 118.0, 76.8, 34.8, 24.6, 7.7. HRMS (ESI) Calcd for $\mathrm{C}_{11} \mathrm{H}_{18} \mathrm{NO}\left([\mathrm{M}+\mathrm{H}]^{+}\right): 180.1383$; Found: 180.1386 .

\section{4-(Dicyclohexylhydroxymethyl)-2-methylpyridine (S1c)}<smiles>Cc1cc(C(O)(Cl)C2CCCCC2)ccn1</smiles>

Red oil, $1.4 \mathrm{~g}, 74 \%$ yield. ${ }^{1} \mathrm{H}$ NMR (400 MHz, $\left.\mathrm{CDCl}_{3}\right) \delta: 8.40(\mathrm{~d}, J=5.4 \mathrm{~Hz}, 1 \mathrm{H}), 7.16$ (s, 1H), $7.05(\mathrm{~s}, 1 \mathrm{H}), 2.57$ (s, 3H), 1.94-1.80 (m, 4H), 1.80-1.58 (m, 6H), $1.44(\mathrm{~d}, J=$ $12.8 \mathrm{~Hz}, 2 \mathrm{H}), 1.32-1.15(\mathrm{~m}, 4 \mathrm{H}), 1.10-0.88(\mathrm{~m}, 4 \mathrm{H}), 0.81-0.66(\mathrm{~m}, 2 \mathrm{H}) .{ }^{13} \mathrm{C}$ NMR $\left(101 \mathrm{MHz}, \mathrm{CDCl}_{3}\right) \delta: 157.6,153.4,148.2,121.2,118.9,80.3,43.9,27.3,26.7,26.6$, 26.5(2), 24.7. HRMS (ESI) Calcd for $\mathrm{C}_{19} \mathrm{H}_{30} \mathrm{NO}\left([\mathrm{M}+\mathrm{H}]^{+}\right)$: 288.2322; Found: 288.2323. 
<smiles>Cc1cc(C(O)(c2ccccc2)c2ccccc2)ccn1</smiles>

White solid, mp $170-171{ }^{\circ} \mathrm{C} .1 .45 \mathrm{~g}, 80 \%$ yield. ${ }^{1} \mathrm{H}$ NMR (400 MHz, $\left.\mathrm{CDCl}_{3}\right) \delta: 8.32$ $(\mathrm{d}, J=5.2 \mathrm{~Hz}, 1 \mathrm{H}), 7.37-7.22(\mathrm{~m}, 10 \mathrm{H}), 7.17(\mathrm{~s}, 1 \mathrm{H}), 7.01(\mathrm{~d}, J=4.8 \mathrm{~Hz}, 1 \mathrm{H}), 3.40(\mathrm{~s}$, 1H), 2.49 (s, 3H). ${ }^{13} \mathrm{C}$ NMR (101 MHz, $\left.\mathrm{CDCl}_{3}\right) \delta: 158.3,155.7,148.7,145.7,128.2$, 127.8, 127.7, 122.0, 120.0, 81.3, 24.5. HRMS (ESI) Calcd for $\mathrm{C}_{19} \mathrm{H}_{18} \mathrm{NO}\left([\mathrm{M}+\mathrm{H}]^{+}\right)$: 276.1383; Found: 276.1385 .

\section{4-[Di(2,6-dimethylphenyl)hydroxymethyl]-2-methylpyridine (S1e)}<smiles>[Y10]C(O)([AlH2])c1ccnc([NH+]([O])O)c1</smiles>

Yellow solid, mp 190-191 ${ }^{\circ} \mathrm{C} .1 .1 \mathrm{~g}, 51 \%$ yield. ${ }^{1} \mathrm{H}$ NMR (400 MHz, $\left.\mathrm{CDCl}_{3}\right) \delta: 8.39$ (d, $J=5.2 \mathrm{~Hz}, 1 \mathrm{H}), 7.20(\mathrm{~d}, J=1.7 \mathrm{~Hz}, 1 \mathrm{H}), 7.04-6.99(\mathrm{~m}, 1 \mathrm{H}), 6.94(\mathrm{~s}, 2 \mathrm{H}), 6.83(\mathrm{~s}, 4 \mathrm{H})$, $2.53(\mathrm{~s}, 3 \mathrm{H}), 2.27$ (s, $12 \mathrm{H}) .{ }^{13} \mathrm{C}$ NMR $\left(101 \mathrm{MHz}, \mathrm{CDCl}_{3}\right) \delta 158.0,156.2,148.4,145.9$, 137.5, 129.2, 125.7, 122.0, 120.2, 81.1, 24.5, 21.5. HRMS (ESI) Calcd for $\mathrm{C}_{23} \mathrm{H}_{26} \mathrm{NO}$ $\left([\mathrm{M}+\mathrm{H}]^{+}\right)$: 332.2009; Found: 332.2013 .

\section{4-(Di(2,6-dimethylphenyl)hydroxymethyl)-2-methylpyridine (S1f)}<smiles>Cc1cc(C(O)(Br)Br)ccn1</smiles>
White solid, mp $177-178{ }^{\circ} \mathrm{C} .2 .4 \mathrm{~g}, 74 \%$ yield. ${ }^{1} \mathrm{H}$ NMR $\left(400 \mathrm{MHz}, \mathrm{CDCl}_{3}\right) \delta: 8.33$ (d, $J=5.3 \mathrm{~Hz}, 1 \mathrm{H}), 7.34(\mathrm{~s}, 2 \mathrm{H}), 7.28(\mathrm{~s}, 1 \mathrm{H}), 7.06(\mathrm{~s}, 4 \mathrm{H}), 6.98(\mathrm{~d}, J=5.4 \mathrm{~Hz}, 1 \mathrm{H}), 3.11$ (s, $1 \mathrm{H}), 2.51(\mathrm{~s}, 3 \mathrm{H}), 1.23(\mathrm{~d}, J=1.8 \mathrm{~Hz}, 36 \mathrm{H}) .{ }^{13} \mathrm{C} \mathrm{NMR}\left(101 \mathrm{MHz}, \mathrm{CDCl}_{3}\right) \delta: 158.0$, 156.6, 150.3, 148.4, 144.9, 122.4, 122.0, 121.2, 120.1, 82.1, 34.9, 31.4, 24.6. HRMS (ESI) Calcd for $\mathrm{C}_{35} \mathrm{H}_{50} \mathrm{NO}\left([\mathrm{M}+\mathrm{H}]^{+}\right)$: 500.3887; Found: 500.3889 .

\section{Synthesis of 4-substituted 2-pyridinecarbaldehyde S2}

General procedure: To the solution of 4-substituted 2-methylpyridine $\mathbf{S 1}(1.0 \mathrm{mmol})$ in dioxane (10 $\mathrm{mL}$ ) was added $\mathrm{SeO}_{2}(0.66 \mathrm{~g}, 6.00 \mathrm{mmol})$. The resulting reaction mixture was then heated to $100{ }^{\circ} \mathrm{C}$ and stirred for $12 \mathrm{~h}$. After the reaction was completed, the reaction mixture was cooled to room temperature, and the formed precipitates were removed by filtration. The solvents were then concentrated in vacuo, and the residue was chromatographed on silica gel column with ethyl acetate/petroleum ether (1:5) as an eluent to offer the corresponding compounds as white/yellow solids.

\section{4-(Diethylhydroxymethyl)-2-pyridinecarbaldehyde (S2a)}<smiles>CCC(O)(O)c1ccnc(C=O)c1</smiles>

Yellow solid, mp $95-96{ }^{\circ} \mathrm{C} .29 \mathrm{mg}, 15 \%$ yield. ${ }^{1} \mathrm{H}$ NMR $\left(400 \mathrm{MHz}, \mathrm{CDCl}_{3}\right) \delta: 10.12$ (s, 1H), $8.75(\mathrm{~d}, J=5.2 \mathrm{~Hz}, 1 \mathrm{H}), 7.95(\mathrm{~s}, 1 \mathrm{H}), 7.61(\mathrm{dd}, J=5.2,2.0 \mathrm{~Hz}, 1 \mathrm{H}), 1.95-$ $1.83(\mathrm{~m}, 4 \mathrm{H}), 1.79-1.71(\mathrm{~m}, 1 \mathrm{H}), 0.75(\mathrm{t}, J=7.4 \mathrm{~Hz}, 6 \mathrm{H}) .{ }^{13} \mathrm{C} \mathrm{NMR}\left(101 \mathrm{MHz}, \mathrm{CDCl}_{3}\right)$ $\delta: 193.8,156.8,152.7,150.1,125.3,118.8,100.0,34.9,7.6$. HRMS (ESI) Calcd for $\mathrm{C}_{11} \mathrm{H}_{16} \mathrm{NO}_{2}\left([\mathrm{M}+\mathrm{H}]^{+}\right)$: 194.1176; Found: 194.1175 .

\section{4-(Dicyclohexylhydroxymethyl)-2-pyridinecarbaldehyde (S2b)}<smiles>O=Cc1cc(C(O)(O)C2CCCCC2)ccn1</smiles>

Yellow solid, mp 129-130 ${ }^{\circ} \mathrm{C} .54 \mathrm{mg}, 18 \%$ yield. ${ }^{1} \mathrm{H}$ NMR (400 MHz, $\left.\mathrm{CDCl}_{3}\right) \delta$ : $10.12(\mathrm{~s}, 1 \mathrm{H}), 8.72(\mathrm{~d}, J=5.2 \mathrm{~Hz}, 1 \mathrm{H}), 7.93(\mathrm{~s}, 1 \mathrm{H}), 7.58(\mathrm{~s}, 1 \mathrm{H}), 1.98-1.84(\mathrm{~m}, 4 \mathrm{H})$, $1.81-1.60(\mathrm{~m}, 6 \mathrm{H}), 1.41(\mathrm{~d}, J=12.7 \mathrm{~Hz}, 2 \mathrm{H}), 1.32-1.15(\mathrm{~m}, 4 \mathrm{H}), 1.06-0.88(\mathrm{~m}, 4 \mathrm{H})$, 0.74-0.61 (m, 2H). ${ }^{13} \mathrm{C}$ NMR (101 MHz, $\left.\mathrm{CDCl}_{3}\right) \delta: 193.9,155.0,152.2,149.6,126.2$, 119.57, 80.5, 44.0, 27.3, 26.6, 26.5, 26.3. HRMS (ESI) Calcd for $\mathrm{C}_{19} \mathrm{H}_{28} \mathrm{NO}_{2}$ $\left([\mathrm{M}+\mathrm{H}]^{+}\right): 302.2115$; Found: 302.2118 .

\section{4-(Diphenylhydroxymethyl)-2-pyridinecarbaldehyde (S2c)}


<smiles>O=Cc1cc(C(O)(c2ccccc2)c2ccccc2)ccn1</smiles>

White solid, mp $153-154{ }^{\circ} \mathrm{C} .35 \mathrm{mg}, 12 \%$ yield. ${ }^{1} \mathrm{H}$ NMR $\left(400 \mathrm{MHz}, \mathrm{CDCl}_{3}\right) \delta 10.04$ (s, 1H), 8.69 (d, $J=5.2 \mathrm{~Hz}, 1 \mathrm{H}), 7.99$ (d, $J=1.6 \mathrm{~Hz}, 1 \mathrm{H}), 7.57$ (dd, $J=5.2,2.0 \mathrm{~Hz}$, 1H), 7.42-7.29 (m, 6H), 7.27-7.15 (m, 4H), 3.12 (s, 1H). ${ }^{13} \mathrm{C} \mathrm{NMR}\left(101 \mathrm{MHz}, \mathrm{CDCl}_{3}\right)$ $\delta: 193.3,157.1,152.7,150.0,145.0,128.5,128.1,127.7,126.6,120.6,81.2$. HRMS (ESI) Calcd for $\mathrm{C}_{19} \mathrm{H}_{16} \mathrm{NO}_{2}\left([\mathrm{M}+\mathrm{H}]^{+}\right)$: 290.1176; Found: 290.1176.

\section{4-[Di(2,6-dimethylphenyl)hydroxymethyl]-2-pyridinecarbaldehyde (S2d)}<smiles>[Y10]C([Y19])(O)c1ccnc(C=O)c1</smiles>

White solid, mp $169-170{ }^{\circ} \mathrm{C} .59 \mathrm{mg}, 17 \%$ yield. ${ }^{1} \mathrm{H}$ NMR (400 $\left.\mathrm{MHz}, \mathrm{CDCl}_{3}\right) \delta$ : $10.05(\mathrm{~s}, 1 \mathrm{H}), 8.69$ (d, $J=5.1 \mathrm{~Hz}, 1 \mathrm{H}), 8.00(\mathrm{~s}, 1 \mathrm{H}), 7.56(\mathrm{dd}, J=5.2,2.0 \mathrm{~Hz}, 1 \mathrm{H})$, $6.94(\mathrm{~s}, 2 \mathrm{H}), 6.82(\mathrm{~s}, 4 \mathrm{H}), 3.06(\mathrm{br}, 1 \mathrm{H}), 2.26$ (s, 12H). ${ }^{13} \mathrm{C} \mathrm{NMR}\left(101 \mathrm{MHz}, \mathrm{CDCl}_{3}\right)$ $\delta: 193.4,157.5,152.5,149.9,145.2,138.0,129.7,126.8,125.5,120.7,81.2,21.5$. HRMS (ESI) Calcd for $\mathrm{C}_{23} \mathrm{H}_{24} \mathrm{NO}_{2}\left([\mathrm{M}+\mathrm{H}]^{+}\right)$: 346.1802; Found: 346.1800 .

\section{4-[Di(2,6-dimethylphenyl)hydroxymethyl]-2-pyridinecarbaldehyde (S2e)}<smiles>[B-][10B]C(O)(Br)c1ccnc(C=O)c1</smiles>

White solid, mp169-170 ${ }^{\circ} \mathrm{C} .93 \mathrm{mg}, 18 \%$ yield. ${ }^{1} \mathrm{H}$ NMR (400 MHz, $\left.\mathrm{CDCl}_{3}\right) \delta: 10.09$ $(\mathrm{s}, 1 \mathrm{H}), 8.72(\mathrm{dd}, J=5.2,0.8 \mathrm{~Hz}, 1 \mathrm{H}), 8.07(\mathrm{dd}, J=1.6,0.8 \mathrm{~Hz}, 1 \mathrm{H}), 7.56(\mathrm{dd}, J=$ $5.2,2.0 \mathrm{~Hz}, 1 \mathrm{H}), 7.36(\mathrm{t}, J=1.8 \mathrm{~Hz}, 2 \mathrm{H}), 7.04(\mathrm{~d}, J=1.6 \mathrm{~Hz}, 4 \mathrm{H}), 2.77(\mathrm{~s}, 1 \mathrm{H}), 1.24$ $(\mathrm{s}, 36 \mathrm{H}) .{ }^{13} \mathrm{C} \mathrm{NMR}\left(101 \mathrm{MHz}, \mathrm{CDCl}_{3}\right) \delta: 193.4,158.3,152.5,150.7,149.7,144.2$, 126.8, 122.2, 121.7, 120.7, 82.1, 35.0, 31.4. HRMS (ESI) Calcd for $\mathrm{C}_{35} \mathrm{H}_{48} \mathrm{NO}_{2}$ $\left([\mathrm{M}+\mathrm{H}]^{+}\right): 514.3680$; Found: 514.3676 .

\section{Synthesis of 4-substituted 2-pyridinecarbaldehydes S2f and S2g}

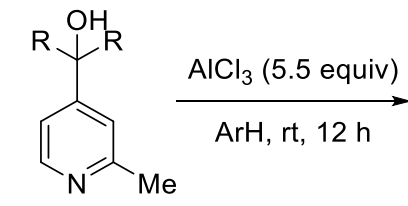

S1a and S1d<smiles>[R]C([R])([Al])c1ccnc(C)c1</smiles>

s3

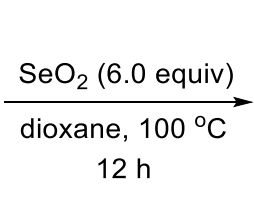

S2f, $\mathrm{R}=\mathrm{Me}, \mathrm{Ar}=\mathrm{C}_{6} \mathrm{H}_{5}$ S2g, $\mathrm{R}=\mathrm{Ph}, \mathrm{Ar}=4-\mathrm{MeOC}_{6} \mathrm{H}_{4}$

General procedure for synthesis of S3: To a mixture of $\mathrm{AlCl}_{3}(1.45 \mathrm{~g}, 10.9 \mathrm{mmol})$ in $30 \mathrm{~mL}$ benzene or anisole was added S1a or S1d $(1.98 \mathrm{mmol})$. The resulting mixture was stirred at room temperature overnight. The mixture was poured onto ice $(100 \mathrm{~mL})$, diluted with EtOAc $(20 \mathrm{~mL})$, and neutralized with saturated sodium hydroxide solution. The residue was extracted with EtOAc $(20 \mathrm{~mL} \times 3)$. The combined organic layer was washed with brine, dried over anhydrous $\mathrm{MgSO}_{4}$, and concentrated in vacuo. The residue was chromatographed on silica gel column with ethyl acetate/petroleum ether (1:5) as an eluent to offer the corresponding products as a yellow oil or a white solid.

\section{4-(2-Phenylpropan-2-yl)-2-methylpyridine (S3a)}

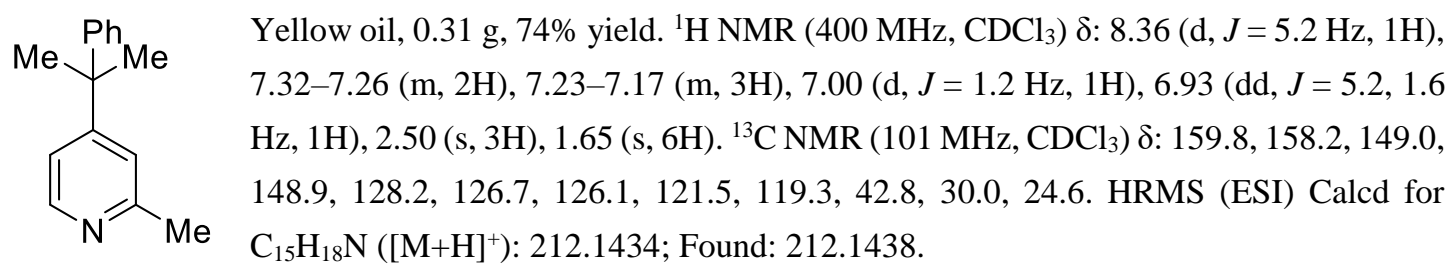




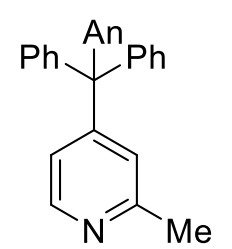

White solid, mp $169-170{ }^{\circ} \mathrm{C} .0 .68 \mathrm{~g}, 94 \%$ yield. ${ }^{1} \mathrm{H}$ NMR (400 MHz, $\left.\mathrm{CDCl}_{3}\right) \delta: 8.37$ $(\mathrm{d}, J=5.2 \mathrm{~Hz}, 1 \mathrm{H}), 7.29-7.16(\mathrm{~m}, 10 \mathrm{H}), 7.10-7.05(\mathrm{~m}, 2 \mathrm{H}), 7.05-7.01(\mathrm{~m}, 1 \mathrm{H}), 6.95$ $(\mathrm{dd}, J=5.2,1.6 \mathrm{~Hz}, 1 \mathrm{H}), 6.83-6.74(\mathrm{~m}, 2 \mathrm{H}), 3.79(\mathrm{~s}, 3 \mathrm{H}), 2.48(\mathrm{~s}, 3 \mathrm{H}) .{ }^{13} \mathrm{C}$ NMR $(101$ $\mathrm{MHz}_{\mathrm{CDCl}}$ ) $\delta: 157.9,157.8,156.2,148.5,145.7,137.6,132.0,130.9,127.7,126.3$, 125.2, 123.4, 113.0, 64.0, 55.2, 24.8. HRMS (ESI) Calcd for $\mathrm{C}_{26} \mathrm{H}_{24} \mathrm{NO}\left([\mathrm{M}+\mathrm{H}]^{+}\right)$: 366.1852; Found: 366.1859.

4-Substituted 2-pyridinecarbaldehydes $\mathbf{S 2 f}$ and $\mathbf{S 2 g}$ were synthesized according to the general procedure for the synthesis of $\mathbf{S 2}$.

\section{4-(2-Phenylpropan-2-yl)-2-pyridinecarbaldehyde (S2f)}

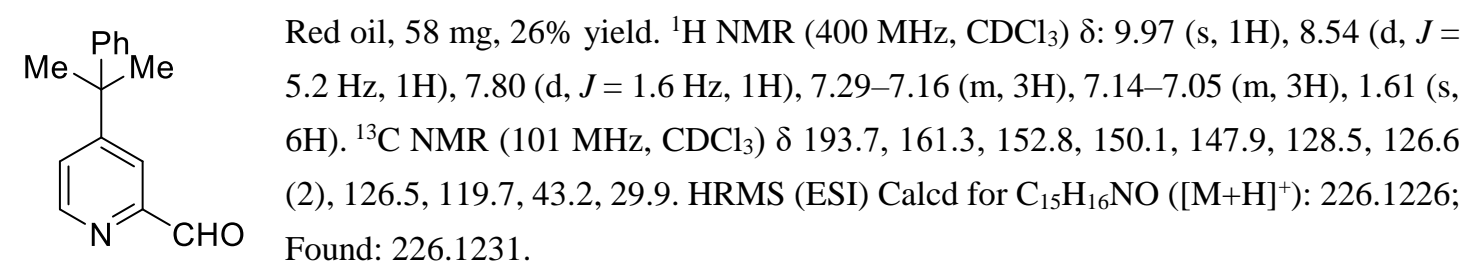

\section{4-[(4-Methoxyphenyl)diphenyl]-2-pyridinecarbaldehyde (S2g)}

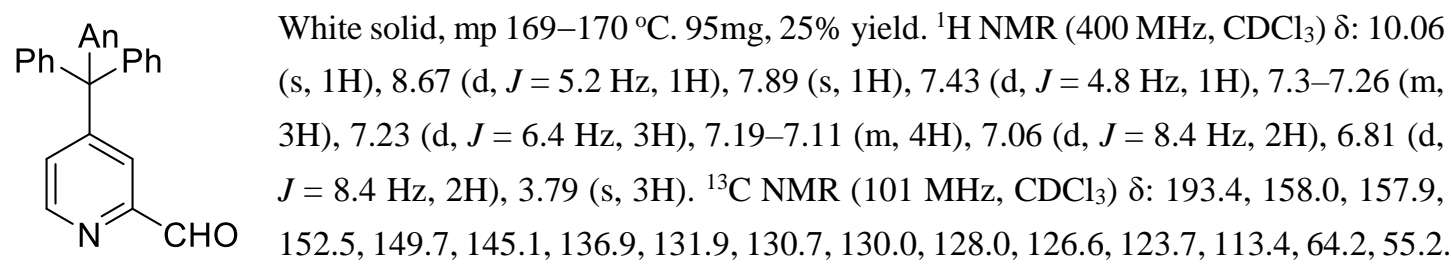

HRMS (ESI) Calcd for $\mathrm{C}_{26} \mathrm{H}_{22} \mathrm{NO}_{2}\left([\mathrm{M}+\mathrm{H}]^{+}\right)$: 380.1645; Found: 380.1639 .

\section{Synthesis of 4-substituted 2-pyridinecarbaldehydes S2h and S2i}<smiles>[R]C([R])(O)c1ccnc(C)c1</smiles>

S1b and S1c

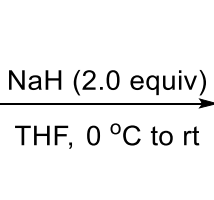<smiles>[R]C([R])([R])c1ccnc(C)c1</smiles>

S4

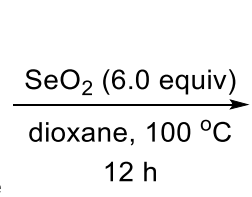

$12 \mathrm{~h}$

-

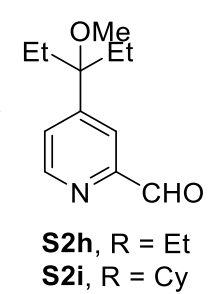

General procedure for synthesis of S4: To a solution of S1b or S1c $(6 \mathrm{mmol})$ in THF (30 mL) was added sodium hydride $(288 \mathrm{mg}, 12 \mathrm{mmol})$ at $0{ }^{\circ} \mathrm{C}$ under argon atmosphere, and the resulting reaction mixture was stirred for at room temperature for $1 \mathrm{~h} . \mathrm{CH}_{3} \mathrm{I}(1.7 \mathrm{~g}, 12 \mathrm{mmol})$ was then added to the reaction mixture and was allowed to warm to room temperature for $2 \mathrm{~h}$. The reaction mixture was then quenched with saturated $\mathrm{NH}_{4} \mathrm{Cl}$ solution $(25 \mathrm{~mL})$ and the aqueous phase was extracted with EtOAc $(20 \mathrm{~mL} \times 3)$. The combined organic layer was washed with brine, dried over anhydrous $\mathrm{MgSO}_{4}$, and concentrated in vacuo. The residue was chromatographed on silica gel column with Ethyl acetate/petroleum ether (1:5) as an eluent to offer $\mathbf{S} \mathbf{4}$ as a yellow oil. 
<smiles>CCOC(CC)(OC)c1ccnc(C)c1</smiles>

Yellow oil, 0.52 g. $45 \%$ yield. ${ }^{1} \mathrm{H}$ NMR $\left(400 \mathrm{MHz}, \mathrm{CDCl}_{3}\right) \delta: 8.44(\mathrm{~d}, J=5.6 \mathrm{~Hz}, 1 \mathrm{H})$, $7.16(\mathrm{~s}, 1 \mathrm{H}), 7.06$ (dd, $J=5.2,1.6 \mathrm{~Hz}, 1 \mathrm{H}), 3.12$ (s, 3H), 2.57 (s, 3H), 1.93-1.83 (m, $2 \mathrm{H}), 1.80-1.67(\mathrm{~m}, 2 \mathrm{H}), 0.67$ (t, $J=7.4 \mathrm{~Hz}, 6 \mathrm{H}) .{ }^{13} \mathrm{C} \mathrm{NMR}\left(101 \mathrm{MHz}, \mathrm{CDCl}_{3}\right) \delta$ : 158.0, 153.8, 148.8, 121.1, 118.8, 80.8, 49.2, 28.2, 24.6, 7.2. HRMS (ESI) Calcd for $\mathrm{C}_{12} \mathrm{H}_{20} \mathrm{NO}\left([\mathrm{M}+\mathrm{H}]^{+}\right):$194.1539; Found: 194.1540 .

\section{4-[Dicyclohexyl(methoxy)methyl]-2-methylpyridine (S4b)}<smiles>COC(Cl)(Cl)c1ccnc(C)c1</smiles>

Used KH instead of NaH. Yellow oil, 0.90 g. $50 \%$ yield. ${ }^{1} \mathrm{H}$ NMR $\left(400 \mathrm{MHz}, \mathrm{CDCl}_{3}\right)$ $\delta: 8.40(\mathrm{~d}, J=5.2 \mathrm{~Hz}, 1 \mathrm{H}), 7.17(\mathrm{~s}, 1 \mathrm{H}), 7.05(\mathrm{~d}, J=5.2 \mathrm{~Hz}, 1 \mathrm{H}), 3.49$ (s, 3H), 2.57 (s, 3H), 2.11-2.01 (m, 2H), 1.90-1.59 (m, 8H), 1.48 (d, $J=12.8 \mathrm{~Hz}, 2 \mathrm{H}), 1.30-1.15$ (m, 4H), 1.09-0.97 (m, 4H), 0.92-0.79 (m, 2H). $\left.{ }^{13} \mathrm{C} \mathrm{NMR} \mathrm{(101} \mathrm{MHz,} \mathrm{CDCl}_{3}\right)$ \&: 157.2, $152.8,147.8,122.4,119.9,84.4,52.8,41.9,28.2,27.8,27.2,27.1,26.7,24.7$. HRMS (ESI) Calcd for $\mathrm{C}_{20} \mathrm{H}_{32} \mathrm{NO}\left([\mathrm{M}+\mathrm{H}]^{+}\right)$: 302.2478; Found: 302.2480 .

4-Substituted 2-pyridinecarbaldehydes $\mathbf{S 2 h}$ and $\mathbf{S 2 i}$ were synthesized according to the general procedure for the synthesis of $\mathbf{S 2}$.

\section{4-(3-Methoxypentan-3-yl)picolinaldehyde (S2h)}<smiles>CCOC(CC)(OC)c1ccnc(C=O)c1</smiles>

Yellow oil, $31 \mathrm{mg} .15 \%$ yield. ${ }^{1} \mathrm{H}$ NMR $\left(400 \mathrm{MHz}, \mathrm{CDCl}_{3}\right) \delta: 10.12(\mathrm{~s}, 1 \mathrm{H}), 8.75$ $(\mathrm{d}, J=5.2 \mathrm{~Hz}, 1 \mathrm{H}), 7.94(\mathrm{~s}, 1 \mathrm{H}), 7.59(\mathrm{dd}, J=5.2,2.0 \mathrm{~Hz}, 1 \mathrm{H}), 3.17(\mathrm{~s}, 3 \mathrm{H}), 1.97$ $(\mathrm{dq}, J=22.0,7.6 \mathrm{~Hz}, 2 \mathrm{H}), 1.77(\mathrm{dq}, J=22.0,7.6 \mathrm{~Hz}, 2 \mathrm{H}), 0.64(\mathrm{t}, J=7.4 \mathrm{~Hz}$, $6 \mathrm{H}) .{ }^{13} \mathrm{C}$ NMR $\left(101 \mathrm{MHz}, \mathrm{CDCl}_{3}\right) \delta: 193.8,155.7,152.7,150.0,126.1,119.6$, 80.9, 49.2, 28.6, 7.2. HRMS (ESI) Calcd for $\mathrm{C}_{12} \mathrm{H}_{18} \mathrm{NO}_{2}\left([\mathrm{M}+\mathrm{H}]^{+}\right)$: 208.1332; Found: 208.1333 .

\section{4-[Dicyclohexyl(methoxy)methyl]picolinaldehyde (S2i)}<smiles>O=Cc1cc(C(O)([Al])C2CCCCC2)ccn1</smiles>

Yellow oil, $32 \mathrm{mg} .10 \%$ yield. ${ }^{1} \mathrm{H}$ NMR (400 MHz, $\left.\mathrm{CDCl}_{3}\right) \delta: 10.12(\mathrm{~s}, 1 \mathrm{H}), 8.71$ $(\mathrm{d}, J=5.2 \mathrm{~Hz}, 1 \mathrm{H}), 7.95(\mathrm{~s}, 1 \mathrm{H}), 7.59(\mathrm{~s}, 1 \mathrm{H}), 3.52(\mathrm{~s}, 3 \mathrm{H}), 2.11(\mathrm{~m}, 2 \mathrm{H}), 1.90$ $1.65(\mathrm{~m}, 8 \mathrm{H}), 1.44(\mathrm{~d}, J=13.2 \mathrm{~Hz}, 2 \mathrm{H}), 1.30-1.17(\mathrm{~m}, 4 \mathrm{H}), 1.08-0.94(\mathrm{~m}, 4 \mathrm{H})$, 0.86-0.75 (m, 2H). ${ }^{13} \mathrm{C}$ NMR (101 MHz, $\left.\mathrm{CDCl}_{3}\right) \delta: 194.1,154.6,152.0,149.4$, 127.2, 120.6, 84.3, 52.7, 41.7, 28.2, 27.8, 27.0, 26.6. HRMS (ESI) Calcd for $\mathrm{C}_{20} \mathrm{H}_{30} \mathrm{NO}_{2}\left([\mathrm{M}+\mathrm{H}]^{+}\right): 316.2271$; Found: 316.2274 .

\section{Synthesis of new SpiroPAP ligands. ${ }^{2}$}

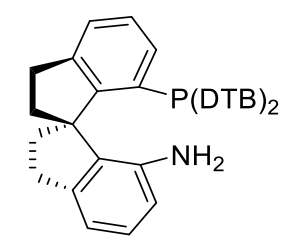

(R)-DTB-SpiroAP

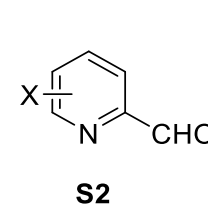

S2

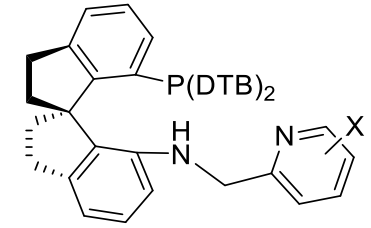

S5

General Procedure: A solution of $(R)$-SpiroAP $(160 \mathrm{mg}, 0.25 \mathrm{mmol})$ and picolinaldehyde S2 $(0.40$ mmol) dissolved in $30 \mathrm{~mL} \mathrm{MeOH}$ was treated with $\mathrm{AcOH}(45 \mathrm{mg}, 0.75 \mathrm{mmol})$ under argon atmosphere. After stirred at $40{ }^{\circ} \mathrm{C}$ for $2 \mathrm{~h}, \mathrm{NaBH}_{3} \mathrm{CN}(0.50 \mathrm{mmol})$ was added. Then the mixture was stirred at $40{ }^{\circ} \mathrm{C}$ until the TLC test showed the reaction was complete. Saturated aqueous $\mathrm{Na}_{2} \mathrm{CO}_{3}(10 \mathrm{~mL})$ was added into 
the reaction mixture. The mixture was extracted with EtOAc $(30 \mathrm{~mL} \times 3)$. The organic layer was dried over anhydrous $\mathrm{Na}_{2} \mathrm{SO}_{4}$ and concentrated in vacuo to afford the crude product. After chromatography on silica-gel column with ethyl acetate/petroleum ether $(\mathrm{v} / \mathrm{v}=1: 20)$ as eluent to offer the corresponding new SpiroPAP ligands as white solid.

\section{Ligand $(R)-S 5 g\left(3-{ }^{i} \mathrm{Pr}\right)$}

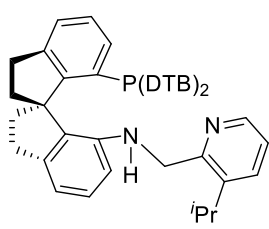

White solid, mp $196-197{ }^{\circ} \mathrm{C} .188 \mathrm{mg}, 97 \%$ yield. $[\alpha]_{\mathrm{D}}^{27}=156.8\left(c=0.5, \mathrm{CH}_{3} \mathrm{Cl}\right)$. ${ }^{1} \mathrm{H}$ NMR $\left(400 \mathrm{MHz}, \mathrm{CDCl}_{3}\right) \delta 7.82(\mathrm{dd}, J=4.8,2.0 \mathrm{~Hz}, 1 \mathrm{H}), 7.42(\mathrm{dd}, J=7.8,2.0$ $\mathrm{Hz}, 1 \mathrm{H}), 7.36(\mathrm{~d}, J=7.2 \mathrm{~Hz}, 1 \mathrm{H}), 7.25-7.16(\mathrm{~m}, 3 \mathrm{H}), 7.12-7.05(\mathrm{~m}, 2 \mathrm{H}), 6.98(\mathrm{dd}$, $J=7.8,4.8 \mathrm{~Hz}, 1 \mathrm{H}), 6.81(\mathrm{dd}, J=7.6,2.0 \mathrm{~Hz}, 2 \mathrm{H}), 6.73-6.63(\mathrm{~m}, 3 \mathrm{H}), 6.32(\mathrm{~d}, J$ $=7.8 \mathrm{~Hz}, 1 \mathrm{H}), 5.58(\mathrm{~d}, J=6.0 \mathrm{~Hz}, 1 \mathrm{H}), 4.26(\mathrm{dd}, J=15.6,6.4 \mathrm{~Hz}, 1 \mathrm{H}), 3.70(\mathrm{~d}, J$ $=16.2 \mathrm{~Hz}, 1 \mathrm{H}), 3.13-2.87(\mathrm{~m}, 4 \mathrm{H}), 2.72(\mathrm{dd}, J=15.6,9.2 \mathrm{~Hz}, 1 \mathrm{H}), 2.50-2.39(\mathrm{~m}, 1 \mathrm{H}), 2.16-1.95(\mathrm{~m}$, 3H), 1.26-1.11 (m, 24H), $0.94(\mathrm{~s}, 18 \mathrm{H}) .{ }^{13} \mathrm{C}$ NMR $\left(101 \mathrm{MHz}, \mathrm{CDCl}_{3}\right) \delta$ 153.0, 152.3, 152.1, 149.8, 149.7, 149.3 (2), 145.5 (2), 144.5, 144.3, 144.2 , 144.1, 140.1, 138.1, 138.0, 136.8, 136.7, 134.9, 134.7, 133.3 (2), 132.4 (2), 132.2, 128.5, 128.3, 128.2, 128.0, 127.8, 126.5, 125.2, 121.6, 121.4, 121.2, 112.9, 107.7, 61.7, 61.6, 44.5, 38.4, 38.3, 35.9, 34.7, 34.5, 31.3, 31.2, 31.1, 30.9, 27.5, 23.3, 22.7. ${ }^{31} \mathrm{P}$ NMR (162 MHz, $\mathrm{CDCl}_{3}$ ) $\delta$ : -17.02. HRMS (ESI) calcd for $\mathrm{C}_{54} \mathrm{H}_{70} \mathrm{~N}_{2} \mathrm{P}[\mathrm{M}+\mathrm{H}]^{+}:$777.5271; Found: 777.5274.

\section{Ligand (R)-S5j (4-Et2(HO)C)}

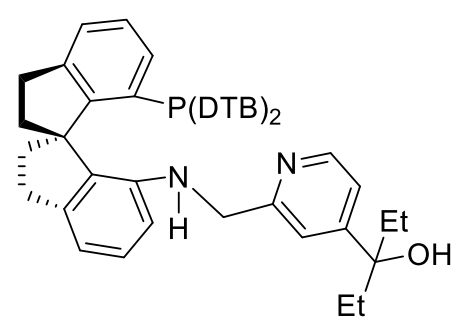

White solid, mp $99-100{ }^{\circ} \mathrm{C} .164 \mathrm{mg}, 80 \%$ yield. $[\alpha]_{\mathrm{D}}^{25}+151.6(c 1.0$, $\left.\mathrm{CHCl}_{3}\right) .{ }^{1} \mathrm{H}$ NMR $\left(400 \mathrm{MHz}, \mathrm{CDCl}_{3}\right) \delta: 8.23(\mathrm{~d}, J=4.8 \mathrm{~Hz}, 1 \mathrm{H}), 7.32$ (d, $J=7.2 \mathrm{~Hz}, 1 \mathrm{H}), 7.24(\mathrm{~s}, 1 \mathrm{H}), 7.22-7.17(\mathrm{~m}, 2 \mathrm{H}), 7.12-7.05(\mathrm{~m}$, $2 \mathrm{H}), 7.02(\mathrm{~d}, J=4.4 \mathrm{~Hz}, 1 \mathrm{H}), 6.91(\mathrm{~s}, 1 \mathrm{H}), 6.85(\mathrm{~d}, J=6.8 \mathrm{~Hz}, 2 \mathrm{H})$, 6.76-6.65 (m, 3H), $6.11(\mathrm{~d}, J=8.0 \mathrm{~Hz}, 1 \mathrm{H}), 4.19(\mathrm{br}, 1 \mathrm{H}), 4.00(\mathrm{dd}$, $J=16.0,6.8 \mathrm{~Hz}, 1 \mathrm{H}), 3.57(\mathrm{~d}, J=16.0 \mathrm{~Hz}, 1 \mathrm{H}), 3.11-2.82(\mathrm{~m}, 4 \mathrm{H})$, 2.55-2.42 (m, 1H), 2.25-2.17 (m, 2H), 2.16-2.07 (m, 1H), 1.73-1.66 (m, 4H), 1.14 (s, 18H), 1.07 (s, 18H), 0.70-0.60 (m, 6H). $\left.{ }^{13} \mathrm{C} \mathrm{NMR} \mathrm{(101} \mathrm{MHz,} \mathrm{CDCl}_{3}\right) \delta 158.5,155.3$ (2), 152.7, 150.0 (2), 149.9 (2), 148.7, 144.5, 144.3, 144.2, 144.1, 144.1, 138.5, 138.4, 136.1, 136.0, 135.0, 134.8, 134.1 (2), 133.2, 133.1, 128.4, 128.2, 128.1, 128.0 (2), 126.9, 125.8, 122.3, 121.5, 119.0, 117.6, 114.0, 108.9, 77.1, 67.2, 61.9, 61.8, 48.4, 39.0, 38.9, 35.9, 34.8 (2), 34.7, 31.4, 31.3, 31.0, 29.9, 7.8, 7.7. ${ }^{31} \mathrm{P}$ NMR (162 MHz, $\left.\mathrm{CDCl}_{3}\right) \delta:-18.40$. HRMS (MALDI) Calcd for $\mathrm{C}_{56} \mathrm{H}_{74} \mathrm{~N}_{2} \mathrm{OP}\left([\mathrm{M}+\mathrm{H}]^{+}\right)$: 821.5533; Found: 821.5538 .

\section{Ligand (R)-S5k (4-Et2 $2(\mathrm{MeO}) \mathrm{C})$}

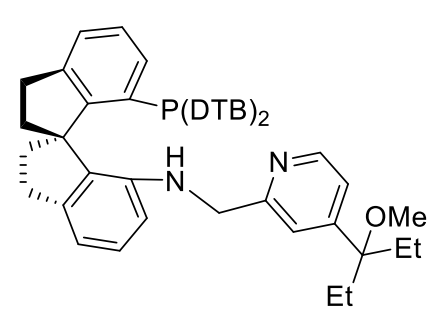

White solid, mp $96-97{ }^{\circ} \mathrm{C} .166 \mathrm{mg}, 80 \%$ yield. $[\alpha]_{\mathrm{D}}^{25}+153.8(c$ 1.0, $\left.\mathrm{CHCl}_{3}\right) .{ }^{1} \mathrm{H}$ NMR $\left(400 \mathrm{MHz}, \mathrm{CDCl}_{3}\right) \delta: 8.22(\mathrm{~d}, J=5.2 \mathrm{~Hz}, 1 \mathrm{H}), 7.32$ (d, $J=7.2 \mathrm{~Hz}, 1 \mathrm{H}), 7.24(\mathrm{~s}, 1 \mathrm{H}), 7.22-7.17(\mathrm{~m}, 2 \mathrm{H}), 7.12-7.04(\mathrm{~m}$, $2 \mathrm{H}), 7.04-7.01(\mathrm{~m}, 1 \mathrm{H}), 6.93(\mathrm{~s}, 1 \mathrm{H}), 6.85(\mathrm{dd}, J=8.0,1.6 \mathrm{~Hz}, 2 \mathrm{H})$, $6.72(\mathrm{dd}, J=7.6,1.6 \mathrm{~Hz}, 2 \mathrm{H}), 6.68(\mathrm{~d}, J=7.6 \mathrm{~Hz}, 1 \mathrm{H}), 6.09(\mathrm{~d}, J=7.6$ $\mathrm{Hz}, 1 \mathrm{H}), 4.25(\mathrm{~d}, J=4.4 \mathrm{~Hz}, 1 \mathrm{H}), 4.04-3.93(\mathrm{~m}, 1 \mathrm{H}), 3.56(\mathrm{dd}, J=$ $16.0,2.4 \mathrm{~Hz}, 1 \mathrm{H}), 3.09-3.03(\mathrm{~m}, 1 \mathrm{H}), 3.01(\mathrm{~s}, 3 \mathrm{H}), 2.98-2.85(\mathrm{~m}, 2 \mathrm{H})$, 2.54-2.44 (m, 1H), 2.26-2.17 (m, 2H), 2.15-2.08 (m, 1H), 1.82-1.72 (m, 2H), 1.67-1.60 (m, 2H), 1.14 (s, 18H), 1.07 (s, 18H), 0.65-0.56 (m, 6H). ${ }^{13} \mathrm{C}$ NMR (101 MHz, $\left.\mathrm{CDCl}_{3}\right) \delta 158.3,153.9,152.9,152.7$, 149.9 (2), 148.6, 144.4, 144.3, 144.2, 144.1, 144.1, 138.6, 138.5, 136.2, 136.1, 134.9, 134.7, 134.1 (2), 
133.2, 133.1, 129.0, 128.4, 128.2, 128.1, 128.0, 127.9, 126.9, 125.8, 122.2, 121.4, 119.8, 118.7, 113.9, 108.9, 81.0, 61.9, 61.8, 49.5, 48.4, 39.0 (2), 35.9, 35.0 (2), 34.8 (2), 34.6, 31.4, 31.0, 28.3, 7.4 (2). ${ }^{31} \mathrm{P}$ NMR (162 MHz, $\left.\mathrm{CDCl}_{3}\right) \delta:-19.00$. HRMS (MALDI) Calcd for $\mathrm{C}_{57} \mathrm{H}_{76} \mathrm{~N}_{2} \mathrm{OP}\left([\mathrm{M}+\mathrm{H}]^{+}\right): 835.5690$; Found: 835.5695.

\section{Ligand (R)-S5I (4-Cy2 $(\mathrm{HO}) \mathrm{C})$}

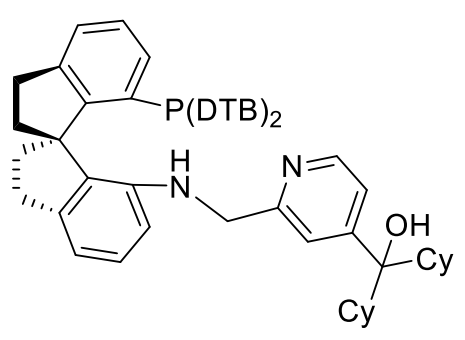

White solid, mp $132-133^{\circ} \mathrm{C} .209 \mathrm{mg}, 90 \%$ yield. $[\alpha]_{\mathrm{D}}^{25}+138.4(c 1.0$, $\left.\mathrm{CHCl}_{3}\right) .{ }^{1} \mathrm{H} \mathrm{NMR}\left(400 \mathrm{MHz}, \mathrm{CDCl}_{3}\right) \delta: 8.24(\mathrm{~d}, J=5.2 \mathrm{~Hz}, 1 \mathrm{H}), 7.32$ (d, $J=7.4 \mathrm{~Hz}, 1 \mathrm{H}), 7.29-7.18(\mathrm{~m}, 3 \mathrm{H}), 7.14-7.07(\mathrm{~m}, 1 \mathrm{H}), 7.05-6.95$ (m, 3H), $6.90(\mathrm{~d}, J=8.4 \mathrm{~Hz}, 2 \mathrm{H}), 6.74(\mathrm{~d}, J=7.6 \mathrm{~Hz}, 2 \mathrm{H}), 6.65(\mathrm{~d}, J$ $=7.2 \mathrm{~Hz}, 1 \mathrm{H}), 5.98(\mathrm{~d}, J=8.0 \mathrm{~Hz}, 1 \mathrm{H}), 4.13(\mathrm{~s}, 1 \mathrm{H}), 3.91(\mathrm{dd}, J=$ $16.4,6.0 \mathrm{~Hz}, 1 \mathrm{H}), 3.69(\mathrm{~d}, J=16.0 \mathrm{~Hz}, 1 \mathrm{H}), 3.18-2.84(\mathrm{~m}, 4 \mathrm{H}), 2.51$ (d, $J=10.9 \mathrm{~Hz}, 1 \mathrm{H}), 2.30-2.19(\mathrm{~m}, 2 \mathrm{H}), 2.17-2.06(\mathrm{~m}, 1 \mathrm{H}), 1.78-$ $1.55(\mathrm{~m}, 11 \mathrm{H}), 1.34-1.19(\mathrm{~m}, 4 \mathrm{H}), 1.14(\mathrm{~d}, J=8.2 \mathrm{~Hz}, 36 \mathrm{H}), 1.04-0.58(\mathrm{~m}, 7 \mathrm{H}) .{ }^{13} \mathrm{C}$ NMR $(101 \mathrm{MHz}$, $\left.\mathrm{CDCl}_{3}\right) \delta: 158.1,153.3,152.9,152.6,149.9,149.8,149.8,149.8,148.2,144.2,144.2,144.0,143.9,143.6$, $143.5,138.5,138.3,136.1,136.0,134.9,134.7,134.1,134.1,132.9,132.9,128.2,128.1,128.0,127.9$, $127.8,126.9,125.7,122.1,121.3,119.7,118.5,113.9,109.0,80.3,61.8,61.7,48.3,44.1,43.7,39.0$, $38.9,35.8,34.8,34.7,31.4,31.3,31.2,30.9,27.2,27.1,26.8,26.7,26.6,26.6,26.5,26.3 .{ }^{31} \mathrm{P}$ NMR (162 $\left.\mathrm{MHz}, \mathrm{CDCl}_{3}\right) \delta$ : -18.98 . HRMS (ESI) Calcd for $\mathrm{C}_{64} \mathrm{H}_{86} \mathrm{~N}_{2} \mathrm{OP}\left([\mathrm{M}+\mathrm{H}]^{+}\right)$: 929.6472 ; Found: 929.6476.

\section{Ligand (R)-S5m (4-Cy $2(\mathrm{MeO}) \mathrm{C})$}

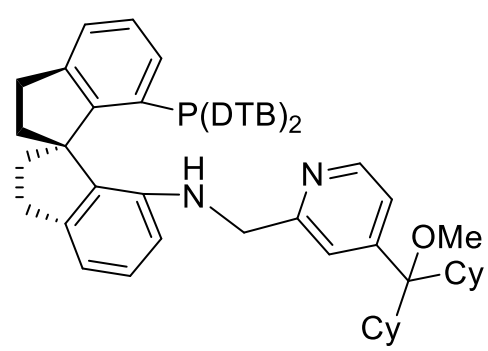

White solid, mp $119-120{ }^{\circ} \mathrm{C} .202 \mathrm{mg}, 86 \%$ yield. $[\alpha]_{\mathrm{D}}^{25}+120.6(c$ $\left.1.0, \mathrm{CHCl}_{3}\right) .{ }^{1} \mathrm{H}$ NMR $\left(400 \mathrm{MHz}, \mathrm{CDCl}_{3}\right) \delta: 8.24(\mathrm{~d}, J=5.2 \mathrm{~Hz}$, 1H), 7.33-7.29 (m, 1H), 7.29-7.26 (m, 1H), 7.24-7.17 (m, 2H), 7.22-7.19 (m, 1H), 7.04-6.97 (m, 2H), 6.97-6.94 (m, 1H), 6.91 $(\mathrm{dd}, J=8.0,1.6 \mathrm{~Hz}, 2 \mathrm{H}), 6.75(\mathrm{dd}, J=7.6,1.6 \mathrm{~Hz}, 2 \mathrm{H}), 6.62(\mathrm{~d}, J$ $=7.6 \mathrm{~Hz}, 1 \mathrm{H}), 5.95(\mathrm{~d}, J=8.0 \mathrm{~Hz}, 1 \mathrm{H}), 4.19-4.11(\mathrm{~m}, 1 \mathrm{H}), 3.94-$ $3.82(\mathrm{~m}, 1 \mathrm{H}), 3.82-3.71(\mathrm{~m}, 1 \mathrm{H}), 3.39(\mathrm{~s}, 3 \mathrm{H}), 3.17-2.88(\mathrm{~m}, 4 \mathrm{H})$,

$2.51(\mathrm{q}, J=11.2 \mathrm{~Hz}, 1 \mathrm{H}), 2.26-2.19(\mathrm{~m}, 2 \mathrm{H}), 2.17-2.10(\mathrm{~m}, 1 \mathrm{H}), 1.92(\mathrm{q}, J=11.2 \mathrm{~Hz}, 2 \mathrm{H}), 1.78-1.52$ (m, 10H), 1.17-1.14 (m, 36H), 1.07-0.79 (m, 10H). ${ }^{13} \mathrm{C}$ NMR (101 MHz, $\left.\mathrm{CDCl}_{3}\right) \delta 157.9,152.9,152.7$, 152.3, 149.7, 149.9, 149.9 (2), 148.1, 144.3, 144.2, 144.0, 143.9, 143.6 (2), 138.6, 138.5, 136.3, 136.2, $135.0,134.8,134.2,134.2,132.8,132.8,131.0,128.8,128.2,128.2,128.1,128.0,127.9,127.0,125.8$, $122.1,121.4,121.1,119.9,113.8,109.0,84.7,61.8,61.8,53.1,48.4,42.3,41.9,39.1,39.0,35.9,34.9$, $34.8,31.6,31.4,31.3,31.0,28.4,28.1,27.8,27.4$ (2), 27.3, 27.2, 26.8, 26.7. ${ }^{31} \mathrm{P}$ NMR (162 MHz, $\mathrm{CDCl}_{3}$ )

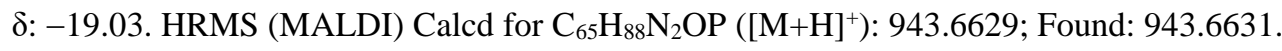

\section{Ligand (R)-S5n (4-Ph $2(\mathrm{HO}) \mathrm{C})$}

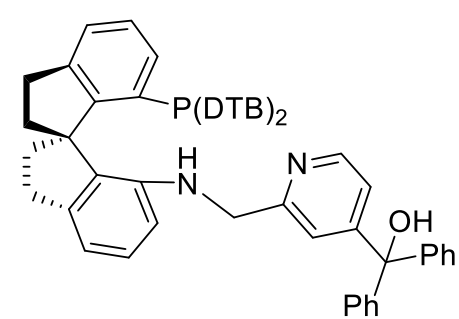

White solid. mp 108-109 ${ }^{\circ} \mathrm{C} .137 \mathrm{mg}, 60 \%$ yield. $[\alpha]_{\mathrm{D}}^{25}+173(c 0.2$, $\left.\mathrm{CHCl}_{3}\right){ }^{1} \mathrm{H}$ NMR $\left(400 \mathrm{MHz}, \mathrm{CDCl}_{3}\right) \delta: 8.21(\mathrm{~d}, J=5.2 \mathrm{~Hz}, 1 \mathrm{H}), 7.32$ $7.28(\mathrm{~m}, 3 \mathrm{H}), 7.28-7.26(\mathrm{~m}, 1 \mathrm{H}), 7.25-7.22(\mathrm{~m}, 3 \mathrm{H}), 7.21-7.14(\mathrm{~m}$, $4 \mathrm{H}), 7.14-7.09$ (m, 3H), 7.08-7.02 (m, 2H), 6.99-6.94 (m, 2H), 6.85 $(\mathrm{d}, J=1.6 \mathrm{~Hz}, 1 \mathrm{H}), 6.83(\mathrm{~d}, J=1.6 \mathrm{~Hz}, 1 \mathrm{H}), 6.72(\mathrm{~d}, J=2.0 \mathrm{~Hz}, 1 \mathrm{H})$, $6.70(\mathrm{~d}, J=2.0 \mathrm{~Hz}, 1 \mathrm{H}), 6.67(\mathrm{~d}, J=7.2 \mathrm{~Hz}, 1 \mathrm{H}), 6.02(\mathrm{~d}, J=8.0 \mathrm{~Hz}$, $1 \mathrm{H}), 4.24-4.18(\mathrm{~m}, 1 \mathrm{H}), 3.91(\mathrm{dd}, J=16.0,6.4 \mathrm{~Hz}, 1 \mathrm{H}), 3.60(\mathrm{dd}, J=$ 
16.0, 3.6 Hz, 1H), 3.04-2.82 (m, 4H), $2.61(\mathrm{~s}, 1 \mathrm{H}), 2.31-2.13(\mathrm{~m}, 3 \mathrm{H}), 2.01-1.93(\mathrm{~m}, 1 \mathrm{H}), 1.14(\mathrm{~s}, 18 \mathrm{H})$, 1.07 (s, 18H). ${ }^{13} \mathrm{C}$ NMR $\left(101 \mathrm{MHz}, \mathrm{CDCl}_{3}\right) \delta: 158.5,155.4,152.8,152.5,149.9$ (2) , 149.8(2), 148.6, 145.7, 145.4, 144.2 (2), 144.1(2), 143.7 (2), 138.4, 138.3, 136.1, 136.0, 135.0, 134.7, 134.0, 133.9, 133.0 (2), 128.3, 128.1 (2), 128.0, 127.9, 127.8 (2), 127.7, 127.6, 126.8, 125.6, 122.1, 121.3, 120.6, 119.5, 113.9, 108.8, 81.2, 77.4, 77.1, 76.8, 61.7, 61.6, 48.2, 38.9, 38.8, 35.8, 34.7, 31.4, 31.2, 30.8. ${ }^{31} \mathrm{P}$ NMR $\left(162 \mathrm{MHz}, \mathrm{CDCl}_{3}\right) \delta:-18.84$. HRMS (MALDI) Calcd for $\mathrm{C}_{64} \mathrm{H}_{74} \mathrm{~N}_{2} \mathrm{OP}\left([\mathrm{M}+\mathrm{H}]^{+}\right)$: 917.5533 Found: 917.5537.

\section{Ligand $(R)-\mathrm{S5o}\left(4-\left(3,5-\mathrm{Me}_{2} \mathrm{C}_{6} \mathrm{H}_{3}\right)_{2}(\mathrm{HO}) \mathrm{C}\right)$}

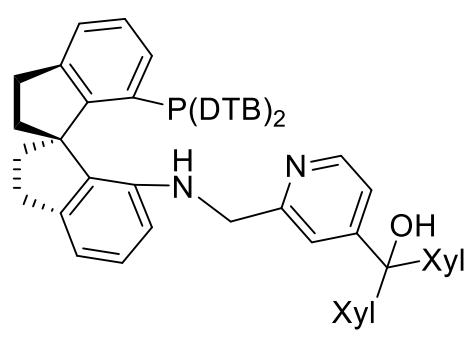

White solid, $\mathrm{mp} 119-120{ }^{\circ} \mathrm{C} .211 \mathrm{mg}, 87 \%$ yield. $[\alpha]_{\mathrm{D}}^{25}+170(c 0.2$, $\left.\mathrm{CHCl}_{3}\right) .{ }^{1} \mathrm{H}$ NMR $\left(400 \mathrm{MHz}, \mathrm{CDCl}_{3}\right) \delta: 8.17(\mathrm{~d}, J=5.2 \mathrm{~Hz}, 1 \mathrm{H})$, 7.25-7.17 (m, 3H), $7.13(\mathrm{t}, J=7.4 \mathrm{~Hz}, 1 \mathrm{H}), 7.09-7.02(\mathrm{~m}, 2 \mathrm{H}), 6.98$ (s, 1H), $6.95(\mathrm{~d}, J=5.2 \mathrm{~Hz}, 1 \mathrm{H}), 6.91(\mathrm{~d}, J=7.2 \mathrm{~Hz}, 2 \mathrm{H}), 6.84(\mathrm{dd}$, $J=8.0,2.0 \mathrm{~Hz}, 2 \mathrm{H}), 6.79-6.70(\mathrm{~m}, 6 \mathrm{H}), 6.66(\mathrm{~d}, J=7.6 \mathrm{~Hz}, 1 \mathrm{H})$, $6.06(\mathrm{~d}, J=7.6 \mathrm{~Hz}, 1 \mathrm{H}), 4.35-4.28(\mathrm{~m}, 1 \mathrm{H}), 3.91(\mathrm{dd}, J=16.0,6.4$ $\mathrm{Hz}, 1 \mathrm{H}), 3.68-3.55(\mathrm{~m}, 1 \mathrm{H}), 3.12-2.76(\mathrm{~m}, 4 \mathrm{H}), 2.62(\mathrm{~s}, 1 \mathrm{H}), 2.35-$ $2.28(\mathrm{~m}, 1 \mathrm{H}), 2.23(\mathrm{~d}, J=11.2 \mathrm{~Hz}, 12 \mathrm{H}), 2.18-2.15(\mathrm{~m}, 1 \mathrm{H}), 2.06-1.99(\mathrm{~m}, 1 \mathrm{H}), 1.15(\mathrm{~s}, 18 \mathrm{H}), 1.07(\mathrm{~s}$, 18H). ${ }^{13} \mathrm{C}$ NMR (101 MHz, $\left.\mathrm{CDCl}_{3}\right) \delta: 158.3,155.6,152.7,152.4,149.8,149.7,148.3,145.7,145.5$, 144.1 (2), 144.0, 143.9(2), 138.4, 138.2, 137.5 (2), 136.0 (2), 134.9, 134.6, 133.8(2), 132.9, 132.8, 129.3, 129.2, 128.2, 128.0 (2), 127.8 (d), 126.7, 125.5 (2), 122.0, 121.3, 120.8, 119.4, 113.7, 108.5, 81.1, 61.6 (2), 48.3, 38.8 (2), 35.8, 34.7, 34.6, 31.3, 21.5, 21.4. ${ }^{31} \mathrm{P} \mathrm{NMR}\left(162 \mathrm{MHz}, \mathrm{CDCl}_{3}\right) \delta:-18.67$. HRMS (ESI) Calcd for $\mathrm{C}_{68} \mathrm{H}_{82} \mathrm{~N}_{2} \mathrm{OP}\left([\mathrm{M}+\mathrm{H}]^{+}\right)$: 973.6159 ; Found: 973.6176.

\section{Ligand $(R)-\mathrm{S} 5 n\left(4-\left(3,5-(t \mathrm{Bu})_{2} \mathrm{C}_{6} \mathrm{H}_{3}\right)_{2}(\mathrm{HO}) \mathrm{C}\right)$}

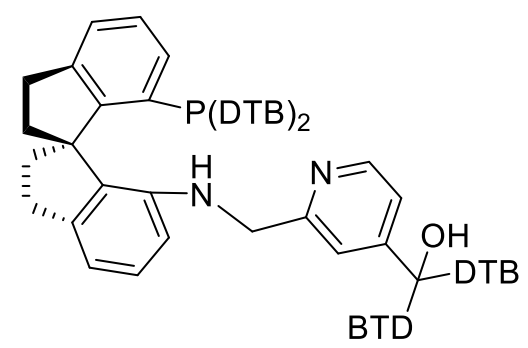

White solid, mp $130-131^{\circ} \mathrm{C} .248 \mathrm{mg}, 87 \%$ yield. $[\alpha]_{\mathrm{D}}^{25}+134(c$ $\left.0.1, \mathrm{CHCl}_{3}\right){ }^{1} \mathrm{H} \mathrm{NMR}\left(400 \mathrm{MHz}, \mathrm{CDCl}_{3}\right) \delta: 8.19(\mathrm{~d}, J=5.2 \mathrm{~Hz}$, 1H), 7.35-7.30 (m, 2H), 7.23 (s, 1H), 7.21-7.17 (m, 2H), 7.14 $(\mathrm{t}, J=7.4 \mathrm{~Hz}, 1 \mathrm{H}), 7.11-6.99(\mathrm{~m}, 4 \mathrm{H}), 6.98(\mathrm{~s}, 3 \mathrm{H}), 6.91-6.83$ $(\mathrm{m}, 3 \mathrm{H}), 6.72(\mathrm{dd}, J=7.6,1.6 \mathrm{~Hz}, 2 \mathrm{H}), 6.64(\mathrm{~d}, J=7.2 \mathrm{~Hz}, 1 \mathrm{H})$, $6.06(\mathrm{~d}, J=7.6 \mathrm{~Hz}, 1 \mathrm{H}), 4.19-4.12(\mathrm{~m}, 1 \mathrm{H}), 3.88(\mathrm{dd}, J=15.6$, $6.4 \mathrm{~Hz}, 1 \mathrm{H}), 3.58(\mathrm{dd}, J=15.6,3.6 \mathrm{~Hz}, 1 \mathrm{H}), 3.06-2.81(\mathrm{~m}, 4 \mathrm{H})$,

2.57 (s, 1H), 2.39-2.14 (m, 3H), 2.07-1.95 (m, 1H), 1.21 (d, $J=3.2 \mathrm{~Hz}, 36 \mathrm{H}), 1.14(\mathrm{~s}, 18 \mathrm{H}), 1.08$ (s, 18H). ${ }^{13} \mathrm{C}$ NMR (101 MHz, $\mathrm{CDCl}_{3}$ ) $\delta: 158.6,156.6,152.8$ (2), 152.6, 150.2, 150.1 (2), 149.8, 149.7(2), 148.1, 144.8, 144.7, 144.1, 144.0, 143.9, 138.5, 138.4, 136.0, 135.9, 134.8, 134.6, 134.0, 133.9, 132.9 (2), 128.3, 128.1, 127.9 (2), 127.7, 126.7, 125.6, 122.3, 122.2, 122.1, 121.2 (2), 121.1, 121.0, 119.2, 113.7, 108.4, 82.1, 61.6 (d), 48.4, 39.0 (2), 35.8, 34.9, 34.7, 31.4, 31.3, 31.2, 30.8. ${ }^{31}$ P NMR (162 MHz, $\left.\mathrm{CDCl}_{3}\right) \delta$ : -18.94. HRMS (ESI) Calcd for $\mathrm{C}_{80} \mathrm{H}_{106} \mathrm{~N}_{2} \mathrm{OP}\left([\mathrm{M}+\mathrm{H}]^{+}\right)$: 1141.8037; Found: 1141.8042.

\section{Ligand $(R)$-S5q $\left(4-(\mathrm{Me})_{2}\left(\mathrm{C}_{6} \mathrm{H}_{5}\right) \mathrm{C}\right)$}




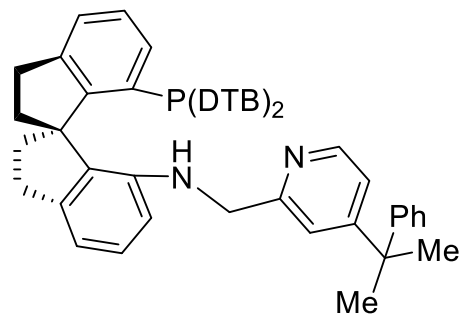

White solid, mp 68-69 ${ }^{\circ} \mathrm{C} .143 \mathrm{mg}, 67 \%$ yield. $[\alpha]_{\mathrm{D}}^{25}+157.2(c 0.5$, $\left.\mathrm{CHCl}_{3}\right)^{1} \mathrm{H}$ NMR $\left(400 \mathrm{MHz}, \mathrm{CDCl}_{3}\right) \delta: 8.13(\mathrm{~d}, J=5.2 \mathrm{~Hz}, 1 \mathrm{H})$, 7.29 (s, 1H), 7.28-7.16 (m, 6H), 7.16-7.05 (m, 4H), $6.91(\mathrm{~s}, 1 \mathrm{H})$, 6.88-6.80 (m, 3H), $6.75(\mathrm{dd}, J=7.6,2.0 \mathrm{~Hz}, 2 \mathrm{H}), 6.70(\mathrm{~d}, J=7.4$ $\mathrm{Hz}, 1 \mathrm{H}), 6.10(\mathrm{~d}, J=7.6 \mathrm{~Hz}, 1 \mathrm{H}), 4.42-4.35(\mathrm{~m}, 1 \mathrm{H}), 3.97(\mathrm{dd}, J=$ 16.0, $6.8 \mathrm{~Hz}, 1 \mathrm{H}), 3.59$ (dd, $J=15.8,3.2 \mathrm{~Hz}, 1 \mathrm{H}), 3.15-2.83$ (m, $4 \mathrm{H}), 2.47-2.35(\mathrm{~m}, 1 \mathrm{H}), 2.24-2.14(\mathrm{~m}, 2 \mathrm{H}), 2.13-2.04(\mathrm{~m}, 1 \mathrm{H}), 1.56$ (s, 6H), 1.17 (s, 18H), 1.09 (s, 18H). ${ }^{13} \mathrm{C}$ NMR (101 MHz, $\left.\mathrm{CDCl}_{3}\right) \delta 159.6,158.2,152.8,152.6,149.9$ (3), 149.8, 148.9, 148.7, 144.3 (2), 144.2, 144.1, 138.5, 138.4, 136.3, 136.2, 136.1 (2), 134.9, 134.7, 134.6, 134.0, 133.0 (2), 128.3 (2), 128.2, 128.1, 128.0, 127.9, 126.9, 126.8, 126.2, 125.8, 122.1, 121.4, 120.4, 119.0, 113.8, 108.7, 61.8 (2), 48.3, 42.9, 38.9, 38.8, 35.9 (2), 34.8 (2), 31.4, 31.0, 30.1, 30.0, 29.8. ${ }^{31} \mathrm{P}$ NMR $\left(162 \mathrm{MHz}, \mathrm{CDCl}_{3}\right) \delta:-18.53$. HRMS (ESI) Calcd for $\mathrm{C}_{60} \mathrm{H}_{74} \mathrm{~N}_{2} \mathrm{P}\left([\mathrm{M}+\mathrm{H}]^{+}\right):$853.5584; Found: 853.5594 .

\section{Ligand $(R)-\mathrm{S} 5 r\left(4-\left(\mathrm{C}_{6} \mathrm{H}_{5}\right)_{2}\left(4-\mathrm{MeOC}_{6} \mathrm{H}_{4}\right) \mathrm{C}\right)$}

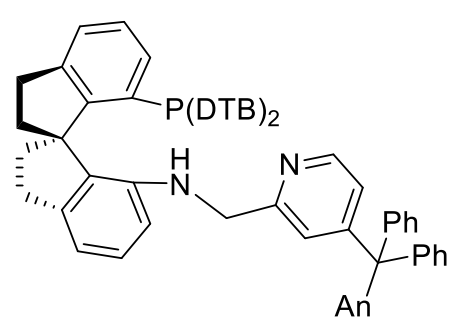

White solid, mp $113-114{ }^{\circ} \mathrm{C} .201 \mathrm{mg}, 80 \%$ yield. $[\alpha]_{\mathrm{D}}^{25}+171.6(c 0.5$, $\left.\mathrm{CHCl}_{3}\right)^{1} \mathrm{H}$ NMR $\left(400 \mathrm{MHz}, \mathrm{CDCl}_{3}\right) \delta: 8.19(\mathrm{~d}, J=5.2 \mathrm{~Hz}, 1 \mathrm{H}), 7.25-$ $7.22(\mathrm{~m}, 2 \mathrm{H}), 7.21-7.12(\mathrm{~m}, 8 \mathrm{H}), 7.11-6.83(\mathrm{~m}, 13 \mathrm{H}), 6.76-6.63(\mathrm{~m}$, $5 \mathrm{H}), 5.91(\mathrm{~d}, J=8.0 \mathrm{~Hz}, 1 \mathrm{H}), 4.16-4.10(\mathrm{~m}, 1 \mathrm{H}), 3.78(\mathrm{~s}, 3 \mathrm{H}), 3.74$ $(\mathrm{d}, J=5.2 \mathrm{~Hz}, 1 \mathrm{H}), 3.67-3.62(\mathrm{~m}, 1 \mathrm{H}), 3.08-2.77(\mathrm{~m}, 4 \mathrm{H}), 2.24-2.03$ (m, 3H), $1.92-1.81(\mathrm{~m}, 1 \mathrm{H}), 1.13(\mathrm{~d}, J=10.3 \mathrm{~Hz}, 36 \mathrm{H}) .{ }^{13} \mathrm{C} \mathrm{NMR}$ (101 MHz, $\left.\mathrm{CDCl}_{3}\right)$ 8: 158.4, 157.6, 156.0, 152.8, 152.5, 149.8 (d), 148.4, 145.7, 144.2 (2), 144.0 (2), 143.5 (2), 138.5, 138.4 137.6, 136.2, 136.0 (2), 134.8 (2), 134.6 (2), 134.0 (2), 132.8 (2), 131.9, 130.8, 128.2, 128.1, 128.0, 127.9, 127.8, 127.7, 126.8, 126.1, 125.7, 123.8, 123.3, 122.1, 121.3, 113.7, 113.0, 108.7, 64.0, 61.6, 61.6, 55.2, 48.2, 39.0, 38.9, 35.6, 34.7, 31.5, 31.4, 31.3, 30.8. ${ }^{31} \mathrm{P}$ NMR (162 MHz, $\left.\mathrm{CDCl}_{3}\right) \delta$ : -18.92 . HRMS (ESI) Calcd for $\mathrm{C}_{71} \mathrm{H}_{80} \mathrm{~N}_{2} \mathrm{OP}\left([\mathrm{M}+\mathrm{H}]^{+}\right)$: 1007.6003; Found: 1007.6008.

\section{(B) Preparation of Racemic $\alpha$-Arylaminated Lactones}

The racemic $\alpha$-arylaminated $\gamma$-butyrolactones and $\delta$-valerolactones were prepared according to the literature method illustrated below.

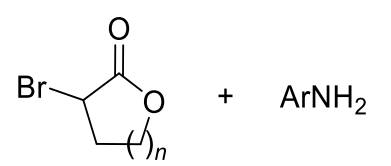

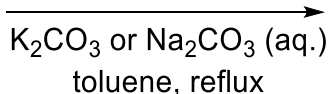<smiles>O=NC1CCOC1=O</smiles>

$$
6(n=1), 8(n=2)
$$

Method A: ${ }^{3}$ To a solution of arylamine $(75.0 \mathrm{mmol})$ in toluene $(40 \mathrm{~mL})$ was added $\alpha$-bromo- $\gamma$ butyrolactone $(12.2 \mathrm{~g}, 75.0 \mathrm{mmol})$. The resulting reaction mixture was heated to $80{ }^{\circ} \mathrm{C}$ and then an aqueous solution of $\mathrm{K}_{2} \mathrm{CO}_{3}$ solution ( $15 \mathrm{~mL}, 37.5 \mathrm{mmol}$ ) was added slowly via a syringe pump over $5 \mathrm{~h}$. After the addition, the reaction mixture was stirred at $80^{\circ} \mathrm{C}$ for $17 \mathrm{~h}$ and then cooled to room temperature. The reaction mixture was extracted with EtOAc $(50 \mathrm{~mL} \times 3)$, and the combined organic layer was washed with $5 \% \mathrm{~K}_{2} \mathrm{CO}_{3}$ solution $(50 \mathrm{~mL})$, water $(50 \mathrm{~mL})$, brine, dried over anhydrous $\mathrm{MgSO}_{4}$, and concentrated in vacuo to yield a residue. The residue was chromatographed on silica gel column with ethyl acetate/petroleum ether (from $3: 1$ to $1: 1$ ) as an eluent to offer the corresponding racemic $\alpha$ arylaminated lactones. 
Method B: To a solution of arylamine $(30.0 \mathrm{mmol})$ and $\mathrm{K}_{2} \mathrm{CO}_{3}(4.1 \mathrm{~g}, 30.0 \mathrm{mmol})$ in toluene $(50 \mathrm{~mL})$ was added $\alpha$-bromo- $\gamma$-butyrolactone $\left(4.9 \mathrm{~g}, 30.0 \mathrm{mmol}\right.$ ). The result mixture was heated to $80{ }^{\circ} \mathrm{C}$ for 17 h. The reaction mixture was cooled to room temperature, filtered to remove the solid deposit, and concentrated under vacuo to give a residue. The residue was chromatographed on silica gel column with EtOAc/petroleum ether (from 3:1 to 1:1) as an eluent to offer the corresponding racemic $\alpha$-arylaminated $\gamma$-butyrolactones.

Method C: To a solution of arylamine $(20.0 \mathrm{mmol})$ and $\alpha$-bromolactone $(42 \mathrm{mmol})$ in DMF $(20 \mathrm{~mL})$ was added $\mathrm{Na}_{2} \mathrm{CO}_{3}(4.2 \mathrm{~g}, 40 \mathrm{mmol})$. The resulting reaction mixture was stirred at $80^{\circ} \mathrm{C}$ for 17 hours and then cooled to room temperature. The reaction mixture was diluted with water and extracted with ether $(50 \mathrm{~mL} \times 3)$. The ether extract was dried over anhydrous $\mathrm{MgSO}_{4}$, and concentrated in vacuo to give a residue. The residue was chromatographed on silica gel column with ethyl acetate/petroleum ether (from $3: 1$ to $1: 1$ ) as an eluent to offer the corresponding racemic $\alpha$-arylaminated $\gamma$-butyrolactones/ $\delta$ valerolactones.

\section{3-(Phenylamino)dihydrofuran-2-one (6a)}<smiles>O=C1OCCC1Nc1ccccc1</smiles>

Method A. White solid, mp 80-81 ${ }^{\circ} \mathrm{C}, 10.6 \mathrm{~g}, 80 \%$ yield, ${ }^{1} \mathrm{H}$ NMR $(400 \mathrm{MHz}$, $\left.\mathrm{CDCl}_{3}\right) \delta: 7.25-7.22(\mathrm{~m}, 2 \mathrm{H}), 6.85(\mathrm{t}, J=7.4 \mathrm{~Hz}, 1 \mathrm{H}), 6.77-6.63(\mathrm{~m}, 2 \mathrm{H}), 4.58-$ $4.44(\mathrm{~m}, 1 \mathrm{H}), 4.36(\mathrm{~m}, 2 \mathrm{H}), 4.16(\mathrm{dd}, J=11.2,8.0 \mathrm{~Hz}, 1 \mathrm{H}), 3.07-2.75(\mathrm{~m}, 1 \mathrm{H})$, $2.21(\mathrm{~m}, 1 \mathrm{H}) .{ }^{13} \mathrm{C} \mathrm{NMR}\left(101 \mathrm{MHz}, \mathrm{CDCl}_{3}\right) \delta: 176.4,146.6,129.5,119.0,113.6$, 65.8, 53.0, 32.2. HRMS (ESI) Calcd for $\mathrm{C}_{10} \mathrm{H}_{12} \mathrm{NO}_{2}\left([\mathrm{M}+\mathrm{H}]^{+}\right)$: 178.0863; Found: 178.0863 .

\section{3-(4-Tolylamino)dihydrofuran-2-one (6b)}<smiles>Cc1ccc(NC2CCOC2=O)cc1</smiles>

Method A. White solid, mp $109-110{ }^{\circ} \mathrm{C}, 9.7 \mathrm{~g}, 68 \%$ yield. ${ }^{1} \mathrm{H}$ NMR (400 $\left.\mathrm{MHz} \mathrm{CDCl}_{3}\right) \delta: 7.07(\mathrm{~d}, J=8.2 \mathrm{~Hz}, 2 \mathrm{H}), 6.63-6.57(\mathrm{~m}, 2 \mathrm{H}), 4.57-4.49(\mathrm{~m}$, $1 \mathrm{H}), 4.36$ (m, 1H), 4.21 (br, 1H) 4.13 (dd, $J=11.2,8.0 \mathrm{~Hz}, 1 \mathrm{H}), 2.98-2.82$ $(\mathrm{m}, 1 \mathrm{H}), 2.29(\mathrm{~s}, 3 \mathrm{H}), 2.26-2.15(\mathrm{~m}, 1 \mathrm{H}) .{ }^{13} \mathrm{C} \mathrm{NMR}\left(101 \mathrm{MHz}, \mathrm{CDCl}_{3}\right) \delta:$ 176.6, 144.3, 129.9, 128.3, 113.8, 65.8, 53.3, 32.2, 20.5. HRMS (ESI) Calcd for $\mathrm{C}_{11} \mathrm{H}_{14} \mathrm{NO}_{2}\left([\mathrm{M}+\mathrm{H}]^{+}\right)$: 192.1019; Found: 192.1020.

\section{3-((4-Methoxyphenyl)amino)dihydrofuran-2-one (6c)}<smiles>COc1ccc(NC2CCOC2=O)cc1</smiles>

Method A. White solid, mp $100-101{ }^{\circ} \mathrm{C}, 12.7 \mathrm{~g}, 82 \%$ yield. ${ }^{1} \mathrm{H}$ NMR (400 $\left.\mathrm{MHz} \mathrm{CDCl}_{3}\right) \delta: 6.87-6.77(\mathrm{~m}, 2 \mathrm{H}), 6.70-6.61(\mathrm{~m}, 2 \mathrm{H}), 4.49(\mathrm{t}, J=9.0 \mathrm{~Hz}$, $1 \mathrm{H}), 4.32(\mathrm{~m}, 1 \mathrm{H}), 4.18-4.00(\mathrm{~m}, 2 \mathrm{H}), 3.76(\mathrm{~s}, 3 \mathrm{H}), 2.84(\mathrm{dd}, J=12.6,6.4$ $\mathrm{Hz}, 1 \mathrm{H}), 2.37-2.08(\mathrm{~m}, 1 \mathrm{H}) .{ }^{13} \mathrm{C} \mathrm{NMR}\left(101 \mathrm{MHz}, \mathrm{CDCl}_{3}\right) \delta: 176.8,153.1$, 140.7, 115.0, 114.9, 65.8, 55.7, 53.7, 31.7. HRMS (ESI) Calcd for $\mathrm{C}_{11} \mathrm{H}_{14} \mathrm{NO}_{3}\left([\mathrm{M}+\mathrm{H}]^{+}\right): 208.0968$; Found: 208.0969 .

\section{3-((4-Fluorophenyl)amino)dihydrofuran-2-one (6d)}<smiles>O=C1OCCC1Nc1ccc(F)cc1</smiles>

Method C. Colorless oil, $2.7 \mathrm{~g}, 68 \%$ yield. ${ }^{1} \mathrm{H}$ NMR (400 MHz, DMSO- $\left.d_{6}\right) \delta$ : $7.01-6.93(\mathrm{~m}, 2 \mathrm{H}), 6.74-6.59(\mathrm{~m}, 2 \mathrm{H}), 5.92(\mathrm{~d}, J=8.0 \mathrm{~Hz}, 1 \mathrm{H}), 4.49-4.32(\mathrm{~m}$, 2H), 4.29-4.16 (m, 1H), 2.66-2.55 (m, 1H), 2.15-1.95 (m, 1H). ${ }^{13} \mathrm{C} \mathrm{NMR} \mathrm{(101}$ MHz, DMSO- $\left.d_{6}\right) \delta: 176.9,156.4$, , 154.1, , 144.6, 115.8, 115.6, 114.1, 114.0, 
65.8, , 52.3, 30.0. HRMS (ESI) Calcd for $\mathrm{C}_{10} \mathrm{H}_{11} \mathrm{FNO}_{2}\left([\mathrm{M}+\mathrm{H}]^{+}\right)$: 196.0768; Found: 196.0771 .

\section{3-((4-Chlorophenyl)amino)dihydrofuran-2-one (6e)}<smiles>O=C1OCCC1Nc1ccc(Cl)cc1</smiles>

Method B. White solid, mp $109-110{ }^{\circ} \mathrm{C}, 2.4 \mathrm{~g}, 38 \%$ yield. ${ }^{1} \mathrm{H}$ NMR (400 $\left.\mathrm{MHz}, \mathrm{CDCl}_{3}\right)$ : $: 7.23-7.12(\mathrm{~m}, 2 \mathrm{H}), 6.67-6.55(\mathrm{~m}, 2 \mathrm{H}), 4.56-4.47(\mathrm{~m}, 1 \mathrm{H})$, $4.33(\mathrm{~m}, 2 \mathrm{H}), 4.10(\mathrm{~m}, 1 \mathrm{H}), 2.87(\mathrm{~m}, 1 \mathrm{H}), 2.17(\mathrm{~m}, 1 \mathrm{H}) .{ }^{13} \mathrm{C}$ NMR $(101 \mathrm{MHz}$, $\left.\mathrm{CDCl}_{3}\right) \delta: 176.0,145.1,129.3,123.8,114.7,65.7,53.0,31.9$. HRMS (ESI)

Calcd for $\mathrm{C}_{10} \mathrm{H}_{11} \mathrm{ClNO}_{2}\left([\mathrm{M}+\mathrm{H}]^{+}\right)$: 212.0473; Found: 212.0476.

\section{3-((4-Bromophenyl)amino)dihydrofuran-2-one (6f)}<smiles>O=C1OCCC1Nc1ccc(Br)cc1</smiles>

Method B. Yellow solid, mp $108-109{ }^{\circ} \mathrm{C}, 4.6 \mathrm{~g}, 60 \%$ yield. ${ }^{1} \mathrm{H}$ NMR $(400$ $\left.\mathrm{MHz}, \mathrm{CDCl}_{3}\right)$ : $7.35-7.27(\mathrm{~m}, 2 \mathrm{H}), 6.60-6.52(\mathrm{~m}, 2 \mathrm{H}), 4.58-4.45(\mathrm{~m}, 1 \mathrm{H})$, $4.33(\mathrm{~m}, 2 \mathrm{H}), 4.10(\mathrm{dd}, J=11.2,8.0 \mathrm{~Hz}, 1 \mathrm{H}), 2.93-2.79(\mathrm{~m}, 1 \mathrm{H}), 2.25-2.10$ $(\mathrm{m}, 1 \mathrm{H}) .{ }^{13} \mathrm{C}$ NMR $\left(101 \mathrm{MHz}, \mathrm{CDCl}_{3}\right) \delta: 176.1,176.0,145.5,132.2,115.1$, 110.8, 110.8, 65.8, 52.9, 31.9, 31.8. HRMS (ESI) Calcd for $\mathrm{C}_{10} \mathrm{H}_{11} \mathrm{BrNO}_{2}\left([\mathrm{M}+\mathrm{H}]^{+}\right)$: 255.9968; Found: 255.9969.

\section{3-(m-Tolylamino)dihydrofuran-2-one $(6 \mathrm{~g})$}<smiles>Cc1cccc(NC2CCOC2=O)c1</smiles>

Method A. White solid, mp $48-49^{\circ} \mathrm{C}, 8.6 \mathrm{~g}, 60 \%$ yield. ${ }^{1} \mathrm{H} \mathrm{NMR}(400 \mathrm{MHz}$, $\left.\mathrm{CDCl}_{3}\right) \delta: 7.14(\mathrm{t}, J=7.6 \mathrm{~Hz}, 1 \mathrm{H}), 6.67(\mathrm{~d}, J=7.6 \mathrm{~Hz}, 1 \mathrm{H}), 6.56-6.46(\mathrm{~m}$, $2 \mathrm{H}), 4.52(\mathrm{~m}, 1 \mathrm{H}), 4.35(\mathrm{~m}, 1 \mathrm{H}), 4.15(\mathrm{dd}, J=11.2,8.0 \mathrm{~Hz}, 1 \mathrm{H}), 2.91(\mathrm{~m}$, $1 \mathrm{H}), 2.33(\mathrm{~s}, 3 \mathrm{H}), 2.20(\mathrm{~m}, 1 \mathrm{H}) .{ }^{13} \mathrm{C} \mathrm{NMR}\left(101 \mathrm{MHz}, \mathrm{CDCl}_{3}\right) \delta:$ 176.4, 146.6, 139.3, 129.3, 119.9, 114.4, 110.6, 65.7, 53.0, 32.2, 21.6. HRMS (ESI) Calcd for $\mathrm{C}_{11} \mathrm{H}_{14} \mathrm{NO}_{2}\left([\mathrm{M}+\mathrm{H}]^{+}\right)$: 192.1019; Found: 192.1019.

\section{3-((3-Methoxyphenyl)amino)dihydrofuran-2-one (6h)}<smiles>COc1cccc(NC2CCOC2=O)c1</smiles>

Method A. White solid, mp $71-72{ }^{\circ} \mathrm{C}, 12.6 \mathrm{~g}, 81 \%$ yield. ${ }^{1} \mathrm{H}$ NMR $(400$ $\left.\mathrm{MHz} \mathrm{CDCl}_{3}\right) \delta: 7.12(\mathrm{t}, J=8.0 \mathrm{~Hz}, 1 \mathrm{H}), 6.36(\mathrm{~d}, J=8.0 \mathrm{~Hz}, 1 \mathrm{H}), 6.28(\mathrm{~d}$, $J=8.0 \mathrm{~Hz}, 1 \mathrm{H}), 6.24-6.18(\mathrm{~m}, 1 \mathrm{H}), 4.45(\mathrm{t}, J=9.0 \mathrm{~Hz}, 1 \mathrm{H}), 4.36(\mathrm{br}, 1 \mathrm{H})$, 4.33-4.24 (m, 1H), 4.11 (dd, $J=11.2,8.0 \mathrm{~Hz}, 1 \mathrm{H}) 3.77$ (s, 3H), 2.99-2.74 (m, 1H), 2.24-2.07 (m, 1H). ${ }^{13} \mathrm{C}$ NMR (101 MHz, $\left.\mathrm{CDCl}_{3}\right)$ 8: 176.4, 160.9, 148.0, 130.3, 106.5, 103.9, 99.8, 65.8, 55.2, 53.0, 32.1. HRMS (ESI) Calcd for $\mathrm{C}_{11} \mathrm{H}_{14} \mathrm{NO}_{3}\left([\mathrm{M}+\mathrm{H}]^{+}\right)$: 208.0968; Found: 208.0971.

\section{3-((3-Fluorophenyl)amino)dihydrofuran-2-one (6i)}<smiles>O=C1OCCC1Nc1cccc(F)c1</smiles>

Method C. White solid, mp $75-76{ }^{\circ} \mathrm{C}, 3.2 \mathrm{~g}, 81 \%$ yield. ${ }^{1} \mathrm{H}$ NMR $(400 \mathrm{MHz}$, DMSO- $\left.d_{6}\right) \delta$ 7.19-7.04 (m, 1H), 6.68-6.47 (m, 2H), 6.44-6.23 (m, 2H), 4.63$4.47(\mathrm{~m}, 1 \mathrm{H}), 4.38(\mathrm{t}, J=8.6 \mathrm{~Hz}, 1 \mathrm{H}), 4.31-4.15(\mathrm{~m}, 1 \mathrm{H}), 2.75-2.55(\mathrm{~m}, 1 \mathrm{H})$, 2.21-1.97 (m, 1H). ${ }^{13} \mathrm{C}$ NMR (101 MHz, DMSO) $\delta 176.15,164.56,162.17$, $149.69,149.59,130.24,130.14,108.99,102.76,102.55,99.11,98.85,65.29,51.30,29.40$. HRMS (ESI) Calcd for $\mathrm{C}_{10} \mathrm{H}_{11} \mathrm{FNO}_{2}\left([\mathrm{M}+\mathrm{H}]^{+}\right)$: 196.0768 ; Found: 196.0770 .

\section{3-((3-Chlorophenyl)amino)dihydrofuran-2-one (6j)}


<smiles>O=C1OCCC1Nc1cccc(Cl)c1</smiles>

Method B. White solid, mp 84-85 ${ }^{\circ} \mathrm{C}, 5.0 \mathrm{~g}, 79 \%$ yield. ${ }^{1} \mathrm{H}$ NMR $(400 \mathrm{MHz}$, $\left.\mathrm{CDCl}_{3}\right) \delta: 7.11(\mathrm{t}, J=8.0 \mathrm{~Hz}, 1 \mathrm{H}), 6.76(\mathrm{dd}, J=8.0,1.0 \mathrm{~Hz}, 1 \mathrm{H}), 6.63(\mathrm{t}, J$ $=2.0 \mathrm{~Hz}, 1 \mathrm{H}), 6.55(\mathrm{dd}, J=8.0,2.0 \mathrm{~Hz}, 1 \mathrm{H}), 4.54-4.40(\mathrm{~m}, 1 \mathrm{H}), 4.37-4.28$ $(\mathrm{m}, 1 \mathrm{H}), 4.12(\mathrm{dd}, J=11.2,8.0 \mathrm{~Hz}, 1 \mathrm{H}), 2.94-2.75(\mathrm{~m}, 1 \mathrm{H}), 2.15(\mathrm{~m}, 1 \mathrm{H})$. ${ }^{13} \mathrm{C}$ NMR (101 MHz, $\left.\mathrm{CDCl}_{3}\right) \delta: 176.1,147.8,135.1,130.5,118.8,113.1,112.0,65.8,52.8,31.8$. HRMS (ESI) Calcd for $\mathrm{C}_{10} \mathrm{H}_{11} \mathrm{ClNO}_{2}\left([\mathrm{M}+\mathrm{H}]^{+}\right)$: 212.0473; Found: 212.0473.

\section{3-(o-Tolylamino)dihydrofuran-2-one (6k)}<smiles>Cc1ccccc1NC1CCOC1=O</smiles>

Method A. White solid, mp 104-105 ${ }^{\circ} \mathrm{C}, 12.2 \mathrm{~g}, 85 \%$ yield. ${ }^{1} \mathrm{H}$ NMR (400 MHz, $\left.\mathrm{CDCl}_{3}\right) \delta: 7.23-7.08(\mathrm{~m}, 2 \mathrm{H}), 6.80(\mathrm{t}, J=7.2 \mathrm{~Hz}, 1 \mathrm{H}), 6.62(\mathrm{~d}, J=8.0 \mathrm{~Hz}, 1 \mathrm{H})$, $4.55-4.48(\mathrm{~m}, 1 \mathrm{H}), 4.40-4.29(\mathrm{~m}, 1 \mathrm{H}), 4.23(\mathrm{br}, 1 \mathrm{H}), 4.18(\mathrm{dd}, J=11.2,8.0 \mathrm{~Hz}$, 1H), 3.01-2.87 (m, 1H), $2.24(\mathrm{~s}, 3 \mathrm{H}), 2.23-2.15(\mathrm{~m}, 1 \mathrm{H}) .{ }^{13} \mathrm{C}$ NMR (101 MHz, $\left.\mathrm{CDCl}_{3}\right) \delta: 176.7,144.7,130.6,127.2,123.3,118.7,110.4,65.9,52.9,32.2,17.5 .46 .4,28.1,21.9$. HRMS (ESI) Calcd for $\mathrm{C}_{11} \mathrm{H}_{14} \mathrm{NO}_{2}\left([\mathrm{M}+\mathrm{H}]^{+}\right)$: 192.1019; Found: 192.1021.

\section{3-((2-Methoxyphenyl)amino)dihydrofuran-2-one (61)}

Method A. White solid, mp 61-62 ${ }^{\circ} \mathrm{C}, 9.8 \mathrm{~g}, 63 \%$ yield. ${ }^{1} \mathrm{H}$ NMR $\left(400 \mathrm{MHz}, \mathrm{CDCl}_{3}\right) \delta 6.91-6.84(\mathrm{~m}$,<smiles>COc1ccccc1NC1CCOC1=O</smiles>
$1 \mathrm{H}), 6.83-6.75(\mathrm{~m}, 2 \mathrm{H}), 6.61(\mathrm{~d}, J=7.8 \mathrm{~Hz}, 1 \mathrm{H}), 4.85(\mathrm{br}, 1 \mathrm{H}), 4.57-4.43(\mathrm{~m}$, $1 \mathrm{H}), 4.40-4.22(\mathrm{~m}, 1 \mathrm{H}), 4.12(\mathrm{dd}, J=11.0,8.2 \mathrm{~Hz}, 1 \mathrm{H}), 3.85(\mathrm{~s}, 3 \mathrm{H}), 2.92-2.80$ $(\mathrm{m}, 1 \mathrm{H}), 2.26-2.10(\mathrm{~m}, 1 \mathrm{H}) .{ }^{13} \mathrm{C} \mathrm{NMR}\left(101 \mathrm{MHz}, \mathrm{CDCl}_{3}\right) \delta: 176.4,147.3,136.5$, 121.1, 118.4, 110.6, 109.8, 65.8, 55.5, 52.8, 32.0. HRMS (ESI) Calcd for $\mathrm{C}_{11} \mathrm{H}_{14} \mathrm{NO}_{3}\left([\mathrm{M}+\mathrm{H}]^{+}\right)$: 208.0968; Found: 208.0972.

\section{3-((2,4-Dimethoxyphenyl)amino)dihydrofuran-2-one (6m)}<smiles>COc1ccc(NC2CCOC2=O)c(OC)c1</smiles>

Method A. White solid, mp 94-95 ${ }^{\circ} \mathrm{C}, 15.5 \mathrm{~g}, 87 \%$ yield. ${ }^{1} \mathrm{H}$ NMR (400 $\left.\mathrm{MHz}, \mathrm{CDCl}_{3}\right) \delta: 6.54(\mathrm{~d}, J=8.8 \mathrm{~Hz}, 1 \mathrm{H}), 6.48(\mathrm{~d}, J=2.8 \mathrm{~Hz}, 1 \mathrm{H}), 6.40$ $(\mathrm{dd}, J=8.4,2.8 \mathrm{~Hz}, 1 \mathrm{H}), 4.58-4.43(\mathrm{~m}, 2 \mathrm{H}), 4.32(\mathrm{~m}, 1 \mathrm{H}), 4.06(\mathrm{t}, J=8.3$ $\mathrm{Hz}, 1 \mathrm{H}), 3.84(\mathrm{~s}, 3 \mathrm{H}), 3.77(\mathrm{~s}, 3 \mathrm{H}), 2.84(\mathrm{~m}, 1 \mathrm{H}), 2.33-2.07(\mathrm{~m}, 1 \mathrm{H}) .{ }^{13} \mathrm{C}$ NMR (101 MHz, $\left.\mathrm{CDCl}_{3}\right) \delta: 176.6,153.2,148.5,130.7,111.3,103.6,99.3,65.7,55.8,55.5,53.5,32.0$. HRMS (ESI) Calcd for $\mathrm{C}_{12} \mathrm{H}_{16} \mathrm{NO}_{4}\left([\mathrm{M}+\mathrm{H}]^{+}\right)$: 238.1074; Found: 238.1076 .

\section{3-(Naphthalen-1-ylamino)dihydrofuran-2-one (6n)}<smiles>O=C1OCCC1Nc1cccc2ccccc12</smiles>

Method C. White solid, mp $123-124{ }^{\circ} \mathrm{C}, 3.2 \mathrm{~g}, 70 \%$ yield. ${ }^{1} \mathrm{H}$ NMR (400 MHz, DMSO- $\left.d_{6}\right) \delta: ~ 8.28-8.00(\mathrm{~m}, 1 \mathrm{H}), 7.86-7.72(\mathrm{~m}, 1 \mathrm{H}), 7.52-7.37(\mathrm{~m}, 2 \mathrm{H}), 7.31(\mathrm{t}$, $J=8.0 \mathrm{~Hz}, 1 \mathrm{H}), 7.30-7.17(\mathrm{~m}, 1 \mathrm{H}), 6.84-6.74(\mathrm{~m}, 1 \mathrm{H}), 6.53-6.33(\mathrm{~m}, 1 \mathrm{H}), 4.79$ $(\mathrm{q}, J=9.2 \mathrm{~Hz}, 1 \mathrm{H}), 4.47(\mathrm{t}, J=8.8 \mathrm{~Hz}, 1 \mathrm{H}), 4.40-4.24(\mathrm{~m}, 1 \mathrm{H}), 2.73-2.55(\mathrm{~m}$, $1 \mathrm{H}), 2.46-2.24(\mathrm{~m}, 1 \mathrm{H}) .{ }^{13} \mathrm{C}$ NMR (101 MHz, DMSO- $\left.d_{6}\right) \delta: 176.5,142.8,134.1$, $128.1,126.6,125.7,124.3,123.2,121.6,116.7,104.6,65.4,51.6,29.0$. HRMS (ESI) Calcd for $\mathrm{C}_{14} \mathrm{H}_{14} \mathrm{NO}_{2}\left([\mathrm{M}+\mathrm{H}]^{+}\right)$: 228.1019; Found: 228.1022 .

\section{3-(Benzo $[d][1,3]$ dioxol-5-ylamino)dihydrofuran-2-one (6o)}<smiles>O=C1OCCC1Nc1ccc2c(c1)OCO2</smiles>

Method C. White solid, mp 102-103 ${ }^{\circ} \mathrm{C}, 3.1 \mathrm{~g}, 70 \%$ yield. ${ }^{1} \mathrm{H}$ NMR (400 MHz, DMSO-d $\left.d_{6}\right) \delta: 6.68(\mathrm{~d}, J=8.4 \mathrm{~Hz}, 1 \mathrm{H}), 6.42(\mathrm{~s}, 1 \mathrm{H}), 6.22-6.07(\mathrm{~m}, 1 \mathrm{H})$, $5.85(\mathrm{~s}, 2 \mathrm{H}), 5.68(\mathrm{~d}, J=7.2 \mathrm{~Hz}, 1 \mathrm{H}), 4.47-4.32(\mathrm{~m}, 2 \mathrm{H}), 4.29-4.15(\mathrm{~m}, 1 \mathrm{H})$, 
2.73-2.54 (m, 1H), 2.14-1.91 (m, 1H). ${ }^{13} \mathrm{C}$ NMR (101 MHz, DMSO- $\left.d_{6}\right) \delta: 177.0,148.2,143.7,139.1$, 108.9, 104.8, 100.6, 96.3, 65.8, 52.7, 30.2. HRMS (ESI) Calcd for $\mathrm{C}_{11} \mathrm{H}_{12} \mathrm{NO}_{4}\left([\mathrm{M}+\mathrm{H}]^{+}\right): 222.0761$; Found: 222.0763.

\section{3-(6-Methylpyridin-2-ylamino)dihydrofuran-2-one (6p)}<smiles>Cc1cccc(NC2CCOC2=O)n1</smiles>

Method C. Pale oil, $2.3 \mathrm{~g}, 60 \%$ yield. ${ }^{1} \mathrm{H}$ NMR $\left(400 \mathrm{MHz}, \mathrm{CDCl}_{3}\right) \delta 7.32(\mathrm{t}$, $J=7.6 \mathrm{~Hz}, 1 \mathrm{H}), 6.52(\mathrm{~d}, J=7.2 \mathrm{~Hz}, 1 \mathrm{H}), 6.32(\mathrm{~d}, J=8.2 \mathrm{~Hz}, 1 \mathrm{H}), 4.89(\mathrm{~s}$, $1 \mathrm{H}), 4.64-4.55(\mathrm{~m}, 1 \mathrm{H}), 4.52(\mathrm{t}, J=9.0 \mathrm{~Hz}, 1 \mathrm{H}), 4.41-4.31(\mathrm{~m}, 1 \mathrm{H}), 3.00-$ $2.89(\mathrm{~m}, 1 \mathrm{H}), 2.38(\mathrm{~s}, 3 \mathrm{H}), 2.29-2.17(\mathrm{~m}, 1 \mathrm{H}) .{ }^{13} \mathrm{C} \mathrm{NMR}\left(101 \mathrm{MHz}, \mathrm{CDCl}_{3}\right)$ $\delta$ 177.2, 156.5, 156.4, 137.7, 113.2, 105.8, 66.2, 51.0, 30.7, 24.3. HRMS (ESI) Calcd for $\mathrm{C}_{10} \mathrm{H}_{13} \mathrm{~N}_{2} \mathrm{O}_{2}$ $\left([\mathrm{M}+\mathrm{H}]^{+}\right)$: 193.0972; Found: 193.0971.

\section{3-(Phenylamino)tetrahydropyran-2-one (8a)}<smiles>O=C1OCCCC1Nc1ccccc1</smiles>

Method C. White solid, mp 137-138 ${ }^{\circ} \mathrm{C}, 2.4 \mathrm{~g}, 64 \%$ yield. ${ }^{1} \mathrm{H}$ NMR $(400 \mathrm{MHz}$, $\left.\mathrm{CDCl}_{3}\right) \delta: 7.19(\mathrm{t}, J=8.0 \mathrm{~Hz}, 2 \mathrm{H}), 6.75(\mathrm{t}, J=8.0 \mathrm{~Hz}, 1 \mathrm{H}), 6.63(\mathrm{~d}, J=8.0 \mathrm{~Hz}$, $2 \mathrm{H}), 4.64(\mathrm{~s}, 1 \mathrm{H}), 4.37(\mathrm{t}, J=6.0 \mathrm{~Hz}, 2 \mathrm{H}), 4.05-4.19(\mathrm{~m}, 1 \mathrm{H}), 2.69-2.56(\mathrm{~m}, 1 \mathrm{H})$, 2.07- $2.00(\mathrm{~m}, 2 \mathrm{H}), 1.68-1.59(\mathrm{~m}, 1 \mathrm{H}) .{ }^{13} \mathrm{C}$ NMR $\left(101 \mathrm{MHz}, \mathrm{CDCl}_{3}\right) \delta: 173.5$, 146.4, 129.4, 118.4, 113.3, 67.3, 51.9, 26.0, 21.1. HRMS (ESI) Calcd for $\mathrm{C}_{11} \mathrm{H}_{14} \mathrm{NO}_{2}\left([\mathrm{M}+\mathrm{H}]^{+}\right)$: 192.1019 ; Found: 192.1020 .

\section{3-(p-Tolylamino)tetrahydropyran-2-one (8b)}<smiles>Cc1ccc(NC2CCCOC2=O)cc1</smiles>

Method C. White solid. mp $115-116{ }^{\circ} \mathrm{C}, 3.3 \mathrm{~g}, 80 \%$ yield. ${ }^{1} \mathrm{H}$ NMR (400 $\left.\mathrm{MHz}, \mathrm{CDCl}_{3}\right)$ : $7.05-6.99(\mathrm{~m}, 2 \mathrm{H}), 6.61-6.55(\mathrm{~m}, 2 \mathrm{H}), 4.54(\mathrm{~s}, 1 \mathrm{H}), 4.45-$ $4.37(\mathrm{~m}, 2 \mathrm{H}), 4.12(\mathrm{dd}, J=11.2,8.0 \mathrm{~Hz}, 1 \mathrm{H}), 2.72-2.58(\mathrm{~m}, 1 \mathrm{H}), 2.25(\mathrm{~s}$, $3 \mathrm{H}), 2.14-2.02(\mathrm{~m}, 2 \mathrm{H}), 1.75-1.60(\mathrm{~m}, 1 \mathrm{H}) .{ }^{13} \mathrm{C} \mathrm{NMR}\left(101 \mathrm{MHz}, \mathrm{CDCl}_{3}\right) \delta$ : 173.6, 144.1, 129.9, 127.6, 113.6, 67.3, 52.3, 26.1, 21.2, 20.4. HRMS (ESI) Calcd for $\mathrm{C}_{12} \mathrm{H}_{16} \mathrm{NO}_{2}$ $\left([\mathrm{M}+\mathrm{H}]^{+}\right):$206.1176; Found: 206.1174.

\section{3-((4-Methoxyphenyl)amino)tetrahydropyran-2-one (8c)}<smiles>COc1ccc(NC2CCCOC2=O)cc1</smiles>
Method C. White solid, mp $133-134{ }^{\circ} \mathrm{C}, 2.3 \mathrm{~g}, 53 \%$ yield. ${ }^{1} \mathrm{H}$ NMR (400 $\left.\mathrm{MHz}, \mathrm{CDCl}_{3}\right) \delta: 6.82-6.76(\mathrm{~m}, 2 \mathrm{H}), 6.66-6.59(\mathrm{~m}, 2 \mathrm{H}), 4.45-4.32(\mathrm{~m}$, $3 \mathrm{H}), 4.10-4.01(\mathrm{~m}, 1 \mathrm{H}), 3.73(\mathrm{~s}, 3 \mathrm{H}), 2.65-2.52(\mathrm{~m}, 1 \mathrm{H}), 2.10-1.99(\mathrm{~m}$, 2H), 1.74-1.59 (m, 1H). $\left.{ }^{13} \mathrm{C} \mathrm{NMR} \mathrm{(101} \mathrm{MHz,} \mathrm{CDCl}_{3}\right) \delta: 173.7,152.8$, 140.6, 115.1, 114.9, 67.3, 55.8, 53.06, 26.7, 21.2. HRMS (ESI) Calcd for $\mathrm{C}_{12} \mathrm{H}_{16} \mathrm{NO}_{3}\left([\mathrm{M}+\mathrm{H}]^{+}\right)$: 222.1125; Found: 222.1129.

\section{3-((4-Fluorophenyl)amino)tetrahydropyran-2-one (8d)}<smiles>O=C1OCCCC1Nc1ccc(F)cc1</smiles>

Method C. White solid, $110-111{ }^{\circ} \mathrm{C}, 1.7 \mathrm{~g}, 41 \%$ yield. ${ }^{1} \mathrm{H}$ NMR $(400 \mathrm{MHz}$, $\left.\mathrm{CDCl}_{3}\right) \delta$ : 6.97-6.85 (m, 2H), 6.64-6.54 (m, 2H), $4.50(\mathrm{~s}, 1 \mathrm{H}), 4.41(\mathrm{dd}, J=$ $7.2,5.1 \mathrm{~Hz}, 2 \mathrm{H}), 4.07(\mathrm{dd}, J=11.6,7.6 \mathrm{~Hz}, 1 \mathrm{H}), 2.69-2.56(\mathrm{~m}, 1 \mathrm{H}), 2.15-$ $2.02(\mathrm{~m}, 2 \mathrm{H}), 1.76-1.61(\mathrm{~m}, 1 \mathrm{H}) .{ }^{13} \mathrm{C}$ NMR $\left(101 \mathrm{MHz}, \mathrm{CDCl}_{3}\right) \delta: 173.4,157.5$, 155.1, 142.8, 115.9, 115.7, 114.5, 114.4, 67.3, 52.6, 26.1, 21.2. HRMS (ESI) Calcd for $\mathrm{C}_{11} \mathrm{H}_{13} \mathrm{FNO}_{2}$ $\left([\mathrm{M}+\mathrm{H}]^{+}\right): 210.0925$; Found: 210.0923. 
3-((4-Chlorophenyl)amino)tetrahydropyran-2-one (8e)<smiles>O=C1OCCCC1Nc1ccc(Cl)cc1</smiles>

Method C. White solid, $127-128{ }^{\circ} \mathrm{C}, 3.1 \mathrm{~g}, 68 \%$ yield. ${ }^{1} \mathrm{H}$ NMR (400 MHz, $\left.\mathrm{CDCl}_{3}\right)$ 8: 7.23-7.09 (m, 2H), 6.62-6.52 (m, 2H), $4.65(\mathrm{~s}, 1 \mathrm{H}), 4.42(\mathrm{dd}, J=$ $7.2,5.2 \mathrm{~Hz}, 2 \mathrm{H}), 4.10(\mathrm{dd}, J=11.6,7.6 \mathrm{~Hz}, 1 \mathrm{H}), 2.70-2.58(\mathrm{~m}, 1 \mathrm{H}), 2.17-$ $2.03(\mathrm{~m}, 2 \mathrm{H}), 1.74-1.62(\mathrm{~m}, 1 \mathrm{H}) .{ }^{13} \mathrm{C} \mathrm{NMR}\left(101 \mathrm{MHz}, \mathrm{CDCl}_{3}\right) \delta: 173.1$, 144.9, 129.2, 123.0, 114.4, 67.3, 52.0 25.8, 21.1. HRMS (ESI) Calcd for $\mathrm{C}_{11} \mathrm{H}_{13} \mathrm{ClNO}_{2}\left([\mathrm{M}+\mathrm{H}]^{+}\right)$: 226.0629; Found: 226.0631.

3-([1,1'-Biphenyl]-4-ylamino)tetrahydropyran-2-one (8f)<smiles>O=C1OCCCC1Nc1ccc(-c2ccccc2)cc1</smiles>

Method C. Yellow solid, mp $154-155^{\circ} \mathrm{C}, 2.9 \mathrm{~g}, 54 \%$ yield. ${ }^{1} \mathrm{H}$ NMR (400 $\left.\mathrm{MHz}, \mathrm{CDCl}_{3}\right)$ ס: 7.57-7.49 (m, 2H), 7.48-7.43 (m, 2H), 7.42-7.35 (m, 2H), $7.30-7.23(\mathrm{~m}, 1 \mathrm{H}), 6.75-6.65(\mathrm{~m}, 2 \mathrm{H}), 4.73(\mathrm{~s}, 1 \mathrm{H}), 4.43-4.35(\mathrm{~m}, 2 \mathrm{H}), 4.18$ $(\mathrm{dd}, J=11.6,7.6 \mathrm{~Hz}, 1 \mathrm{H}), 2.75-2.61(\mathrm{~m}, 1 \mathrm{H}), 2.12-2.00(\mathrm{~m}, 2 \mathrm{H}), 1.76-1.64$ (m, 1H). ${ }^{13} \mathrm{C}$ NMR (101 MHz, $\left.\mathrm{CDCl}_{3}\right) \delta: 173.3,145.7,141.0,131.4,128.7,128.1,126.2,126.3,113.6$, 67.3, 51.9, 26.0, 21.1. HRMS (ESI) Calcd for $\mathrm{C}_{17} \mathrm{H}_{18} \mathrm{NO}_{2}\left([\mathrm{M}+\mathrm{H}]^{+}\right)$: 268.1332; Found: 268.1337.

3-(m-Tolylamino)tetrahydro-2H-pyran-2-one (8g)<smiles>Cc1cccc(NC2CCCOC2=O)c1</smiles>

Method C. White solid, mp $89-90{ }^{\circ} \mathrm{C}, 3.3 \mathrm{~g}, 80 \%$ yield. ${ }^{1} \mathrm{H}$ NMR $(400 \mathrm{MHz}$, $\left.\mathrm{CDCl}_{3}\right) \delta: 7.09(\mathrm{t}, J=7.8 \mathrm{~Hz}, 1 \mathrm{H}), 6.60(\mathrm{~d}, J=7.2 \mathrm{~Hz}, 1 \mathrm{H}), 6.46(\mathrm{~d}, J=7.6$ $\mathrm{Hz}, 2 \mathrm{H}), 4.62(\mathrm{~s}, 1 \mathrm{H}), 4.45-4.40(\mathrm{~m}, 2 \mathrm{H}), 4.14(\mathrm{dd}, J=11.6,7.6 \mathrm{~Hz}, 1 \mathrm{H})$, 2.72-2.62 (m, 1H), $2.29(\mathrm{~s}, 3 \mathrm{H}), 2.13-2.04(\mathrm{~m}, 2 \mathrm{H}), 1.74-1.63(\mathrm{~m}, 1 \mathrm{H}) .{ }^{13} \mathrm{C}$ NMR (101 MHz, $\left.\mathrm{CDCl}_{3}\right) \delta:$ 173.5, 146.4, 139.2, 129.3, 119.3, 114.1, 110.4, 67.2, 51.9, 26.1, 21.7, 21.1. HRMS (ESI) Calcd for $\mathrm{C}_{12} \mathrm{H}_{16} \mathrm{NO}_{2}\left([\mathrm{M}+\mathrm{H}]^{+}\right)$: 206.1176; Found: 206.1176 .

3-((3-Methoxyphenyl)amino)tetrahydropyran-2-one (8h)<smiles>COc1cccc(NC2CCCOC2=O)c1</smiles>

Method C. Pale yellow oil, $2.3 \mathrm{~g}, 53 \%$ yield. ${ }^{1} \mathrm{H}$ NMR $\left(400 \mathrm{MHz}, \mathrm{CDCl}_{3}\right)$ $\delta: 7.13(\mathrm{t}, J=8.0 \mathrm{~Hz}, 1 \mathrm{H}), 6.34(\mathrm{dd}, J=8.2,2.4 \mathrm{~Hz}, 1 \mathrm{H}), 6.28(\mathrm{dd}, J=8.0$, $2.4 \mathrm{~Hz}, 1 \mathrm{H}), 6.21(\mathrm{t}, J=2.4 \mathrm{~Hz}, 1 \mathrm{H}), 4.72(\mathrm{~d}, J=4.8 \mathrm{~Hz}, 1 \mathrm{H}), 4.46-4.36$ $(\mathrm{m}, 2 \mathrm{H}), 4.21-4.11(\mathrm{~m}, 1 \mathrm{H}), 3.77(\mathrm{~s}, 3 \mathrm{H}), 2.74-2.62(\mathrm{~m}, 1 \mathrm{H}), 2.14-2.03$ (m, 2H), 1.73-1.63 (m, 1H). ${ }^{13} \mathrm{C}$ NMR (101 MHz, $\left.\mathrm{CDCl}_{3}\right) \delta: 173.4,160.8,147.7,130.2,106.3,103.2$, 99.5, 67.2, 55.2, 51.9, 25.9, 21.1. HRMS (ESI) Calcd for $\mathrm{C}_{12} \mathrm{H}_{16} \mathrm{NO}_{3}\left([\mathrm{M}+\mathrm{H}]^{+}\right)$: 222.1125; Found: 222.1128.

\section{3-((3-Chlorophenyl)amino)tetrahydropyran-2-one (8i)}<smiles>O=C1OCCCC1Nc1cccc(Cl)c1</smiles>

Method C. White solid, mp $106-107^{\circ} \mathrm{C}, 3.1 \mathrm{~g}, 69 \%$ yield. ${ }^{1} \mathrm{H}$ NMR (400 $\left.\mathrm{MHz}, \mathrm{CDCl}_{3}\right) \delta: 7.10(\mathrm{t}, J=8.0 \mathrm{~Hz}, 1 \mathrm{H}), 6.72(\mathrm{dd}, J=8.0,2.0 \mathrm{~Hz}, 1 \mathrm{H})$, $6.59(\mathrm{t}, J=2.0 \mathrm{~Hz}, 1 \mathrm{H}), 6.52(\mathrm{dd}, J=8.0,2.4 \mathrm{~Hz}, 1 \mathrm{H}), 4.75(\mathrm{~d}, J=4.8 \mathrm{~Hz}$, $1 \mathrm{H}), 4.47-4.35(\mathrm{~m}, 2 \mathrm{H}), 4.19-4.06(\mathrm{~m}, 1 \mathrm{H}), 2.74-2.59(\mathrm{~m}, 1 \mathrm{H}), 2.18-2.02$ (m, 2H), 1.74-1.61 (m, 1H). ${ }^{13} \mathrm{C}$ NMR (101 MHz, $\left.\mathrm{CDCl}_{3}\right) \delta: 173.1,147.5,135.0,118.1,112.7,111.9$, 67.3, 51.6, 25.7, 21.0. HRMS (ESI) Calcd for $\mathrm{C}_{11} \mathrm{H}_{13} \mathrm{ClNO}_{2}\left([\mathrm{M}+\mathrm{H}]^{+}\right)$: 226.0629; Found: 226.0628.

\section{3-(o-Tolylamino)tetrahydropyran-2-one $(8 \mathbf{j})$}


<smiles>Cc1ccccc1NC1CCCOC1=O</smiles>

Method C. White solid, mp $105-106{ }^{\circ} \mathrm{C}, 2.6 \mathrm{~g}, 64 \%$ yield. ${ }^{1} \mathrm{H}$ NMR $(400 \mathrm{MHz}$, $\left.\mathrm{CDCl}_{3}\right) \delta: 7.16-7.06(\mathrm{~m}, 2 \mathrm{H}), 6.72(\mathrm{t}, J=6.8 \mathrm{~Hz}, 1 \mathrm{H}), 6.54(\mathrm{~d}, J=8.0 \mathrm{~Hz}, 1 \mathrm{H})$, $4.66(\mathrm{~s}, 1 \mathrm{H}), 4.47-4.40(\mathrm{~m}, 2 \mathrm{H}), 4.18(\mathrm{dd}, J=11.6,7.6 \mathrm{~Hz}, 1 \mathrm{H}), 2.76-2.66(\mathrm{~m}$, 1H), $2.22(\mathrm{~s}, 3 \mathrm{H}), 2.14-2.05(\mathrm{~m}, 2 \mathrm{H}), 1.76-1.64(\mathrm{~m}, 1 \mathrm{H}) .{ }^{13} \mathrm{C}$ NMR $(101 \mathrm{MHz}$, $\left.\mathrm{CDCl}_{3}\right) \delta:$ 173.6, 144.4, 130.5, 127.0, 123.1, 118.0, 109.9, 67.3, 52.0, 26.1, 21.1, 17.5. HRMS (ESI) Calcd for $\mathrm{C}_{12} \mathrm{H}_{16} \mathrm{NO}_{2}\left([\mathrm{M}+\mathrm{H}]^{+}\right)$: 206.1176; Found: 206.1179 .

\section{3-((2-Ethylphenyl)amino)tetrahydropyran-2-one (8k)}<smiles>CCc1ccccc1NC1CCCOC1=O</smiles>

Method C. White solid. mp $61-62{ }^{\circ} \mathrm{C}, 2.7 \mathrm{~g}, 62 \%$ yield. ${ }^{1} \mathrm{H}$ NMR $(400 \mathrm{MHz}$, $\left.\mathrm{CDCl}_{3}\right) \delta: 7.14(\mathrm{t}, J=7.6 \mathrm{~Hz}, 2 \mathrm{H}), 6.78(\mathrm{t}, J=7.6 \mathrm{~Hz}, 1 \mathrm{H}), 6.57(\mathrm{~d}, J=8.0 \mathrm{~Hz}$, $1 \mathrm{H}), 4.80(\mathrm{~d}, J=4.0 \mathrm{~Hz}, 1 \mathrm{H}), 4.46-4.40(\mathrm{~m}, 2 \mathrm{H}), 4.23-4.14(\mathrm{~m}, 1 \mathrm{H}), 2.77-2.67$ $(\mathrm{m}, 1 \mathrm{H}), 2.59(\mathrm{q}, J=7.6 \mathrm{~Hz}, 2 \mathrm{H}), 2.15-2.04(\mathrm{~m}, 2 \mathrm{H}), 1.74-1.64(\mathrm{~m}, 1 \mathrm{H}), 1.29(\mathrm{t}$, $J=7.5 \mathrm{~Hz}, 3 \mathrm{H}) .{ }^{13} \mathrm{C} \mathrm{NMR}\left(101 \mathrm{MHz}, \mathrm{CDCl}_{3}\right) \delta: 173.7,143.9,128.8,128.4,126.9$, 118.2, 110.1, 67.3, 52.1, 26.2, 23.9, 21.1, 13.0. HRMS (ESI) Calcd for $\mathrm{C}_{13} \mathrm{H}_{18} \mathrm{NO}_{2}\left([\mathrm{M}+\mathrm{H}]^{+}\right)$: 220.1332; Found: 220.1335 .

\section{3-((2-Isopropylphenyl)amino)tetrahydropyran-2-one (81)}<smiles>CCCc1ccccc1NC1CCCOC1=O</smiles>

Method C. Colorless oil, $2.9 \mathrm{~g}, 63 \%$ yield. ${ }^{1} \mathrm{H}$ NMR $\left(400 \mathrm{MHz}, \mathrm{CDCl}_{3}\right) \delta: 7.19$ (d, $J=7.2,1.5 \mathrm{~Hz}, 1 \mathrm{H}), 7.11(\mathrm{t}, J=7.2, \mathrm{~Hz}, 1 \mathrm{H}), 6.80(\mathrm{~d}, J=7.2 \mathrm{~Hz}, 1 \mathrm{H}), 6.56(\mathrm{~d}, J$ $=8.0 \mathrm{~Hz}, 1 \mathrm{H}), 4.91(\mathrm{~d}, J=4.0 \mathrm{~Hz}, 1 \mathrm{H}), 4.47-4.37(\mathrm{~m}, 2 \mathrm{H}), 4.23-4.10(\mathrm{~m}, 1 \mathrm{H})$, $3.05-2.96(\mathrm{~m}, 1 \mathrm{H}), 2.77-2.65(\mathrm{~m}, 1 \mathrm{H}), 2.15-2.04(\mathrm{~m}, 2 \mathrm{H}), 1.75-1.64(\mathrm{~m}, 1 \mathrm{H}), 1.28$ (dd, $J=10.4,6.8 \mathrm{~Hz}, 6 \mathrm{H}) .{ }^{13} \mathrm{C} \mathrm{NMR}\left(101 \mathrm{MHz}, \mathrm{CDCl}_{3}\right) \delta: 173.8,143.2,133.4,126.6,125.5,118.4$, 110.5, 67.2, 52.2, 27.3, 26.2, 22.5, 22.3, 21.1. HRMS (ESI) Calcd for $\mathrm{C}_{14} \mathrm{H}_{20} \mathrm{NO}_{2}\left([\mathrm{M}+\mathrm{H}]^{+}\right): 234.1489$; Found: 234.1496.

\section{3-((2-Methoxyphenyl)amino)tetrahydropyran-2-one (8m)}<smiles>COc1ccccc1NC1CCCOC1=O</smiles>

Method C. Pale yellow oil. $3.1 \mathrm{~g}, 71 \%$ yield. ${ }^{1} \mathrm{H}$ NMR $\left(400 \mathrm{MHz}, \mathrm{CDCl}_{3}\right) \delta: 6.85$ $(\mathrm{td}, J=7.6,1.6 \mathrm{~Hz}, 1 \mathrm{H}), 6.79(\mathrm{dd}, J=8.0,1.6 \mathrm{~Hz}, 1 \mathrm{H}), 6.72(\mathrm{td}, J=8.0,1.6 \mathrm{~Hz}$, $1 \mathrm{H}), 6.55(\mathrm{dd}, J=8.0,1.6 \mathrm{~Hz}, 1 \mathrm{H}), 5.11(\mathrm{br}, 1 \mathrm{H}), 4.43-4.35(\mathrm{~m}, 2 \mathrm{H}), 4.14(\mathrm{dd}, J$ $=11.6,7.6 \mathrm{~Hz}, 1 \mathrm{H}), 3.85(\mathrm{~s}, 3 \mathrm{H}), 2.70-2.58(\mathrm{~m}, 1 \mathrm{H}), 2.11-2.01(\mathrm{~m}, 2 \mathrm{H}), 1.77-$ $1.66(\mathrm{~m}, 1 \mathrm{H}) .{ }^{13} \mathrm{C}$ NMR $\left(101 \mathrm{MHz}, \mathrm{CDCl}_{3}\right) \delta: 173.2,147.2,136.3,121.0,117.7,110.0,109.8,67.3,55.5$, 51.8, 26.1, 21.2. HRMS (ESI) Calcd for $\mathrm{C}_{12} \mathrm{H}_{16} \mathrm{NO}_{3}\left([\mathrm{M}+\mathrm{H}]^{+}\right)$: 222.1125; Found: 222.1128 .

\section{3-((2-Fluorophenyl)amino)tetrahydropyran-2-one (8n)}<smiles>O=C1OCCCC1Nc1ccccc1F</smiles>

Method C. White solid. mp 109-110 ${ }^{\circ} \mathrm{C}, 2.5 \mathrm{~g}, 60 \%$ yield. ${ }^{1} \mathrm{H}$ NMR $(400 \mathrm{MHz}$, $\left.\mathrm{CDCl}_{3}\right)$ ): 7.05-6.95 (m, 2H), 6.75-6.60 (m, 2H), 4.84-4.69 (br, 1H), 4.46-4.33 $(\mathrm{m}, 2 \mathrm{H}), 4.21-4.08(\mathrm{~m}, 1 \mathrm{H}), 2.71-2.54(\mathrm{~m}, 1 \mathrm{H}), 2.17-1.98(\mathrm{~m}, 2 \mathrm{H}), 1.80-1.62(\mathrm{~m}$, 1H). ${ }^{13} \mathrm{C}$ NMR $\left(101 \mathrm{MHz}, \mathrm{CDCl}_{3}\right) \delta: 173.0,152.9,150.5,135.0,134.8,124.6$, 124.5, 118.0, 117.9, 115.0, 114.8, 112.6, 112.6, 67.4, 51.7, 26.0, 21.2. HRMS (ESI) Calcd for $\mathrm{C}_{11} \mathrm{H}_{13} \mathrm{FNO}_{2}\left([\mathrm{M}+\mathrm{H}]^{+}\right)$: 210.0925; Found: 210.0929.

\section{3-((2,4-Dimethoxyphenyl)amino)tetrahydropyran-2-one (80)}


<smiles>COc1ccc(NC2CCCOC2=O)c(OC)c1</smiles>

Method C. Colorless oil, $3.0 \mathrm{~g}, 60 \%$ yield. ${ }^{1} \mathrm{H}$ NMR (400 MHz, $\mathrm{CDCl}_{3}$ )

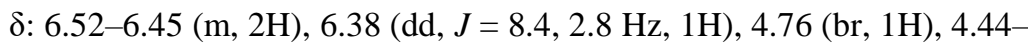
$4.37(\mathrm{~m}, 2 \mathrm{H}), 4.07(\mathrm{dd}, J=11.4,7.6 \mathrm{~Hz}, 1 \mathrm{H}), 3.84(\mathrm{~s}, 3 \mathrm{H}), 3.76(\mathrm{~s}, 3 \mathrm{H})$, $2.66-2.56(\mathrm{~m}, 1 \mathrm{H}), 2.12-2.02(\mathrm{~m}, 2 \mathrm{H}), 1.80-1.70(\mathrm{~m}, 1 \mathrm{H}) .{ }^{13} \mathrm{C} \mathrm{NMR}(101$ $\left.\mathrm{MHz}, \mathrm{CDCl}_{3}\right) \delta: 173.4,152.8,148.5,130.6,111.0,103.6,99.4,67.3,55.8,55.6,52.7,26.4,21.3$. HRMS (ESI) Calcd for $\mathrm{C}_{13} \mathrm{H}_{18} \mathrm{NO}_{4}\left([\mathrm{M}+\mathrm{H}]^{+}\right)$: 252.1230: Found: 252.1235 .

\section{3-(Naphthalen-1-ylamino)tetrahydropyran-2-one (8p)}<smiles>O=C1OCCCC1Nc1cccc2ccccc12</smiles>

Method C. White solid, mp $138-139{ }^{\circ} \mathrm{C}, 2.9 \mathrm{~g}, 61 \%$ yield. ${ }^{1} \mathrm{H}$ NMR $(400$ $\left.\mathrm{MHz}, \mathrm{CDCl}_{3}\right) 8:$ 7.99-7.92 (m, 1H), 7.84-7.77 (m, 1H), 7.52-7.43 (m, 2H), $7.39-7.29(\mathrm{~m}, 2 \mathrm{H}),, 6.56(\mathrm{~d}, J=6.8 \mathrm{~Hz}, 1 \mathrm{H}), 5.55(\mathrm{~s}, 1 \mathrm{H}), 4.52-4.44(\mathrm{~m}, 2 \mathrm{H})$, $4.32(\mathrm{t}, J=9.6 \mathrm{~Hz}, 1 \mathrm{H}), 2.89-2.77(\mathrm{~m}, 1 \mathrm{H}), 2.20-2.08(\mathrm{~m}, 2 \mathrm{H}), 1.83-1.68(\mathrm{~m}$, 1H). ${ }^{13} \mathrm{C}$ NMR (101 MHz, $\left.\mathrm{CDCl}_{3}\right) \delta: 173.5,141.4,134.4,128.6,126.2,126.07,125.2,123.9,120.4$, 118.6, 104.7, 67.4, 52.0, 25.5, 21.1. HRMS (ESI) Calcd for $\mathrm{C}_{15} \mathrm{H}_{16} \mathrm{NO}_{2}\left([\mathrm{M}+\mathrm{H}]^{+}\right)$: 242.1176; Found: 242.1179 .

\section{(C) Asymmetric Hydrogenation of Racemic $\alpha$-Arylaminated Lactones}<smiles>NCCNC1CCOC1=O</smiles>

$6(n=1), 8(n=2)$

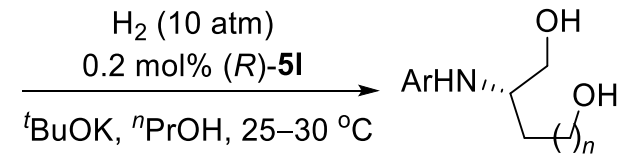

$$
7(n=1), 9(n=2)
$$

General procedure: To a $20 \mathrm{~mL}$ hydrogenation vessel in an autoclave was added racemic substrates 6 or $7(1.0 \mathrm{mmol})$, chiral iridium catalyst $(R)-5 \mathrm{l}(2.3 \mathrm{mg}, 0.2 \mu \mathrm{mmol}), t \mathrm{BuOK}(11.2 \mathrm{mg}, 0.1 \mathrm{mmol})$ and $n \mathrm{PrOH}(5.0 \mathrm{~mL})$. The autoclave was purged with hydrogen by pressurizing to $5 \mathrm{~atm}$ and releasing the pressure. This procedure was repeated three times and then pressurized to $10 \mathrm{~atm}$ of $\mathrm{H}_{2}$. The reaction mixture was stirred at room temperature $\left(25-30{ }^{\circ} \mathrm{C}\right)$ until no obvious hydrogen pressure drop was observed. The reaction mixture was then quenched with saturated $\mathrm{NH}_{4} \mathrm{Cl}(5 \mathrm{~mL})$ and extracted with EtOAc $(5 \mathrm{~mL} \times 3)$. The combined extracts were washed with brine, dried over anhydrous $\mathrm{MgSO}_{4}$ and concentrated in vacuo. The residue was purified by flash column chromatography on silical gel with petroleum ether/ethyl acetate (from 1:1 to 0:1) as an eluent to afford the chiral diols $\mathbf{7}$ or $\mathbf{9}$.

\section{(S)-2-(Phenylamino)butane-1,4-diol (7a)}<smiles>OCC[C@H](CO)Nc1ccccc1</smiles>

White solid, $6 \mathrm{~h}, \mathrm{mp} 80-81{ }^{\circ} \mathrm{C}, 163 \mathrm{mg}, 90 \%$ yield, $90 \%$ ee. $[\alpha]_{\mathrm{D}}^{25}-54.2(c 1.0$, MeOH). ${ }^{1} \mathrm{H}$ NMR (400 MHz, $\mathrm{CDCl}_{3}$ ) $\delta: 7.17$ (t, $\left.J=8.0 \mathrm{~Hz}, 2 \mathrm{H}\right), 6.72$ (t, $J=7.2$ $\mathrm{Hz}, 1 \mathrm{H}), 6.66(\mathrm{~d}, J=8.0 \mathrm{~Hz}, 2 \mathrm{H}), 3.88-3.58(\mathrm{~m}, 6 \mathrm{H}), 3.01(\mathrm{br}, 1 \mathrm{H}), 1.93-1.85$ (m, 1H), 1.85-1.74 (m, 1H). $\left.{ }^{13} \mathrm{C} \mathrm{NMR} \mathrm{(101} \mathrm{MHz,} \mathrm{CDCl}_{3}\right) \delta: 147.2,129.5,118.0$, 113.9, 64.4, 59.8, 53.1, 34.5. HRMS (ESI) Calcd for $\mathrm{C}_{10} \mathrm{H}_{16} \mathrm{NO}_{2}\left([\mathrm{M}+\mathrm{H}]^{+}\right)$: 182.1176; Found: 182.1178. HPLC conditions: Chiralcel IC-3 column $(25 \mathrm{~cm} \times 0.46 \mathrm{~cm} \mathrm{ID}) ; n$-hexane $/ 2-$ propanol $=75: 25$; temp, rt; flow rate $=1.0 \mathrm{~mL} / \mathrm{min} ; 254 \mathrm{~nm} \mathrm{UV}$ detector; $t_{\mathrm{R}}(R)=5.6 \mathrm{~min}\left(\right.$ minor); $t_{\mathrm{R}}(S)$ $=7.5 \mathrm{~min}$ (major).

The product 7a (20 mg) was dissolved in mixture solvents of dichloromethane $(1 \mathrm{~mL})$ and hexane $(1 \mathrm{~mL})$. After slowly evaporation of solvents at ambient temperautre, the fine crystals which were suitable for the 
$\mathrm{X}$-ray diffraction analyses were obtained. The data of X-ray diffraction analysis of $\mathbf{7 a}$ are summarized in Table S1.

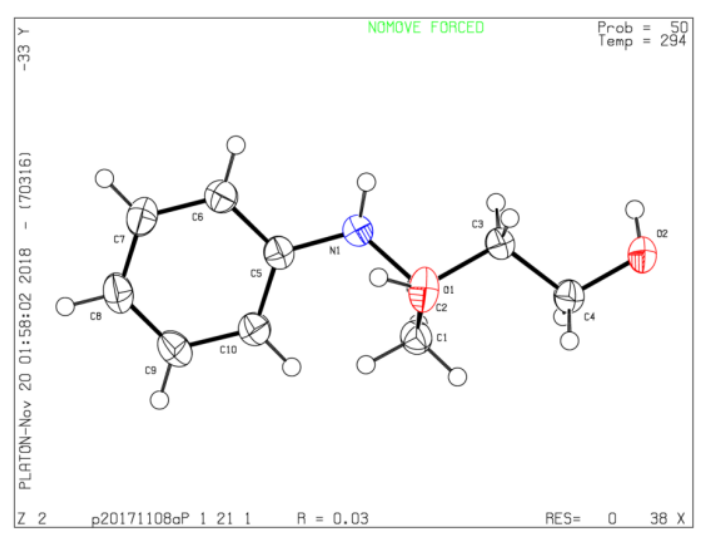

Table S1. Crystal data and structure refinement for $7 \mathbf{a}$.

\begin{tabular}{|c|c|}
\hline Identification code & P20171108a \\
\hline Empirical formula & $\mathrm{C}_{10} \mathrm{H}_{15} \mathrm{NO}_{2}$ \\
\hline Formula weight & 181.23 \\
\hline Temperature & $294 \mathrm{~K}$ \\
\hline Crystal system & monoclinic \\
\hline Space group & $\mathrm{P} 21_{1}$ \\
\hline $\mathrm{a} / \AA$ & $7.16950(10)$ \\
\hline $\mathrm{b} / \AA$ & $6.37400(10)$ \\
\hline $\mathrm{c} / \AA$ & $10.56690(10)$ \\
\hline$\alpha /^{\circ}$ & 90 \\
\hline$\beta /{ }^{\circ}$ & $99.0540(10)$ \\
\hline$\gamma /{ }^{\circ}$ & 90 \\
\hline Volume $/ \AA^{3}$ & $476.874(11)$ \\
\hline $\mathrm{Z}$ & 2 \\
\hline$\rho_{\text {calc }} g / \mathrm{cm}^{3}$ & 1.262 \\
\hline$\mu / \mathrm{mm}^{-1}$ & 0.709 \\
\hline $\mathrm{F}(000)$ & 196.0 \\
\hline Crystal size $/ \mathrm{mm}^{3}$ & $0.34 \times 0.26 \times 0.1$ \\
\hline Radiation & $\operatorname{CuK} \alpha(\lambda=1.54184)$ \\
\hline $2 \Theta$ range for data collection $/^{\circ}$ & 8.474 to 158.554 \\
\hline Index ranges & $-8 \leq \mathrm{h} \leq 9,-5 \leq \mathrm{k} \leq 7,-13 \leq \mathrm{l} \leq 13$ \\
\hline Reflections collected & 7625 \\
\hline Independent reflections & $1746\left[\mathrm{R}_{\mathrm{int}}=0.0160, \mathrm{R}_{\mathrm{sigma}}=0.0091\right]$ \\
\hline Data/restraints/parameters & $1746 / 1 / 131$ \\
\hline Goodness-of-fit on $\mathrm{F}^{2}$ & 0.883 \\
\hline Final $R$ indices $[I>2 \sigma(I)]$ & $\mathrm{R} 1=0.0258, \mathrm{wR} 2=0.0691$ \\
\hline Final R indices (all data) & $\mathrm{R} 1=0.0258, \mathrm{wR} 2=0.0691$ \\
\hline
\end{tabular}


<smiles>Cc1ccc(N[C@@H](CO)CCO)cc1</smiles>

Colorless oil, 6 h, $183 \mathrm{mg}, 94 \%$ yield, $89 \%$ ee. $[\alpha]_{\mathrm{D}}^{25}-31.4\left(c 1.0, \mathrm{CHCl}_{3}\right)$. ${ }^{1} \mathrm{H}$ NMR (400 MHz, $\mathrm{CDCl}_{3}$ ) $\delta: 7.02(\mathrm{~d}, J=8.4 \mathrm{~Hz}, 2 \mathrm{H}), 6.64(\mathrm{~d}, J=8.4 \mathrm{~Hz}$, $2 \mathrm{H}), 3.10$ (br, 3H), 3.84-3.78 (m, 2H), 3.75 (dd, $J=10.3,4.2 \mathrm{~Hz}, 1 \mathrm{H}), 3.66$ $(\mathrm{d}, J=8.2 \mathrm{~Hz}, 2 \mathrm{H}), 2.27(\mathrm{~s}, 3 \mathrm{H}), 1.99-1.73(\mathrm{~m}, 2 \mathrm{H}) .{ }^{13} \mathrm{C}$ NMR $(101 \mathrm{MHz}$, $\left.\mathrm{CDCl}_{3}\right) \delta: 144.8,130.0,127.5,114.4,64.3,59.8,53.7,34.5,20.4$. HRMS

(ESI) Calcd for $\mathrm{C}_{11} \mathrm{H}_{18} \mathrm{NO}_{2}\left([\mathrm{M}+\mathrm{H}]^{+}\right)$: 196.1332; Found: 196.1334. HPLC conditions: Chiralcel IC-3 column $(25 \mathrm{~cm} \times 0.46 \mathrm{~cm}$ ID); $n$-hexane/2-propanol $=75: 25$; temp, rt; flow rate $=1.0 \mathrm{~mL} / \mathrm{min} ; 210 \mathrm{~nm}$ $\mathrm{UV}$ detector; $t_{\mathrm{R}}(R)=6.1 \min \left(\right.$ minor); $t_{\mathrm{R}}(S)=9.0 \min$ (major).

\section{(S)-2-((4-Methoxyphenyl)amino)butane-1,4-diol (7c)}<smiles>COc1ccc(N[C@@H](CO)CCO)cc1</smiles>

White solid, $6 \mathrm{~h}$, mp $108-109{ }^{\circ} \mathrm{C}, 194 \mathrm{mg}, 92 \%$ yield, $92 \%$ ee. $[\alpha]_{\mathrm{D}}^{25}-37.0$ (c 1.0, $\mathrm{MeOH}) .{ }^{1} \mathrm{H}$ NMR $\left(400 \mathrm{MHz}, \mathrm{CDCl}_{3}\right) \delta: 6.83-6.78(\mathrm{~m}, 2 \mathrm{H}), 6.73-$ $6.67(\mathrm{~m}, 2 \mathrm{H}), 3.82-3.76(\mathrm{~m}, 2 \mathrm{H}), 3.74(\mathrm{~s}, 3 \mathrm{H}), 3.72-3.69(\mathrm{~m}, 1 \mathrm{H}), 3.67-$ $3.57(\mathrm{~m}, 2 \mathrm{H}), 3.52(\mathrm{br}, 3 \mathrm{H}) 1.90-1.78(\mathrm{~m}, 2 \mathrm{H}) .{ }^{13} \mathrm{C} \mathrm{NMR}(101 \mathrm{MHz}$, $\left.\mathrm{CDCl}_{3}\right) \delta: 152.8,141.0,116.0,115.0,64.2,60.2,55.8,55.0,34.3$. HRMS (ESI) Calcd for $\mathrm{C}_{11} \mathrm{H}_{18} \mathrm{NO}_{3}\left([\mathrm{M}+\mathrm{H}]^{+}\right)$: 212.1281; Found: 212.1281. HPLC conditions: Chiralcel OD-3 column $(25 \mathrm{~cm} \times 0.46 \mathrm{~cm} \mathrm{ID}) ; n$-hexane/2-propanol $=93: 7$; temp, rt; flow rate $=1.0 \mathrm{~mL} / \mathrm{min} ; 254 \mathrm{~nm}$ $\mathrm{UV}$ detector; $t_{\mathrm{R}}(R)=27.8 \mathrm{~min}$ (minor); $t_{\mathrm{R}}(S)=31.8 \mathrm{~min}$ (major).

\section{(S)-2-((4-Fluorophenyl)amino)butane-1,4-diol (7d)}<smiles>OCC[C@H](CO)Nc1ccc(F)cc1</smiles>

White solid, $17 \mathrm{~h}, \mathrm{mp} 85-86{ }^{\circ} \mathrm{C}, 189 \mathrm{mg}, 95 \%$ yield, $89 \%$ ee. $[\alpha]_{\mathrm{D}}^{25}-43.2(\mathrm{c}$ $1.0, \mathrm{MeOH}) .{ }^{1} \mathrm{H}$ NMR $\left(400 \mathrm{MHz}, \mathrm{DMSO}-d_{6}\right) \delta: 6.87(\mathrm{t}, J=8.8 \mathrm{~Hz}, 2 \mathrm{H}), 6.61-$ $6.52(\mathrm{~m}, 2 \mathrm{H}), 5.17(\mathrm{~d}, J=8.0 \mathrm{~Hz}, 1 \mathrm{H}), 4.64(\mathrm{~s}, 1 \mathrm{H}), 4.46(\mathrm{~s}, 1 \mathrm{H}), 3.54-3.45$ $(\mathrm{m}, 2 \mathrm{H}), 3.44-3.39(\mathrm{~m}, 1 \mathrm{H}), 3.32-3.26(\mathrm{~m}, 1 \mathrm{H}), 1.82-1.69(\mathrm{~m}, 1 \mathrm{H}), 1.58-1.45$ $(\mathrm{m}, 1 \mathrm{H}) .{ }^{13} \mathrm{C}$ NMR $\left(101 \mathrm{MHz}, \mathrm{DMSO}-d_{6}\right) \delta: 155.5,153.3,145.8,115.7,115.5$, 113.5, 113.4, 63.4, 58.5, 52.3, 35.3. HRMS (ESI) Calcd for $\mathrm{C}_{10} \mathrm{H}_{15} \mathrm{FNO}_{2}\left([\mathrm{M}+\mathrm{H}]^{+}\right)$: 200.1081; Found: 200.1085. HPLC conditions: Chiralcel IC-3 column $(25 \mathrm{~cm} \times 0.46 \mathrm{~cm} \mathrm{ID})$; -hexane/2-propanol = 90:10; temp, $\mathrm{rt}$; flow rate $=1.0 \mathrm{~mL} / \mathrm{min} ; 220 \mathrm{~nm} \mathrm{UV} \mathrm{detector;} t_{\mathrm{R}}(R)=10.8 \mathrm{~min}($ minor $) ; t_{\mathrm{R}}(S)=13.2 \mathrm{~min}$ (major).

\section{(S)-2-((4-Chlorophenyl)amino)butane-1,4-diol (7e)}<smiles>OCC[C@H](CO)Nc1ccc(Cl)cc1</smiles>
Colorless oil, $6 \mathrm{~h}, 194 \mathrm{mg}, 90 \%$ yield, $90 \%$ ee. $[\alpha]_{\mathrm{D}}^{25}-15.2\left(c 1.0, \mathrm{CHCl}_{3}\right)$. ${ }^{1} \mathrm{H} \mathrm{NMR}\left(400 \mathrm{MHz}, \mathrm{CDCl}_{3}\right) \delta: 7.15-7.06(\mathrm{~m}, 2 \mathrm{H}), 6.63-6.53(\mathrm{~m}, 2 \mathrm{H}), 3.79-$ $3.58(\mathrm{~m}, 5 \mathrm{H}), 3.16(\mathrm{br}, 2 \mathrm{H}), 1.95-1.84(\mathrm{~m}, 1 \mathrm{H}), 1.83-1.74(\mathrm{~m}, 1 \mathrm{H}) .{ }^{13} \mathrm{C} \mathrm{NMR}$ $\left(101 \mathrm{MHz}, \mathrm{CDCl}_{3}\right) \delta: 145.8,129.2,122.4,114.9,64.3,59.6,53.1,34.3$. HRMS (ESI) Calcd for $\mathrm{C}_{10} \mathrm{H}_{15} \mathrm{ClNO}_{2}\left([\mathrm{M}+\mathrm{H}]^{+}\right)$: 216.0786 ; Found: 216.0787. HPLC conditions: Chiralcel IF-3 column $(25 \mathrm{~cm} \times 0.46 \mathrm{~cm}$ ID); $n$-hexane/2-propanol = 95:5; temp, rt; flow rate $=1.0 \mathrm{~mL} / \mathrm{min} ; 254 \mathrm{~nm} \mathrm{UV} \mathrm{detector;} t_{\mathrm{R}}(S)=28.0 \mathrm{~min}$ (major); $t_{\mathrm{R}}(R)=33.0 \mathrm{~min}$ (minor). 


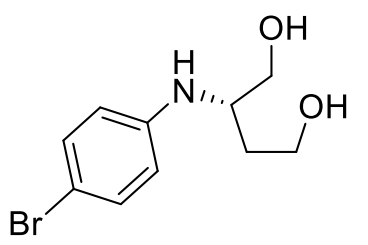

Yellow solid, $24 \mathrm{~h}, \mathrm{mp} 76-77^{\circ} \mathrm{C}, 233 \mathrm{mg}, 90 \%$ yield, $90 \%$ ee. $[\alpha]_{\mathrm{D}}^{25}-41.2(\mathrm{c}$ 1.0, MeOH). ${ }^{1} \mathrm{H}$ NMR (400 MHz, DMSO- $\left.d_{6}\right) \delta: 7.20-7.12(\mathrm{~m}, 2 \mathrm{H}), 6.59-$ $6.51(\mathrm{~m}, 2 \mathrm{H}), 5.51(\mathrm{~d}, J=8.0 \mathrm{~Hz}, 1 \mathrm{H}), 4.66(\mathrm{t}, J=5.6 \mathrm{~Hz}, 1 \mathrm{H}), 4.46(\mathrm{t}, J=$ $4.8 \mathrm{~Hz}, 1 \mathrm{H}), 3.56-3.37(\mathrm{~m}, 4 \mathrm{H}), 3.33-3.26(\mathrm{~m}, 1 \mathrm{H}), 1.84-1.66(\mathrm{~m}, 1 \mathrm{H})$, $1.58-1.43(\mathrm{~m}, 1 \mathrm{H}) .{ }^{13} \mathrm{C}$ NMR (100 MHz, DMSO-d $\left.d_{6}\right) \delta: 148.4,131.7,114.5$, 105.9, 63.7, 58.4, 51.6, 35.2. HRMS (ESI) Calcd for $\mathrm{C}_{10} \mathrm{H}_{15} \mathrm{BrNO}_{2}\left([\mathrm{M}+\mathrm{H}]^{+}\right)$: 260.0281; Found: 260.0278. HPLC conditions: Chiralcel IC-3 column $(25 \mathrm{~cm} \times 0.46 \mathrm{~cm} \mathrm{ID}) ; n$-hexane $/ 2$-propanol = 90:10; temp, $\mathrm{rt}$; flow rate $=1.0 \mathrm{~mL} / \mathrm{min} ; 254 \mathrm{~nm} \mathrm{UV}$ detector; $t_{\mathrm{R}}(R)=10.8 \mathrm{~min}\left(\right.$ minor); $t_{\mathrm{R}}(S)=13.9 \mathrm{~min}$ (major).

\section{(S)-2-(m-Tolylamino)butane-1,4-diol (7g)}<smiles>Cc1cccc(N[C@@H](CO)CCO)c1</smiles>

Colorless oil, 6h, $176 \mathrm{mg}, 90 \%$ yield, $90 \%$ ee. $[\alpha]_{\mathrm{D}}^{25}-22.2\left(c 1.0, \mathrm{CHCl}_{3}\right)$. ${ }^{1} \mathrm{H}$ NMR $\left(400 \mathrm{MHz}, \mathrm{CDCl}_{3}\right) \delta: 7.05(\mathrm{t}, J=7.6 \mathrm{~Hz}, 1 \mathrm{H}), 6.55(\mathrm{~d}, J=7.2$ $\mathrm{Hz}, 1 \mathrm{H}), 6.46$ (d, J = 8.0 Hz, 2H), 3.78-3.60 (m, 5H), 3.40 (br, 2H), 2.26 $(\mathrm{s}, 3 \mathrm{H}), 1.91-1.83(\mathrm{~m}, 1 \mathrm{H}), 1.82-1.71(\mathrm{~m}, 1 \mathrm{H}) .{ }^{13} \mathrm{C}$ NMR $(101 \mathrm{MHz}$, $\left.\mathrm{CDCl}_{3}\right) \delta: 147.2,139.3,129.3,119.0,114.8,111.0,64.4,59.8,53.2,34.5$, 21.6. HRMS (ESI) Calcd for $\mathrm{C}_{11} \mathrm{H}_{18} \mathrm{NO}_{2}\left([\mathrm{M}+\mathrm{H}]^{+}\right)$: 196.1332; Found: 196.1336. HPLC conditions: Chiralcel IC-3 column $(25 \mathrm{~cm} \times 0.46 \mathrm{~cm} \mathrm{ID}) ; n$-hexane/2-propanol $=90: 10$; temp, rt; flow rate $=1.0$ $\mathrm{mL} / \mathrm{min} ; 254 \mathrm{~nm} \mathrm{UV} \mathrm{detector;} t_{\mathrm{R}}(R)=13.7 \mathrm{~min}\left(\right.$ minor); $t_{\mathrm{R}}(S)=19.0 \mathrm{~min}$ (major).

\section{(S)-2-((3-Methoxyphenyl)amino)butane-1,4-diol (7h)}<smiles>COc1cccc(N[C@@H](CO)CCO)c1</smiles>

Colorless oil, 6 h, $198 \mathrm{mg}, 94 \%$ yield, $90 \%$ ee. $[\alpha]_{\mathrm{D}}^{25}-16.4\left(c 1.0, \mathrm{CHCl}_{3}\right)$. ${ }^{1} \mathrm{H}$ NMR (400 MHz, $\left.\mathrm{CDCl}_{3}\right) \delta: 7.06(\mathrm{t}, J=8.1 \mathrm{~Hz}, 1 \mathrm{H}), 6.27(\mathrm{t}, J=7.5$ $\mathrm{Hz}, 2 \mathrm{H}), 6.21$ (s, 1H), 3.79-3.59 (m, 8H), 3.38 (br, 2H), 1.95-1.82 (m, 1H), $1.80-1.66(\mathrm{~m}, 1 \mathrm{H}) .{ }^{13} \mathrm{C} \mathrm{NMR}\left(101 \mathrm{MHz}, \mathrm{CDCl}_{3}\right) \delta: 160.8,148.7$, 130.2, 106.9, 102.8, 99.8, 64.3, 59.6, 55.2, 52.8, 34.5. HRMS (ESI)

Calcd for $\mathrm{C}_{11} \mathrm{H}_{18} \mathrm{NO}_{3}\left([\mathrm{M}+\mathrm{H}]^{+}\right): 212.1281$, Found: 212.1282. HPLC conditions: Chiralcel AD-H column $(25 \mathrm{~cm} \times 0.46 \mathrm{~cm} \mathrm{ID}) ; n$-hexane $/ 2$-propanol $=90: 10$; temp, rt; flow rate $=1.0 \mathrm{~mL} / \mathrm{min} ; 210 \mathrm{~nm} \mathrm{UV}$ detector; $t_{\mathrm{R}}(R)=15.2 \mathrm{~min}$ (minor); $t_{\mathrm{R}}(S)=16.6 \mathrm{~min}$ (major).

\section{(S)-2-((3-Fluorophenyl)amino)butane-1,4-diol (7i)}<smiles>OCC[C@H](CO)Nc1cccc(F)c1</smiles>

White solid, $17 \mathrm{~h}$, mp $96-97{ }^{\circ} \mathrm{C}, 189 \mathrm{mg}, 95 \%$ yield, $90 \%$ ee. $[\alpha]_{\mathrm{D}}^{25}-54.4(c$ $1.0, \mathrm{MeOH}) .{ }^{1} \mathrm{H}$ NMR (400 MHz, DMSO-d $) \delta: 7.02$ (q, $\left.J=8.0 \mathrm{~Hz}, 1 \mathrm{H}\right)$, $6.47-6.32(\mathrm{~m}, 2 \mathrm{H}), 6.22(\mathrm{td}, J=8.4,2.4 \mathrm{~Hz}, 1 \mathrm{H}), 5.62(\mathrm{~d}, J=7.6 \mathrm{~Hz}, 1 \mathrm{H})$, $4.66(\mathrm{t}, J=5.4 \mathrm{~Hz}, 1 \mathrm{H}), 4.47(\mathrm{td}, J=5.0,1.0 \mathrm{~Hz}, 1 \mathrm{H}), 3.57-3.37(\mathrm{~m}, 4 \mathrm{H})$, $3.34-3.23(\mathrm{~m}, 1 \mathrm{H}), 1.85-1.71(\mathrm{~m}, 1 \mathrm{H}), 1.58-1.44(\mathrm{~m}, 1 \mathrm{H}) .{ }^{13} \mathrm{C}$ NMR $(101$ MHz, DMSO) $\delta: 164.7,162.3,150.8,150.7,130.1,130.0,108.5,101.0,100.8,98.2,98.0,63.0,57.9$, 51.2, 34.7. HRMS (ESI) Calcd for $\mathrm{C}_{10} \mathrm{H}_{15} \mathrm{FNO}_{2}\left([\mathrm{M}+\mathrm{H}]^{+}\right)$: 200.1081; Found: 200.1083. HPLC conditions: Chiralcel IC-3 column $(25 \mathrm{~cm} \times 0.46 \mathrm{~cm}$ ID $) ; n$-hexane/2-propanol $=85: 15$; temp, rt; flow rate $=1.0$ $\mathrm{mL} / \mathrm{min} ; 254 \mathrm{~nm}$ UV detector; $t_{\mathrm{R}}(R)=6.3 \mathrm{~min}$ (minor); $t_{\mathrm{R}}(S)=9.8 \mathrm{~min}$ (major).

\section{(S)-2-((3-Chlorophenyl)amino)butane-1,4-diol (7j)}


<smiles>OCC[C@H](CO)Nc1cccc(Cl)c1</smiles>

Colorless oil, $6 \mathrm{~h}, 202 \mathrm{mg}, 94 \%$ yield, $91 \%$ ee. $[\alpha]_{\mathrm{D}}^{25}-13.5\left(c 1.0, \mathrm{CHCl}_{3}\right)$. ${ }^{1} \mathrm{H}$ NMR $\left(400 \mathrm{MHz}, \mathrm{CDCl}_{3}\right) \delta: 7.05(\mathrm{t}, J=8.0 \mathrm{~Hz}, 1 \mathrm{H}), 6.73-6.57(\mathrm{~m}, 2 \mathrm{H})$, $6.50(\mathrm{dd}, J=8.4,2.4 \mathrm{~Hz}, 1 \mathrm{H}), 4.11(\mathrm{br}, 1 \mathrm{H}), 3.84-3.57$ (m, 5H), 3.36 (br, 1H), 3.03 (br, 1H), 1.99-1.85 (m, 1H), 1.84-1.73 (m, 1H). ${ }^{13} \mathrm{C}$ NMR $(101$ $\left.\mathrm{MHz}, \mathrm{CDCl}_{3}\right) \delta: 148.5,135.1,130.4,117.5,113.1,111.9,64.2,59.3,52.5$,

34.3. HRMS (ESI) Calcd for $\mathrm{C}_{10} \mathrm{H}_{15} \mathrm{ClNO}_{2}\left([\mathrm{M}+\mathrm{H}]^{+}\right)$: 216.0786; Found: 216.0787. HPLC conditions: Chiralcel AD-3 column $(25 \mathrm{~cm} \times 0.46 \mathrm{~cm} \mathrm{ID}) ; n$-hexane $/ 2$-propanol $=90: 10$; temp, rt; flow rate $=1.0$ $\mathrm{mL} / \mathrm{min} ; 254 \mathrm{~nm}$ UV detector; $t_{\mathrm{R}}(R)=8.5 \mathrm{~min} ; t_{\mathrm{R}}(S)=9.0 \mathrm{~min}$ (major).

\section{(S)-2-(o-Tolylamino)butane-1,4-diol (7k)}<smiles>Cc1ccccc1N[C@@H](CO)CCO</smiles>

Colorless oil, $6 \mathrm{~h}, 181 \mathrm{mg}, 93 \%$ yield, $98 \%$ ee. $[\alpha]_{\mathrm{D}}^{25}-15.4\left(c 1.0, \mathrm{CHCl}_{3}\right) .{ }^{1} \mathrm{H}$ NMR (400 MHz, $\left.\mathrm{CDCl}_{3}\right) \delta: 7.16-7.03(\mathrm{~m}, 2 \mathrm{H}), 6.73-6.61(\mathrm{~m}, 2 \mathrm{H}), 3.83-3.78$ (m, 2H), 3.78-3.74 (m, 2H), 3.74-3.68 (m, 1H), $2.86(\mathrm{br}, 2 \mathrm{H}), 2.17(\mathrm{~s}, 3 \mathrm{H})$, 2.01-1.92 (m, 1H), 1.91-1.87 (m, 1H). ${ }^{13} \mathrm{C}$ NMR (101 MHz, $\left.\mathrm{CDCl}_{3}\right) \delta: 145.1$, $130.5,127.2,122.7,117.5,110.9,64.4,59.8,52.9,34.5,17.6$. HRMS (ESI) Calcd for $\mathrm{C}_{11} \mathrm{H}_{18} \mathrm{NO}_{2}\left([\mathrm{M}+\mathrm{H}]^{+}\right)$: 196.1332; Found: 196.1335. HPLC conditions: Chiralcel IC-3 column $(25 \mathrm{~cm} \times 0.46 \mathrm{~cm} \mathrm{ID}) ; n$-hexane $/ 2$-propanol $=80: 20$; temp, rt; flow rate $=1.0 \mathrm{~mL} / \mathrm{min} ; 220 \mathrm{~nm} \mathrm{UV}$ detector; $t_{\mathrm{R}}(R)=5.9 \mathrm{~min}\left(\right.$ minor); $t_{\mathrm{R}}(S)=8.6 \mathrm{~min}$ (major).

\section{(S)-2-((2-Methoxyphenyl)amino)butane-1,4-diol (7l)}<smiles>COc1ccccc1N[C@@H](CO)CCO</smiles>

White solid, $6 \mathrm{~h}$, mp $97-98{ }^{\circ} \mathrm{C}, 190 \mathrm{mg}, 90 \%$ yield, $97 \%$ ee. $[\alpha]_{\mathrm{D}}^{25}-44.8(c 1.0$, $\mathrm{MeOH}) .{ }^{1} \mathrm{H} \mathrm{NMR}\left(400 \mathrm{MHz}, \mathrm{CDCl}_{3}\right) \delta: 6.85(\mathrm{td}, J=7.6,1.6 \mathrm{~Hz}, 1 \mathrm{H}), 6.80$ $6.75(\mathrm{~m}, 1 \mathrm{H}), 6.73-6.66(\mathrm{~m}, 2 \mathrm{H}), 3.83(\mathrm{~s}, 3 \mathrm{H}), 3.78-3.60(\mathrm{~m}, 5 \mathrm{H}), 3.09(\mathrm{br}$, 2H), 1.96-1.74 (m, 2H) ${ }^{13} \mathrm{C}$ NMR (101 MHz, $\left.\mathrm{CDCl}_{3}\right) \delta: 147.3,136.9,121.3$, 117.2, 111.2, 109.9, 64.6, 59.8, 55.5, 52.9, 34.8. HRMS (ESI) Calcd for $\mathrm{C}_{11} \mathrm{H}_{18} \mathrm{NO}_{3}\left([\mathrm{M}+\mathrm{H}]^{+}\right)$: 212.1281; Found: 212.1281. HPLC conditions: Chiralcel IG-3 column $(25 \mathrm{~cm} \times$ $0.46 \mathrm{~cm} \mathrm{ID}) ; n$-hexane $/ 2$-propanol $=95: 5 ;$ temp, rt; flow rate $=1.0 \mathrm{~mL} / \mathrm{min} ; 210 \mathrm{~nm} \mathrm{UV}$ detector; $t_{\mathrm{R}}(R)$ $=39.5 \mathrm{~min}\left(\right.$ minor); $t_{\mathrm{R}}(S)=42.5 \mathrm{~min}$ (major).

\section{(S)-2-(2,4-Dimethoxyphenylamino)butane-1,4-diol (7m)}

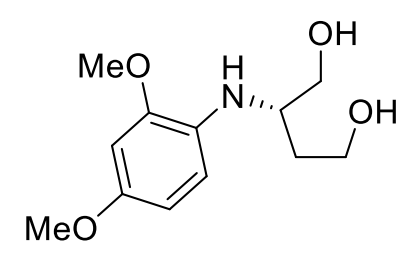

Yellow solid, $6 \mathrm{~h}, \mathrm{mp} 53-54{ }^{\circ} \mathrm{C}, 227 \mathrm{mg}, 94 \%$ yield, $97 \%$ ee. $[\alpha]_{\mathrm{D}}^{25}-18.0$ (c 1.0, $\left.\mathrm{CHCl}_{3}\right) .{ }^{1} \mathrm{H}$ NMR $\left(400 \mathrm{MHz}, \mathrm{CDCl}_{3}\right) \delta: 6.65(\mathrm{~d}, J=8.4 \mathrm{~Hz}, 1 \mathrm{H})$, $6.45(\mathrm{~d}, J=2.0 \mathrm{~Hz}, 1 \mathrm{H}), 6.39(\mathrm{dd}, J=8.4,2.0 \mathrm{~Hz}, 1 \mathrm{H}), 3.81(\mathrm{~s}, 3 \mathrm{H}), 3.79$ $3.72(\mathrm{~m}, 5 \mathrm{H}), 3.69-3.63(\mathrm{~m}, 1 \mathrm{H}), 3.60(\mathrm{~d}, J=4.0 \mathrm{~Hz}, 1 \mathrm{H}), 3.58-3.50(\mathrm{~m}$, 1H), 3.41 (br, 2H), 1.90-1.77 (m, 2H). $\left.{ }^{13} \mathrm{C} \mathrm{NMR} \mathrm{(101} \mathrm{MHz,} \mathrm{CDCl}_{3}\right) \delta$ : 152.6, 148.8, 130.8, 112.9, 104.1, 99.3, 64.3, 60.0, 55.8, 55.58, 54.4, 34.6. HRMS (ESI) Calcd for $\mathrm{C}_{12} \mathrm{H}_{20} \mathrm{NO}_{4}\left([\mathrm{M}+\mathrm{H}]^{+}\right): 242.1387$; Found: 242.1388. HPLC conditions: Chiralcel AD-3 column $(25 \mathrm{~cm} \times$ $0.46 \mathrm{~cm} \mathrm{ID}) ; n$-hexane $/ 2$-propanol $=93: 7$; temp, $\mathrm{rt}$; flow rate $=1.0 \mathrm{~mL} / \mathrm{min} ; 210 \mathrm{~nm} \mathrm{UV}$ detector; $t_{\mathrm{R}}(S)$ $=24.4 \min ($ major $) ; t_{\mathrm{R}}(R)=27.0 \mathrm{~min}$ (minor).

(S)-2-(Naphthalen-1-ylamino)butane-1,4-diol (7n) 


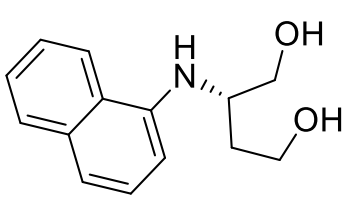

White solid, $20 \mathrm{~h}, \mathrm{mp} 144-145^{\circ} \mathrm{C}, 217 \mathrm{mg}, 94 \%$ yield, $97 \%$ ee. $[\alpha]_{\mathrm{D}}^{25}-70.8$ (c $1.0, \mathrm{MeOH}) .{ }^{1} \mathrm{H}$ NMR $\left(400 \mathrm{MHz}, \mathrm{DMSO}-d_{6}\right) \delta: 8.14(\mathrm{~d}, J=8.0 \mathrm{~Hz}, 1 \mathrm{H})$, $7.74(\mathrm{~d}, J=7.2 \mathrm{~Hz}, 1 \mathrm{H}), 7.40$ (p, $J=6.8 \mathrm{~Hz}, 2 \mathrm{H}), 7.27$ (t, $J=7.6 \mathrm{~Hz}, 1 \mathrm{H})$, $7.07(\mathrm{~d}, J=8.0 \mathrm{~Hz}, 1 \mathrm{H}), 6.62(\mathrm{~d}, J=7.6 \mathrm{~Hz}, 1 \mathrm{H}), 5.69(\mathrm{~d}, J=7.2 \mathrm{~Hz}, 1 \mathrm{H})$, $4.77(\mathrm{t}, J=6.0 \mathrm{~Hz}, 1 \mathrm{H}), 4.58(\mathrm{t}, J=4.8 \mathrm{~Hz}, 1 \mathrm{H}), 3.77-3.53(\mathrm{~m}, 4 \mathrm{H}), 3.52-3.43(\mathrm{~m}, 1 \mathrm{H}), 1.97-1.75(\mathrm{~m}$, $2 \mathrm{H}) .{ }^{13} \mathrm{C}$ NMR $\left(101 \mathrm{MHz}, \mathrm{DMSO}-d_{6}\right) \delta: 143.6,134.1,127.9,126.8,125.5,123.8,122.9,121.4,115.0$, 103.2, 62.6, 58.2, 51.8, 34.2. HRMS (ESI) Calcd for $\mathrm{C}_{14} \mathrm{H}_{18} \mathrm{NO}_{2}\left([\mathrm{M}+\mathrm{H}]^{+}\right)$: 232.1332; Found: 232.1336. HPLC conditions: Chiralcel IG-3 column $(25 \mathrm{~cm} \times 0.46 \mathrm{~cm} \mathrm{ID})$; $n$-hexane/2-propanol = 80:20; temp, rt; flow rate $=1.0 \mathrm{~mL} / \mathrm{min} ; 210 \mathrm{~nm} \mathrm{UV} \mathrm{detector;} t_{\mathrm{R}}(R)=6.5 \mathrm{~min}$ (minor); $t_{\mathrm{R}}(S)=8.2 \mathrm{~min}$ (major).

\section{(S)-2-(Benzo[d][1,3]dioxol-5-ylamino)butane-1,4-diol (7o)}<smiles>OCC[C@H](CO)Nc1ccc2c(c1)OCO2</smiles>
Brown solid, $17 \mathrm{~h}, \mathrm{mp} 117-118^{\circ} \mathrm{C}, 207 \mathrm{mg}, 92 \%$ yield, $88 \%$ ee. $[\alpha]_{\mathrm{D}}^{25}-35.6$ (c 1.0, MeOH). ${ }^{1} \mathrm{H}$ NMR (400 MHz, DMSO- $\left.d_{6}\right) \delta: 6.68(\mathrm{~d}, J=8.4 \mathrm{~Hz}, 1 \mathrm{H})$, $6.30(\mathrm{~d}, J=2.4 \mathrm{~Hz}, 1 \mathrm{H}), 6.02(\mathrm{dd}, J=8.4,2.4 \mathrm{~Hz} 1 \mathrm{H}), 5.86-5.77(\mathrm{~m}, 2 \mathrm{H})$, $4.94(J=7.6 \mathrm{~Hz}, 1 \mathrm{H}), 4.60(\mathrm{t}, J=5.2 \mathrm{~Hz} 1 \mathrm{H}), 4.15(\mathrm{t}, J=4.8 \mathrm{~Hz}, 1 \mathrm{H}), 3.55$ $3.38(\mathrm{~m}, 3 \mathrm{H}), 3.34-3.24(\mathrm{~m}, 2 \mathrm{H}), 1.80-1.68(\mathrm{~m}, 1 \mathrm{H}), 1.57-1.46(\mathrm{~m}, 1 \mathrm{H})$. ${ }^{13} \mathrm{C}$ NMR (101 MHz, DMSO-d $\left.d_{6}\right) \delta: 148.2,145.0,138.0,108.9,104.1,100.3,95.7,63.5,58.6,52.6,35.3$. HRMS (ESI) Calcd for $\mathrm{C}_{11} \mathrm{H}_{16} \mathrm{NO}_{4}\left([\mathrm{M}+\mathrm{H}]^{+}\right)$: 226.1074; Found: 226.1074. HPLC conditions: Chiralcel IH-3 column $(25 \mathrm{~cm} \times 0.46 \mathrm{~cm}$ ID); $n$-hexane/2-propanol $=70: 30$; temp, rt; flow rate $=1.0 \mathrm{~mL} / \mathrm{min} ; 210$ $\mathrm{nm}$ UV detector; $t_{\mathrm{R}}(R)=12.7 \mathrm{~min}$ (minor); $t_{\mathrm{R}}(S)=17.6 \mathrm{~min}$ (major).

\section{(S)-2-(6-Methylpyridin-2-ylamino)butane-1,4-diol (7p)}<smiles>Cc1cccc(N[C@H](CO)CCO)n1</smiles>

White solid, $24 \mathrm{~h}, \mathrm{mp} 90-92{ }^{\circ} \mathrm{C}, 168 \mathrm{mg}, 86 \%$ yield, $93 \%$ ee. $[\alpha]_{\mathrm{D}}^{27}-45.6$ (c 0.25, MeOH). ${ }^{1} \mathrm{H}$ NMR (400 MHz, $\left.\mathrm{CDCl}_{3}\right) \delta 7.36-7.27(\mathrm{~m}, 1 \mathrm{H}), 6.42(\mathrm{~d}$, $J=7.2 \mathrm{~Hz}, 1 \mathrm{H}), 6.27(\mathrm{~d}, J=8.0 \mathrm{~Hz}, 1 \mathrm{H}), 4.73(\mathrm{~s}, 1 \mathrm{H}), 4.21(\mathrm{~s}, 1 \mathrm{H}), 3.90$ $3.80(\mathrm{~m}, 1 \mathrm{H}), 3.77-3.59(\mathrm{~m}, 3 \mathrm{H}), 2.35(\mathrm{~d}, J=2.4 \mathrm{~Hz}, 3 \mathrm{H}), 1.91-1.79(\mathrm{~m}$, 1H), 1.67-1.56 (m, 1H). ${ }^{13} \mathrm{C}$ NMR (101 MHz, $\left.\mathrm{CDCl}_{3}\right) \delta 158.3,155.9,138.1,112.0,106.2,66.5,58.5$, 50.6, 35.4, 23.7. HRMS (ESI) Calcd for $\mathrm{C}_{10} \mathrm{H}_{17} \mathrm{~N}_{2} \mathrm{O}_{2}\left([\mathrm{M}+\mathrm{H}]^{+}\right)$: 197.1285; Found: 197.1289. HPLC conditions: Chiralcel LuX ${ }^{\mathrm{TM}} 5 \mu \mathrm{m}$ Amylose-1(4.6×250 mm, P/N: 00G-4732-E0); $n$ hexane/ethanol/DEA $=93: 7: 0.1$; temp, $\mathrm{rt}$; flow rate $=1.0 \mathrm{~mL} / \mathrm{min} ; 254 \mathrm{~nm} \mathrm{UV}$ detector; $t_{\mathrm{R}}(S)=18.4$ $\min \left(\right.$ major); $t_{\mathrm{R}}(R)=21.1 \min ($ minor).

\section{((S)-1-(Hydroxymethyl)-3-hydroxypropyl) carbamic acid tert-butyl ester (7q) ${ }^{4}$}<smiles>O=C(O)OC(=O)N[C@@H](CO)CCO</smiles>

White solid, 16 h, $199 \mathrm{mg}, 97 \%$ yield, $88 \%$ ee, determined by the corresponding benzoyl ester on HPLC. $[\alpha]_{\mathrm{D}}^{25}-26.8(c 1.0, \mathrm{MeOH}) .\left(\right.$ lit. $\left.^{4}[\alpha]_{\mathrm{D}}^{15}-32.6(c 1.0, \mathrm{MeOH})\right)$ ${ }^{1} \mathrm{H}$ NMR $\left(400 \mathrm{MHz}, \mathrm{CDCl}_{3}\right) \delta 5.06(\mathrm{~d}, J=8.0 \mathrm{~Hz}, 1 \mathrm{H}), 3.94-3.82(\mathrm{~m}, 1 \mathrm{H}), 3.80$ $3.60(\mathrm{~m}, 4 \mathrm{H}), 3.33$ (br, 1H), 2.63 (br, 1H), 1.87-1.74 (m, 1H), 1.67-1.57 (m, 1H), $1.45(\mathrm{~s}, 9 \mathrm{H})$. HPLC conditions: Chiralcel IG-3 column $(25 \mathrm{~cm} \times 0.46 \mathrm{~cm}$ ID $) ; n$-hexane/2-propanol = 70:30; temp, $\mathrm{rt}$; flow rate $=1.0 \mathrm{~mL} / \mathrm{min} ; 210 \mathrm{~nm} \mathrm{UV}$ detector; $t_{\mathrm{R}}(R)=12.2 \mathrm{~min}($ minor $) ; t_{\mathrm{R}}(S)=18.2$ $\min$ (major).

\section{$N$-(3-Hydroxy-1-hydroxymethyl-propyl)-4-methyl-benzenesulfonamide (7r) ${ }^{5}$}


TsHN ".

White solid, $14 \mathrm{~h}, \mathrm{mp} 72-74{ }^{\circ} \mathrm{C}, 166 \mathrm{mg}, 64 \%$ yield, $92 \%$ ee. $[\alpha]_{\mathrm{D}}^{27}-19.2(c 0.25$, MeOH). ${ }^{1} \mathrm{H}$ NMR $\left(400 \mathrm{MHz}, \mathrm{CDCl}_{3}\right) \delta 7.78(\mathrm{~d}, J=8.4 \mathrm{~Hz}, 2 \mathrm{H}), 7.32(\mathrm{~d}, J=8.0 \mathrm{~Hz}$, $2 \mathrm{H}), 5.52(\mathrm{~d}, J=7.2 \mathrm{~Hz}, 1 \mathrm{H}), 3.81-3.73(\mathrm{~m}, 1 \mathrm{H}), 3.70-3.62(\mathrm{~m}, 1 \mathrm{H}), 3.55-3.40(\mathrm{~m}$, 3H), $2.77(\mathrm{~s}, 1 \mathrm{H}), 2.49(\mathrm{~s}, 1 \mathrm{H}), 2.43(\mathrm{~s}, 3 \mathrm{H}), 1.80-1.68(\mathrm{~m}, 2 \mathrm{H}) .{ }^{13} \mathrm{C}$ NMR $\left(101 \mathrm{MHz}, \mathrm{CDCl}_{3}\right) \delta 143.7$, 137.4, 129.8, 127.1, 64.6, 58.8, 53.0, 34.4, 21.6. HPLC conditions: Chiralcel IC-3 column $(25 \mathrm{~cm} \times 0.46$ $\mathrm{cm} \mathrm{ID);} n$-hexane $/ 2$-propanol $=70: 30$; temp, rt; flow rate $=1.0 \mathrm{~mL} / \mathrm{min} ; 210 \mathrm{~nm}$ UV detector; $t_{\mathrm{R}}(S)=$ $18.1 \min$ (major); $t_{\mathrm{R}}(R)=22.2 \min$ (minor).

\section{(S)-2-(2-Phenylamino)pentane-1,5-diol (9a)}

Colorless oil, $16 \mathrm{~h}, 179 \mathrm{mg}, 92 \%$ yield, $92 \%$ ee. $[\alpha]_{\mathrm{D}}^{25}-28.0(c 1.0, \mathrm{MeOH}) .{ }^{1} \mathrm{H}$<smiles>OCCC[C@H](CO)Nc1ccccc1</smiles>
$\operatorname{NMR}\left(400 \mathrm{MHz}, \mathrm{CDCl}_{3}\right) \delta: 7.17(\mathrm{t}, J=8.0 \mathrm{~Hz}, 2 \mathrm{H}), 6.72(\mathrm{t}, J=7.2 \mathrm{~Hz}, 1 \mathrm{H})$, $6.66(\mathrm{~d}, J=8.0 \mathrm{~Hz}, 2 \mathrm{H}), 3.74(\mathrm{dd}, J=10.8,4.4 \mathrm{~Hz}, 1 \mathrm{H}), 3.65(\mathrm{t}, J=5.6 \mathrm{~Hz}$, $2 \mathrm{H}), 3.60-3.49(\mathrm{~m}, 2 \mathrm{H}), 2.04(\mathrm{br}, 1 \mathrm{H}), 1.74-1.59(\mathrm{~m}, 4 \mathrm{H}) .{ }^{13} \mathrm{C} \mathrm{NMR}(101 \mathrm{MHz}$, $\mathrm{CDCl}_{3}$ ) $\delta: 147.6,129.4,117.8,113.7,64.0,62.5,54.8,29.1,28.4$. HRMS (ESI)

Calcd for $\mathrm{C}_{11} \mathrm{H}_{18} \mathrm{NO}_{2}\left([\mathrm{M}+\mathrm{H}]^{+}\right)$: 196.1332; Found: 196.1335. HPLC conditions: Chiralcel IC-3 column $(25 \mathrm{~cm} \times 0.46 \mathrm{~cm} \mathrm{ID}) ; n$-hexane $/ 2$-propanol $=70: 30$; temp, rt; flow rate $=1.0 \mathrm{~mL} / \mathrm{min} ; 254 \mathrm{~nm} \mathrm{UV}$ detector; $t_{\mathrm{R}}(R)=5.2 \mathrm{~min}\left(\right.$ minor); $t_{\mathrm{R}}(S)=6.1 \mathrm{~min}$ (major).

\section{(S)-2-(p-Tolylamino)pentane-1,5-diol (9b)}<smiles>Cc1ccc(N[C@@H](CO)CCCO)cc1</smiles>

Colorless oil, $16 \mathrm{~h}, 192 \mathrm{mg}, 92 \%$ yield, $92 \%$ ee. $[\alpha]_{\mathrm{D}}^{25}-17.6(c 1.0, \mathrm{MeOH})$. ${ }^{1} \mathrm{H}$ NMR $\left(400 \mathrm{MHz}, \mathrm{CDCl}_{3}\right) \delta: 6.99(\mathrm{~d}, J=8.4 \mathrm{~Hz}, 2 \mathrm{H}), 6.60(\mathrm{~d}, J=8.4$ $\mathrm{Hz}, 2 \mathrm{H}), 3.79-3.71(\mathrm{~m}, 1 \mathrm{H}), 3.69-3.62(\mathrm{~m}, 2 \mathrm{H}), 3.54(\mathrm{dd}, J=10.4,5.2 \mathrm{~Hz}$, $1 \mathrm{H}), 3.50-3.43(\mathrm{~m}, 1 \mathrm{H}), 2.24(\mathrm{~s}, 3 \mathrm{H}), 1.73-1.55(\mathrm{~m}, 4 \mathrm{H}) .{ }^{13} \mathrm{C}$ NMR $(101$ $\left.\mathrm{MHz}, \mathrm{CDCl}_{3}\right) \delta 145.3,130.0,127.3,114.2,64.1,62.6,55.5,29.2,28.6,20.5$. HRMS (ESI) Calcd for $\mathrm{C}_{12} \mathrm{H}_{20} \mathrm{NO}_{2}\left([\mathrm{M}+\mathrm{H}]^{+}\right)$: 210.1489; Found: 210.1492. HPLC conditions: Chiralcel IG-3 column $(25 \mathrm{~cm} \times$ $0.46 \mathrm{~cm} \mathrm{ID}) ; n$-hexane $/ 2$-propanol = 90:10; temp, rt; flow rate $=1.0 \mathrm{~mL} / \mathrm{min} ; 254 \mathrm{~nm}$ UV detector; $t_{\mathrm{R}}(R)$ $=15.0 \mathrm{~min}(\mathrm{minor}) ; t_{\mathrm{R}}(S)=18.0 \mathrm{~min}$ (major).

\section{(S)-2-((4-Methoxyphenyl)amino)pentane-1,5-diol (9c)}<smiles>COc1ccc(N[C@@H](CO)CCCO)cc1</smiles>
Colorless oil, $16 \mathrm{~h}, 207 \mathrm{mg}, 92 \%$ yield, $91 \%$ ee. $[\alpha]_{\mathrm{D}}^{25}-1.4\left(\mathrm{c} 1.0, \mathrm{CHCl}_{3}\right)$ ${ }^{1} \mathrm{H}$ NMR $\left(400 \mathrm{MHz}, \mathrm{CDCl}_{3}\right) \delta: 6.79-6.73(\mathrm{~m}, 2 \mathrm{H}), 6.65-6.59(\mathrm{~m}, 2 \mathrm{H})$, $3.73(\mathrm{~s}, 3 \mathrm{H}), 3.69-3.62(\mathrm{~m}, 1 \mathrm{H}), 3.61-3.58(\mathrm{~m}, 2 \mathrm{H}), 3.51$ (dd, $J=10.8$, $4.8 \mathrm{~Hz}, 1 \mathrm{H}), 3.36-3.29(\mathrm{~m}, 1 \mathrm{H}), 3.12(\mathrm{br}, 3 \mathrm{H}), 1.70-1.53(\mathrm{~m}, 4 \mathrm{H}) .{ }^{13} \mathrm{C}$ NMR (101 MHz, $\left.\mathrm{CDCl}_{3}\right) \delta: 152.6,141.5,115.8,115.0,63.9,62.6,56.4,55.8,29.3,28.7$. HRMS (ESI) Calcd for $\mathrm{C}_{12} \mathrm{H}_{20} \mathrm{NO}_{3}\left([\mathrm{M}+\mathrm{H}]^{+}\right)$: 226.1438; Found: 226.1437. HPLC conditions: Chiralcel IC-3 column $(25 \mathrm{~cm} \times 0.46 \mathrm{~cm} \mathrm{ID}) ; n$-hexane $/ 2$-propanol $=75: 25$; temp, $\mathrm{rt}$; flow rate $=1.0 \mathrm{~mL} / \mathrm{min} ; 254 \mathrm{~nm} \mathrm{UV}$ detector; $t_{\mathrm{R}}(R)=9.8 \mathrm{~min}$ (minor); $t_{\mathrm{R}}(S)=11.3 \mathrm{~min}$ (major).

\section{(S)-2-((4-Fluorophenyl)amino)pentane-1,5-diol (9d)}<smiles>OCC(CO)Nc1ccc(F)cc1</smiles>

Colorless oil, 16 h, $200 \mathrm{mg}$, 94\% yield, $92 \%$ ee. $[\alpha]_{\mathrm{D}}^{25}-25.0(c 1.0, \mathrm{MeOH})$ ${ }^{1} \mathrm{H} \mathrm{NMR}\left(400 \mathrm{MHz}, \mathrm{CDCl}_{3}\right) \delta:$ 6.96-6.83 (m, 2H), 6.66-6.55 (m, 2H), 3.71 (dd, $J=10.8,4.0 \mathrm{~Hz}, 1 \mathrm{H}), 3.68-3.61(\mathrm{~m}, 2 \mathrm{H}), 3.60-3.52(\mathrm{~m}, 1 \mathrm{H}), 3.44-$ $3.35(\mathrm{~m}, 1 \mathrm{H}), 2.42(\mathrm{br}, 3 \mathrm{H}), 1.77-1.51(\mathrm{~m}, 4 \mathrm{H}) .{ }^{13} \mathrm{C} \mathrm{NMR}\left(101 \mathrm{MHz}, \mathrm{CDCl}_{3}\right)$ $\delta: 157.1,154.8,143.9,143.9,115.9,115.7,114.8,114.8,63.6,62.4,55.7,29.0,28.3$. HRMS (ESI) Calcd 
for $\mathrm{C}_{11} \mathrm{H}_{17} \mathrm{FNO}_{2}\left([\mathrm{M}+\mathrm{H}]^{+}\right)$: 214.1238; Found: 214.1239. HPLC conditions: Chiralcel IC-3 column (25 $\mathrm{cm} \times 0.46 \mathrm{~cm} \mathrm{ID)}$; $n$-hexane/2-propanol $=85: 15$; temp, rt; flow rate $=1.0 \mathrm{~mL} / \mathrm{min} ; 254 \mathrm{~nm} \mathrm{UV} \mathrm{detector;}$ $t_{\mathrm{R}}(R)=7.8 \min$ (minor); $t_{\mathrm{R}}(S)=8.6 \min$ (major).

\section{(S)-2-((4-Chlorophenyl)amino)pentane-1,5-diol (9e)}<smiles>OCCC[C@H](CO)Nc1ccc(Cl)cc1</smiles>

Colorless oil, $16 \mathrm{~h}, 213 \mathrm{mg}, 93 \%$ yield, $90 \%$ ee. $[\alpha]_{\mathrm{D}}^{25}-28.0(c 1.0, \mathrm{MeOH})$. ${ }^{1} \mathrm{H}$ NMR (400 MHz, $\left.\mathrm{CDCl}_{3}\right) \delta: 7.16-7.08(\mathrm{~m}, 2 \mathrm{H}), 6.62-6.56(\mathrm{~m}, 2 \mathrm{H}), 3.74$ $(\mathrm{dd}, J=10.8,4.0 \mathrm{~Hz}, 1 \mathrm{H}), 3.67(\mathrm{t}, J=5.6 \mathrm{~Hz}, 2 \mathrm{H}), 3.58(\mathrm{dd}, J=10.8,5.2$ $\mathrm{Hz}, 1 \mathrm{H}), 3.50-3.43$ (m, 1H), 2.18 (br, 3H), $1.74-1.58$ (m, 4H). ${ }^{13} \mathrm{C}$ NMR $\left(101 \mathrm{MHz}, \mathrm{CDCl}_{3}\right) \delta:$ 146.2, 129.2, 122.5, 114.8, 64.1, 62.6, 55.3, 29.0, 28.4. HRMS (ESI) Calcd for $\mathrm{C}_{11} \mathrm{H}_{17} \mathrm{ClNO}_{2}\left([\mathrm{M}+\mathrm{H}]^{+}\right): 230.0942$; Found: 230.0945. HPLC conditions: Chiralcel IC-3 column $(25 \mathrm{~cm}$ $\times 0.46 \mathrm{~cm} \mathrm{ID)}$; $n$-hexane/2-propanol = 90:10; temp, rt; flow rate $=1.0 \mathrm{~mL} / \mathrm{min} ; 254 \mathrm{~nm} \mathrm{UV} \mathrm{detector;} t_{\mathrm{R}}$ $(R)=12.3 \min ($ minor $) ; t_{\mathrm{R}}(S)=13.8 \min ($ major $)$.

\section{(S)-2-([1,1'-Biphenyl]-4-ylamino)pentane-1,5-diol (9f)}<smiles>OCCC[C@H](CO)Nc1ccc(-c2ccccc2)cc1</smiles>

Yellow solid, $16 \mathrm{~h}, \mathrm{mp} 72-73{ }^{\circ} \mathrm{C}, 244 \mathrm{mg}, 90 \%$ yield, $90 \%$ ee. $[\alpha]_{\mathrm{D}}^{25}-8.6$ (c 1.0, MeOH). ${ }^{1} \mathrm{H}$ NMR (400 MHz, $\left.\mathrm{CDCl}_{3}\right) \delta: 7.52(\mathrm{~d}, J=7.2 \mathrm{~Hz}, 2 \mathrm{H})$, 7.47-7.34 (m, 4H), 7.29-7.23 (m, 1H), 6.78-6.66 (m, 2H), 3.79-3.70 (m, 1H), 3.69-3.49 (m, 4H), 2.44 (br, 2H), 1.80-1.57 (m, 4H). ${ }^{13} \mathrm{C}$ NMR (101 $\left.\mathrm{MHz}, \mathrm{CDCl}_{3}\right) \delta: 147.0,141.0,130.6,128.7,128.1,126.2,126.2,113.9,64.0,62.5,54.8,29.1,28.4$. HRMS (ESI) Calcd for $\mathrm{C}_{17} \mathrm{H}_{22} \mathrm{NO}_{2}\left([\mathrm{M}+\mathrm{H}]^{+}\right)$: 272.1645; Found: 272.1649. HPLC conditions: Chiralcel IG-3 column $(25 \mathrm{~cm} \times 0.46 \mathrm{~cm} \mathrm{ID}) ; n$-hexane/2-propanol = 90:10; temp, rt; flow rate $=1.0 \mathrm{~mL} / \mathrm{min} ; 210$ $\mathrm{nm}$ UV detector; $t_{\mathrm{R}}(S)=24.6 \mathrm{~min}$ (major); $t_{\mathrm{R}}(R)=26.9 \mathrm{~min}$ (minor).

(S)-2-(m-Tolylamino)pentane-1,5-diol (9g)<smiles>Cc1cccc(N[C@@H](CO)CCCO)c1</smiles>

Colorless oil, $16 \mathrm{~h}, 199 \mathrm{mg}, 95 \%$ yield, $94 \%$ ee. $[\alpha]_{\mathrm{D}}^{25}-25.4(c 1.0, \mathrm{MeOH})$. ${ }^{1} \mathrm{H}$ NMR $\left(400 \mathrm{MHz}, \mathrm{CDCl}_{3}\right) \delta: 7.06(\mathrm{t}, J=7.6 \mathrm{~Hz}, 1 \mathrm{H}), 6.55(\mathrm{~d}, J=7.6 \mathrm{~Hz}$, $1 \mathrm{H}), 6.47(\mathrm{~d}, J=10.0 \mathrm{~Hz}, 2 \mathrm{H}), 3.77-3.68(\mathrm{~m}, 1 \mathrm{H}), 3.68-3.61(\mathrm{~m}, 2 \mathrm{H})$, $3.55(\mathrm{dd}, J=10.4,5.2 \mathrm{~Hz}, 1 \mathrm{H}), 3.52-3.44(\mathrm{~m}, 1 \mathrm{H}), 2.69(\mathrm{br}, 2 \mathrm{H}), 2.27$ (s, 3H), 1.77-1.51 (m, 4H). $\left.{ }^{13} \mathrm{C} \mathrm{NMR} \mathrm{(101} \mathrm{MHz,} \mathrm{CDCl}_{3}\right) \delta: 147.7,139.2,129.3,118.8,114.6,110.9$, 64.0, 62.5, 54.9, 29.1, 28.5, 21.6. HRMS (ESI) Calcd for $\mathrm{C}_{12} \mathrm{H}_{20} \mathrm{NO}_{2}\left([\mathrm{M}+\mathrm{H}]^{+}\right)$: 210.1489; Found: 210.1490. HPLC conditions: Chiralcel IC-3 column ( $25 \mathrm{~cm} \times 0.46 \mathrm{~cm} \mathrm{ID);} n$-hexane/2-propanol = 85:15; temp, rt; flow rate $=1.0 \mathrm{~mL} / \mathrm{min} ; 254 \mathrm{~nm} \mathrm{UV} \mathrm{detector;} t_{\mathrm{R}}(R)=9.1 \mathrm{~min}$ (minor); $t_{\mathrm{R}}(S)=10.8$ min (major).

\section{(S)-2-((3-Methoxyphenyl)amino)pentane-1,5-diol (9h)}

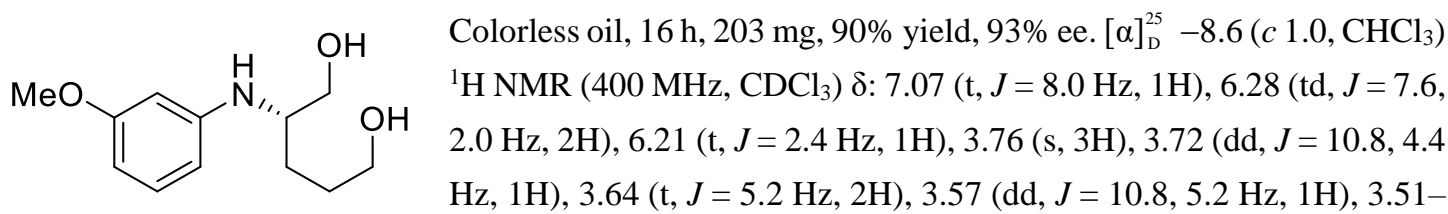
$3.42(\mathrm{~m}, 1 \mathrm{H}), 2.34$ (br, 1H), 1.90-1.55 (m, 4H). ${ }^{13} \mathrm{C}$ NMR (101 MHz, $\left.\mathrm{CDCl}_{3}\right) \delta: 160.8,149.1,130.2$, 106.7, 102.7, 99.7, 64.0, 62.5, 55.1, 54.8, 29.1, 28.4. HRMS (ESI) Calcd for $\mathrm{C}_{12} \mathrm{H}_{20} \mathrm{NO}_{3}\left([\mathrm{M}+\mathrm{H}]^{+}\right)$: 226.1438; Found: 226.1439. HPLC conditions: Chiralcel IC-3 column ( $25 \mathrm{~cm} \times 0.46 \mathrm{~cm}$ ID); $n$-hexane/2propanol $=75: 25$; temp, rt; flow rate $=1.0 \mathrm{~mL} / \mathrm{min} ; 220 \mathrm{~nm} \mathrm{UV} \mathrm{detector;} t_{\mathrm{R}}(R)=9.0 \min (\operatorname{minor}) ; t_{\mathrm{R}}(S)$ $=14.8 \mathrm{~min}$ (major). 


\section{(S)-2-((3-Chlorophenyl)amino)pentane-1,5-diol (9i)}<smiles>OCCC[C@H](CO)Nc1cccc(Cl)c1</smiles>

Colorless oil, $16 \mathrm{~h}, 213 \mathrm{mg}, 93 \%$ yield, $93 \%$ ee. $[\alpha]_{\mathrm{D}}^{25}-21.8(c 1.0, \mathrm{MeOH})$. ${ }^{1} \mathrm{H} \mathrm{NMR}\left(400 \mathrm{MHz}, \mathrm{CDCl}_{3}\right) \delta: 7.05(\mathrm{t}, J=8.0 \mathrm{~Hz}, 1 \mathrm{H}), 6.66(\mathrm{~d}, J=8.0$ $\mathrm{Hz}, 1 \mathrm{H}), 6.60(\mathrm{~s}, 1 \mathrm{H}), 6.49$ (d, $J=7.6 \mathrm{~Hz}, 1 \mathrm{H}), 4.22-3.55(\mathrm{~m}, 4 \mathrm{H}), 3.48-$ $3.39(\mathrm{~m}, 1 \mathrm{H}), 2.61(\mathrm{br}, 2 \mathrm{H}), 1.75-1.51(\mathrm{~m}, 4 \mathrm{H}) .{ }^{13} \mathrm{C}$ NMR $(101 \mathrm{MHz}$, $\left.\mathrm{CDCl}_{3}\right) \delta: 148.9,135.1,130.4,117.5,113.1,111.8,64.0,62.5,54.7,28.9,28.2$. HRMS (ESI) Calcd for $\mathrm{C}_{11} \mathrm{H}_{17} \mathrm{ClNO}_{2}\left([\mathrm{M}+\mathrm{H}]^{+}\right): 230.0942$; Found: 230.0946. HPLC conditions: Chiralcel IC-3 column $(25 \mathrm{~cm}$ $\times 0.46 \mathrm{~cm} \mathrm{ID}$ ); $n$-hexane $/ 2$-propanol $=90: 10$; temp, rt; flow rate $=1.0 \mathrm{~mL} / \mathrm{min} ; 254 \mathrm{~nm} \mathrm{UV}$ detector; $t_{\mathrm{R}}$ $(R)=12.1 \mathrm{~min}\left(\right.$ minor); $t_{\mathrm{R}}(S)=13.7 \mathrm{~min}$ (major).

\section{(S)-2-(o-Tolylamino)pentane-1,5-diol (9j)}<smiles>Cc1ccccc1N[C@@H](CO)CCCO</smiles>

Colorless oil, $16 \mathrm{~h}, 196 \mathrm{mg}, 94 \%$ yield, $96 \%$ ee. $[\alpha]_{\mathrm{D}}^{25}-3.4\left(c 1.0, \mathrm{CHCl}_{3}\right) .{ }^{1} \mathrm{H}$ NMR (400 MHz, $\left.\mathrm{CDCl}_{3}\right)$ 8: 7.16-7.04 (m, 2H), 6.73-6.64 (m, 2H), 3.83-3.74 (m, 1H), 3.71-3.65 (m, 2H), 3.64-3.39 (m, 3H), $2.16(\mathrm{~s}, 3 \mathrm{H}), 1.93(\mathrm{~s}, 1 \mathrm{H}), 1.80$ $1.62(\mathrm{~m}, 4 \mathrm{H}) .{ }^{13} \mathrm{C}$ NMR $\left(101 \mathrm{MHz}, \mathrm{CDCl}_{3}\right) \delta: 145.4,130.5,127.2,122.5,117.4$, 110.8, 64.0, 62.6, 54.6, 29.1, 28.5, 17.6. HRMS (ESI) Calcd for $\mathrm{C}_{12} \mathrm{H}_{20} \mathrm{NO}_{2}\left([\mathrm{M}+\mathrm{H}]^{+}\right)$: 210.1489; Found: 210.1492. HPLC conditions: Chiralcel IG-3 column ( $25 \mathrm{~cm} \times 0.46 \mathrm{~cm}$ ID); $n$-hexane/2-propanol = 90:10; temp, rt; flow rate $=1.0 \mathrm{~mL} / \mathrm{min} ; 210 \mathrm{~nm} \mathrm{UV}$ detector; $t_{\mathrm{R}}(R)=11.7 \mathrm{~min}($ minor $) ; t_{\mathrm{R}}(S)=13.4 \mathrm{~min}$ (major).

\section{(S)-2-((2-Ethylphenyl)amino)pentane-1,5-diol (9k)}<smiles>CCc1ccccc1N[C@@H](CO)CCCO</smiles>

White solid, $16 \mathrm{~h}, \mathrm{mp} 73-74{ }^{\circ} \mathrm{C}, 201 \mathrm{mg}, 90 \%$ yield, $97 \%$ ee. $[\alpha]_{\mathrm{D}}^{25}-32.6(c 1.0$, MeOH). ${ }^{1} \mathrm{H}$ NMR (400 MHz, $\left.\mathrm{CDCl}_{3}\right) \delta:$ 7.14-7.05 (m, 2H), 6.76-6.66 (m, 2H), $3.82-3.73(\mathrm{~m}, 1 \mathrm{H}), 3.70-3.55(\mathrm{~m}, 4 \mathrm{H}), 2.51(\mathrm{q}, J=7.6 \mathrm{~Hz}, 2 \mathrm{H}), 2.02(\mathrm{br}, 1 \mathrm{H})$, $1.79-1.61(\mathrm{~m}, 4 \mathrm{H}), 1.25(\mathrm{t}, J=7.6 \mathrm{~Hz}, 3 \mathrm{H}) .{ }^{13} \mathrm{C} \mathrm{NMR}\left(101 \mathrm{MHz}, \mathrm{CDCl}_{3}\right) \delta$ : 144.8, 128.3, 128.2, 127.0, 117.6, 111.1, 64.0, 62.6, 54.6, 29.1, 28.5, 23.9, 13.0. HRMS (ESI) Calcd for $\mathrm{C}_{13} \mathrm{H}_{22} \mathrm{NO}_{2}\left([\mathrm{M}+\mathrm{H}]^{+}\right): 224.1645$; Found: 224.1649. HPLC conditions: Chiralcel IC-3 column $(25 \mathrm{~cm} \times$ $0.46 \mathrm{~cm} \mathrm{ID}) ; n$-hexane $/ 2$-propanol = 90:10; temp, rt; flow rate $=1.0 \mathrm{~mL} / \mathrm{min} ; 210 \mathrm{~nm}$ UV detector; $t_{\mathrm{R}}(R)$ $=11.3 \min (\operatorname{minor}) ; t_{\mathrm{R}}(S)=17.0 \mathrm{~min}$ (major).

\section{(S)-2-((2-Isopropylphenyl)amino)pentane-1,5-diol (9l)}<smiles>CCCc1ccccc1N[C@@H](CO)CCCO</smiles>

White solid, $16 \mathrm{~h}$, mp $90-91{ }^{\circ} \mathrm{C}, 228 \mathrm{mg}, 96 \%$ yield, $97 \%$ ee. $[\alpha]_{\mathrm{D}}^{25}-27.6$ (c 1.0, MeOH). ${ }^{1} \mathrm{H}$ NMR $\left(400 \mathrm{MHz}, \mathrm{CDCl}_{3}\right) \delta: 7.16(\mathrm{~d}, J=6.8 \mathrm{~Hz}, 1 \mathrm{H}), 7.09$ (td, $J=$ $7.6,1.6 \mathrm{~Hz}, 1 \mathrm{H}), 6.75(\mathrm{t}, J=7.6 \mathrm{~Hz}, 1 \mathrm{H}), 6.70(\mathrm{~d}, J=8.0 \mathrm{~Hz}, 1 \mathrm{H}), 3.84-3.72$ (m, 1H), 3.70-3.55 (m, 4H), 2.93-2.84 (m, 1H), $2.06(\mathrm{~s}, 1 \mathrm{H}), 1.80-1.60(\mathrm{~m}, 4 \mathrm{H})$, $1.26(\mathrm{dd}, J=6.8,2.4 \mathrm{~Hz}, 6 \mathrm{H}) .{ }^{13} \mathrm{C}$ NMR $\left(101 \mathrm{MHz}, \mathrm{CDCl}_{3}\right) \delta: 144.0,132.8,126.8,125.3,117.7,111.5$, 63.9, 62.6, 54.7, 29.1, 28.5, 27.2, 22.6, 22.4. HRMS (ESI) Calcd for $\mathrm{C}_{14} \mathrm{H}_{24} \mathrm{NO}_{2}\left([\mathrm{M}+\mathrm{H}]^{+}\right): 238.1802$; Found: 238.1805. HPLC conditions:Chiralcel IC-3 column ( $25 \mathrm{~cm} \times 0.46 \mathrm{~cm}$ ID); $n$-hexane/2-propanol $=80: 20$; temp, rt; flow rate $=1.0 \mathrm{~mL} / \mathrm{min} ; 210 \mathrm{~nm} \mathrm{UV}$ detector; $t_{\mathrm{R}}(R)=9.0 \mathrm{~min}\left(\right.$ minor); $t_{\mathrm{R}}(S)=11.3$ $\min$ (major).

\section{(S)-2-((2-Methoxyphenyl)amino)pentane-1,5-diol (9m)}


OMe

White solid, $16 \mathrm{~h}, \mathrm{mp} 100-101{ }^{\circ} \mathrm{C}, 207 \mathrm{mg}, 92 \%$ yield, $95 \%$ ee. $[\alpha]_{\mathrm{D}}^{25}-33.4(\mathrm{c}$ $1.0, \mathrm{MeOH}) .{ }^{1} \mathrm{H} \mathrm{NMR}\left(400 \mathrm{MHz}, \mathrm{CDCl}_{3}\right) \delta: 6.86(\mathrm{td}, J=7.6,1.6 \mathrm{~Hz}, 1 \mathrm{H}), 6.82$ $6.77(\mathrm{~m}, 1 \mathrm{H}), 6.73-6.66(\mathrm{~m}, 2 \mathrm{H}), 3.85(\mathrm{~s}, 3 \mathrm{H}), 3.75(\mathrm{dd}, J=10.6,4.0 \mathrm{~Hz}, 1 \mathrm{H})$, $3.65(\mathrm{t}, J=5.6 \mathrm{~Hz}, 2 \mathrm{H}), 3.60-3.49(\mathrm{~m}, 2 \mathrm{H}), 2.09(\mathrm{br}, 1 \mathrm{H}), 1.80-1.59(\mathrm{~m}, 4 \mathrm{H})$. ${ }^{13} \mathrm{C}$ NMR (101 MHz, $\mathrm{CDCl}_{3}$ ) $\delta: 147.2,137.4,121.3,117.1,111.1,109.8,64.6,62.8,55.5,55.0,29.3$, 28.7. HRMS (ESI) Calcd for $\mathrm{C}_{12} \mathrm{H}_{20} \mathrm{NO}_{3}\left([\mathrm{M}+\mathrm{H}]^{+}\right)$: 226.1438; Found: 226.1441. HPLC conditions: Chiralcel IC-3 column $(25 \mathrm{~cm} \times 0.46 \mathrm{~cm}$ ID); $n$-hexane $/ 2$-propanol $=60: 40$; temp, rt; flow rate $=1.0$ $\mathrm{mL} / \mathrm{min} ; 210 \mathrm{~nm} \mathrm{UV}$ detector; $t_{\mathrm{R}}(R)=4.9 \mathrm{~min}$ (minor); $t_{\mathrm{R}}(S)=7.6 \mathrm{~min}$ (major).

The product $9 \mathbf{m}(20 \mathrm{mg})$ was dissolved in mixture solvents of ethyl acetate $(1 \mathrm{~mL})$ and hexane $(1 \mathrm{~mL})$. After slowly evaporation of solvents at ambient temperautre, the fine crystals which were suitable for the X-ray diffraction analyses were obtained. The data of X-ray diffraction analysis of $\mathbf{9 m}$ are summarized in Table S2.

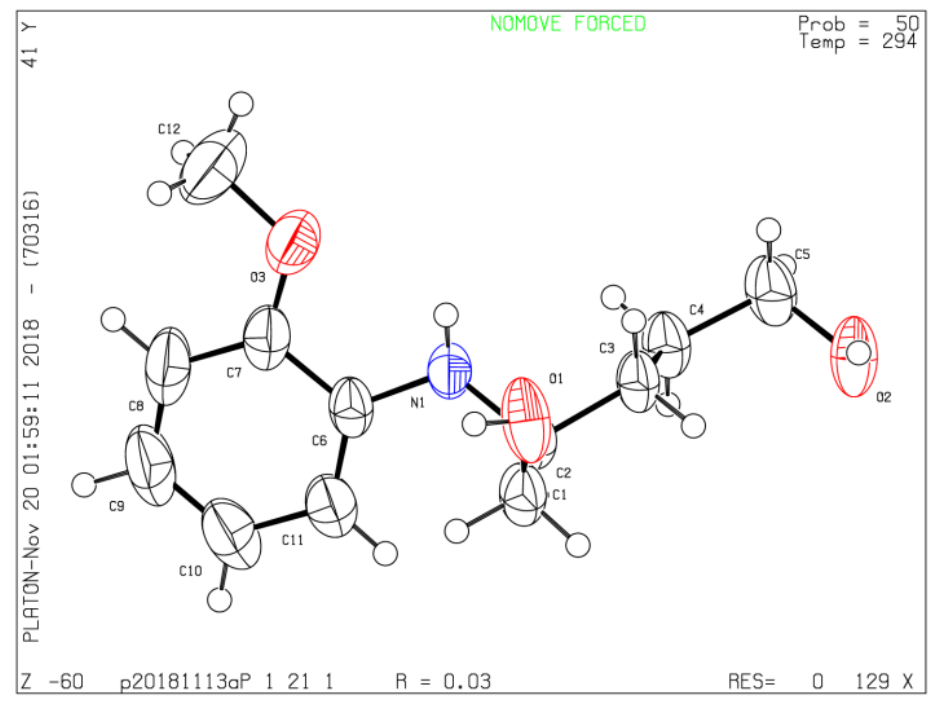

Table S2. Crystal data and structure refinement for $\mathbf{9 m}$.

\begin{tabular}{ll}
\hline Identification code & $\mathrm{P} 20181113 \mathrm{a}$ \\
Empirical formula & $\mathrm{C}_{12} \mathrm{H}_{19} \mathrm{NO}_{3}$ \\
Formula weight & 225.28 \\
Temperature & $294.15 \mathrm{~K}$ \\
Crystal system & monoclinic \\
Space group & $\mathrm{P} 2{ }_{1}$ \\
$\mathrm{a} / \AA$ & $7.49260(10)$ \\
$\mathrm{b} / \AA$ & $8.18990(10)$ \\
$\mathrm{c} / \AA$ & $10.35320(10)$ \\
$\alpha /^{\circ}$ & 90 \\
$\beta /{ }^{\circ}$ & $93.8140(10)$ \\
$\gamma /{ }^{\circ}$ & 90 \\
$\mathrm{Volume} / \AA^{3}$ & $633.903(13)$ \\
$\mathrm{Z}$ & 2 \\
$\rho_{\text {calc }} \mathrm{g} / \mathrm{cm}^{3}$ & 1.180
\end{tabular}




$\begin{array}{ll}\mu / \mathrm{mm}^{-1} & 0.687 \\ \mathrm{~F}(000) & 244.0 \\ \text { Crystal size } / \mathrm{mm}^{3} & 0.32 \times 0.26 \times 0.22 \\ \text { Radiation } & \mathrm{CuK} \alpha(\lambda=1.54184) \\ 2 \Theta \text { range for data collection/ } & \\ \text { Index ranges } & 8.56 \text { to } 158.958 \\ \text { Reflections collected } & -8 \leq \mathrm{h} \leq 8,-10 \leq \mathrm{k} \leq 10,-13 \leq 1 \leq 13 \\ \text { Independent reflections } & 8715 \\ \text { Data/restraints/parameters } & 2573\left[\mathrm{R}_{\mathrm{int}}=0.0184, \mathrm{R}_{\text {sigma }}=0.0126\right] \\ \text { Goodness-of-fit on } \mathrm{F}^{2} & 2573 / 1 / 159 \\ \text { Final R indices }[\mathrm{I}>2 \sigma(\mathrm{I})] & 1.061 \\ \text { Final R indices }(\text { all data }) & \mathrm{R}_{1}=0.0293, \mathrm{wR}_{2}=0.0799 \\ \text { Largest diff. peak and hole e } \AA^{-3} & \mathrm{R}_{1}=0.0295, \mathrm{wR}_{2}=0.0801 \\ \text { Flack parameter } & 0.13 /-0.13\end{array}$

\section{(S)-2-((2-Fluorophenyl)amino)pentane-1,5-diol (9n)}<smiles>OCCC[C@H](CO)Nc1ccccc1F</smiles>

Colorless oil, $16 \mathrm{~h}, 198 \mathrm{mg}, 93 \%$ yield, $95 \%$ ee. $[\alpha]_{\mathrm{D}}^{25}-7.2\left(c 1.0, \mathrm{CHCl}_{3}\right) .{ }^{1} \mathrm{H}$

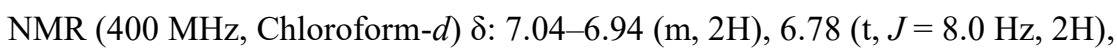
6.71-6.60 (m, 1H), $3.76(\mathrm{dd}, J=10.8,4.4 \mathrm{~Hz}, 1 \mathrm{H}), 3.67(\mathrm{t}, J=5.6 \mathrm{~Hz}, 2 \mathrm{H}), 3.61$ (dd, $J=10.8,5.6 \mathrm{~Hz}, 1 \mathrm{H}), 3.56-3.49$ (m, 1H), 1.98 (br, 1H), 1.81-1.53 (m, 4H). ${ }^{13} \mathrm{C}$ NMR (101 MHz, $\mathrm{CDCl}_{3}$ ) $\delta: 153.0,150.7,136.1,136.0,124.7,124.6,117.2,117.1,114.9,114.7$, 113.1, 113.1, 64.3, 62.6, 54.8, 29.0, 28.4. HRMS (ESI) Calcd for $\mathrm{C}_{11} \mathrm{H}_{17} \mathrm{FNO}_{2}\left([\mathrm{M}+\mathrm{H}]^{+}\right): 214.1238$; Found: 214.1243. HPLC conditions: Chiralcel IC-3 column $(25 \mathrm{~cm} \times 0.46 \mathrm{~cm} \mathrm{ID}) ; n$-hexane/2-propanol $=90: 10$; temp, rt; flow rate $=1.0 \mathrm{~mL} / \mathrm{min} ; 210 \mathrm{~nm} \mathrm{UV}$ detector; $t_{\mathrm{R}}(R)=13.0 \mathrm{~min}($ minor $) ; t_{\mathrm{R}}(S)=21.3$ $\min$ (major).

\section{(S)-2-((2,4-Dimethoxyphenyl)amino)pentane-1,5-diol (9o)}

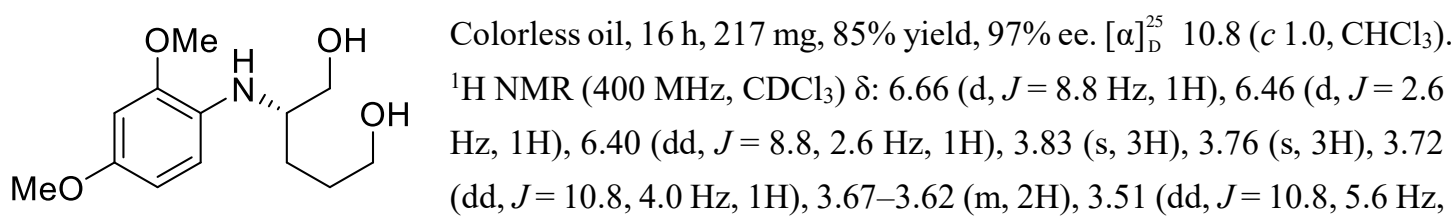
$1 \mathrm{H}), 3.43-3.36(\mathrm{~m}, 1 \mathrm{H}), 2.17$ (s, 1H), 1.75-1.57 (m, 4H). ${ }^{13} \mathrm{C}$ NMR (101 MHz, $\left.\mathrm{CDCl}_{3}\right) \delta: 152.5,148.7$, 131.2, 112.6, 104.1, 99.3, 64.2, 62.6, 56.2, 55.8, 55.6, 29.3, 28.8. HRMS (ESI) Calcd for $\mathrm{C}_{13} \mathrm{H}_{22} \mathrm{NO}_{4}$ $\left([\mathrm{M}+\mathrm{H}]^{+}\right): 256.1543$; Found: 256.1548 . HPLC conditions: Chiralcel IC-3 column $(25 \mathrm{~cm} \times 0.46 \mathrm{~cm}$ ID); $n$-hexane $/ 2$-propanol $=75: 25$; temp, rt; flow rate $=1.0 \mathrm{~mL} / \mathrm{min} ; 210 \mathrm{~nm} \mathrm{UV}$ detector; $t_{\mathrm{R}}(R)=12.1 \mathrm{~min}$ (minor); $t_{\mathrm{R}}(S)=19.8 \mathrm{~min}$ (major).

\section{(S)-2-(Naphthalen-1-ylamino)pentane-1,5-diol (9p)}

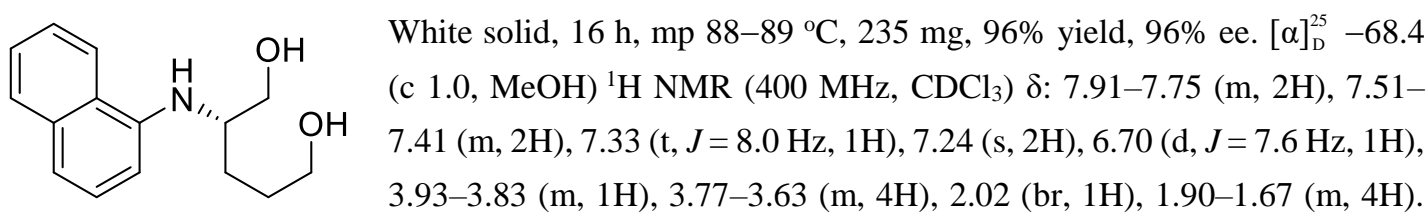

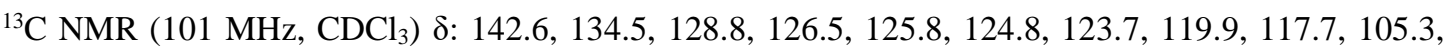


63.9, 62.7, 54.7, 29.1, 28.2. HRMS (ESI) Calcd for $\mathrm{C}_{15} \mathrm{H}_{20} \mathrm{NO}_{2}\left([\mathrm{M}+\mathrm{H}]^{+}\right)$: 246.1489; Found: 246.1491. HPLC conditions: Chiralcel IC-3 column $(25 \mathrm{~cm} \times 0.46 \mathrm{~cm}$ ID); $n$-hexane/2-propanol = 80:20; temp, rt; flow rate $=1.0 \mathrm{~mL} / \mathrm{min} ; 210 \mathrm{~nm} \mathrm{UV} \mathrm{detector} ; t_{\mathrm{R}}(R)=7.6 \mathrm{~min}($ minor $) ; t_{\mathrm{R}}(S)=8.9 \mathrm{~min}$ (major).

\section{(D) Gram-Scale Asymmetric Hydrogenation and Its Applications}

\section{Gram scale asymmetric hydrogenation of $6 \mathrm{~m}$ at $\mathrm{S} / \mathrm{C}=\mathbf{2 0 0 0}$}

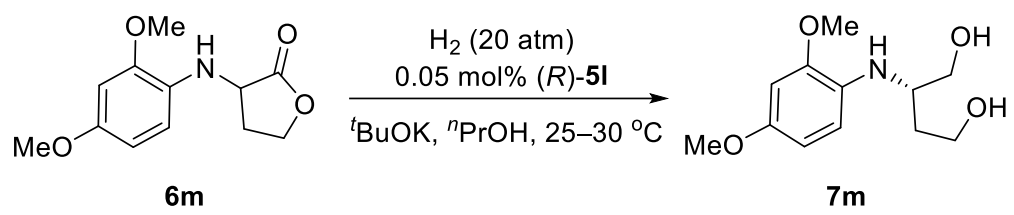

To a $200 \mathrm{~mL}$ hydrogenation vessel in an autoclave was added racemic substrates $6 \mathbf{m}(2.37 \mathrm{~g}, 10 \mathrm{mmol})$, chiral iridium catalyst $(R)-5 \mathbf{l}(5.8 \mathrm{mg}, 5 \mu \mathrm{mol}),{ }^{t} \mathrm{BuOK}(112 \mathrm{mg}, 1 \mathrm{mmol})$ and ${ }^{n} \mathrm{PrOH}(30 \mathrm{~mL})$. The autoclave was purged with hydrogen by pressurizing to $10 \mathrm{~atm}$ and releasing the pressure. This procedure was repeated three times and then pressurized to 20 atm of $\mathrm{H}_{2}$. The reaction mixture was stirred at room temperature $\left(25-30{ }^{\circ} \mathrm{C}\right)$ until no obvious hydrogen pressure drop was observed. The reaction mixture was then quenched with saturated $\mathrm{NH}_{4} \mathrm{Cl}(25 \mathrm{~mL})$ and extracted with EtOAc $(25 \mathrm{~mL} \times 3)$. The combined extracts were washed with brine, dried over anhydrous $\mathrm{MgSO}_{4}$ and concentrated in vacuo. The residue was purified by flash column chromatography on silical gel with petroleum ether/ethyl acetate (from 1:1 to $0: 1)$ as an eluent to afford the chiral diol $7 \mathbf{m}(2.1 \mathrm{~g}, 90 \%$ yield $)$ as a yellow solid. $97 \%$ ee, mp 53-54 ${ }^{\circ} \mathrm{C} .[\alpha]_{\mathrm{D}}^{25}-17.8\left(c 1.0, \mathrm{CHCl}_{3}\right)$.
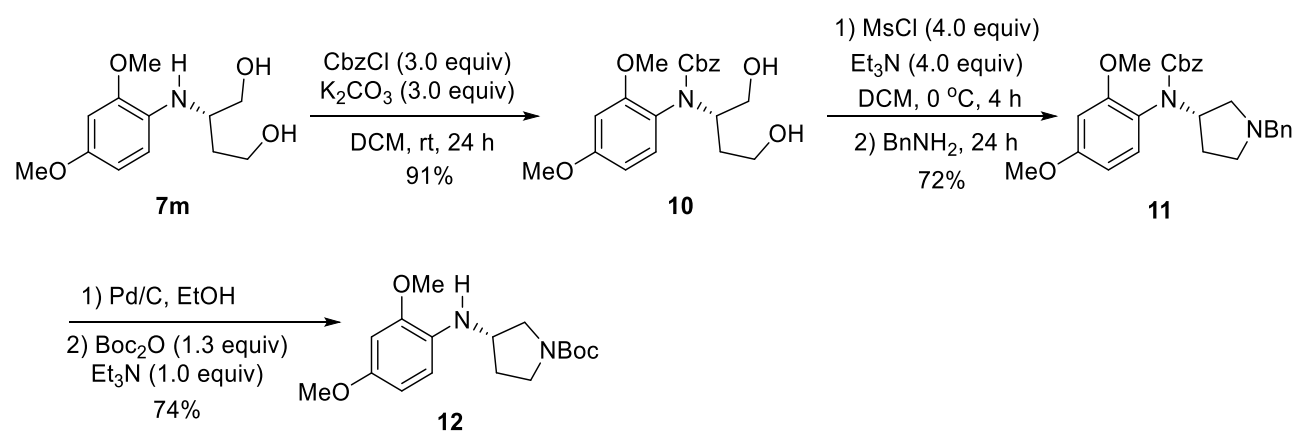

\section{Synthesis of compound 10.6}

To a stirred mixture of $7 \mathbf{m}(241 \mathrm{mg}, 1.0 \mathrm{mmol})$ and $\mathrm{K}_{2} \mathrm{CO}_{3}(690 \mathrm{mg}, 5.0 \mathrm{mmol})$ in $\mathrm{CH}_{2} \mathrm{Cl}_{2}(8 \mathrm{~mL})$ was added benzyl chloroformate $(420 \mathrm{mg}, 2.5 \mathrm{mmol})$. The reaction mixture was allowed to stir at room temperature for $24 \mathrm{~h}$. After the $\mathrm{K}_{2} \mathrm{CO}_{3}$ was removed by filtration through a plug of Celite, concentrated in vacuo to give a residue. The residue was chromatographed on silica gel column with ethyl acetate/petroleum ether (1:1) as an eluent to offer the corresponding product $\mathbf{1 0}(341 \mathrm{mg}, 91 \%$ yield) as a yellowish liquid. $[\alpha]_{\mathrm{D}}^{25}-3.6\left(c 1.0, \mathrm{CHCl}_{3}\right) .{ }^{1} \mathrm{H}$ NMR $\left(400 \mathrm{MHz}, \mathrm{CDCl}_{3}\right) \delta: 7.44-7.21(\mathrm{~m}, 3.8 \mathrm{H}), 7.19$ $7.07(\mathrm{~m}, 1.6 \mathrm{H}), 6.87(\mathrm{~d}, J=8.4 \mathrm{~Hz}, 0.6 \mathrm{H}), 6.60-6.38(\mathrm{~m}, 2.0 \mathrm{H}), 5.32-5.13(\mathrm{~m}, 1.1 \mathrm{H}), 5.02-4.90(\mathrm{~m}$, $0.9 \mathrm{H}), 4.80-4.70(\mathrm{~m}, 0.7 \mathrm{H}), 4.32-4.21(\mathrm{~m}, 0.3 \mathrm{H}), 3.82(\mathrm{~s}, 2.3 \mathrm{H}), 3.81(\mathrm{~s}, 0.7 \mathrm{H}), 3.78-3.73(\mathrm{~m}, 1.6 \mathrm{H})$, $3.72(\mathrm{~s}, 2.4 \mathrm{H}), 3.6(\mathrm{~s}, 0.6 \mathrm{H}), 3.46-3.37(\mathrm{~m}, 1.4 \mathrm{H}), 3.26-3.16(\mathrm{~m}, 0.7 \mathrm{H}), 2.91-2.78(\mathrm{~m}, 0.3 \mathrm{H}), 1.74-1.64$ $(\mathrm{m}, 1.3 \mathrm{H}), 1.56-1.48(\mathrm{~m}, 0.7 \mathrm{H}) .{ }^{13} \mathrm{C} \mathrm{NMR}\left(101 \mathrm{MHz}, \mathrm{CDCl}_{3}\right) \delta: 160.5,160.2,157.8,157.5,156.7,156.4$, 136.9, 136.7, 130.7, 128.3, 128.2, 127.8, 127.6, 127.2, 118.8, 104.9, 104.2, 99.8, 99.2, 67.3, 67.0, 63.8, 


\section{Synthesis of compound $11 .^{7}$}

To a stirred solution of $\mathrm{MsCl}(241 \mathrm{mg}, 4.0 \mathrm{mmol})$ in $5 \mathrm{~mL} \mathrm{CH} \mathrm{Cl}_{2}$, was added 10 (375 mg, $\left.1.0 \mathrm{mmol}\right)$ and triethylamine (402 mg, $4.0 \mathrm{mmol})$ in $\mathrm{CH}_{2} \mathrm{Cl}_{2}(5 \mathrm{~mL})$ dropwise below $5{ }^{\circ} \mathrm{C}$ in an ice-water bath for $0.5 \mathrm{~h}$. Then the reaction was stirred at room temperature under Ar atmosphere for another $2 \mathrm{~h}$. When the reaction was finished, concentrated in vacuo to give a residue. To the residue was added $8 \mathrm{~mL}$ benzylamine and the resulting reaction mixture was stirred at room temperature under Ar atmosphere for another $24 \mathrm{~h}$. The reaction mixture was concentrated in vacuo and the residue was chromatographed on silica gel column with ethyl acetate/petroleum ether (1:1) as an eluent to offer the corresponding product 11 (320 mg, 72\% yield) as a yellowish liquid. $[\alpha]_{\mathrm{D}}^{25}+12.4\left(c 0.5, \mathrm{CHCl}_{3}\right) .{ }^{1} \mathrm{H} \mathrm{NMR}\left(400 \mathrm{MHz}, \mathrm{CDCl}_{3}\right)$ $\delta: 7.45-7.18(\mathrm{~m}, 8 \mathrm{H}), 7.15-7.08(\mathrm{~m}, 2 \mathrm{H}), 7.01(\mathrm{t}, J=8.8 \mathrm{~Hz}, 1 \mathrm{H}), 6.53-6.38(\mathrm{~m}, 2 \mathrm{H}), 5.18(\mathrm{~d}, J=12.0$ $\mathrm{Hz}, 1.2 \mathrm{H}), 4.92-4.87(\mathrm{~m}, 0.8 \mathrm{H}), 4.83-4.60(\mathrm{~m}, 1 \mathrm{H}), 3.83(\mathrm{~s}, 3 \mathrm{H}), 3.78-3.54(\mathrm{~m}, 4 \mathrm{H}), 3.52-3.28(\mathrm{~m}, 1 \mathrm{H})$, 2.96-2.86 (m, 0.5H), 2.75-2.65 (m, 0.5H), 2.62-2.32 (m, 3H), 2.31-2.20 (m, 0.5H), 2.10-1.85 (m, 1H), 1.75-1.59 (m, 0.5H). ${ }^{13} \mathrm{C}$ NMR (101 MHz, $\left.\mathrm{CDCl}_{3}\right) \delta: 160.1,157.0,156.9,156.2,139.1,137.2,131.2$, 131.2, 128.8, 128.6, 128.5, 128.1, 128.1, 127.9, 127.4, 127.4, 127.1, 127.0, 126.8, 126.7, 121.3, 103.6, 99.1, 98.9, 66.4, 60.3, 60.3, 58.7, 57.3, 57.2, 56.3, 55.4, 55.2, 55.1, 53.0, 44.4, 30.5, 27.9. HRMS (ESI) Calcd for $\mathrm{C}_{27} \mathrm{H}_{31} \mathrm{~N}_{2} \mathrm{O}_{4}\left([\mathrm{M}+\mathrm{H}]^{+}\right)$: 447.2278; Found: 447.2285.

\section{Synthesis of compound $12 .^{7}$}

To a solution of 11 (186 mg, $0.42 \mathrm{mmol})$ in ethanol $(6 \mathrm{~mL})$ was added $18.6 \mathrm{mg}$ of $10 \% \mathrm{Pd} / \mathrm{C}$ at room temperature. The mixture was stirred under $1 \mathrm{~atm}$ hydrogen atmosphere overnight. The solid was filtered off and washed with ethanol $(20 \mathrm{~mL})$. The solvent was evaporated under reduced pressure to about $6 \mathrm{~mL}$ and then (Boc) $)_{2} \mathrm{O}(275 \mathrm{mg}, 1.26 \mathrm{mmol})$ and triethylamine (97 mg, $\left.0.97 \mathrm{mmol}\right)$ was added. The resulting reaction mixture was stirred at the room temperature for $12 \mathrm{~h}$. The solvent was evaporated under reduced pressure to give a residue. The residue was chromatographed on silica gel column with ethyl acetate/petroleum ether (1:3) as an eluent to offer the corresponding product 12 (100 $\mathrm{mg}, 74 \%$ yield) as a yellowish liquid. $[\alpha]_{\mathrm{D}}^{25}+3.2\left(c 0.5, \mathrm{CHCl}_{3}\right) .{ }^{1} \mathrm{H} \mathrm{NMR}\left(400 \mathrm{MHz}, \mathrm{CDCl}_{3}\right) \delta: 6.53(\mathrm{~d}, J=8.4 \mathrm{~Hz}, 1 \mathrm{H})$, $6.46(\mathrm{~s}, 1 \mathrm{H}), 6.43-6.35(\mathrm{~m}, 1 \mathrm{H}), 4.01-3.91(\mathrm{~m}, 1 \mathrm{H}), 3.82(\mathrm{~s}, 3 \mathrm{H}), 3.76(\mathrm{~s}, 3 \mathrm{H}), 3.73-3.63(\mathrm{~m}, 1 \mathrm{H}), 3.55-$ $3.40(\mathrm{~m}, 2 \mathrm{H}), 3.30-3.16(\mathrm{~m}, 1 \mathrm{H}), 2.24-2.13(\mathrm{~m}, 1 \mathrm{H}), 1.96-1.83(\mathrm{~m}, 1 \mathrm{H}), 1.46(\mathrm{~s}, 9 \mathrm{H}) .{ }^{13} \mathrm{C} \mathrm{NMR}(101$

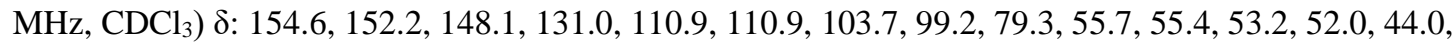
31.6, 28.5. HRMS (ESI) Calcd for $\mathrm{C}_{17} \mathrm{H}_{27} \mathrm{~N}_{2} \mathrm{O}_{4}\left([\mathrm{M}+\mathrm{H}]^{+}\right)$: 323.1965; Found: 323.1970.

\section{The synthesis of compound $(13)^{8}$}

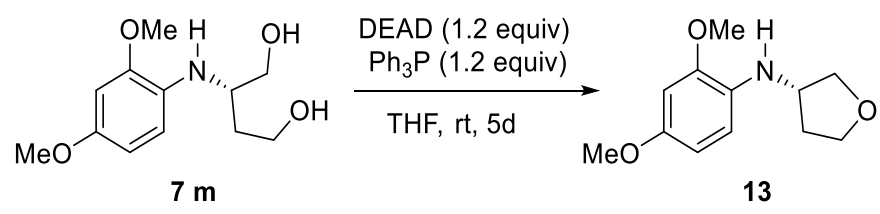

To a solution of diethyl azodicarboxylate (DEAD, $208.8 \mathrm{mg}, 1.2 \mathrm{mmol}$ ) in dried THF (5 mL) was added dropwise a solution of $\mathbf{7 m}(241.0 \mathrm{mg}, 1 \mathrm{mmol})$ and $\mathrm{Ph}_{3} \mathrm{P}(314.4 \mathrm{mg}, 1.2 \mathrm{mmol})$ in THF (10 mL) under Ar atmosphere. The reaction mixture was stirred at room temperature for 5 days. The solvent was concentrated in vacuo to yield a residue. The residue was chromatographed on silica gel column with ethyl acetate/petroleum ether (1:5) as an eluent to offer the corresponding product $\mathbf{1 3}$ (188 $\mathrm{mg}, 84 \%$ yield) 
as a yellowish liquid. $[\alpha]_{\mathrm{D}}^{25}+13.6\left(c 0.5, \mathrm{CHCl}_{3}\right) .{ }^{1} \mathrm{H} \mathrm{NMR}\left(400 \mathrm{MHz}, \mathrm{CDCl}_{3}\right) \delta 6.50(\mathrm{~d}, J=8.4 \mathrm{~Hz}, 1 \mathrm{H})$, $6.46(\mathrm{~d}, J=2.8 \mathrm{~Hz}, 1 \mathrm{H}), 6.40(\mathrm{dd}, J=8.4,2.8 \mathrm{~Hz}, 1 \mathrm{H}), 4.07-4.02(\mathrm{~m}, 1 \mathrm{H}), 4.01-3.93(\mathrm{~m}, 2 \mathrm{H}), 3.88-$ $3.83(\mathrm{~m}, 1 \mathrm{H}), 3.82(\mathrm{~s}, 3 \mathrm{H}), 3.76(\mathrm{~s}, 3 \mathrm{H}), 3.71(\mathrm{dd}, J=8.8,2.8 \mathrm{~Hz}, 1 \mathrm{H}), 2.29-2.20(\mathrm{~m}, 1 \mathrm{H}), 1.92-1.83(\mathrm{~m}$, 1H). ${ }^{13} \mathrm{C}$ NMR $\left(101 \mathrm{MHz}, \mathrm{CDCl}_{3}\right) \delta 152.2,148.3,131.3,110.9,103.7,99.2,73.9,67.2,55.8,55.4,54.3$, 33.4. HRMS (ESI) Calcd for $\mathrm{C}_{12} \mathrm{H}_{18} \mathrm{NO}_{3}\left([\mathrm{M}+\mathrm{H}]^{+}\right)$: 224.1281; Found: 224.1285 .

\section{Reference}

1. Baskar, B.; Dakas, P.-Y.; Kumar, K. Org. Lett. 2011, 13, 1988-1991.

2. Xie, J.-H.; Liu, X.-Y.; Xie, J.-B.; Wang, L.-X.; Zhou, Q.-L. Angew. Chem. Int. Ed. 2011, 50, 7329-7332.

3. Kulig, K.; Więckowski, K.; Więckowska, A.; Gajda, J.; Pochwat, B.; Höfner,G.C.; Wanner, K. T.; Malawska, B. Eur. J. Med. Chem. 2011, 46, 183-190.

4. Xie, X-M.; Stahl, S.-S. J. Am. Chem. Soc. 2015, 137, 3767-3770.

5. Mancey, N. C.; Sandon, N.; Auvinet, A.-L.; Butlin, R. J.; Czechtizkyc, W. ; Harrity, J. P. A. Chem. Commun. 2011, 47, 9804-9806.

6. Xie, J.; Wolfe, A. L.; Boger, D. L. Org. Lett. 2013, 15, 868-870.

7. Han, Z.-J.; Li, Y.-B.; Gu, B.-H.; Li, Y.-M.; Chen, H. Synth. Commun. 2018, 48, 2452-2456.

8. He, L.; Liu, Y.-M.; Zhang, W.; Li, M.; Chen, Q.-H. Tetrahedron. 2005, 61, 8505-8511. 
(E) NMR Spectra of New Compounds

4-(2-Hydroxypropan-2-yl)-2-methylpyridine (S1a)

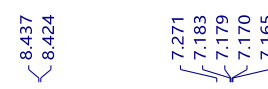<smiles>Cc1cc(C(C)(C)O)ccn1</smiles>

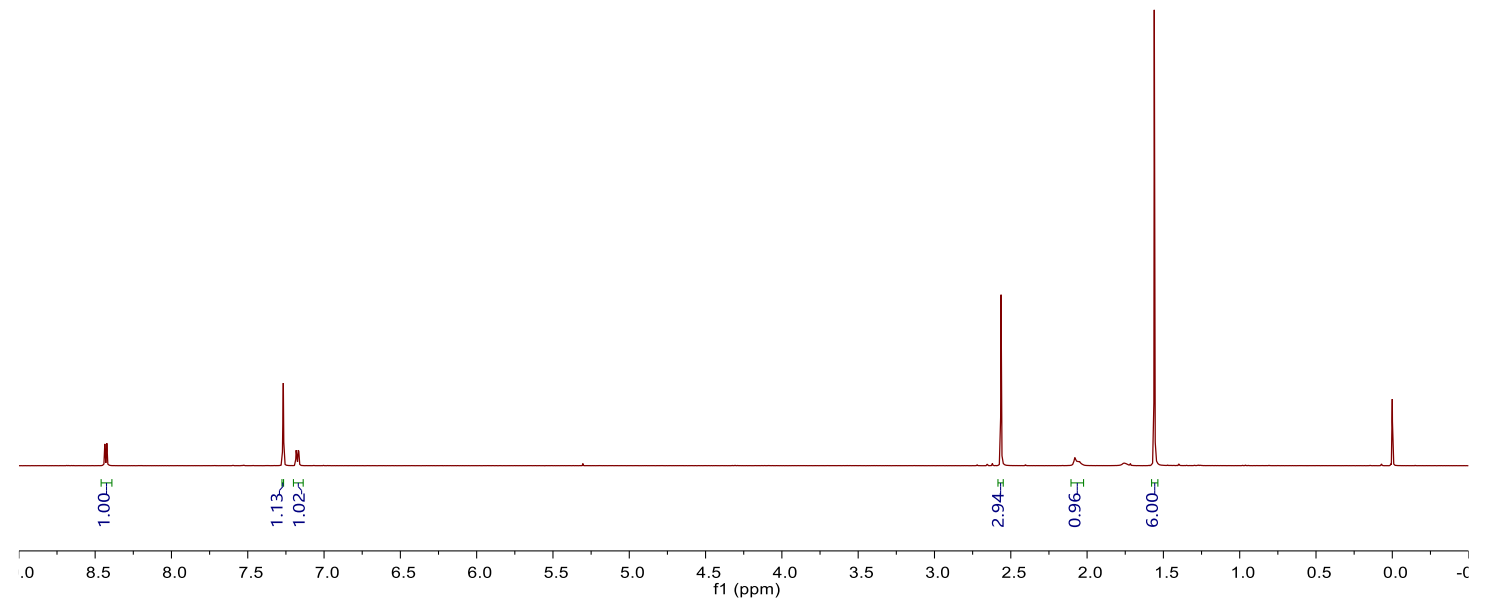

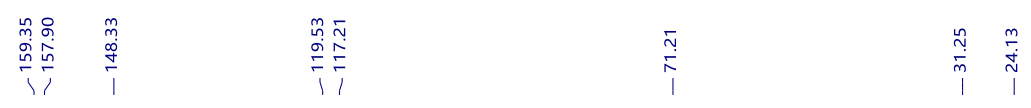<smiles>Cc1cc(C(C)(C)O)ccn1</smiles>

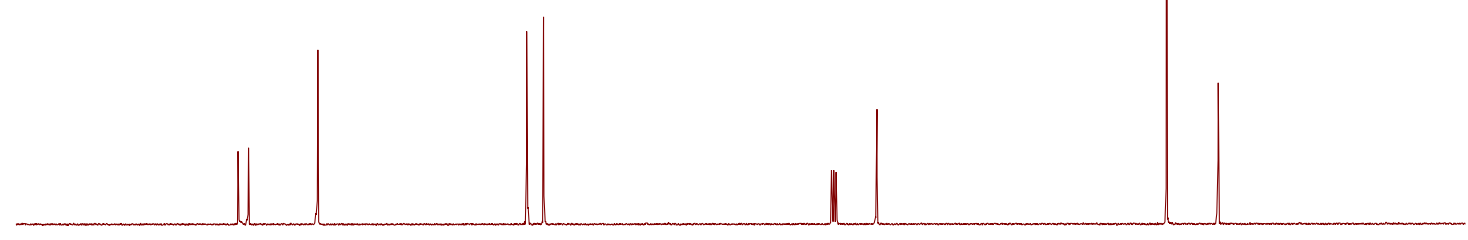

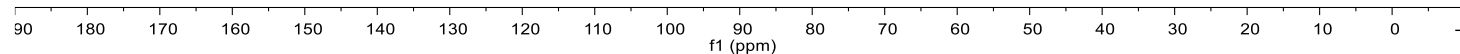


4-(Diethylhydroxymethyl)-2-methylpyridine (S1b)

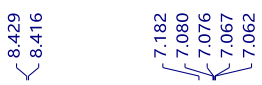<smiles>CCC(O)(CC)c1ccnc(C)c1</smiles>

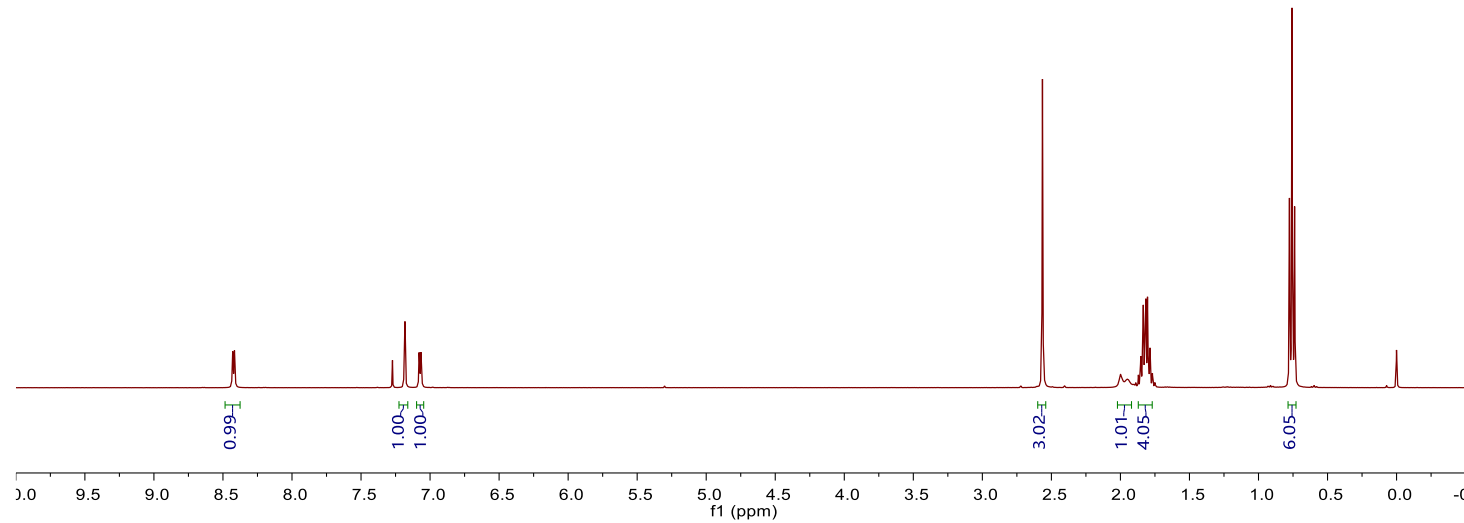

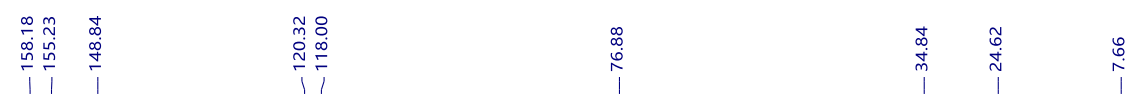<smiles>CCC(O)(CC)c1ccnc(C)c1</smiles>

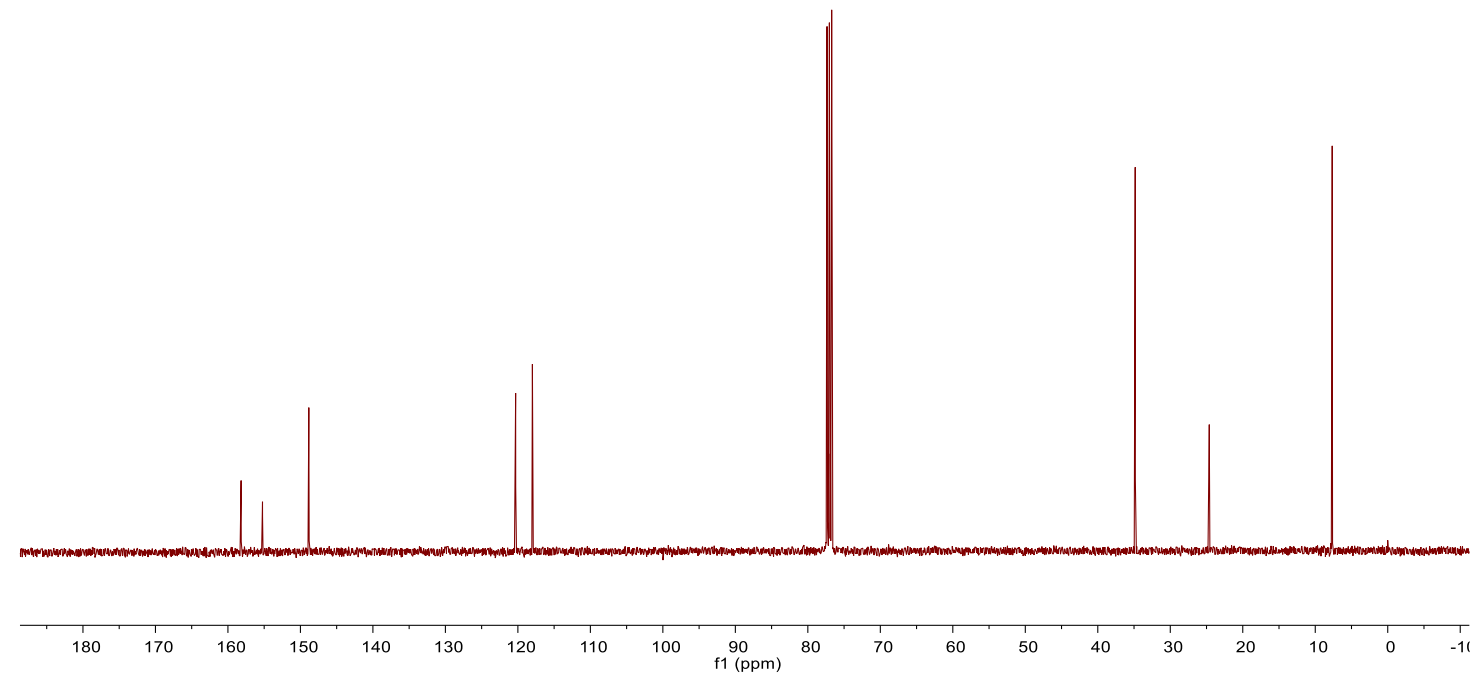


4-(Dicyclohexylhydroxymethyl)-2-methylpyridine (S1c)
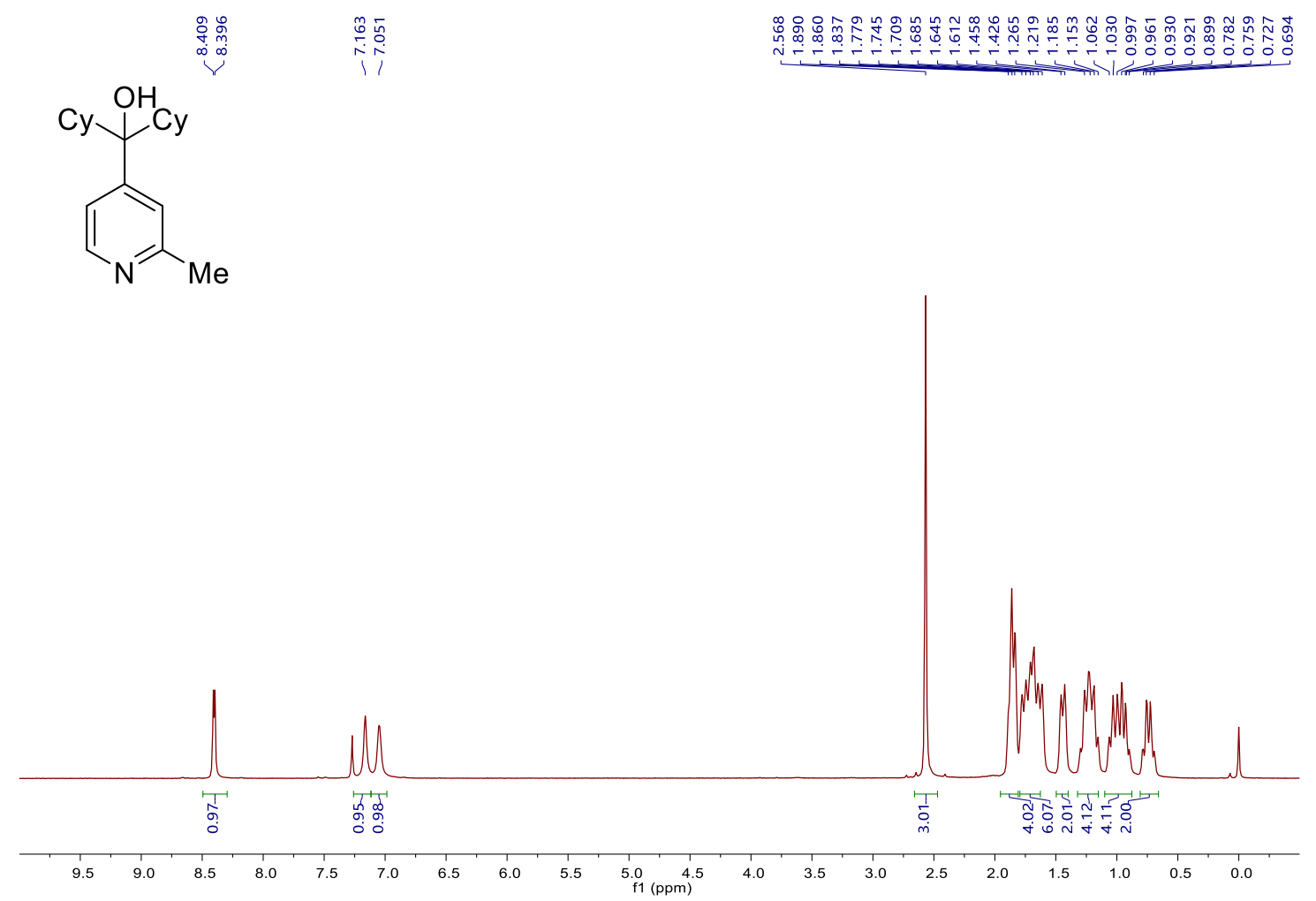

\begin{tabular}{|c|c|c|}
\hline 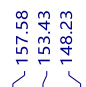 & 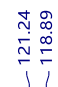 & $\begin{array}{l}\stackrel{n}{N} \\
\stackrel{\infty}{\infty} \\
i\end{array}$ \\
\hline
\end{tabular}

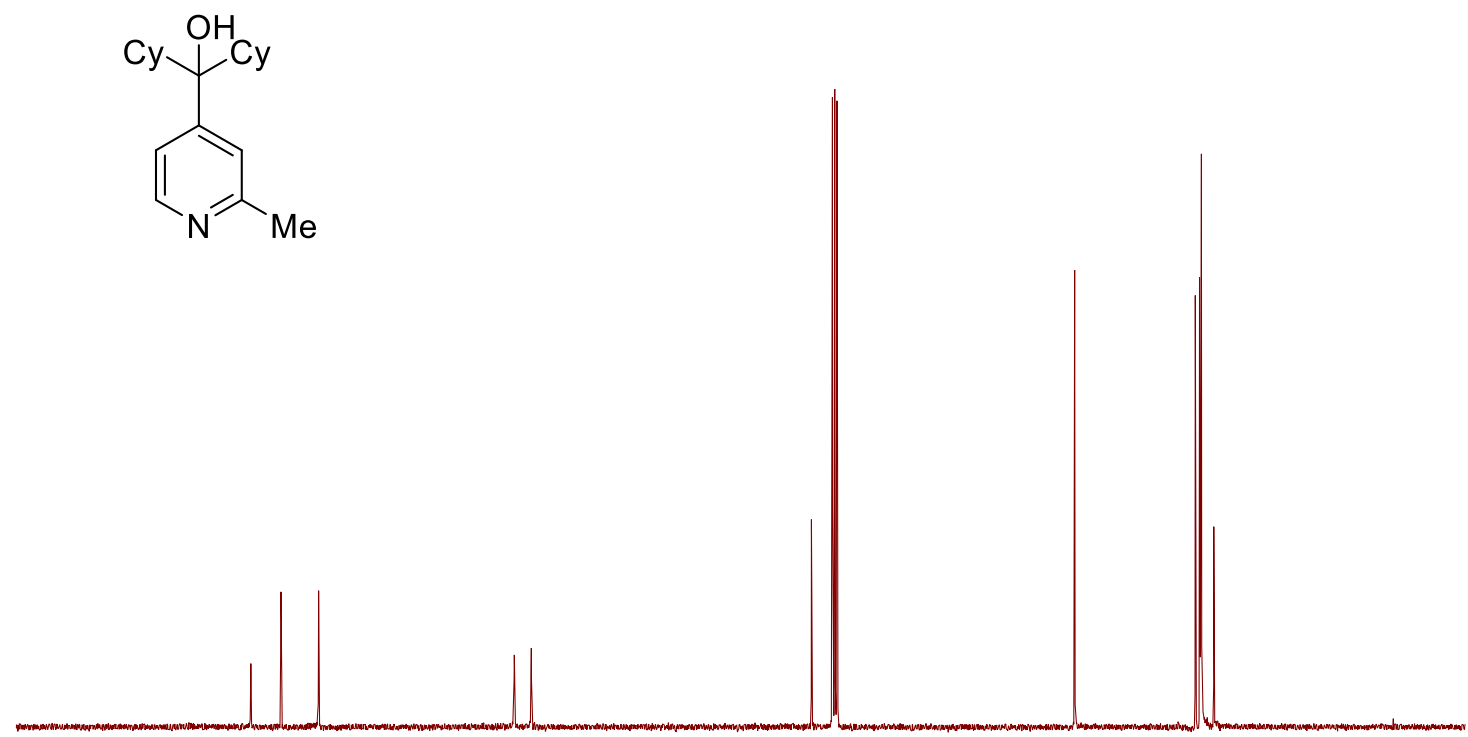

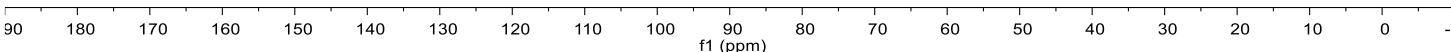


4-(Diphenylhydroxymethyl)-2-methylpyridine (S1d)

$\underbrace{\infty}_{\infty}$<smiles>Cc1cc(C(O)(c2ccccc2)c2ccccc2)ccn1</smiles>

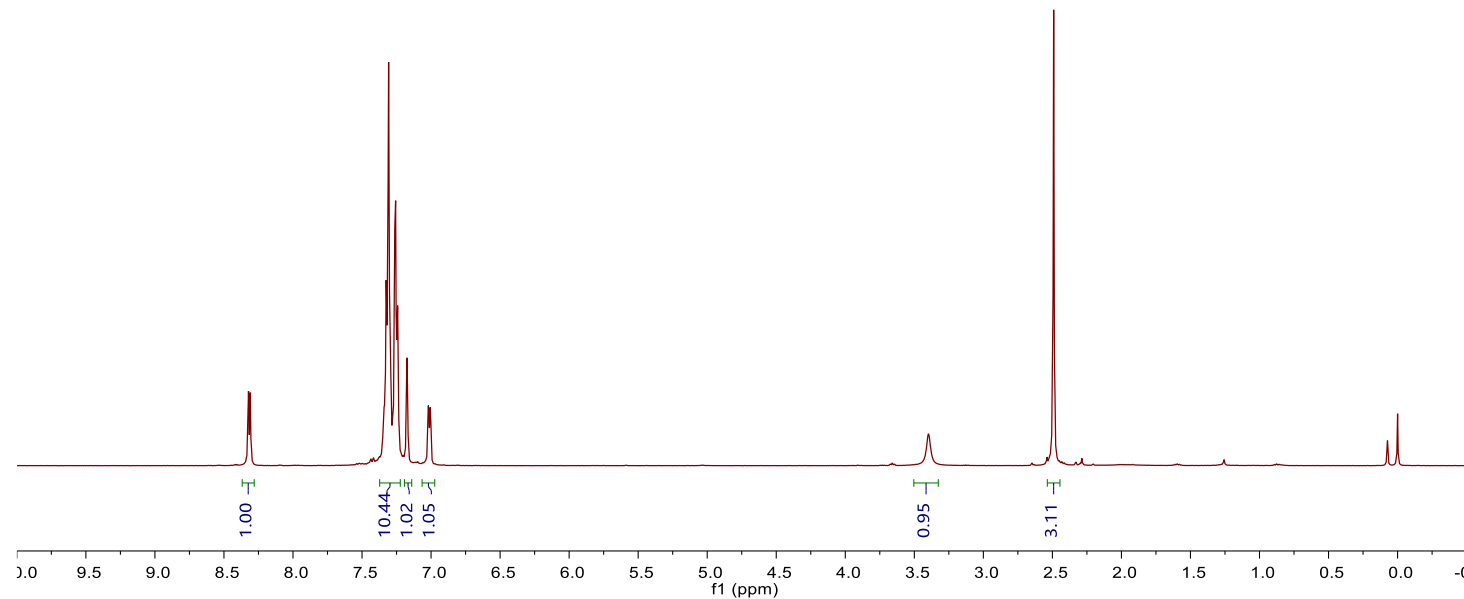

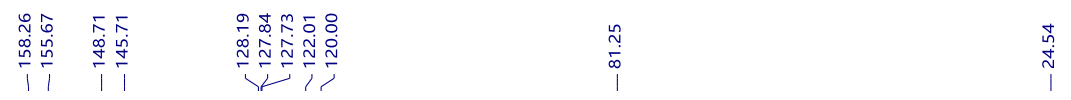<smiles>Cc1cc(C(O)(c2ccccc2)c2ccccc2)ccn1</smiles>

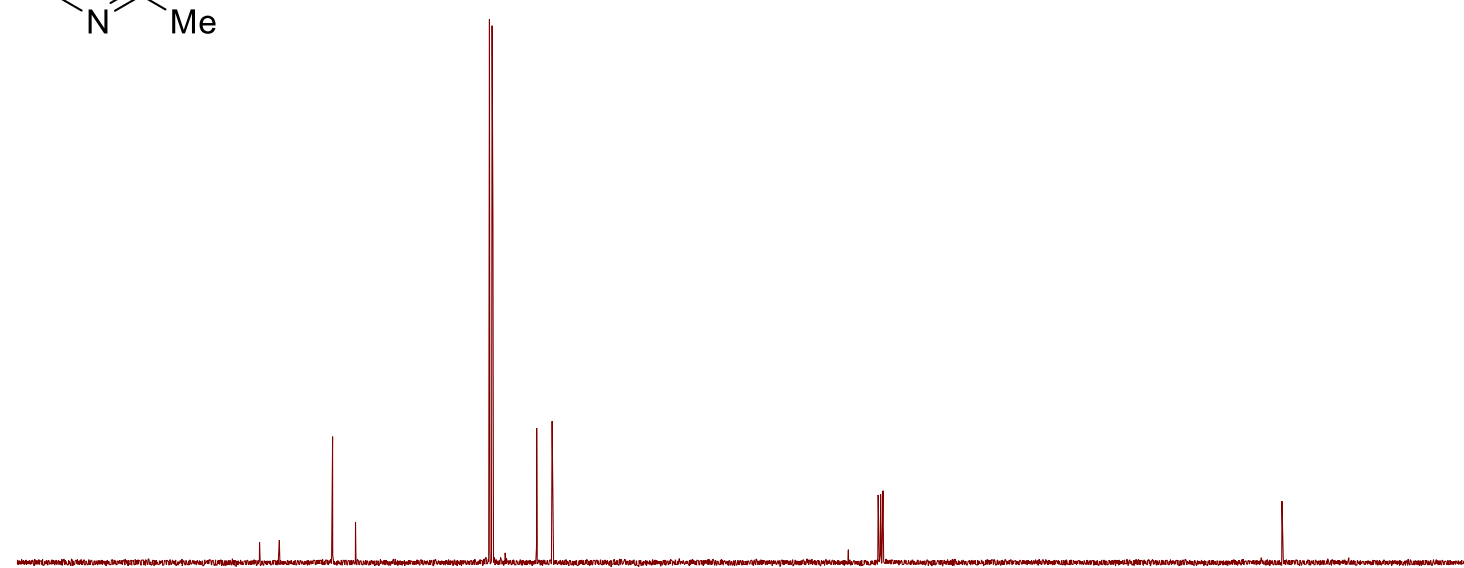

$\begin{array}{llllllllll}90 & 180 & 170 & 160 & 150 & 140 & 130 & 120 & 110 & 100 \\ \mathrm{f} 1(\mathrm{ppm}) & 90\end{array}$ 
4-[Di(2,6-dimethylphenyl)hydroxymethyl]-2-methylpyridine (S1e)

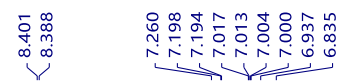

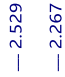<smiles>[Y10]C([Y19])(O)c1ccnc(C)c1</smiles>

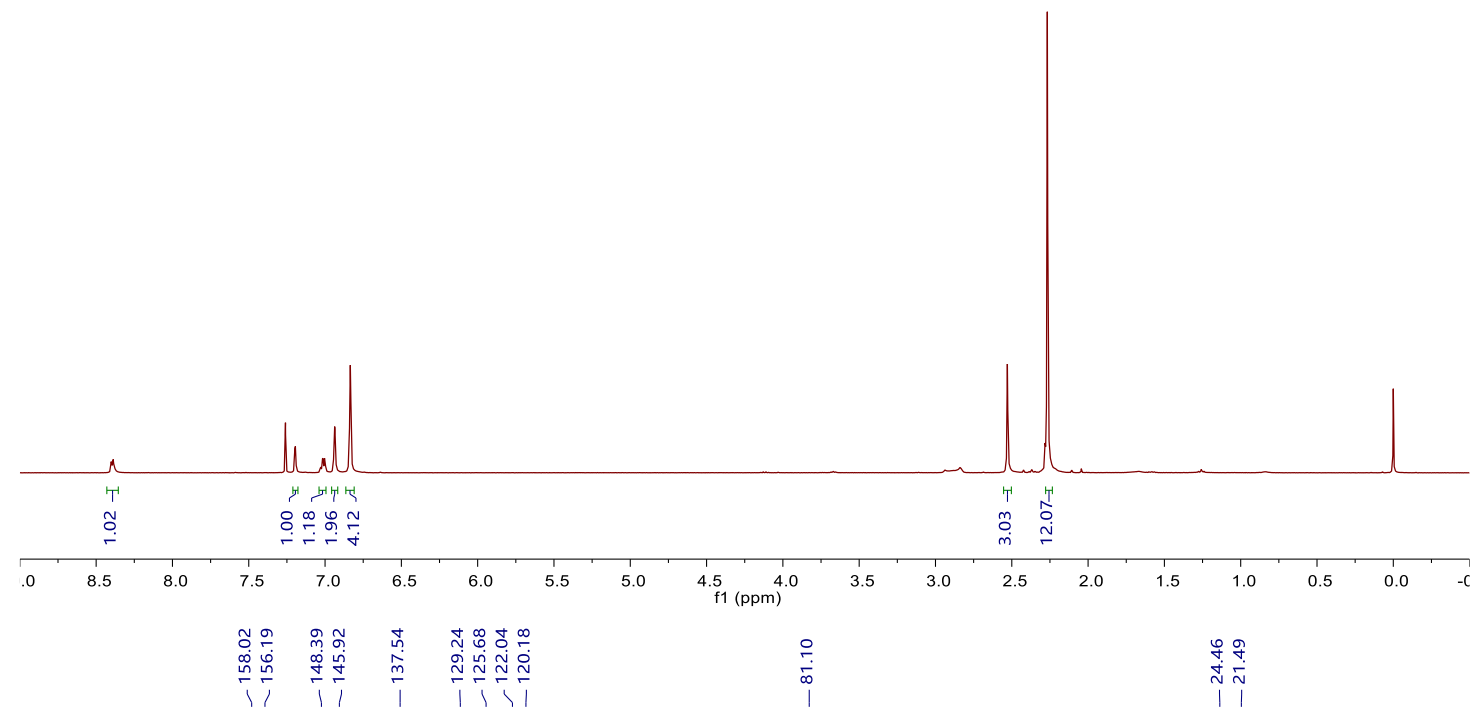<smiles>[Y10]C(O)([AlH2])c1ccnc(C)c1</smiles>

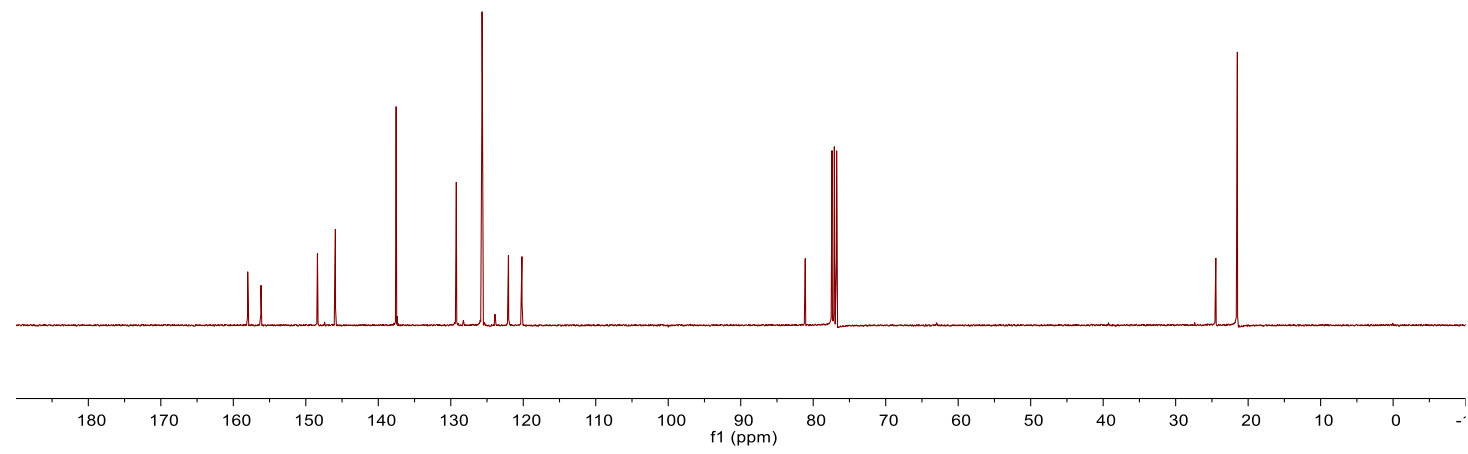


4-(Di(2,6-dimethylphenyl)hydroxymethyl)-2-methylpyridine (S1f)

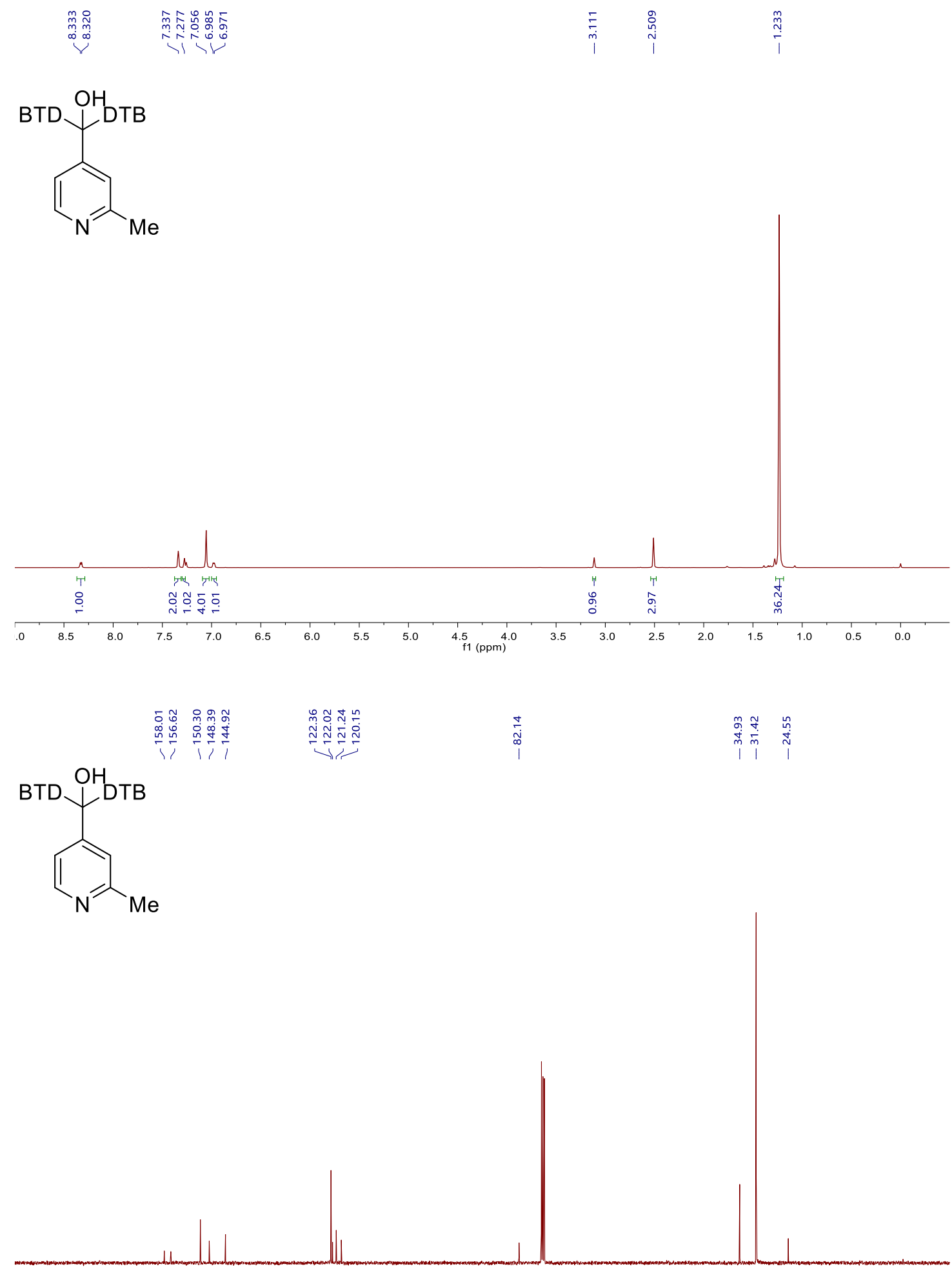

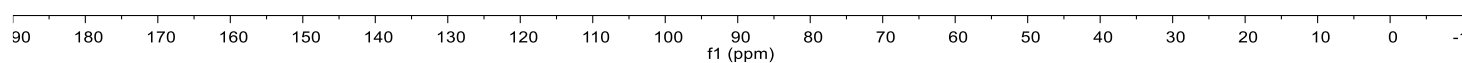


4-(Diethylhydroxymethyl)-2-pyridinecarbaldehyde (S2a)

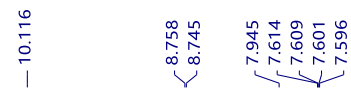

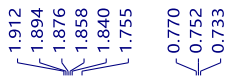<smiles>CCC(O)(CC)c1ccnc(C=O)c1</smiles>

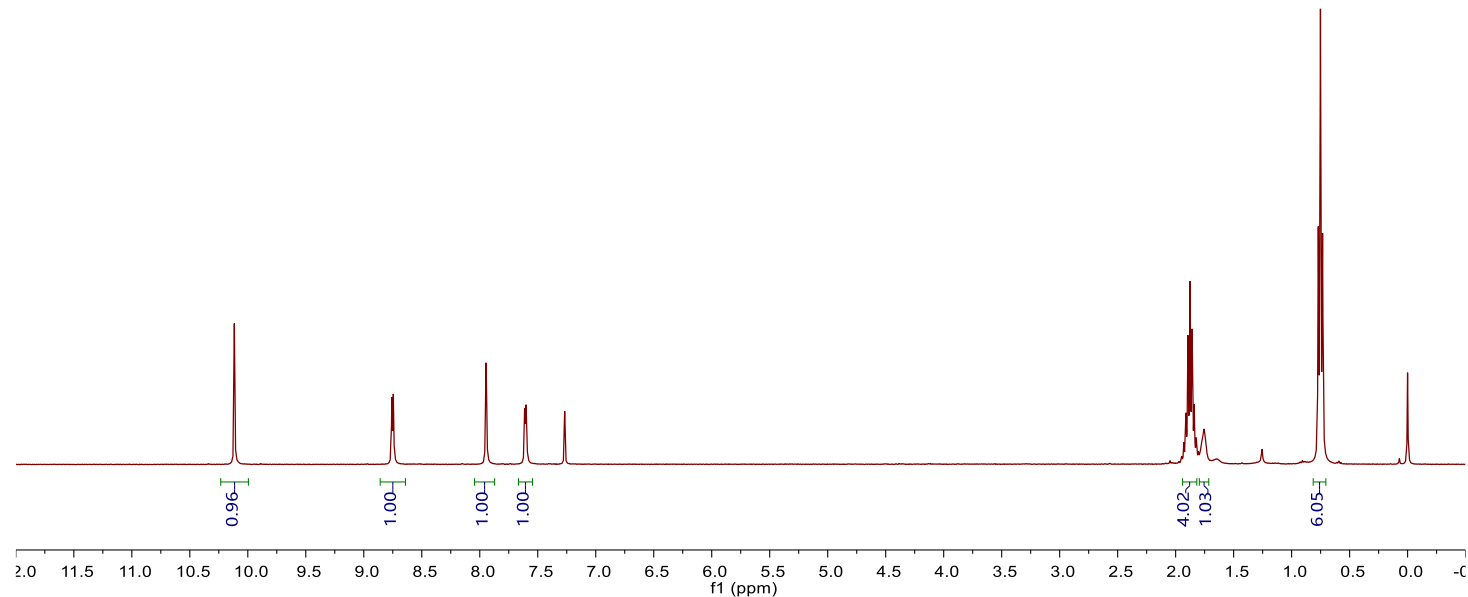

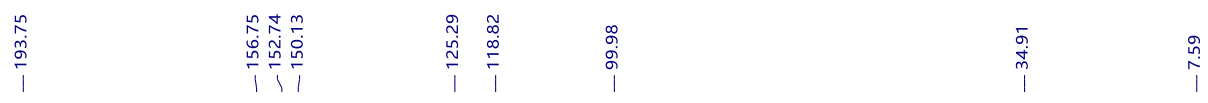<smiles>CCC(O)(CC)c1ccnc(C=O)c1</smiles>

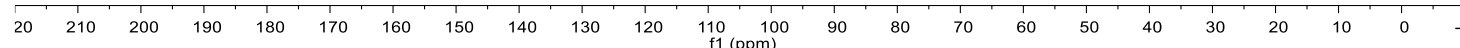


4-(Dicyclohexylhydroxymethyl)-2-pyridinecarbaldehyde (S2b)

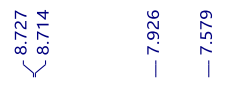

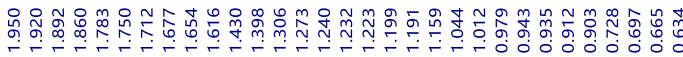<smiles>O=Cc1cc(C(O)(Cl)Cl)ccn1</smiles>

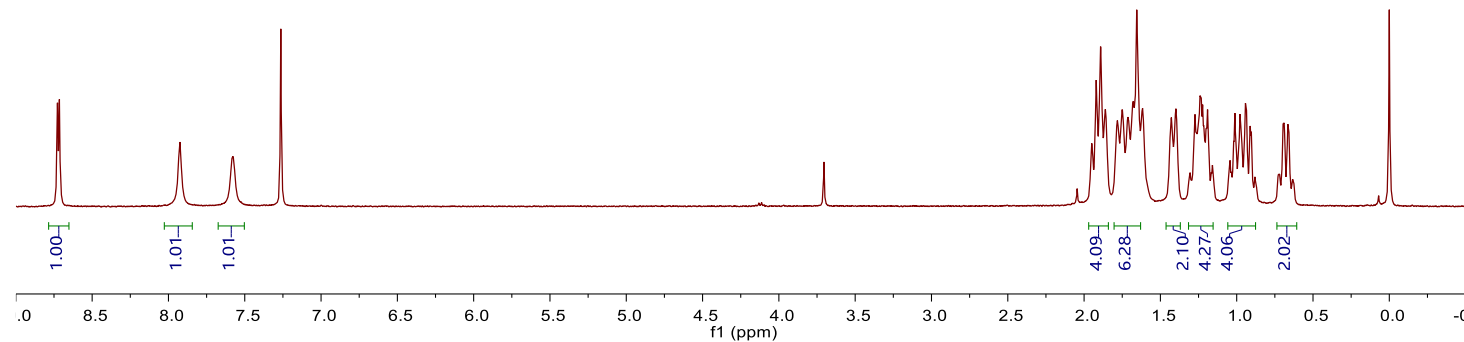

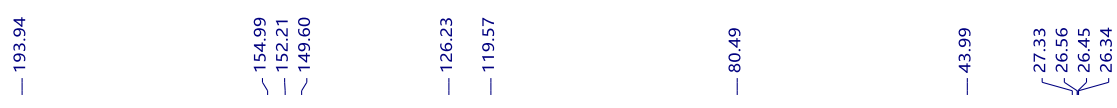<smiles>O=Cc1cc(C(O)(Cl)Cl)ccn1</smiles>
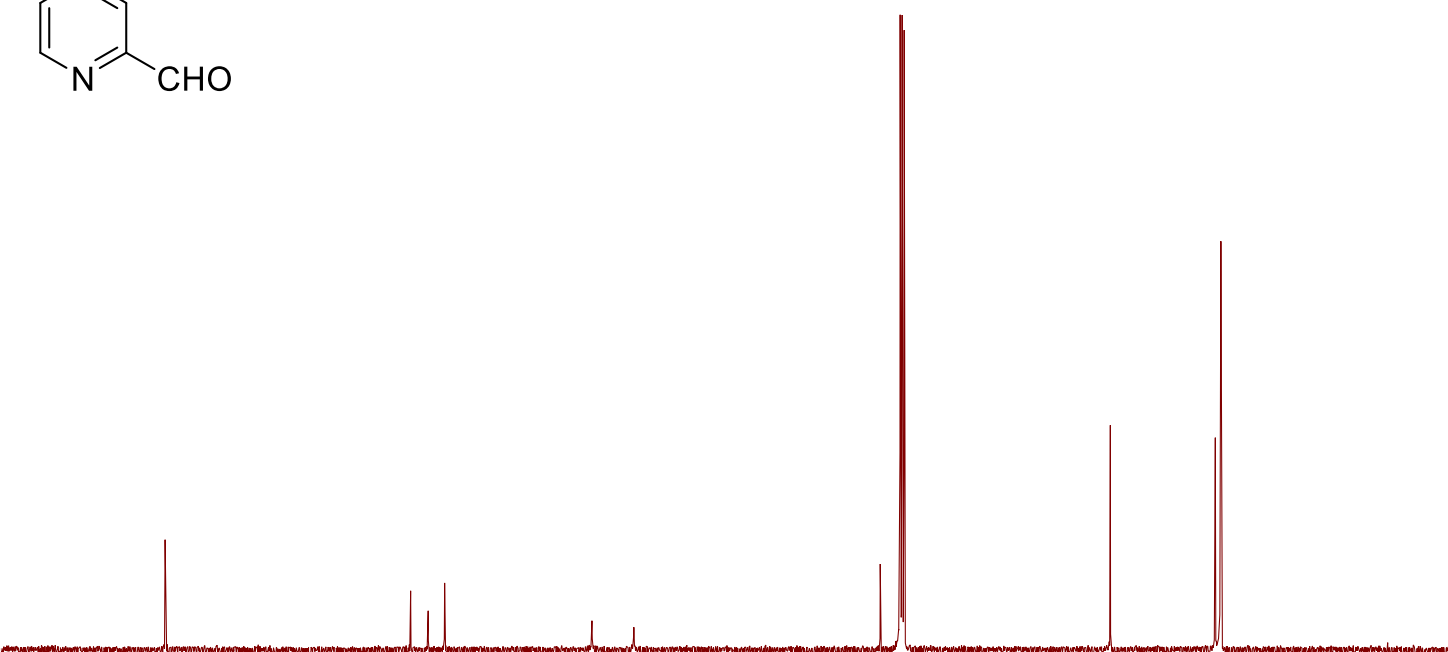

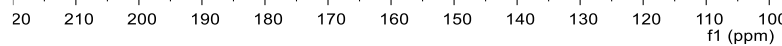


4-(Diphenylhydroxymethyl)-2-pyridinecarbaldehyde (S2c)

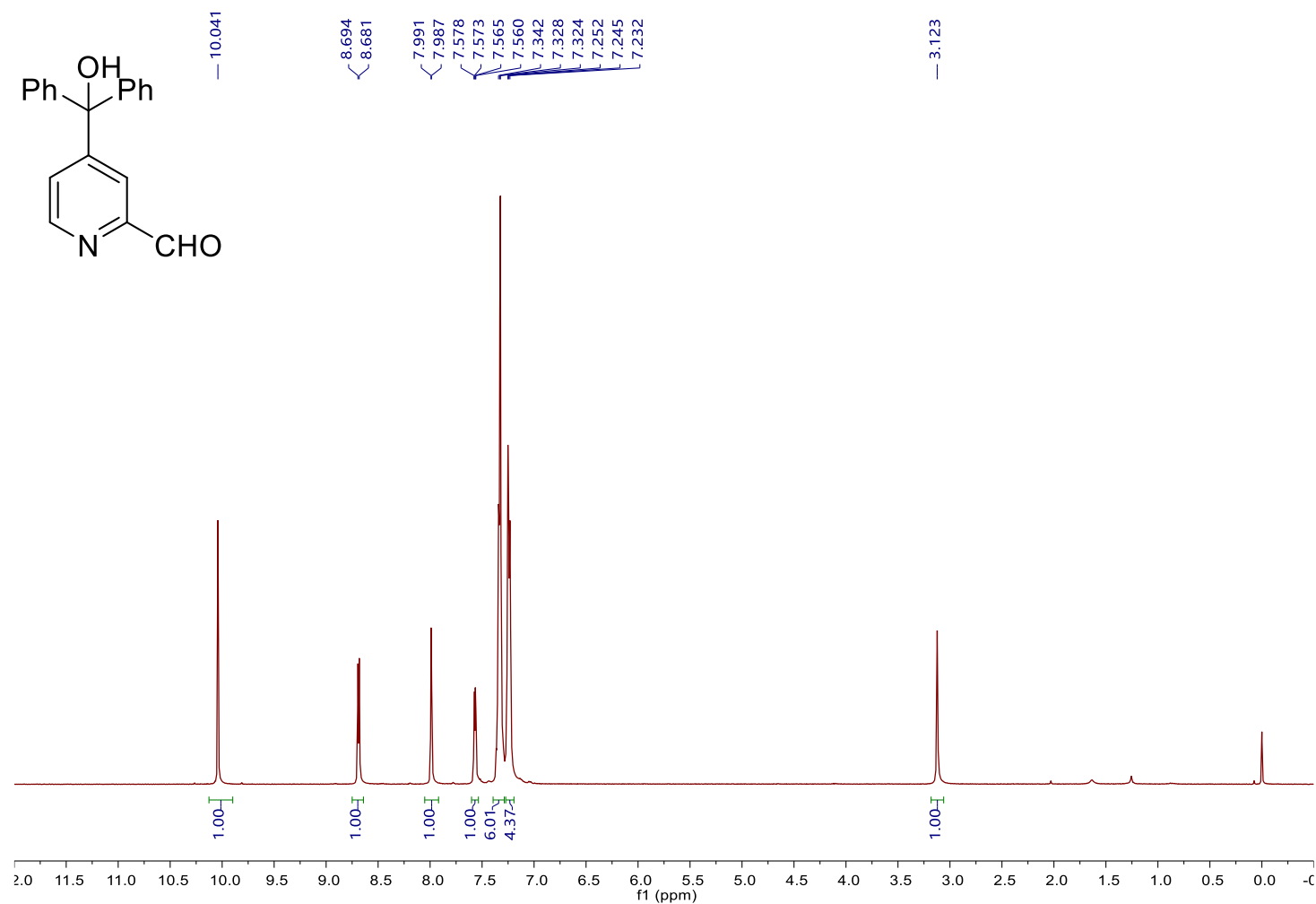

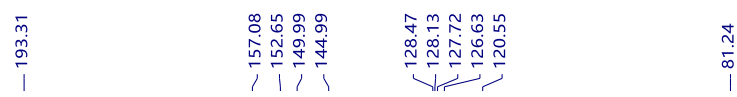<smiles>O=Cc1cc(C(O)(c2ccccc2)c2ccccc2)ccn1</smiles>

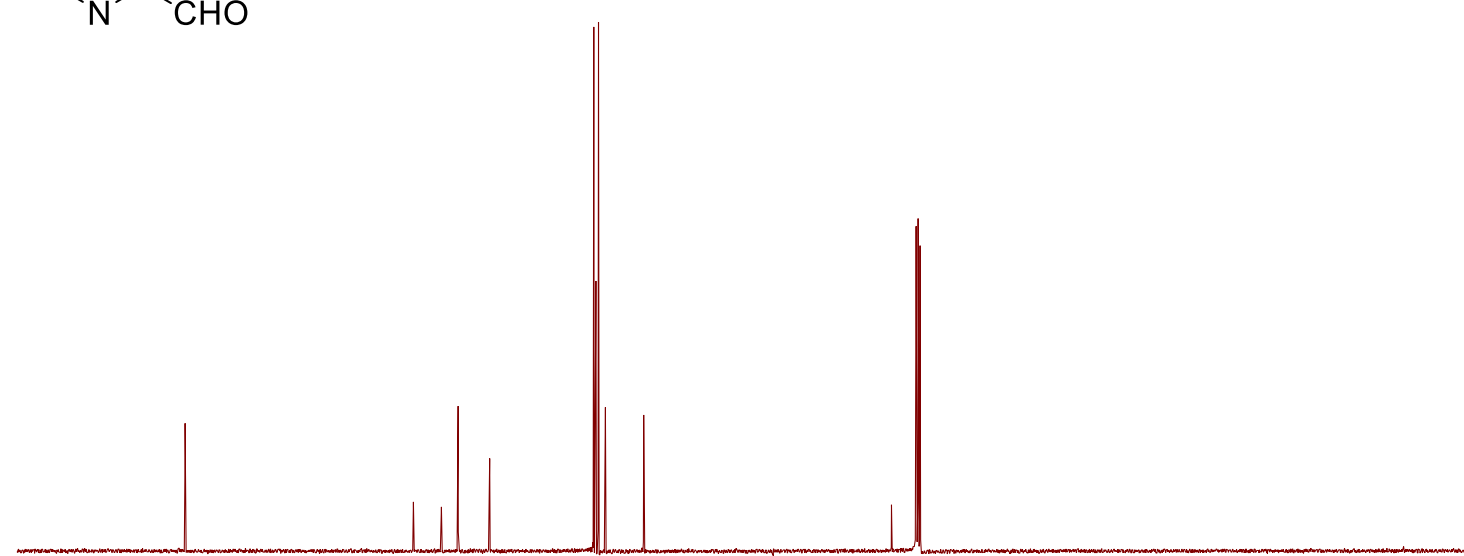

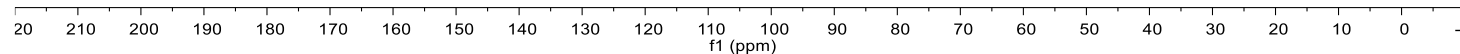


4-[Di(2,6-dimethylphenyl)hydroxymethyl]-2-pyridinecarbaldehyde (S2d)

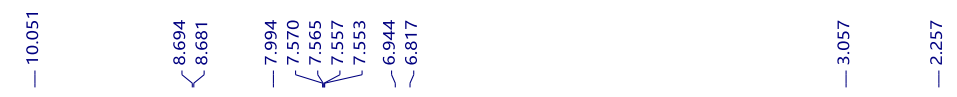<smiles>[Y14]C(O)([AlH2])c1ccnc(C=O)c1</smiles>

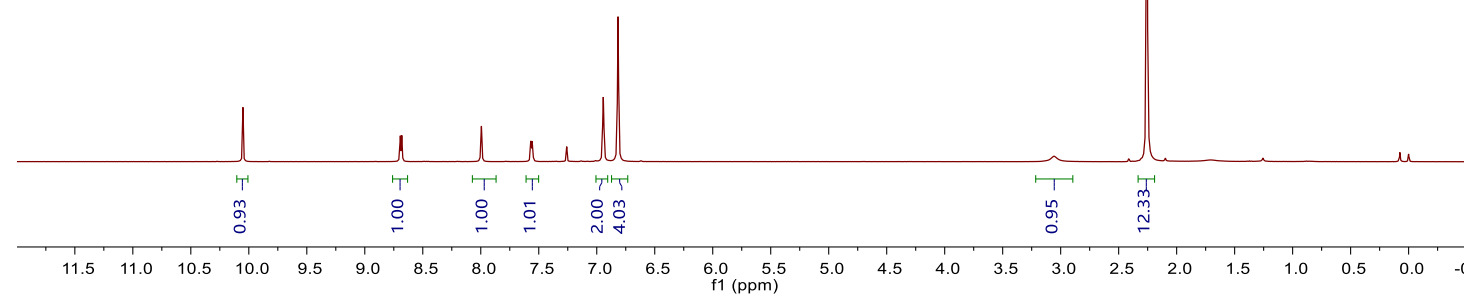

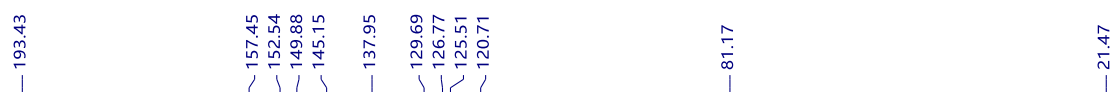<smiles>[Y14]C(O)([AlH2])c1ccnc(C=O)c1</smiles>

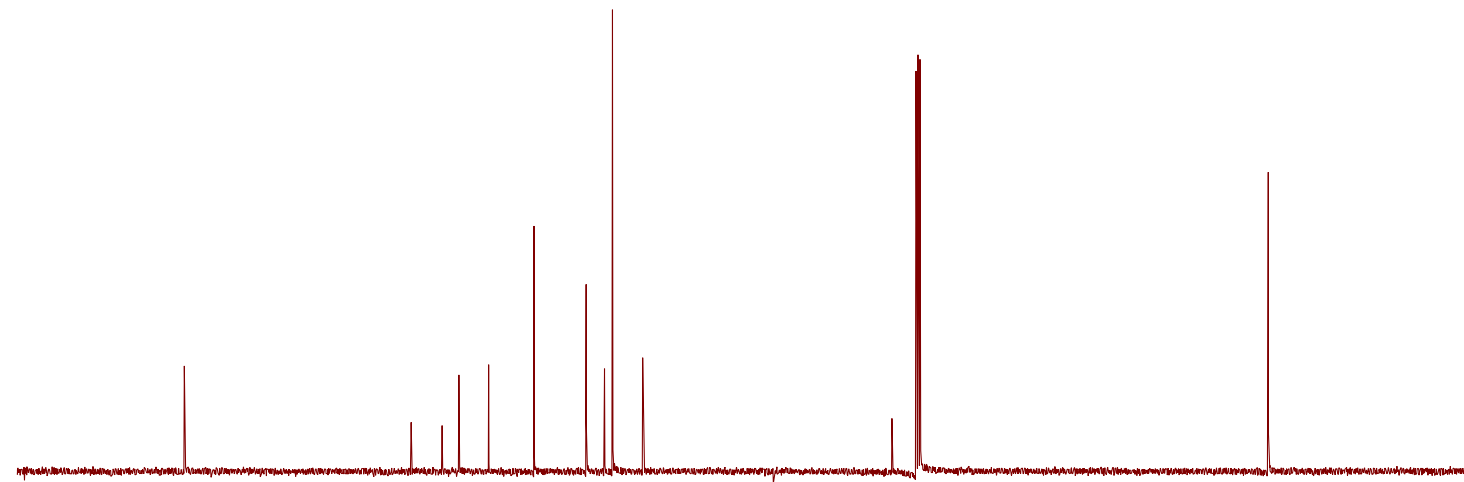

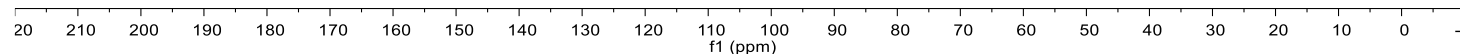


4-[Di(2,6-dimethylphenyl)hydroxymethyl]-2-pyridinecarbaldehyde (S2e)

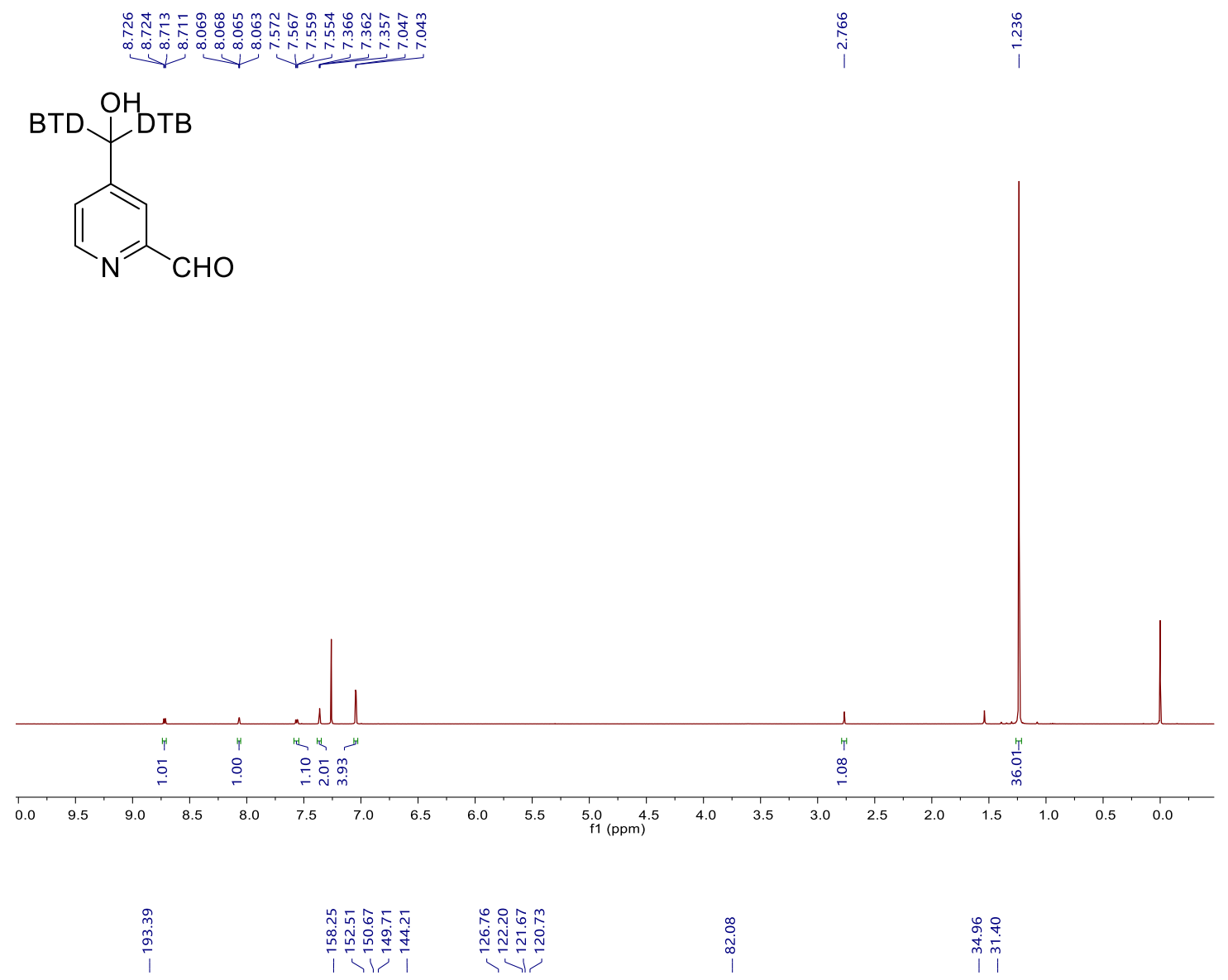<smiles>[B][B]C([B])([B])c1ccnc(C=O)c1</smiles>

$\begin{array}{llllllllllll}210 & 200 & 190 & 180 & 170 & 160 & 150 & 140 & 130 & 120 & \begin{array}{r}110 \\ \mathrm{f} 1(\mathrm{ppm})\end{array}\end{array}$ 
4-(2-Phenylpropan-2-yl)-2-methylpyridine (S3a)
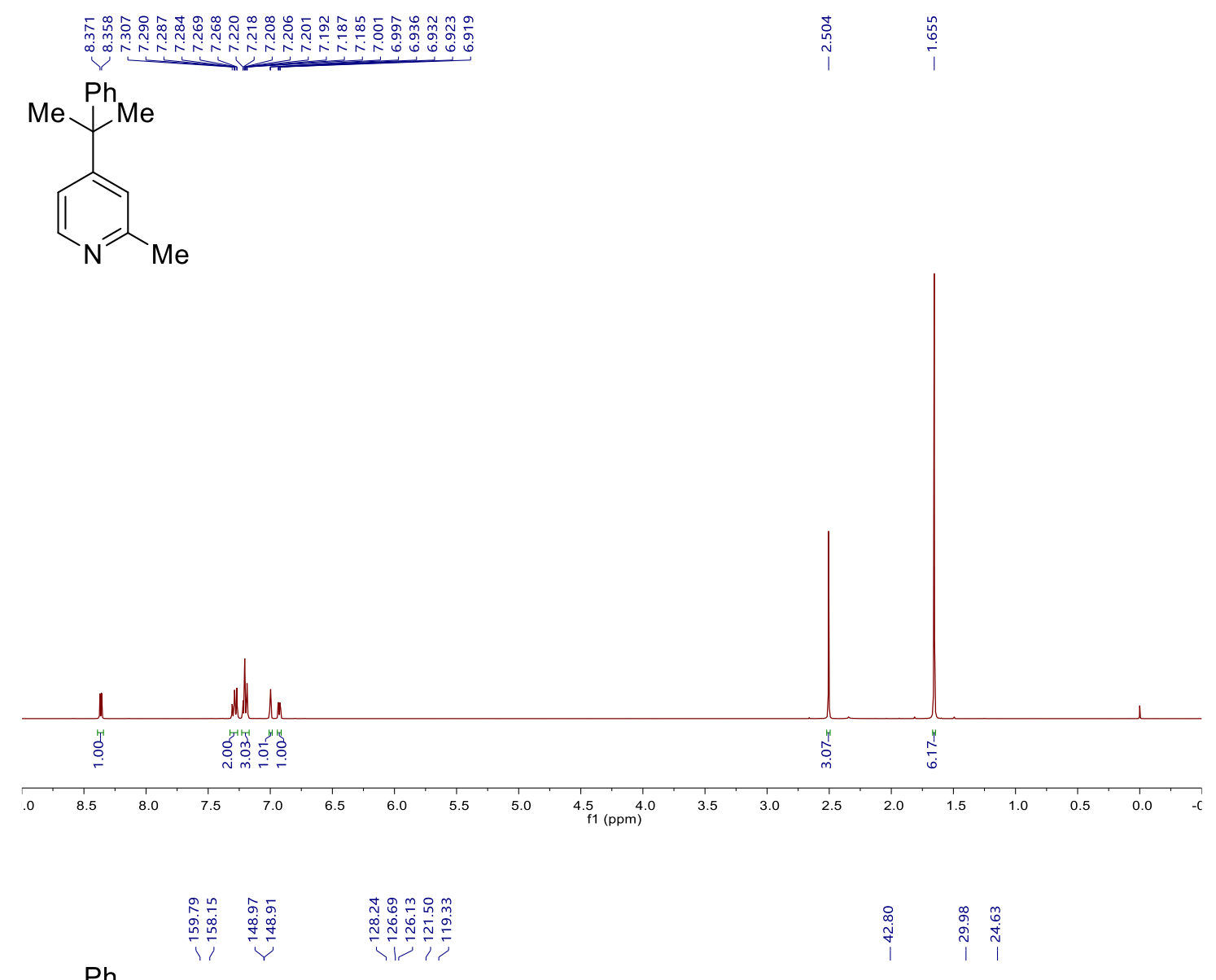<smiles>Cc1cc(C(C)(C)P)ccn1</smiles>

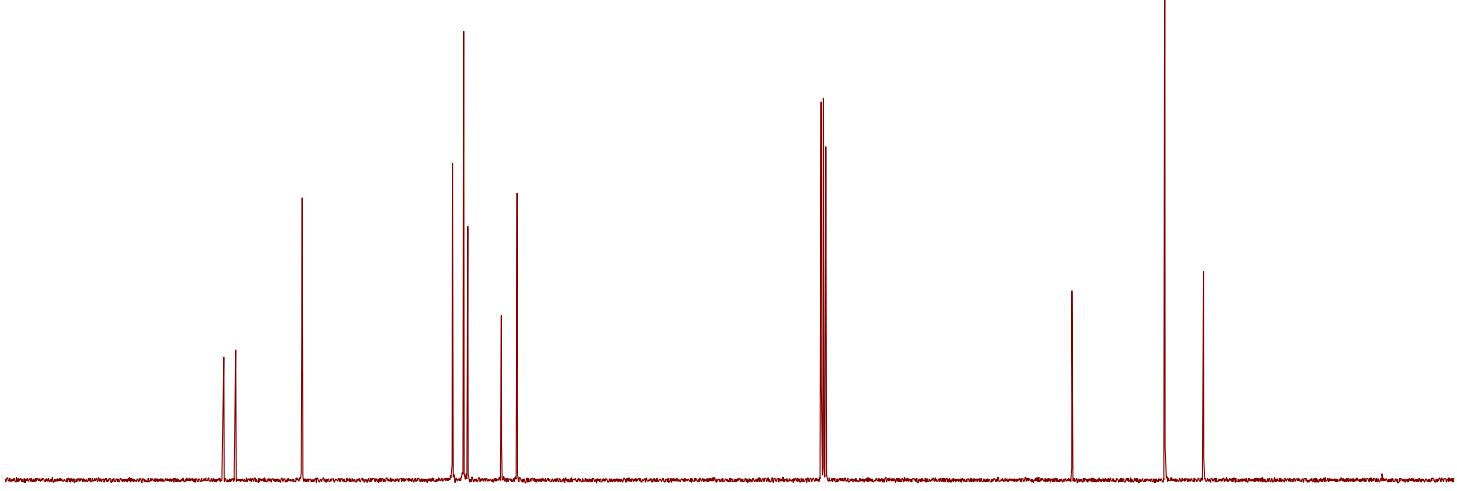

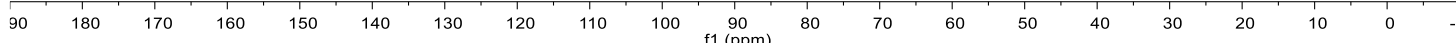


4-[(4-Methoxyphenyl)diphenyl]-2-methylpyridine (S3b)

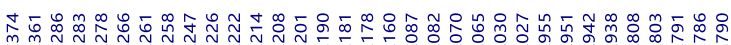

V)<smiles>Cc1cc(C(c2ccccc2)(c2ccccc2)[n+]2ccccc2)ccn1</smiles>

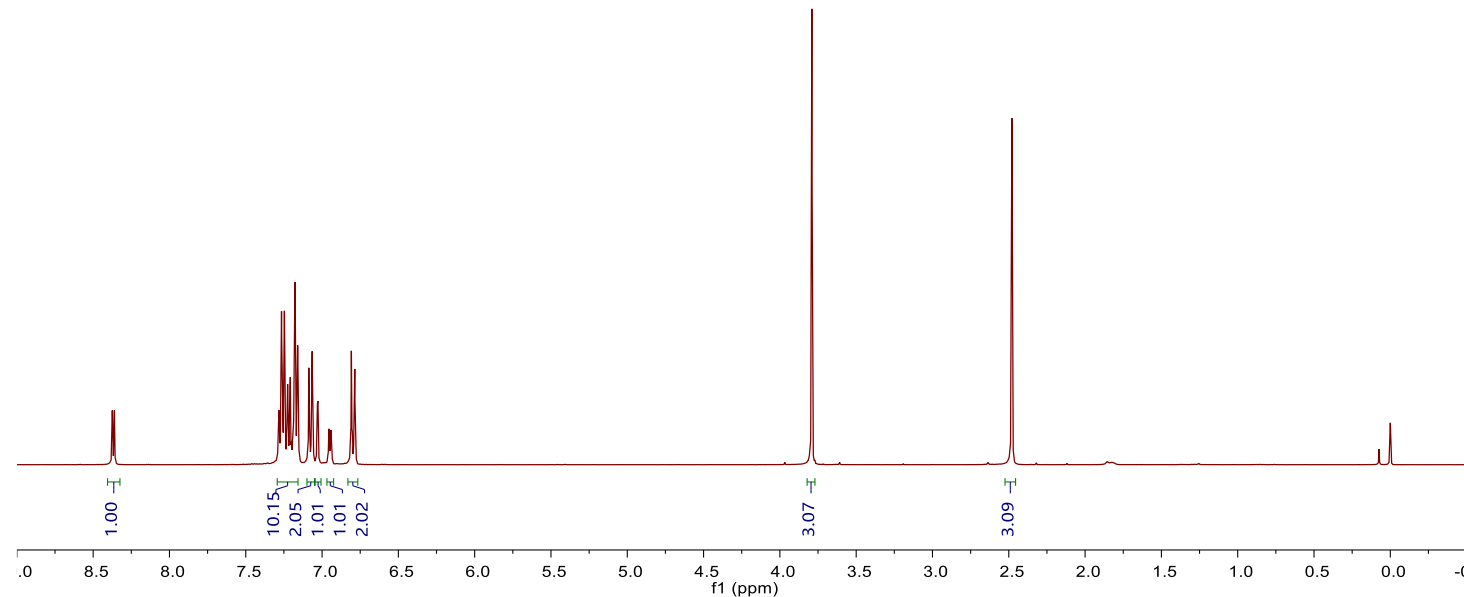

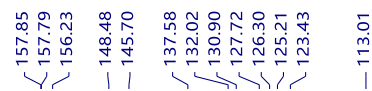

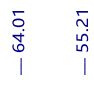

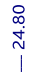<smiles>Cc1cc(C(c2ccccc2)(c2ccccc2)c2ccccc2)ccn1</smiles>

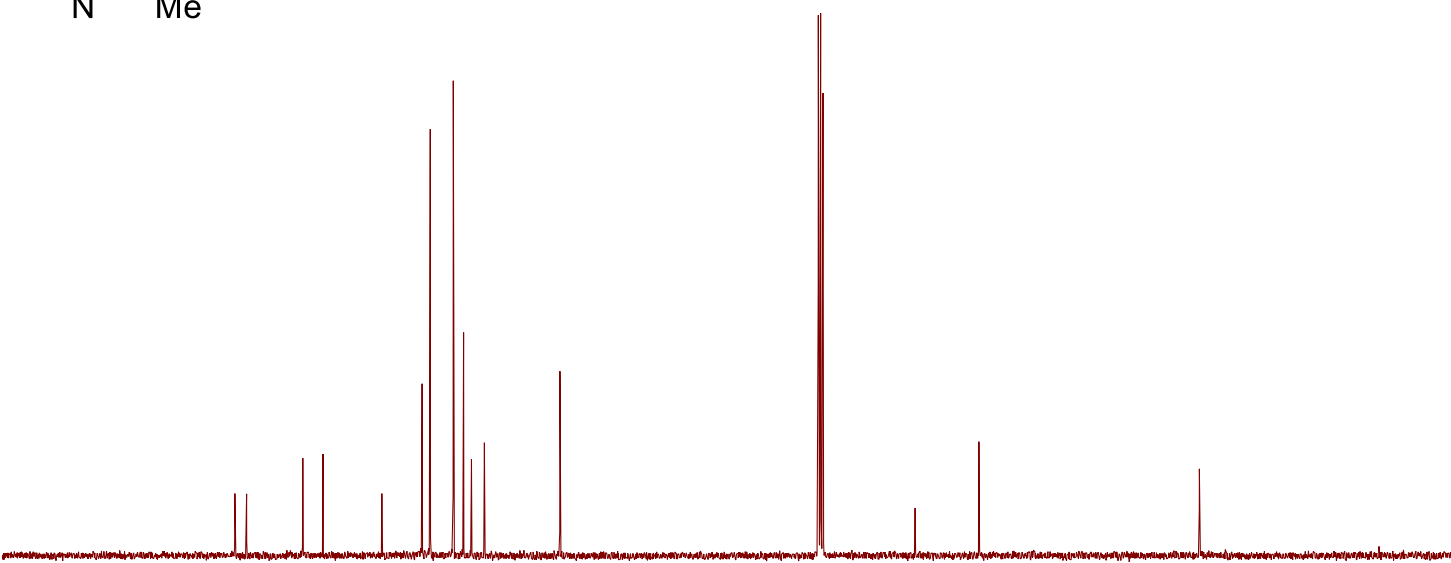

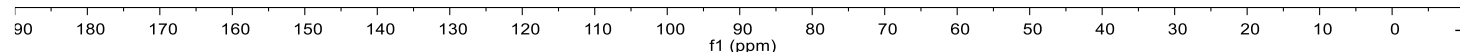


4-(2-Phenylpropan-2-yl)-2-pyridinecarbaldehyde (S2f)

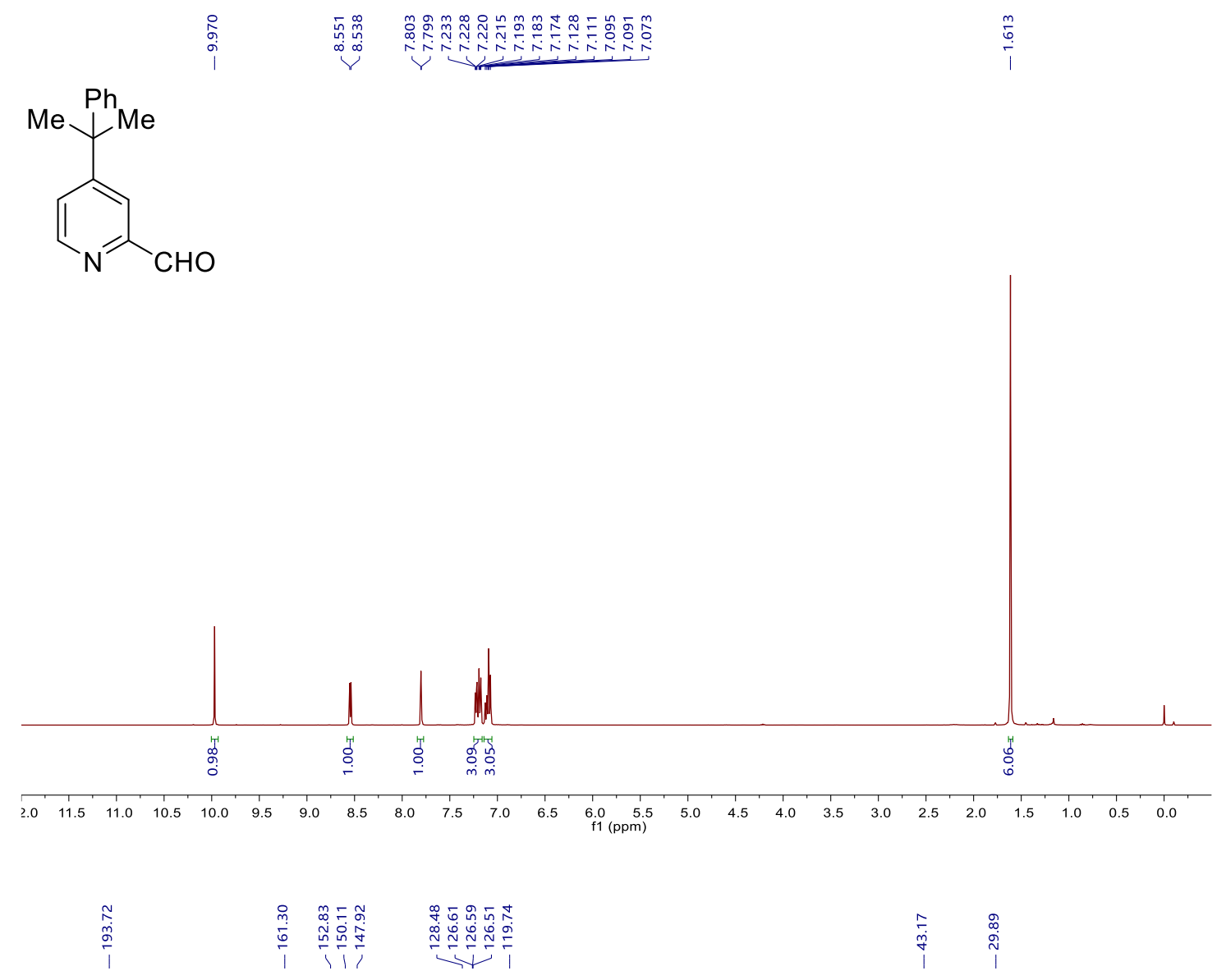<smiles>CC(C)(c1ccccc1)c1ccnc(C=O)c1</smiles>

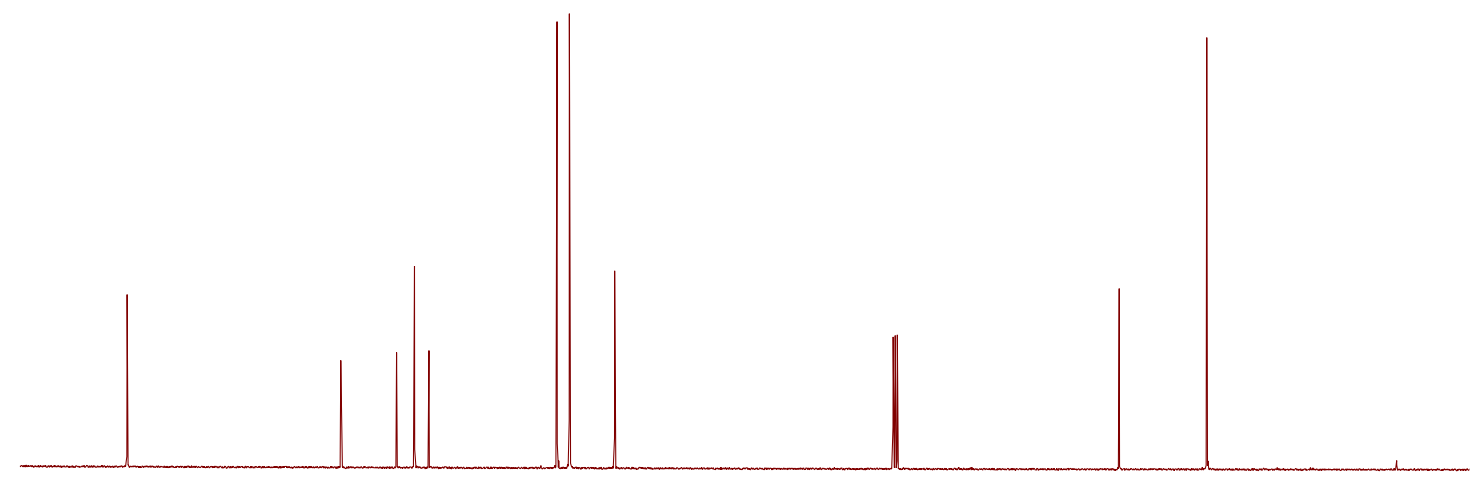

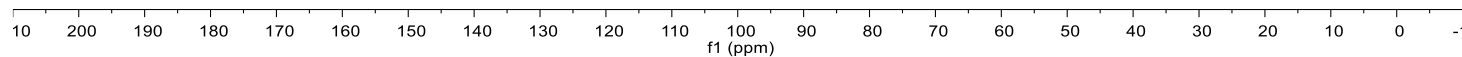


4-[(4-Methoxyphenyl)diphenyl]-2-pyridinecarbaldehyde (S2g)

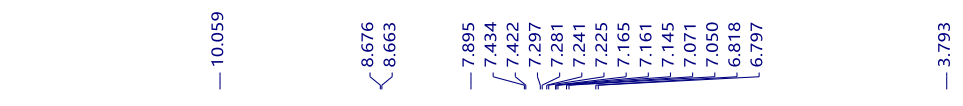<smiles>O=Cc1cc(C(c2ccccc2)(c2ccccc2)[n+]2ccccc2)ccn1</smiles>
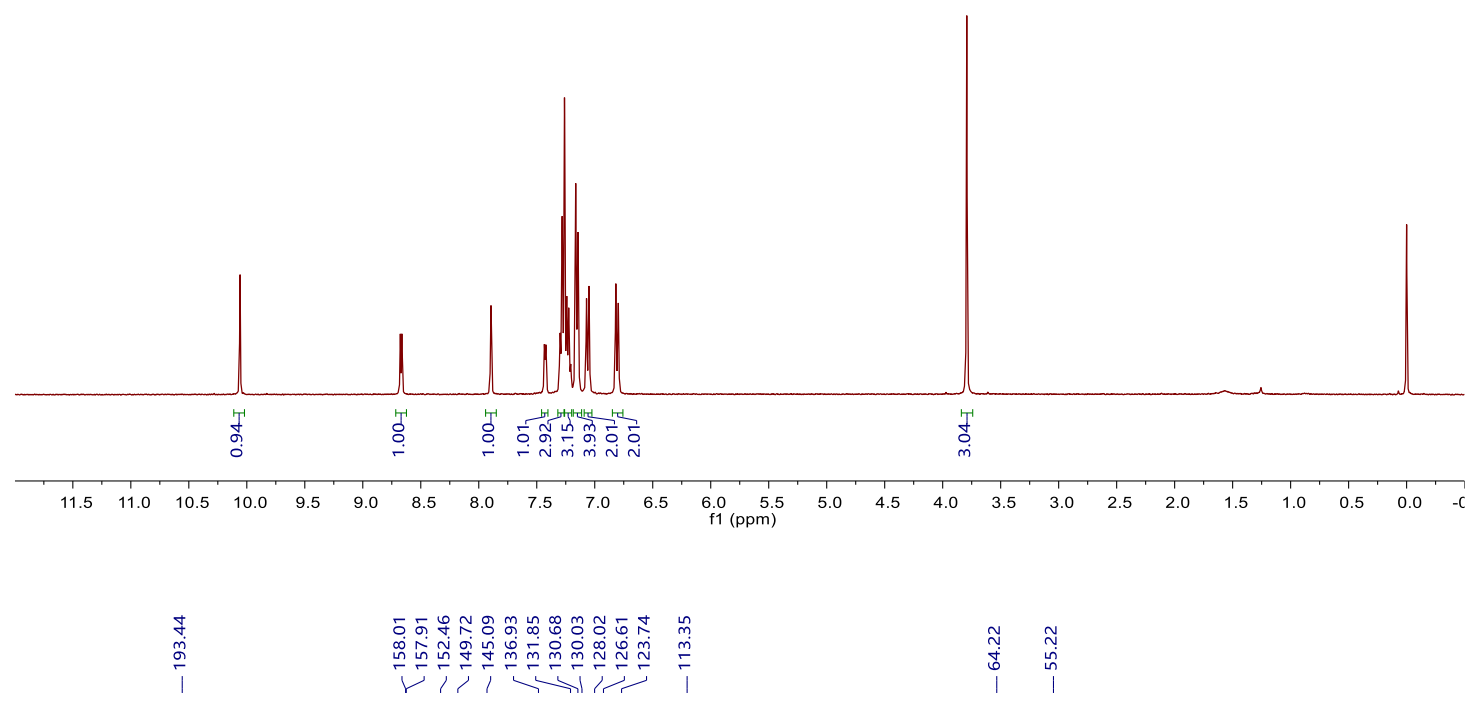<smiles>O=Cc1cc(C(c2ccccc2)(c2ccccc2)[n+]2ccccc2)ccn1</smiles>

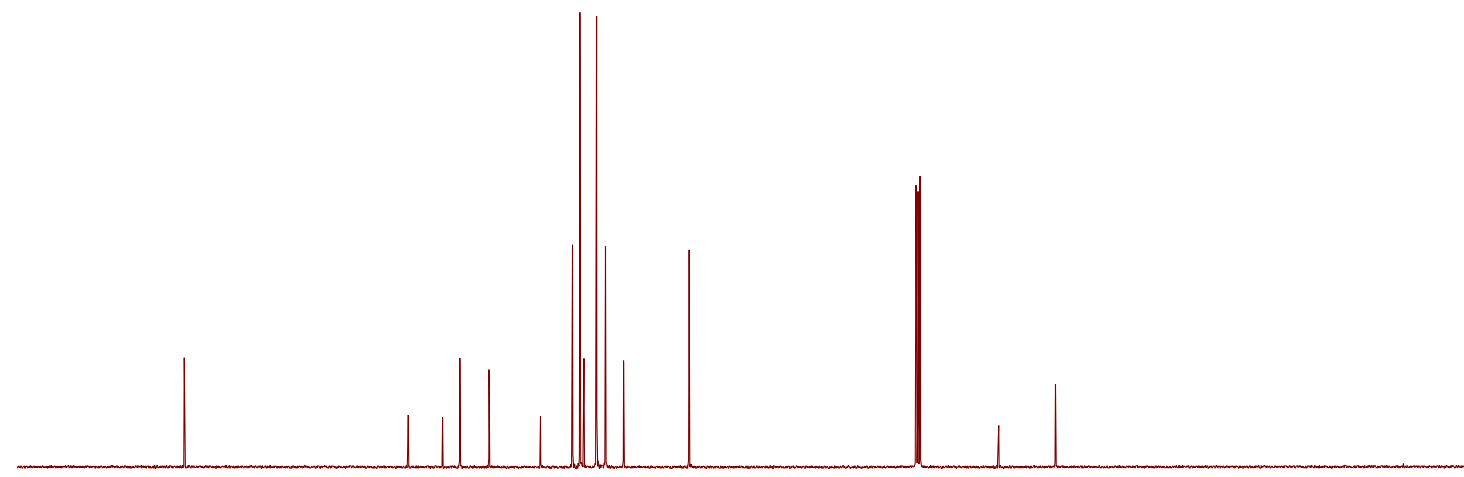

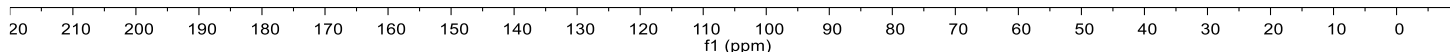


4-(3-Methoxypentan-3-yl)-2-methylpyridine (S4a)

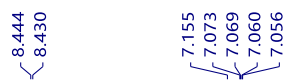

焉<smiles>CCC(CC)(OC)c1ccnc(C)c1</smiles>

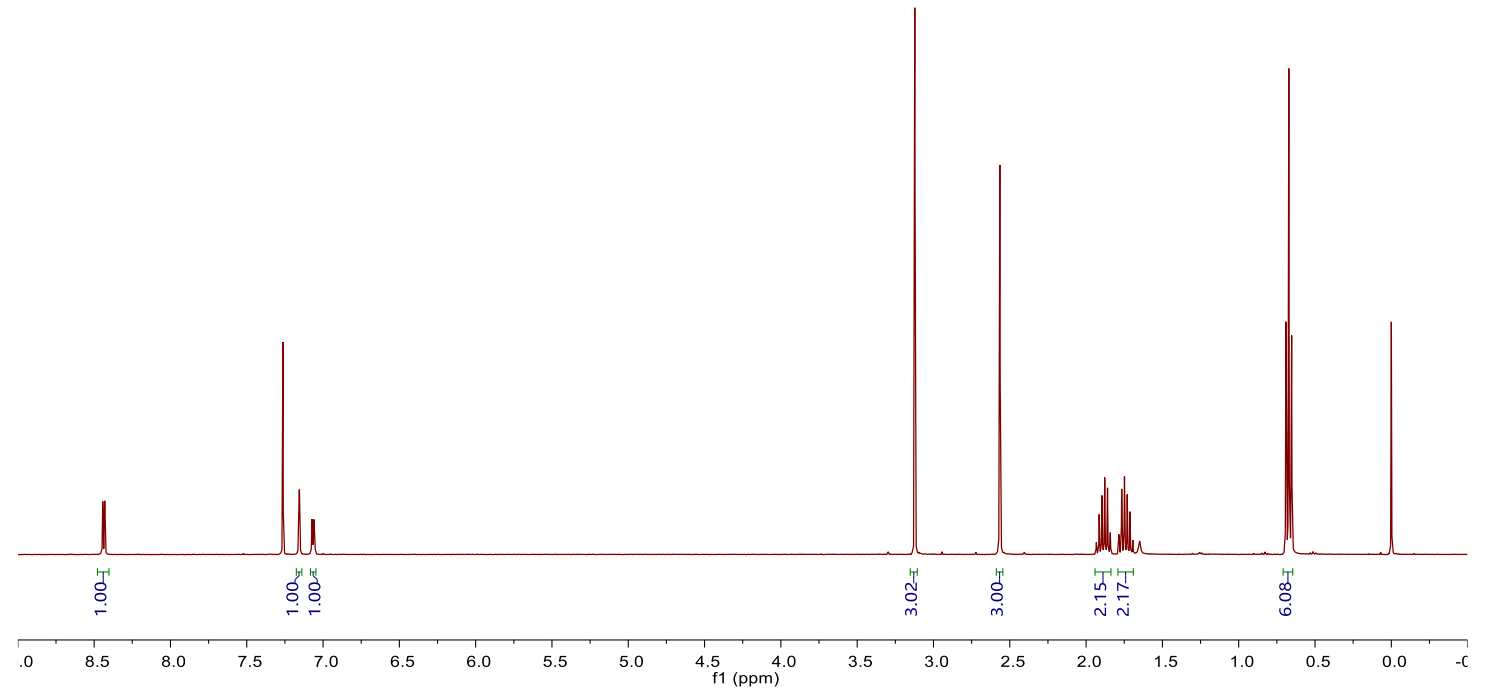

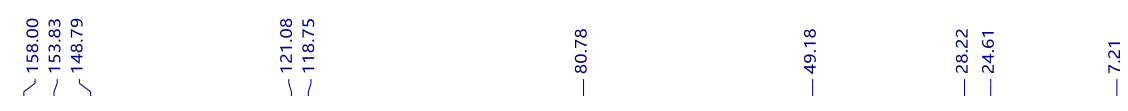

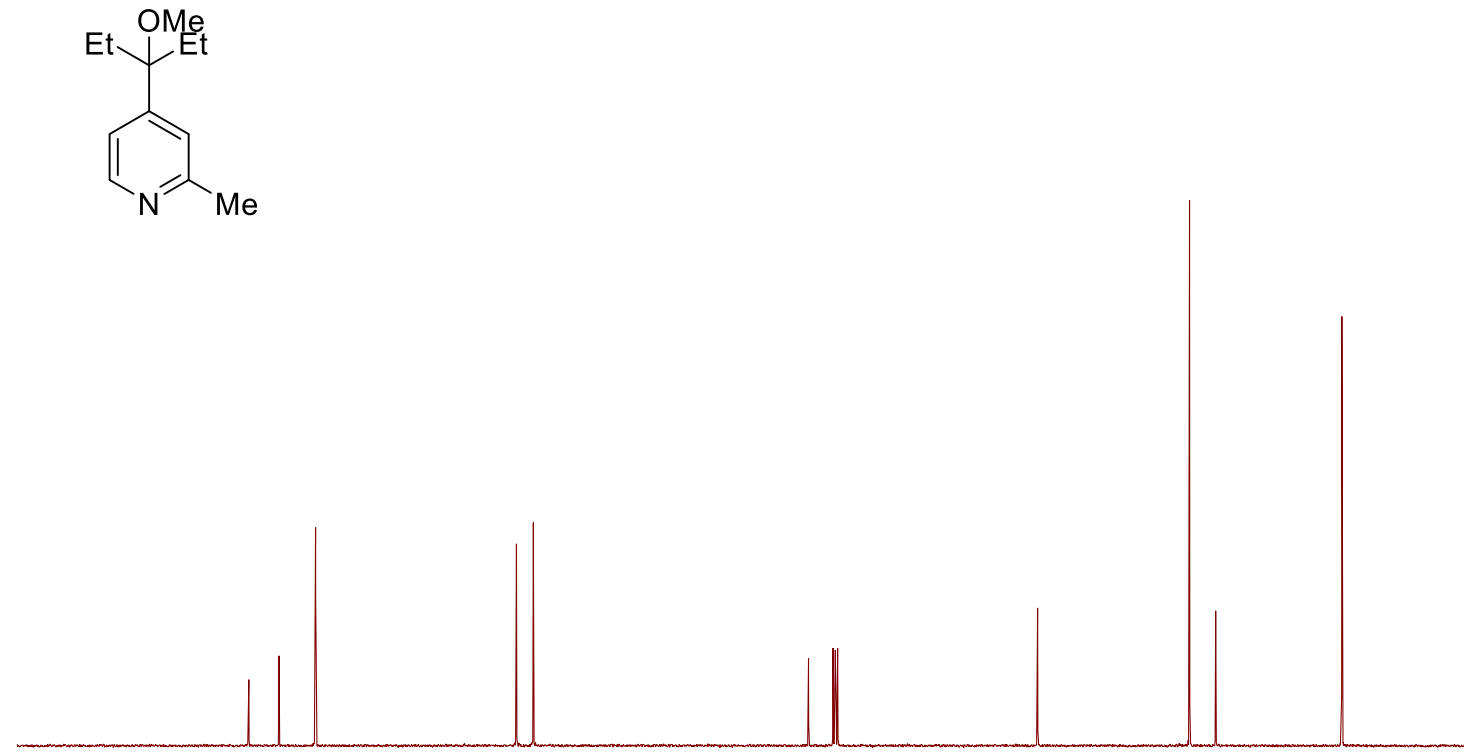

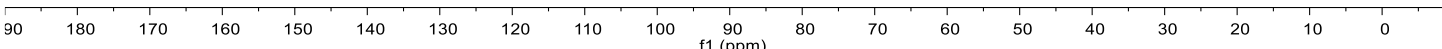


4-[Dicyclohexyl(methoxy)methyl]-2-methylpyridine (S4b)

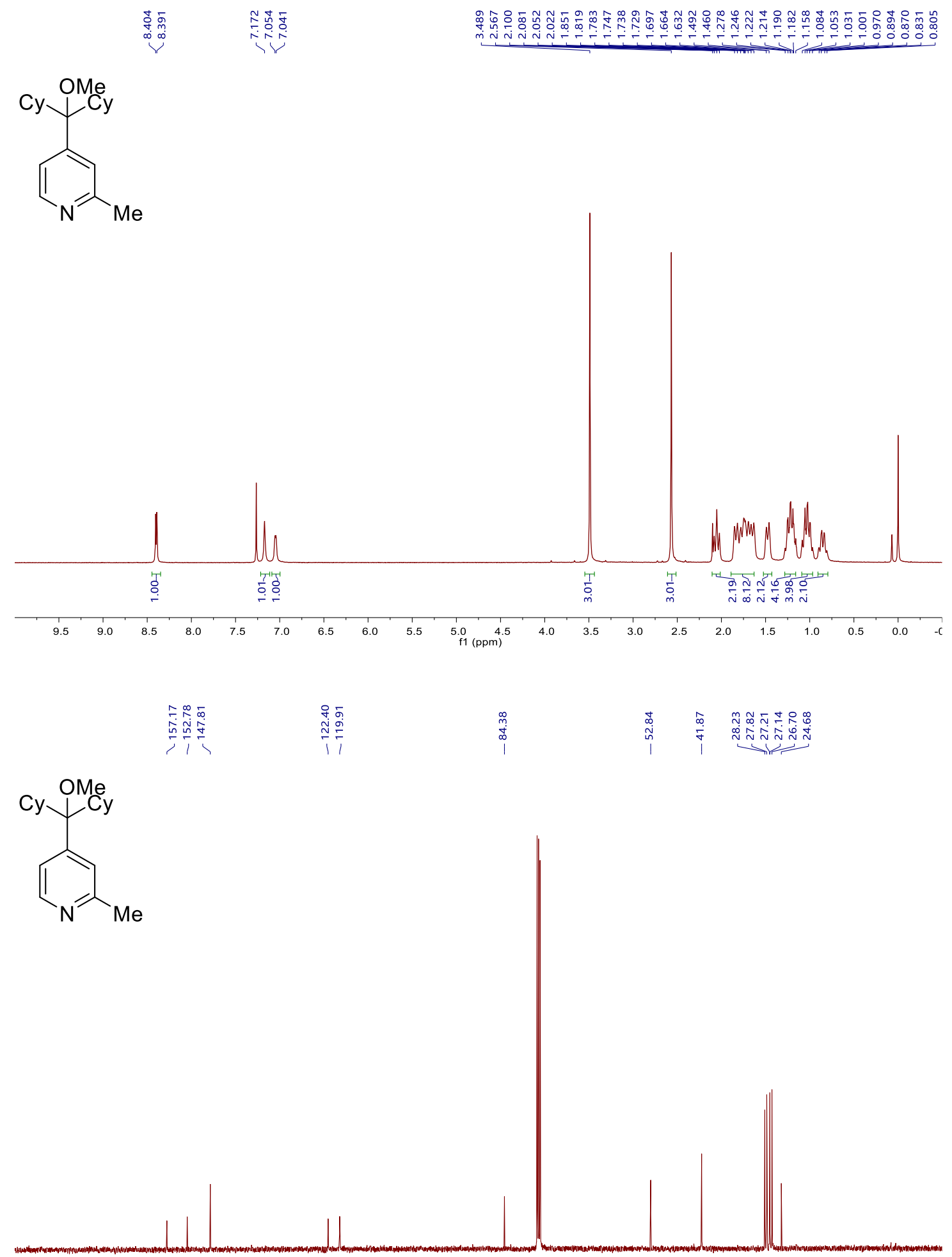

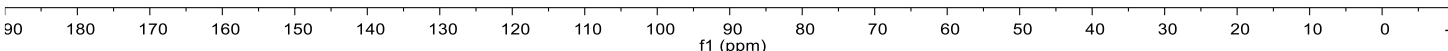


4-(3-Methoxypentan-3-yl)picolinaldehyde (S2h)
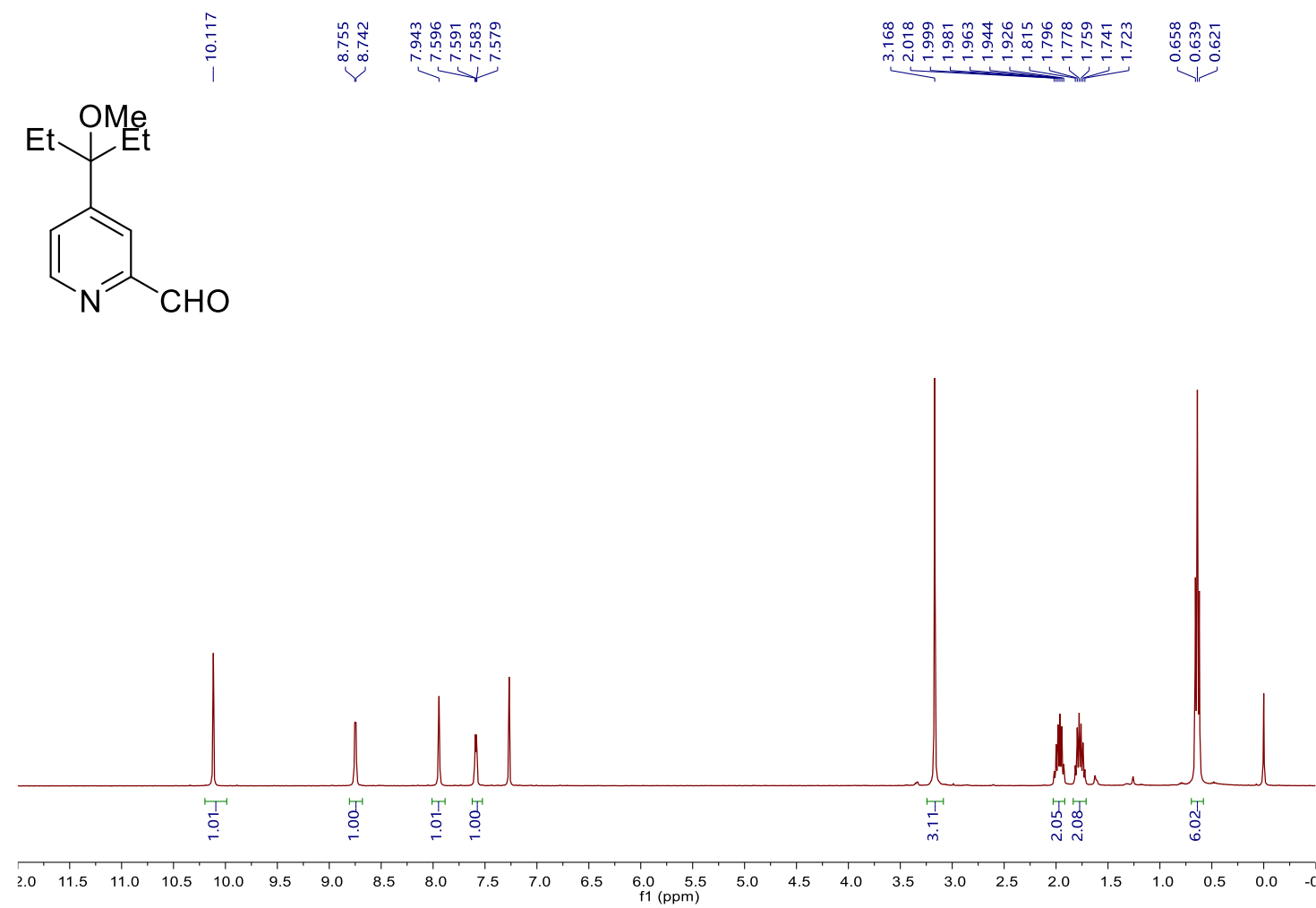

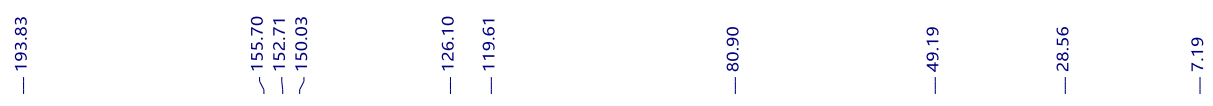<smiles>CCC(CC)(OC)c1ccnc(C=O)c1</smiles>

$\begin{array}{llllllllllll}210 & 200 & 190 & 180 & 170 & 160 & 150 & 140 & 130 & 120 & \begin{array}{l}110 \\ \mathrm{f} 1(\mathrm{ppm})\end{array}\end{array}$ 
4-[Dicyclohexyl(methoxy)methyl]picolinaldehyde (S2i)
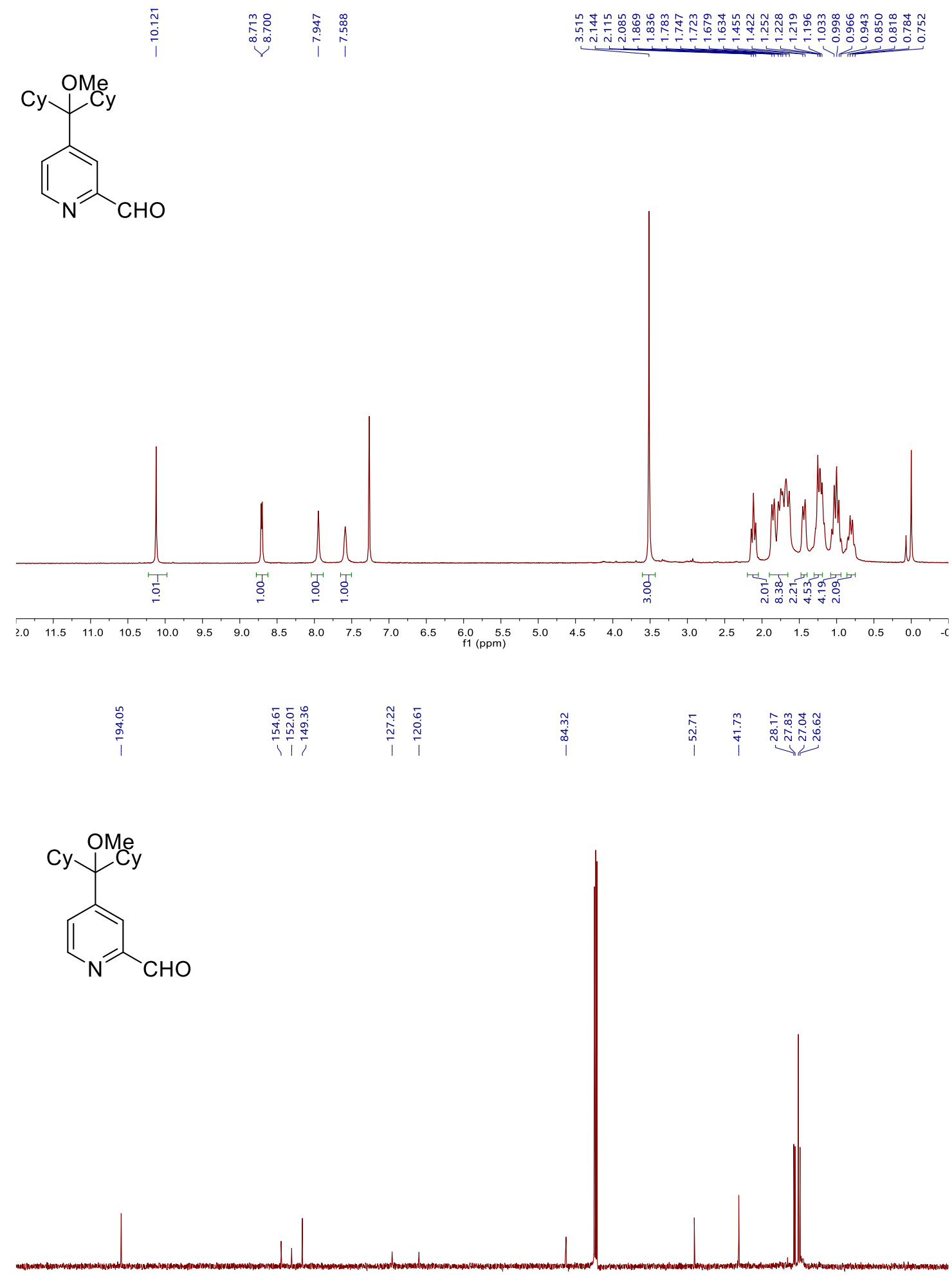

\begin{tabular}{llllllllllllllllllllllll}
\hline & 210 & 200 & 190 & 180 & 170 & 160 & 150 & 140 & 130 & 120 & 110 & 100 & 90 & 80 & 70 & 60 & 50 & 40 & 30 & 20 & 10 & 0 & 1
\end{tabular} 


\section{Ligand $(R)-S 5 g$ (3-iPr)}

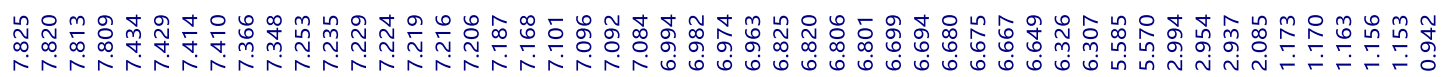

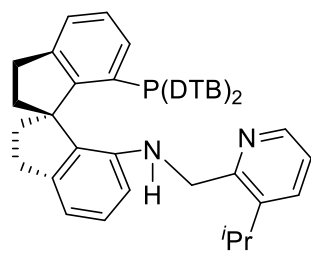

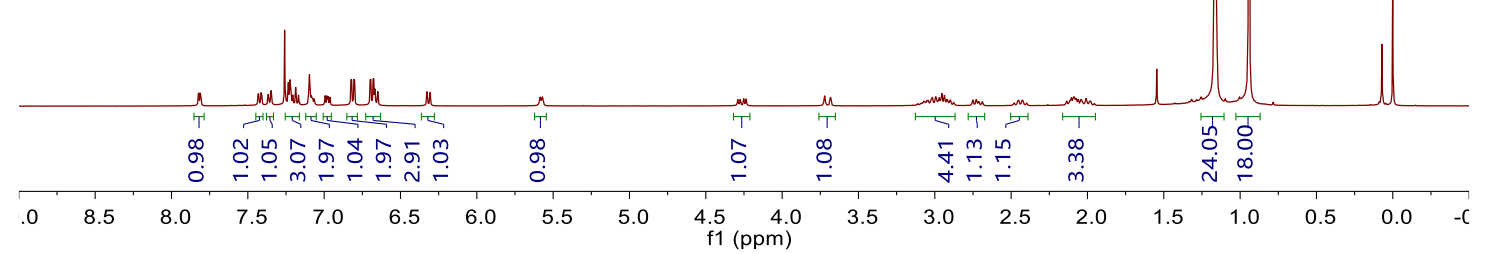

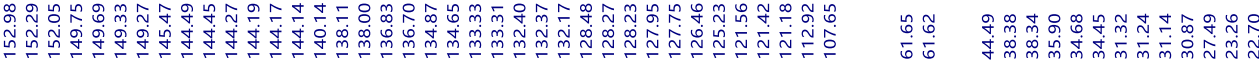
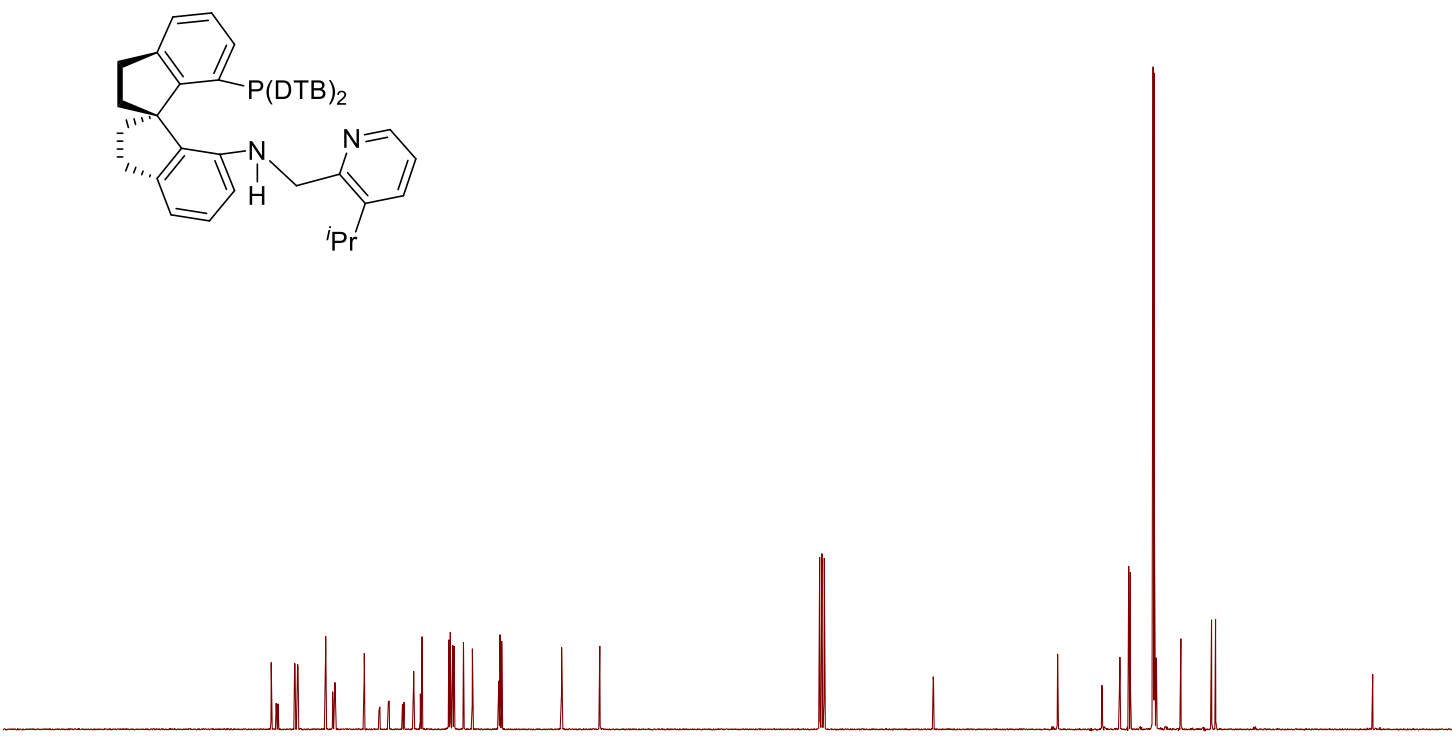

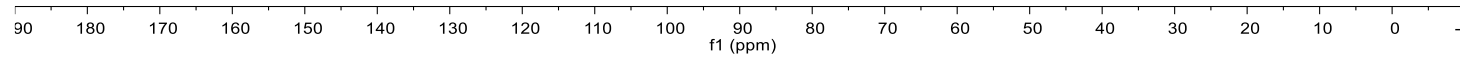



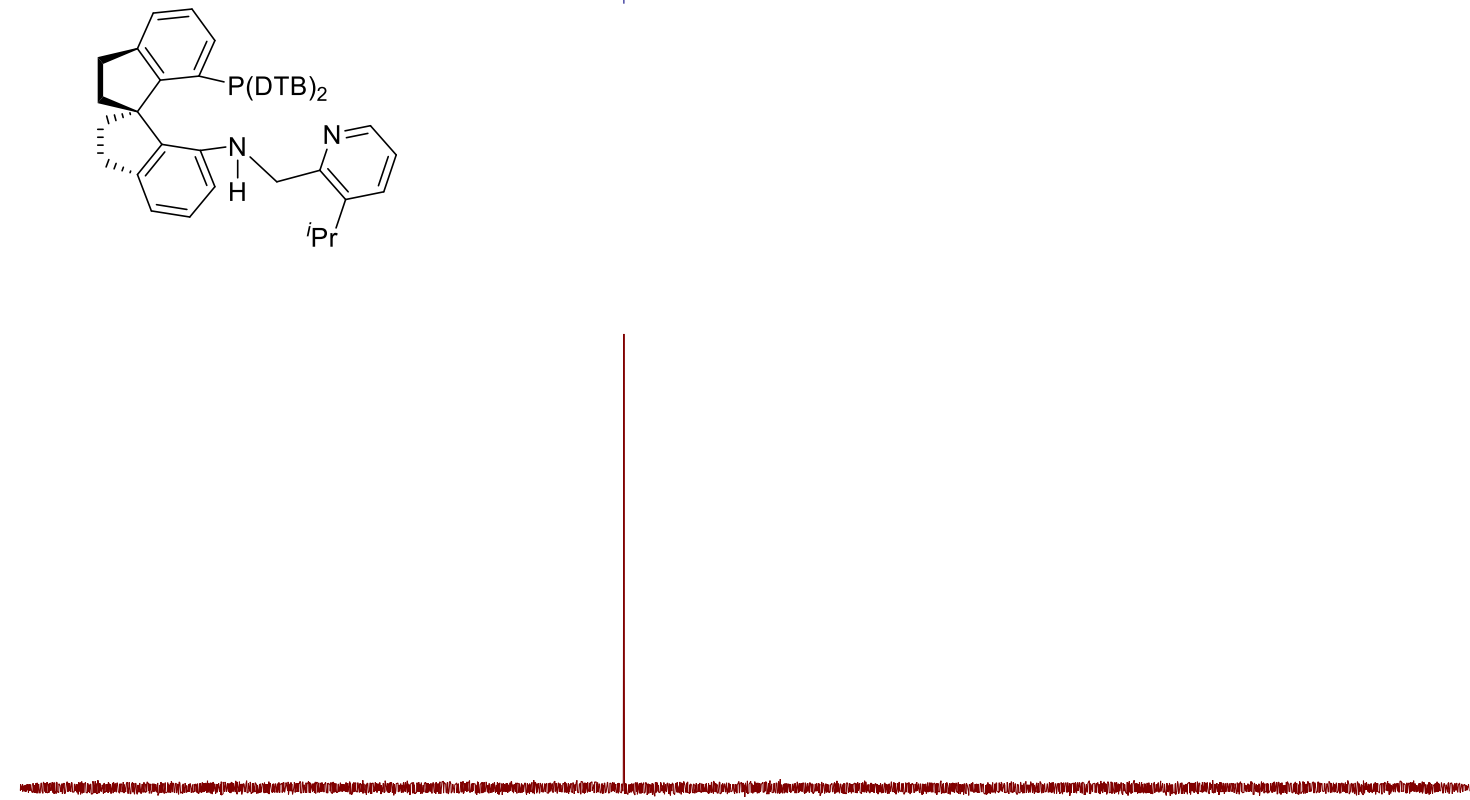

$\begin{array}{llllllllllllllllllllll}140 & 120 & 100 & 80 & 60 & 40 & 20 & 0 & -20 & -40 & -60 & -80 & -100 & -120 & -140 & -160 & -180 & -200 & -220 & -240\end{array}$

\section{Ligand (R)-S5j (4-Et $2(\mathrm{HO}) \mathrm{C})$}

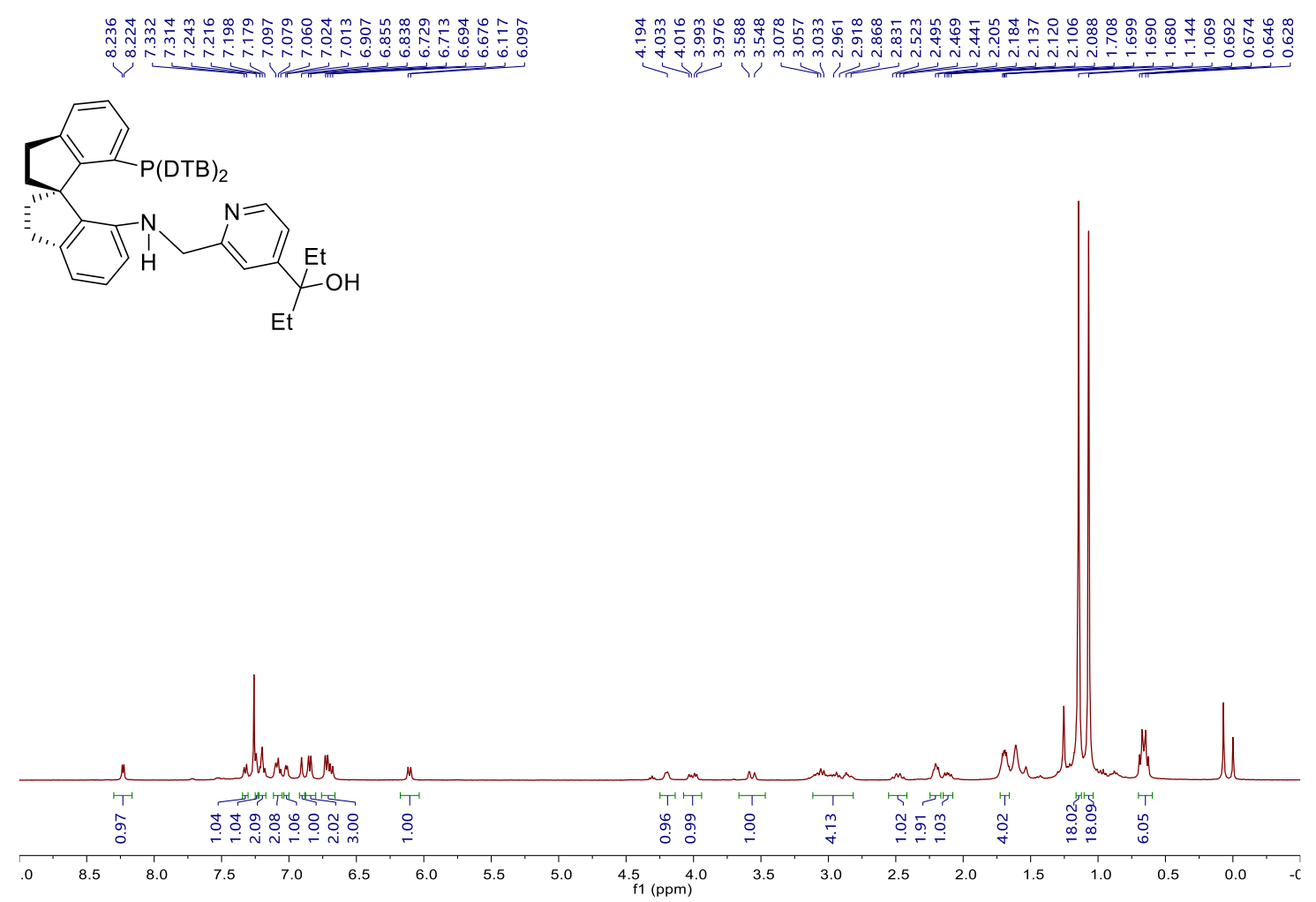



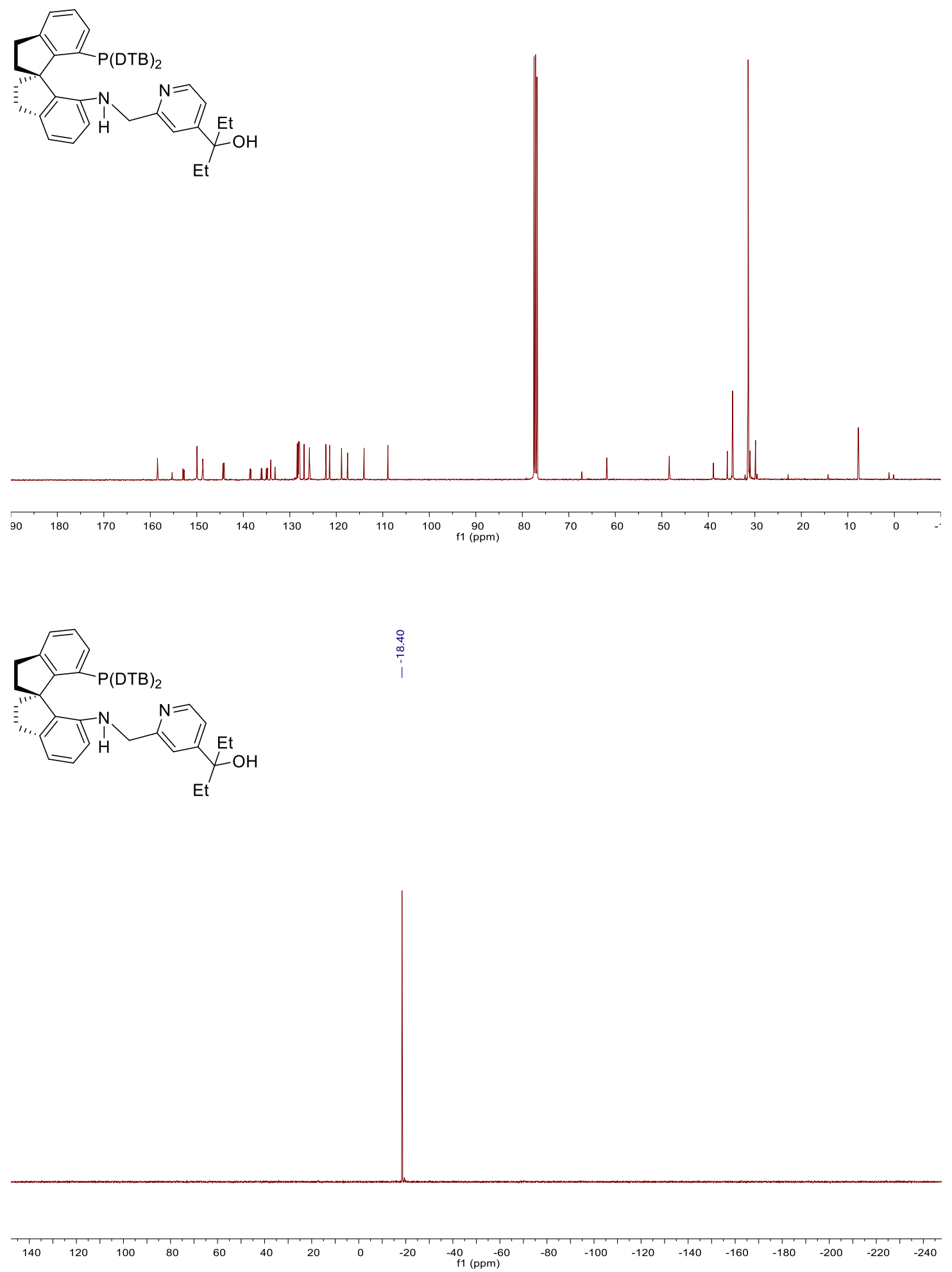
Ligand (R)-S5k (4-Et $2(\mathrm{MeO}) \mathrm{C})$

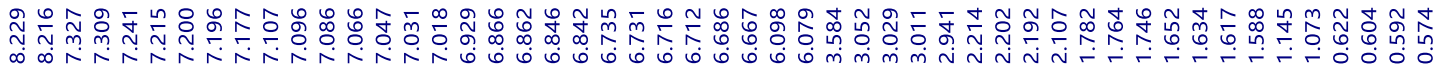

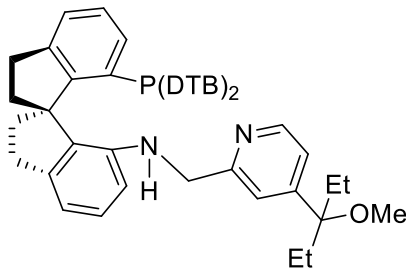

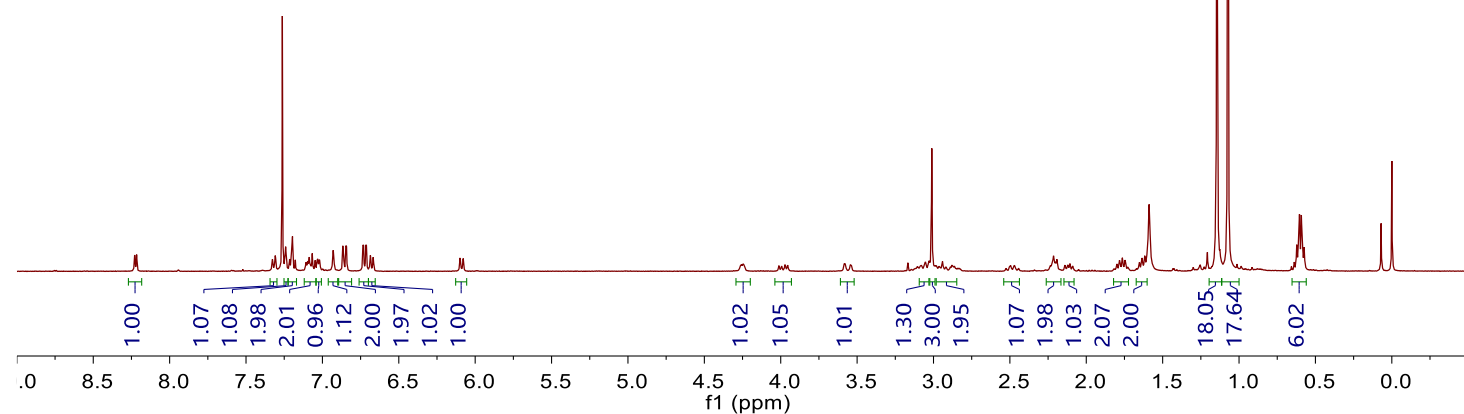
m

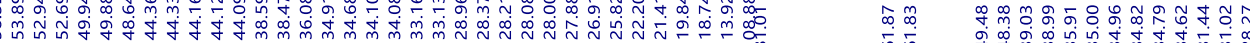
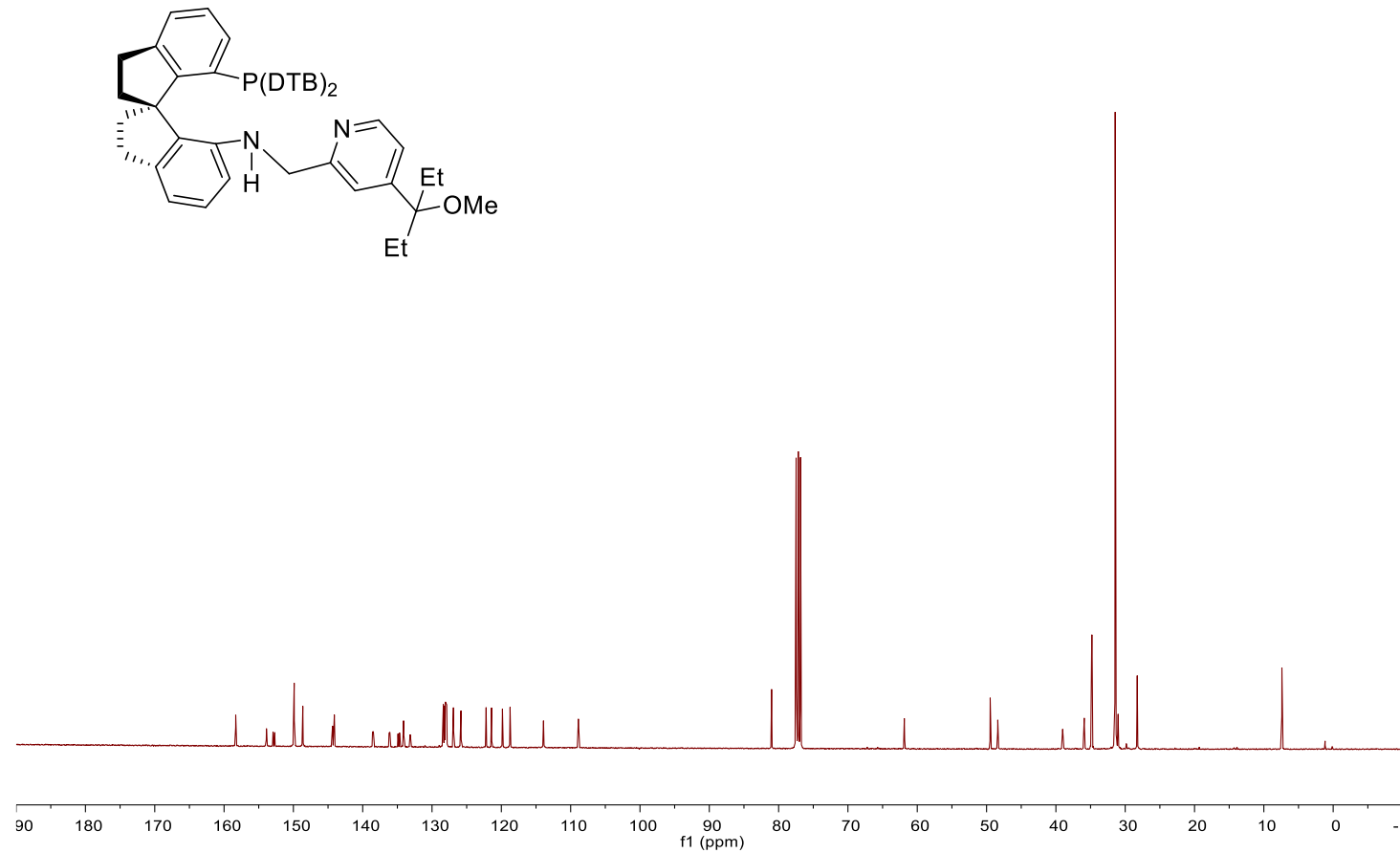


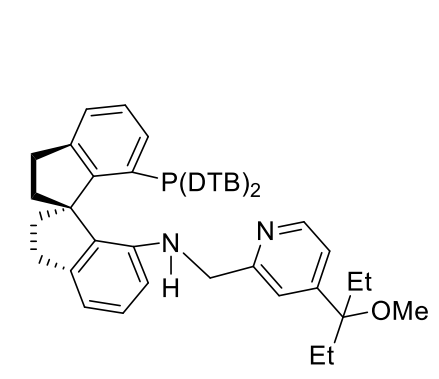

$\frac{8}{i}$ 


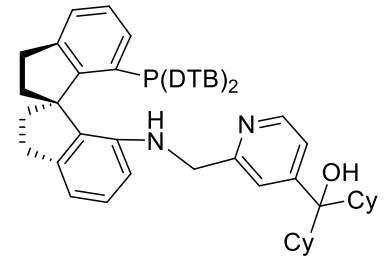

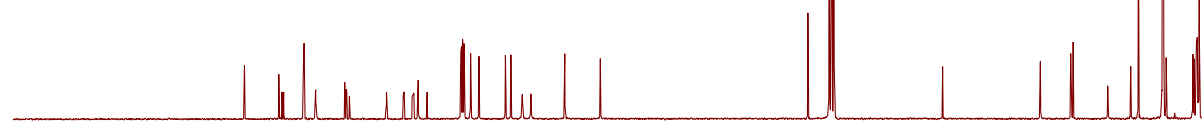

$180 \quad 170 \quad 160$
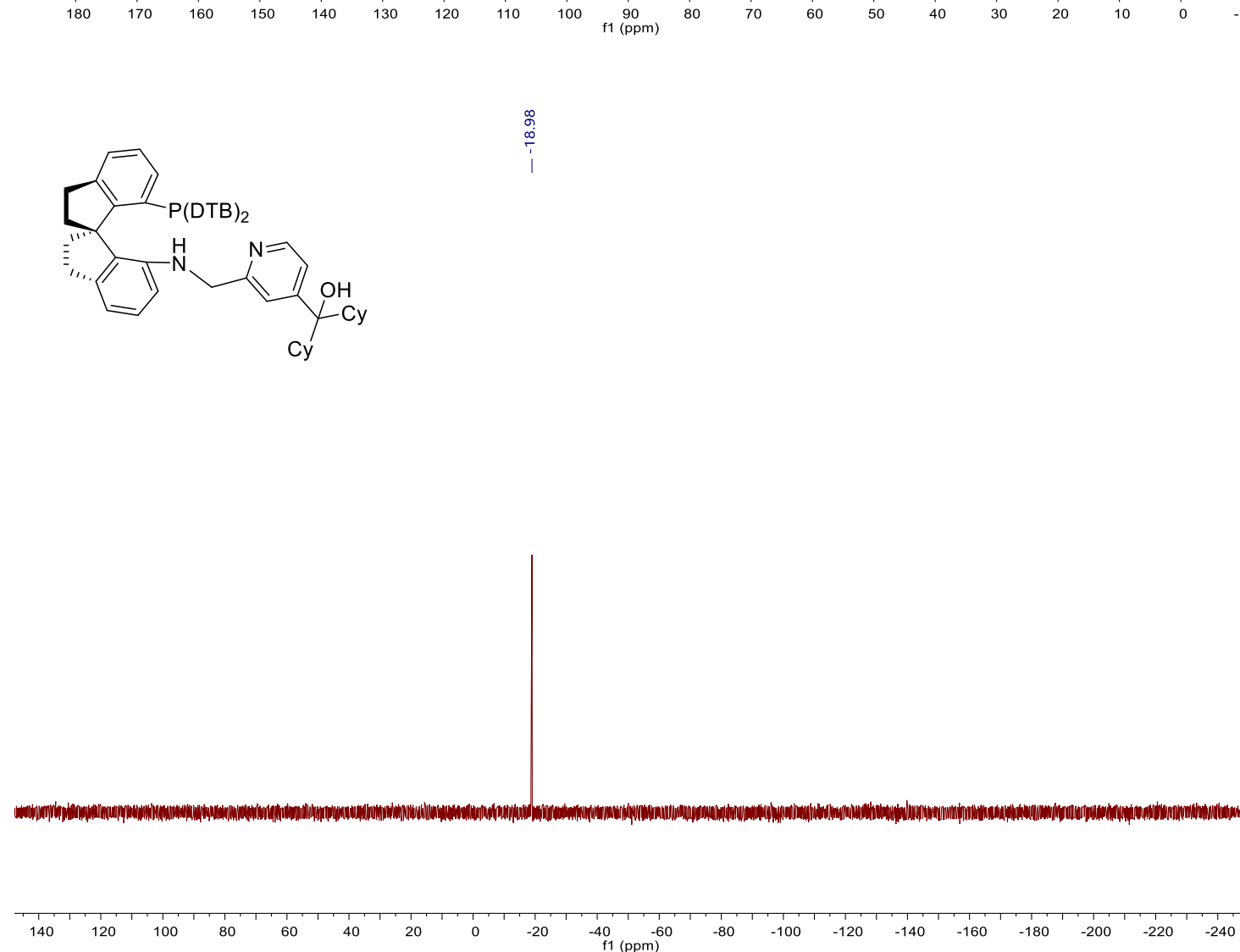


\section{Ligand (R)-S5m (4-Cy2(MeO)C)}

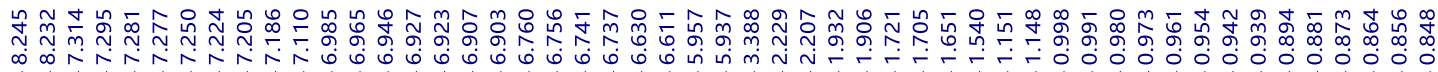<smiles>COC(C)(Cl)c1ccnc(CNc2cccc3c2C2c4ccccc4C[C@@H]2C3)c1</smiles>
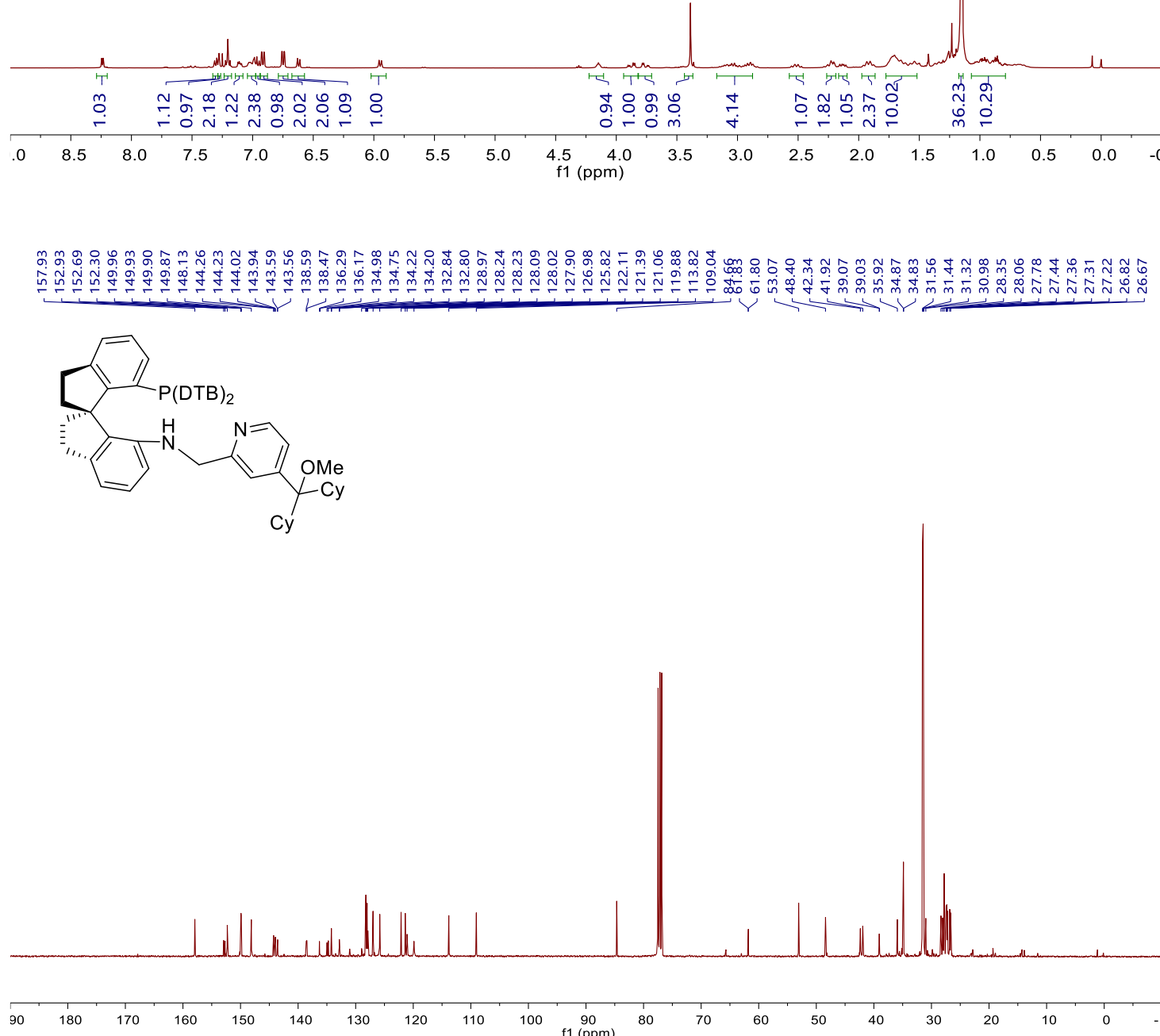

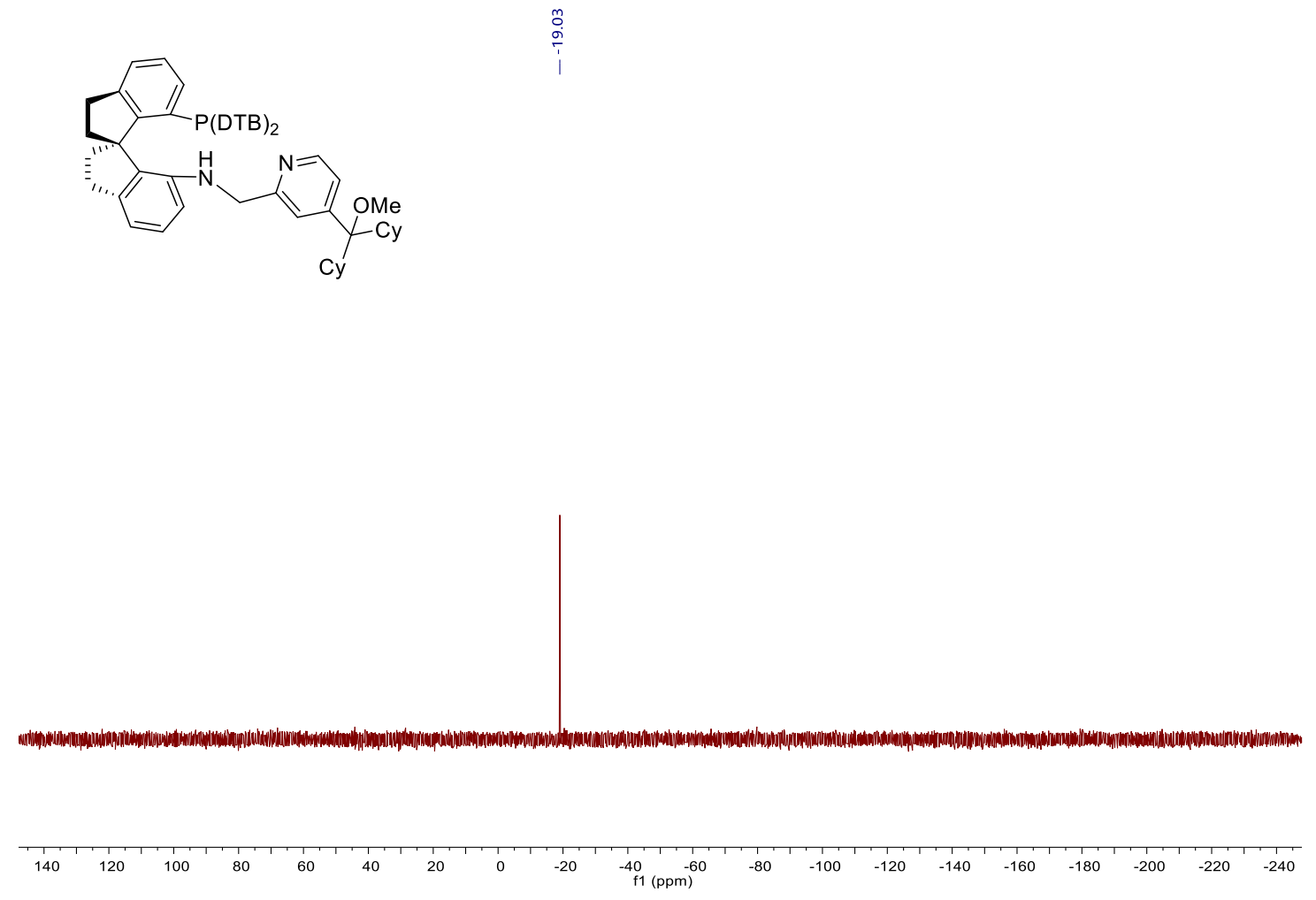

Ligand (R)-S5n (4-Ph $2(\mathrm{HO}) \mathrm{C})$

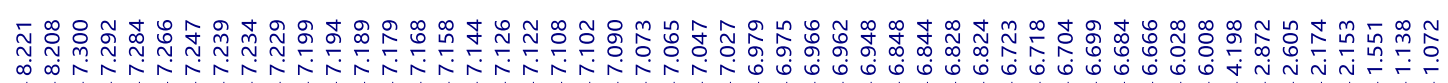

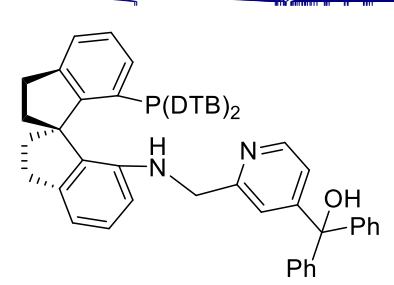

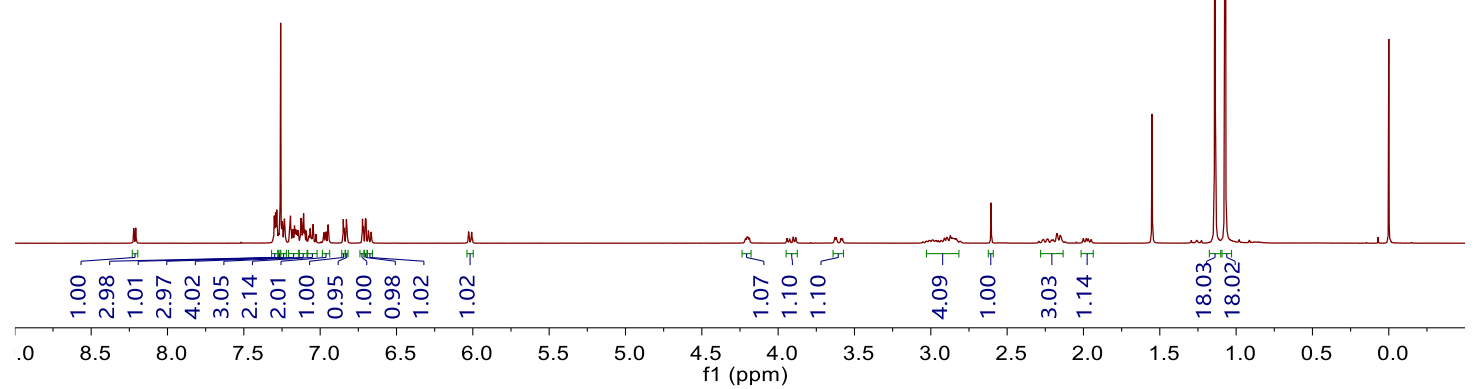



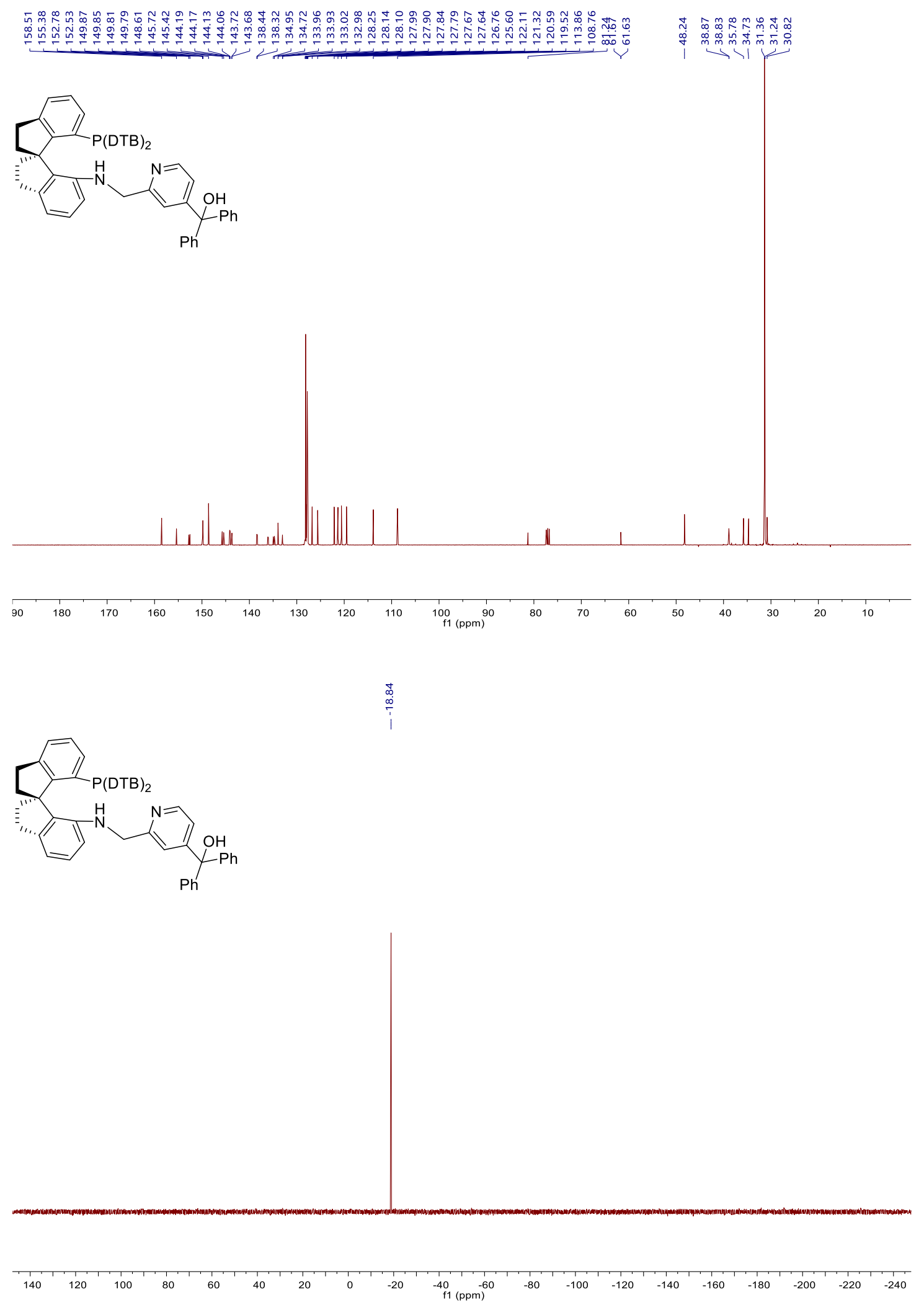


\section{Ligand $(R)-\mathrm{S} 50\left(4-\left(3,5-\mathrm{Me}_{2} \mathrm{C}_{6} \mathrm{H}_{3}\right)_{2}(\mathrm{HO}) \mathrm{C}\right)$}

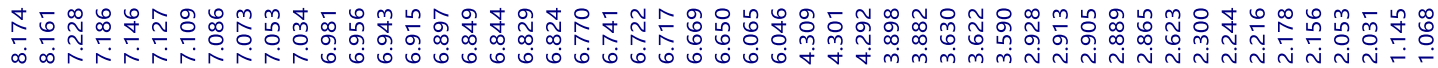

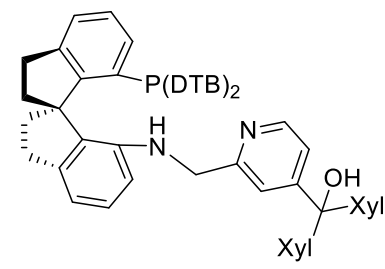

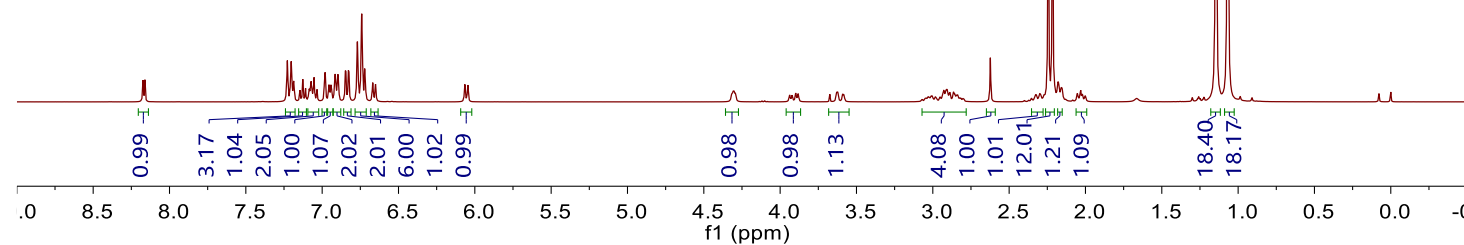

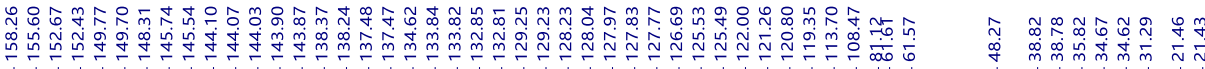<smiles>[Y14]C([Y14])(O)c1ccnc(CNc2cccc3c2[C@@H]2CC[C@H]3C2)c1</smiles>
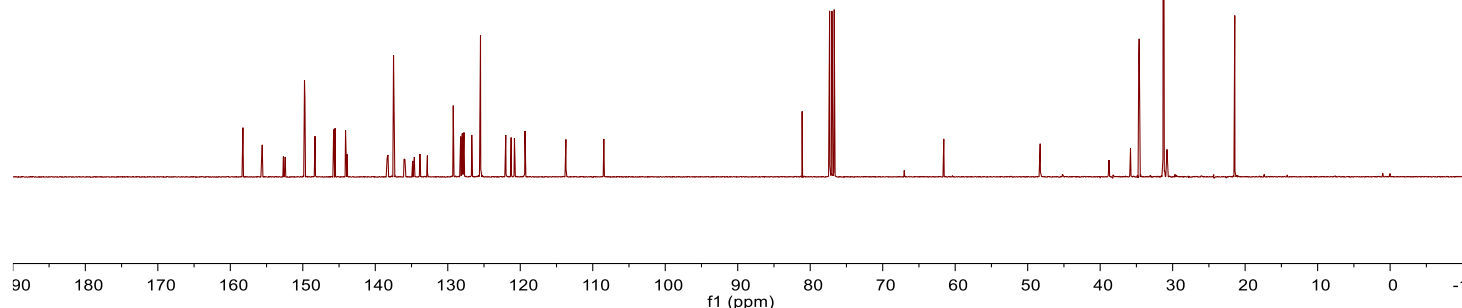

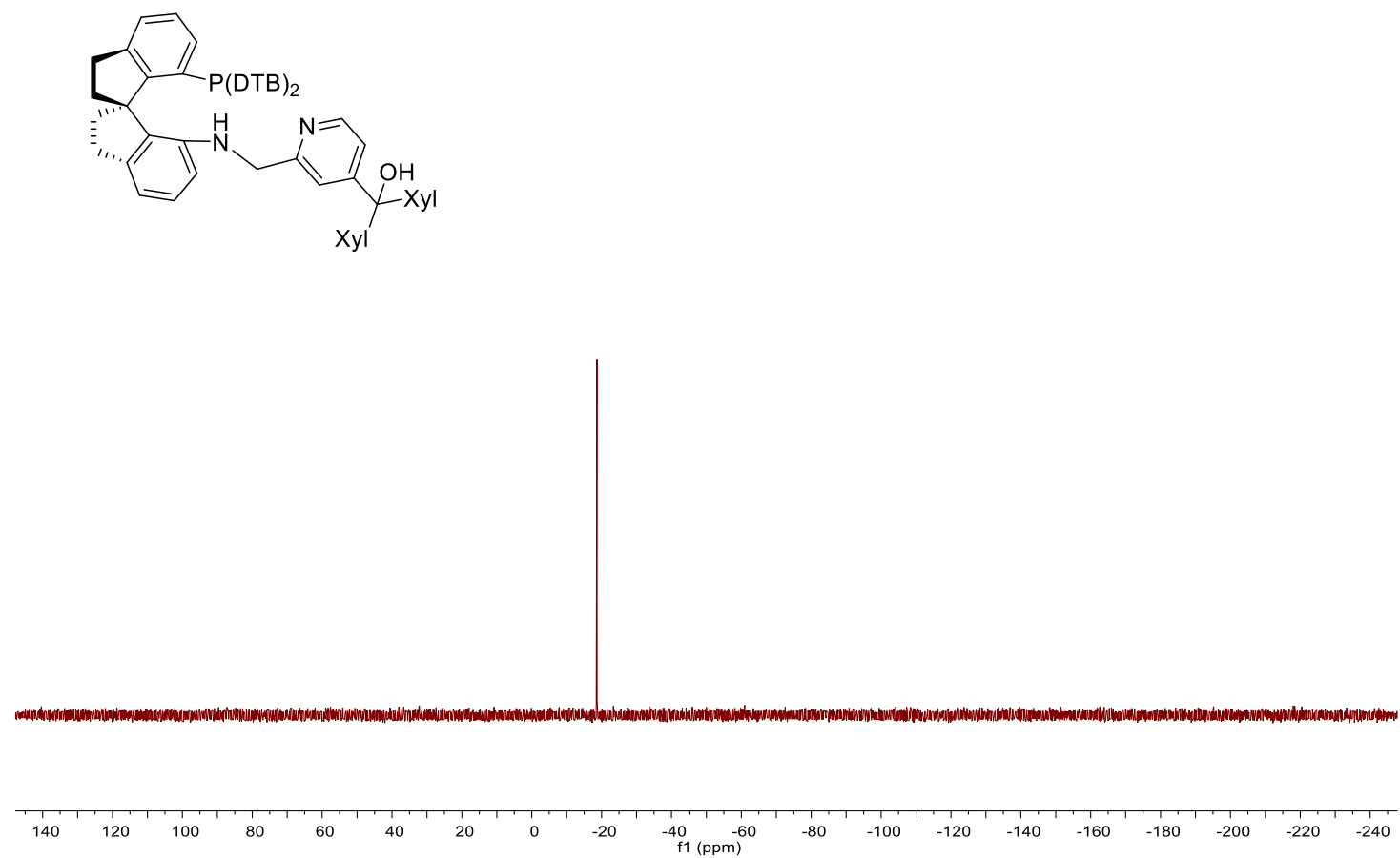

\section{Ligand $(R)-\mathrm{S} 5 \mathrm{p}\left(4-\left(3,5-(t \mathrm{Bu})_{2} \mathrm{C}_{6} \mathrm{H}_{3}\right)_{2}(\mathrm{HO}) \mathrm{C}\right)$}

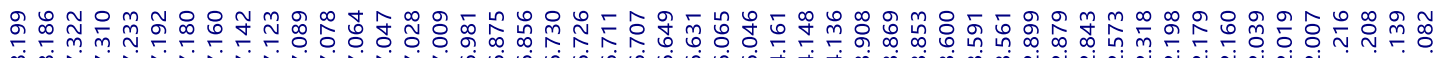
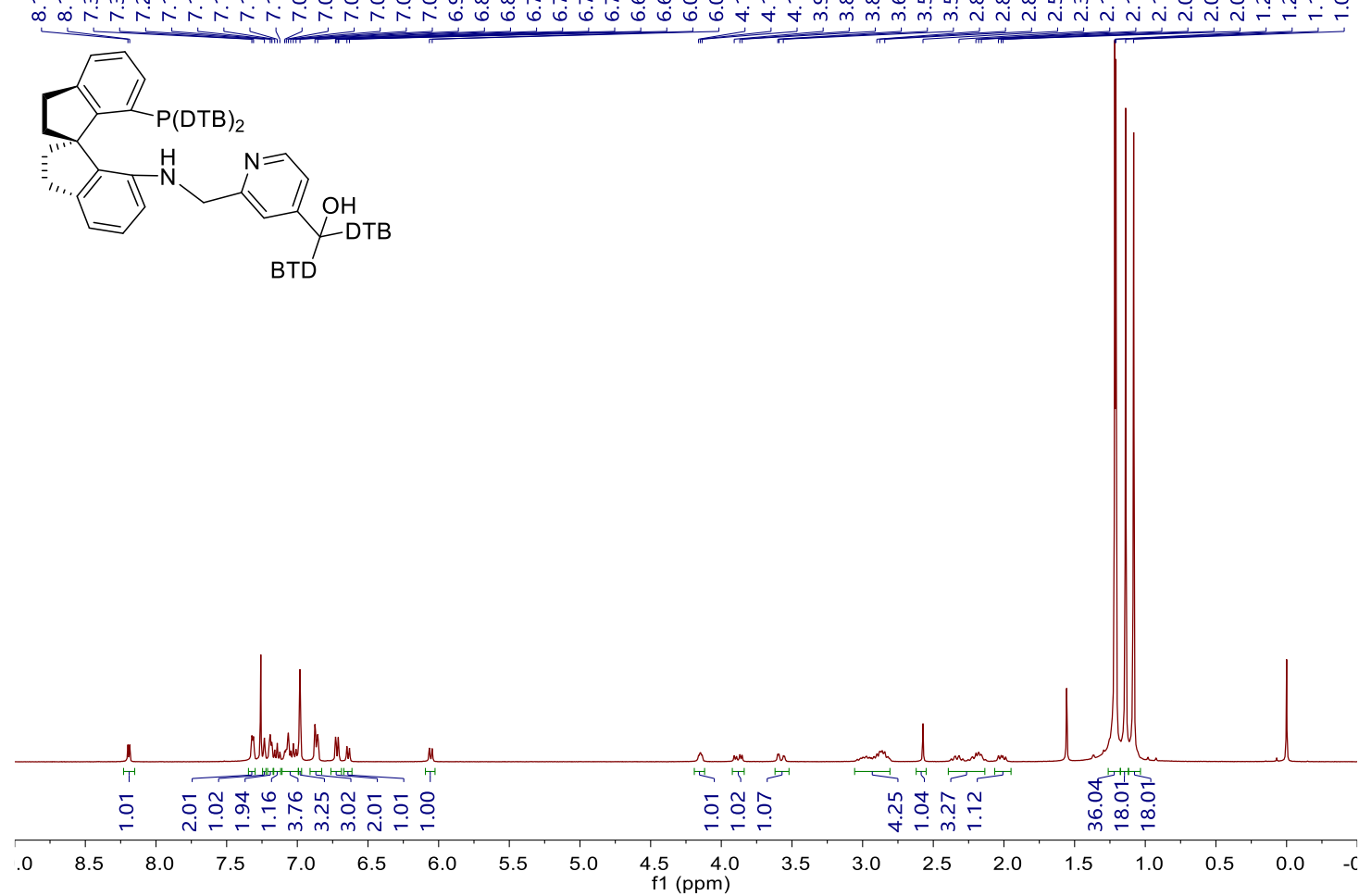

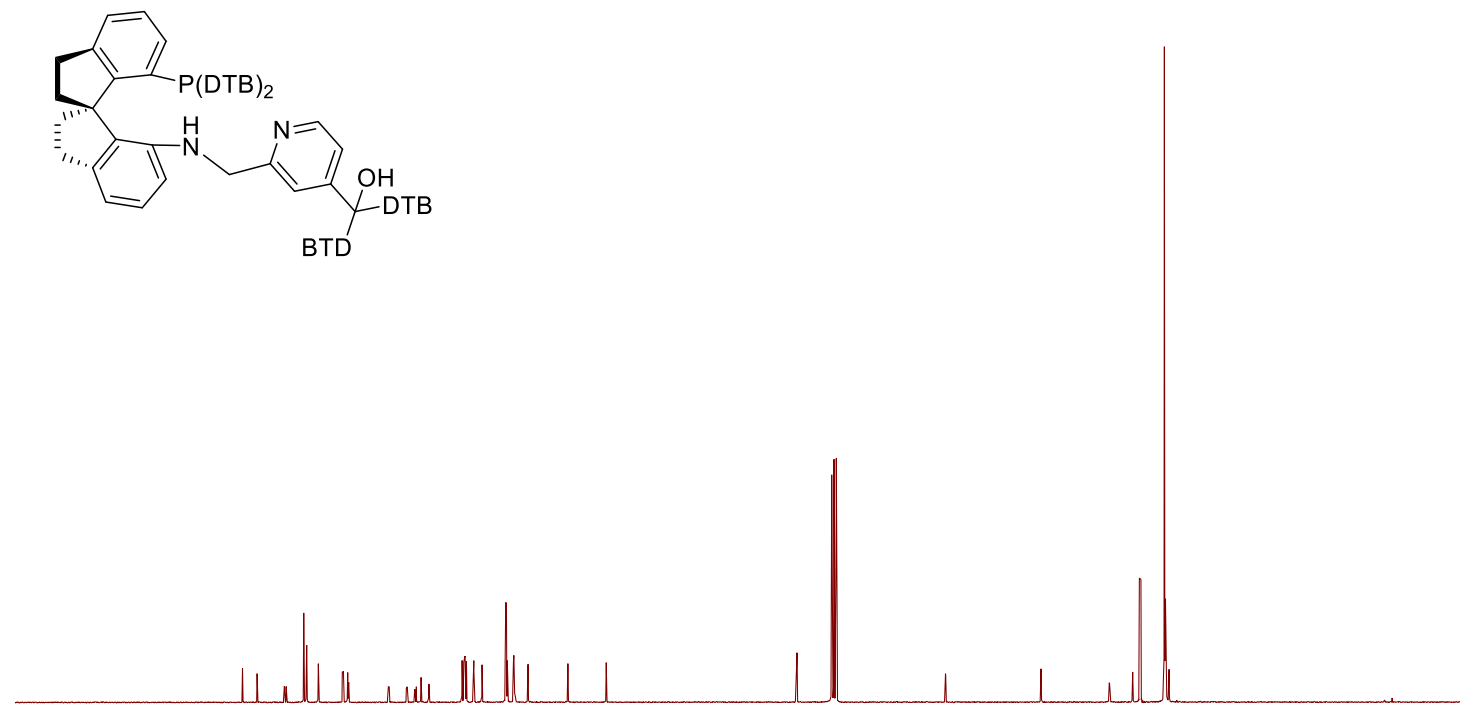

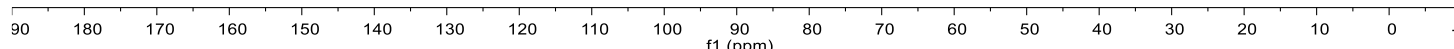

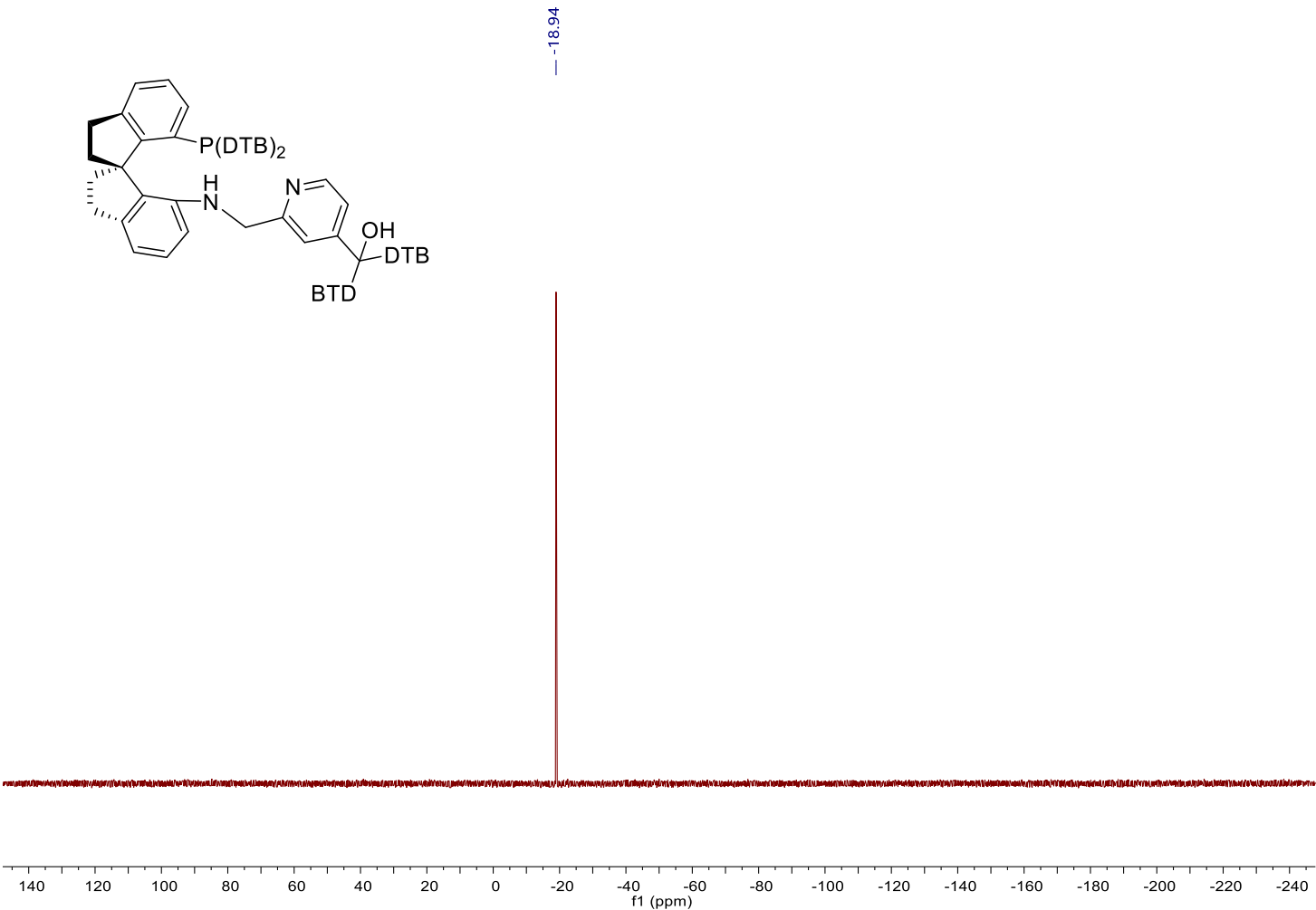




\section{Ligand (R)-S5q (4-(Me) $\left.2\left(\mathrm{C}_{6} \mathrm{H}_{5}\right) \mathrm{C}\right)$}

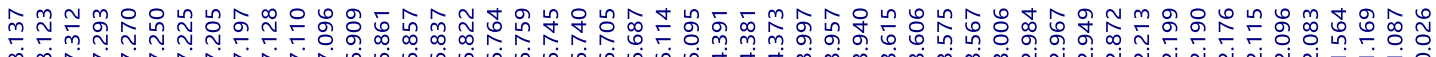

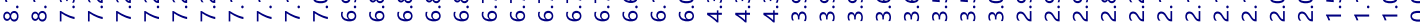

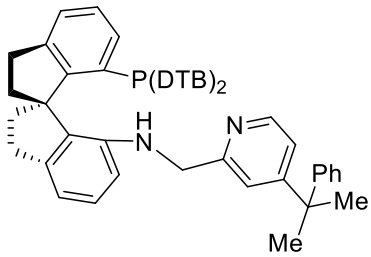
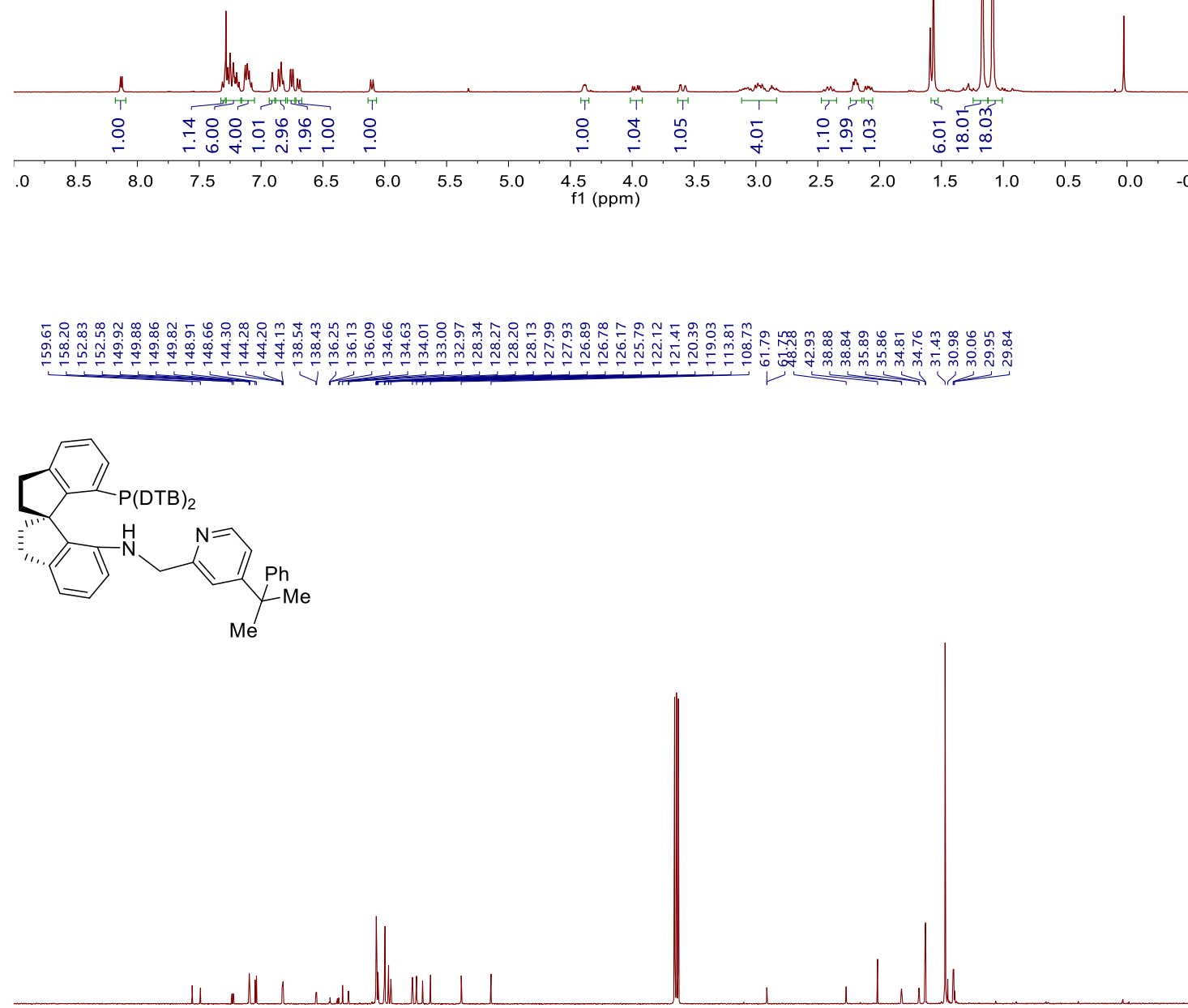

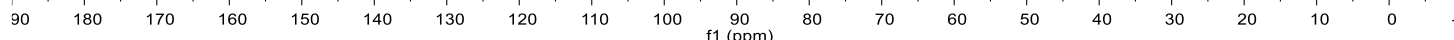



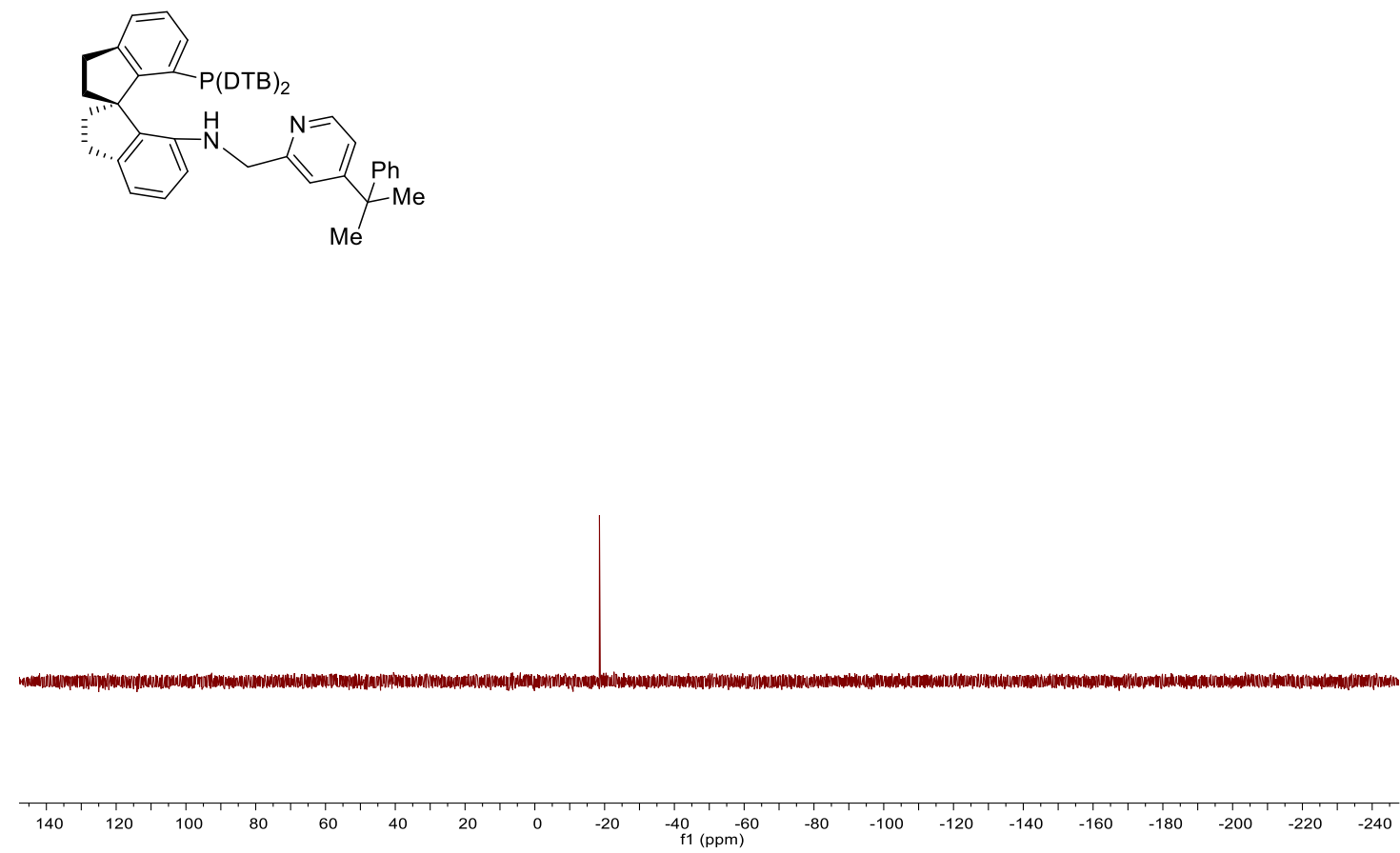

Ligand $(R)-S 5 r\left(4-\left(\mathrm{C}_{6} \mathrm{H}_{5}\right)_{2}\left(4-\mathrm{MeOC}_{6} \mathrm{H}_{4}\right) \mathrm{C}\right)$

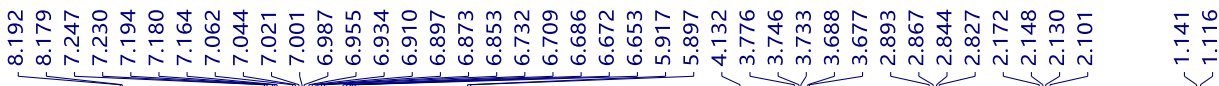
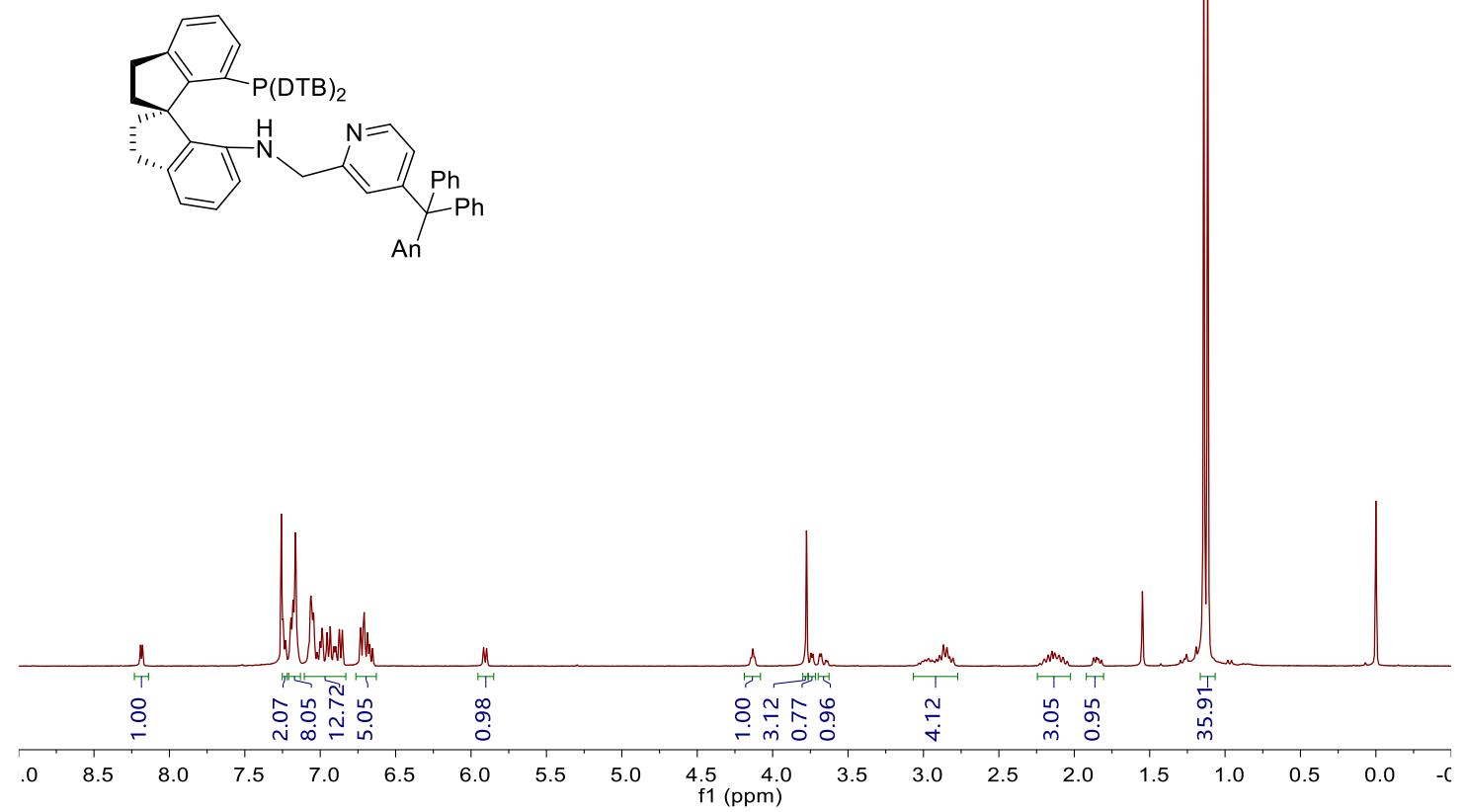

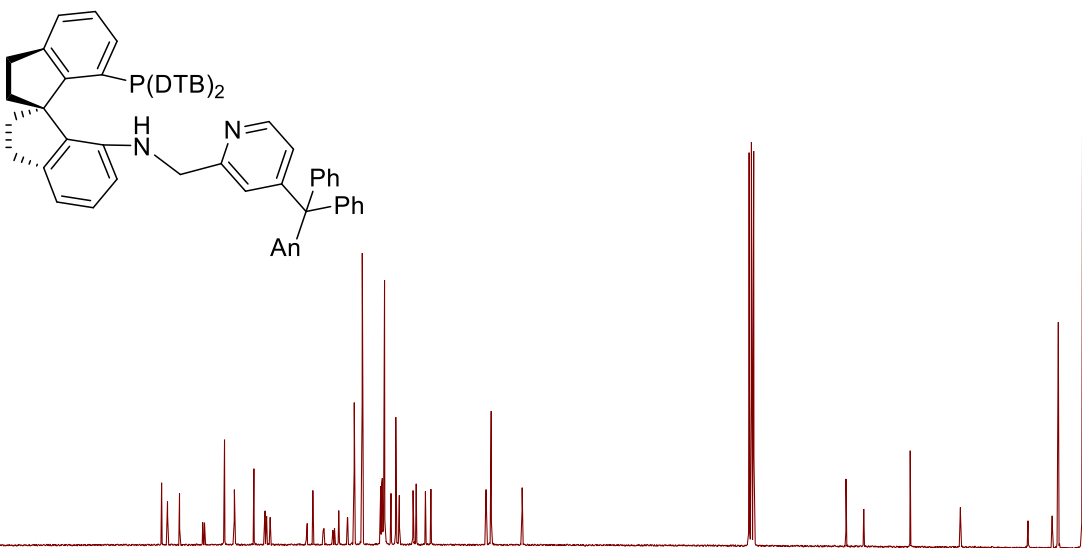

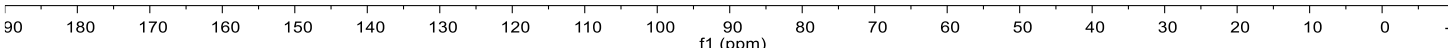<smiles>CCCCc1cccc2c1C1c3c(cccc3NCc3cc(C(c4ccccc4)(c4ccccc4)c4ccccc4)ccn3)CC[C@@H]21</smiles> 
3-(Phenylamino)dihydrofuran-2-one (6a)

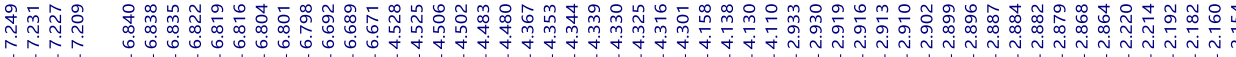<smiles>O=C1OCCC1Nc1ccccc1</smiles>

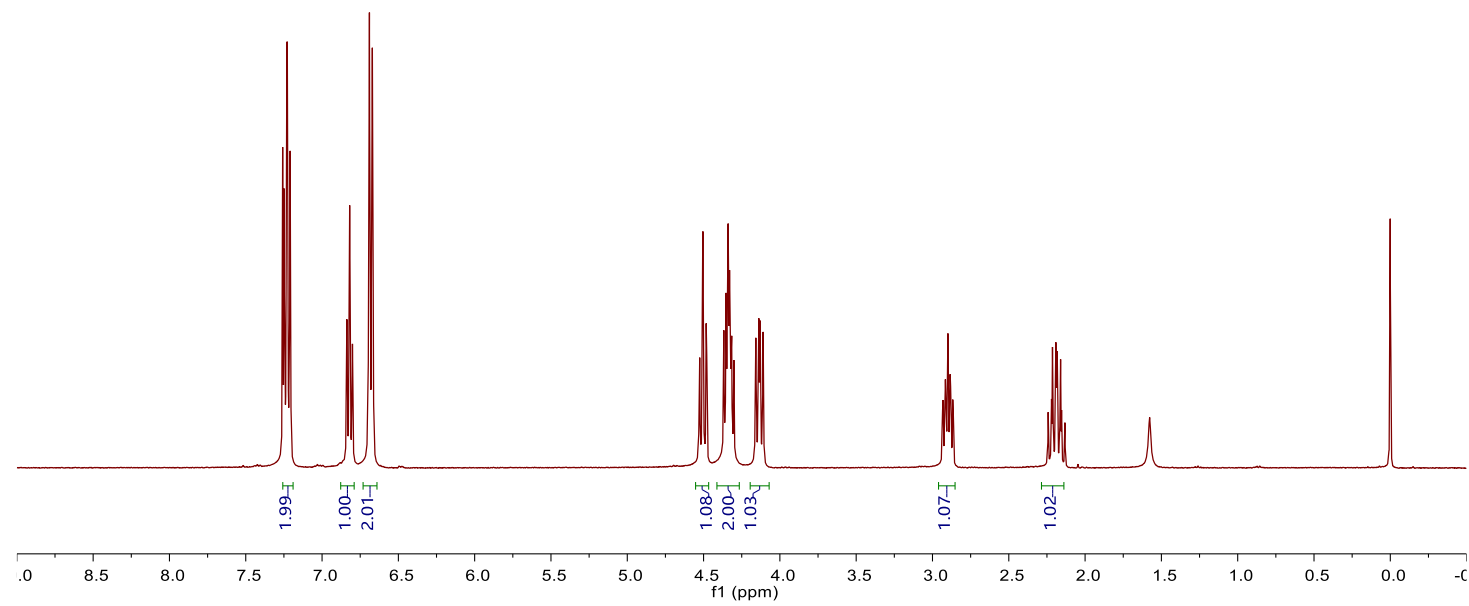

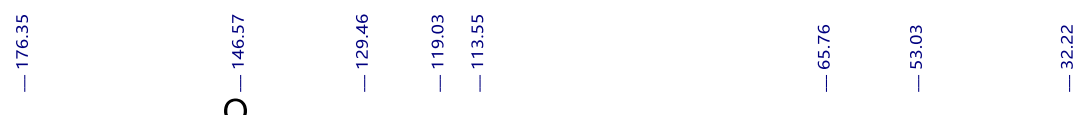<smiles>O=C1OCCC1Nc1ccccc1</smiles>

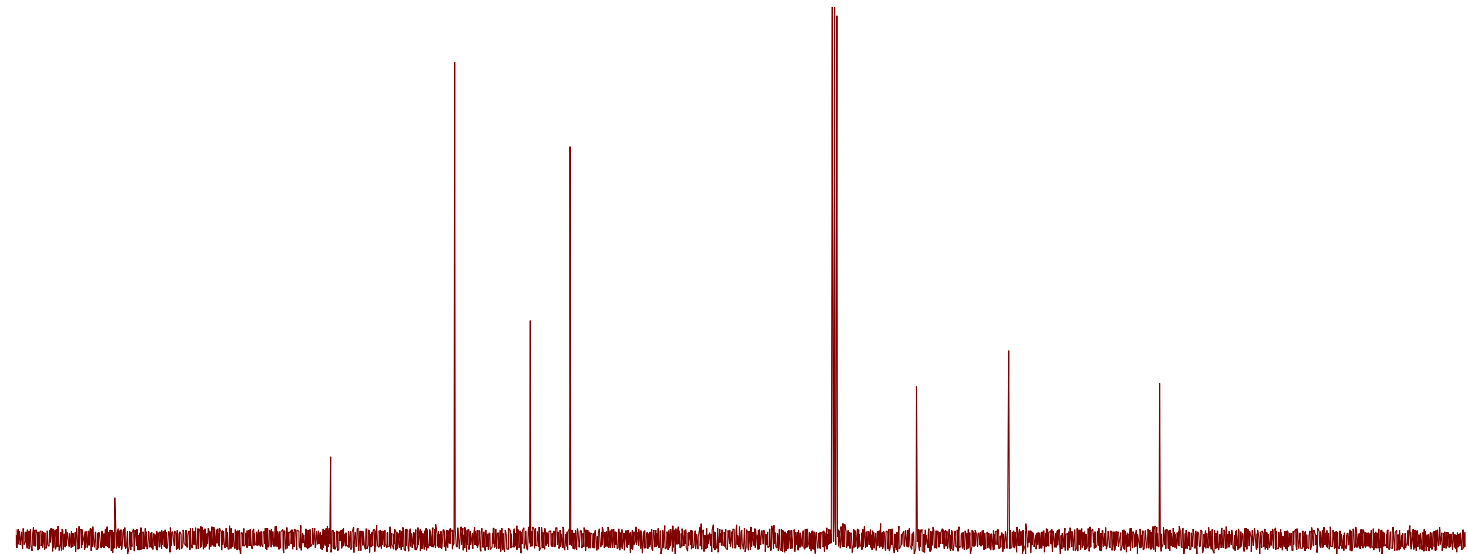

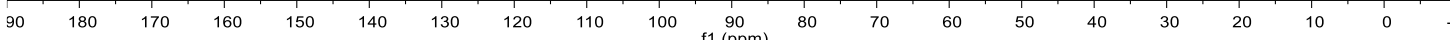


3-(4-Tolylamino)dihydrofuran-2-one (6b)

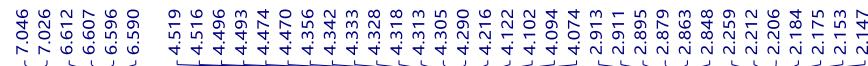

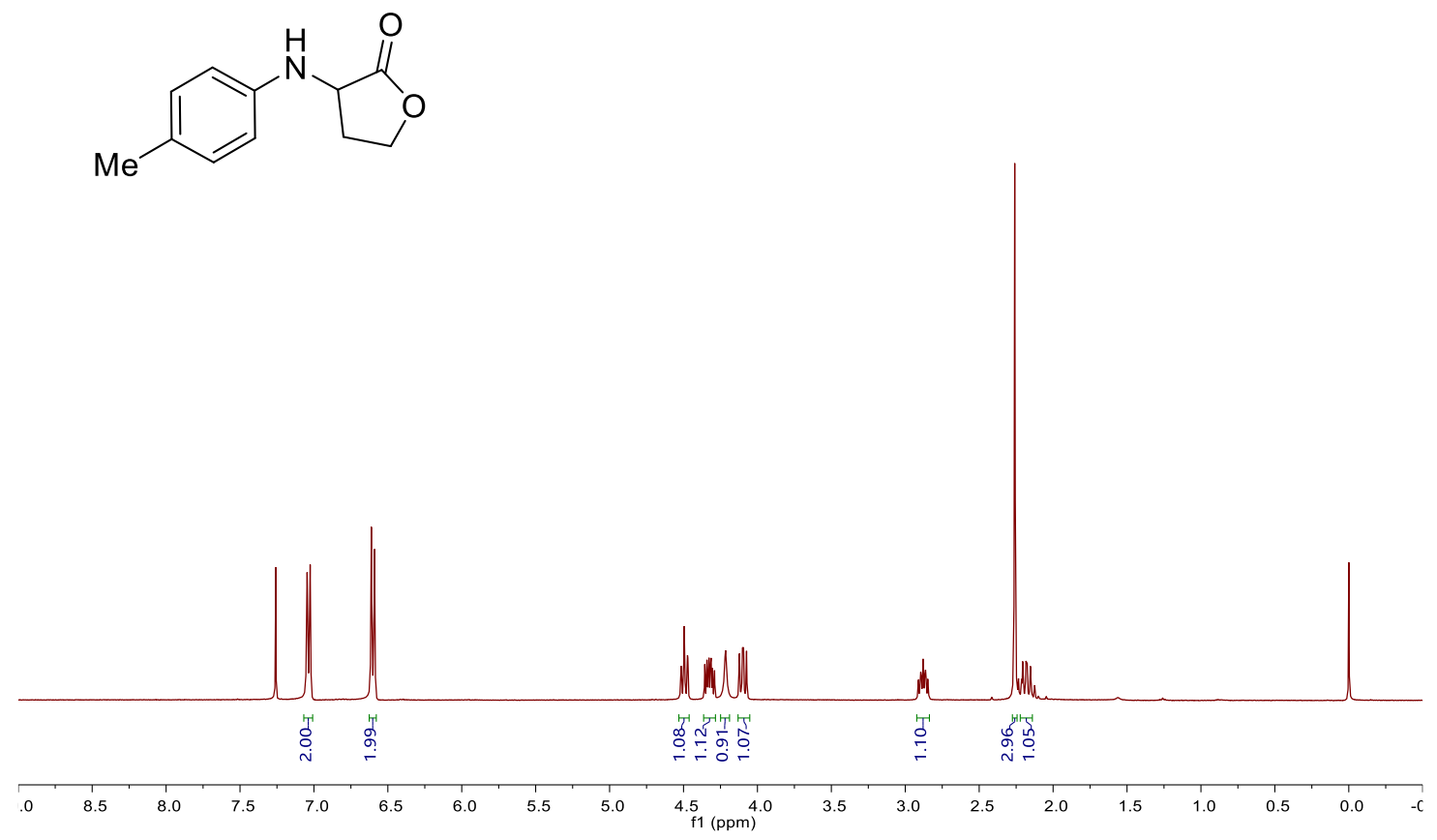

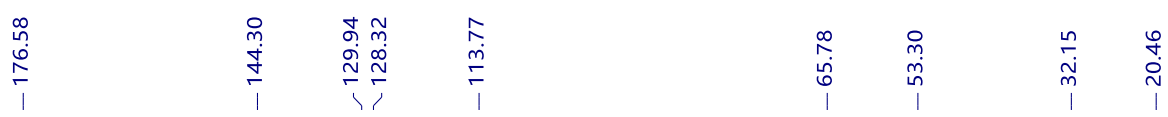<smiles>Cc1ccc(NC2CCOC2=O)cc1</smiles>

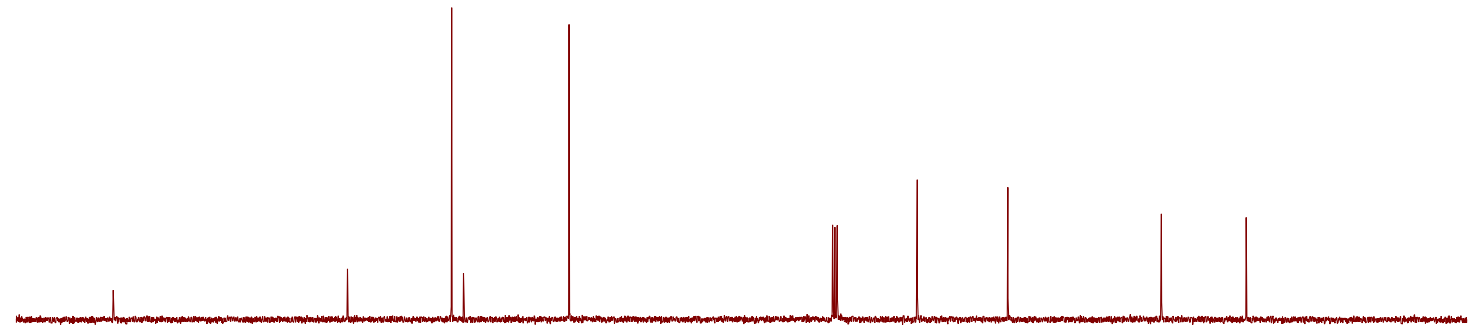

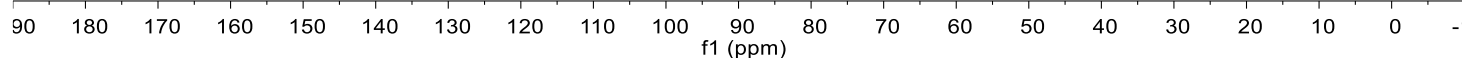


3-((4-Methoxyphenyl)amino)dihydrofuran-2-one (6c)

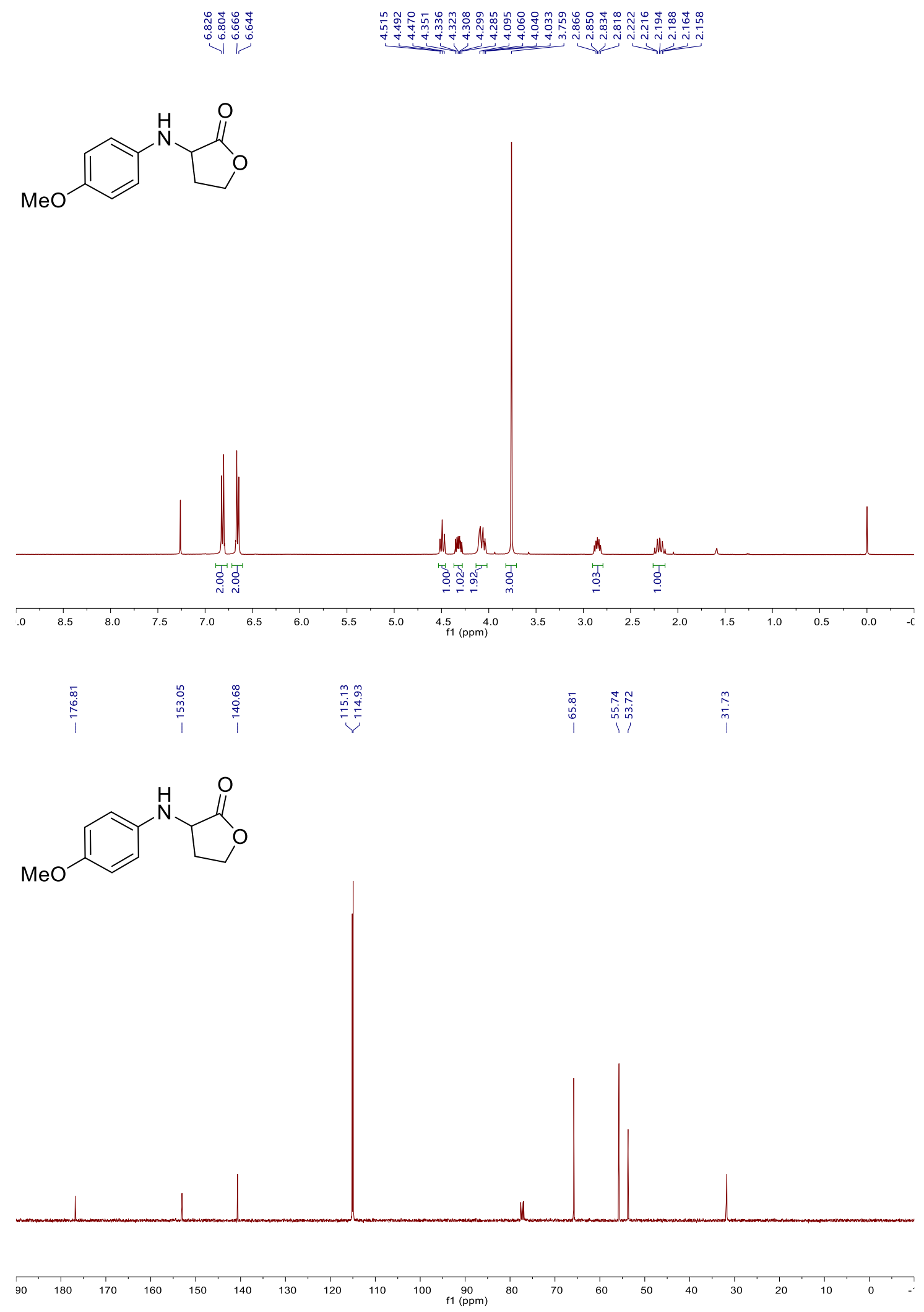


3-((4-Fluorophenyl)amino)dihydrofuran-2-one (6d)
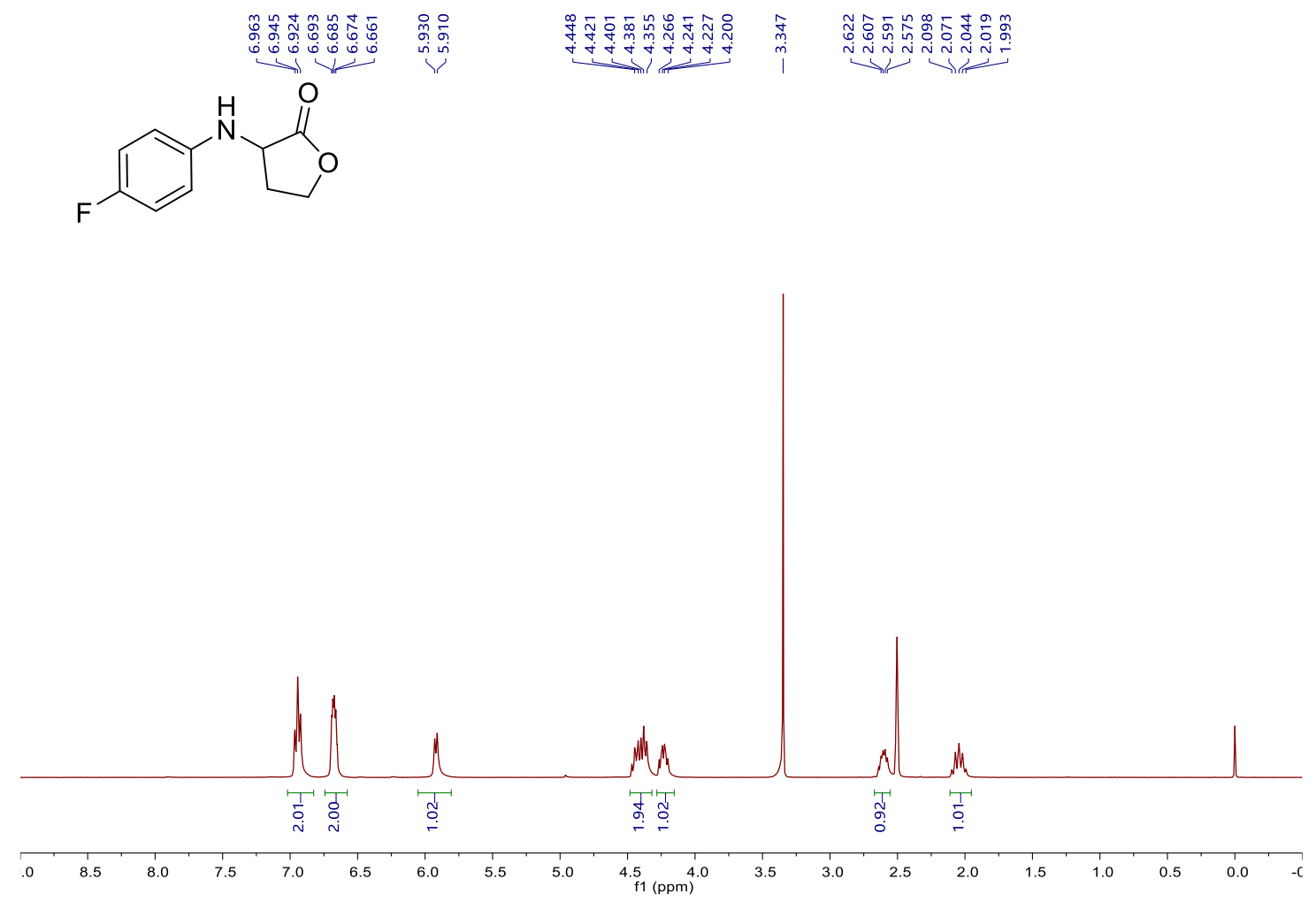

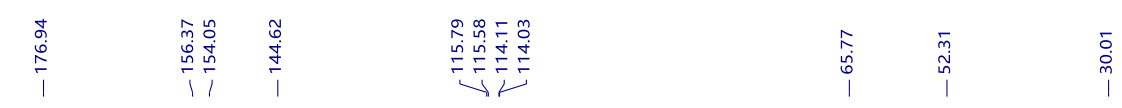<smiles>O=C1OCCC1Nc1ccc(F)cc1</smiles>

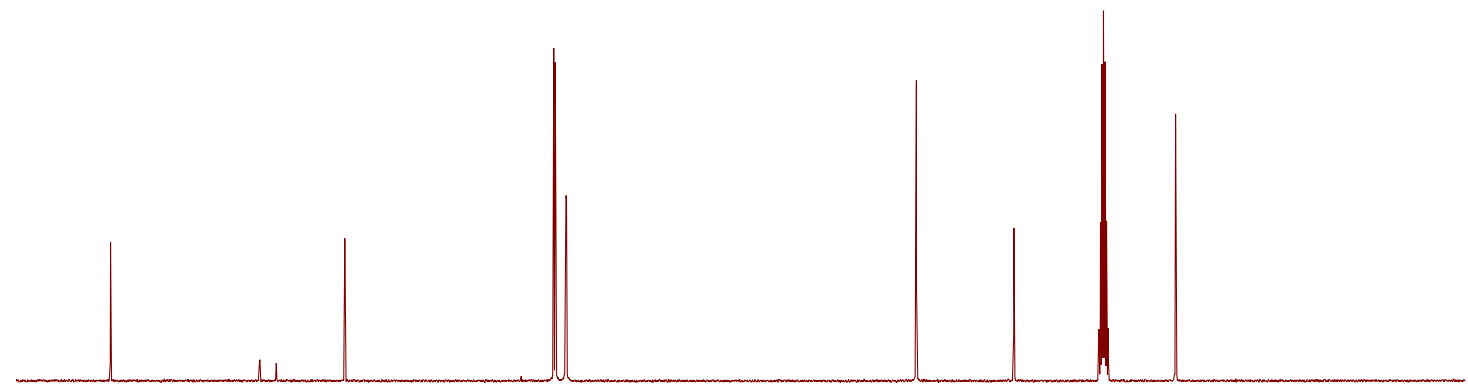

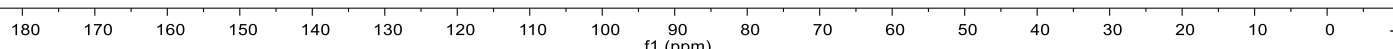


3-((4-Chlorophenyl)amino)dihydrofuran-2-one (6e)

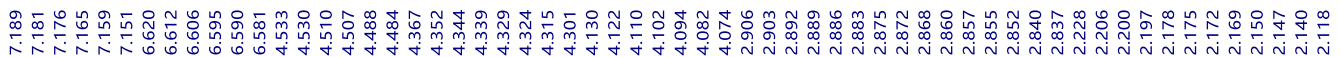<smiles>O=C1OCCC1Nc1ccc(Cl)cc1</smiles>

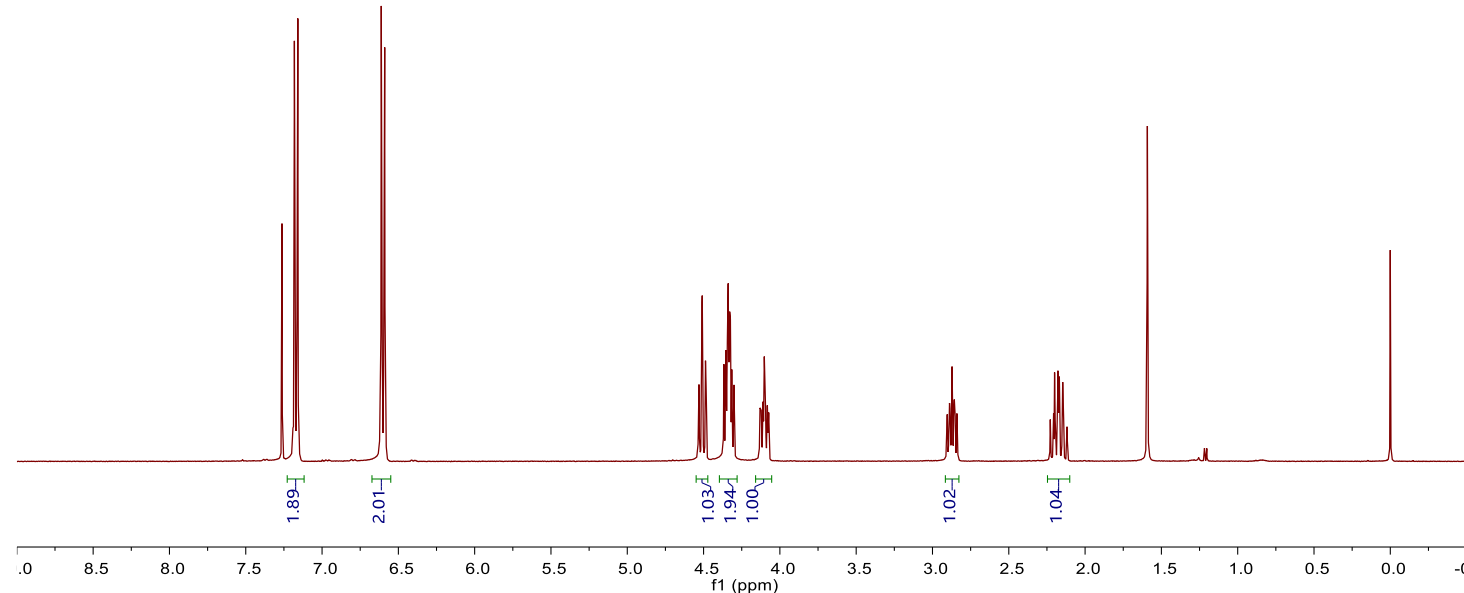

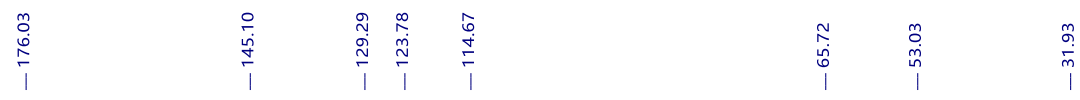<smiles>O=C1OCCC1Nc1ccc(Cl)cc1</smiles>

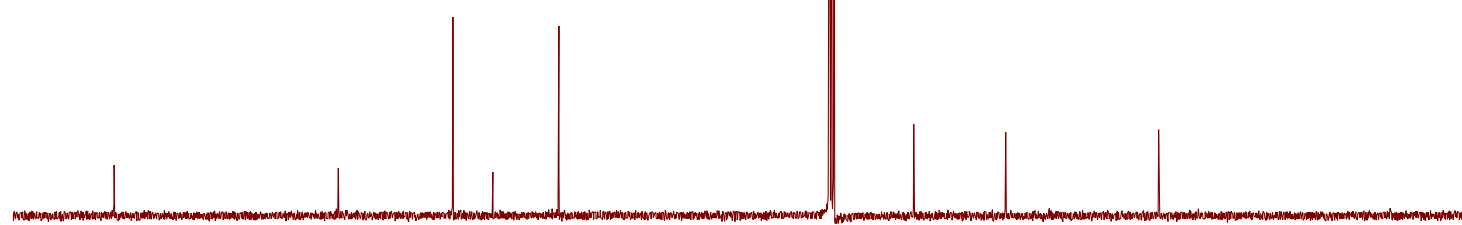

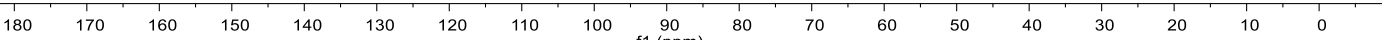


3-((4-Bromophenyl)amino)dihydrofuran-2-one (6f)

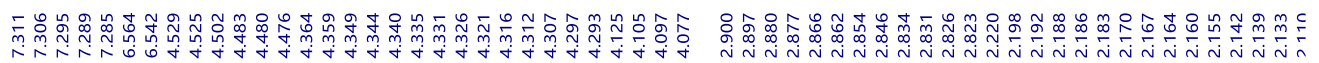<smiles>O=C1OCCC1Nc1ccc(Br)cc1</smiles>

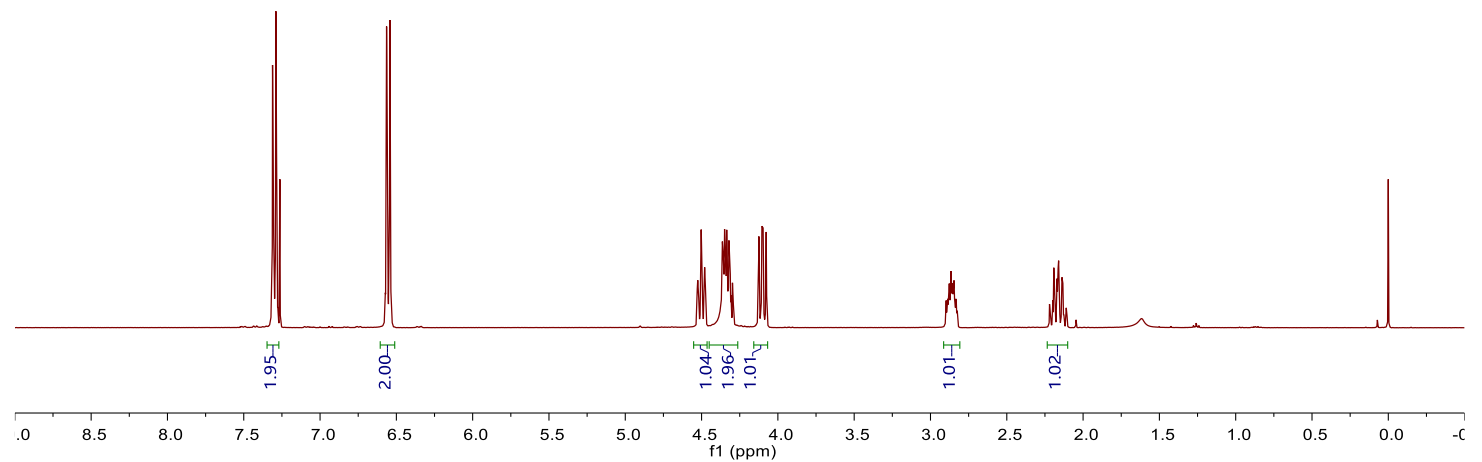

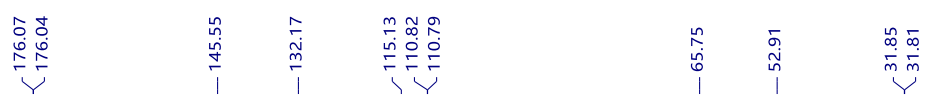

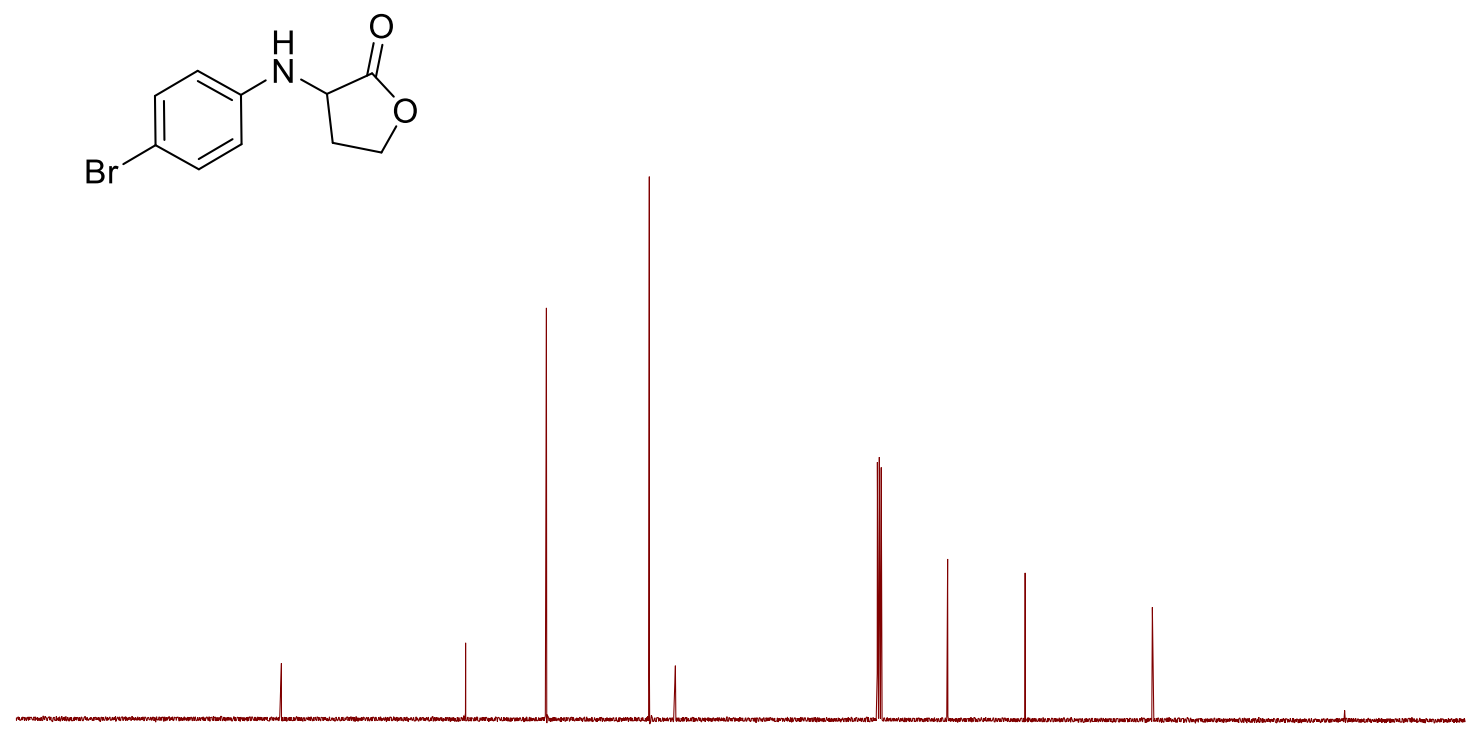

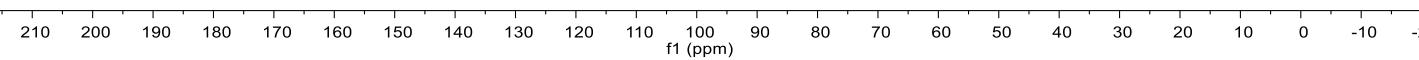


3-(m-Tolylamino)dihydrofuran-2-one $(6 \mathrm{~g})$

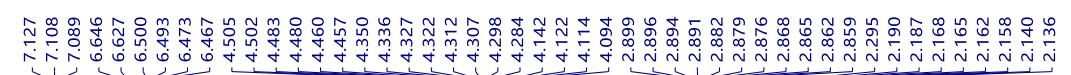

${ }_{1}$

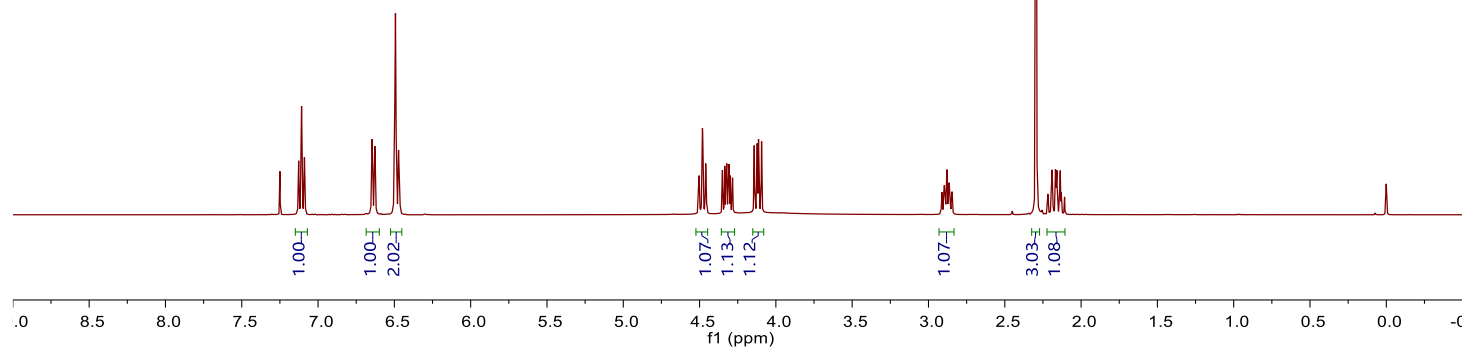

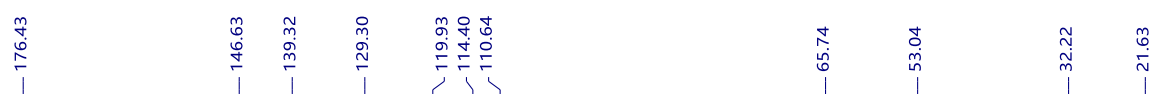<smiles>Cc1cccc(NC2CCOC2=O)c1</smiles>

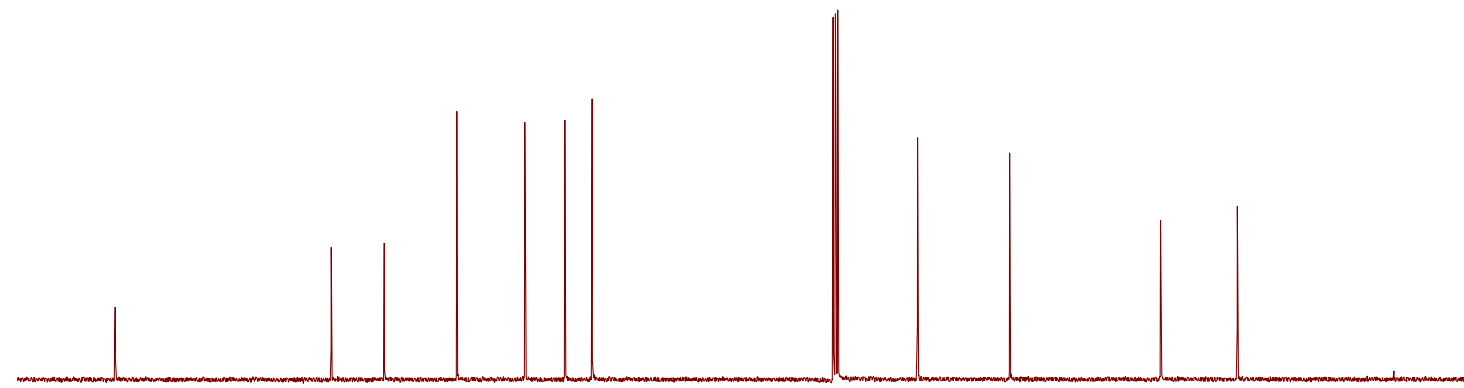

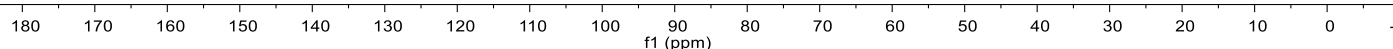


3-((3-Methoxyphenyl)amino)dihydrofuran-2-one (6h)

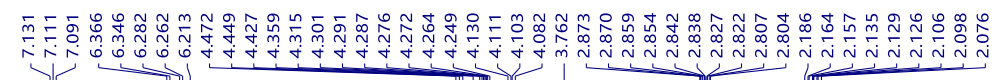

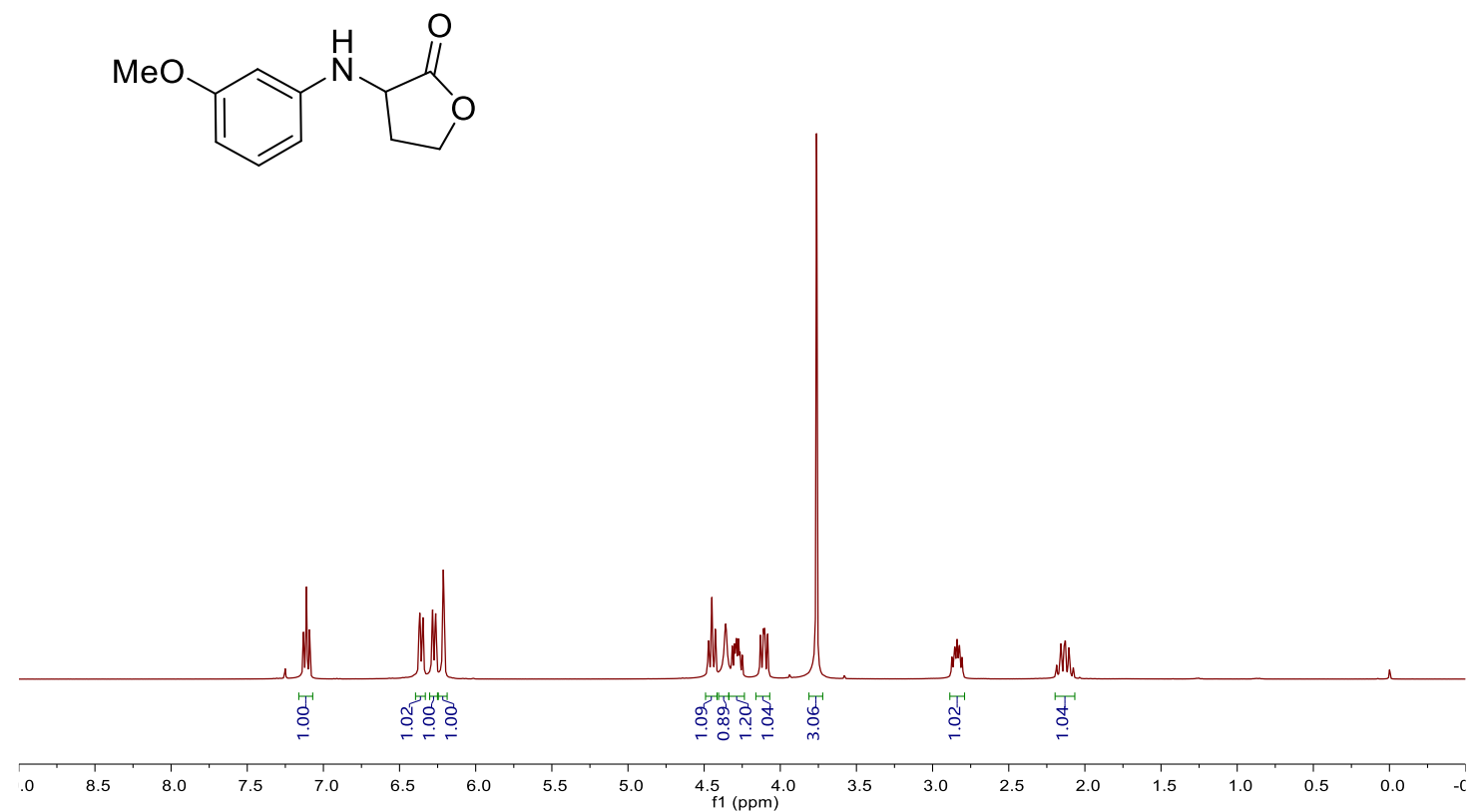

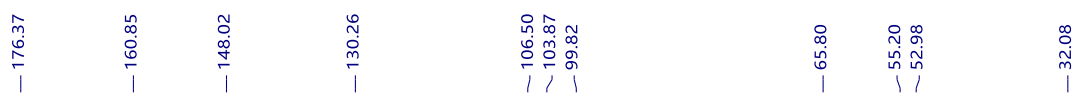<smiles>COc1cccc(NC2CCOC2=O)c1</smiles>

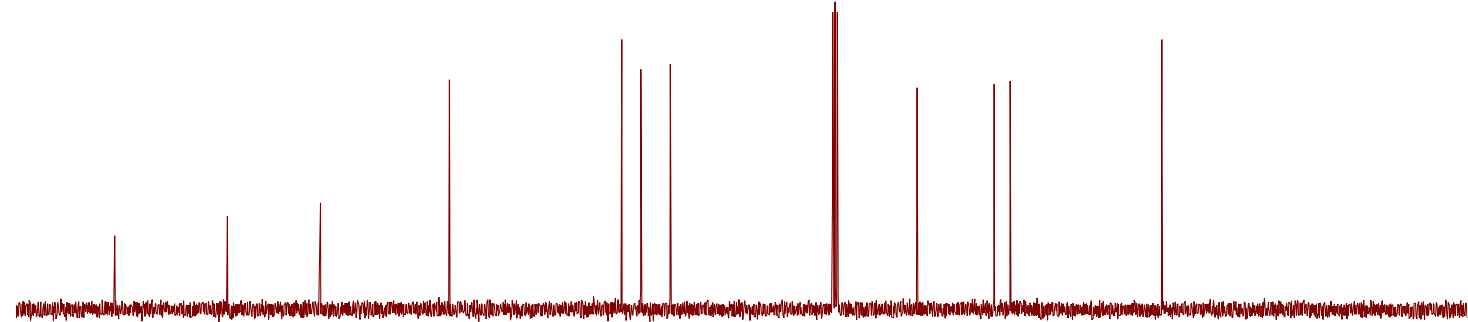

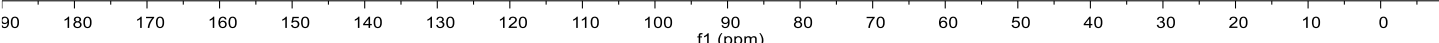


3-((3-Fluorophenyl)amino)dihydrofuran-2-one (6i)

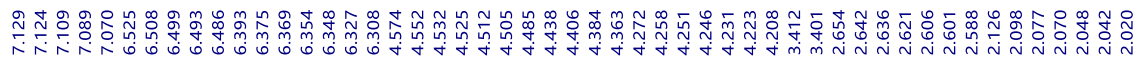

$\overbrace{1}^{\mathrm{N}}$

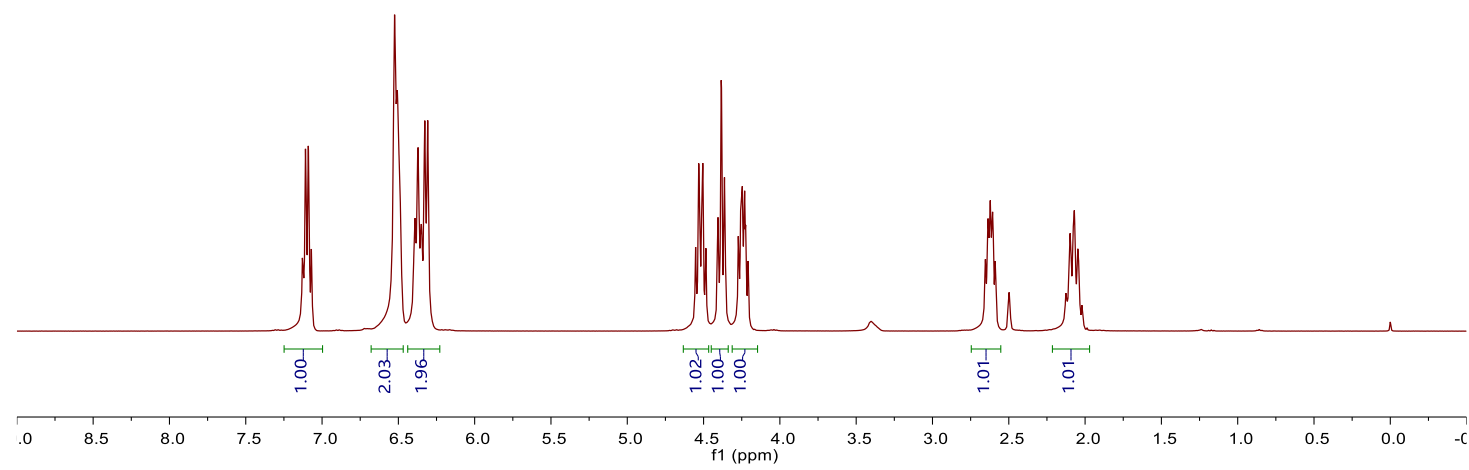

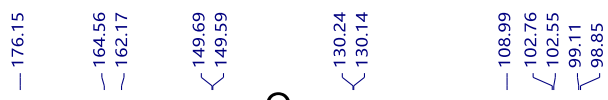

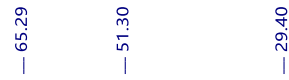<smiles>O=C1OCCC1Nc1cccc(F)c1</smiles>

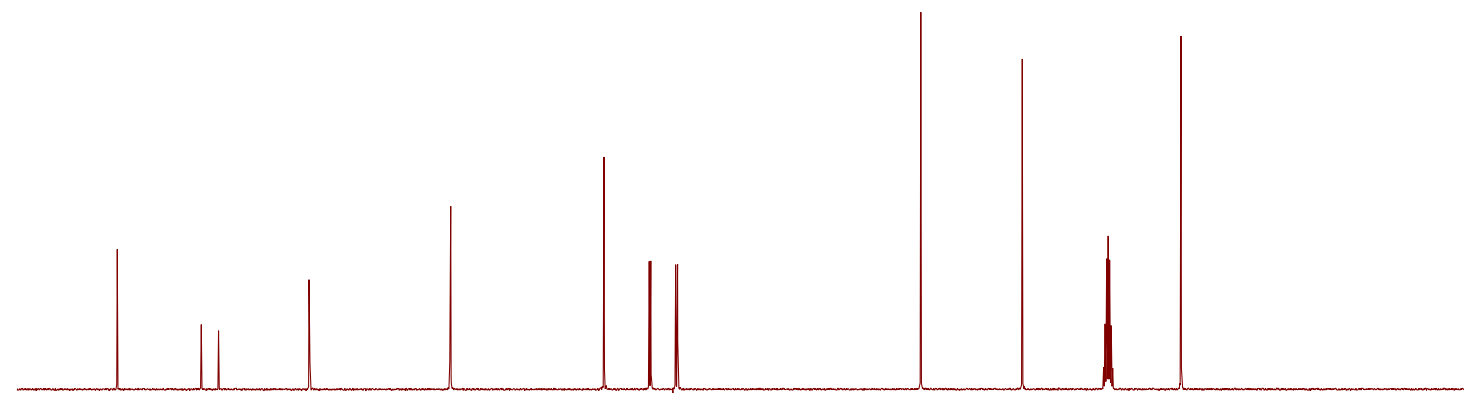

$\begin{array}{lllllllllllllllllllllll}1 & 180 & 170 & 160 & 150 & 140 & 130 & 120 & 110 & 100 & 90 & 80 & 70 & 60 & 50 & 40 & 30 & 20 & 10 & 0 & 10\end{array}$ 
3-((3-Chlorophenyl)amino)dihydrofuran-2-one (6j)

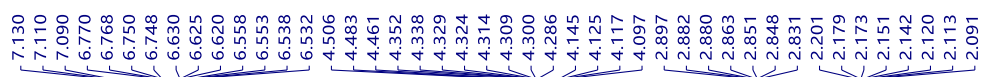<smiles>O=C1OCCC1Nc1cccc(Cl)c1</smiles>

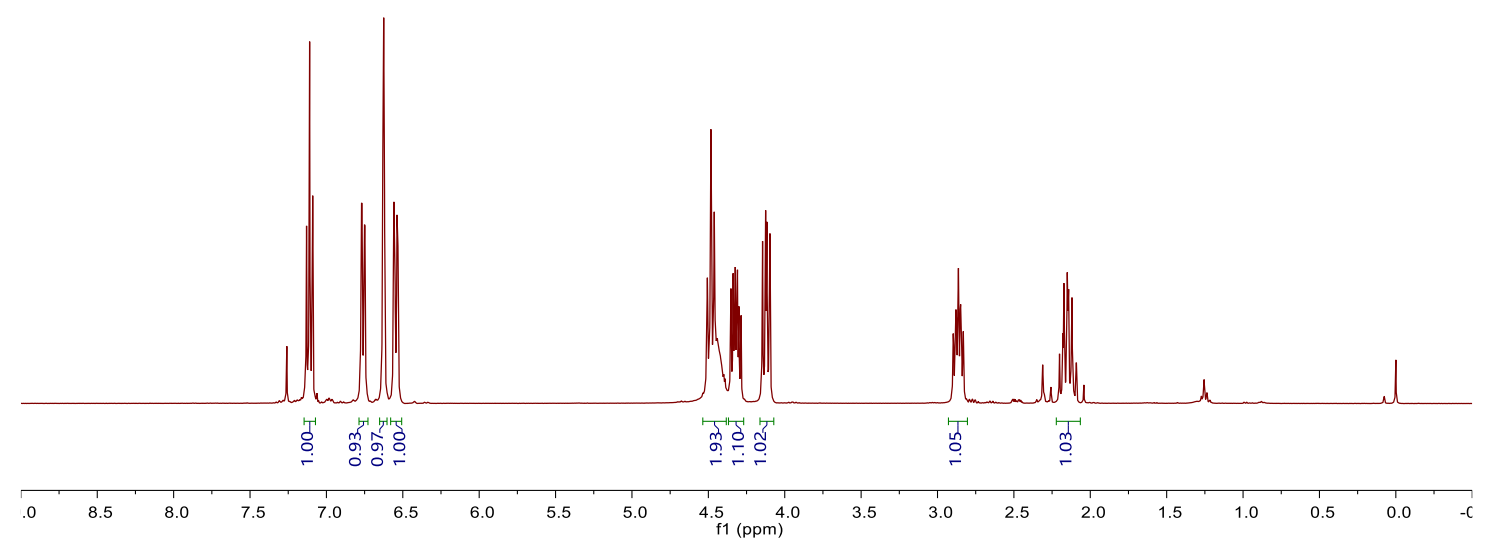

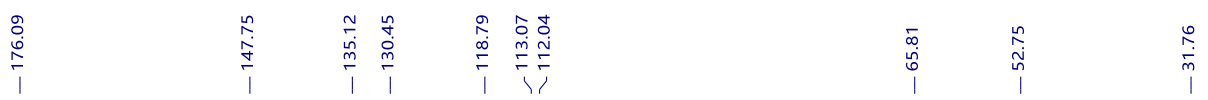<smiles>O=C1OCCC1Nc1cccc(Cl)c1</smiles>

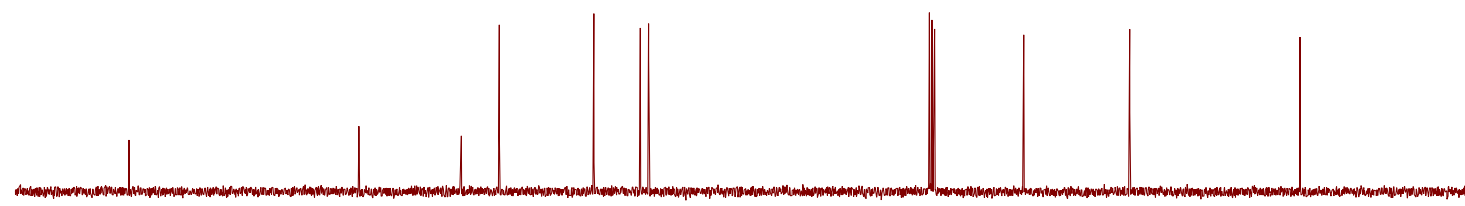

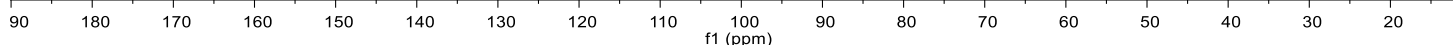


3-(o-Tolylamino)dihydrofuran-2-one (6k)

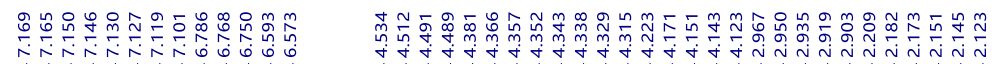

Me

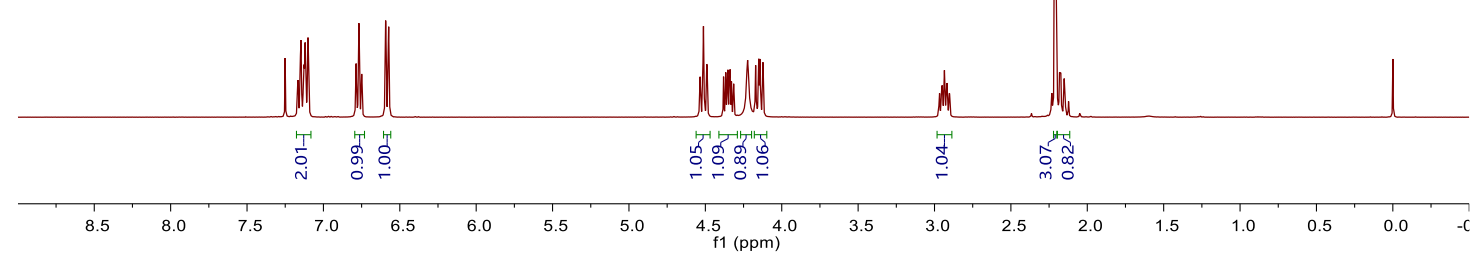

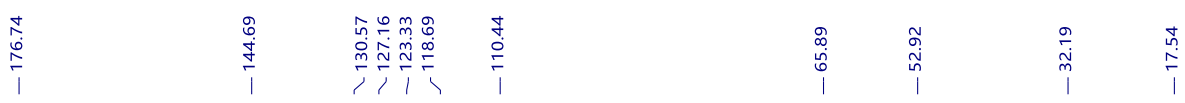

${ }^{M e}$

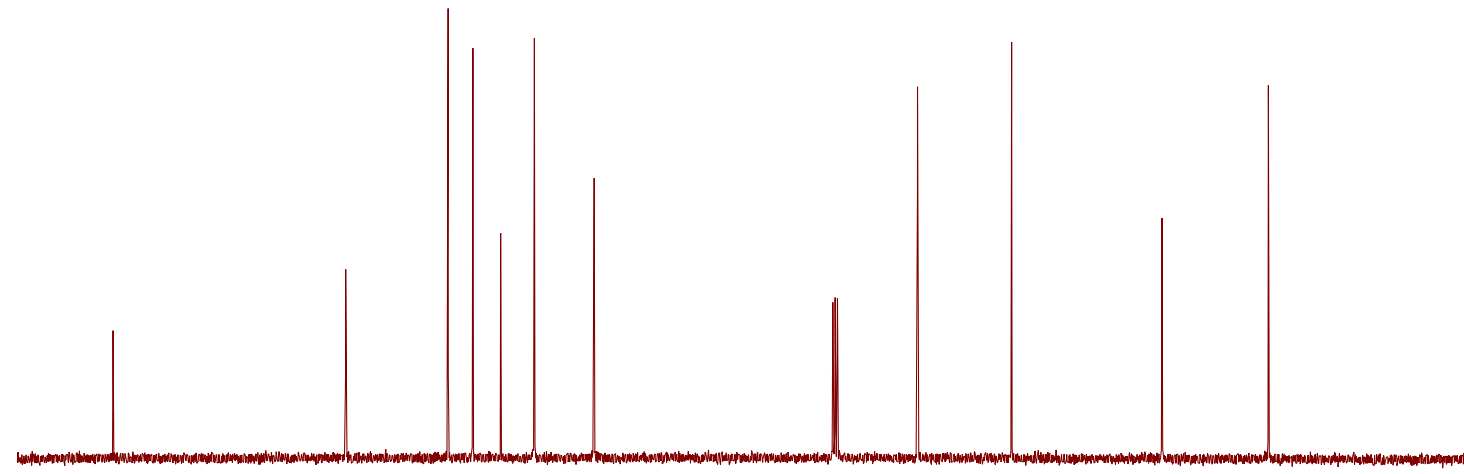

$\begin{array}{llllllllllllllllllllllllllllll} & 180 & 170 & 160 & 150 & 140 & 130 & 120 & 110 & 100 & 90 & 80 & 70 & 60 & 50 & 40 & 30 & 20 & 10 & 0 & -\end{array}$ 
3-((2-Methoxyphenyl)amino)dihydrofuran-2-one (61)

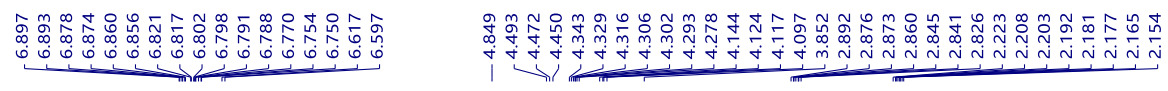

OMe

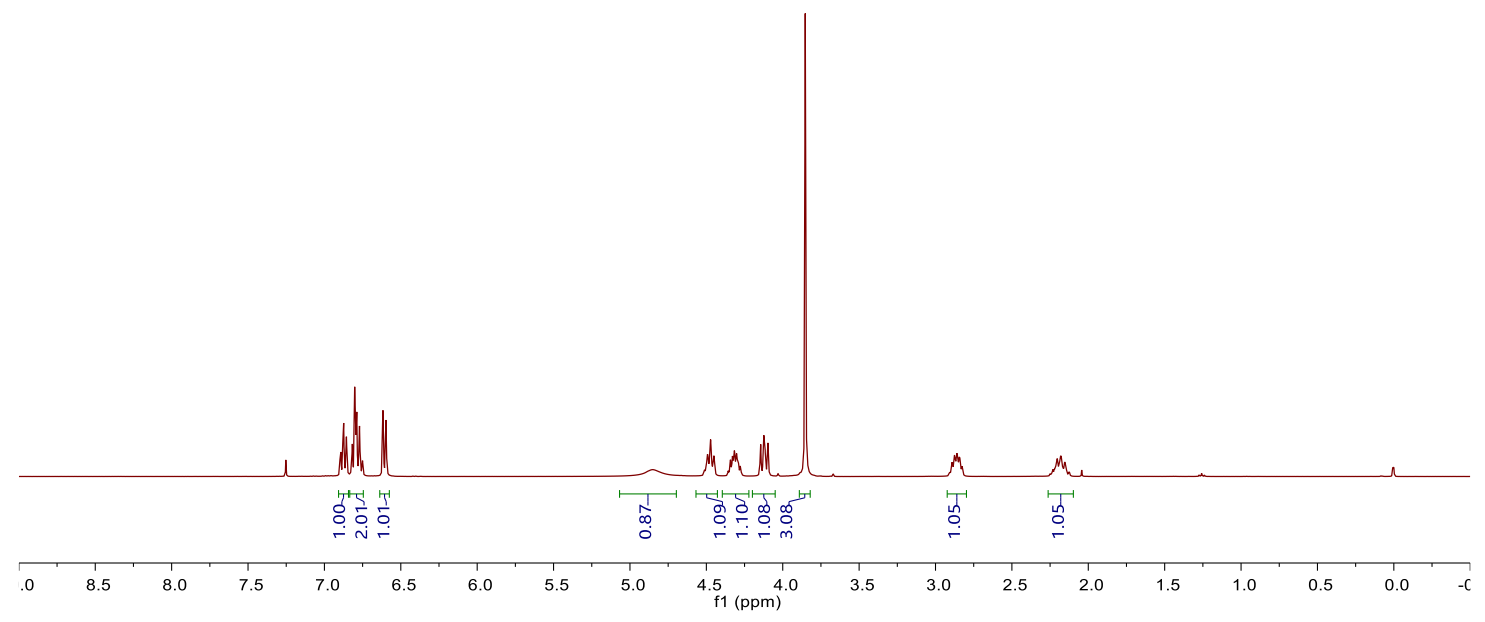

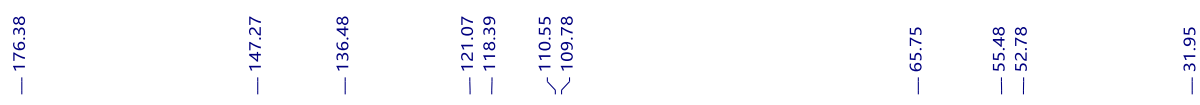<smiles>COc1ccccc1NC1CCOC1=O</smiles>

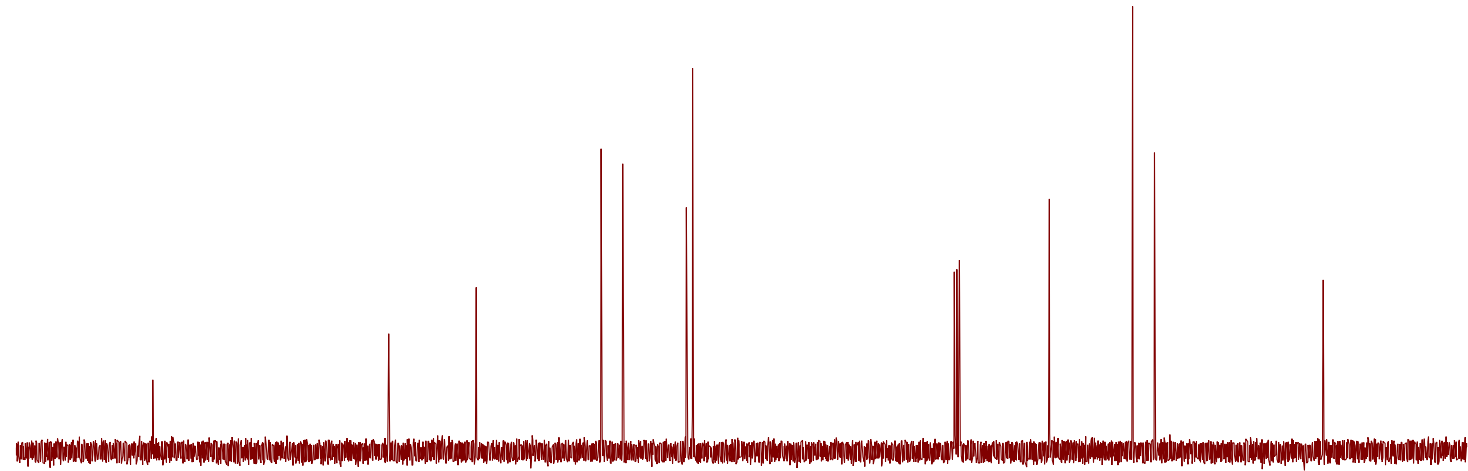

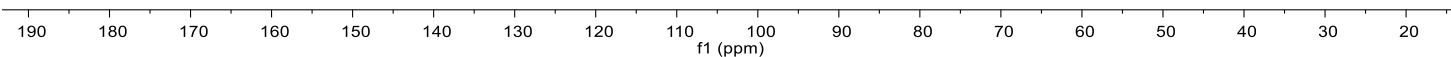


3-((2,4-Dimethoxyphenyl)amino)dihydrofuran-2-one (6m)

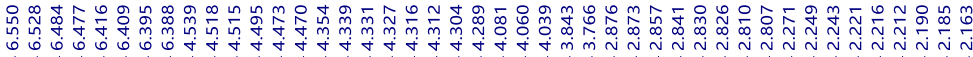<smiles>COc1ccc(NC2CCOC2=O)c(OC)c1</smiles>

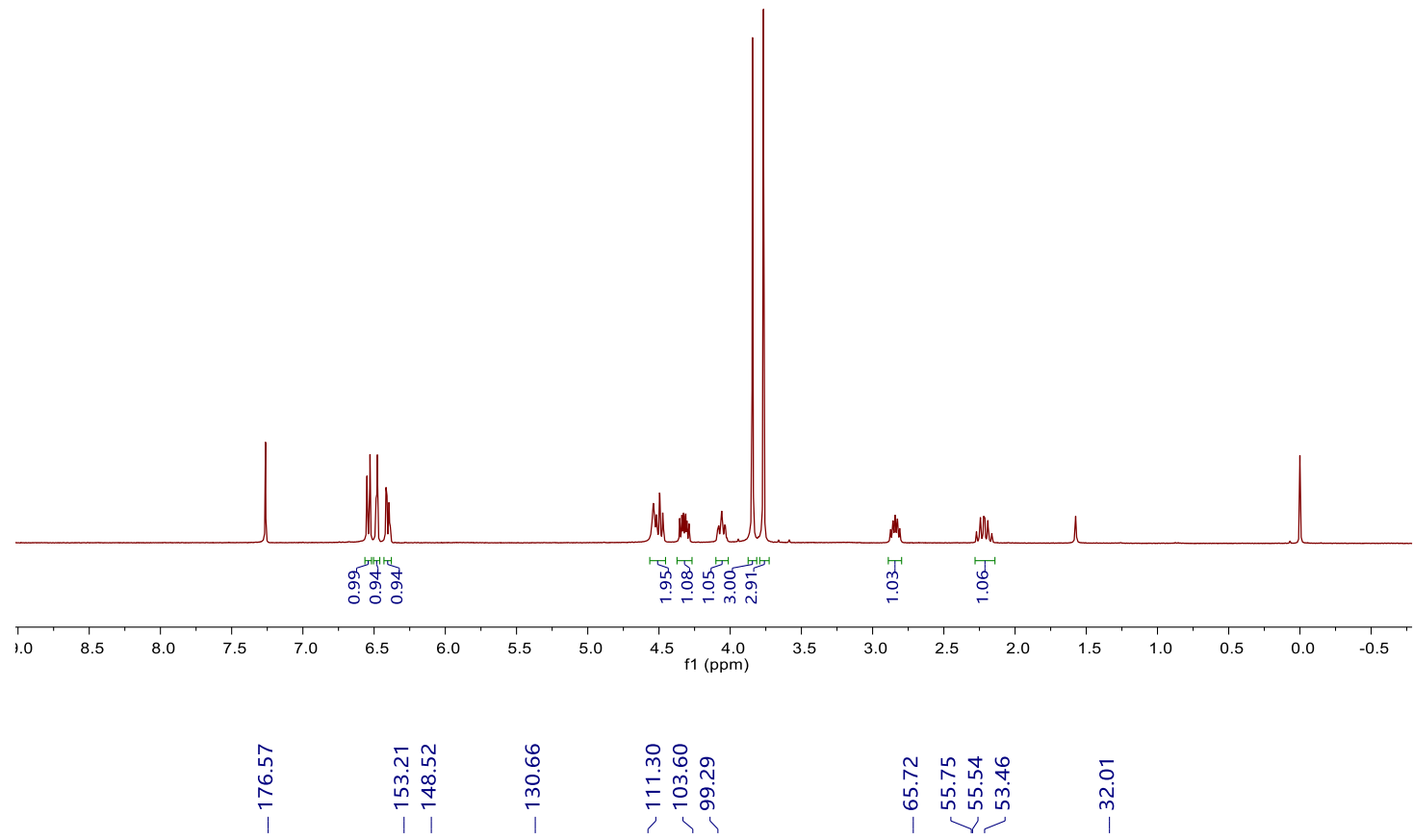<smiles>COc1ccc(NC2CCOC2=O)c(OC)c1</smiles>

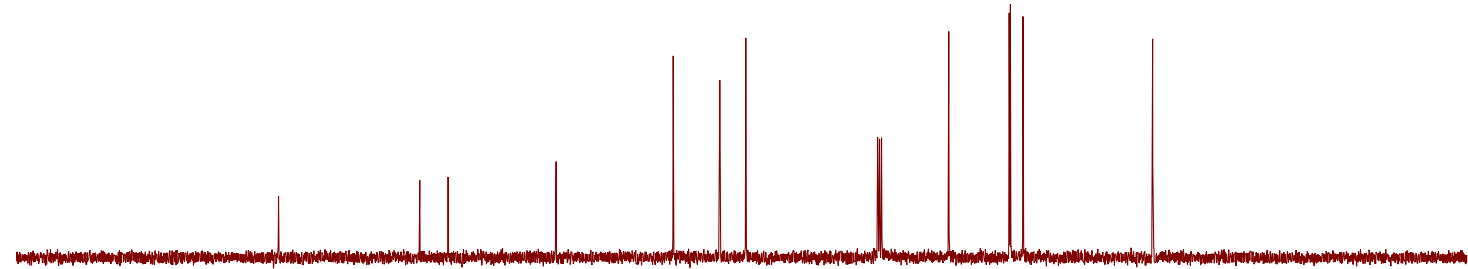

\begin{tabular}{llllllllllllllllllllllllllll}
\hline 20 & 210 & 200 & 190 & 180 & 170 & 160 & 150 & 140 & 130 & 120 & 110 & 100 & 90 & 80 & 70 & 60 & 50 & 40 & 30 & 20 & 10 & 0 & -10 & -4
\end{tabular} 
3-(Naphthalen-1-ylamino)dihydrofuran-2-one (6n)

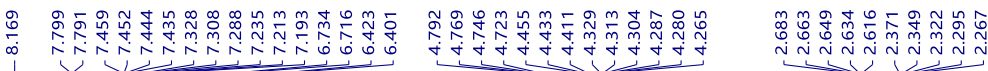

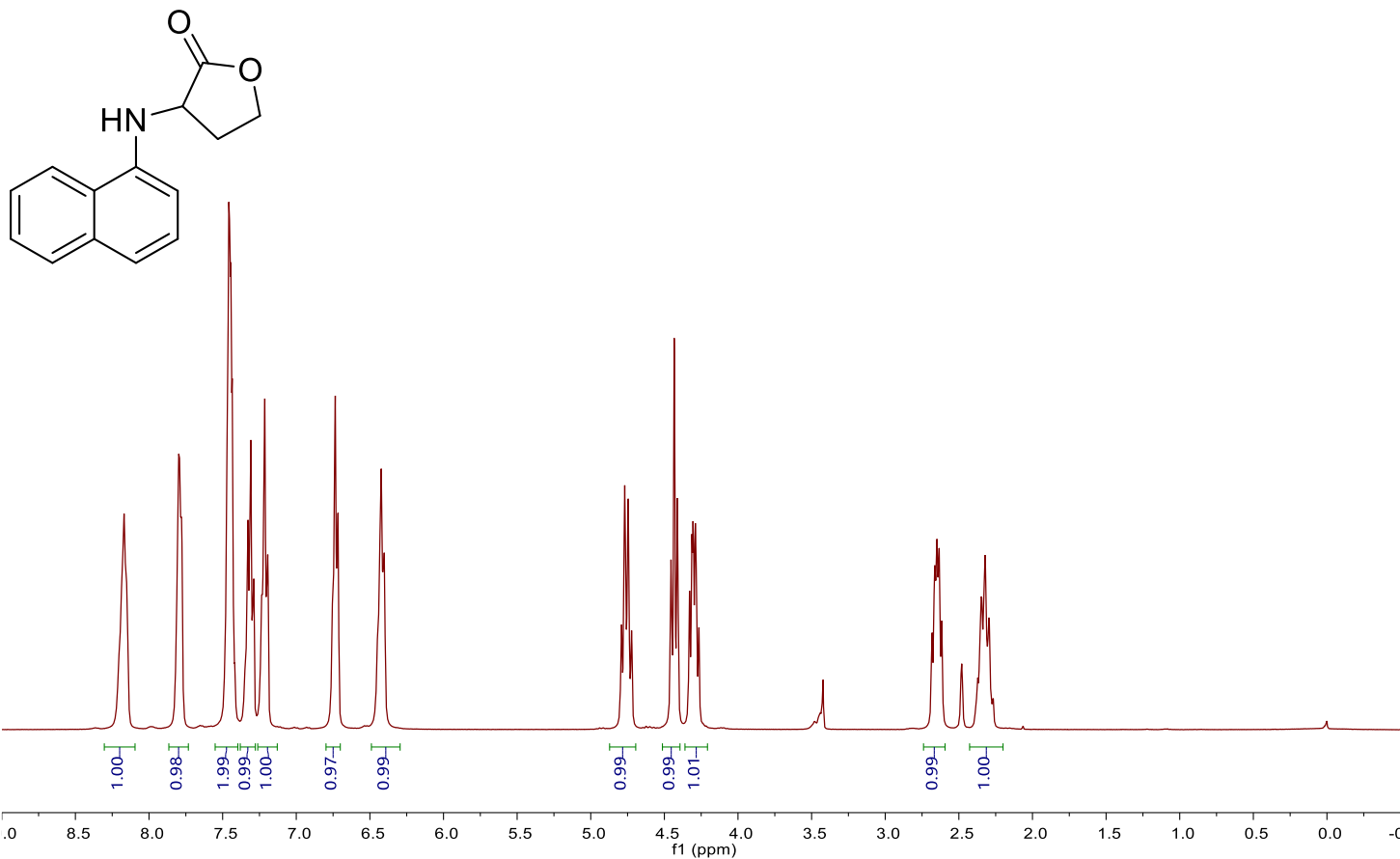

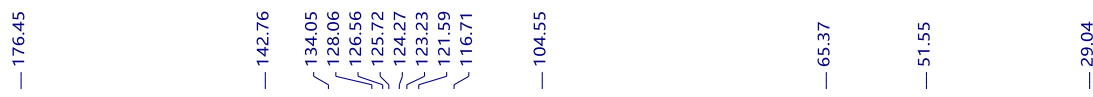

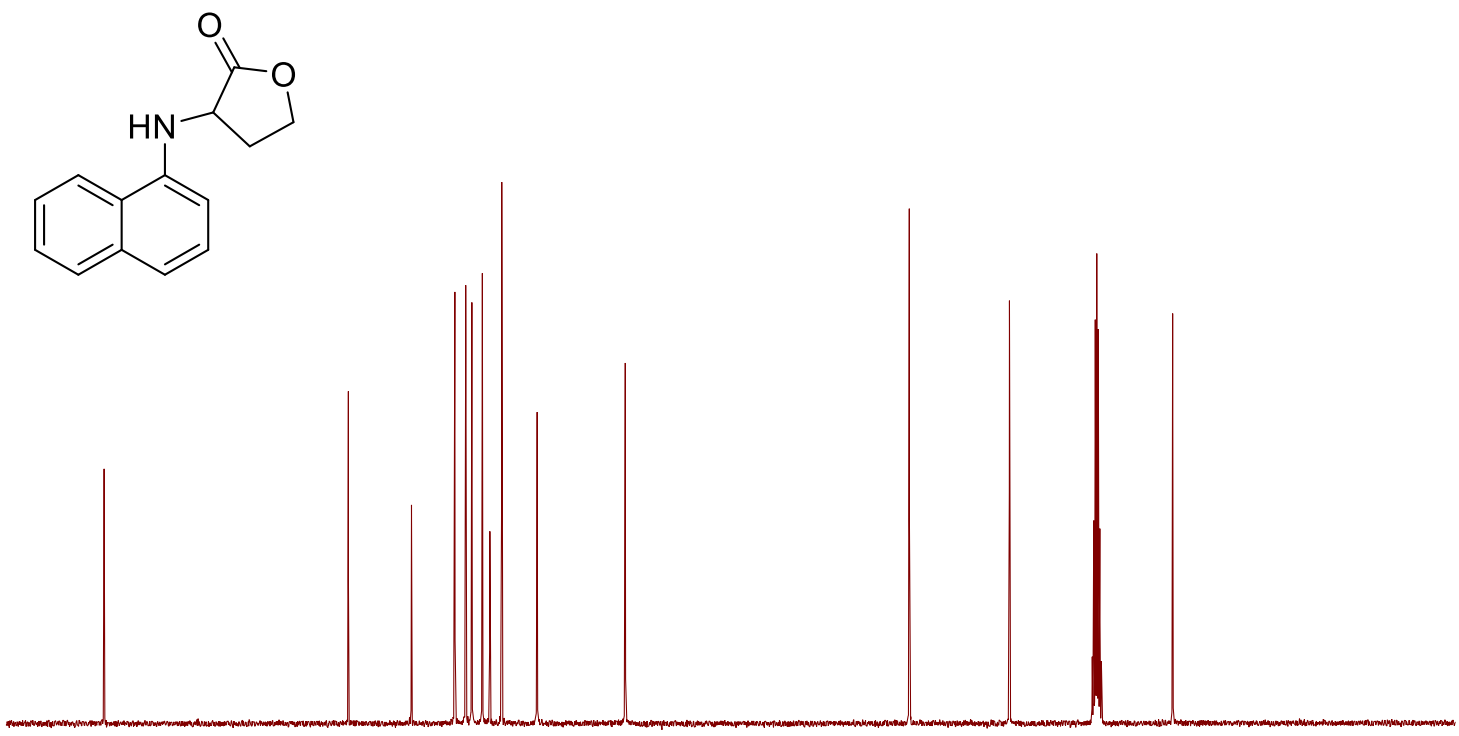

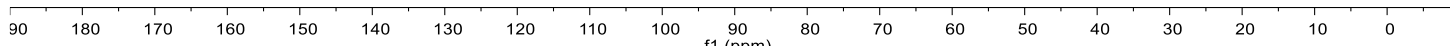


3-(Benzo $[d][1,3]$ dioxol-5-ylamino)dihydrofuran-2-one (6o)

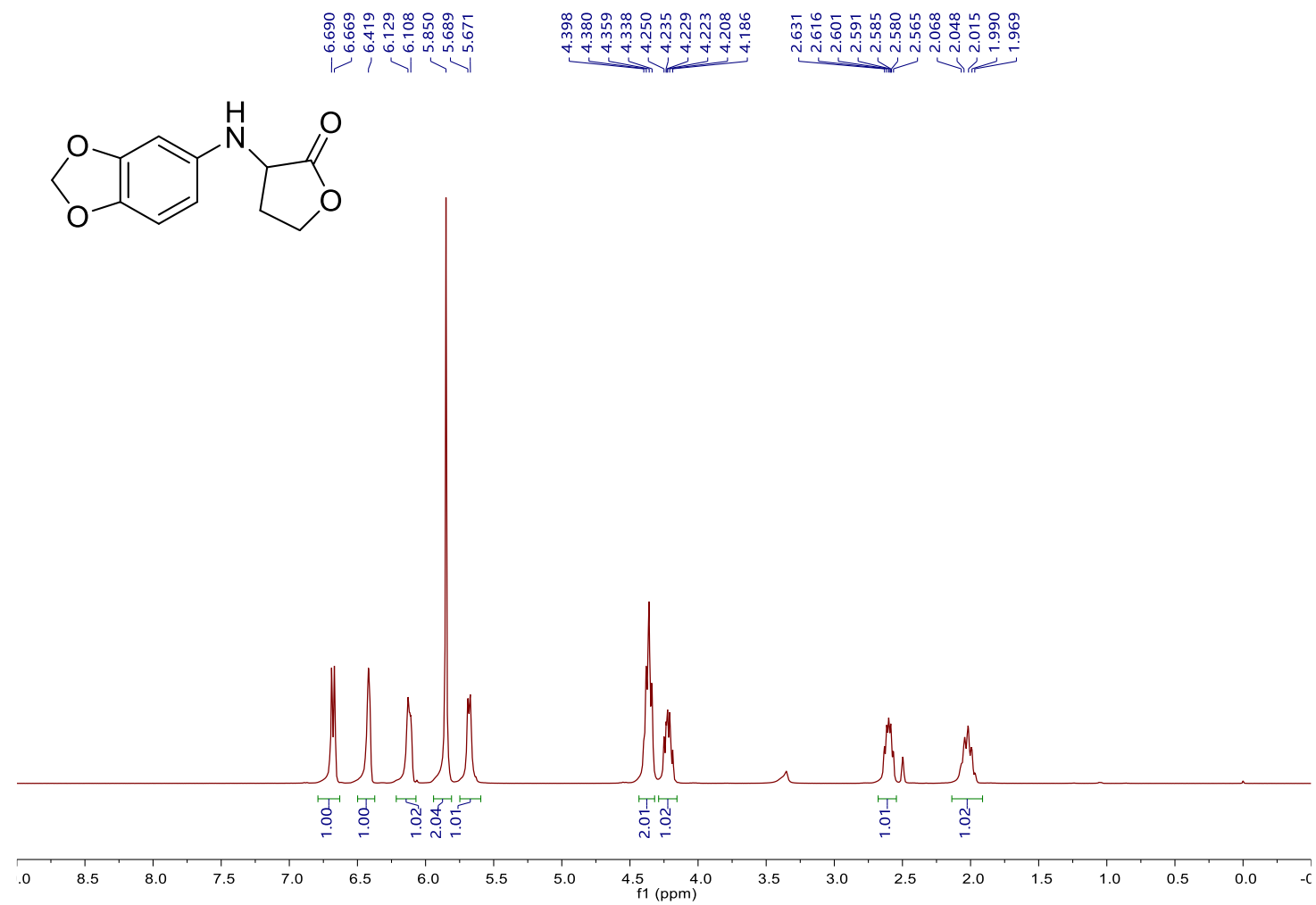

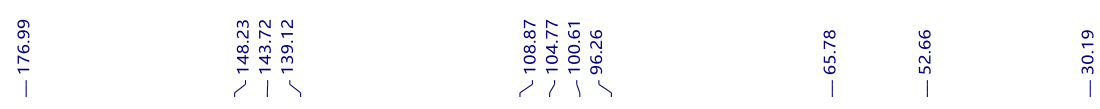<smiles>O=C1OCCC1Nc1ccc2c(c1)OCO2</smiles>

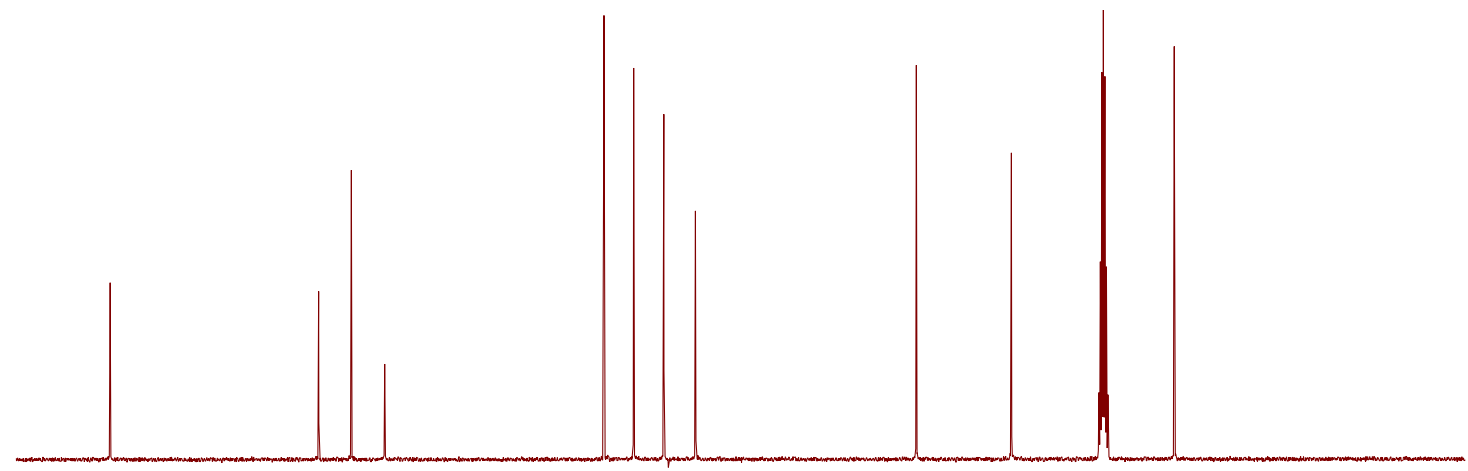

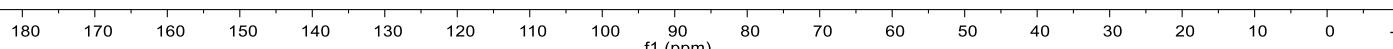


3-(6-Methylpyridin-2-ylamino)dihydrofuran-2-one (6p)

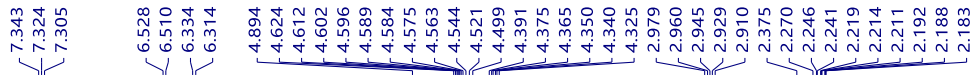<smiles>Cc1cccc(NC2CCOC2=O)n1</smiles>

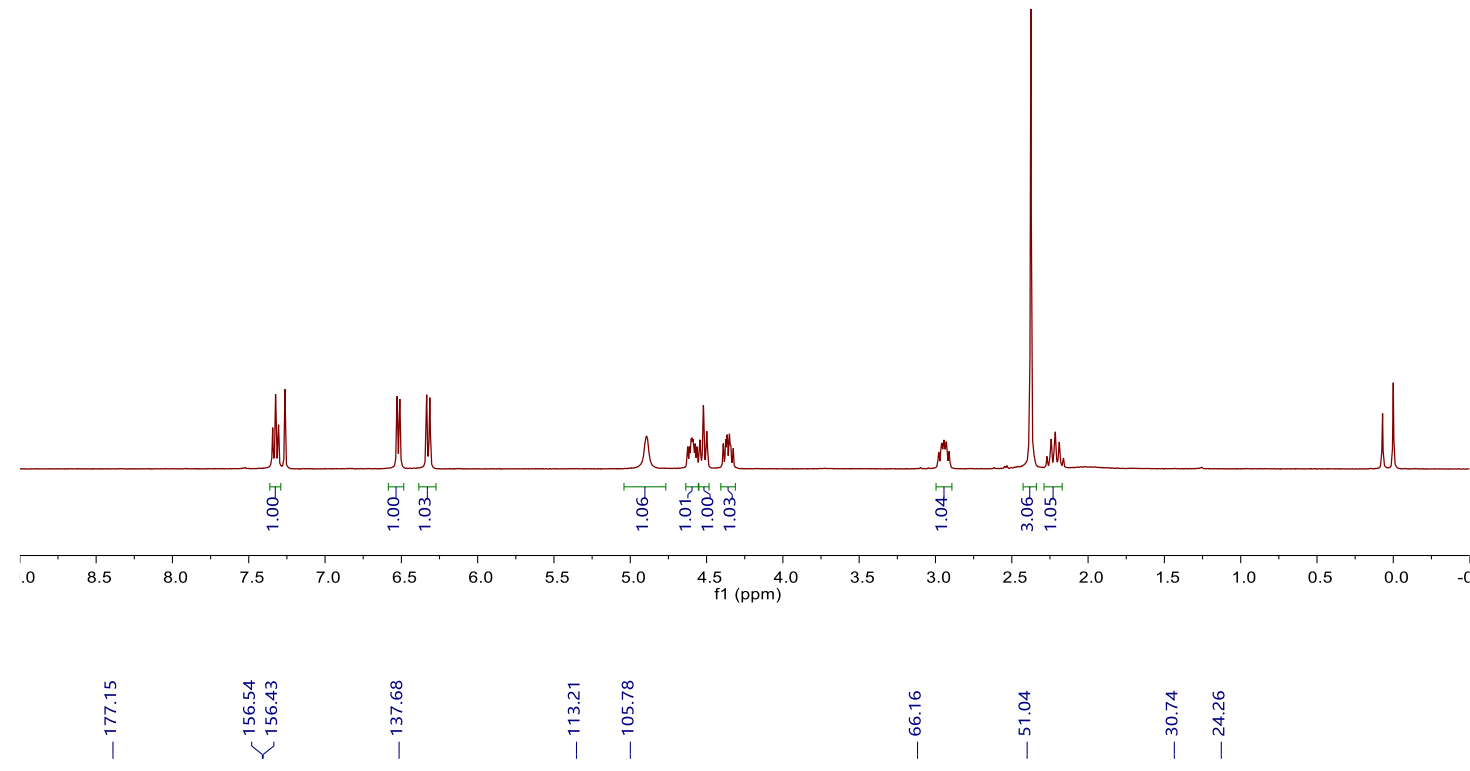<smiles>Cc1cccc(NC2CCOC2=O)n1</smiles>

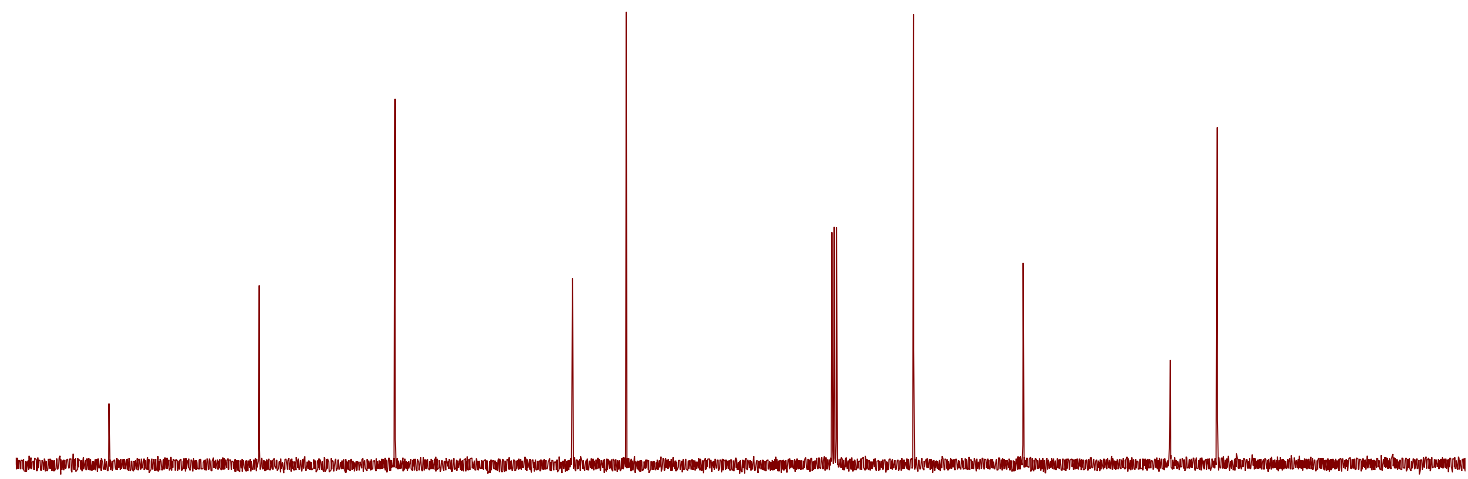

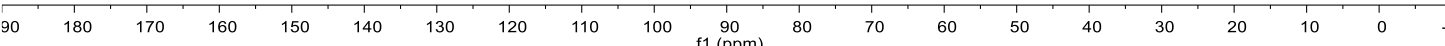


3-(Phenylamino)tetrahydropyran-2-one (8a)

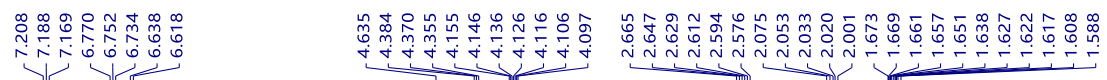

${ }^{N}$

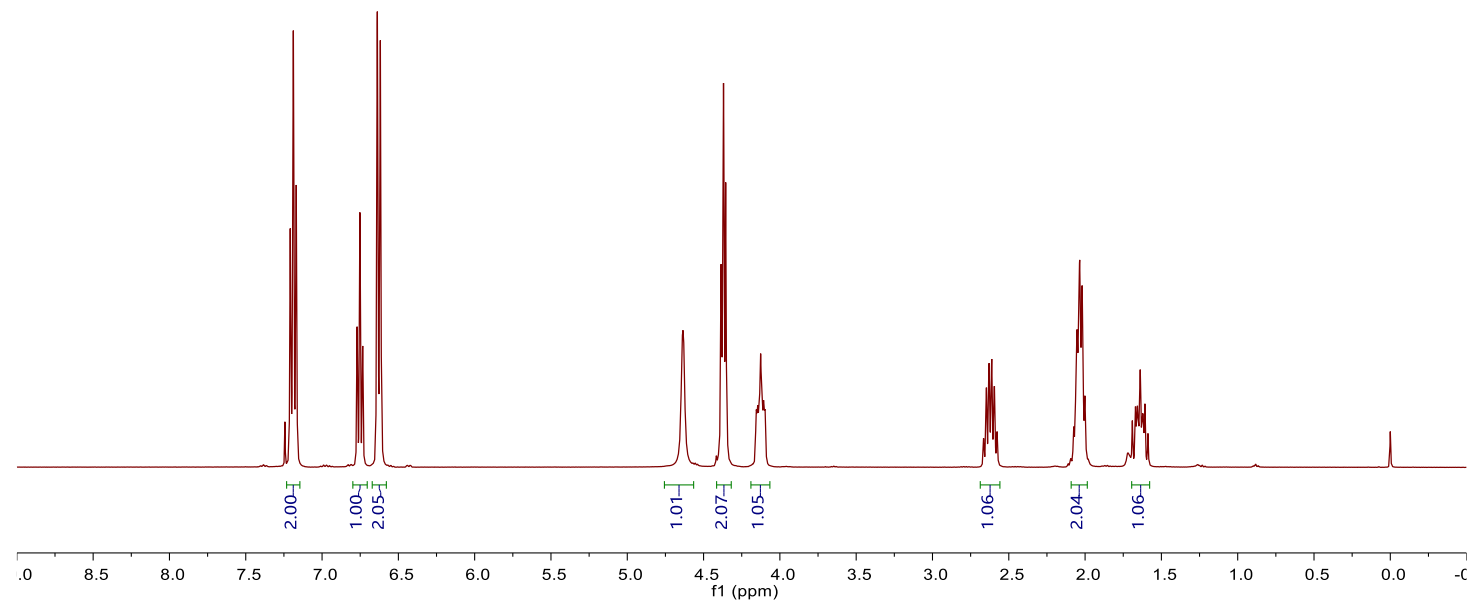

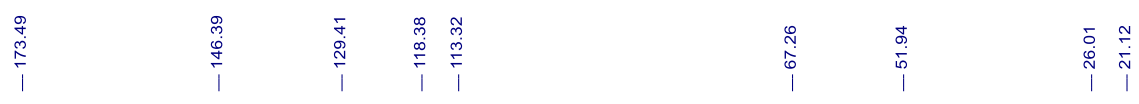<smiles>O=C1OCCCC1Nc1ccccc1</smiles>

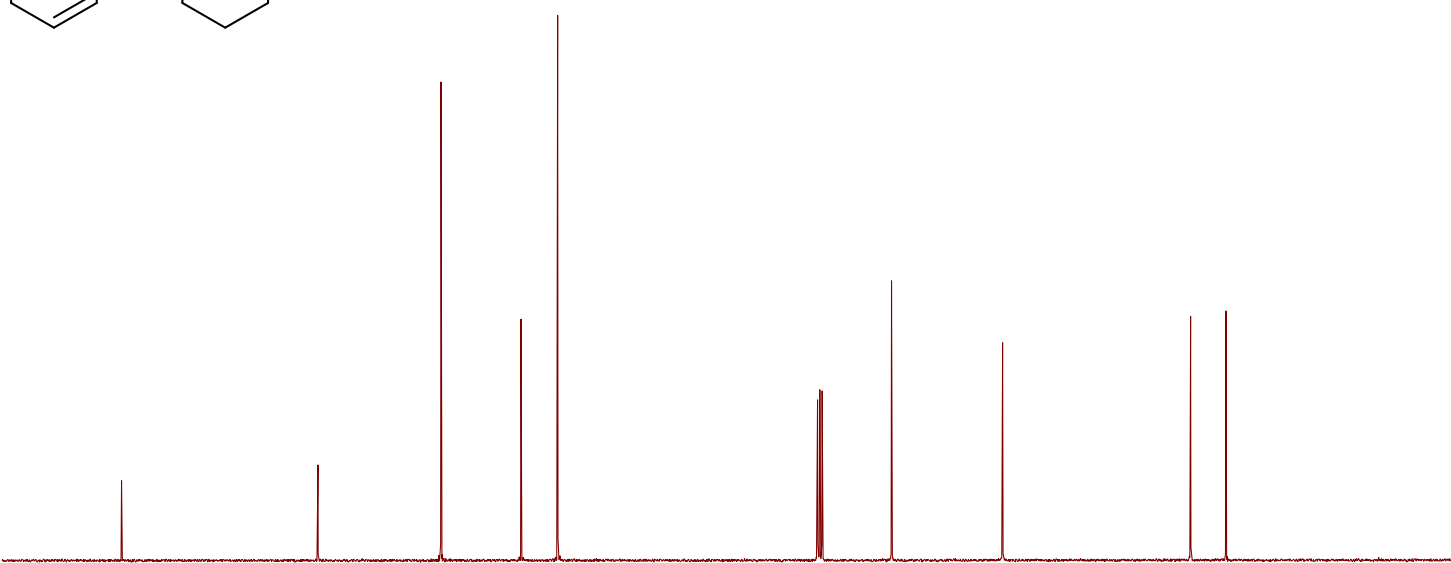

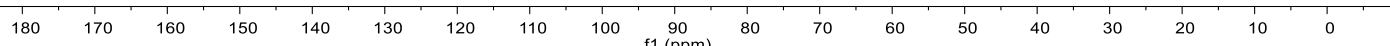


3-(p-Tolylamino)tetrahydropyran-2-one (8b)

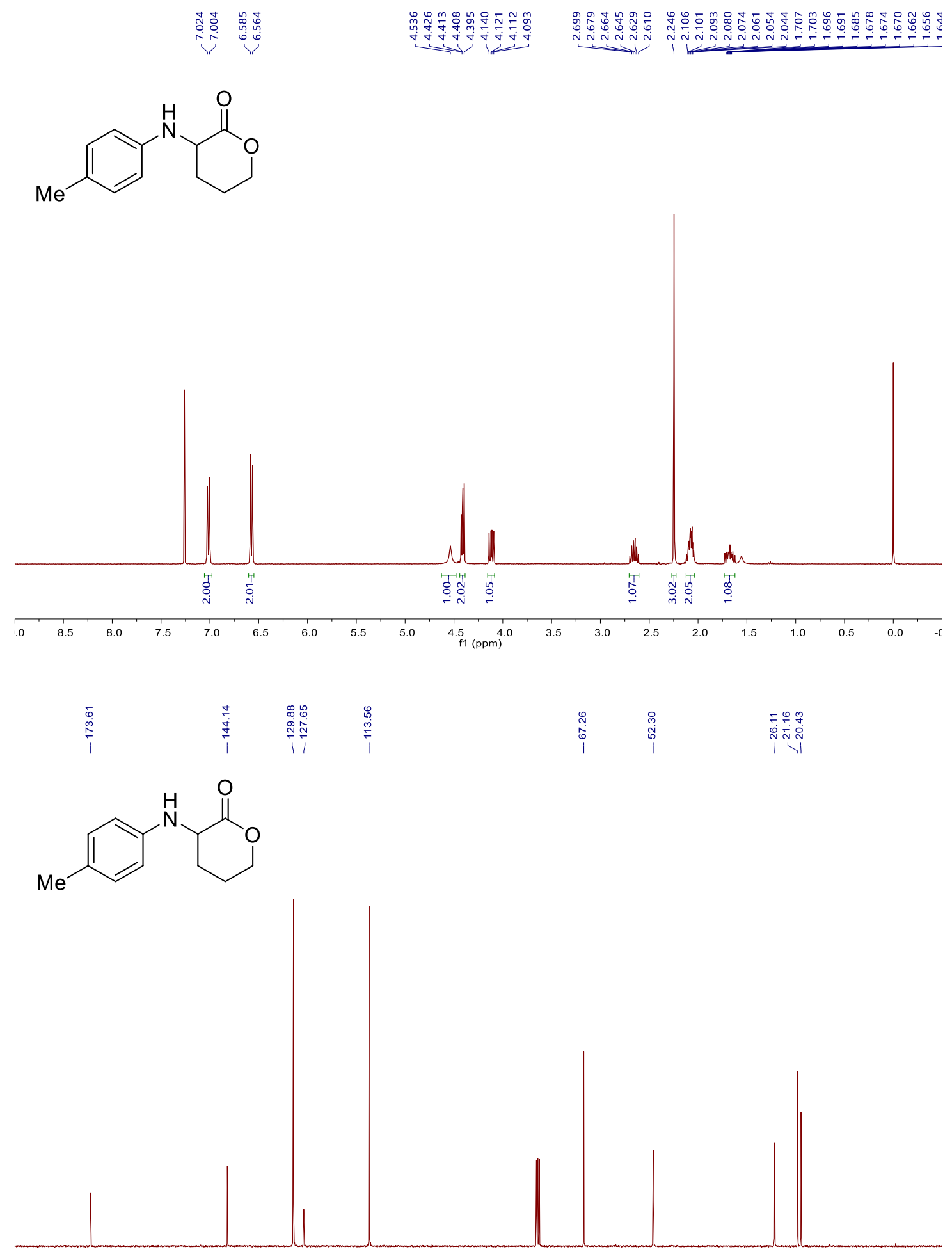

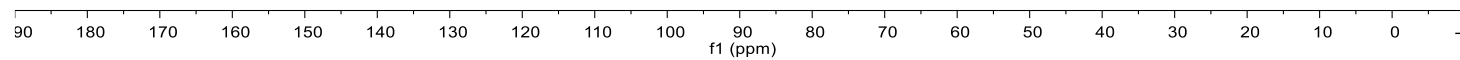


3-((4-Methoxyphenyl)amino)tetrahydropyran-2-one (8c)

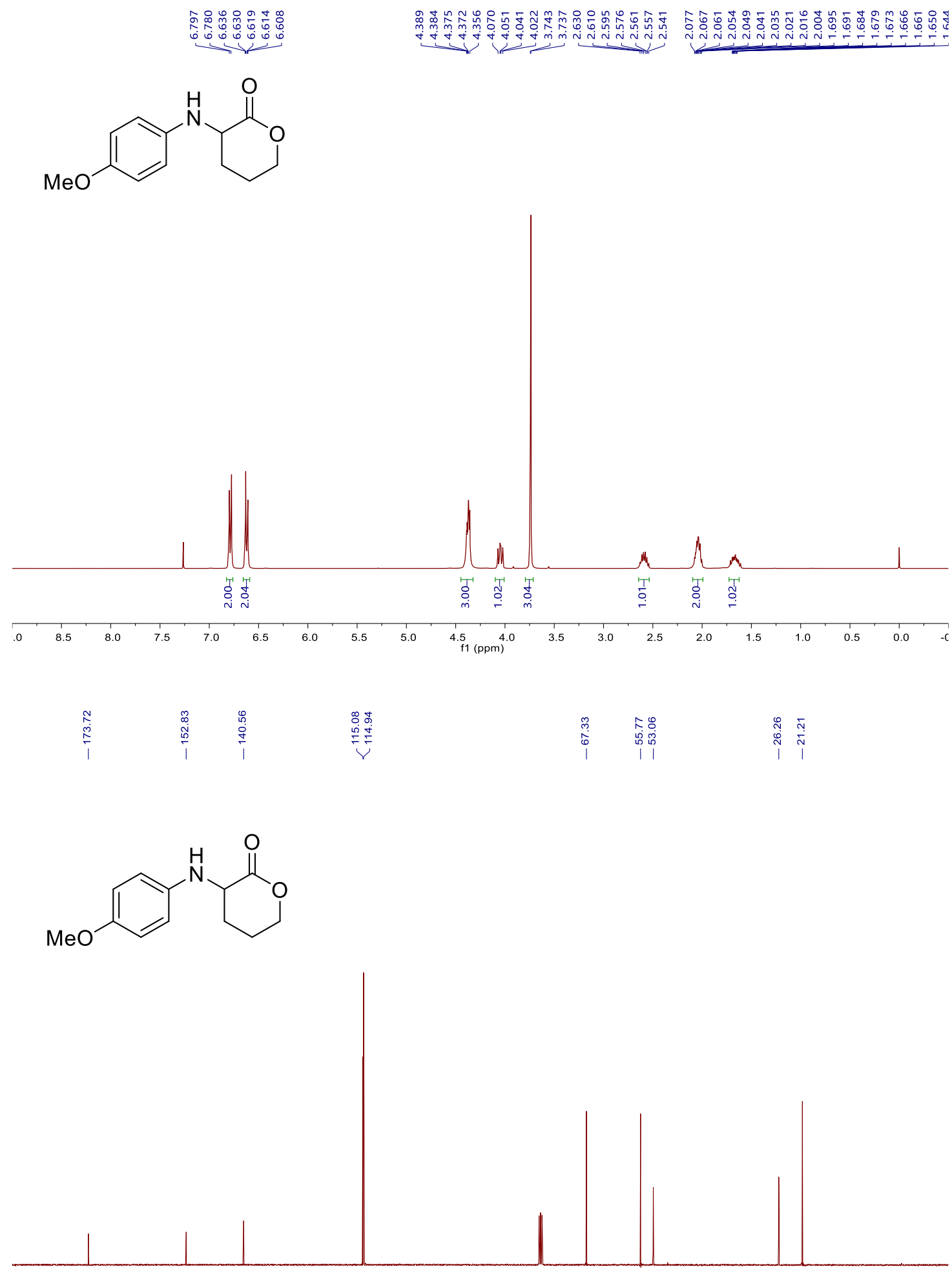

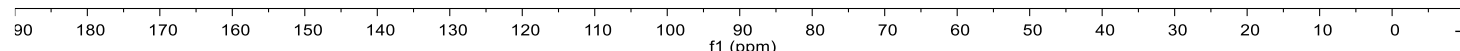


3-((4-Fluorophenyl)amino)tetrahydropyran-2-one (8d)

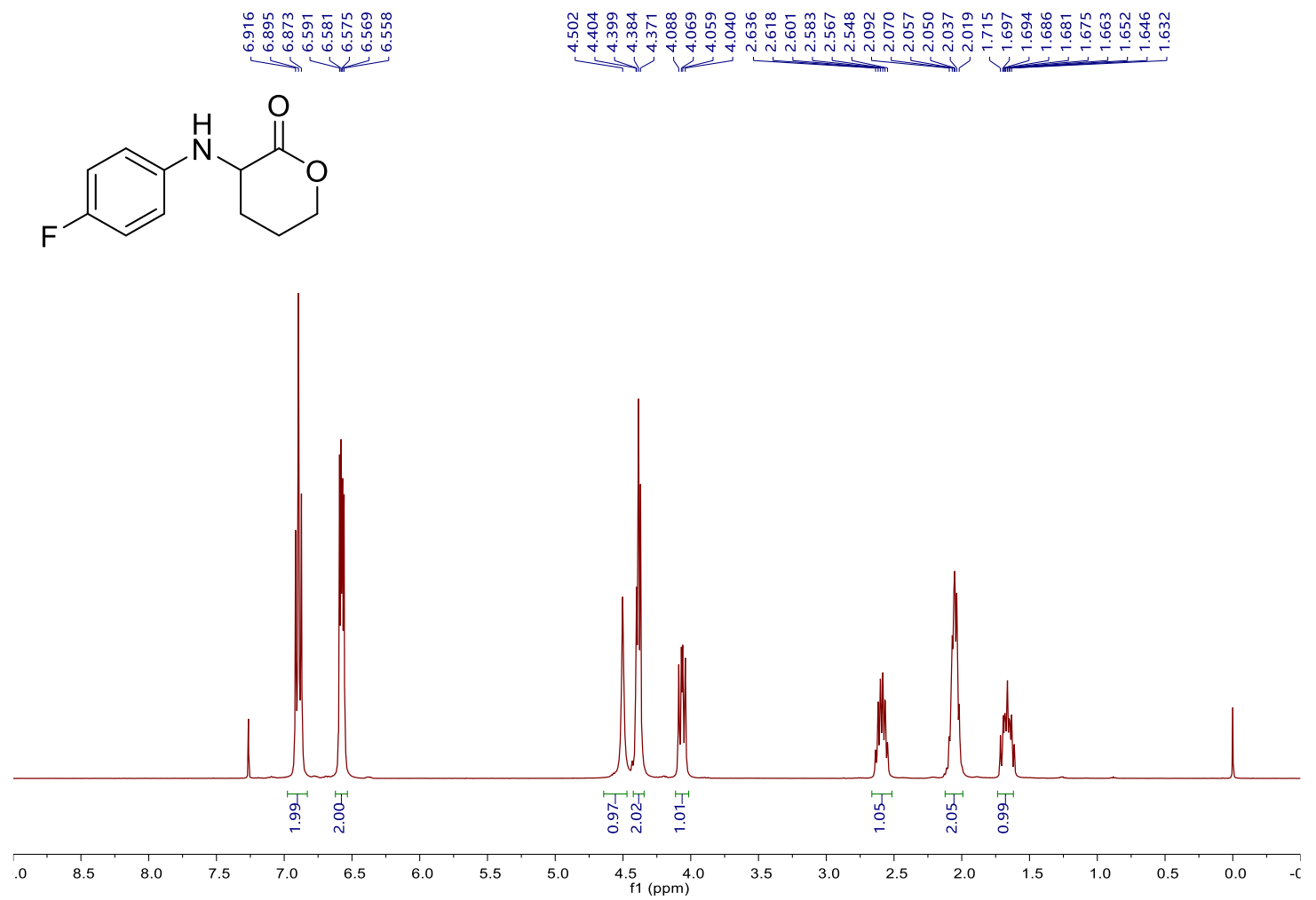

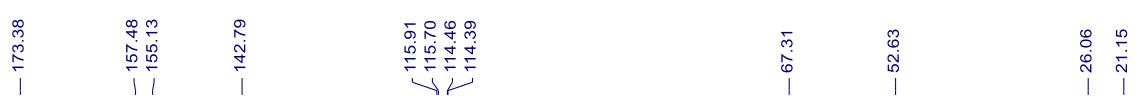<smiles>O=C1OCCCC1Nc1ccc(F)cc1</smiles>

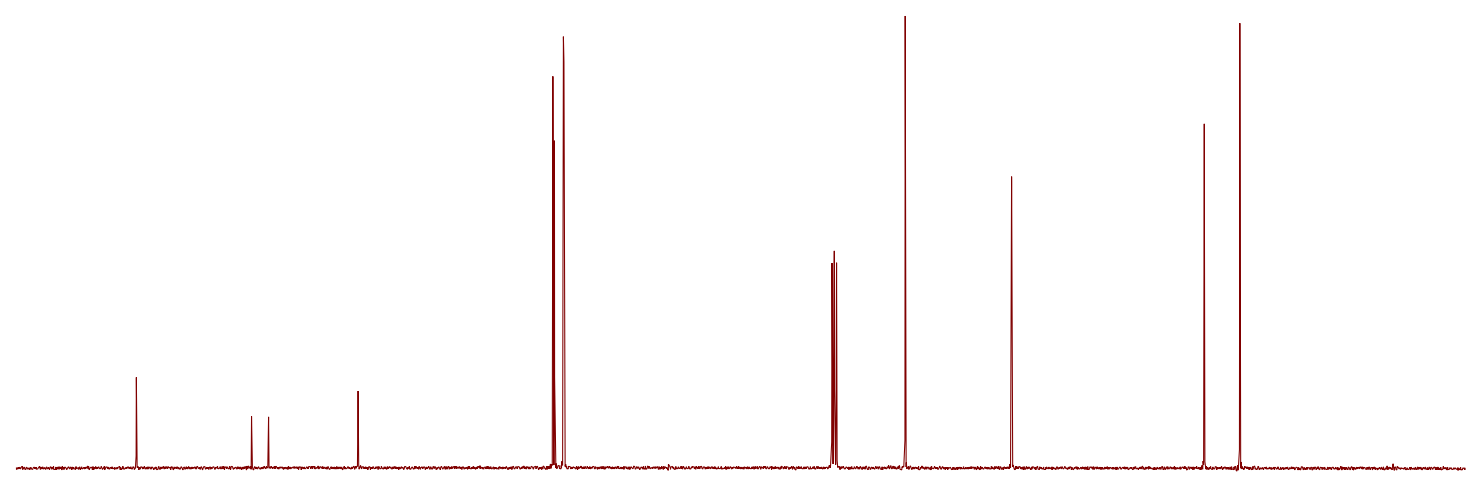

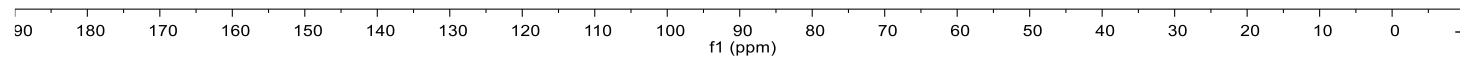


3-((4-Chlorophenyl)amino)tetrahydropyran-2-one (8e)

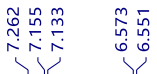

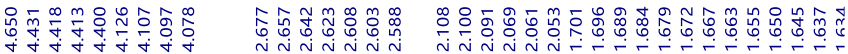<smiles>O=C1OCCCC1Nc1ccc(Cl)cc1</smiles>

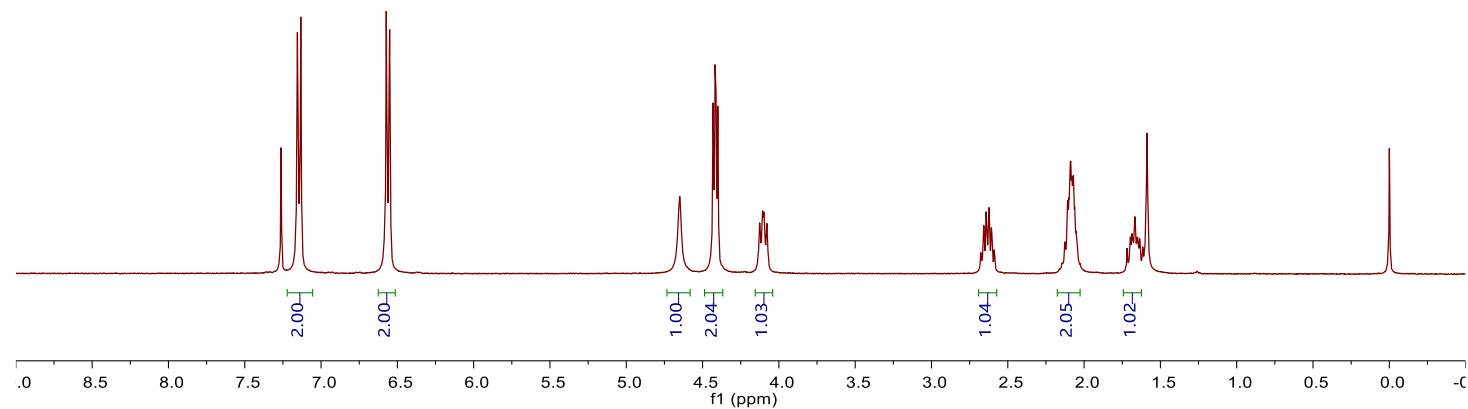<smiles>O=C1OCCCC1Nc1ccc(Cl)cc1</smiles>

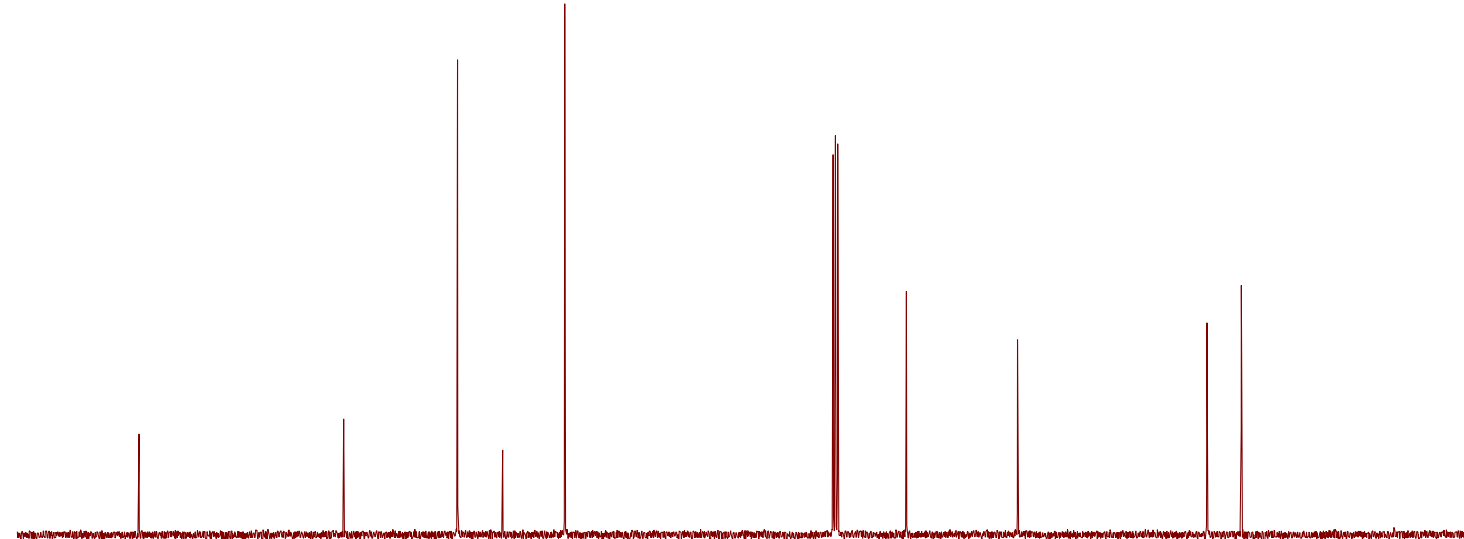

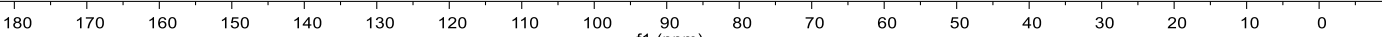


3-([1,1'-Biphenyl]-4-ylamino)tetrahydropyran-2-one (8f)

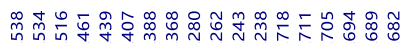

$\begin{array}{lll}0 & 0 \\ 0\end{array}$

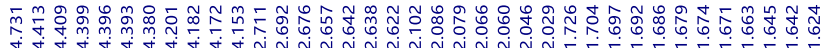

(1)

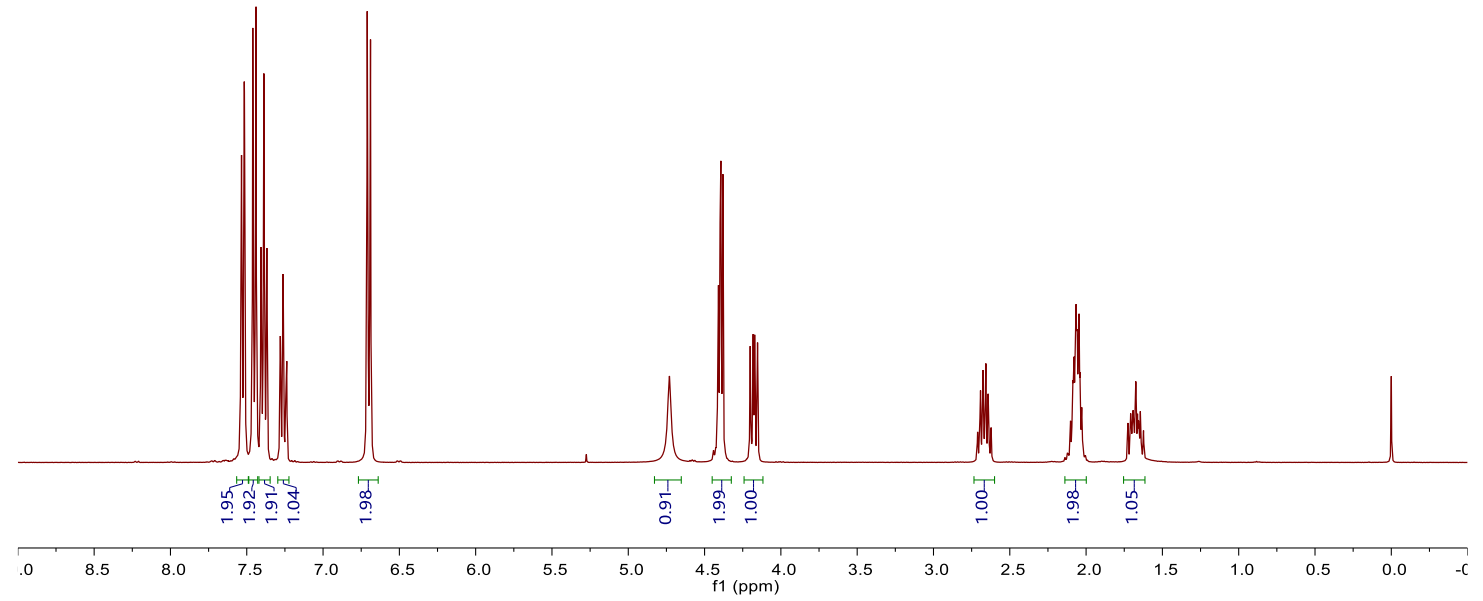

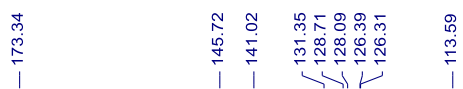

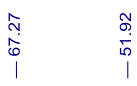

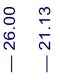<smiles>O=C1OCCCC1Nc1ccc(-c2ccccc2)cc1</smiles>

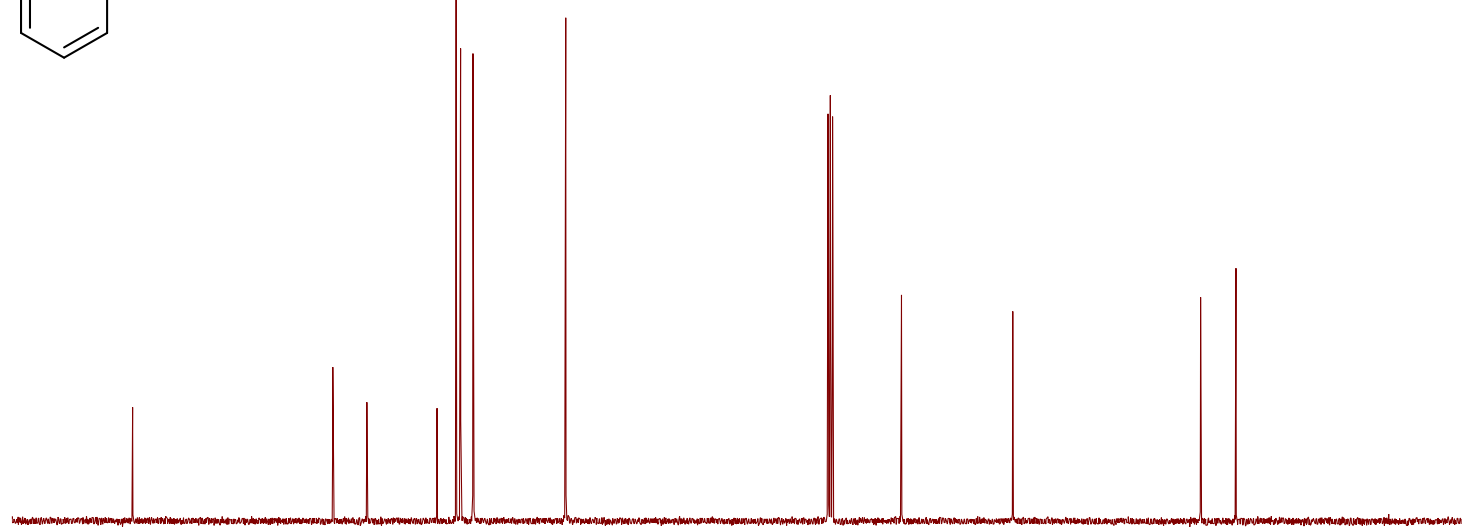

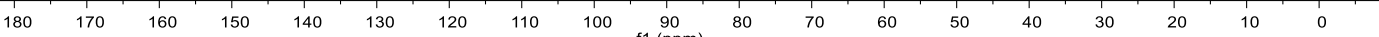


3-(m-Tolylamino)tetrahydro-2H-pyran-2-one (8g)

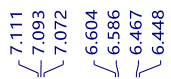

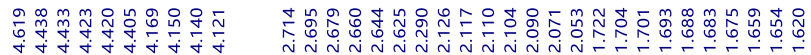

$\mathrm{H}_{\mathrm{C}}^{\mathrm{N}}$

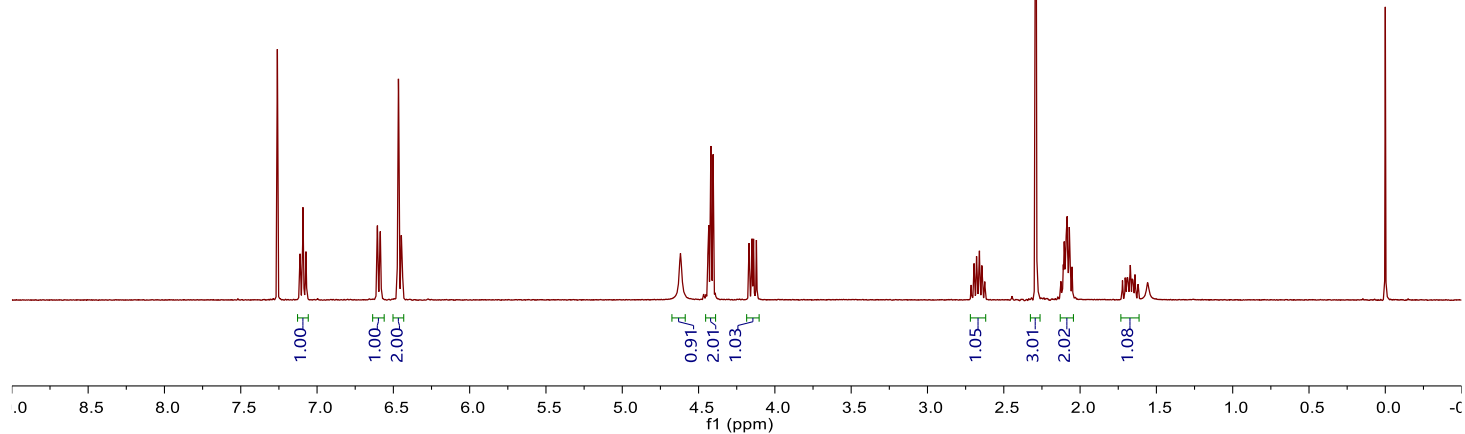

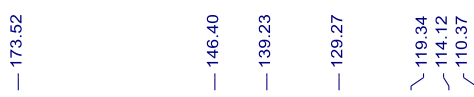

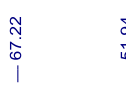

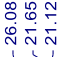<smiles>Cc1cccc(NC2CCCOC2=O)c1</smiles>

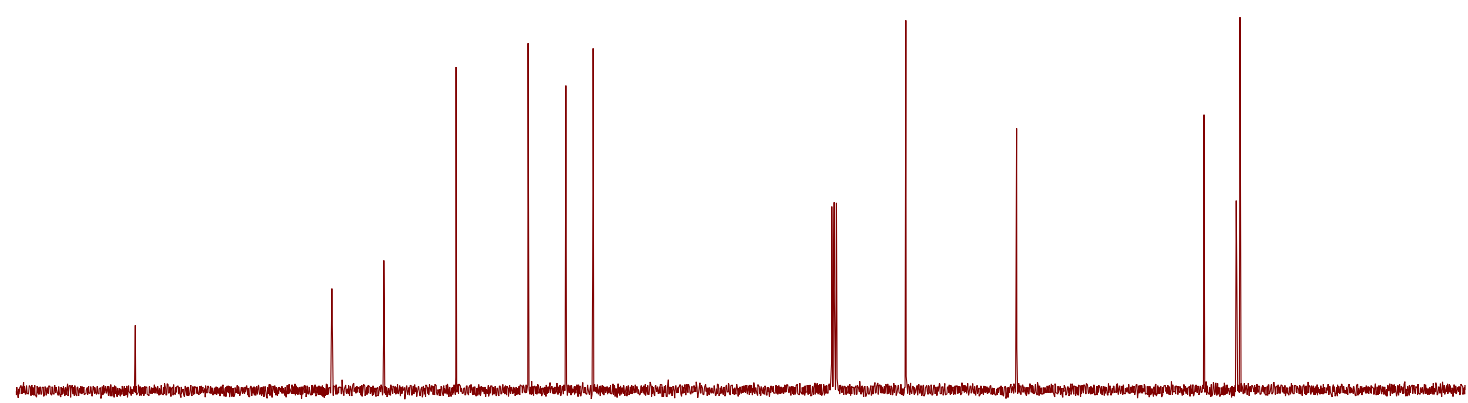

$\begin{array}{lllllllllllllllllllllllllll} & 180 & 170 & 160 & 150 & 140 & 130 & 120 & 110 & 100 & 90 & 90 & 70 & 60 & 50 & 40 & 30 & 20 & 10 & 0 & -\end{array}$ 
<smiles>COc1cccc(NC2CCCOC2=O)c1</smiles>

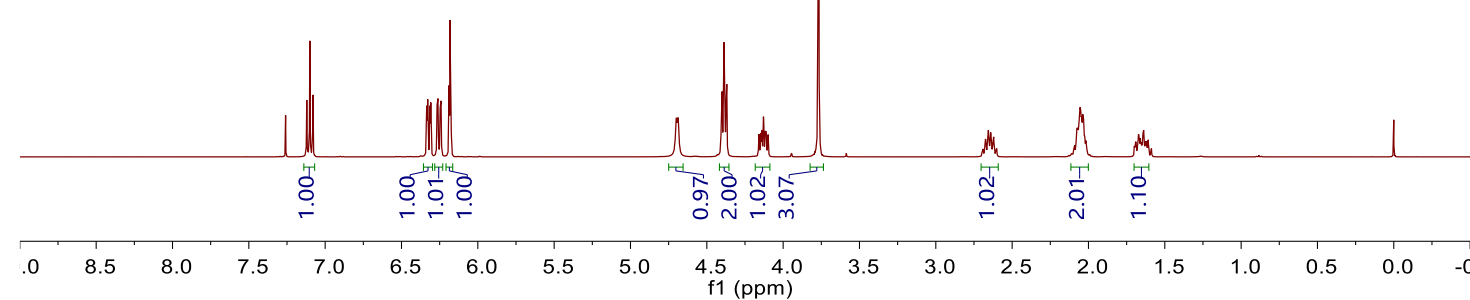<smiles>COc1cccc(NC2CCCOC2=O)c1</smiles>
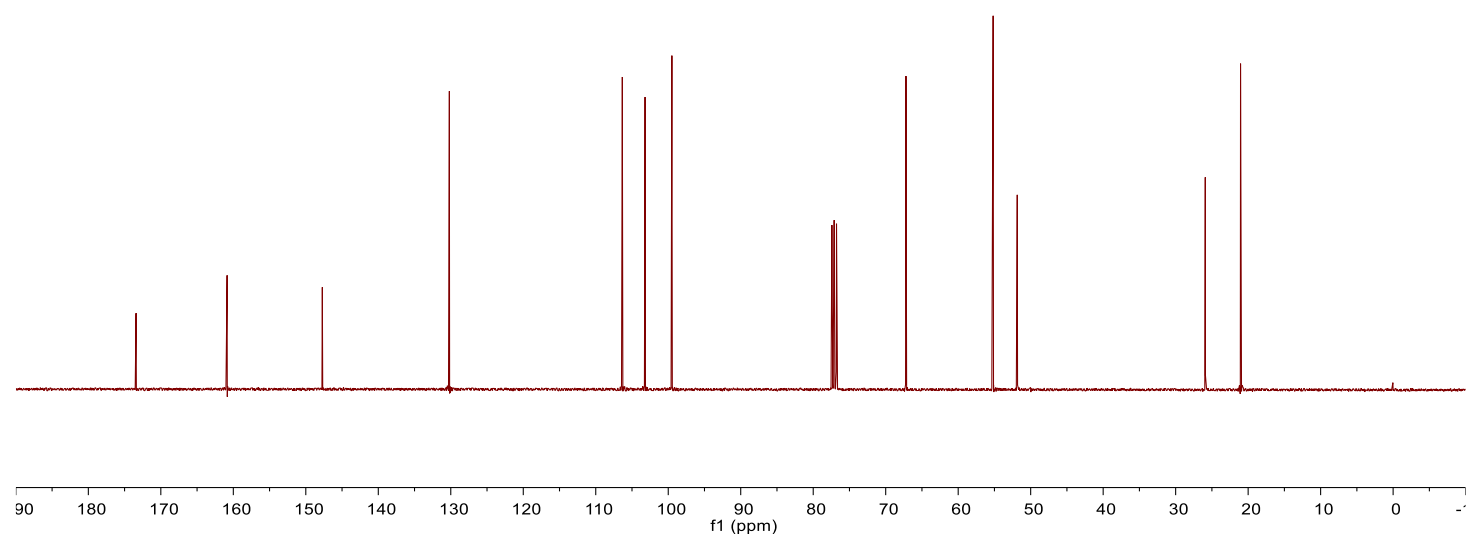
3-((3-Chlorophenyl)amino)tetrahydropyran-2-one (8i)

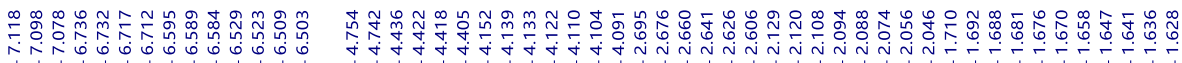<smiles>O=C1OCCCC1Nc1cccc(Cl)c1</smiles>

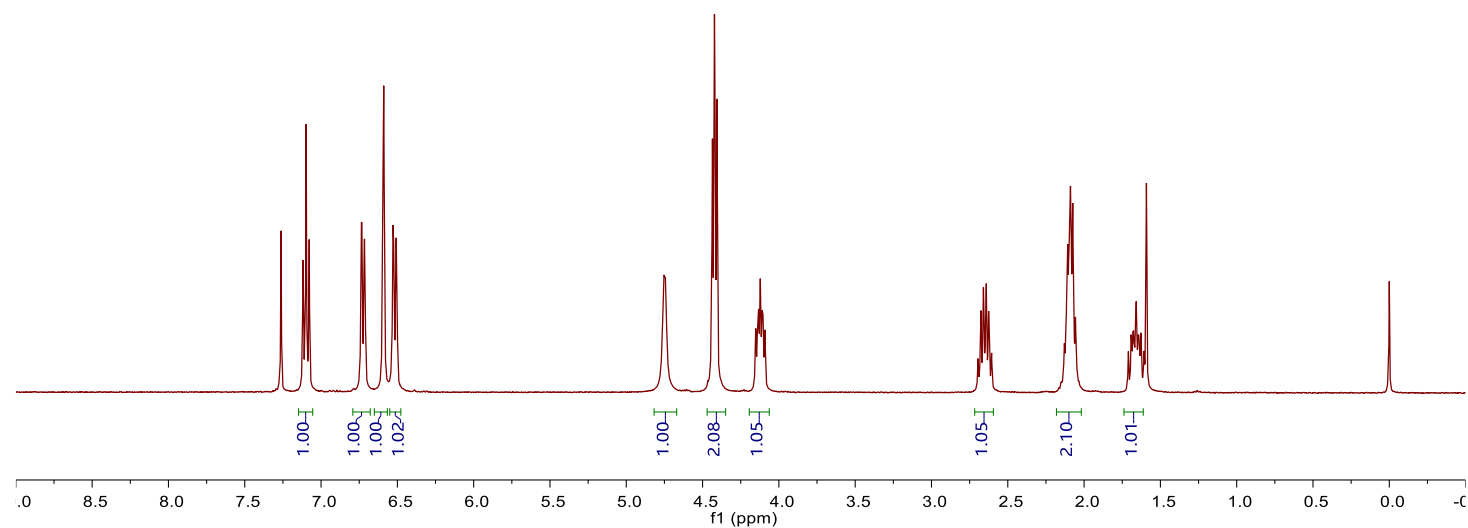

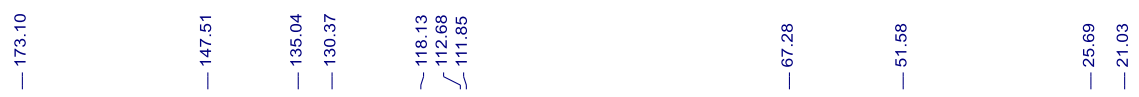<smiles>O=C1OCCCC1Nc1cccc(Cl)c1</smiles>

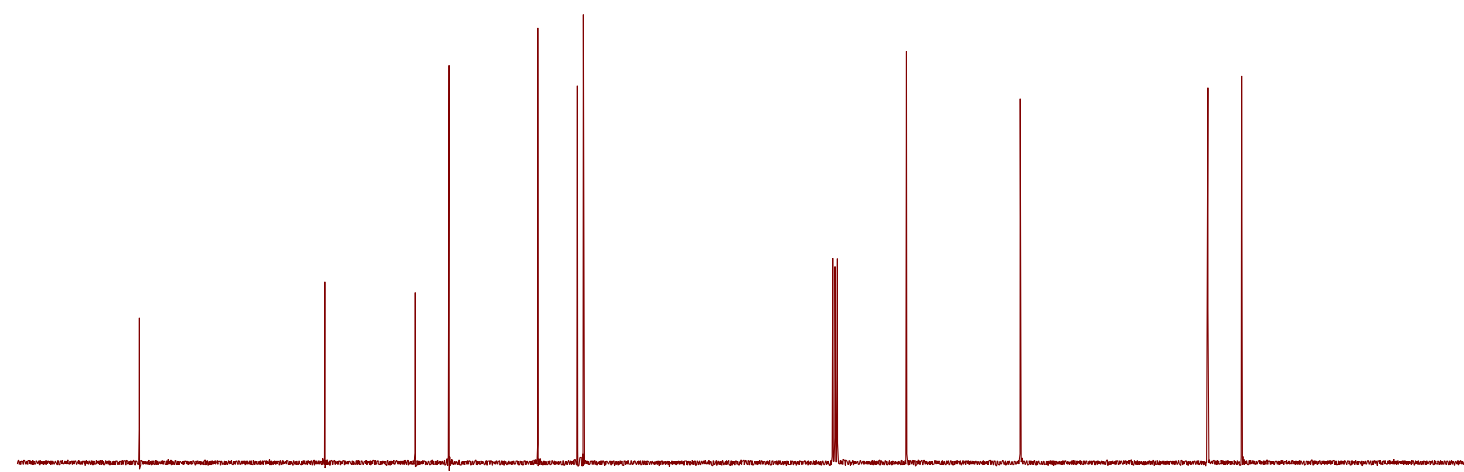

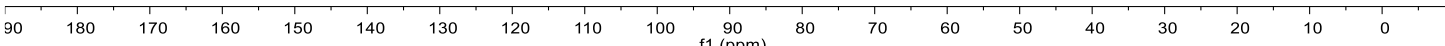


3-(o-Tolylamino)tetrahydropyran-2-one (8j)
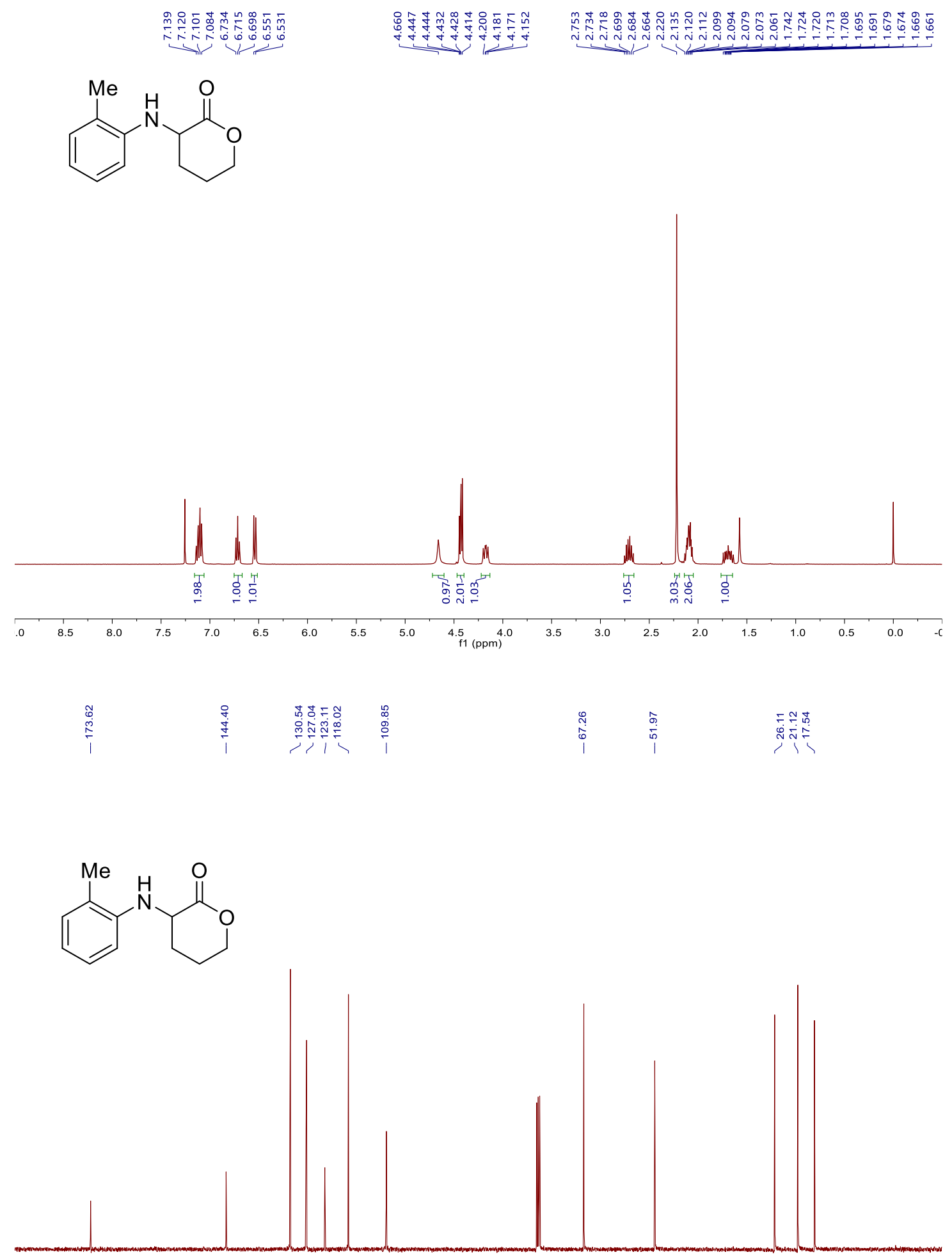

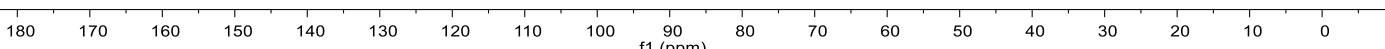


3-((2-Ethylphenyl)amino)tetrahydropyran-2-one (8k)

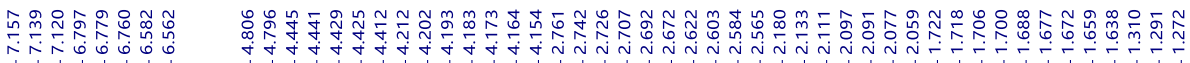

(N)

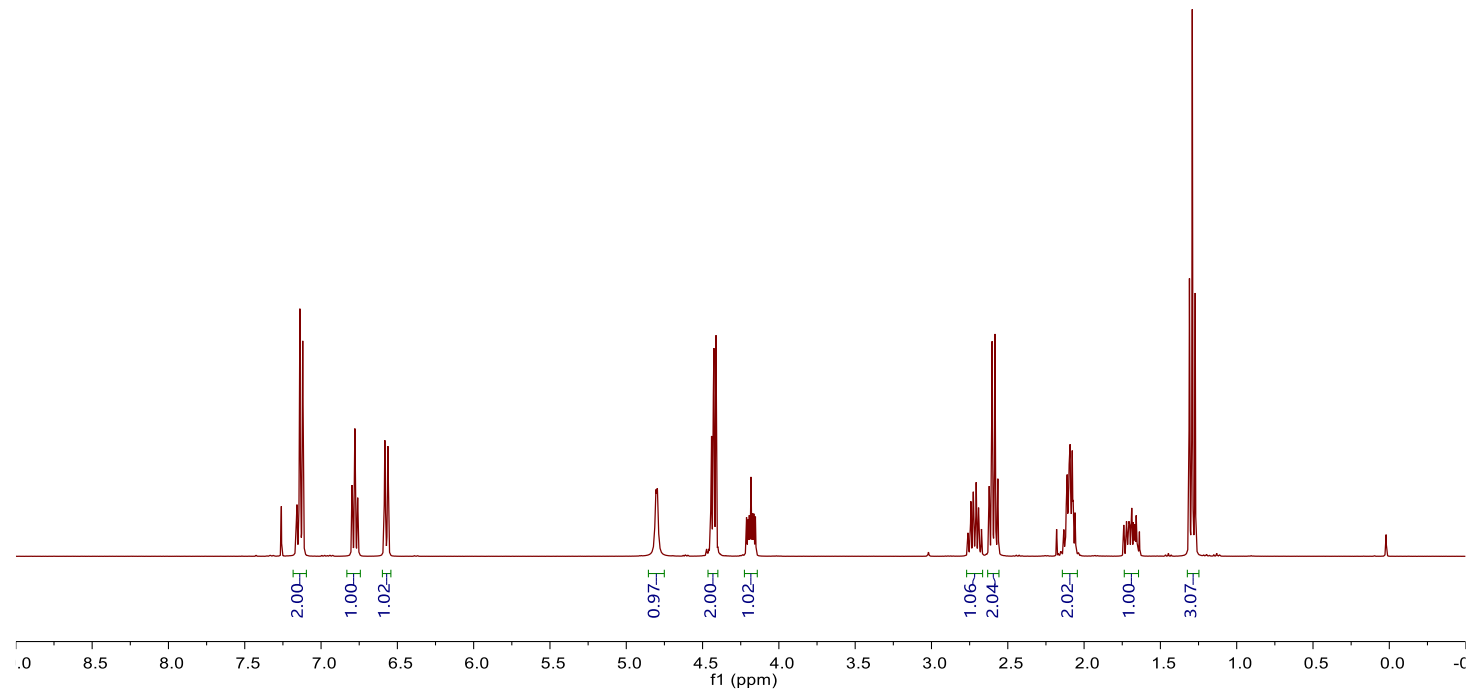

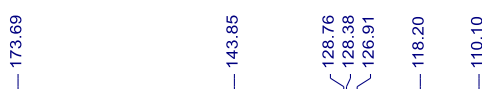

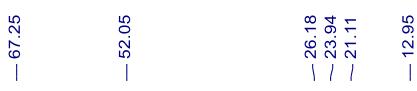

$\mathrm{H}^{\mathrm{E}}$

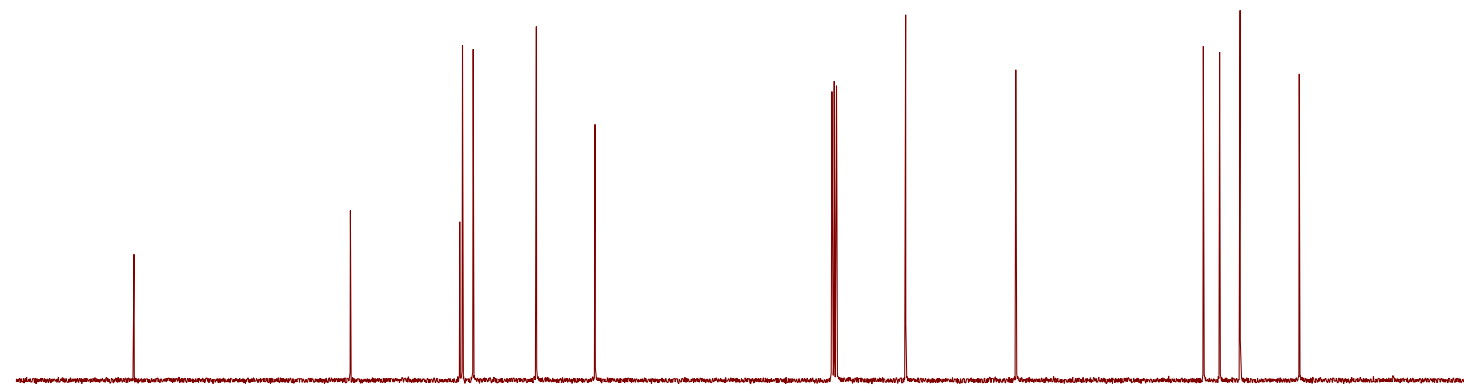

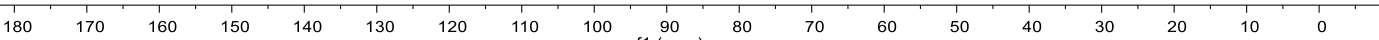


3-((2-Isopropylphenyl)amino)tetrahydropyran-2-one (8I)

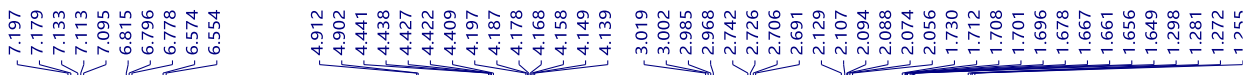<smiles>CCCc1ccccc1NC1CCCOC1=O</smiles>

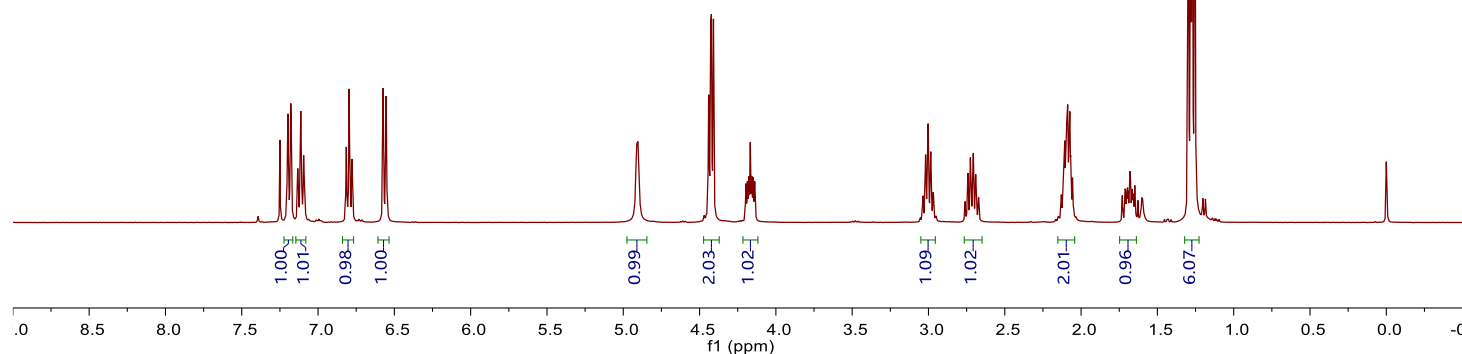

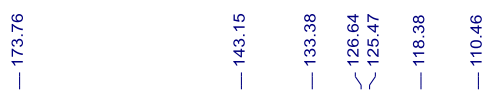

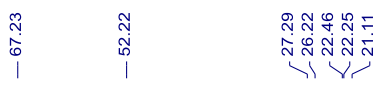

${ }^{i} \mathrm{Pr}$

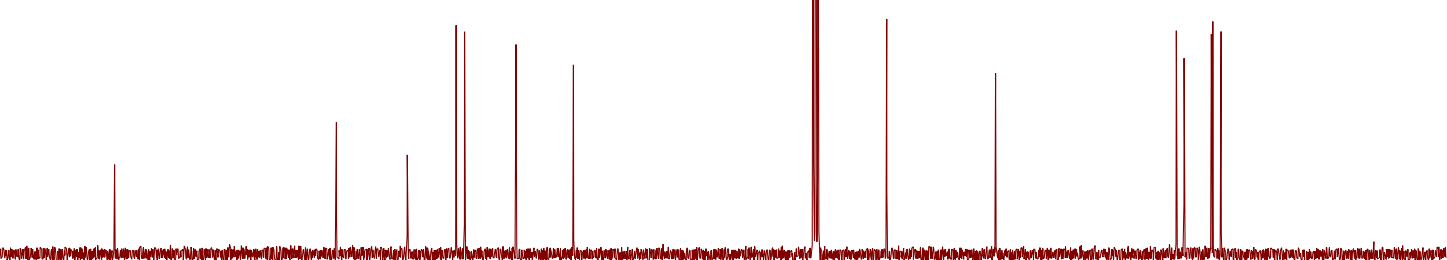

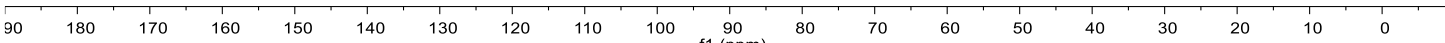


3-((2-Methoxyphenyl)amino)tetrahydropyran-2-one (8m)

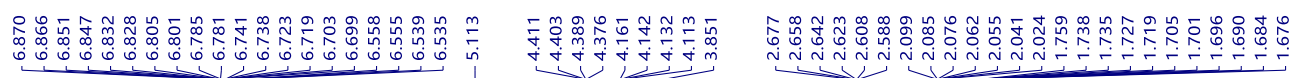

$\underbrace{\mathrm{OMe}}$

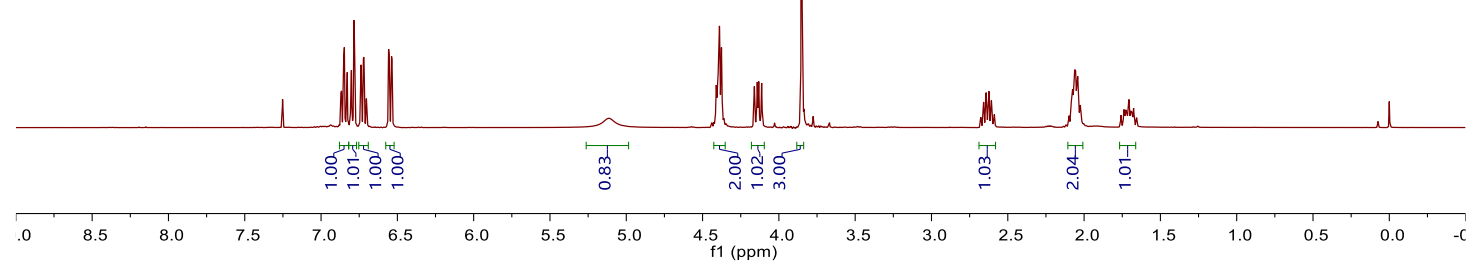

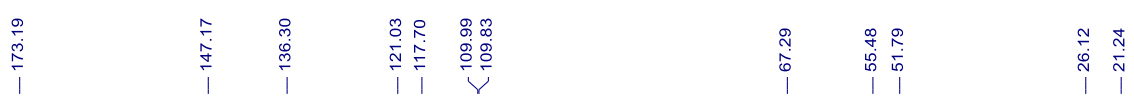<smiles>COc1ccccc1NC1CCCOC1=O</smiles>

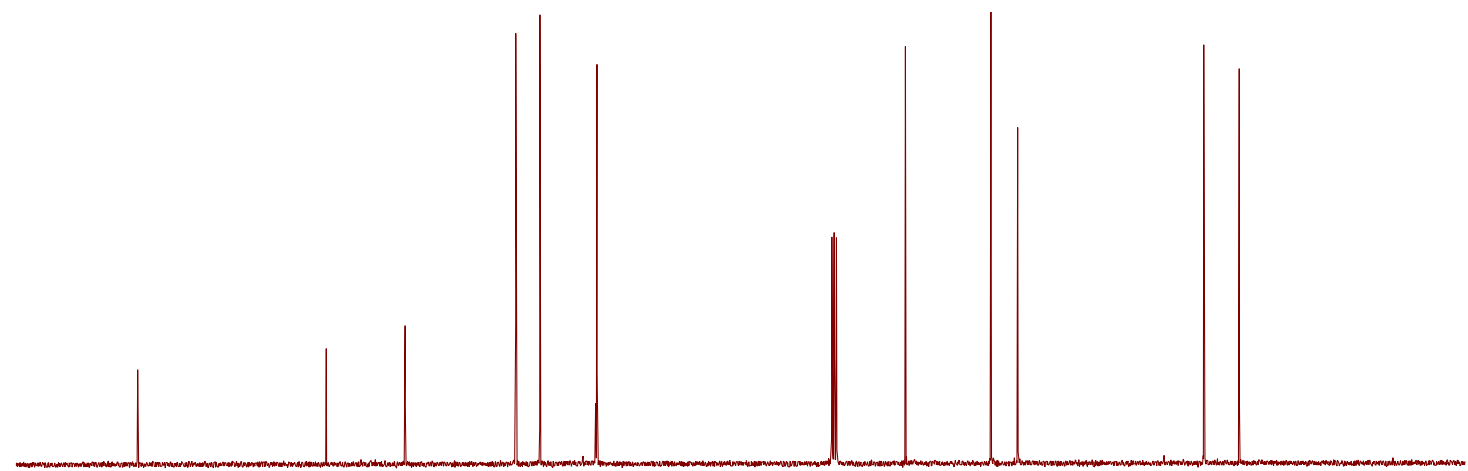

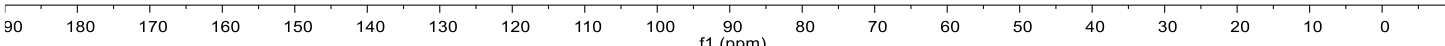


3-((2-Fluorophenyl)amino)tetrahydropyran-2-one (8n)

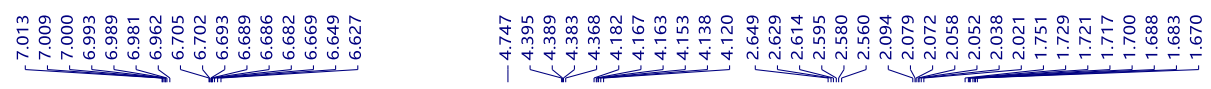

$\mathrm{H}^{\mathrm{N}}$

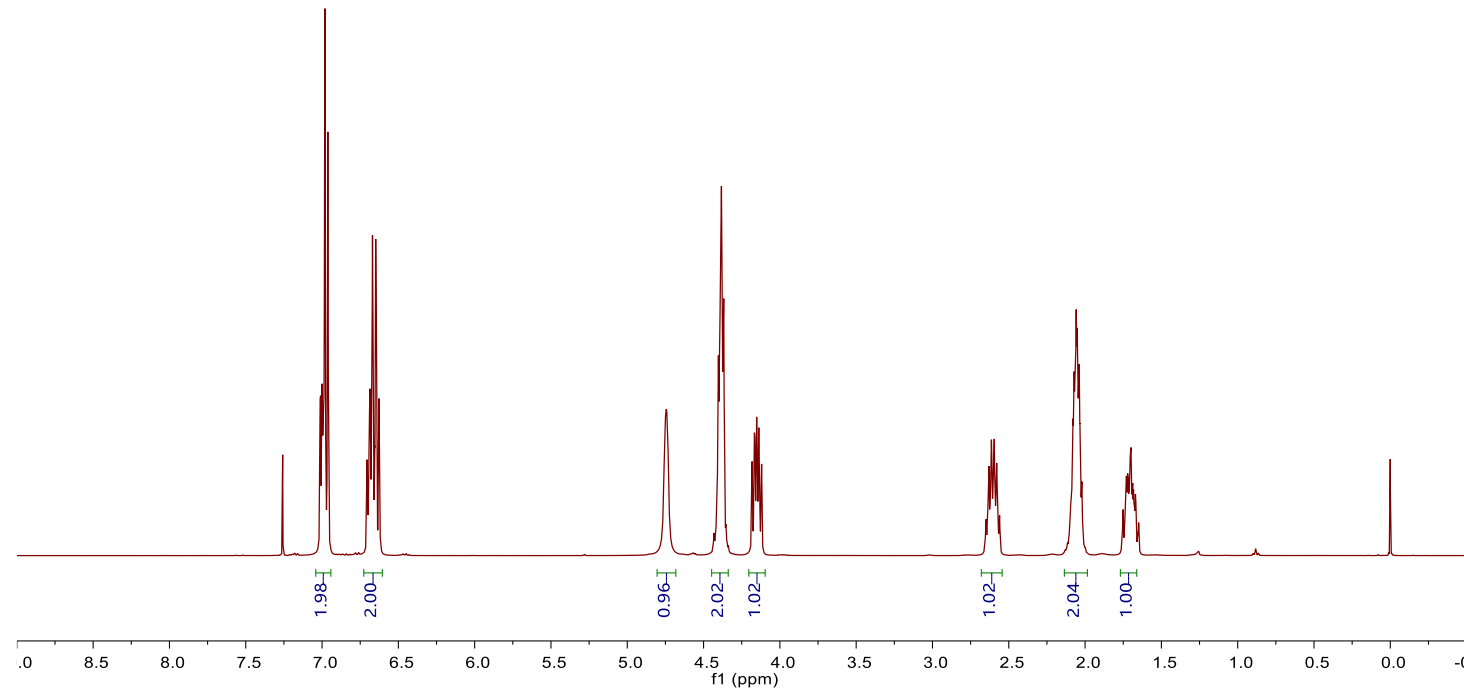

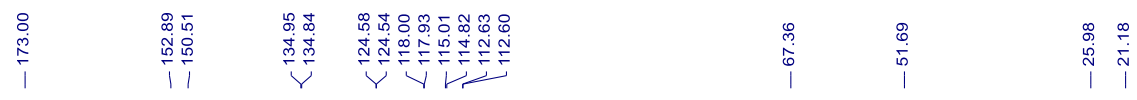<smiles>O=C1OCCCC1Nc1ccccc1F</smiles>

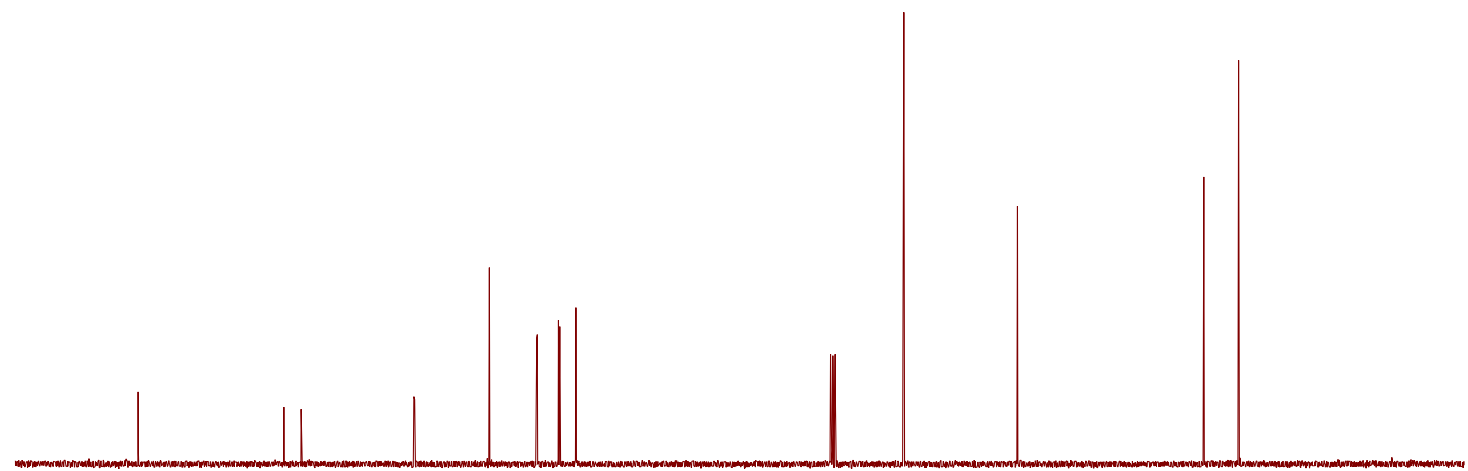

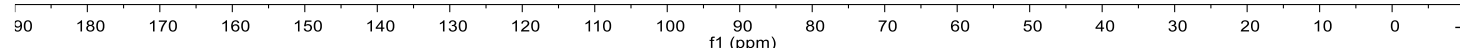


3-((2,4-Dimethoxyphenyl)amino)tetrahydropyran-2-one (80)
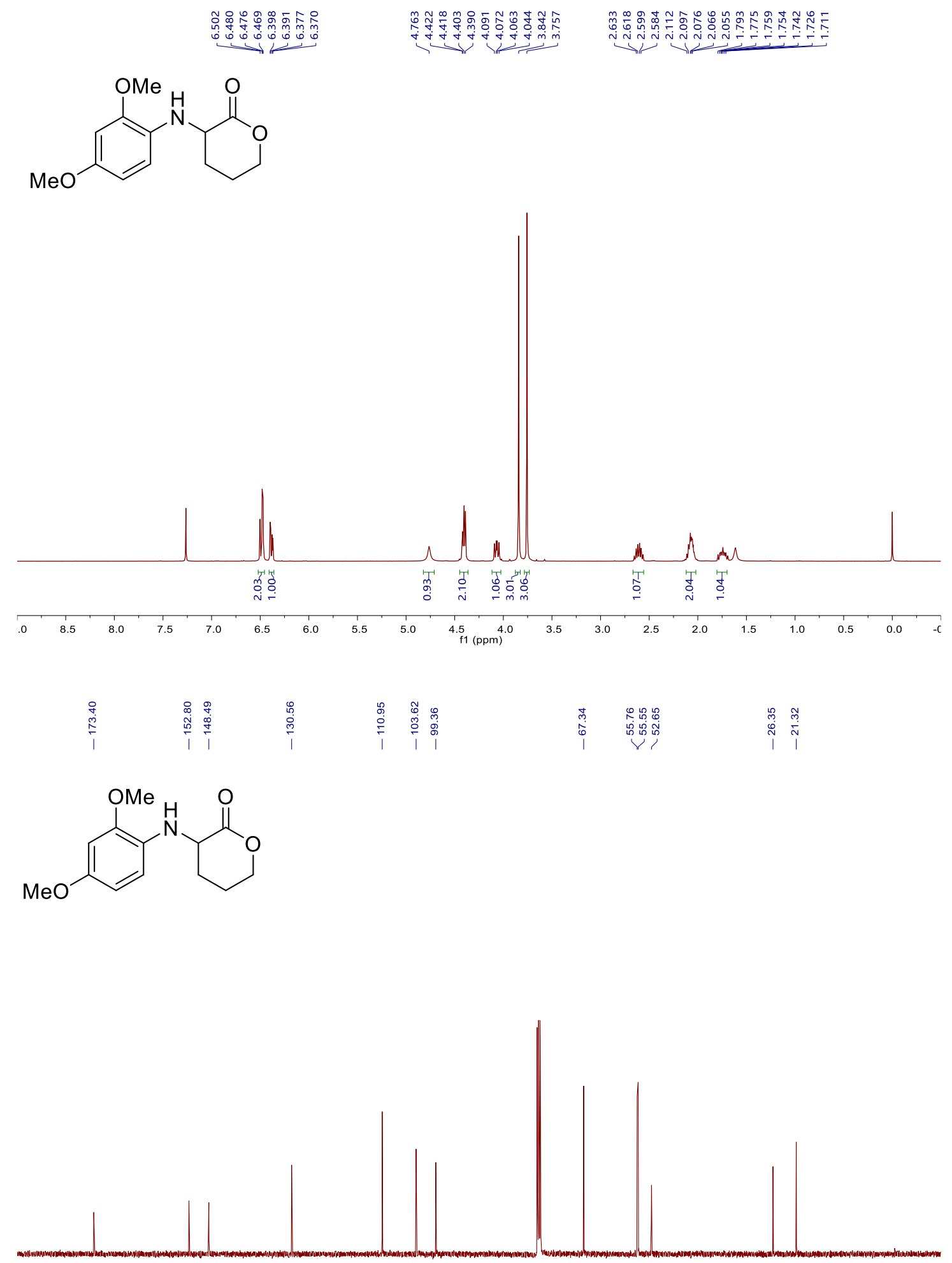

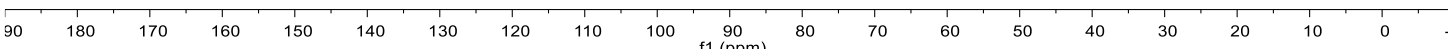


3-(Naphthalen-1-ylamino)tetrahydropyran-2-one (8p)

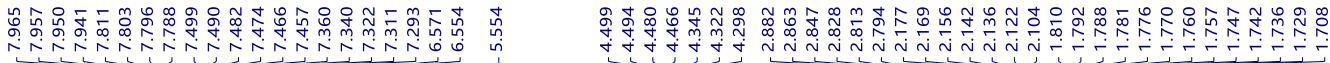

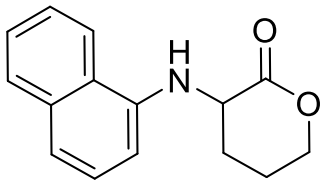

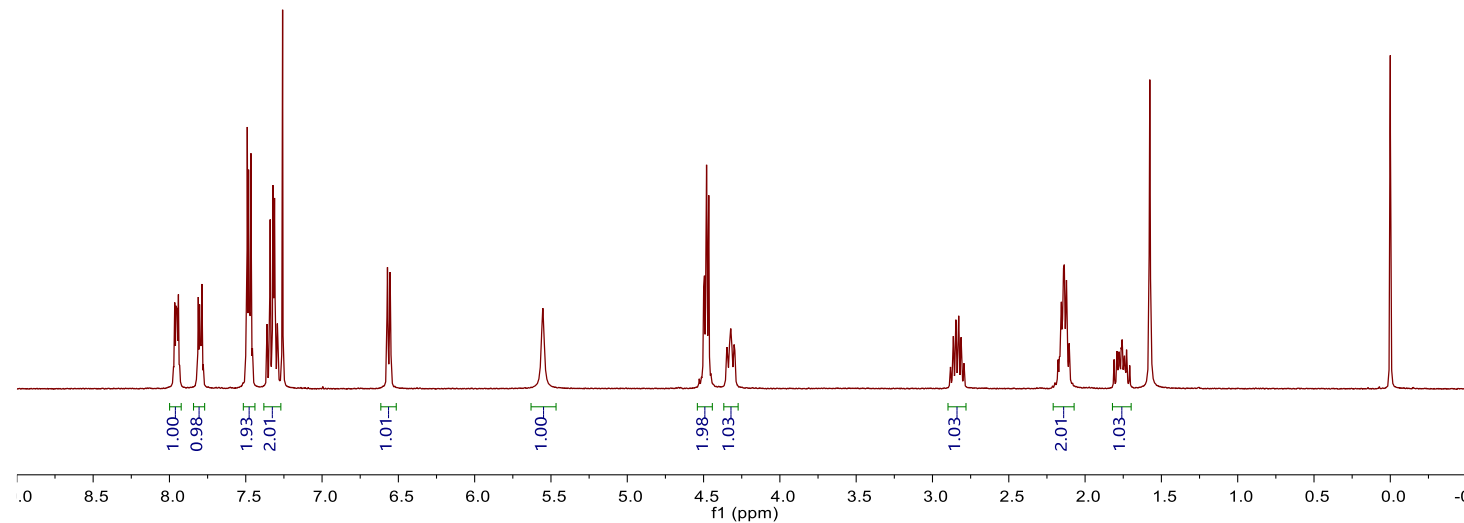

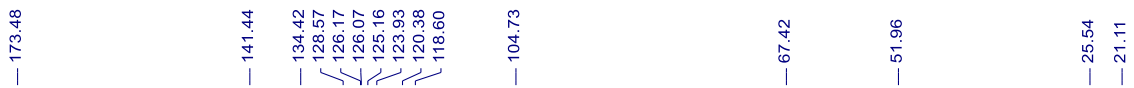<smiles>O=C1OCCCC1Nc1cccc2ccccc12</smiles>

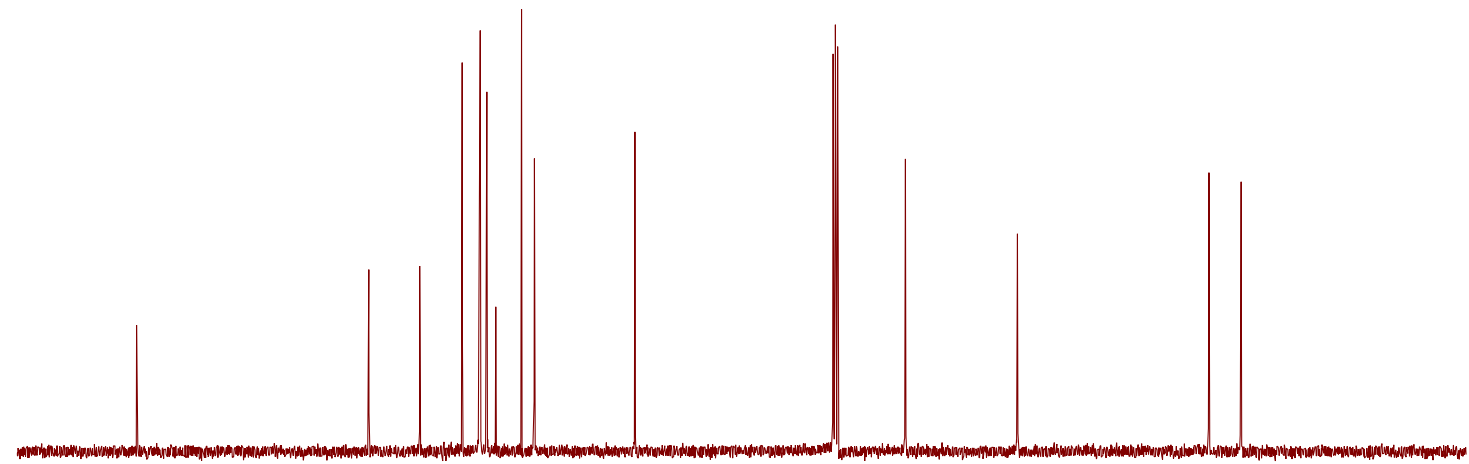

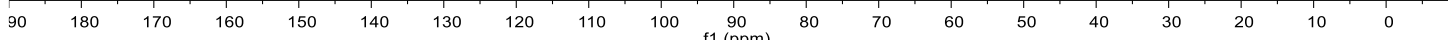


(S)-2-(Phenylamino)butane-1,4-diol (7a)

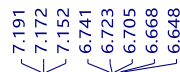<smiles>OCC[C@H](CO)Nc1ccccc1</smiles>

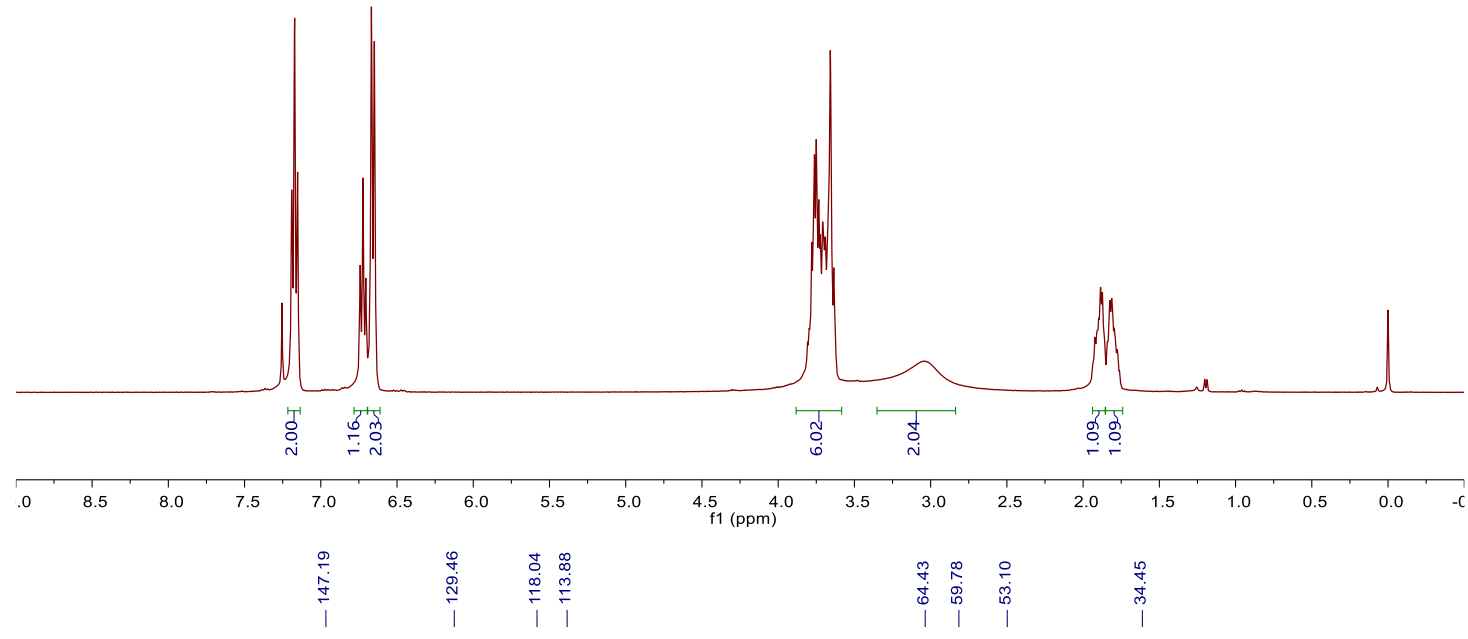<smiles>OCC[C@H](CO)Nc1ccccc1</smiles>

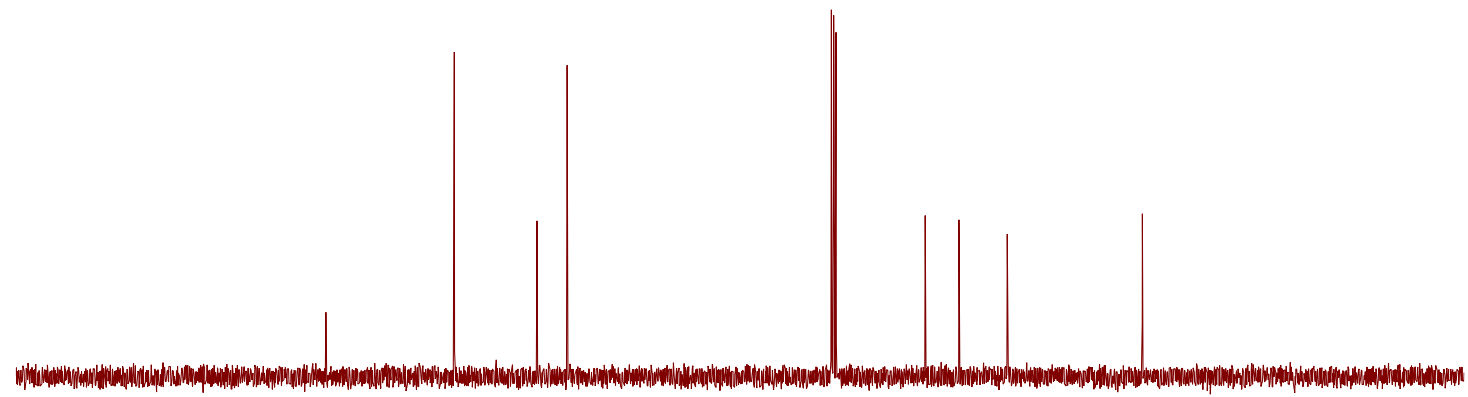

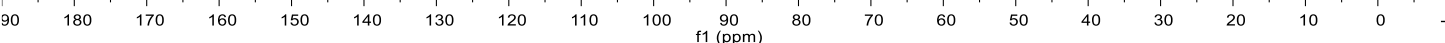


(S)-2-(p-Tolylamino)butane-1,4-diol (7b)
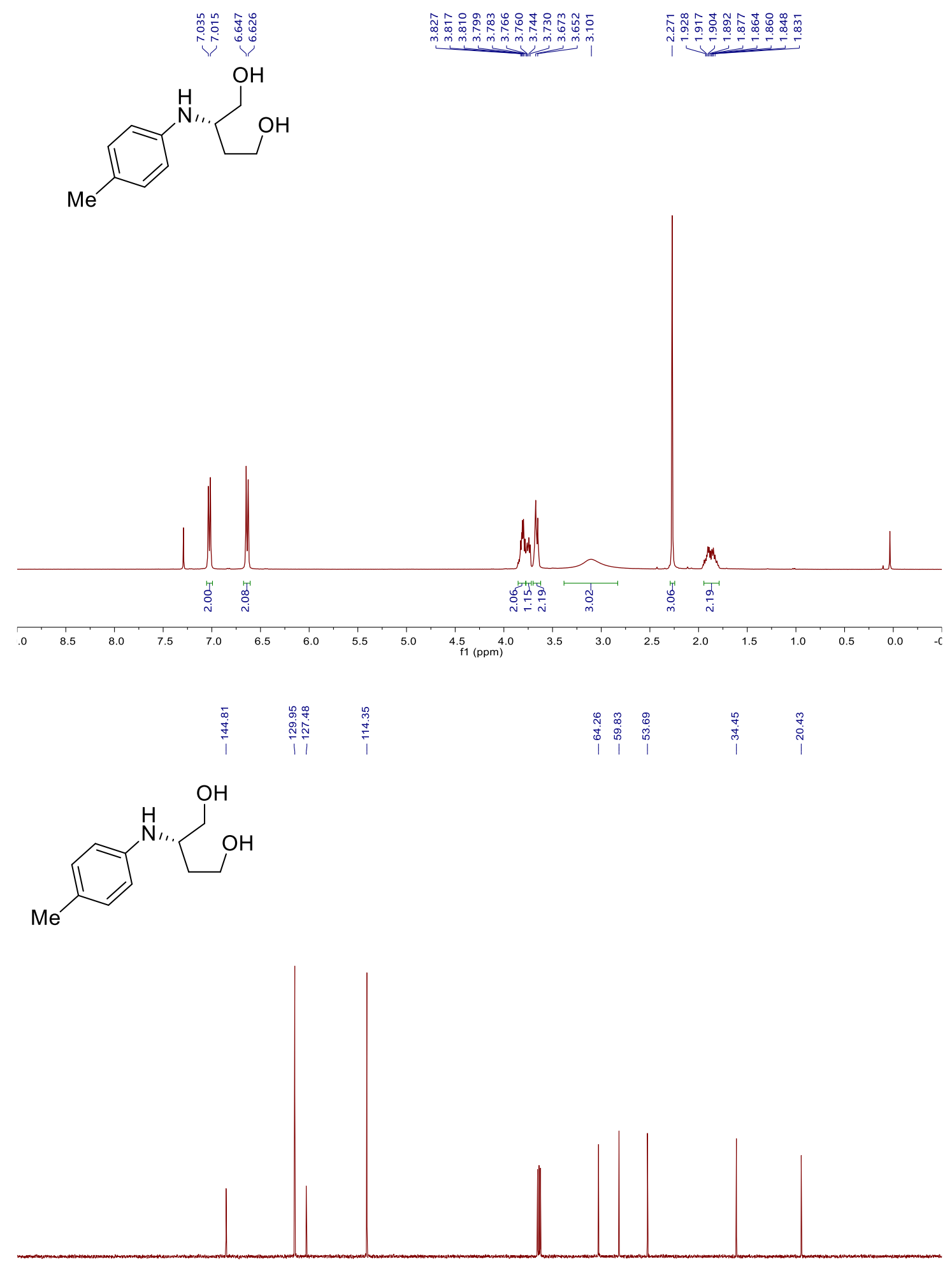

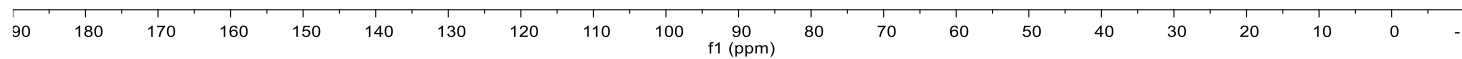


(S)-2-((4-Methoxyphenyl)amino)butane-1,4-diol (7c)

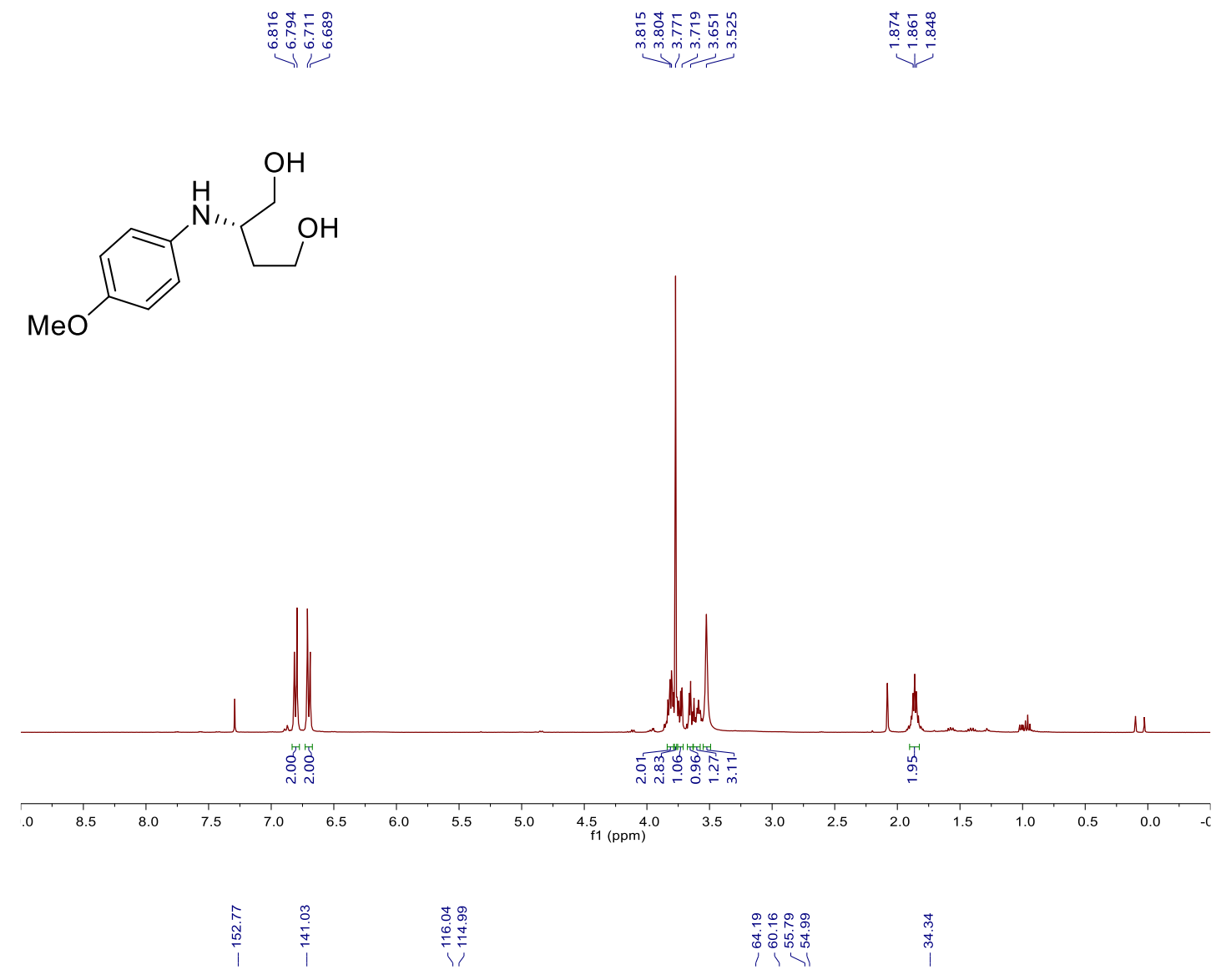

(N)

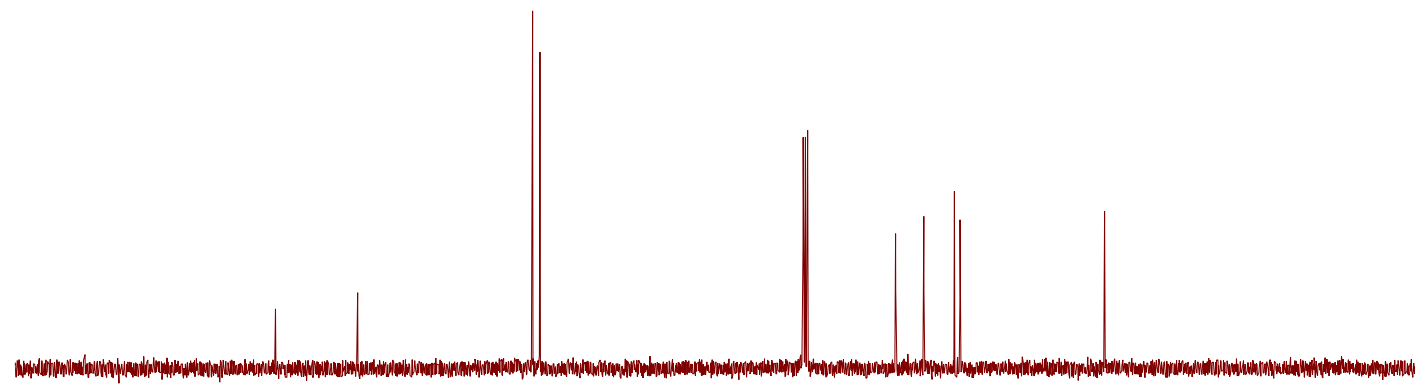

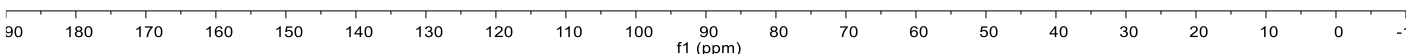


(S)-2-((4-Fluorophenyl)amino)butane-1,4-diol (7d)

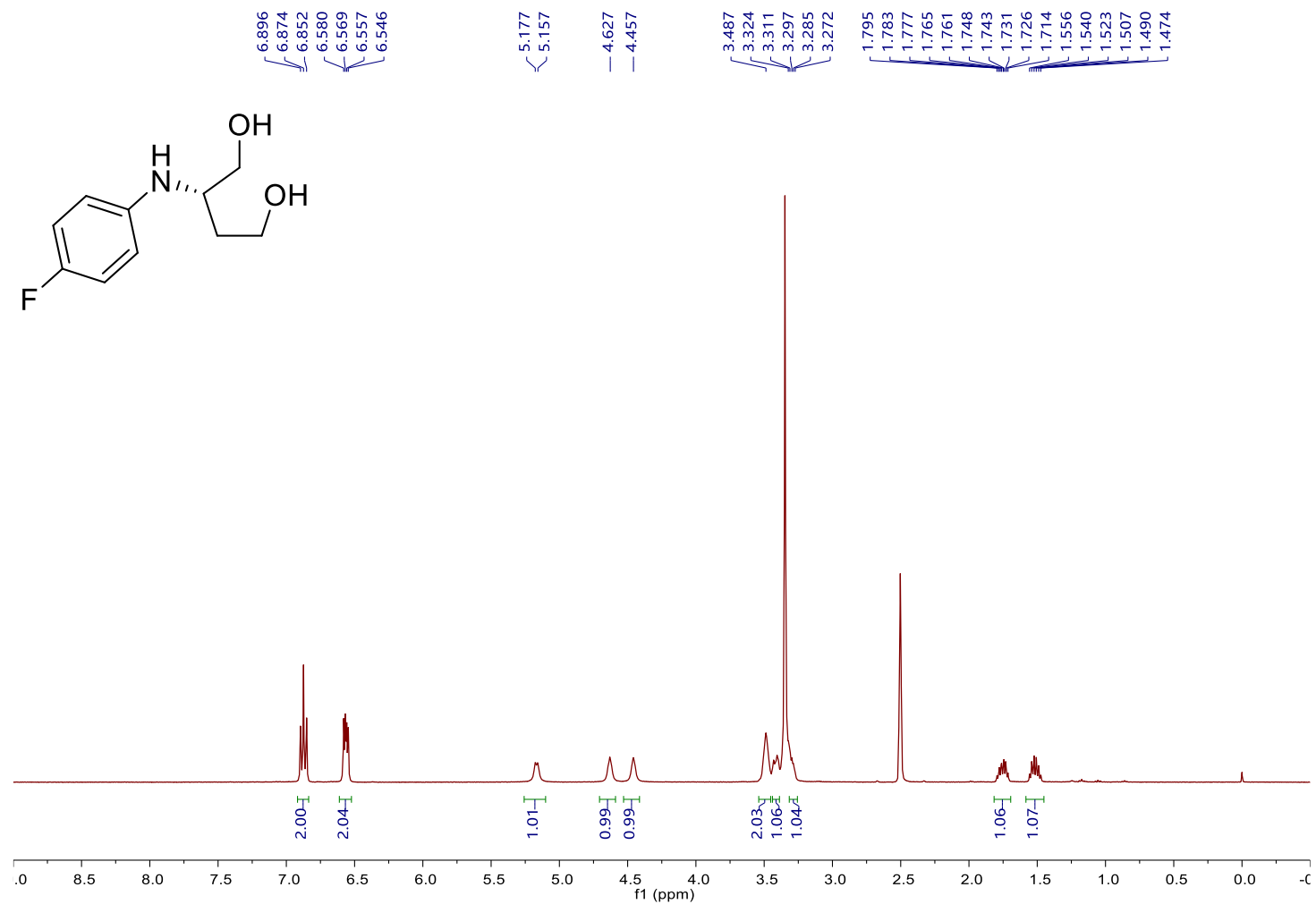

ill<smiles>OCC[C@H](CO)Nc1ccc(F)cc1</smiles>

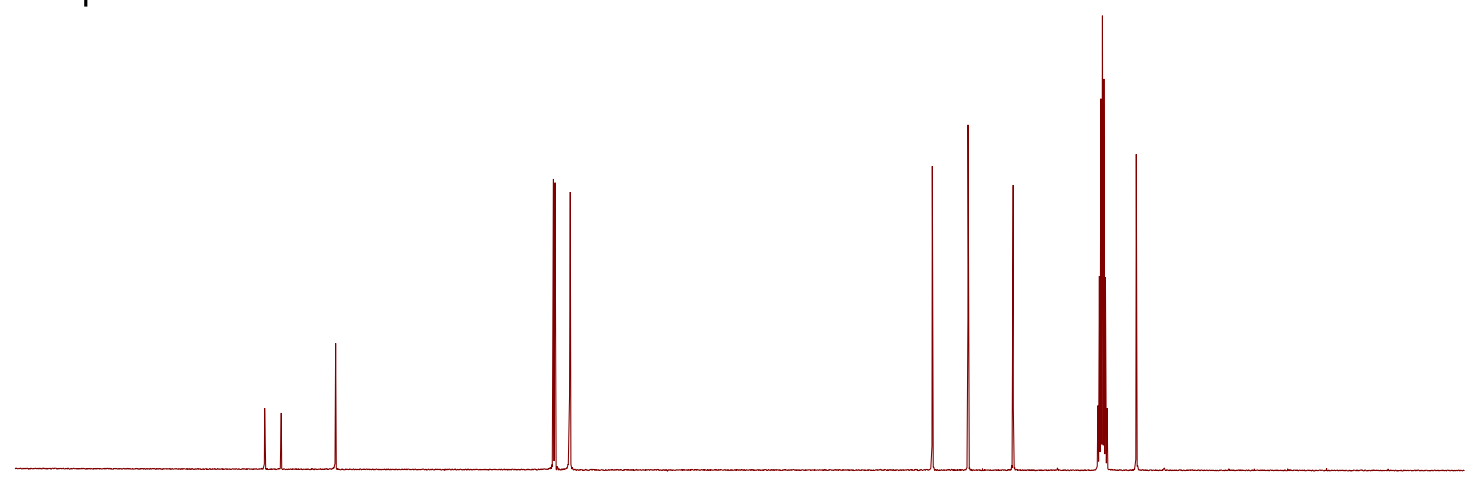

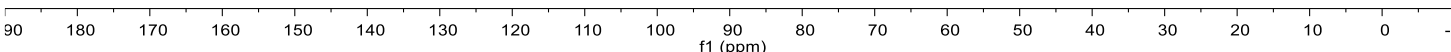


(S)-2-((4-Chlorophenyl)amino)butane-1,4-diol (7e)

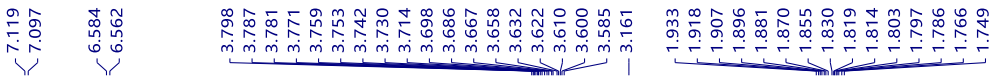<smiles>OCC[C@H](CO)Nc1ccc(Cl)cc1</smiles>

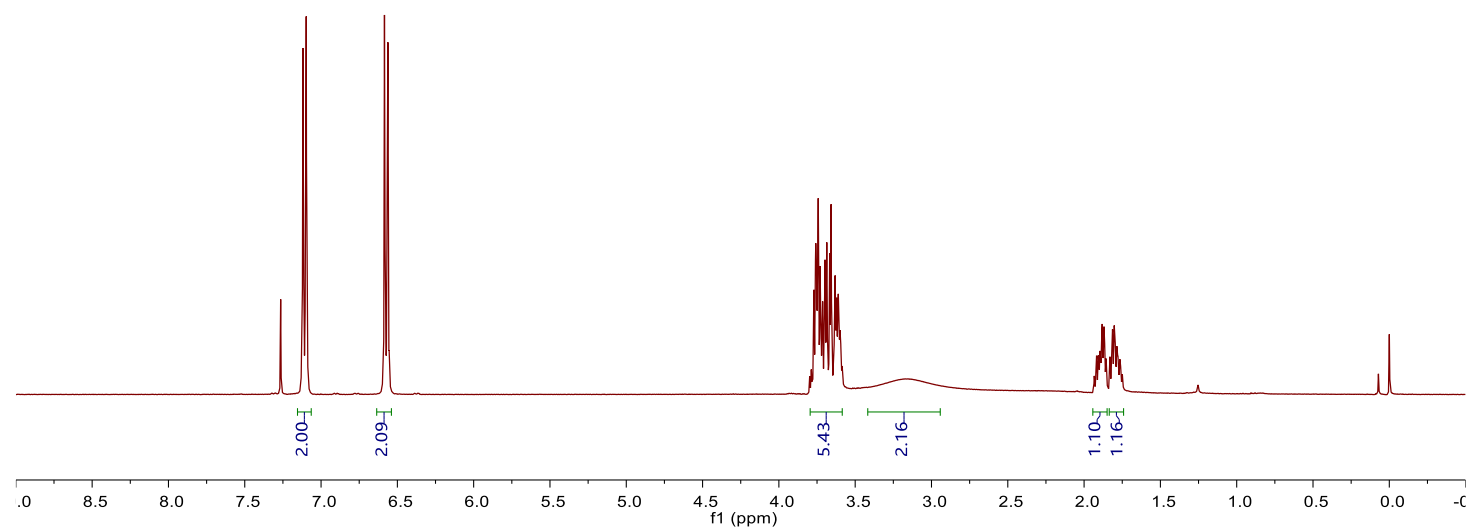

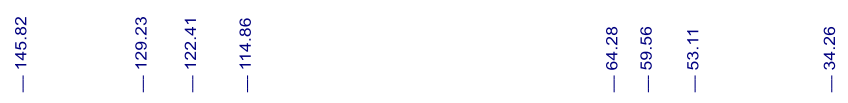

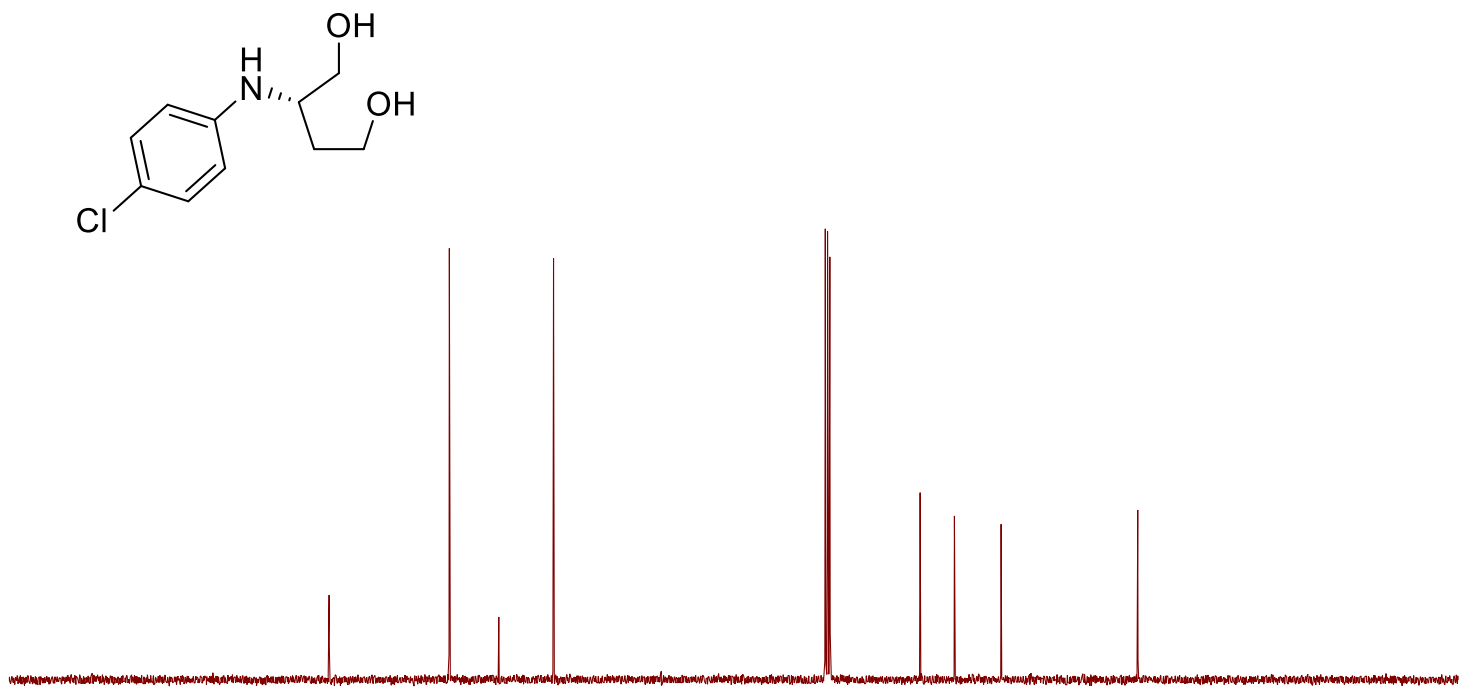

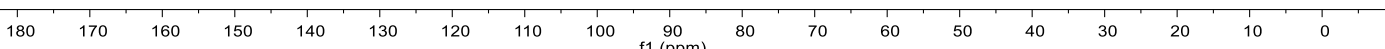


(S)-2-((4-Bromophenyl)amino)butane-1,4-diol (7f)

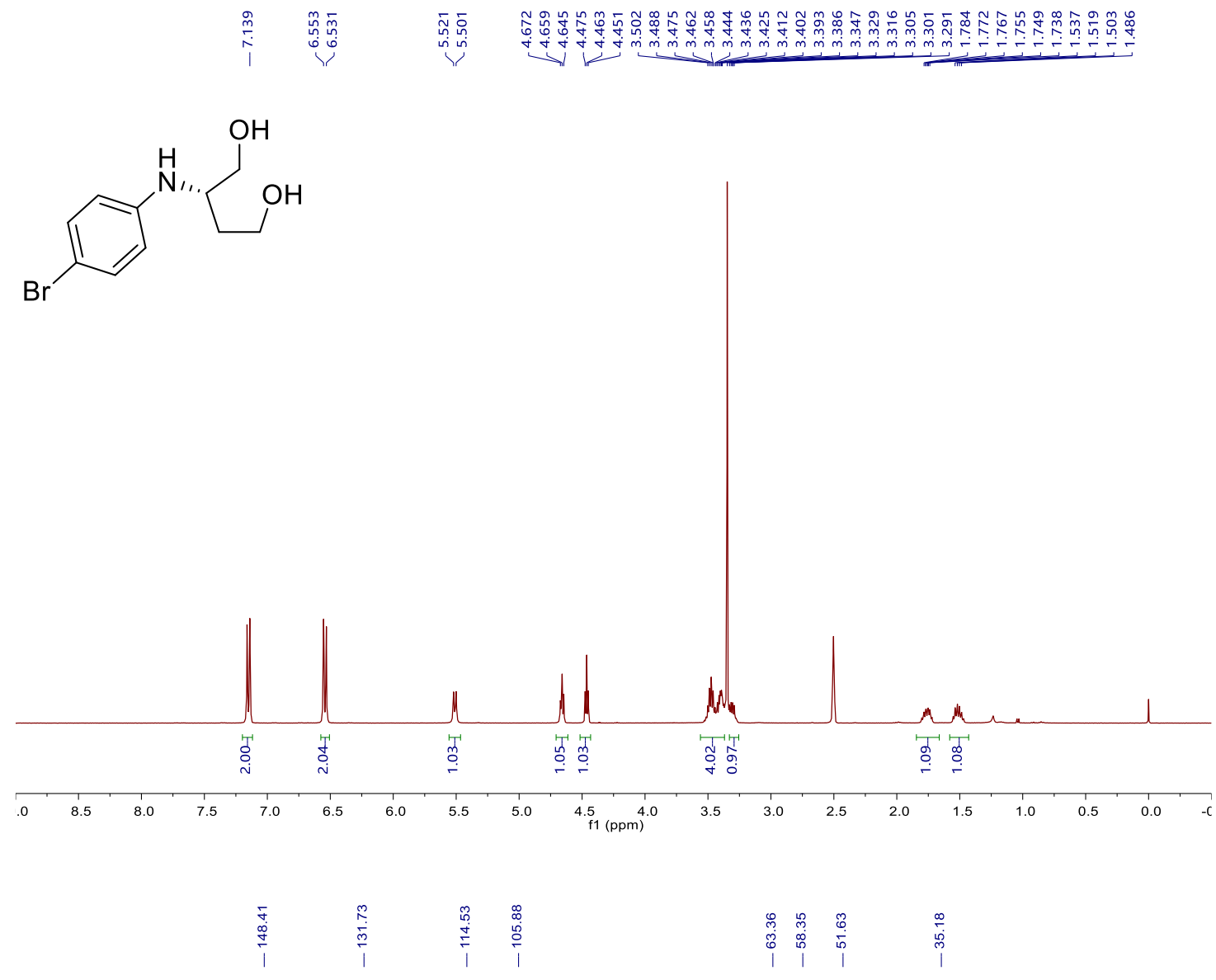<smiles>OCC[C@H](CO)Nc1ccc(Br)cc1</smiles>

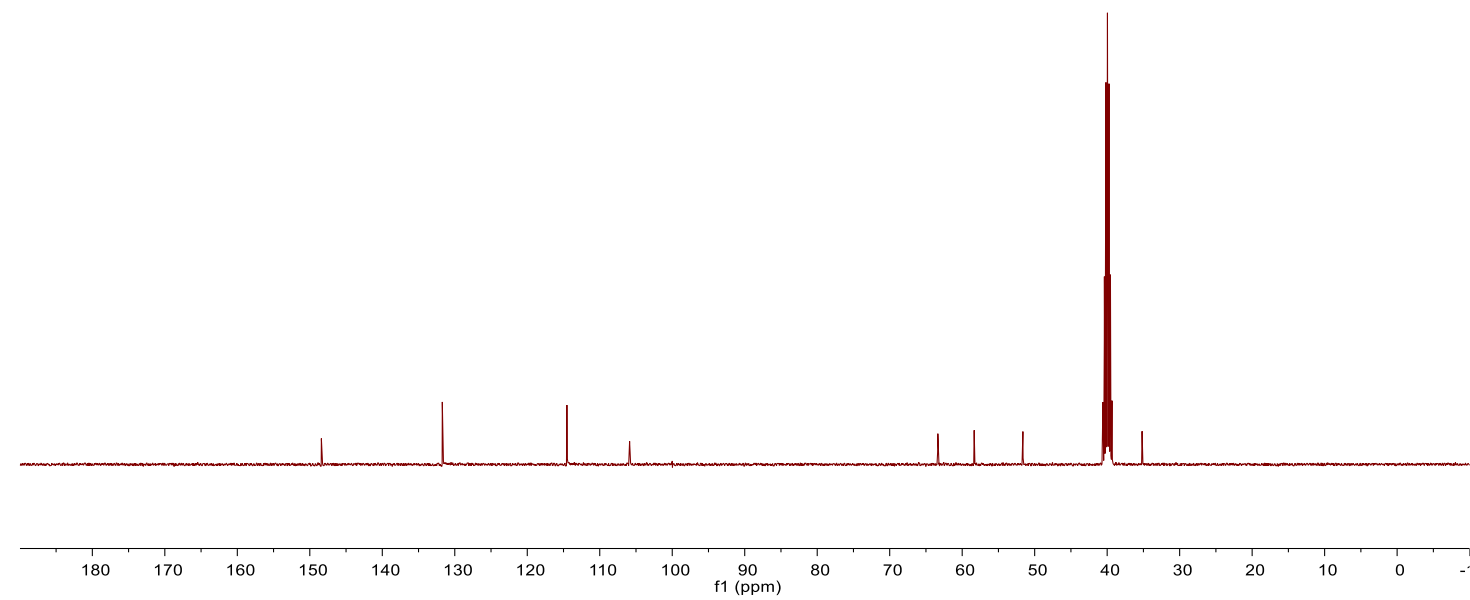


(S)-2-(m-Tolylamino)butane-1,4-diol (7g)

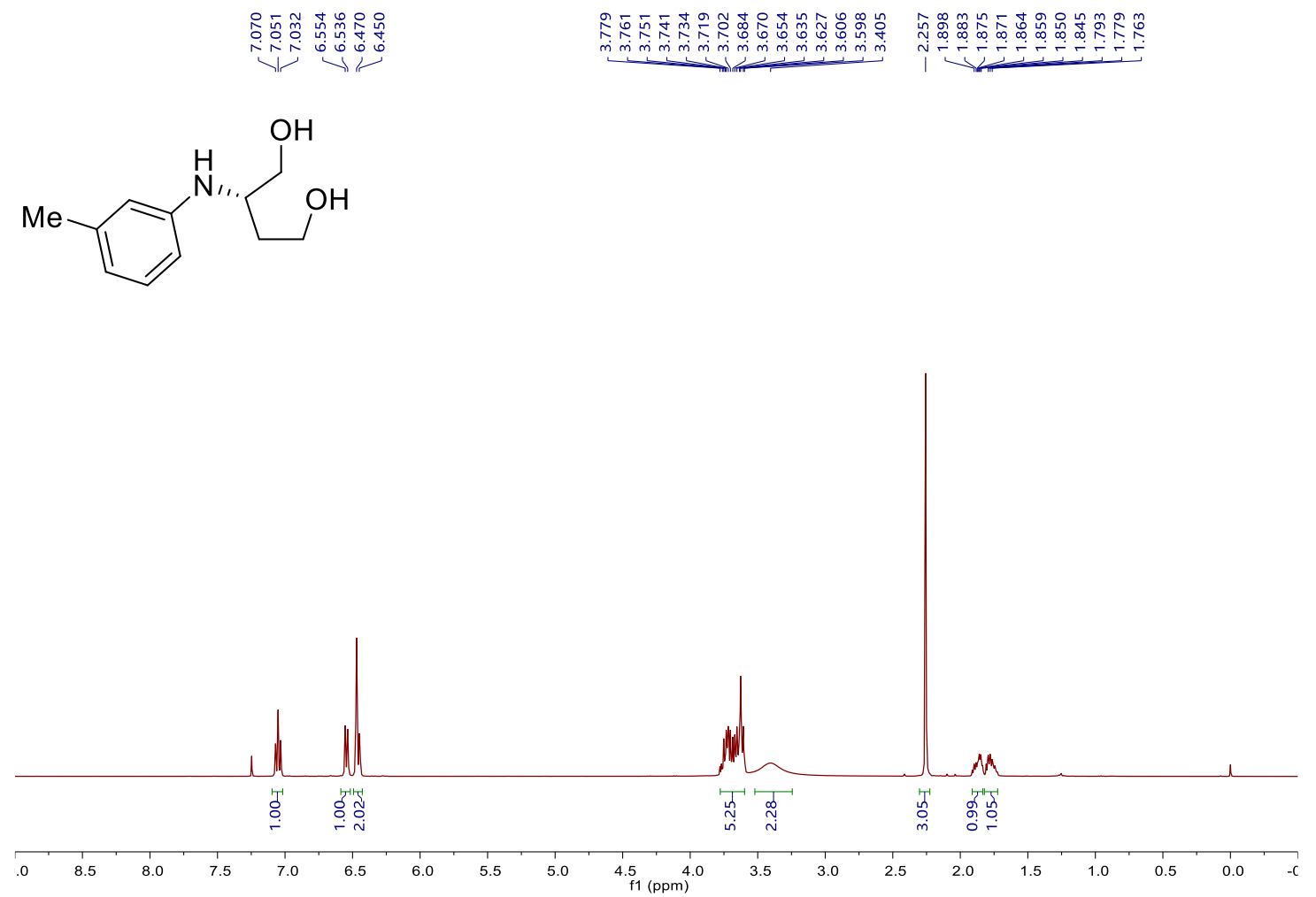

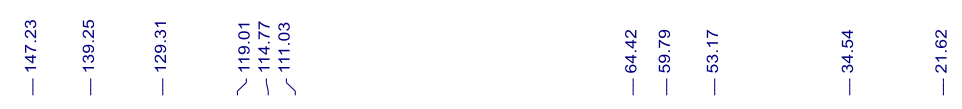<smiles>Cc1cccc(N[C@@H](CO)CCO)c1</smiles>

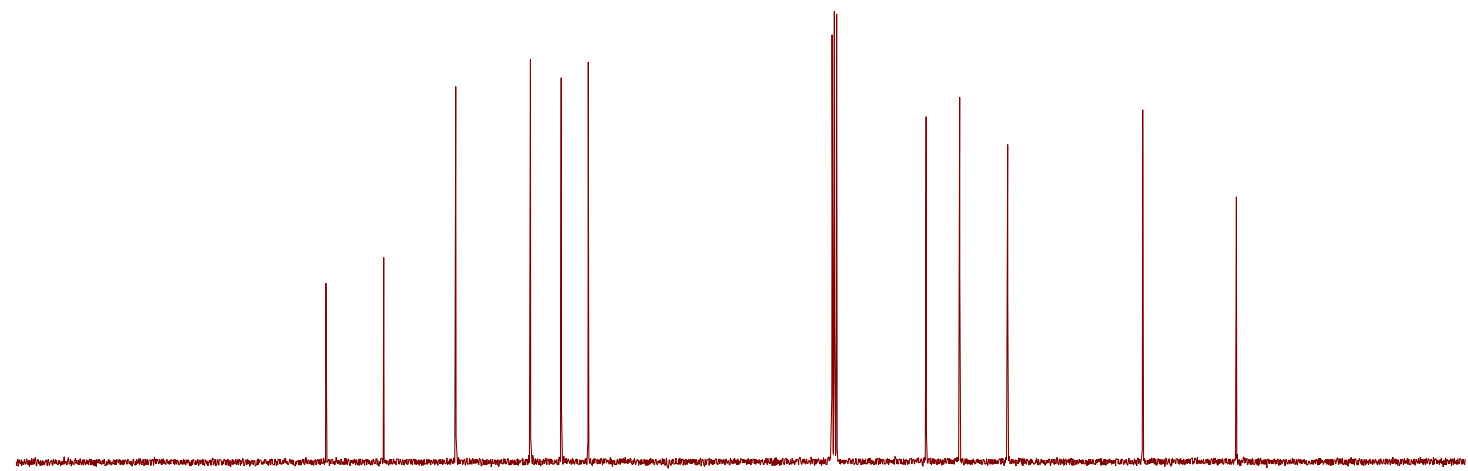

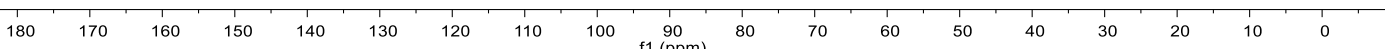


(S)-2-((3-Methoxyphenyl)amino)butane-1,4-diol (7h)
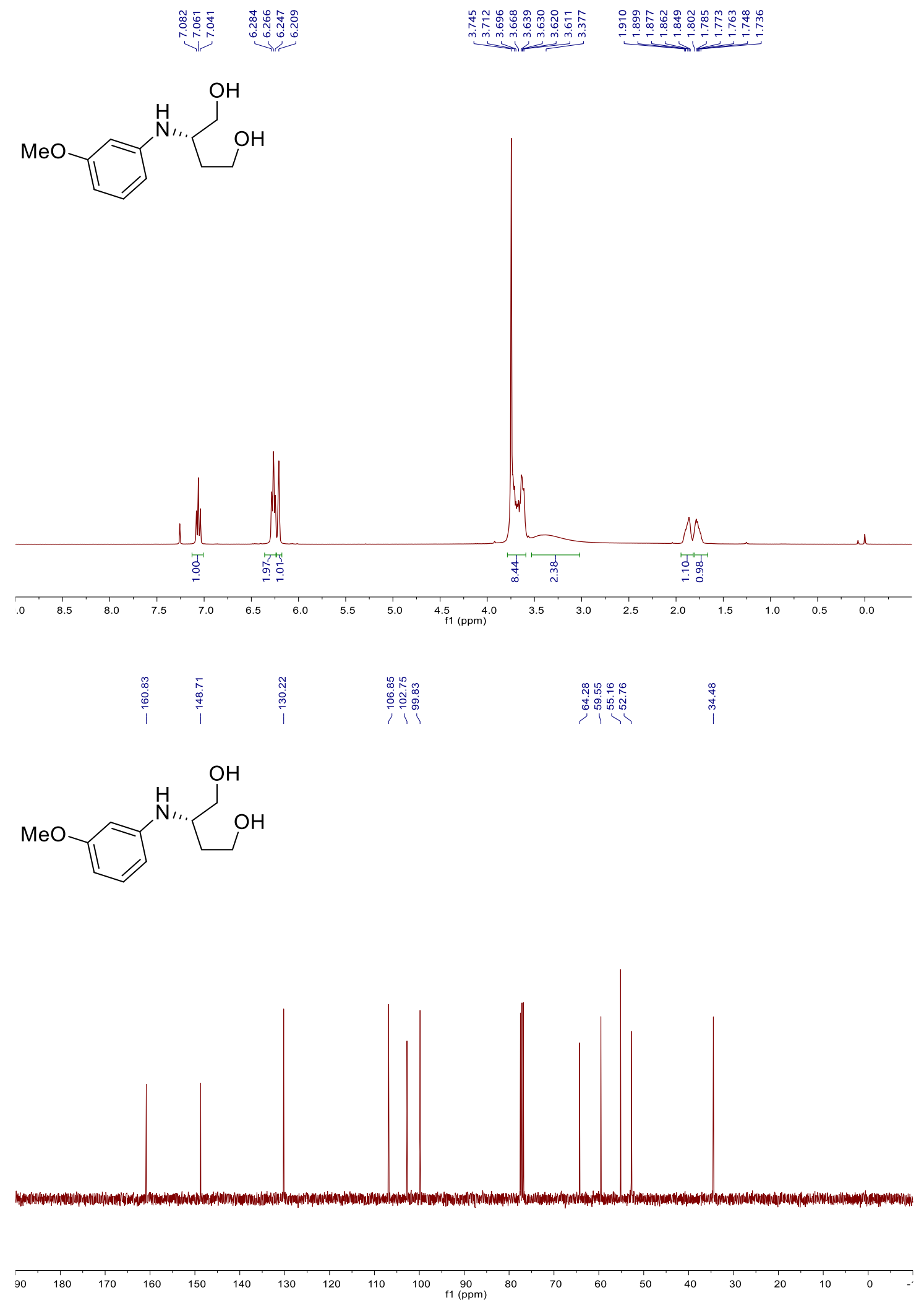
(S)-2-((3-Fluorophenyl)amino)butane-1,4-diol (7i)
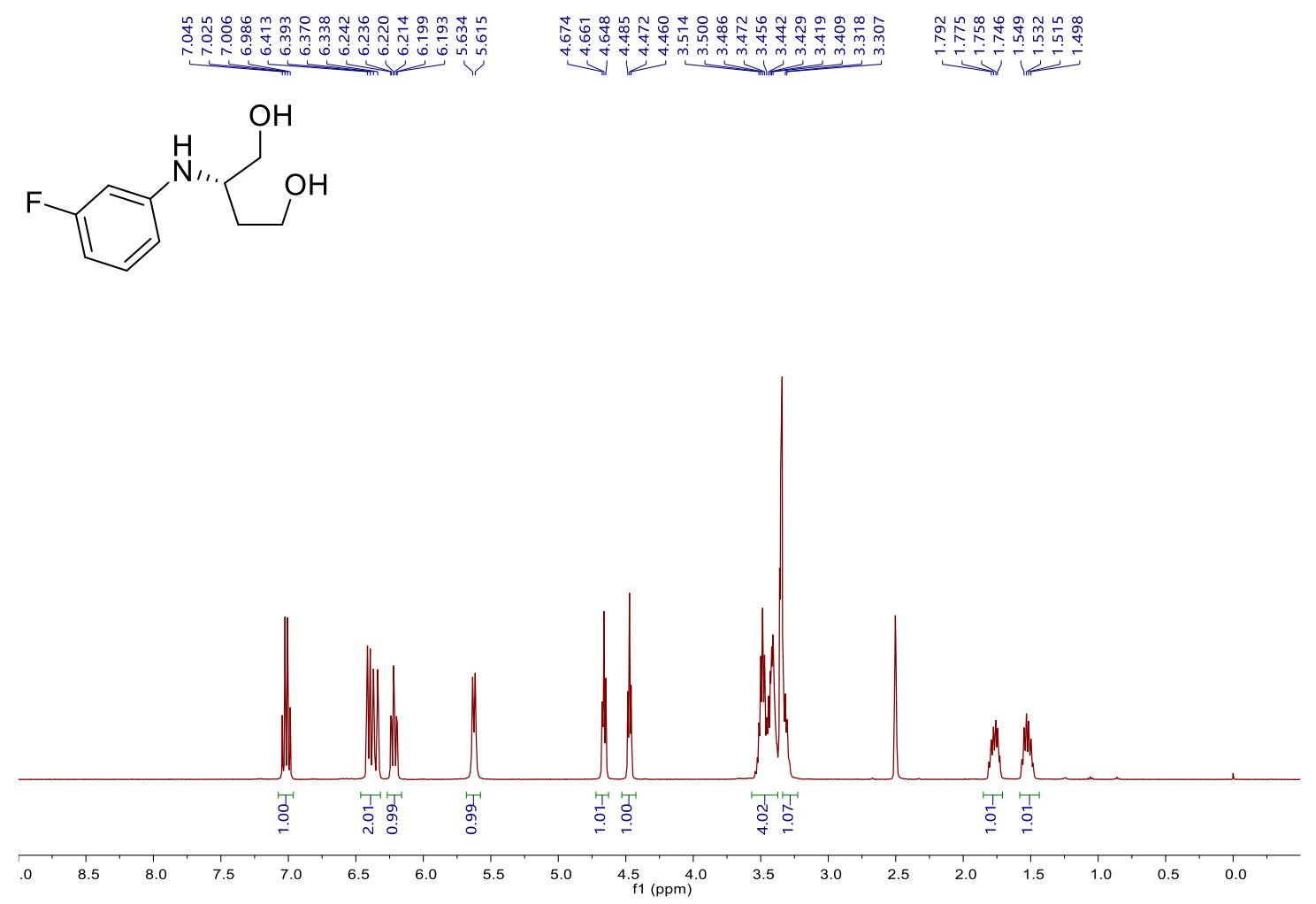

\begin{tabular}{|c|c|c|c|c|c|}
\hline 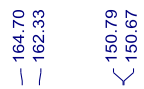 & 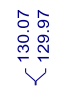 & 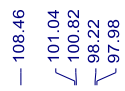 & $\begin{array}{l}\text { o } \\
\substack{0 \\
0 \\
1}\end{array}$ & $\begin{array}{c}\infty \\
\infty \\
\infty \\
\infty \\
1\end{array}$ & 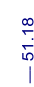 \\
\hline
\end{tabular}<smiles>OCC[C@H](CO)Nc1cccc(F)c1</smiles>

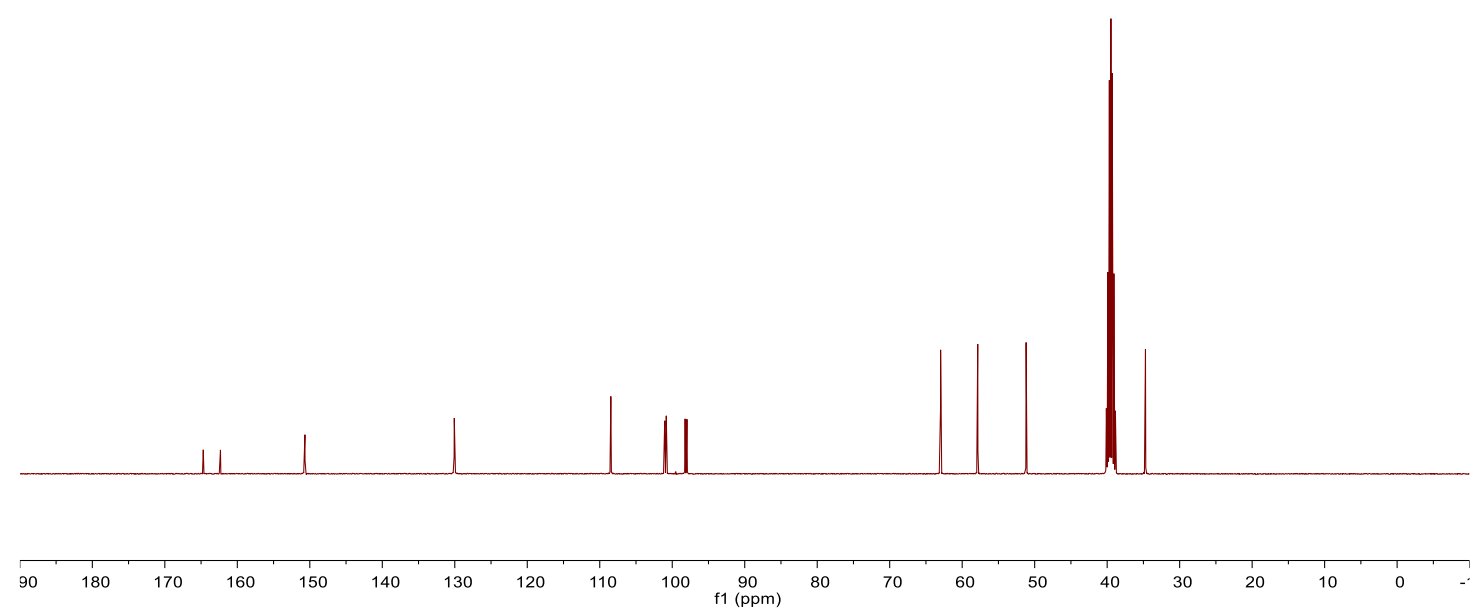


(S)-2-((3-Chlorophenyl)amino)butane-1,4-diol (7j)

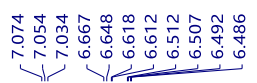

눈

(1)

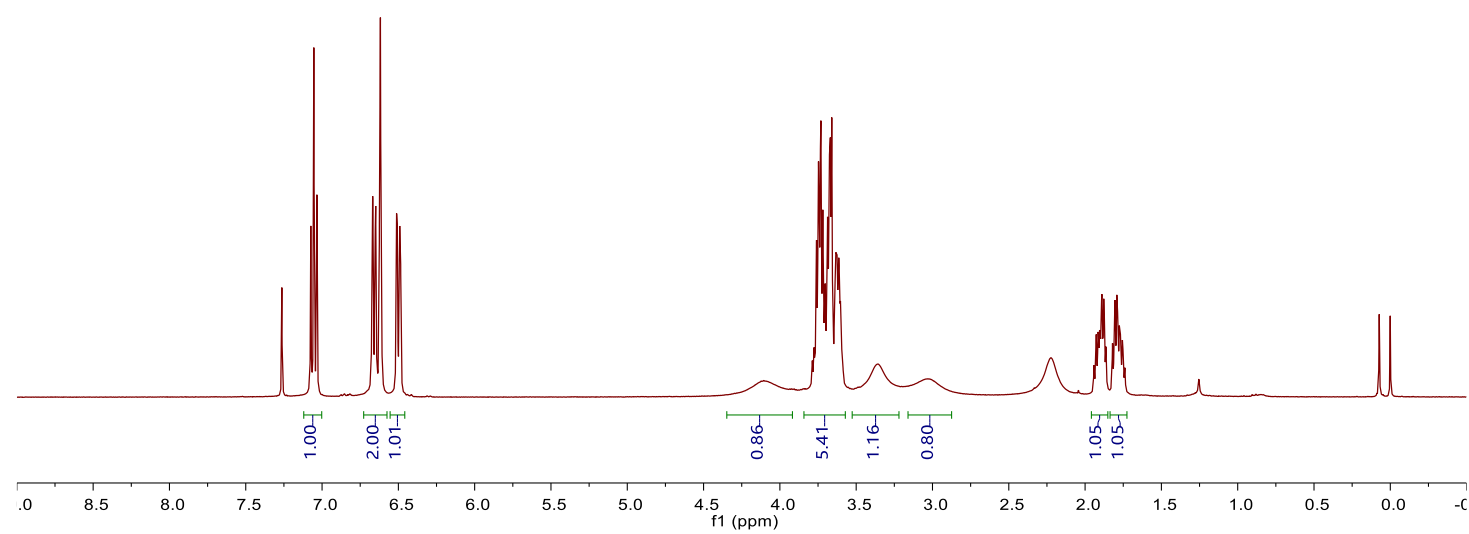

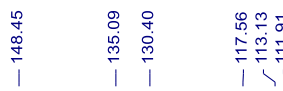

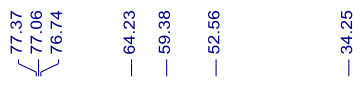<smiles>OCC[C@H](CO)Nc1cccc(Cl)c1</smiles>

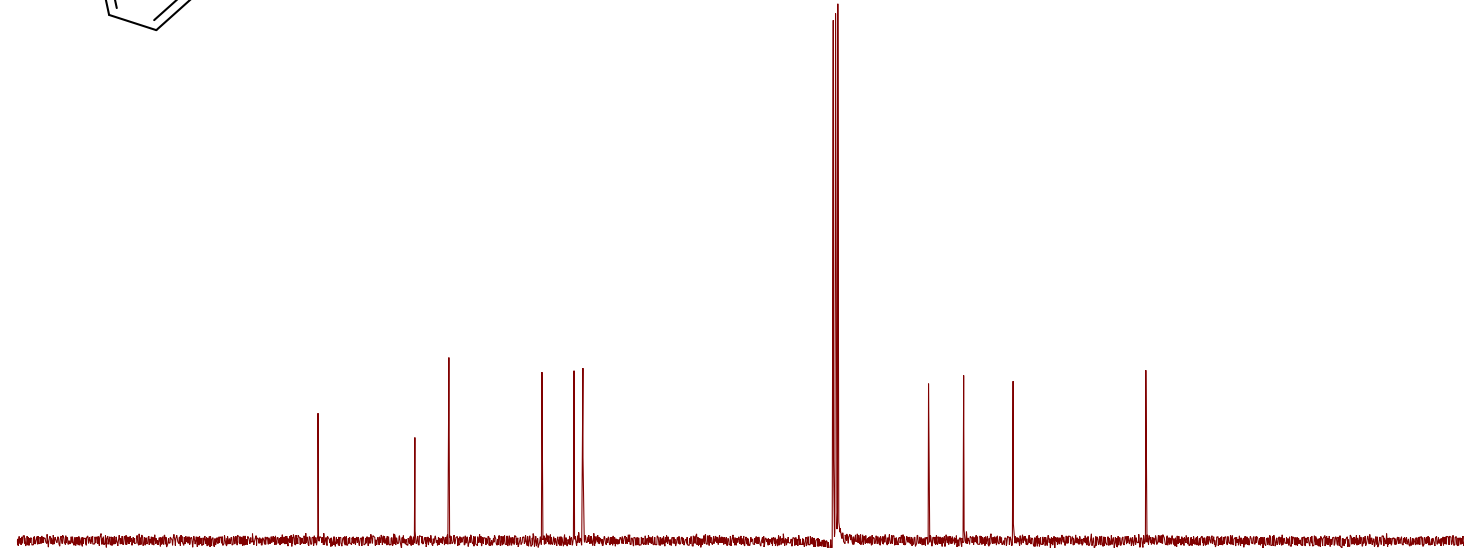

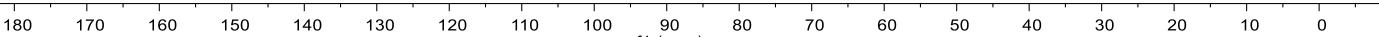


(S)-2-(o-Tolylamino)butane-1,4-diol (7k)

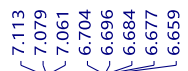

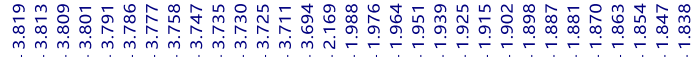

Me

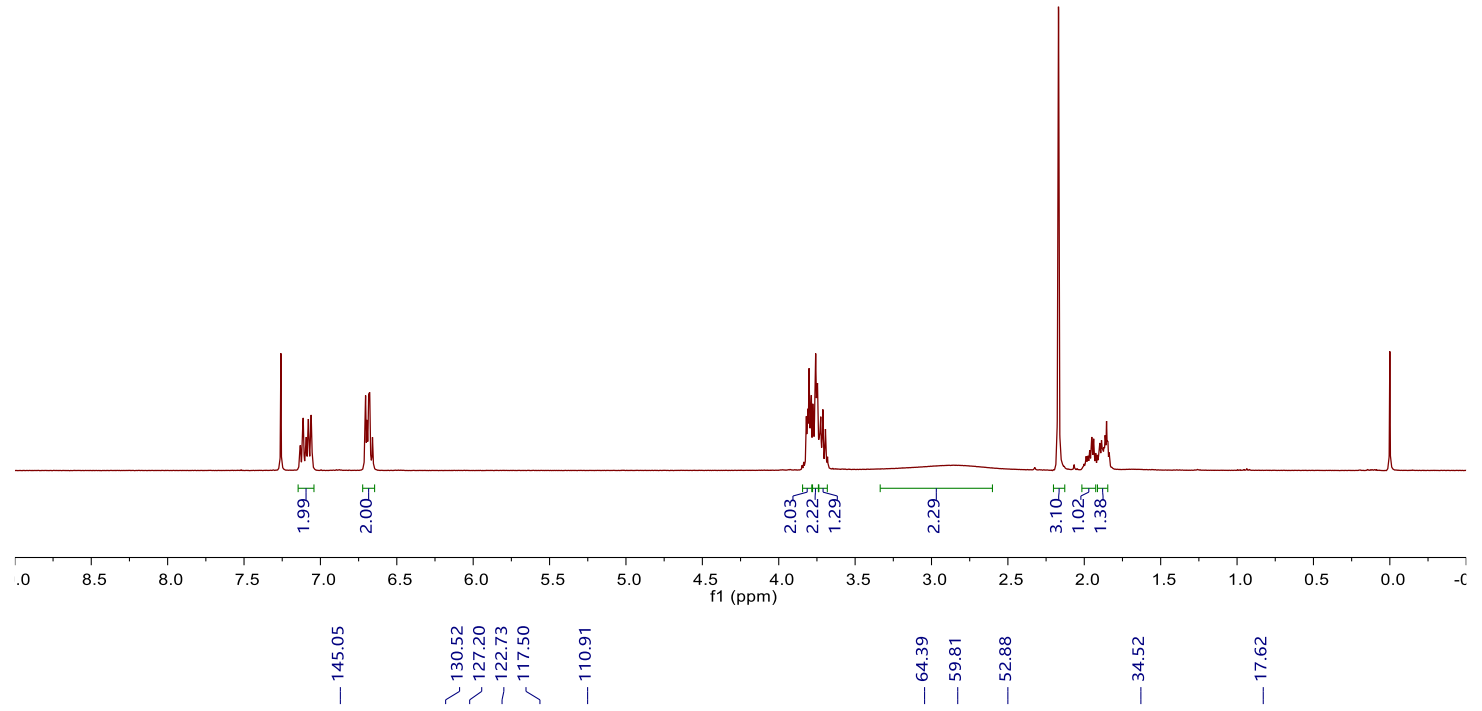<smiles>Cc1ccccc1N[C@H](CO)CCO</smiles>

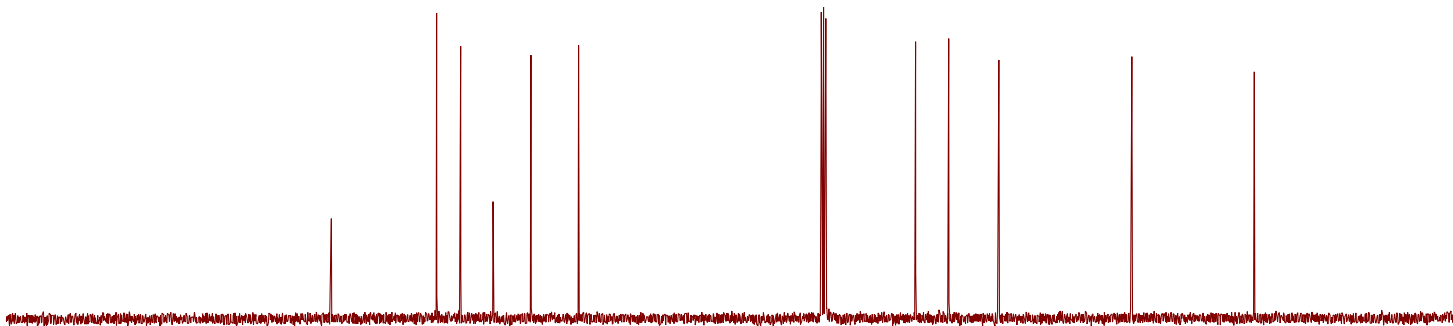

\begin{tabular}{lllllllllllllllllllllllllll}
\hline 90 & 180 & 170 & 160 & 150 & 140 & 130 & 120 & 110 & 100 & 90 & 80 & 70 & 60 & 50 & 40 & 30 & 20 & 10 & 0 & -
\end{tabular} 
(S)-2-((2-Methoxyphenyl)amino)butane-1,4-diol (71)

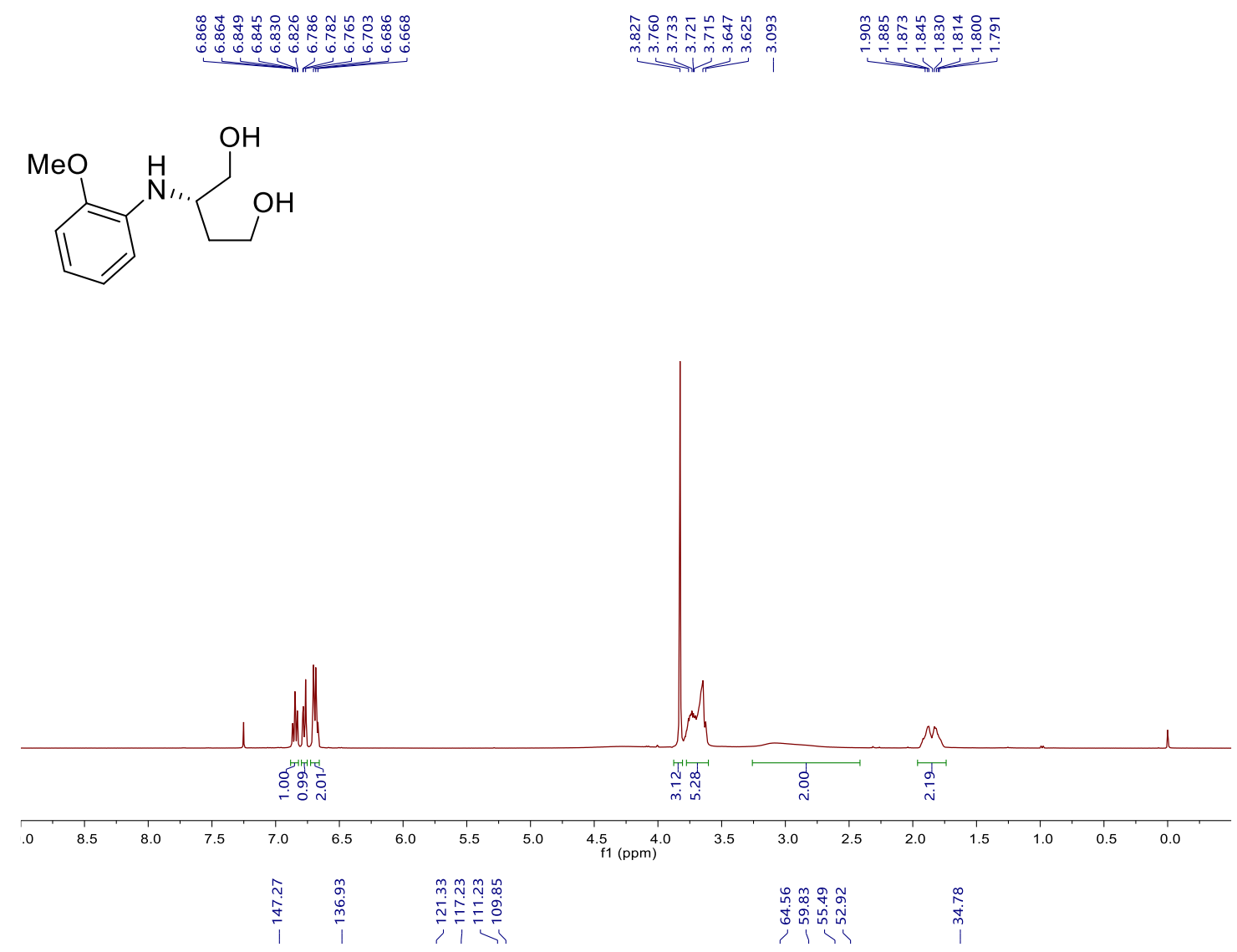<smiles>COc1ccccc1N[C@@H](CO)CCO</smiles>

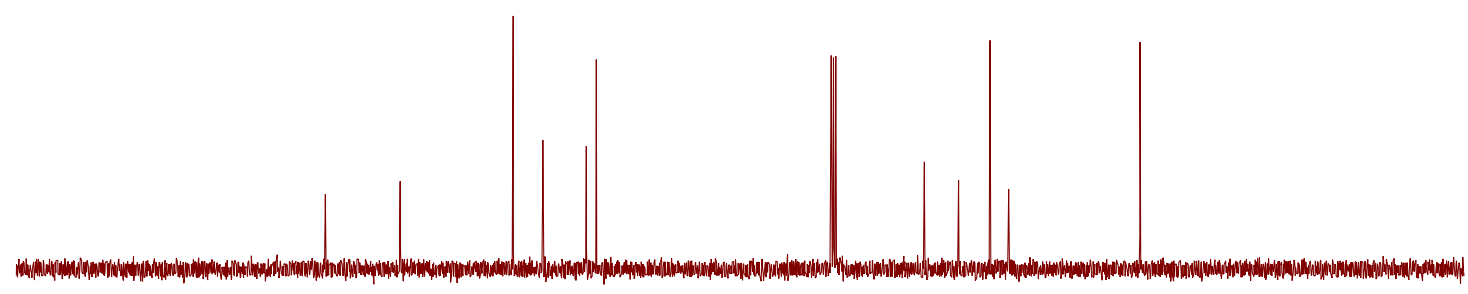

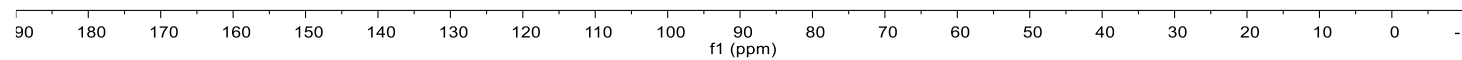


(S)-2-(2,4-Dimethoxyphenylamino)butane-1,4-diol (7 m)

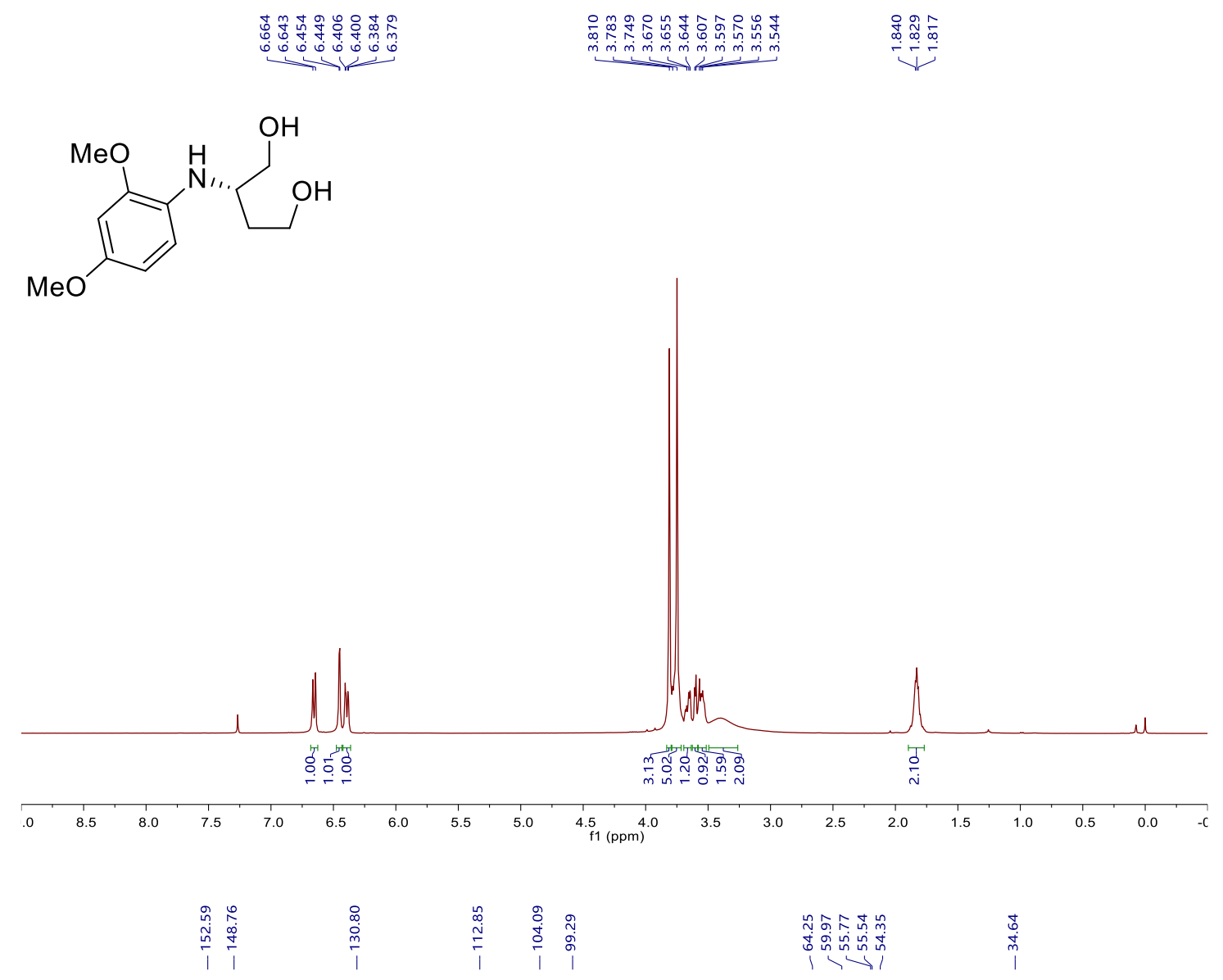

(neOr)

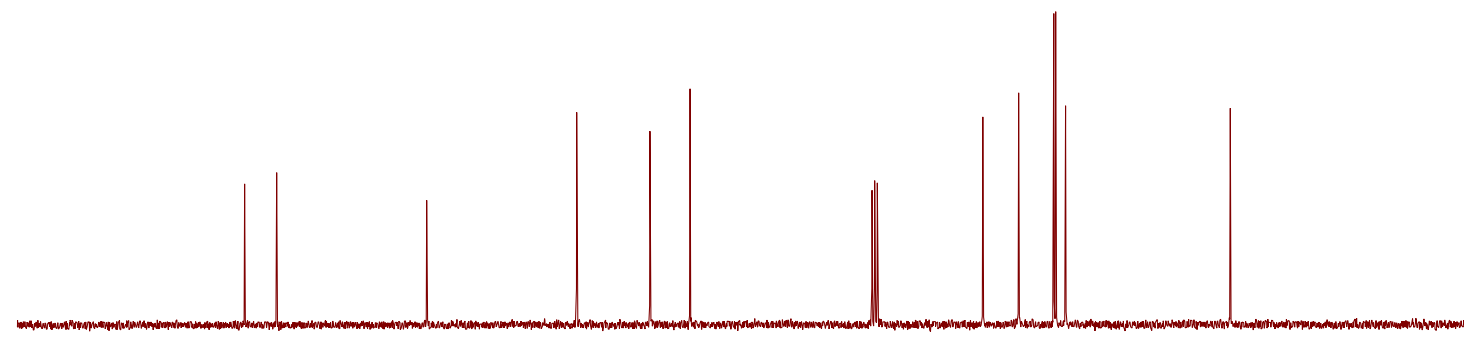


(S)-2-(naphthalen-1-ylamino)butane-1,4-diol (7n)

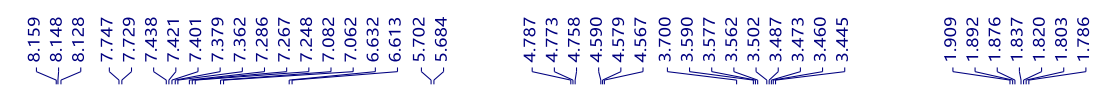<smiles>OCC[C@H](CO)Nc1cccc2ccccc12</smiles>
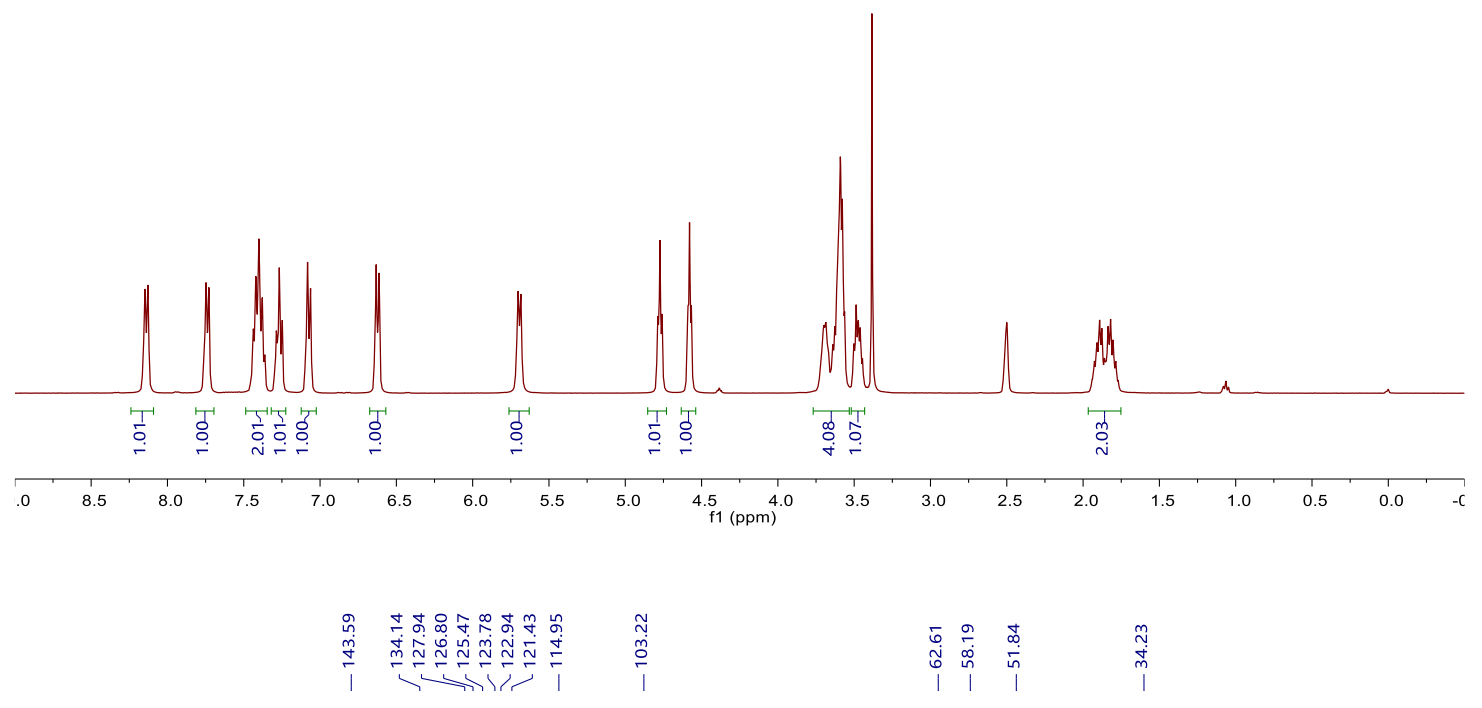<smiles>OCC[C@H](CO)Nc1cccc2ccccc12</smiles>

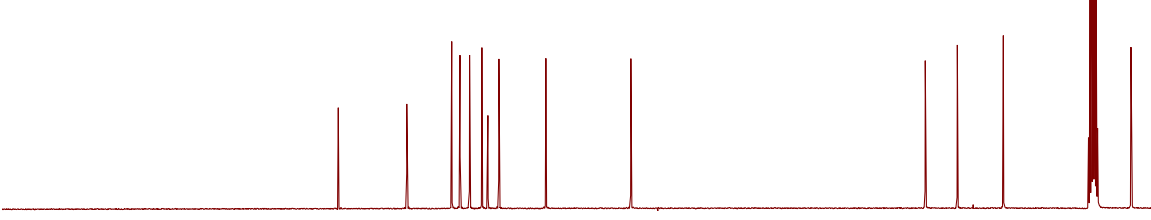

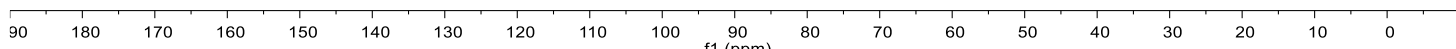


(S)-2-(Benzo[d][1,3]dioxol-5-ylamino)butane-1,4-diol (7o)

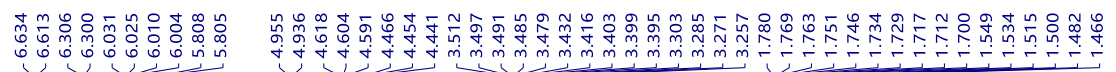

管

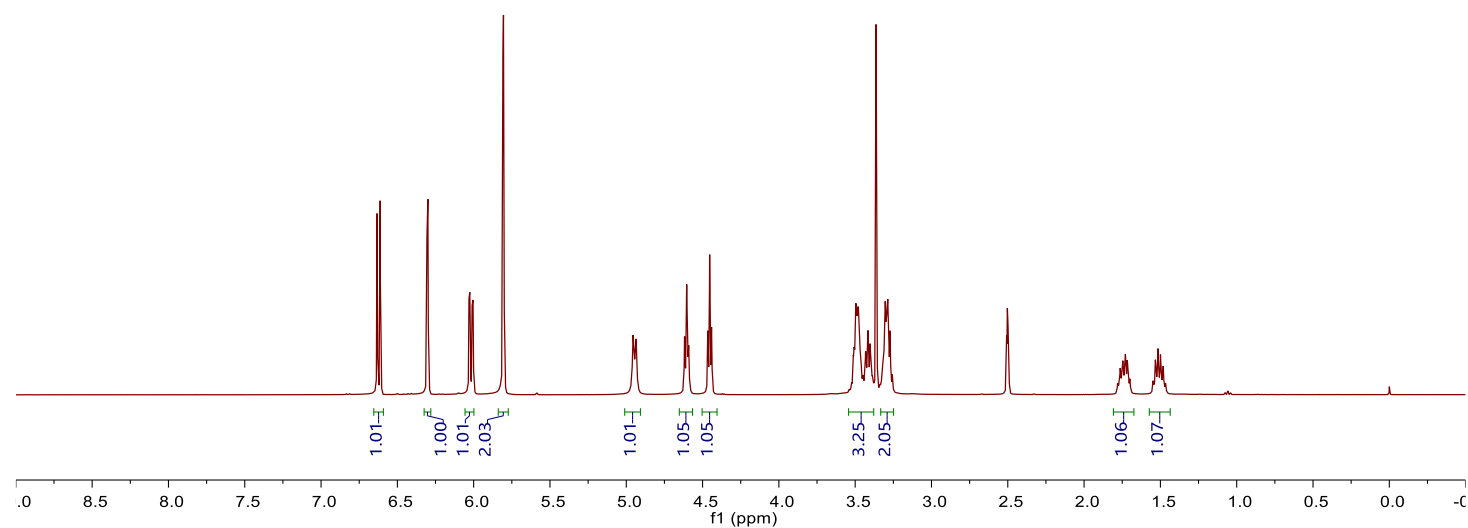

\begin{tabular}{|c|c|c|}
\hline 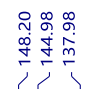 & 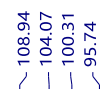 & 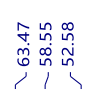 \\
\hline
\end{tabular}<smiles>OCCC(CO)Nc1ccc2c(c1)OCO2</smiles>

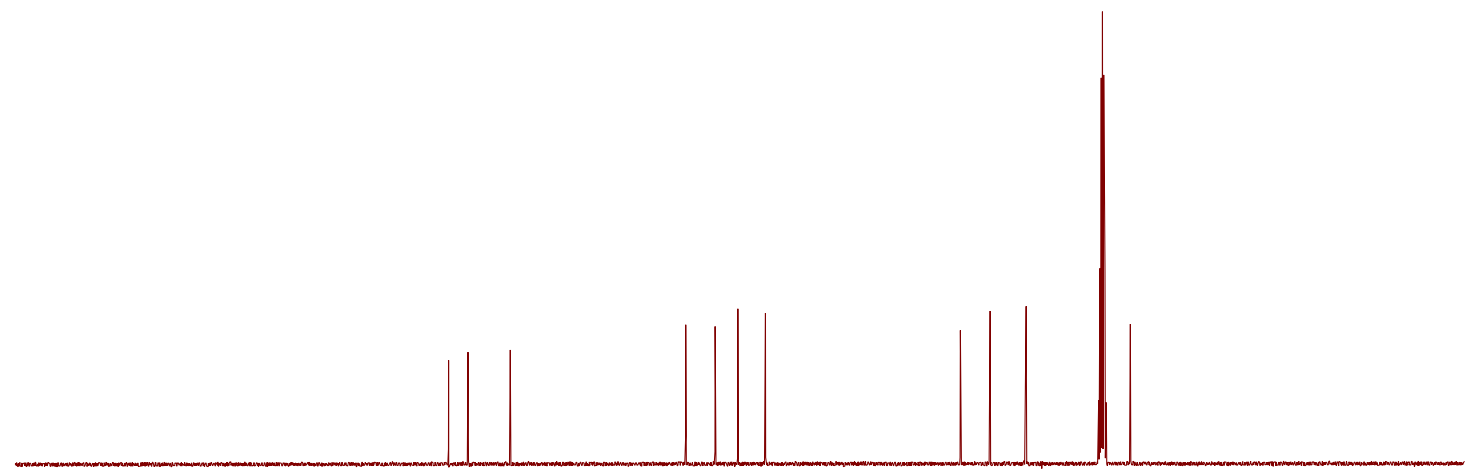

$\begin{array}{lllllllllllllllllllllllllll}1 & 210 & 200 & 190 & 180 & 170 & 160 & 150 & 140 & 130 & 120 & 110 & 100 & 10 & 80 & 70 & 60 & 50 & 40 & 30 & 20 & 10 & 0 & -10 & -:\end{array}$ 
(S)-2-(6-Methylpyridin-2-ylamino)butane-1,4-diol (7p)

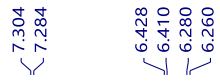

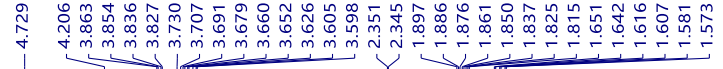<smiles>Cc1cccc(N[C@@H](CO)CCO)n1</smiles>

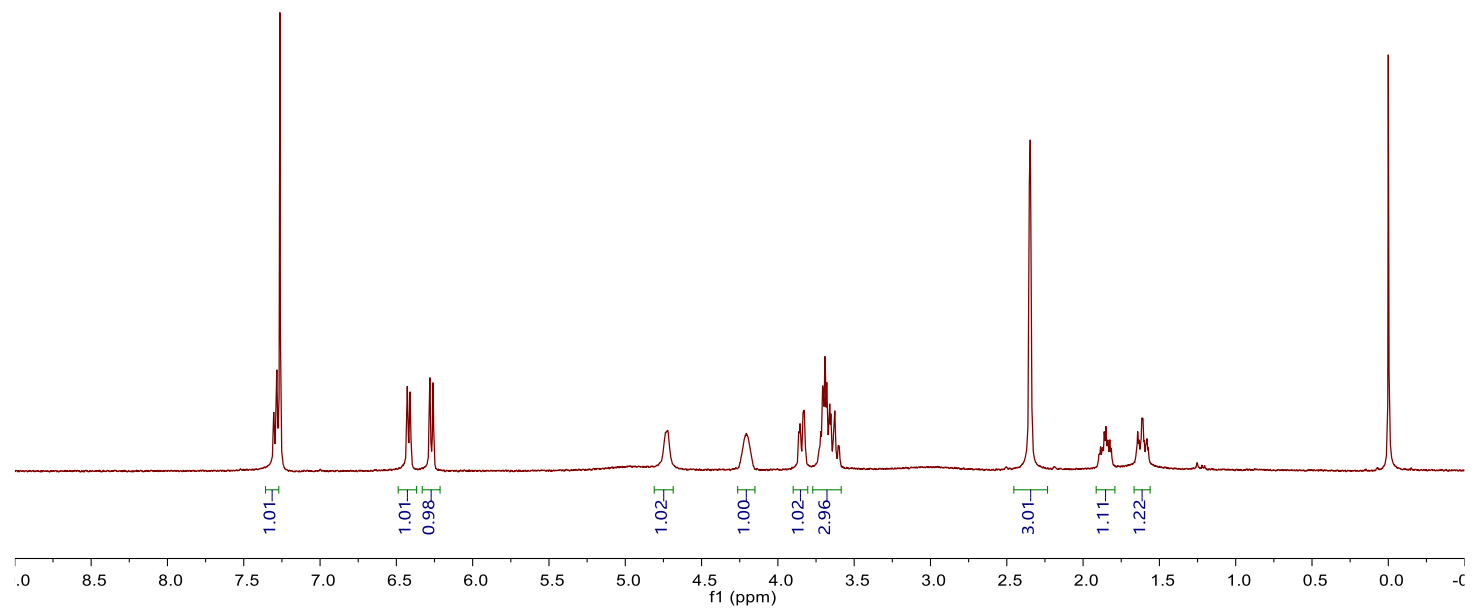

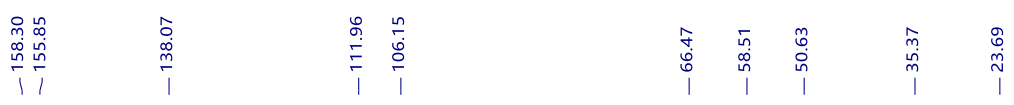<smiles>Cc1cccc(N[C@@H](CO)CCO)n1</smiles>

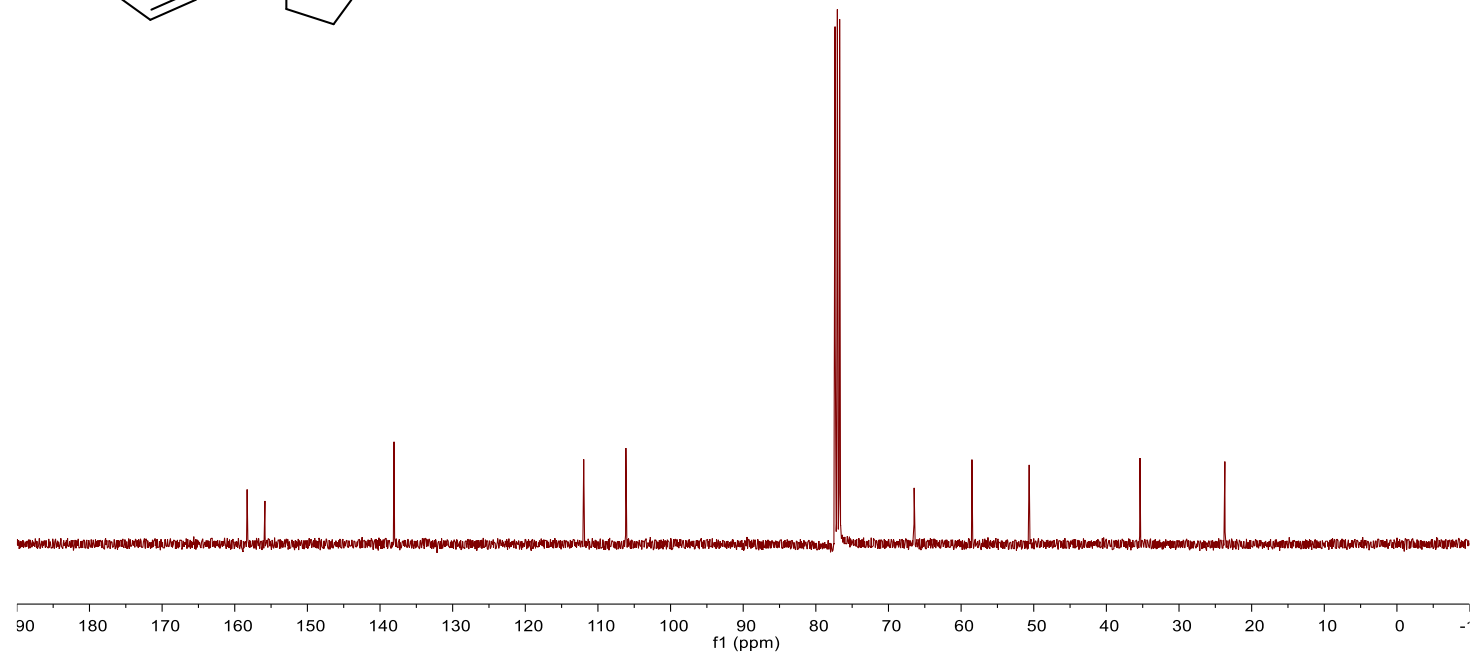


$N$-(3-Hydroxy-1-hydroxymethyl-propyl)-4-methyl-benzenesulfonamide (7r)

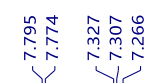

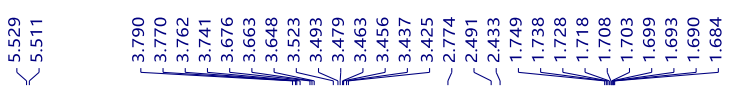

TsHN ".

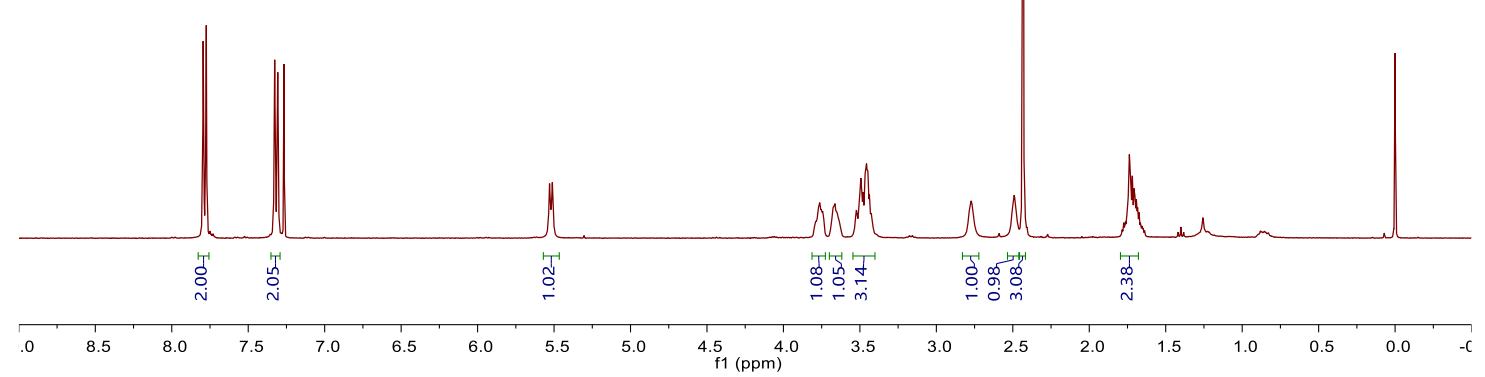

TsHN ,.

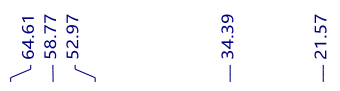

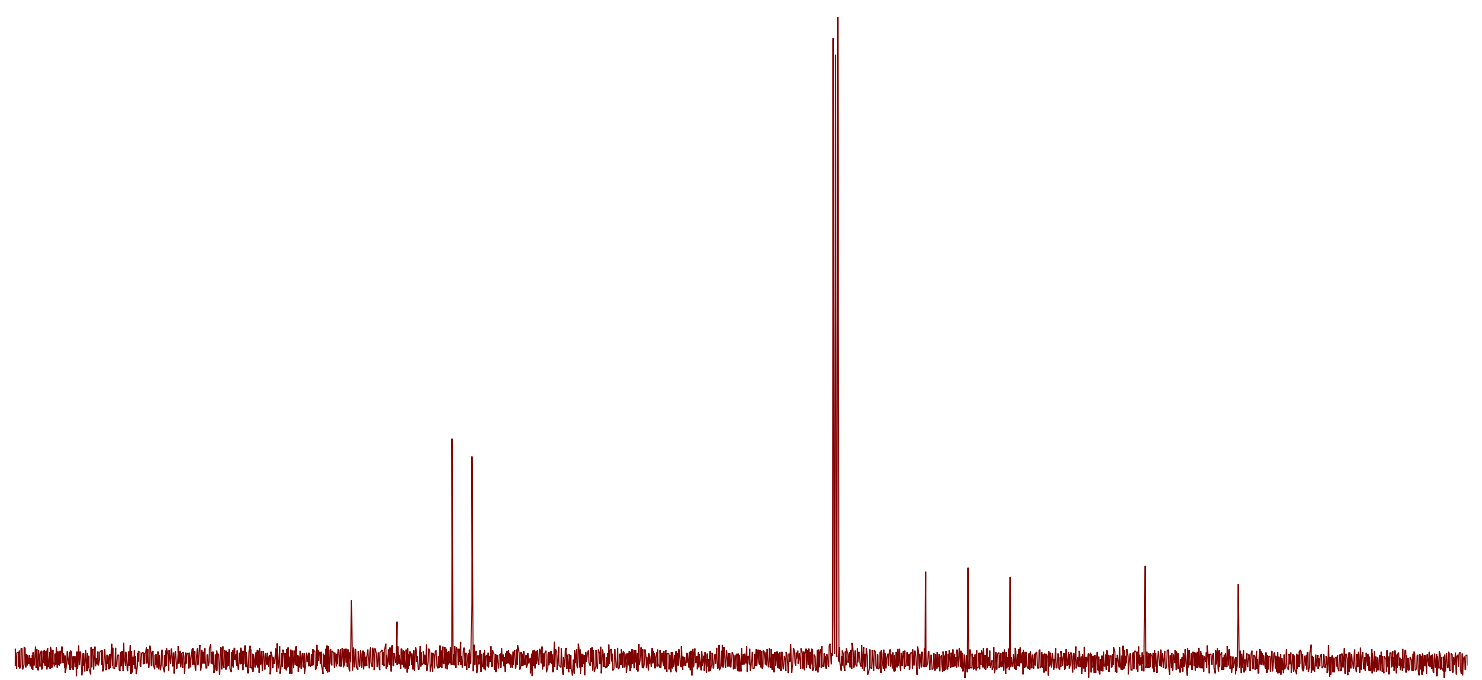

\begin{tabular}{lllllllllllllllllllllllll}
\hline & 180 & 170 & 160 & 150 & 140 & 130 & 120 & 110 & 100 & 90 & 80 & 70 & 60 & 50 & 40 & 30 & 20 & 10 & 0 & 1
\end{tabular} 


\section{(S)-2-(2-Phenylamino)pentane-1,5-diol (9a)}

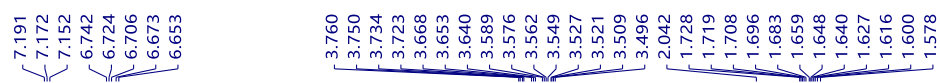<smiles>OCCC[C@H](CO)Nc1ccccc1</smiles>

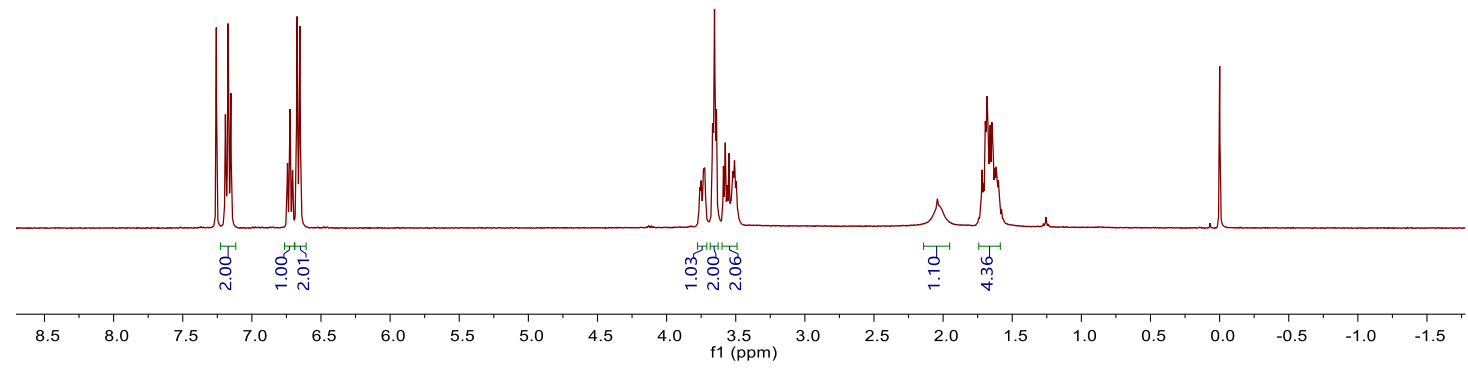

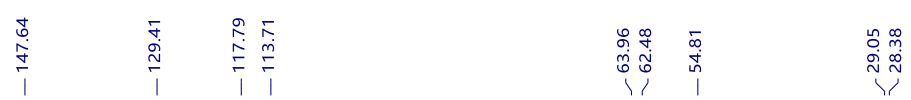<smiles>OCCCC(CO)Nc1ccccc1</smiles>

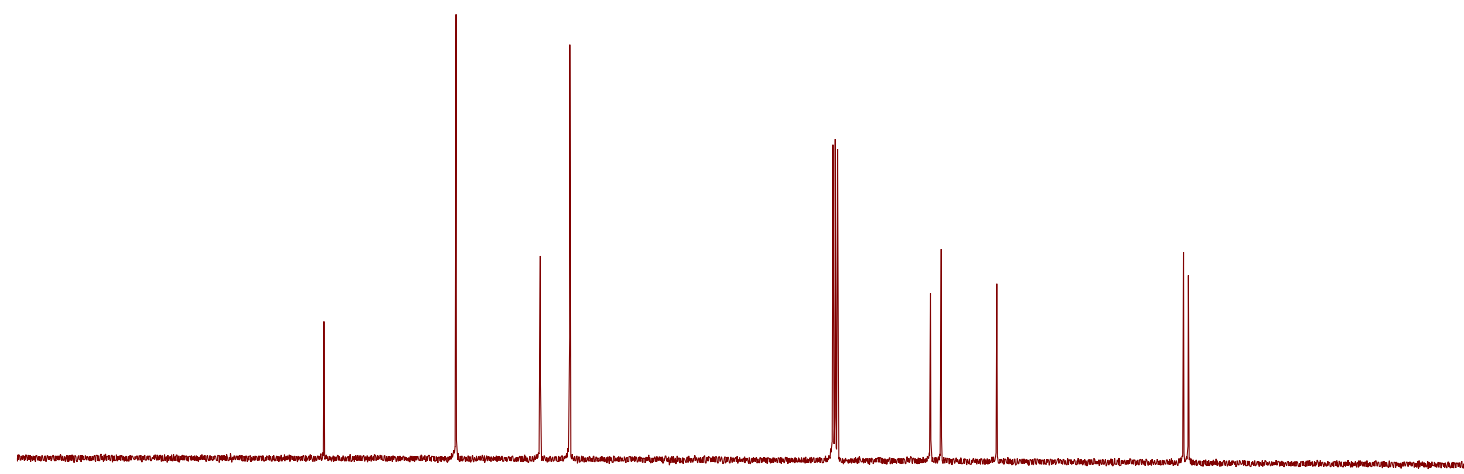

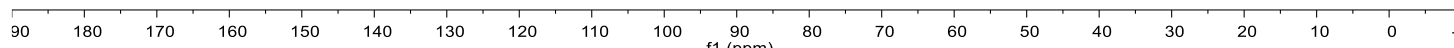


(S)-2-(p-Tolylamino)pentane-1,5-diol (9b)
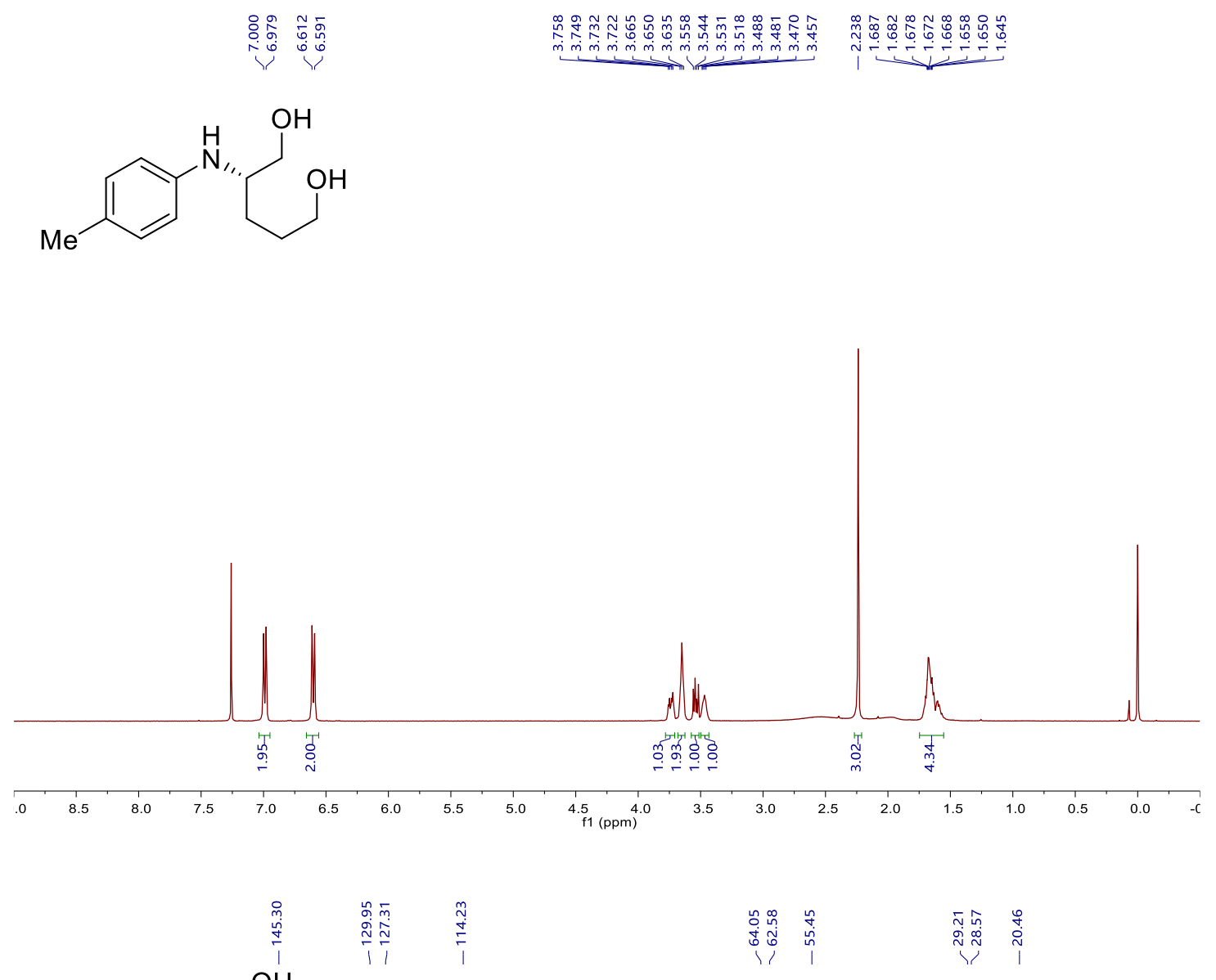<smiles>Cc1ccc(N[C@@H](CO)CCCO)cc1</smiles>

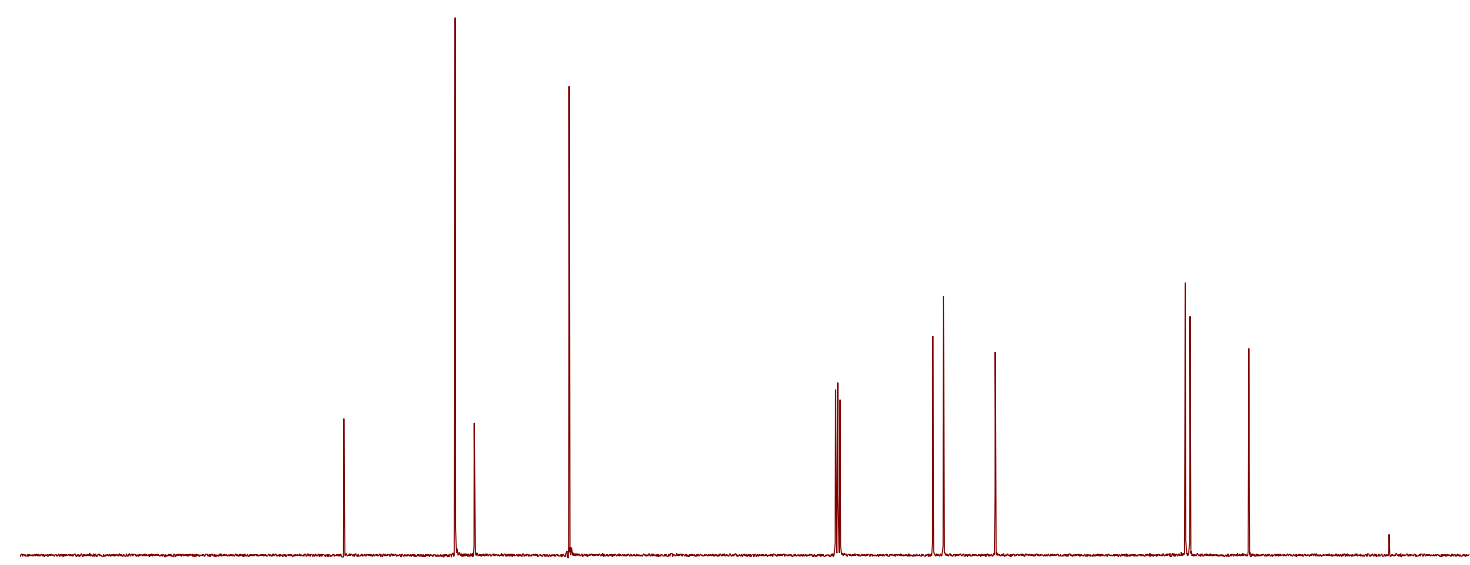

\begin{tabular}{rllllllllllllllllllll}
\hline & 180 & 170 & 160 & 150 & 140 & 130 & 120 & 110 & 100 & $\begin{array}{c}90 \\
\mathrm{f} 1(\mathrm{ppm})\end{array}$ & 80 & 70 & 60 & 50 & 40 & 30 & 20 & 10 & 0 & 1
\end{tabular} 
(S)-2-((4-Methoxyphenyl)amino)pentane-1,5-diol (9c)

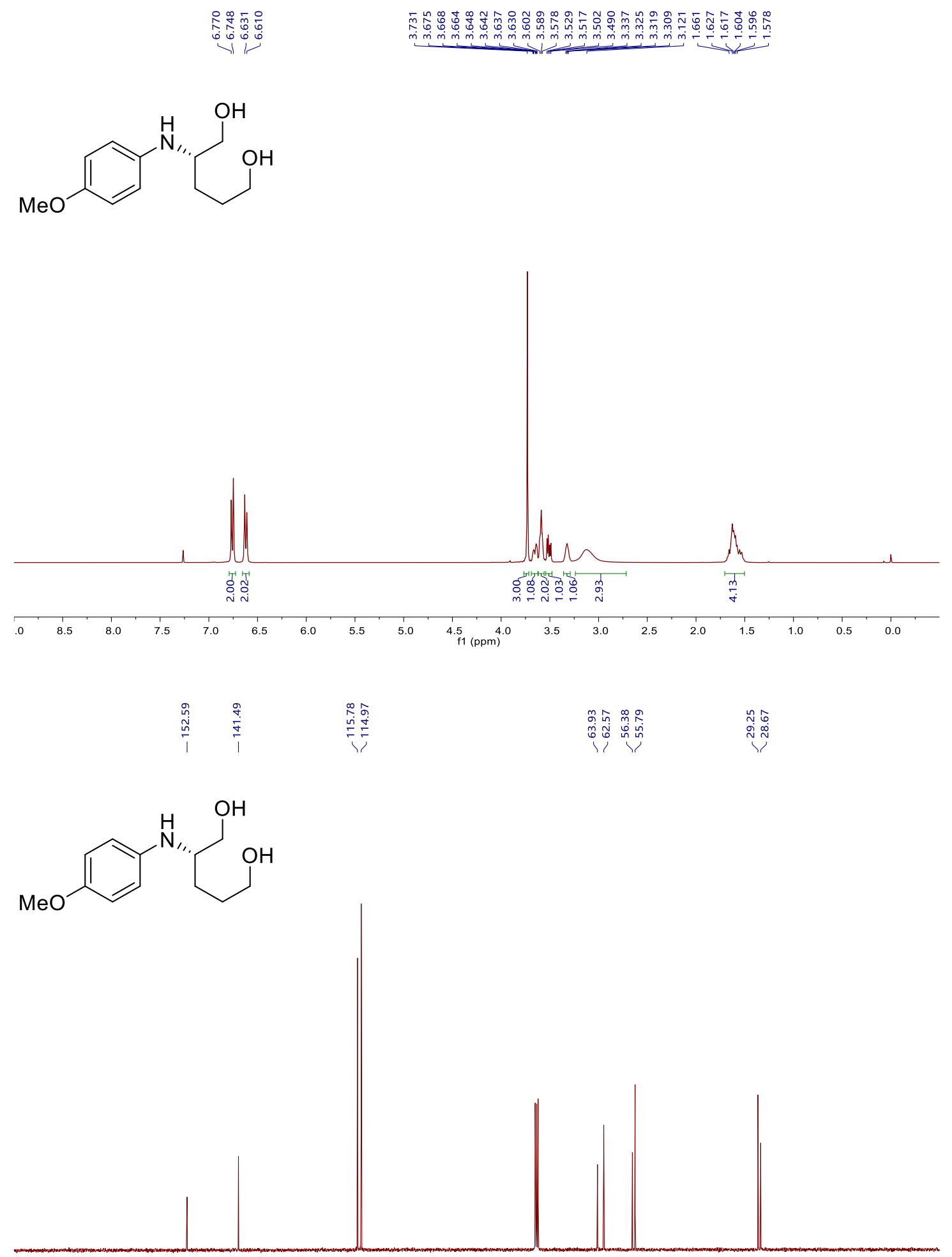

\begin{tabular}{rllllllllllllllllllll}
\hline & 180 & 170 & 160 & 150 & 140 & 130 & 120 & 110 & 100 & $\begin{array}{c}90 \\
\mathrm{f} 1(\mathrm{ppm})\end{array}$ & 80 & 70 & 60 & 50 & 40 & 30 & 20 & 10 & 0 & 1
\end{tabular} 
(S)-2-((4-Fluorophenyl)amino)pentane-1,5-diol (9d)

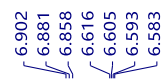

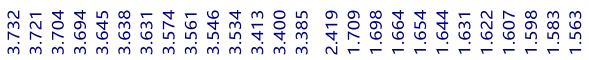

$\overbrace{\mathrm{N}}^{\mathrm{OH}}$

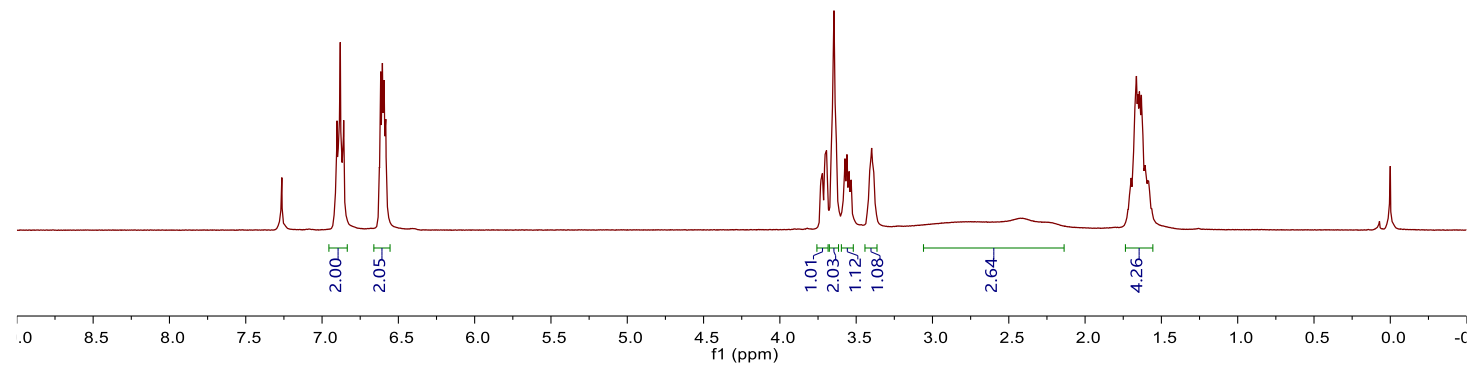

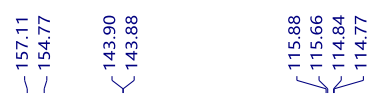

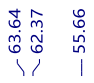

$\stackrel{\substack{\infty \\ \infty}}{\infty} \underset{\infty}{\infty}$<smiles>OCC(CO)Nc1ccc(F)cc1</smiles>

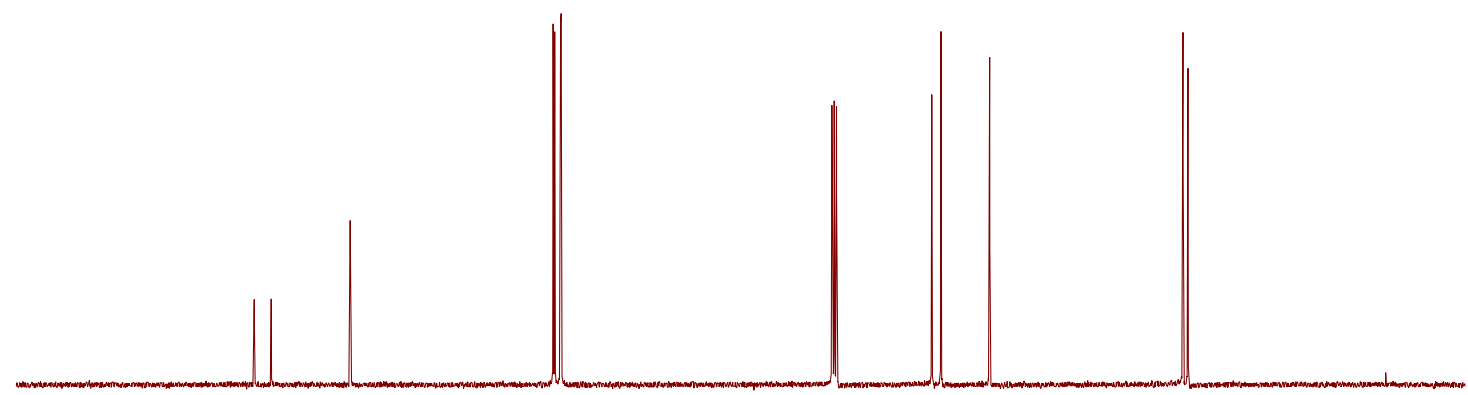

\begin{tabular}{llllllllllllllllllllll}
\hline 90 & 180 & 170 & 160 & 150 & 140 & 130 & 120 & 110 & 100 & $\underset{\mathrm{f} 1}{90}(\mathrm{ppm})$ & 80 & 70 & 60 & 50 & 40 & 30 & 20 & 10 & 0 & -
\end{tabular} 
(S)-2-((4-Chlorophenyl)amino)pentane-1,5-diol (9e)
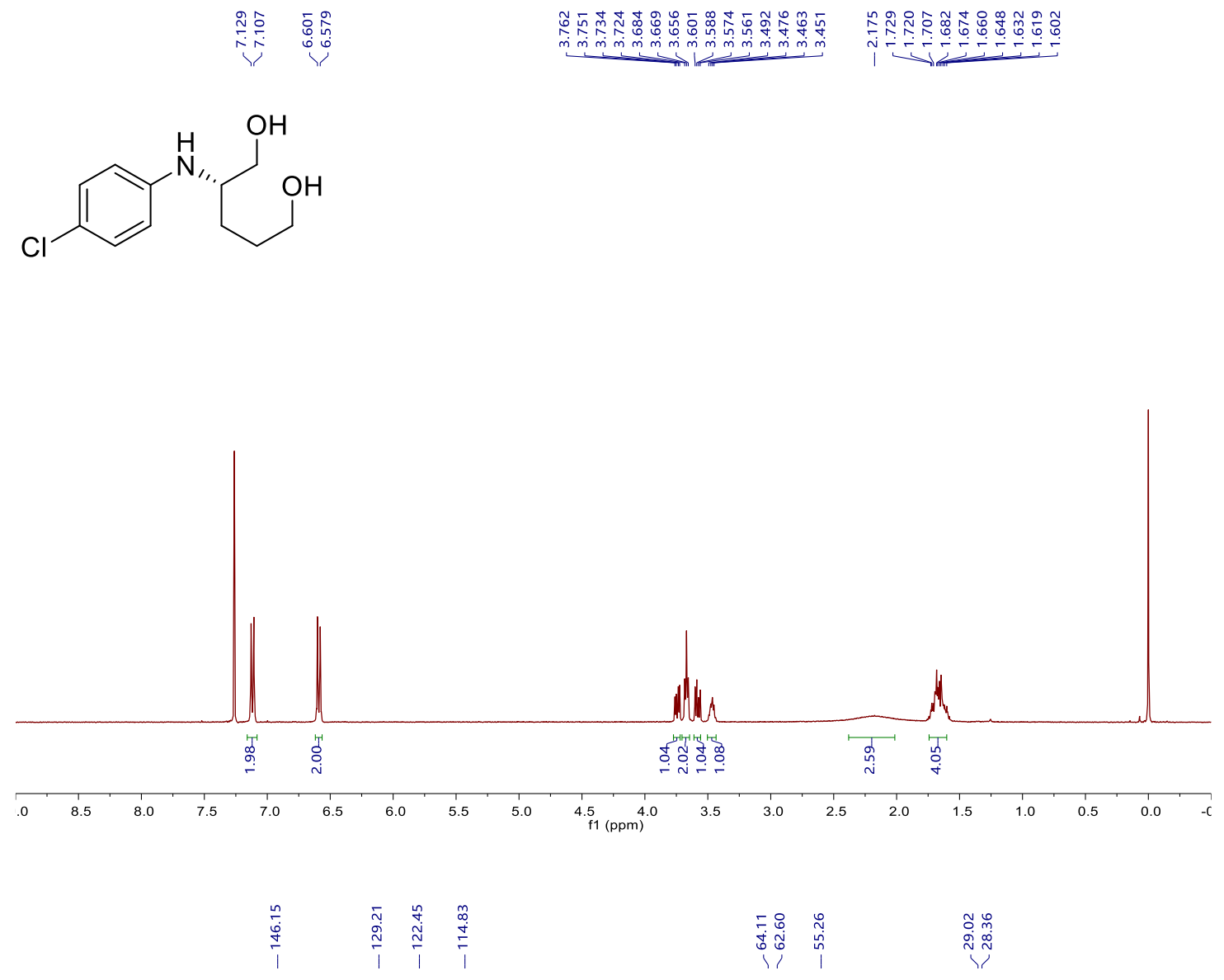<smiles>OCCC[C@H](CO)Nc1ccc(Cl)cc1</smiles>

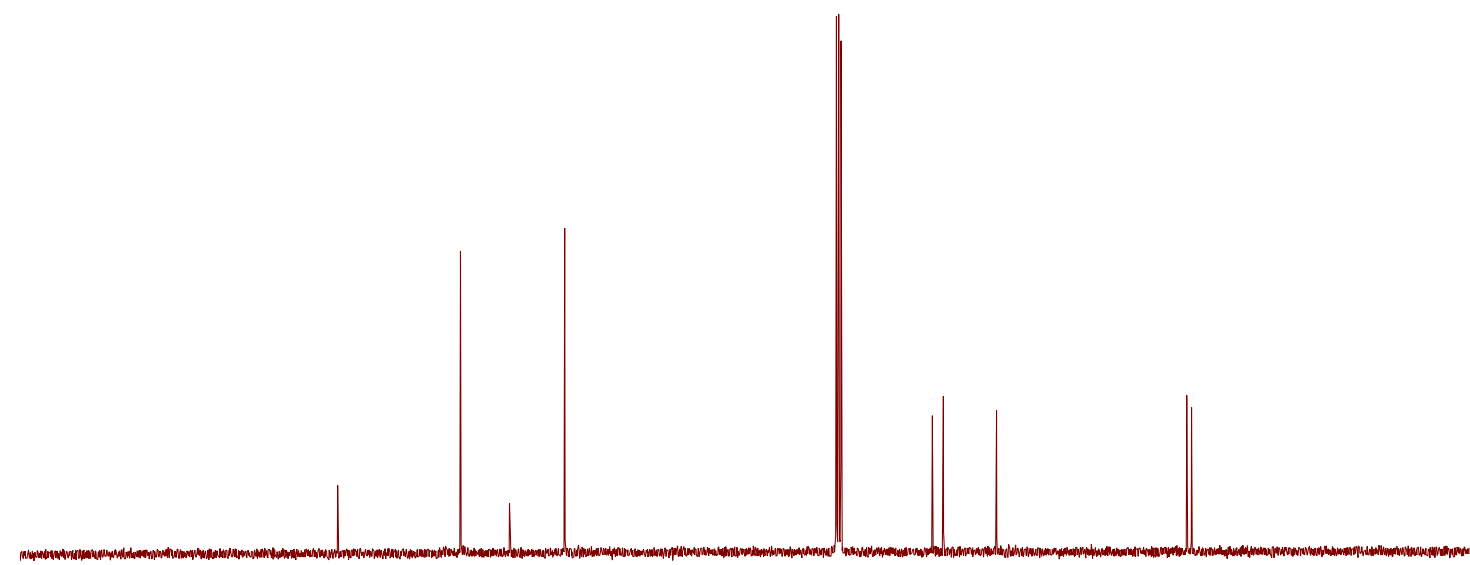

\begin{tabular}{lllllllllllllllllllllll}
\hline & 180 & 170 & 160 & 150 & 140 & 130 & 120 & 110 & 100 & $\underset{f}{9}(\mathrm{ppm})$ & 80 & 70 & 60 & 50 & 40 & 30 & 20 & 10 & 0 & -
\end{tabular} 
(S)-2-([1,1'-Biphenyl]-4-ylamino)pentane-1,5-diol (9f)

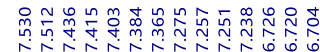

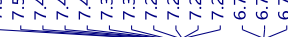

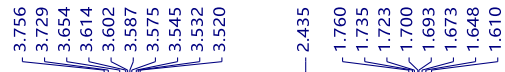

$\overbrace{N}^{\text {OH }}$

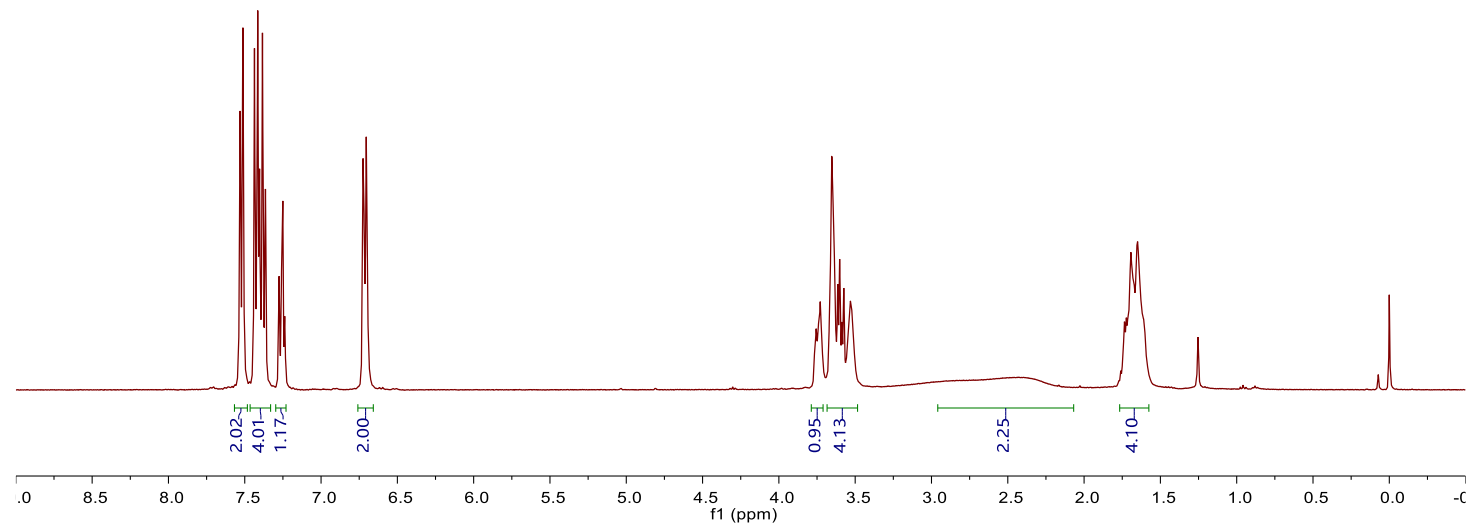<smiles>OCCC[C@H](CO)Nc1ccc(-c2ccccc2)cc1</smiles>
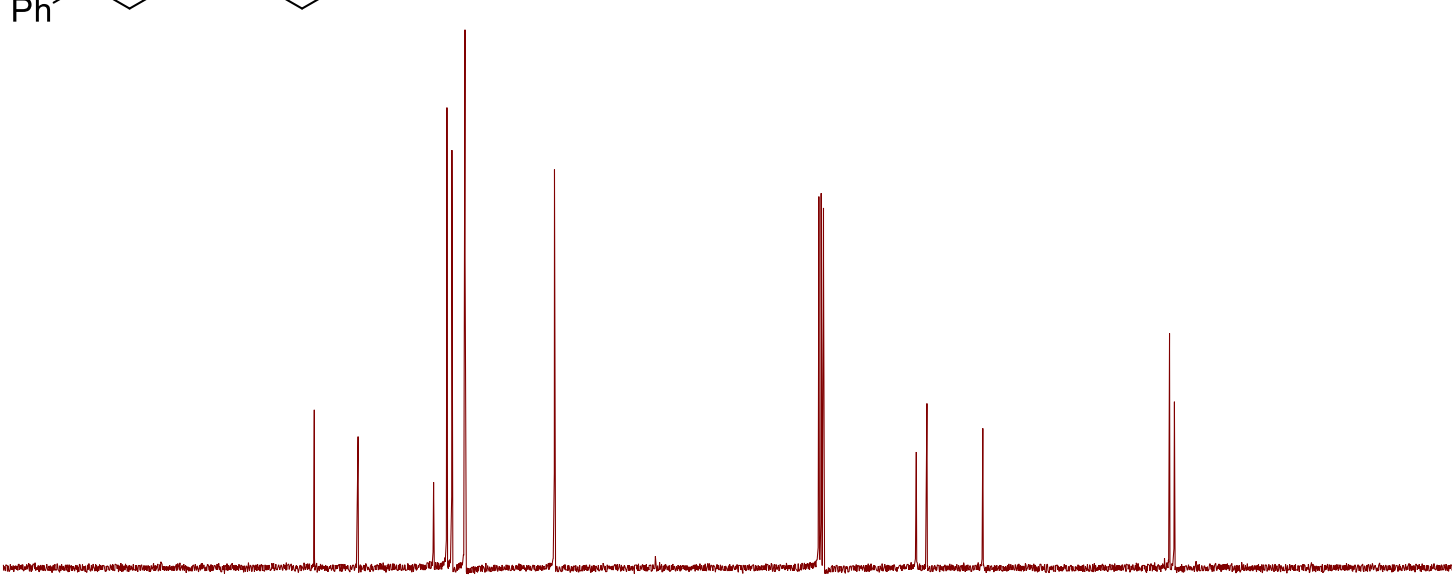

$180 \quad 170 \quad 160$

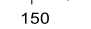

$130 \quad 120$

10090

$\quad 70$

40

10 
(S)-2-(m-Tolylamino)pentane-1,5-diol (9g)

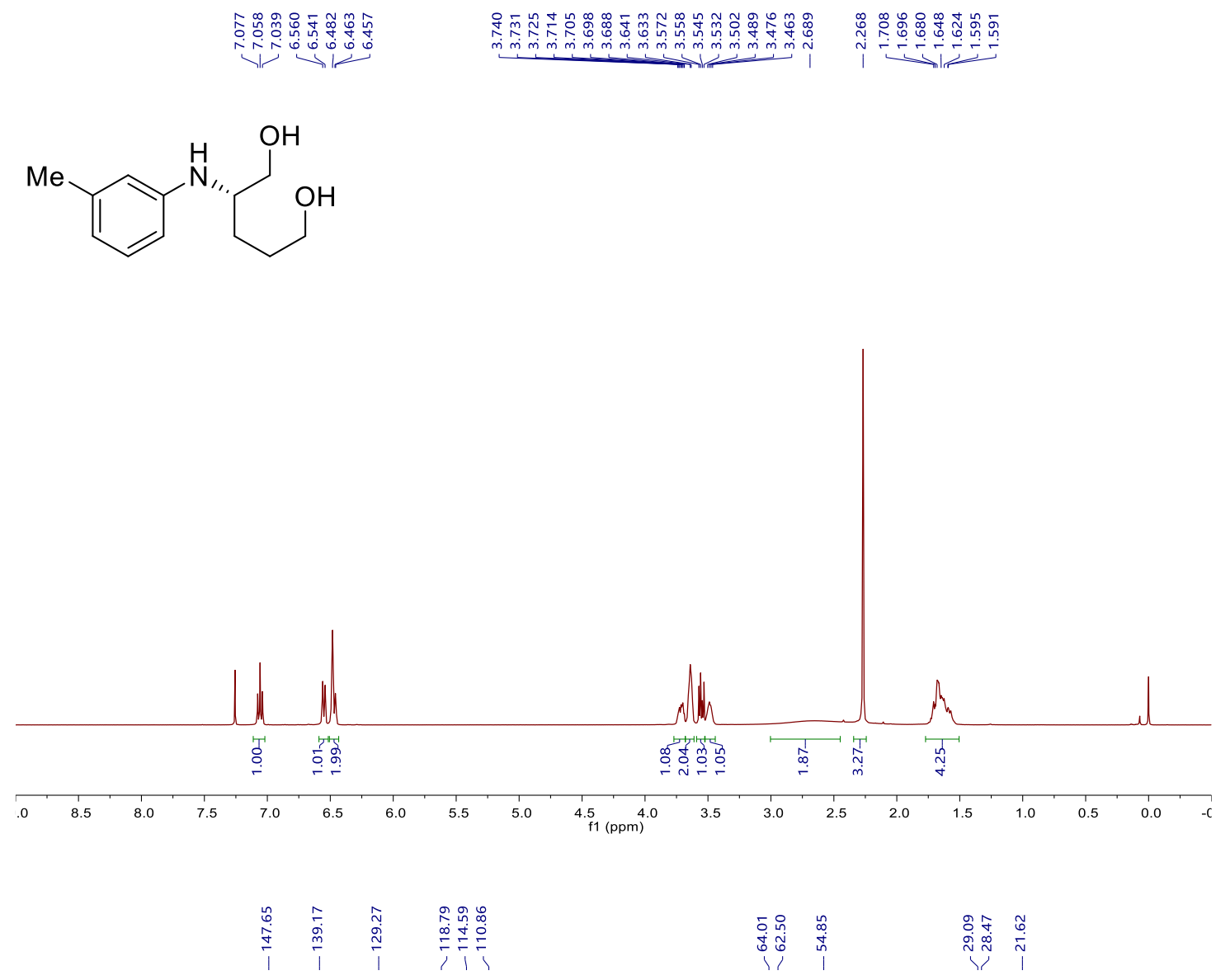<smiles>Cc1cccc(N[C@@H](CO)CCCO)c1</smiles>

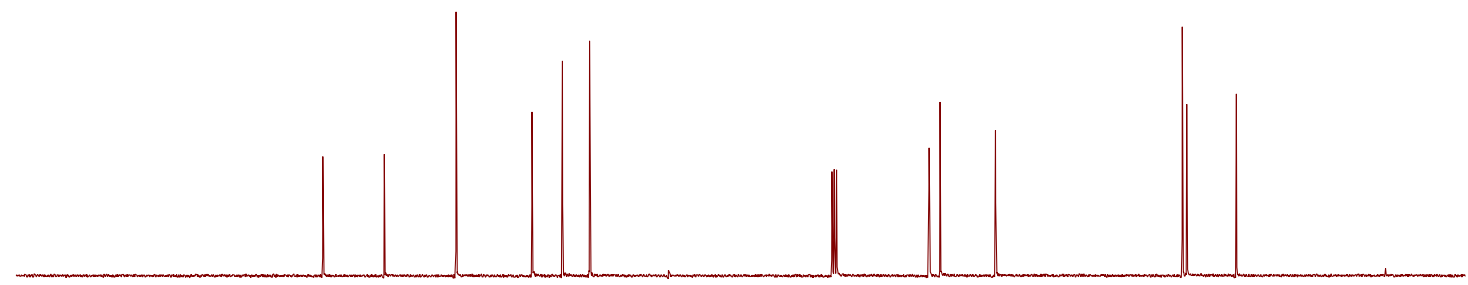

\begin{tabular}{lllllllllllllllllllll}
\hline & 180 & 170 & 160 & 150 & 140 & 130 & 120 & 110 & 100 & $\underset{f 1}{90}(\mathrm{ppm})$ & 80 & 70 & 60 & 50 & 40 & 30 & 20 & 10 & 0 & -
\end{tabular} 
(S)-2-((3-Methoxyphenyl)amino)pentane-1,5-diol (9h)

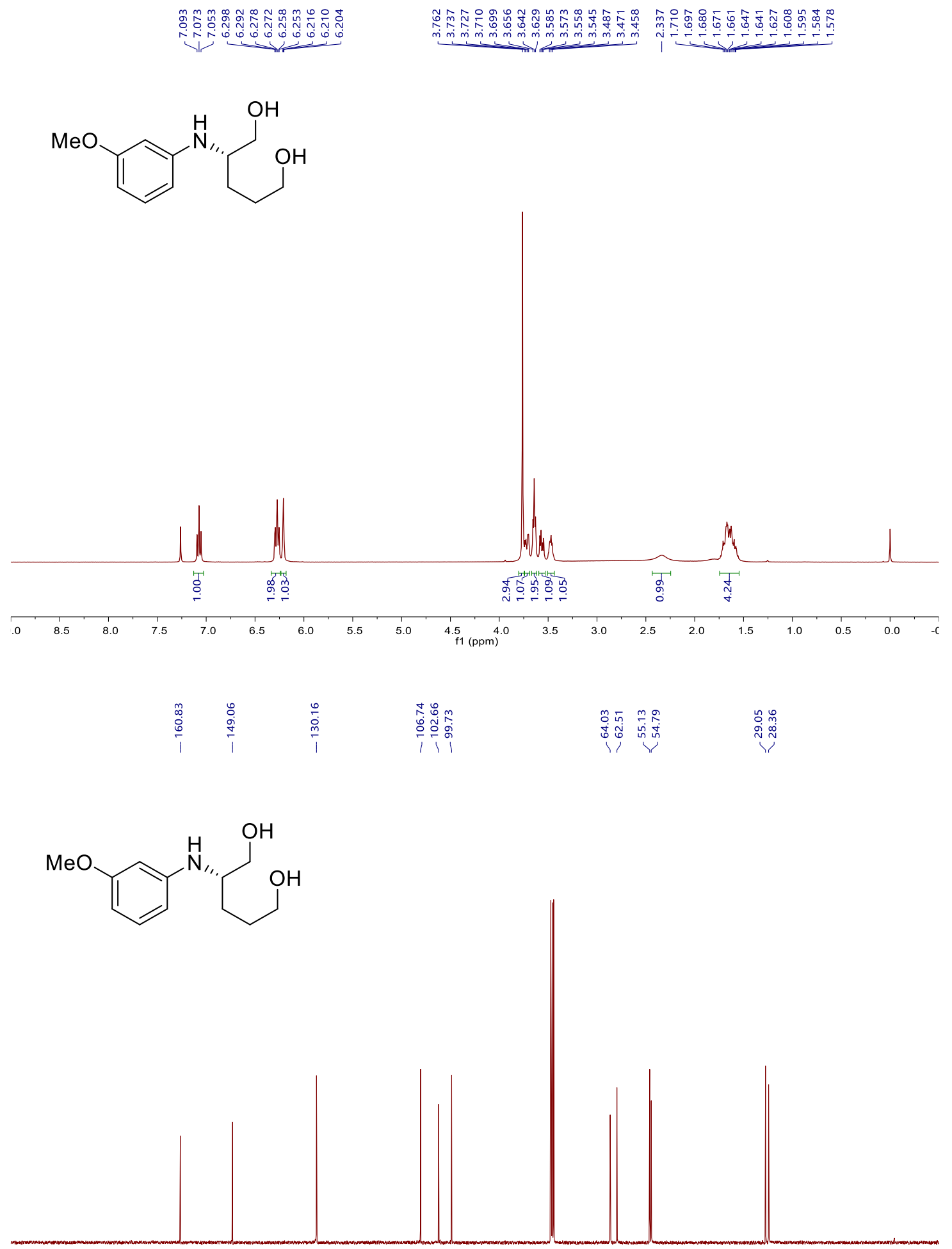

$\begin{array}{llllllllll}190 & 180 & 170 & 160 & 150 & 140 & 130 & 120 & 110 & 100 \\ \mathrm{f} 1(\mathrm{ppm}) & 90\end{array}$ 
(S)-2-((3-Chlorophenyl)amino)pentane-1,5-diol (9i)

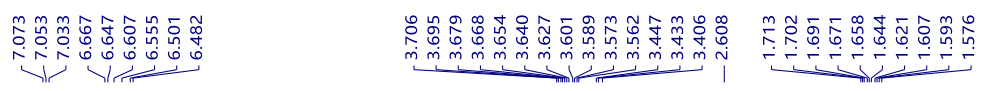

$\mathrm{H}_{\mathrm{OH}}^{\mathrm{OH}}$

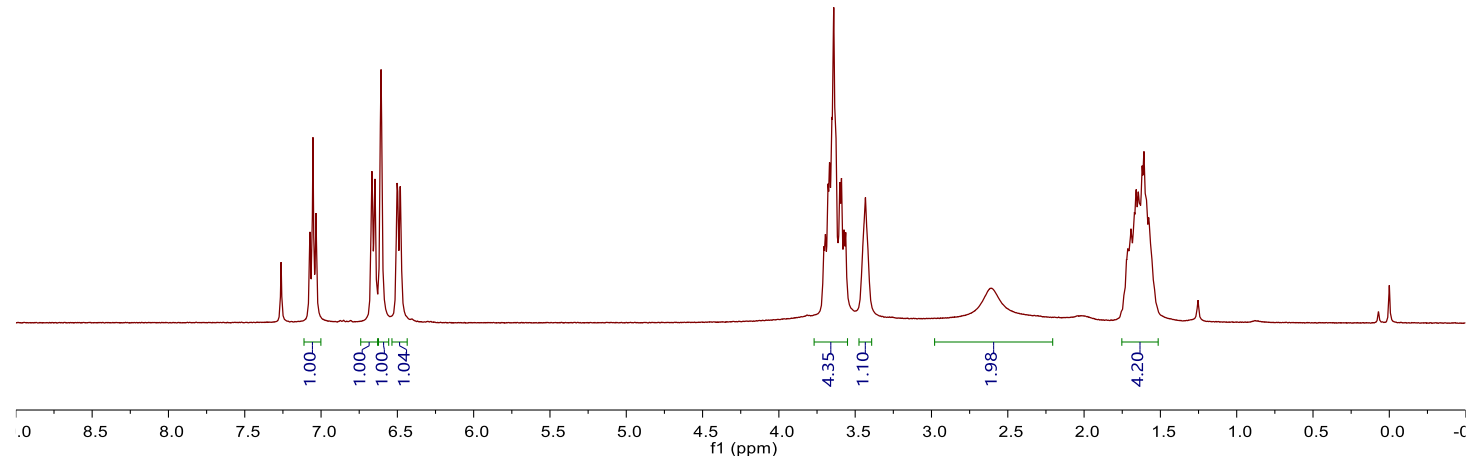

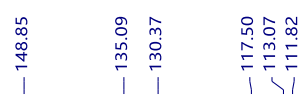

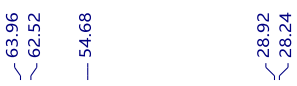<smiles>OCCC[C@H](CO)Nc1cccc(Cl)c1</smiles>

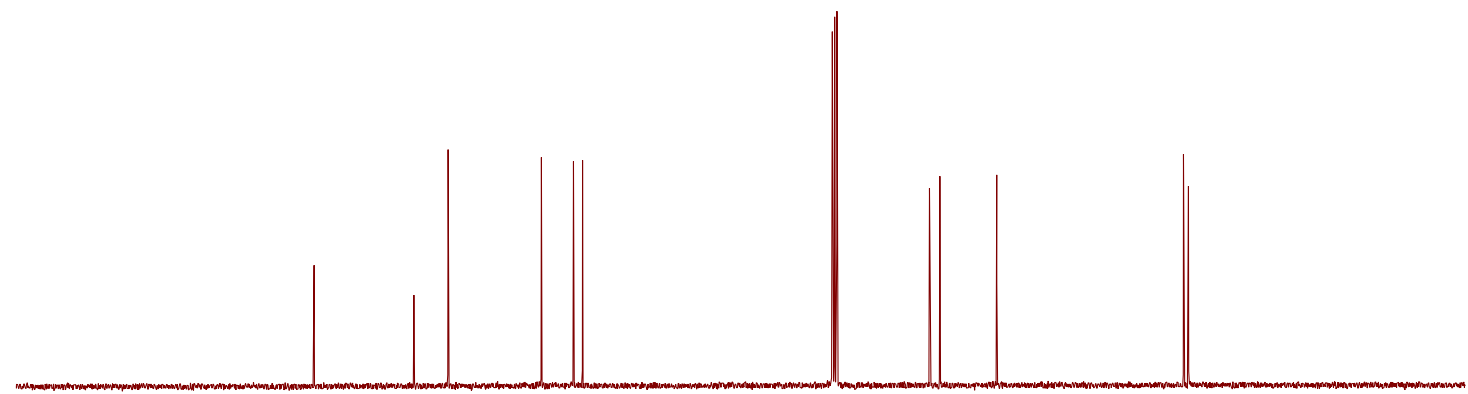

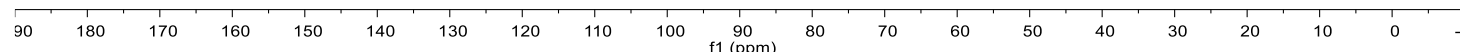


(S)-2-(o-Tolylamino)pentane-1,5-diol (9j)

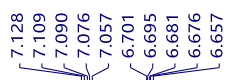

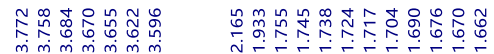

$\mathrm{N}_{\mathrm{OH}}^{\mathrm{Me}}$
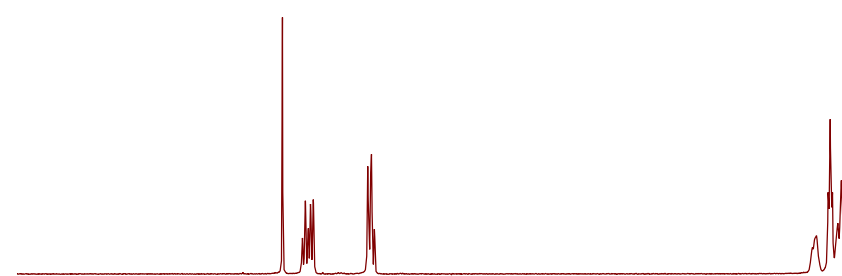

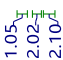
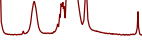

管喜翠

\begin{tabular}{lllllllllll}
\hline .0 & 8.5 & 8.0 & 7.5 & 7.0 & 6.5 & 6.0 & 5.5 & 5.0 & 4.5 & 4.0 \\
$\mathrm{f} 1(\mathrm{ppm})$ &
\end{tabular}

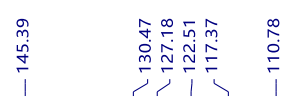

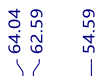

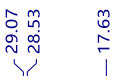

Me

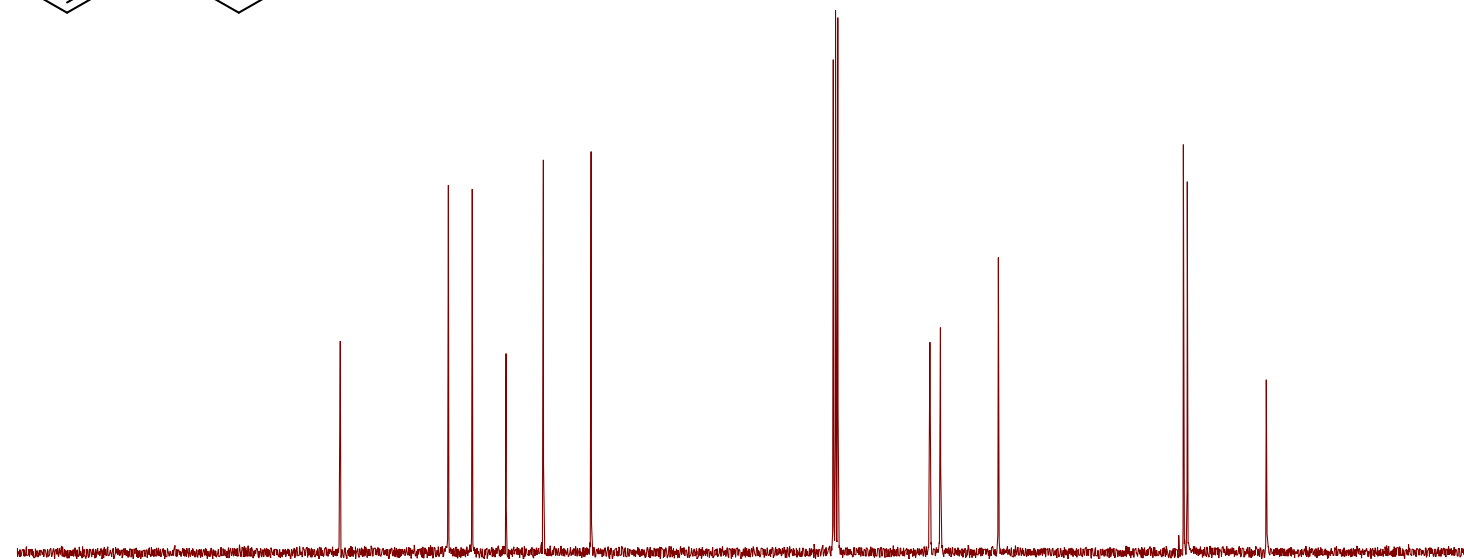

$\begin{array}{llllllllllllllllllllllll}1 & 180 & 170 & 160 & 150 & 140 & 130 & 120 & 110 & 100 & 90 & 80 & 70 & 60 & 50 & 40 & 30 & 20 & 10 & 0 & 1\end{array}$ 
(S)-2-((2-Ethylphenyl)amino)pentane-1,5-diol (9k)

$\underbrace{2}$

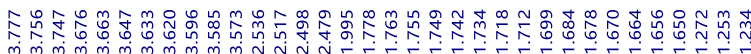

$\mathrm{N}_{\mathrm{N}}^{\mathrm{Et}}$

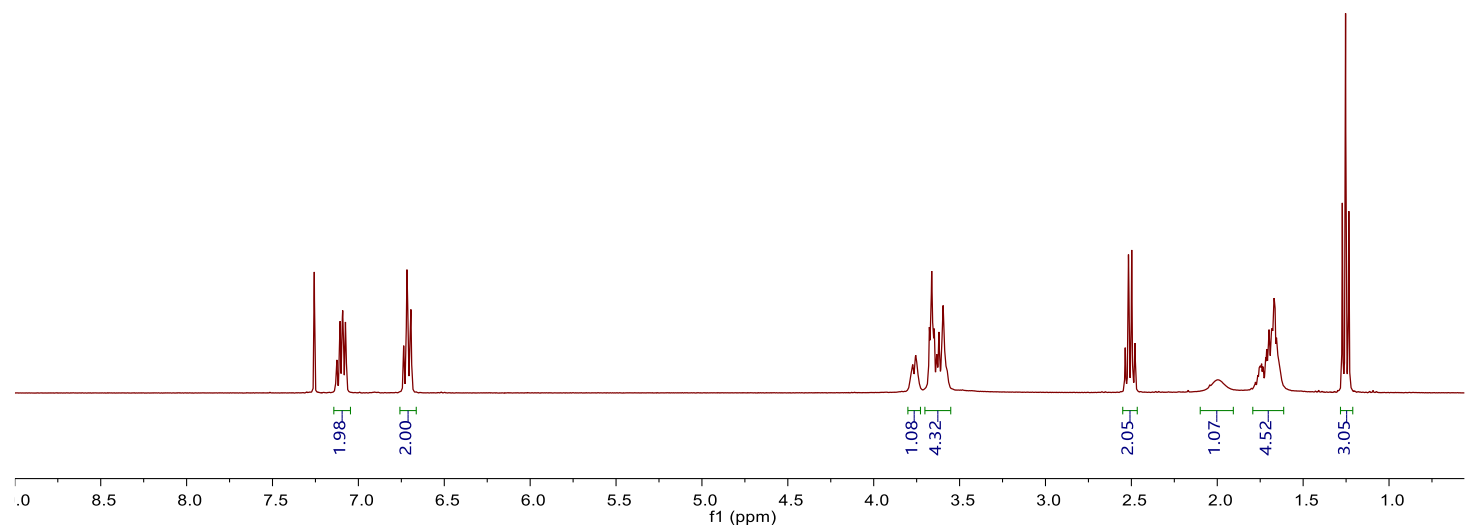

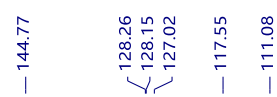

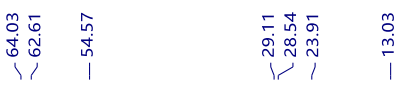<smiles>CCc1ccccc1N[C@H](CO)CCCO</smiles>

$90 \quad 180$

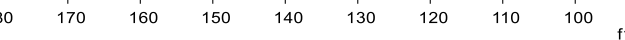

1 (ppm)

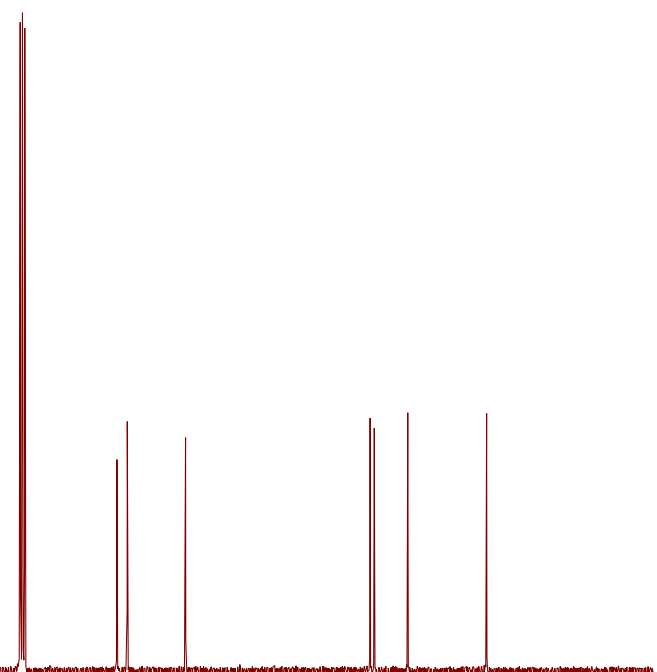


(S)-2-((2-Isopropylphenyl)amino)pentane-1,5-diol (9I)

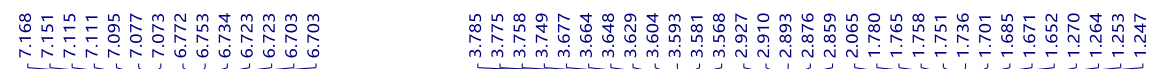

$\mathrm{N}_{\mathrm{N}}^{\mathrm{Pr}}$

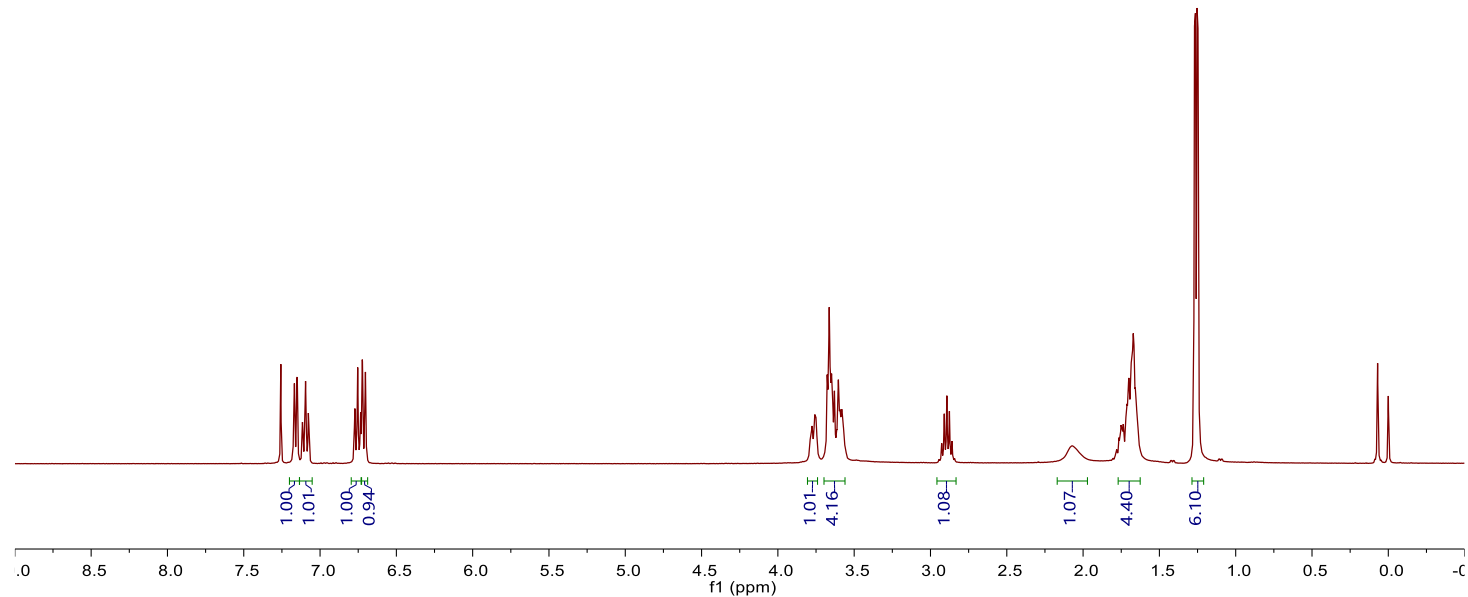

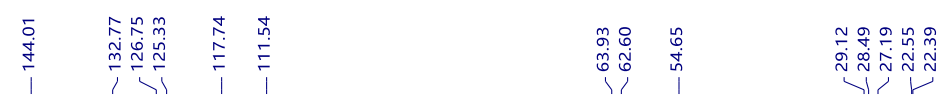

$\mathrm{C}_{\mathrm{N}}^{\mathrm{Pr}}$

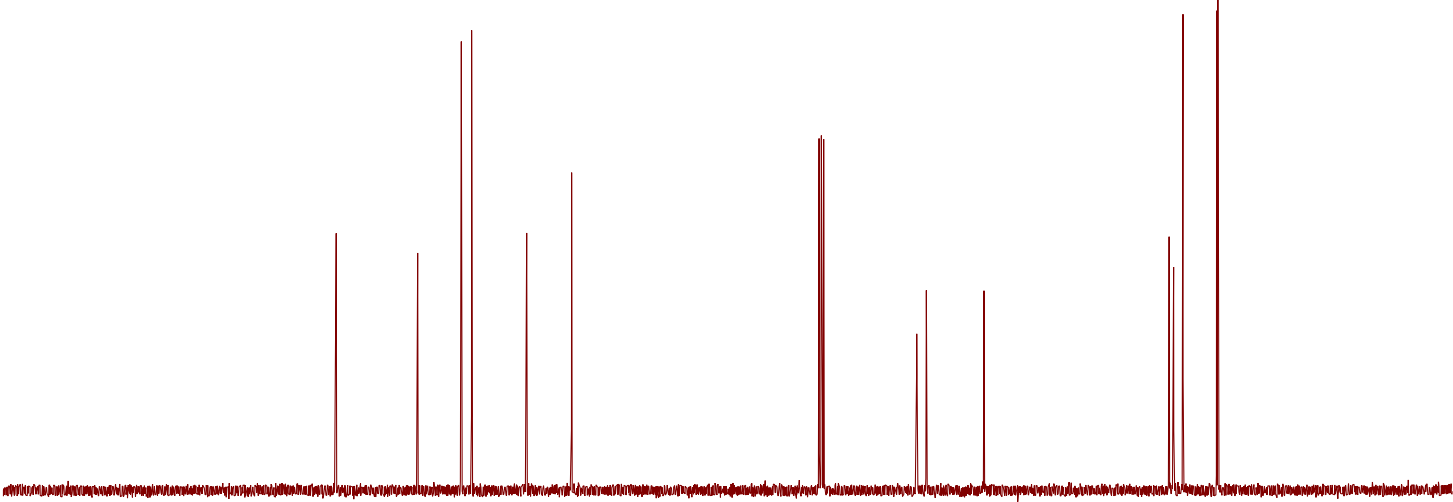

\begin{tabular}{lllllllllllllllllllllllllllll}
\hline & 180 & 170 & 160 & 150 & 140 & 130 & 120 & 110 & 100 & 90 & 80 & 70 & 60 & 50 & 40 & 30 & 20 & 10 & 0 & -
\end{tabular} 
(S)-2-((2-Methoxyphenyl)amino)pentane-1,5-diol (9m)

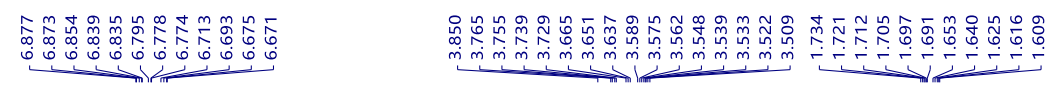

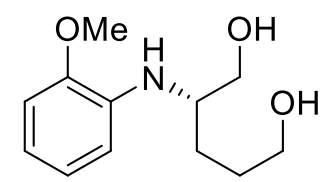

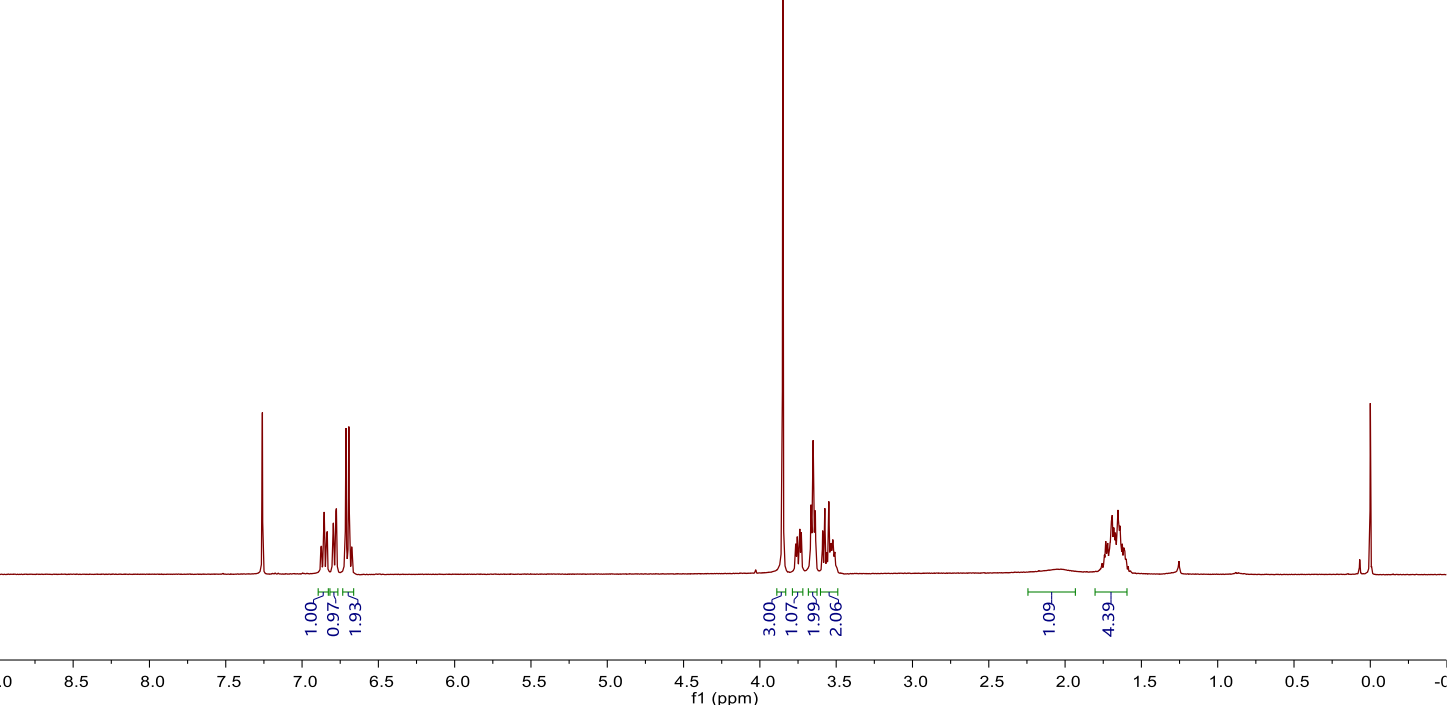

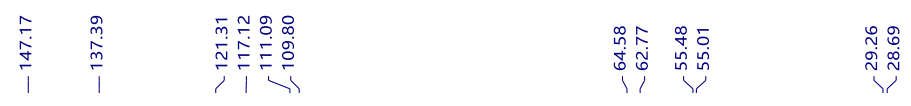<smiles>COc1ccccc1N[C@H](CO)CCCO</smiles>

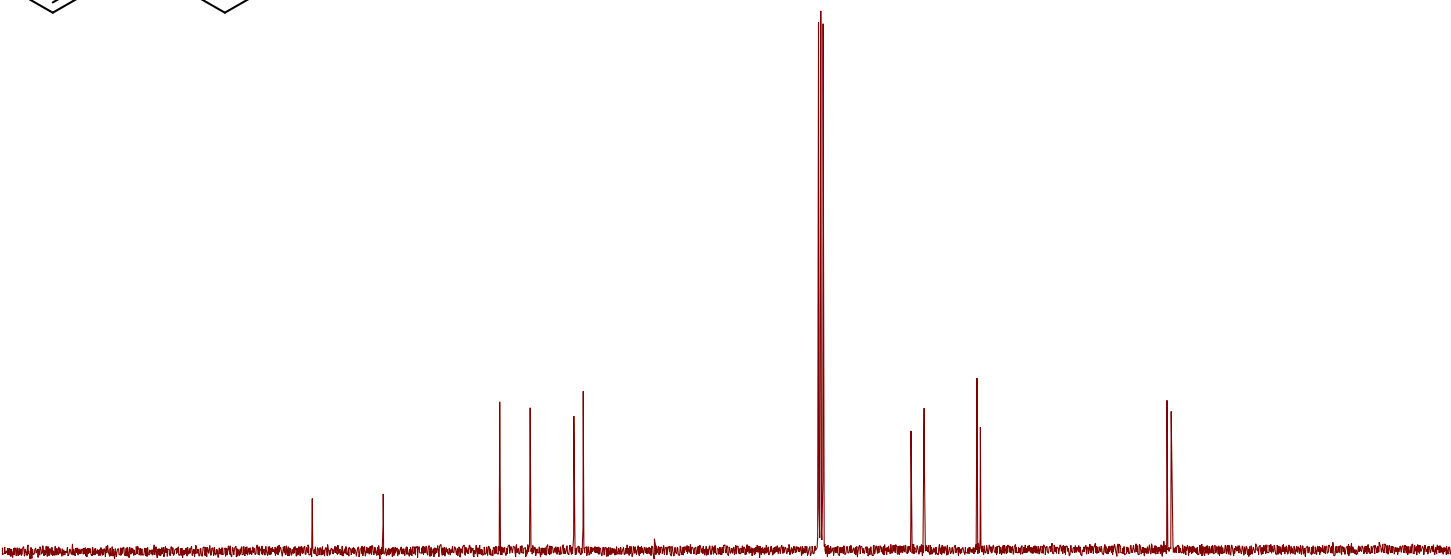

\begin{tabular}{lllllllllllllllllllll}
\hline 90 & 180 & 170 & 160 & 150 & 140 & 130 & 120 & 110 & 100 & 90 & 90 & 1 & 1 & 1 & 1 & 1 & 1 & 1 & 1 & 1 \\
$\mathrm{f} 1(\mathrm{ppm})$ & 80 & 70 & 60 & 50 & 40 & 30 & 20 & 10 & 0 & -
\end{tabular} 
(S)-2-((2-Fluorophenyl)amino)pentane-1,5-diol (9n)

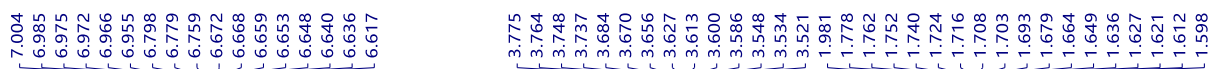

$\mathrm{N}_{\mathrm{OH}}^{\mathrm{OH}}$

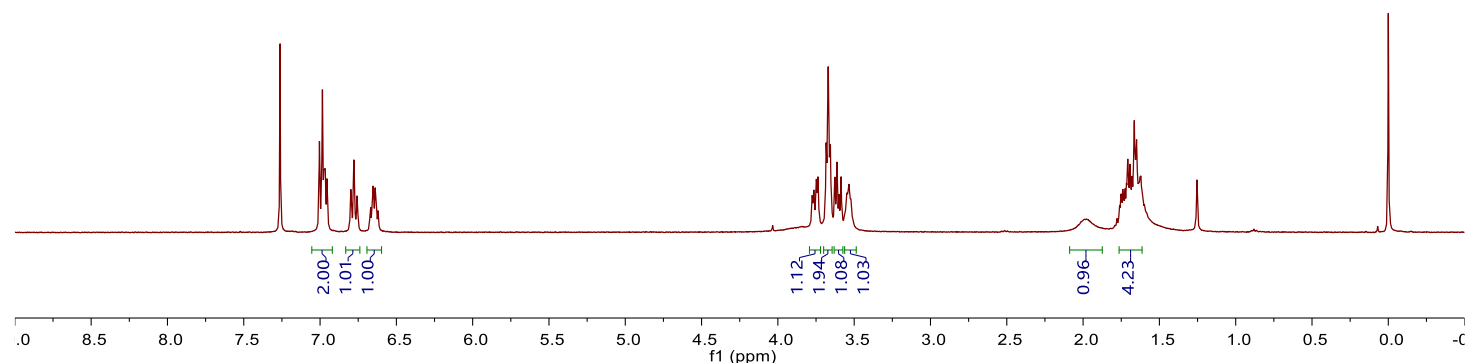

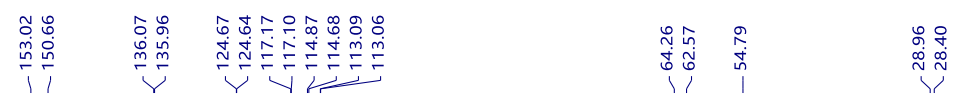

$\mathrm{H}_{\mathrm{N}}^{\mathrm{OH}}$

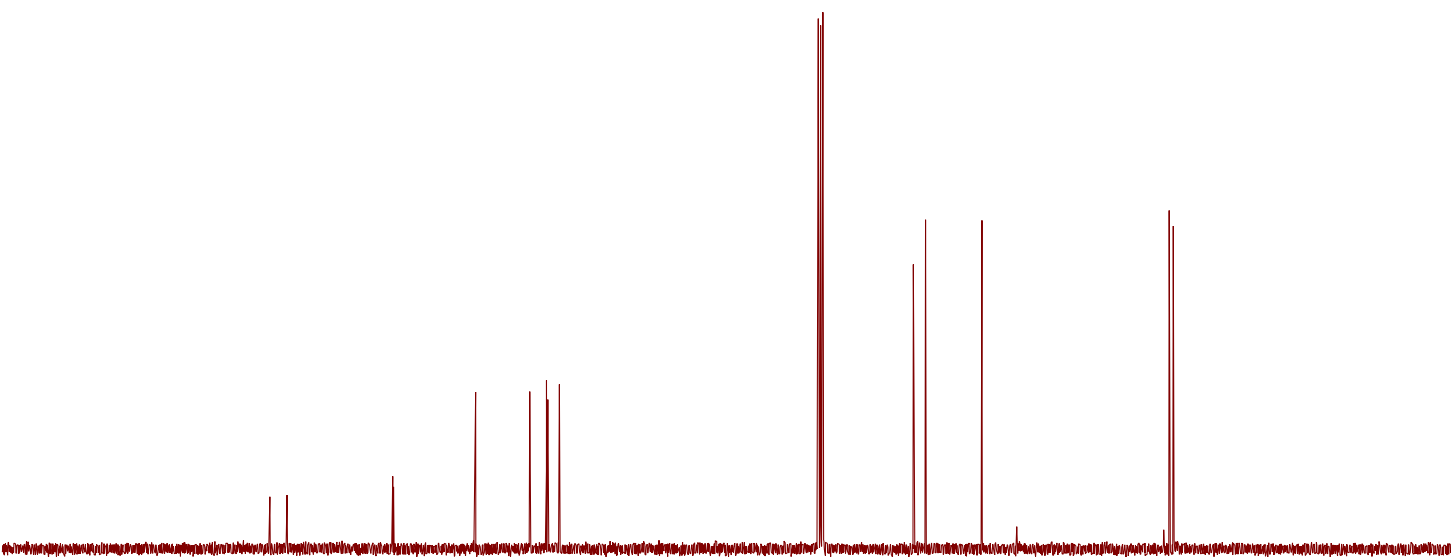

\begin{tabular}{rlllllllllllllllllllllll}
\hline & 180 & 170 & 160 & 150 & 140 & 130 & 120 & 110 & 100 & 90 & 90 & 70 & 60 & 50 & 40 & 30 & 20 & 10 & 0 & -
\end{tabular} 
(S)-2-((2,4-Dimethoxyphenyl)amino)pentane-1,5-diol (9o)

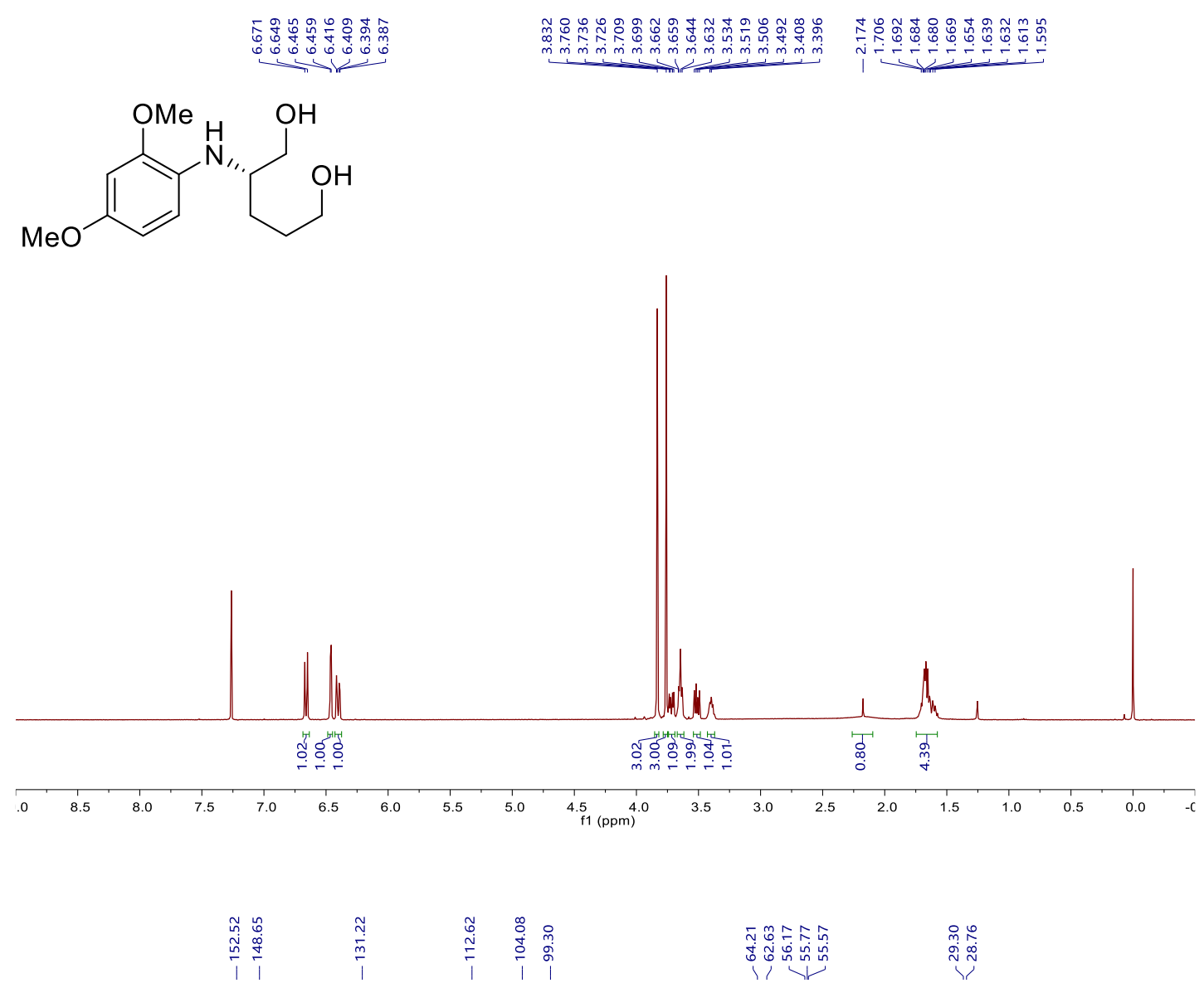<smiles>COc1ccc(N[C@@H](CO)CCCO)c(OC)c1</smiles>

$\mathrm{MeO}$

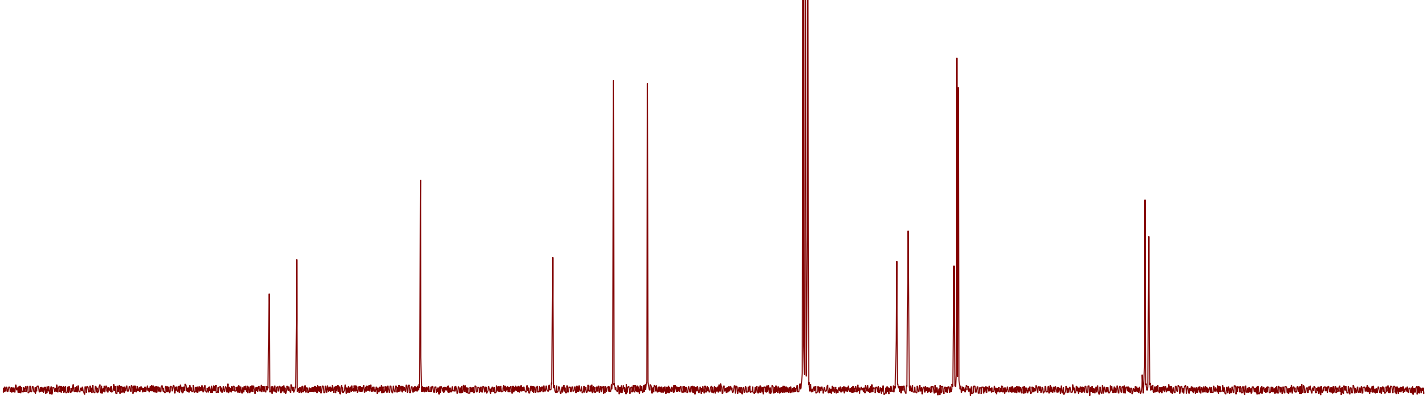

$\begin{array}{lllllllllllllllllllllllllll}180 & 170 & 160 & 150 & 140 & 130 & 120 & 110 & 100 & 90 & 80 & 70 & 60 & 50 & 40 & 30 & 20 & 10 & 0 & -10\end{array}$ 
(S)-2-(Naphthalen-1-ylamino)pentane-1,5-diol (9p)

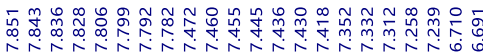

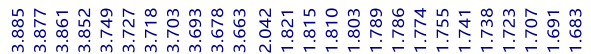

$\mathrm{N}_{\mathrm{N}}^{\mathrm{OH}}$
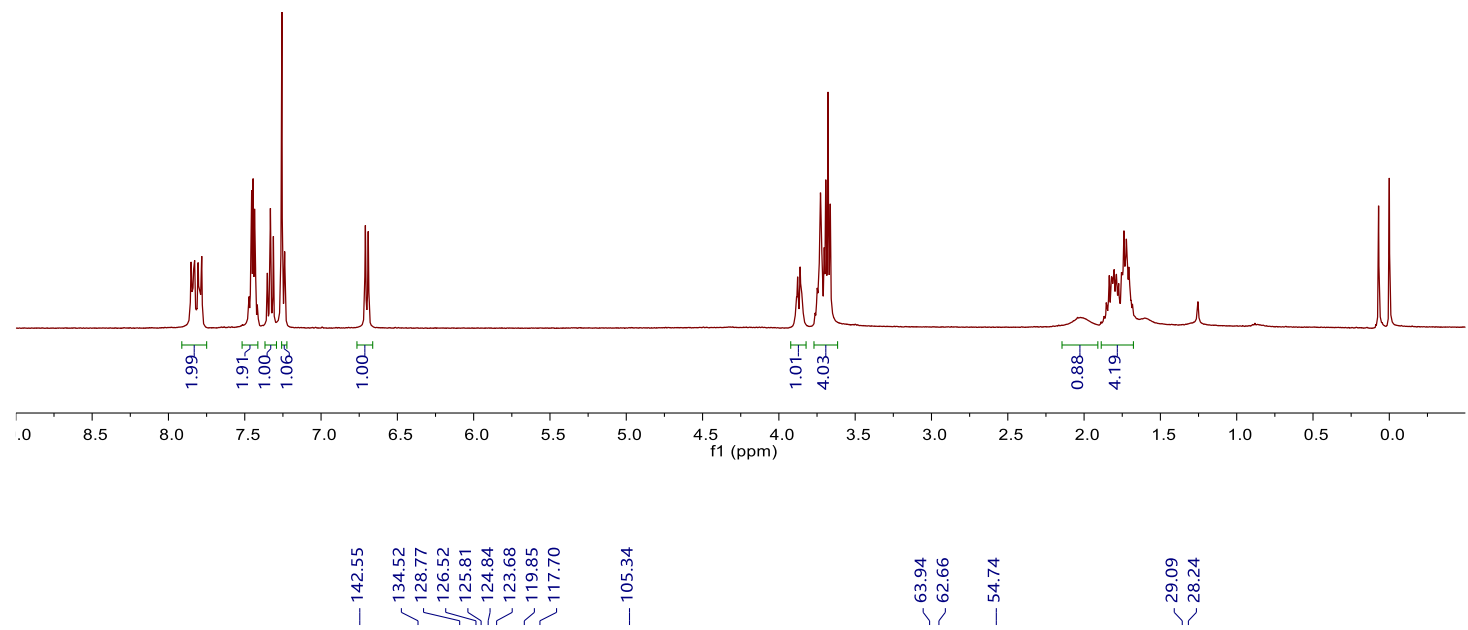

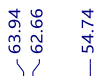

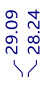<smiles>OCCC[C@H](CO)Nc1cccc2ccccc12</smiles>

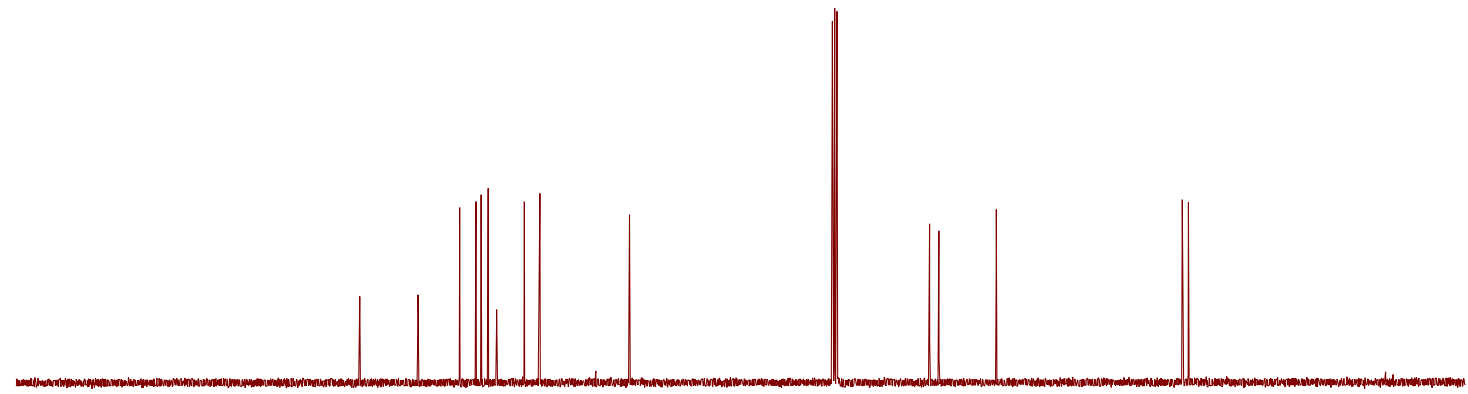

\begin{tabular}{llllllllllllllllllllllll}
\hline & 180 & 170 & 160 & 150 & 140 & 130 & 120 & 110 & 100 & 90 & 80 & 70 & 60 & 50 & 40 & 30 & 20 & 10 & 0 & 1
\end{tabular} 


\section{Compound 10}

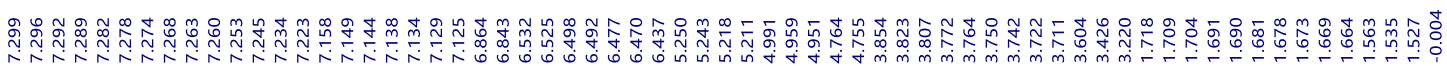<smiles>COc1ccc(N(C(C)=O)C(CO)CCO)c(OC)c1</smiles>

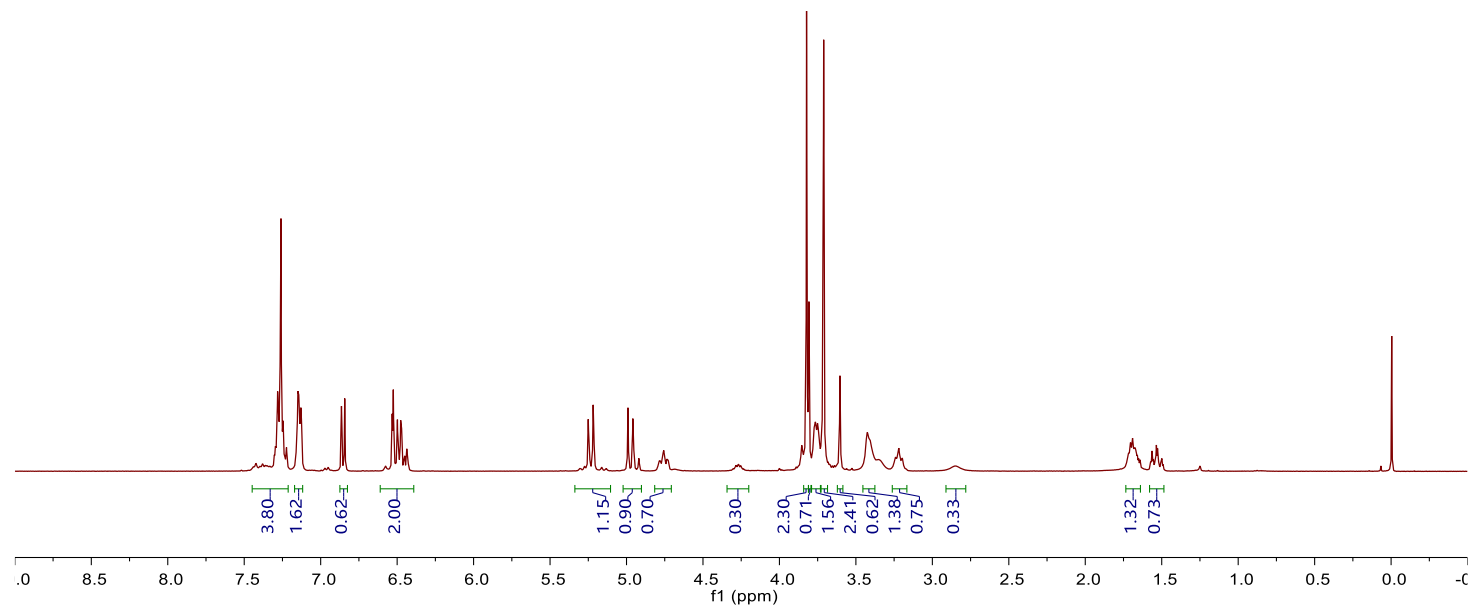

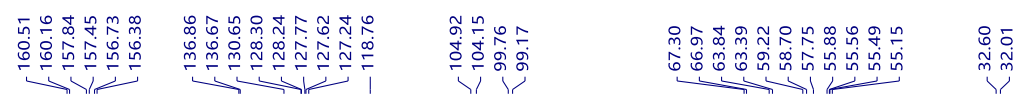<smiles>COc1ccc(N(C(C)=O)C(CO)CCO)c(OC)c1</smiles> 


\section{Compound 11}

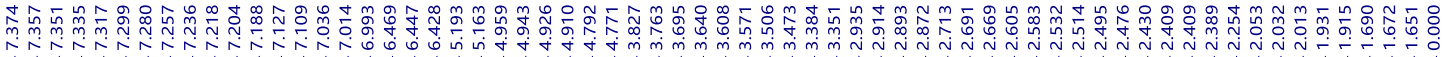<smiles>COc1ccc(N(C(C)=O)C2CC[NH+](Cc3ccccc3)C2)c(OC)c1</smiles>

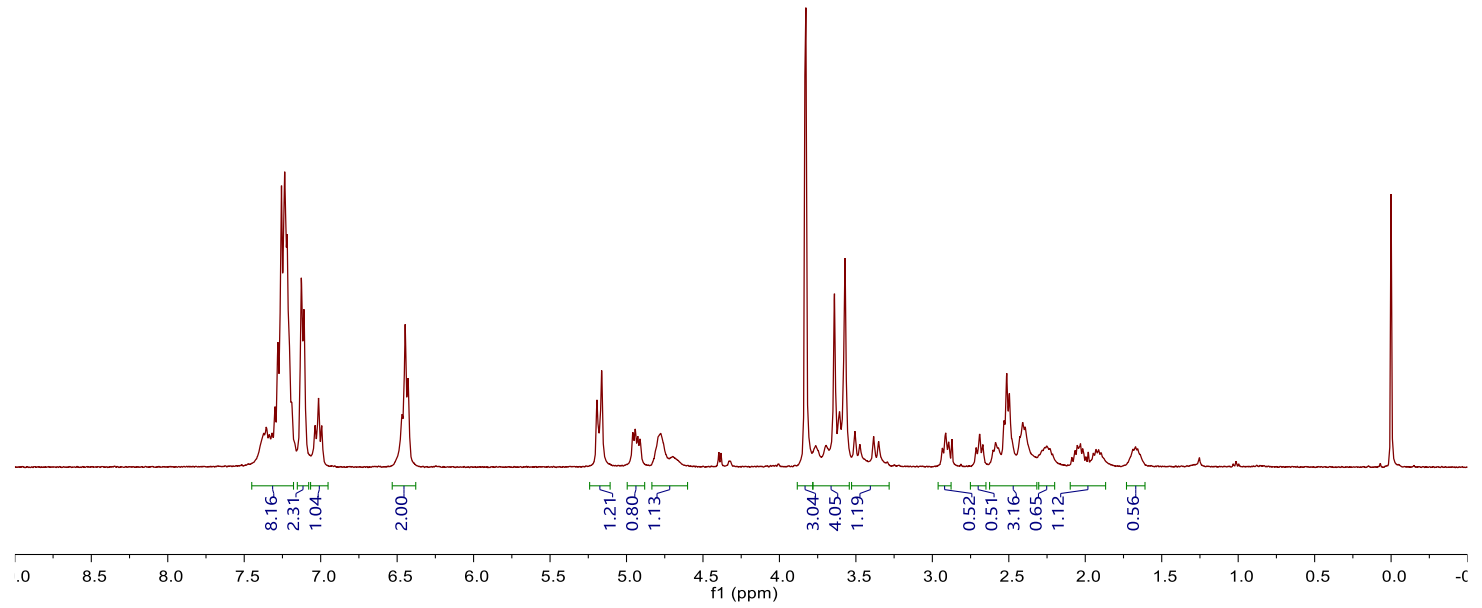

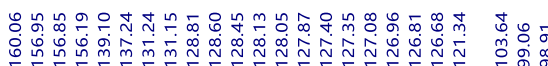<smiles>COc1ccc(N(C(C)=O)C2CC[NH+](Cc3ccccc3)C2)c(OC)c1</smiles>

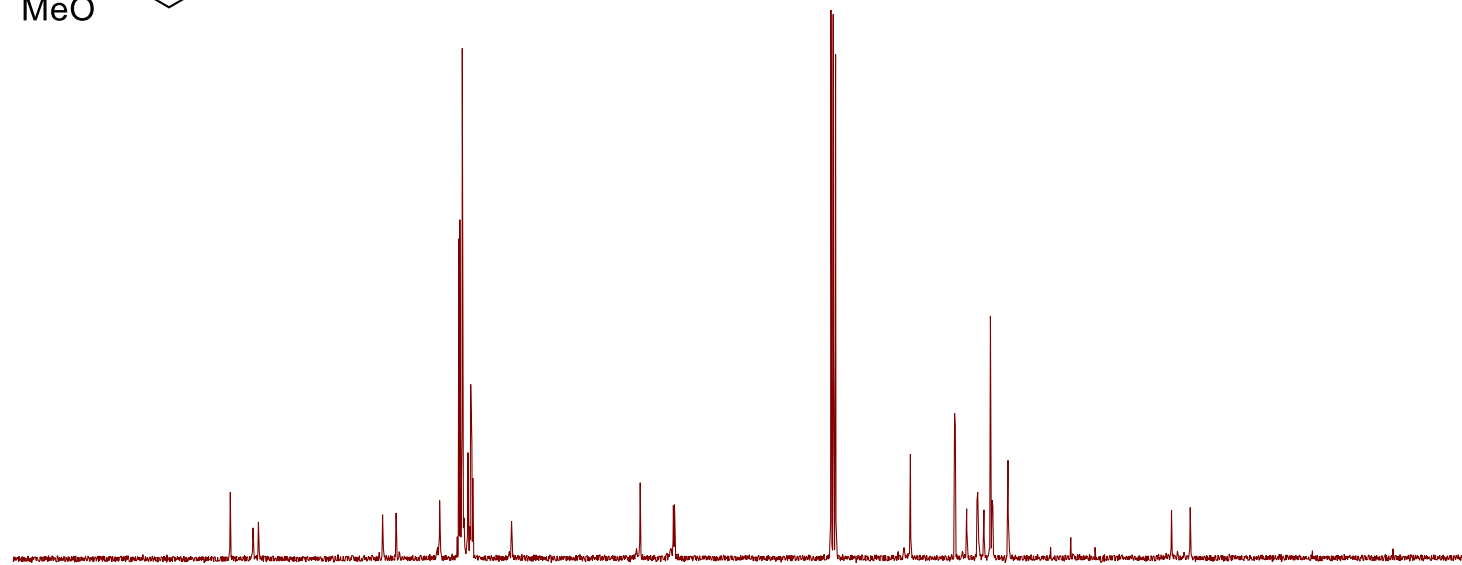

$\begin{array}{lllllllllll}90 & 180 & 170 & 160 & 150 & 140 & 130 & 120 & 110 & 100 & \begin{array}{c}90 \\ \mathrm{f} 1(\mathrm{ppm})\end{array}\end{array}$ 


\section{Compound 12}

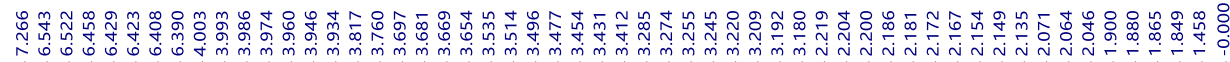<smiles>COc1ccc(N[C@H]2CCN(C(C)(C)C)C2)c(OC)c1</smiles>

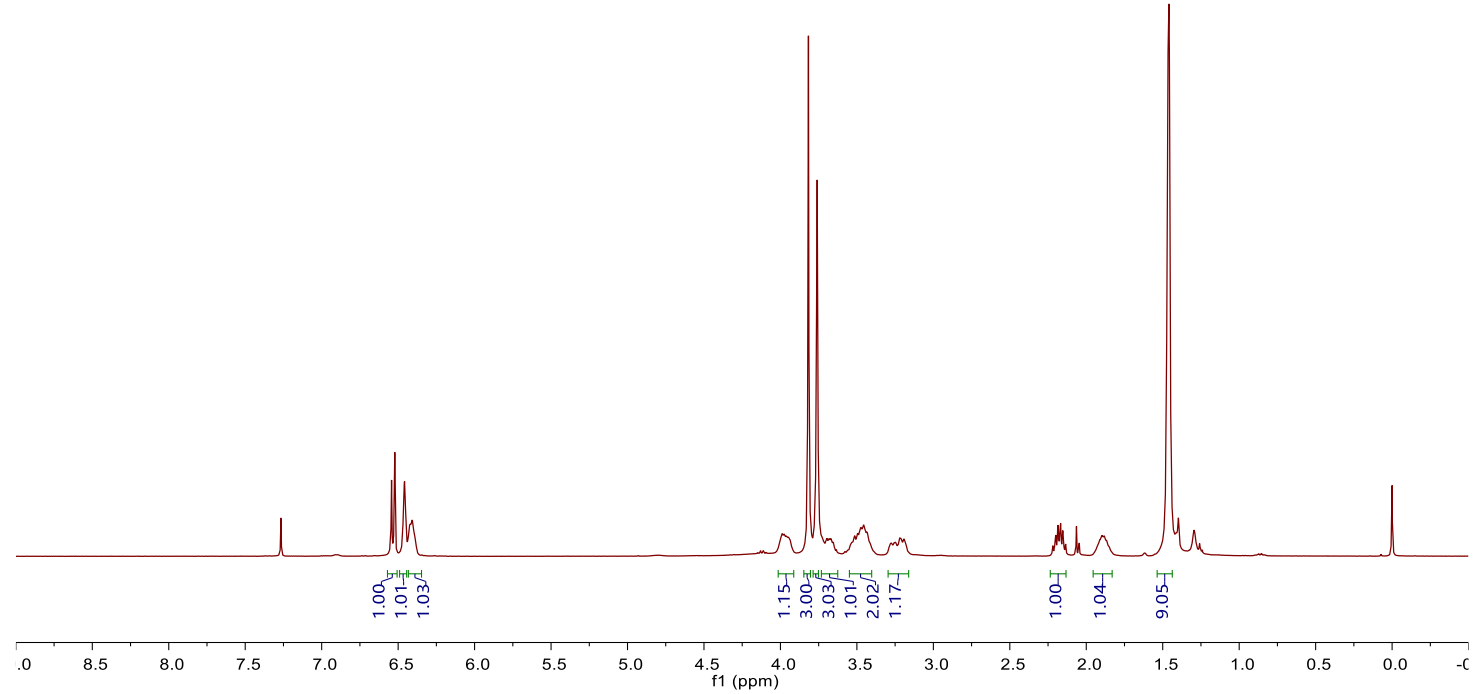

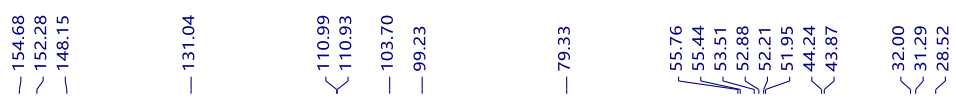<smiles>COc1ccc(NC2CCN(C(C)(C)C)C2)c(OC)c1</smiles>

$\mathrm{MeO}$ NBoc

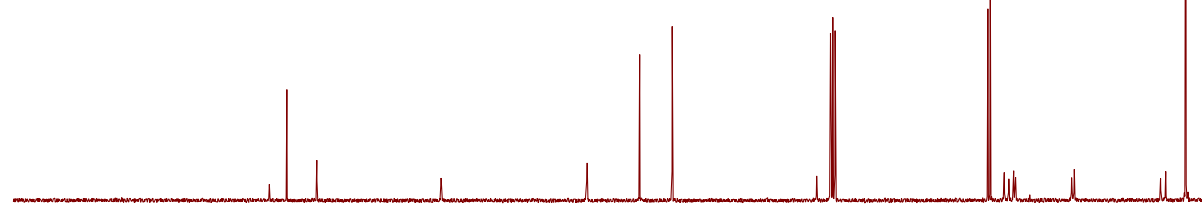

$\begin{array}{lllllllllll}90 & 180 & 170 & 160 & 150 & 140 & 130 & 120 & 110 & 100 & \begin{array}{c}90 \\ \mathrm{f} 1(\mathrm{ppm})\end{array}\end{array}$ 


\section{Compound (13)}

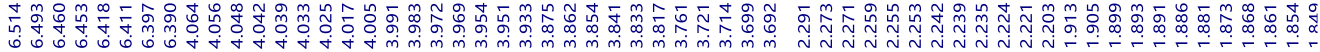<smiles>COc1ccc(NC2CCOC2)c(OC)c1</smiles>

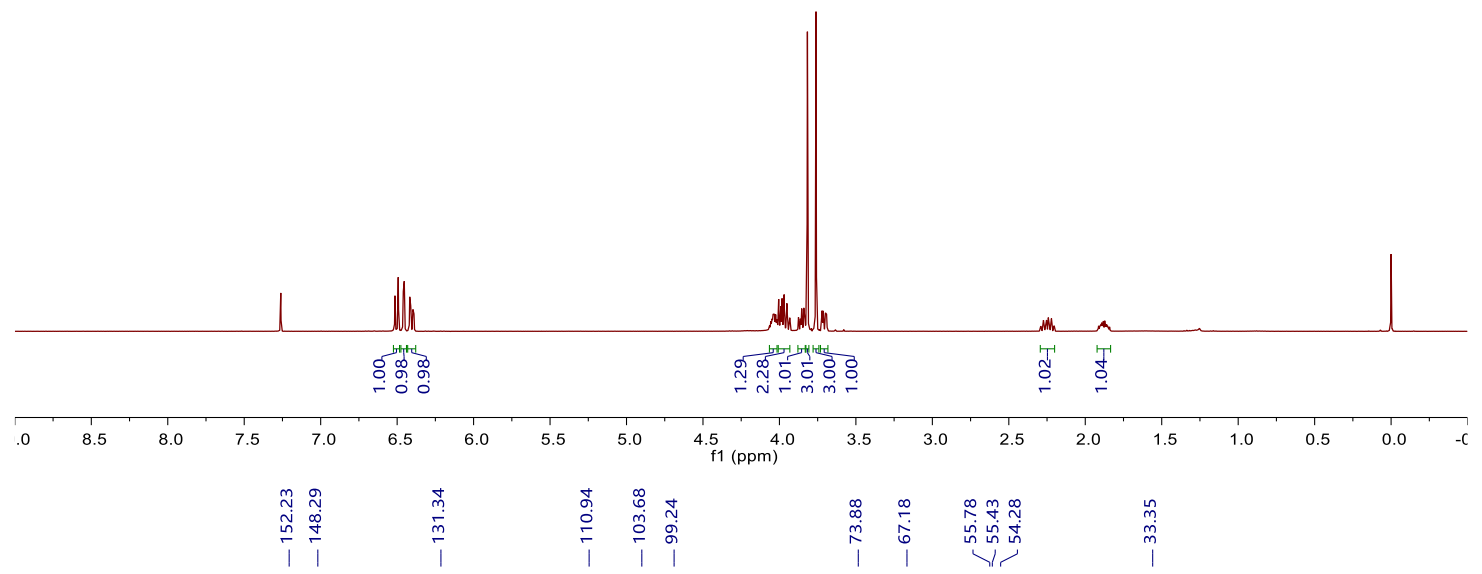<smiles>COc1ccc(NC2CCOC2)c(OC)c1</smiles>

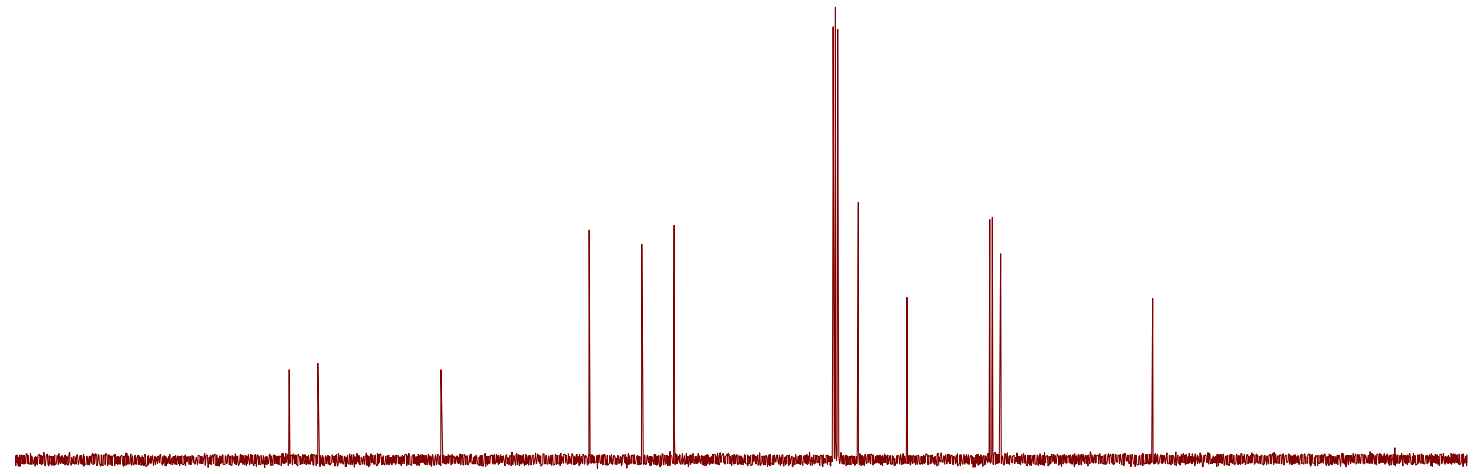

\begin{tabular}{|lllllllllllllllllllll}
\hline 90 & 180 & 170 & 160 & 150 & 140 & 130 & 120 & 110 & 100 & $\underset{\mathrm{f} 1}{90}(\mathrm{ppm})$ & 80 & 70 & 60 & 50 & 40 & 30 & 20 & 10 & 0 & -
\end{tabular} 
(F) HPLC Charts for Hydrogenation Products

\section{(S)-2-(Phenylamino)butane-1,4-diol (7a)}
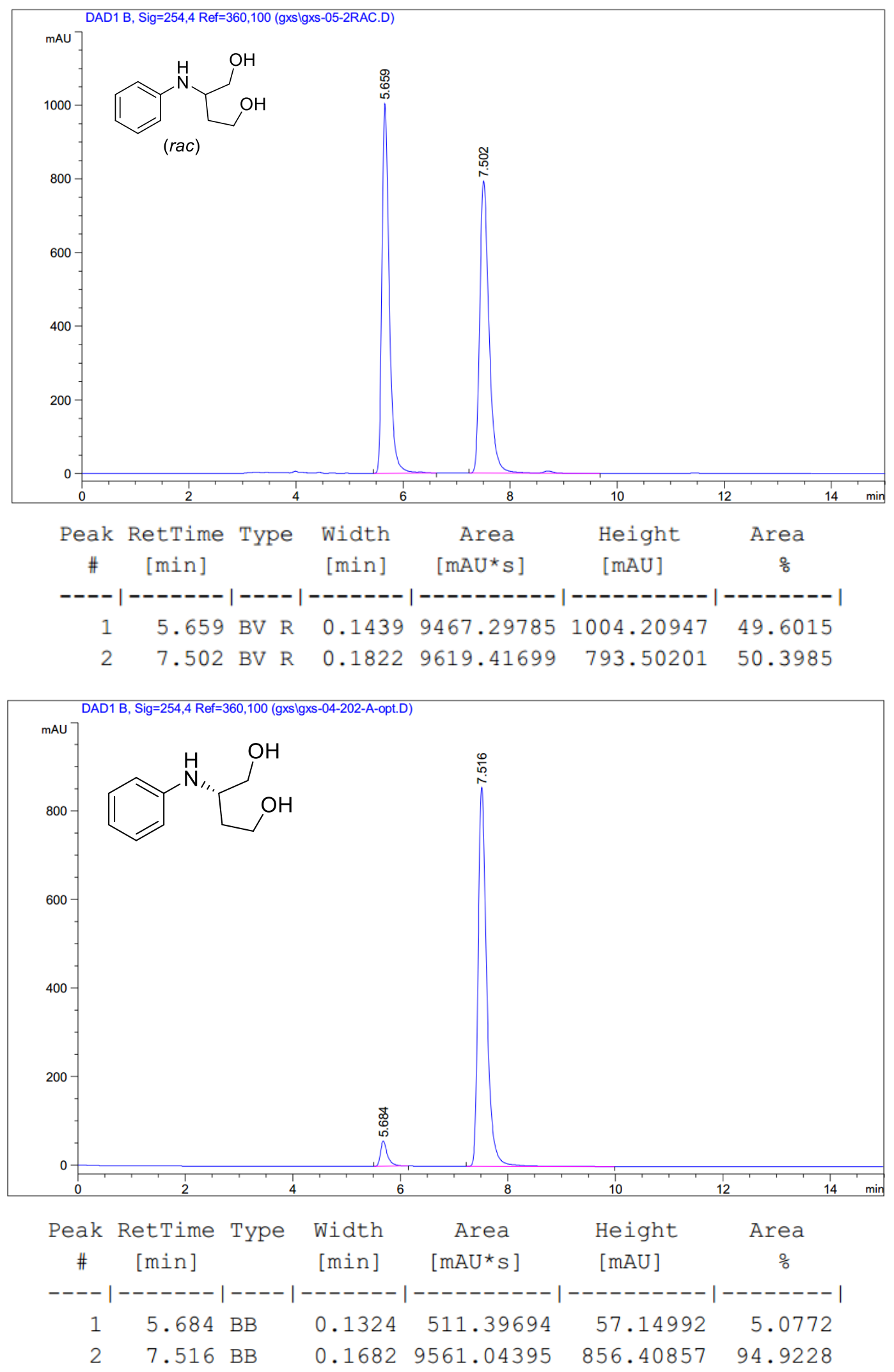
(S)-2-(p-Tolylamino)butane-1,4-diol (7b)
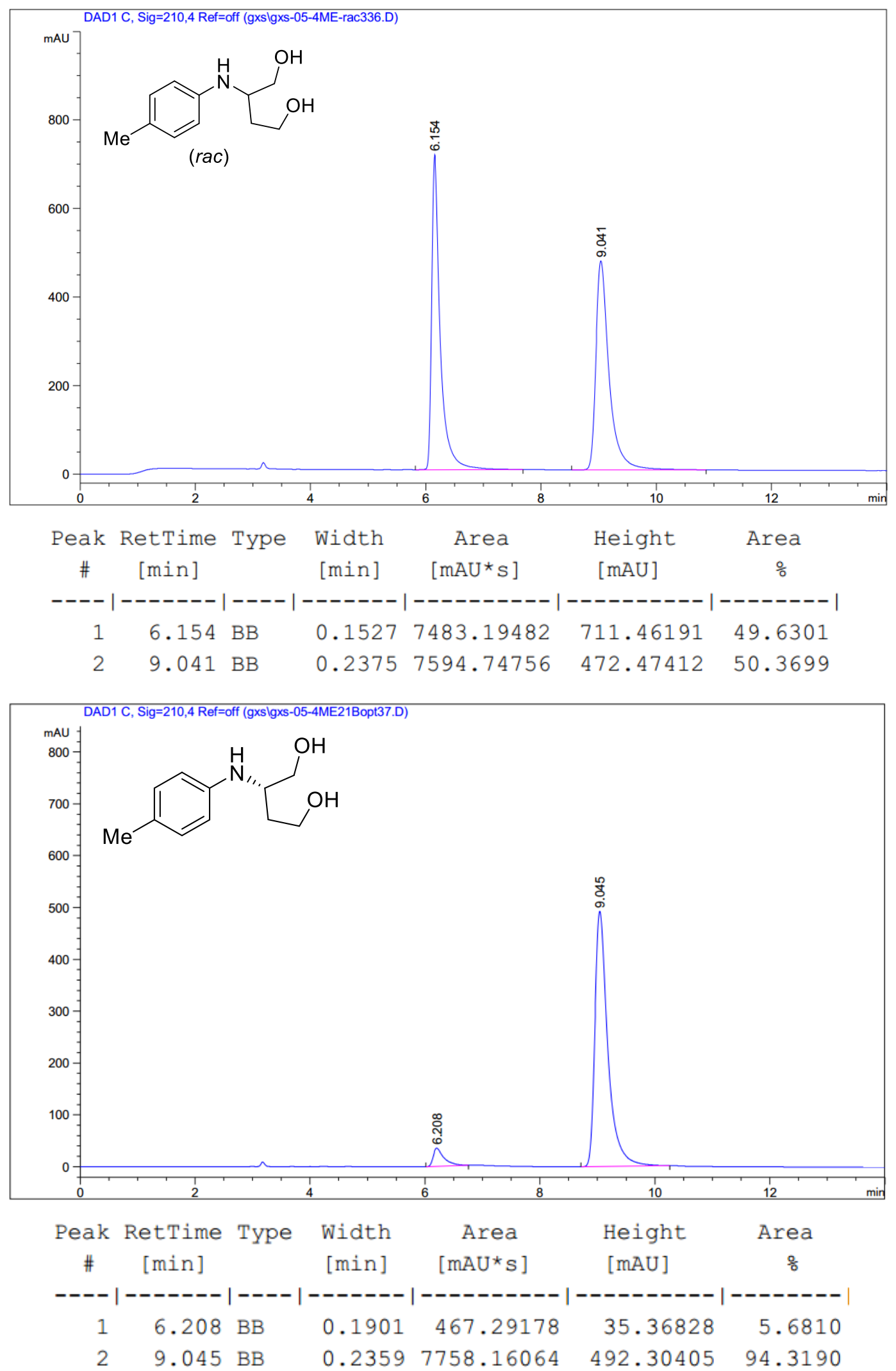


\section{(S)-2-((4-Methoxyphenyl)amino)butane-1,4-diol (7c)}
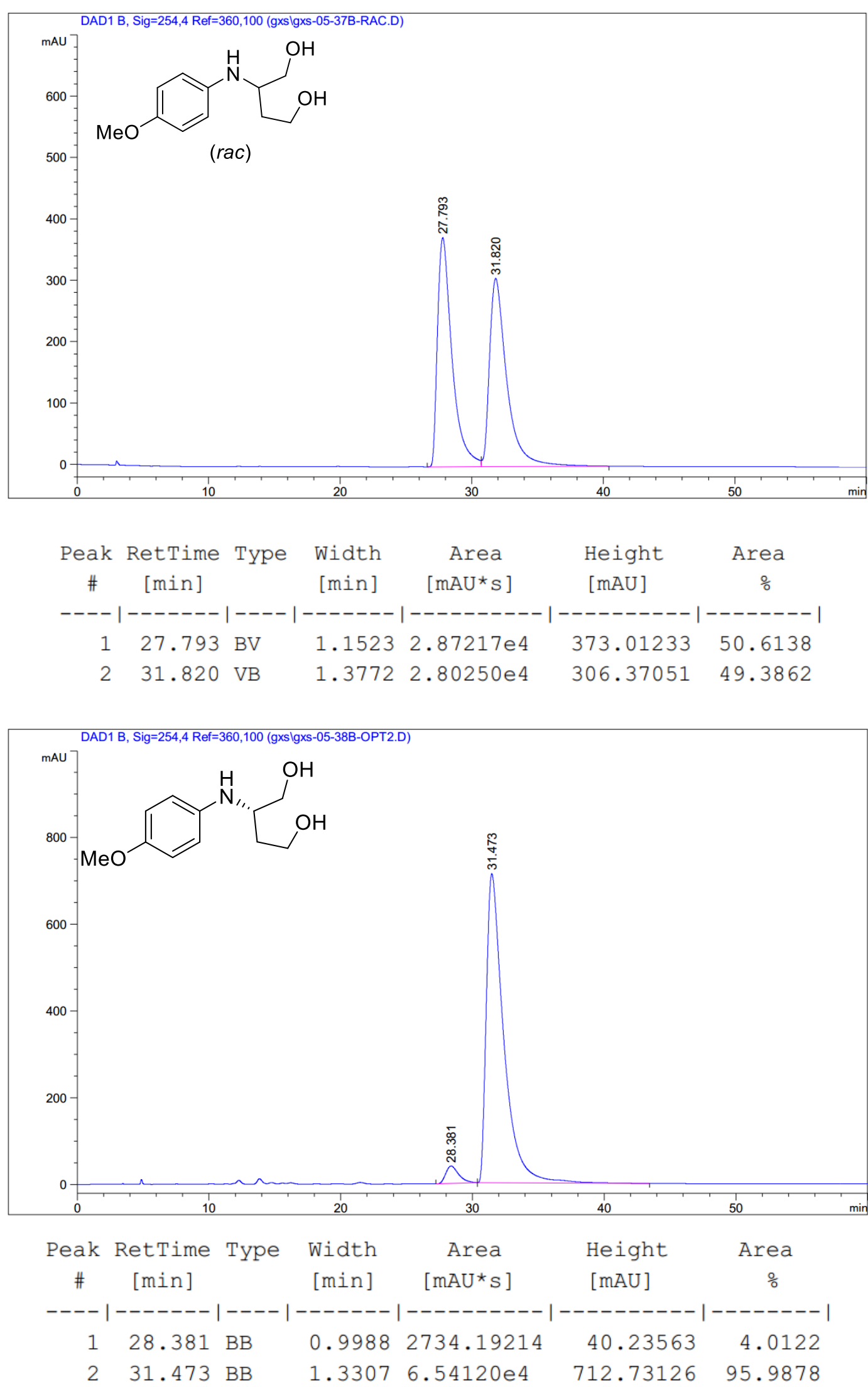
(S)-2-((4-Fluorophenyl)amino)butane-1,4-diol (7d)
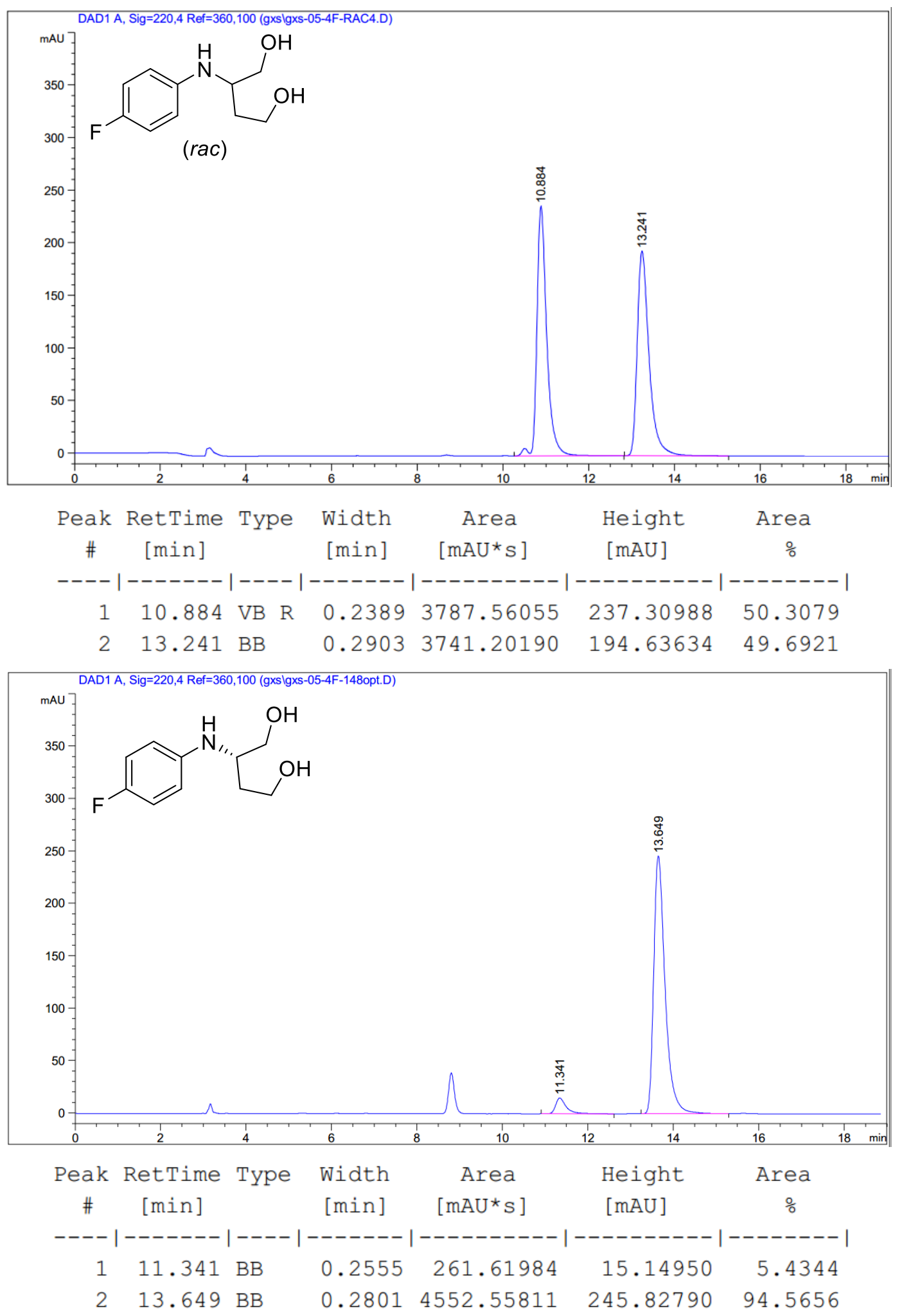


\section{(S)-2-((4-Chlorophenyl)amino)butane-1,4-diol (7e)}
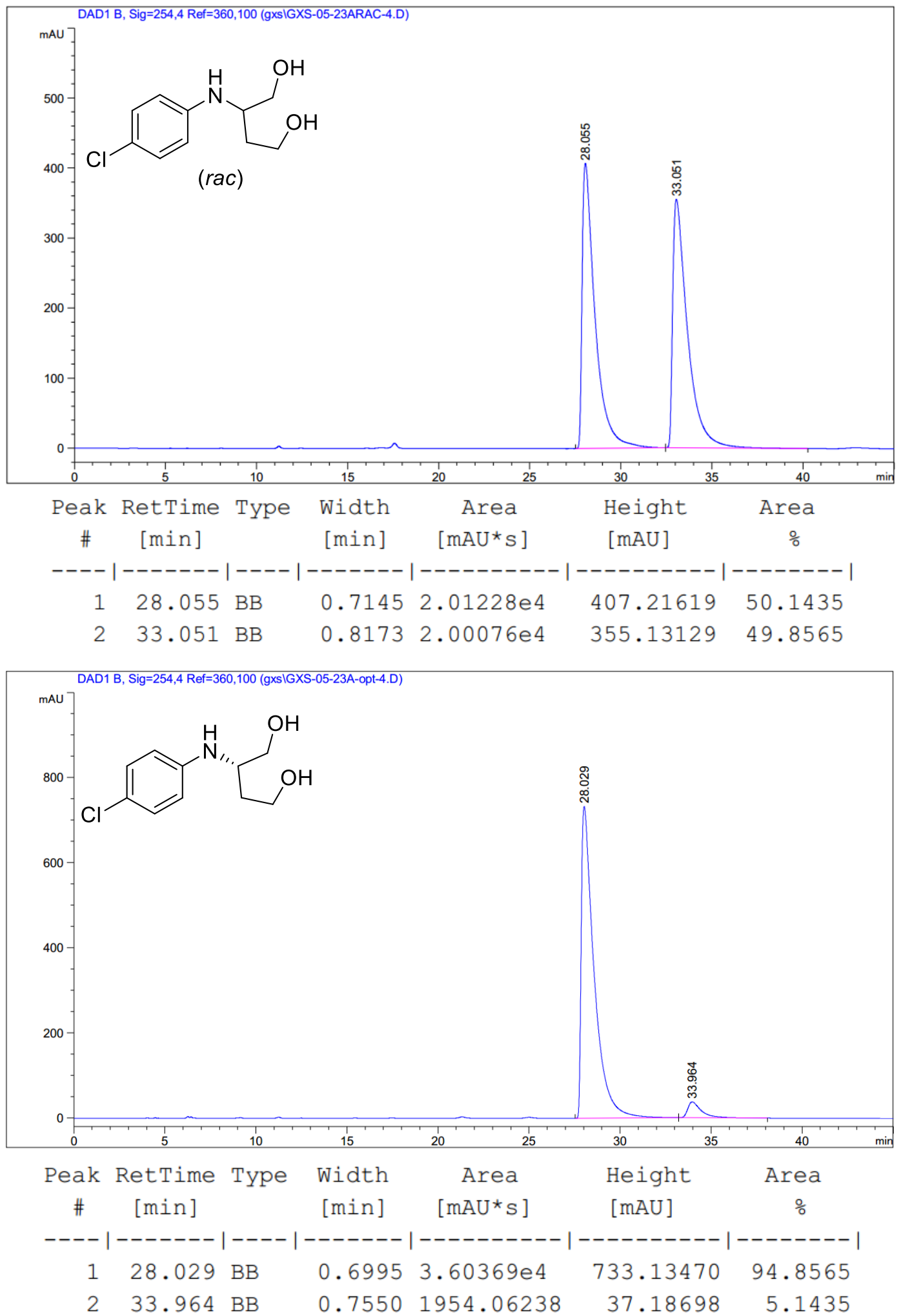
(S)-2-((4-Bromophenyl)amino)butane-1,4-diol (7f)
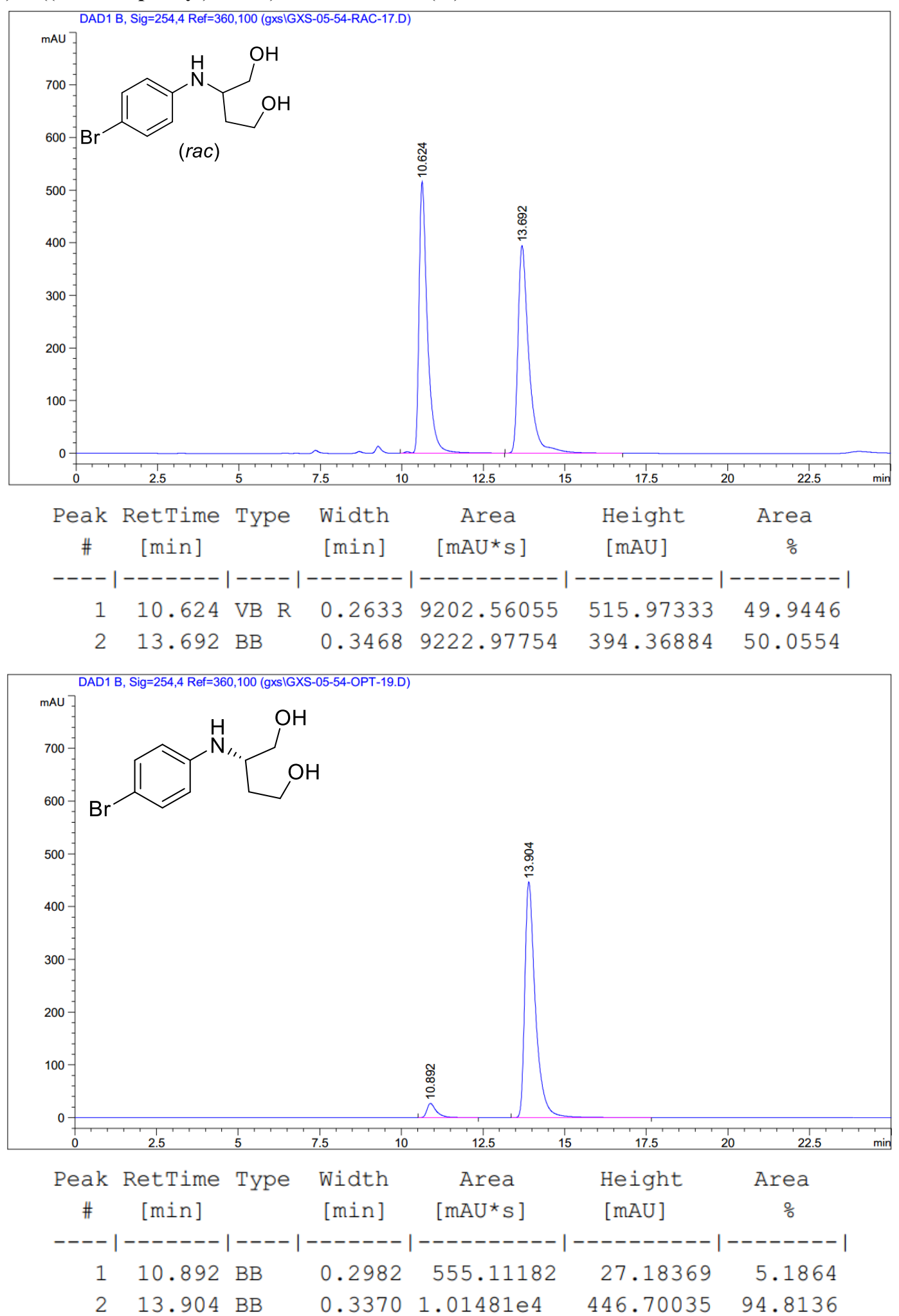


\section{(S)-2-(m-Tolylamino)butane-1,4-diol (7g)}
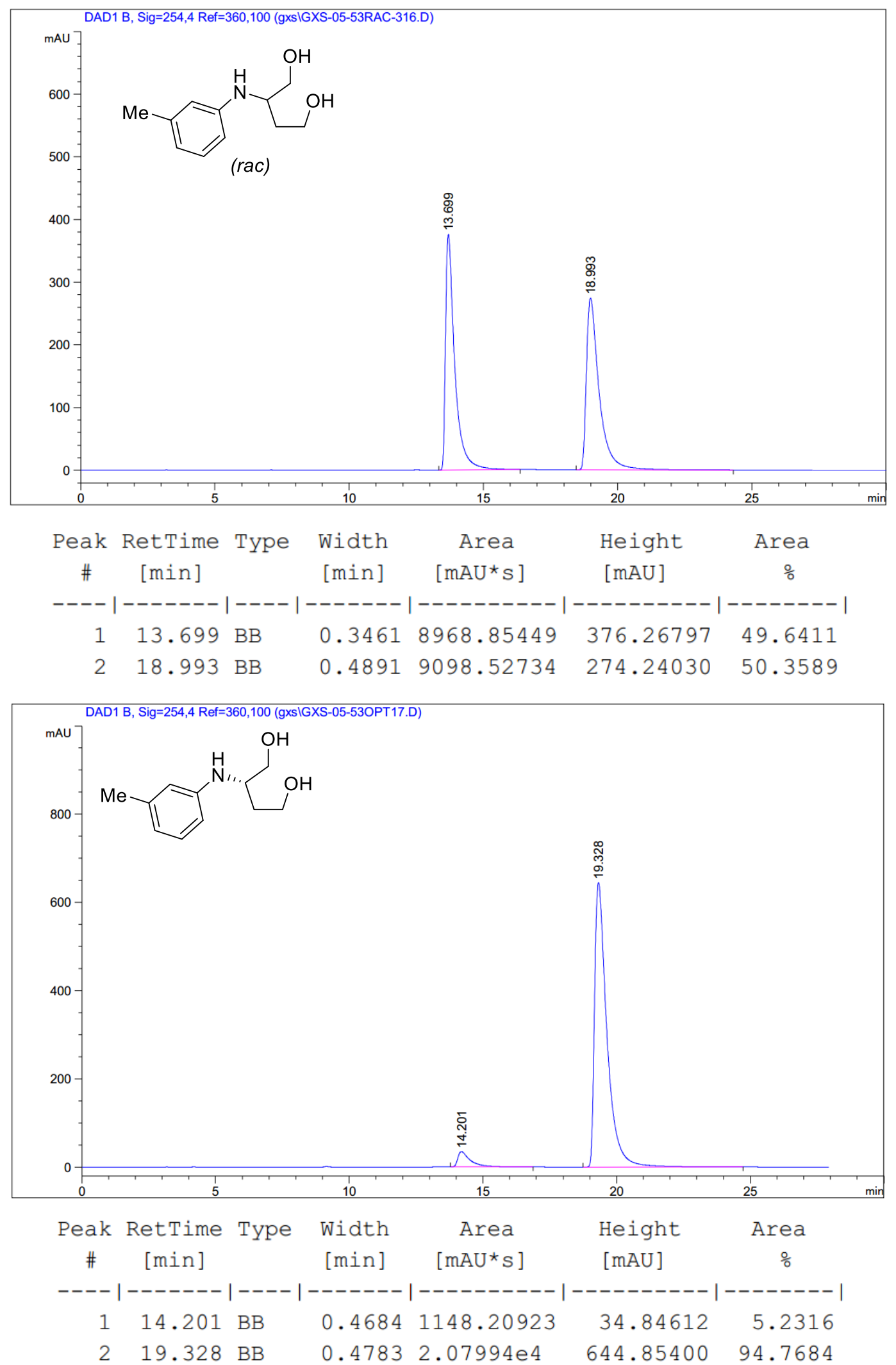


\section{(S)-2-((3-Methoxyphenyl)amino)butane-1,4-diol (7h)}
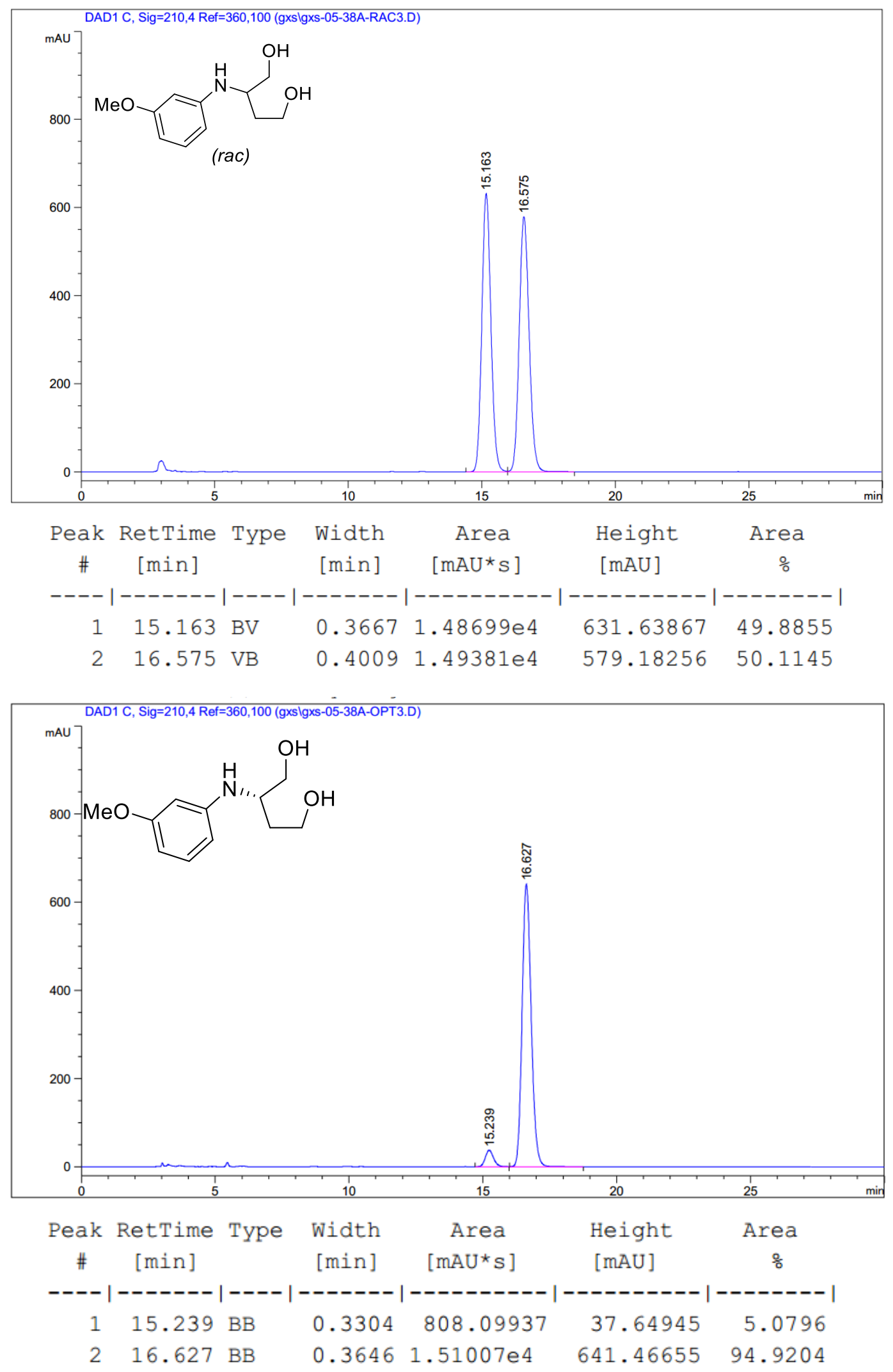


\section{(S)-2-((3-Fluorophenyl)amino)butane-1,4-diol (7i)}
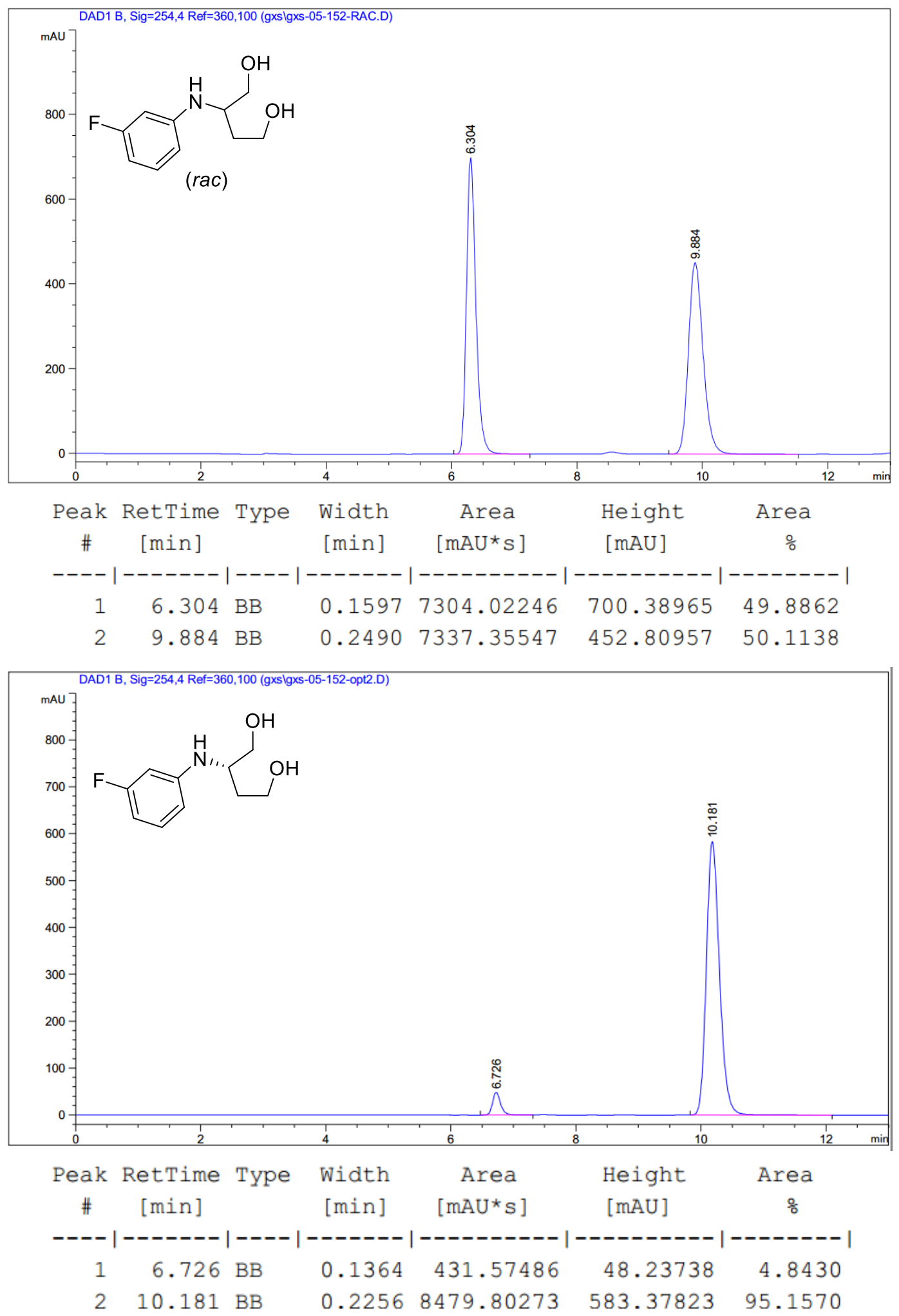
(S)-2-((3-Chlorophenyl)amino)butane-1,4-diol (7j)
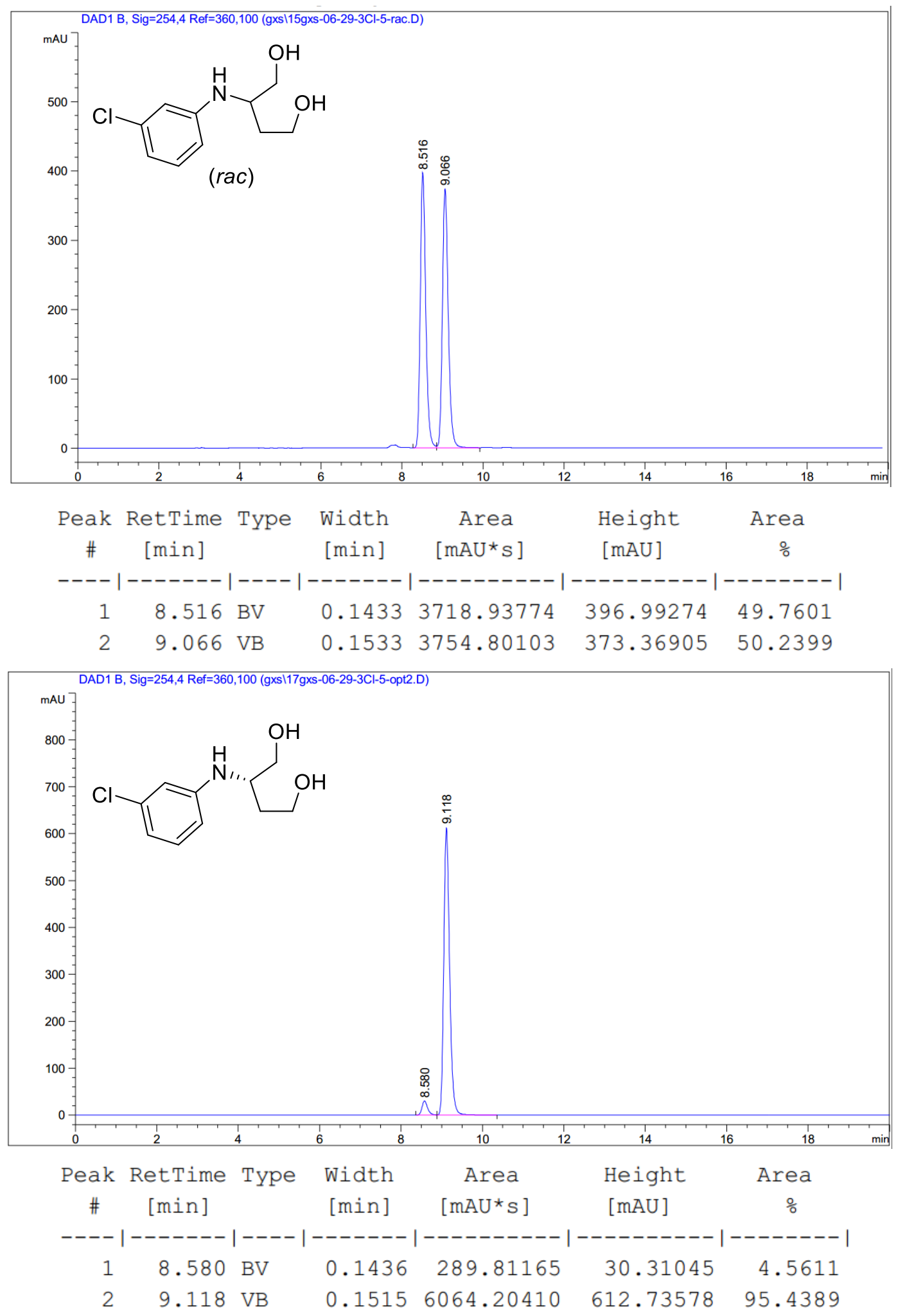


\section{(S)-2-(o-Tolylamino)butane-1,4-diol (7k)}
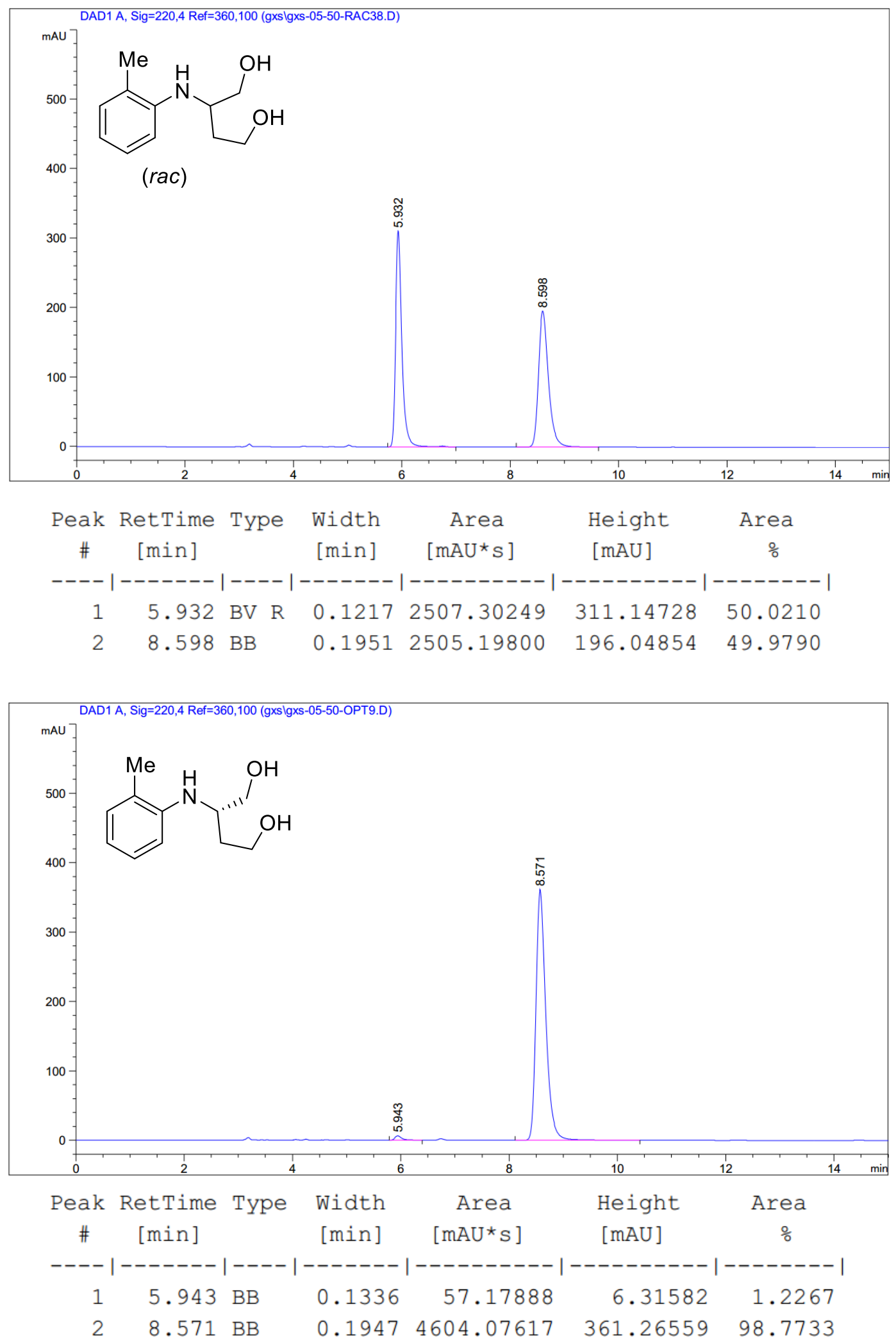
(S)-2-((2-Methoxyphenyl)amino)butane-1,4-diol (7l)
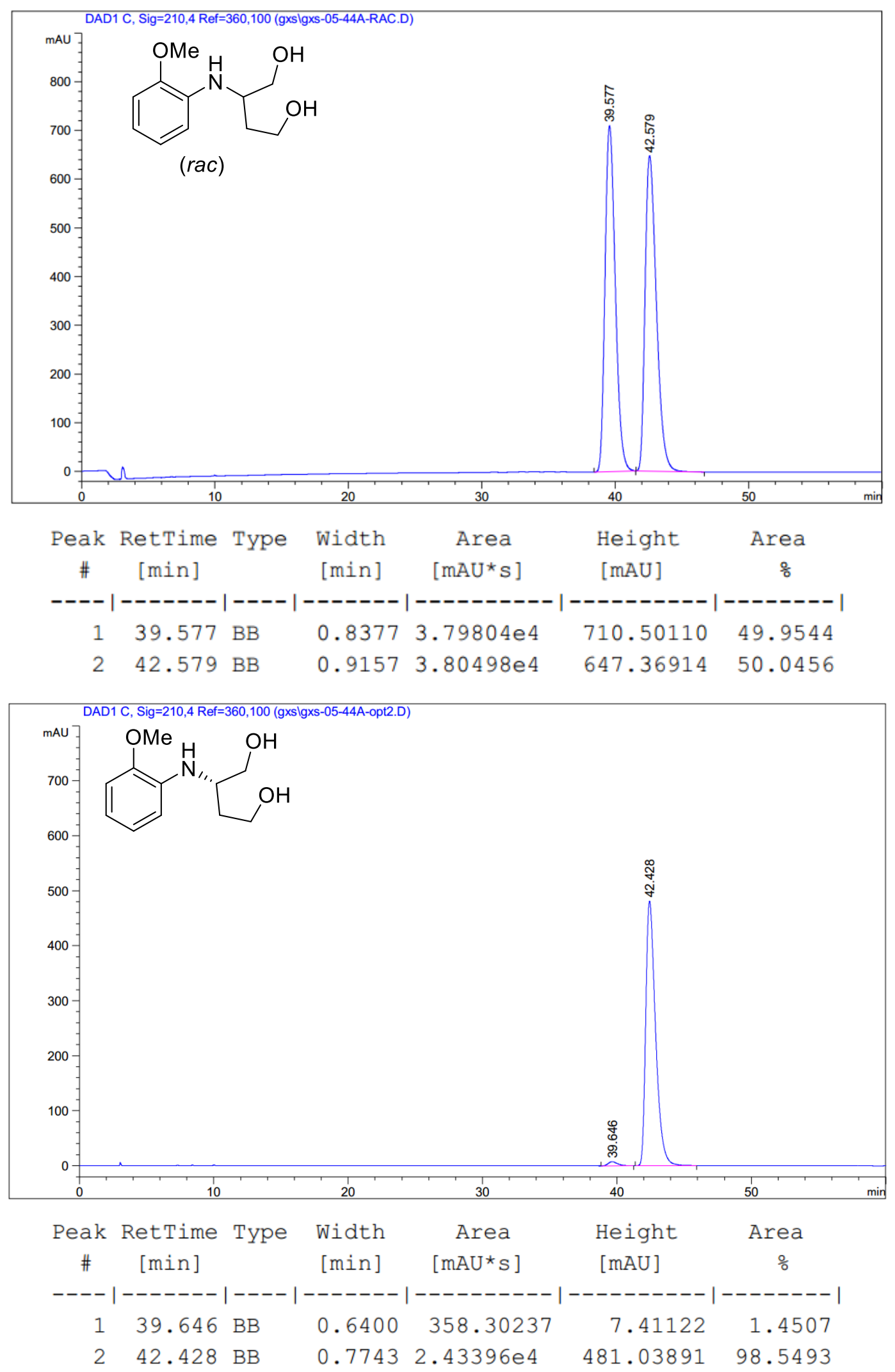


\section{(S)-2-(2,4-Dimethoxyphenylamino)butane-1,4-diol (7m)}
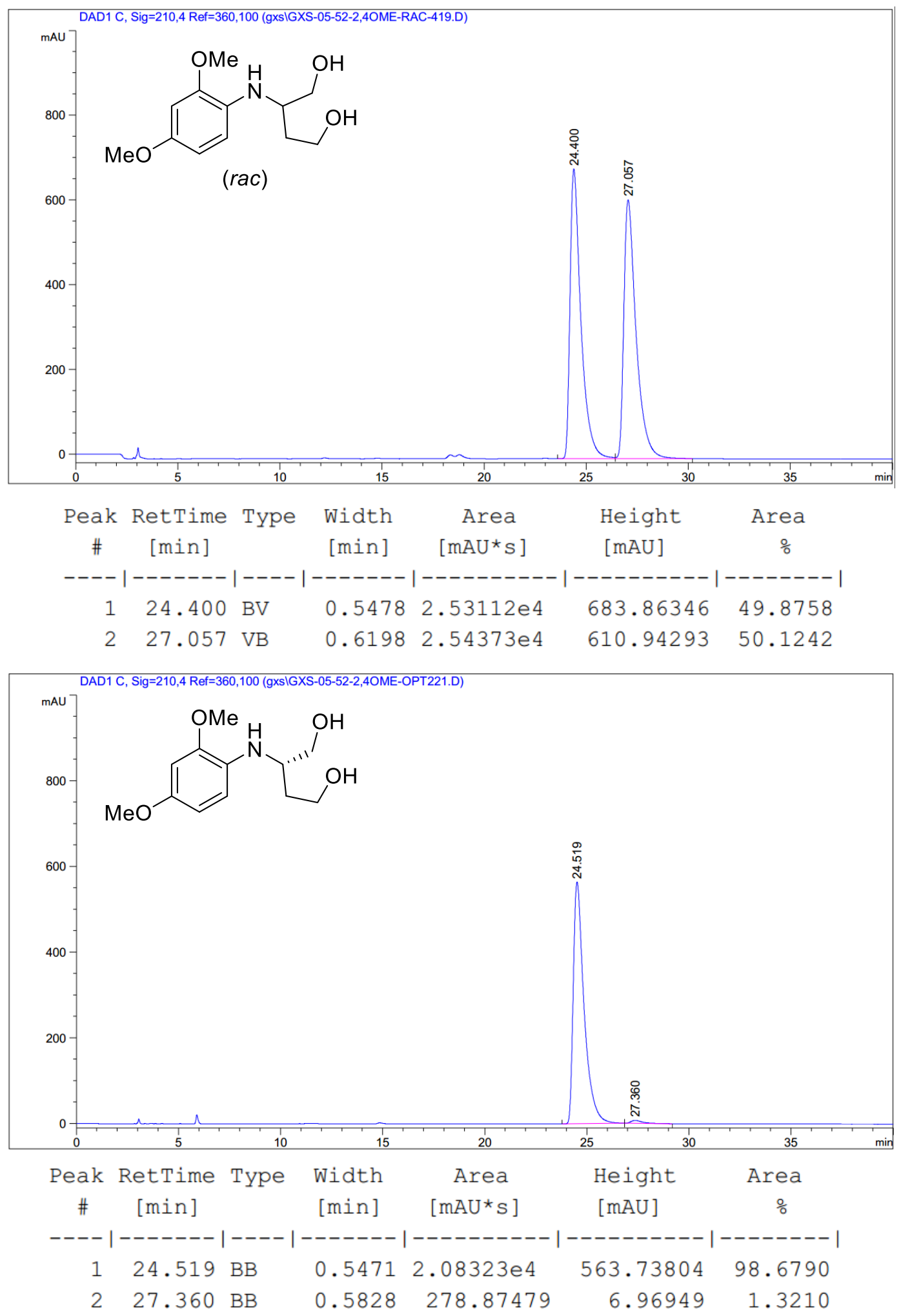


\section{(S)-2-(Naphthalen-1-ylamino)butane-1,4-diol (7n)}
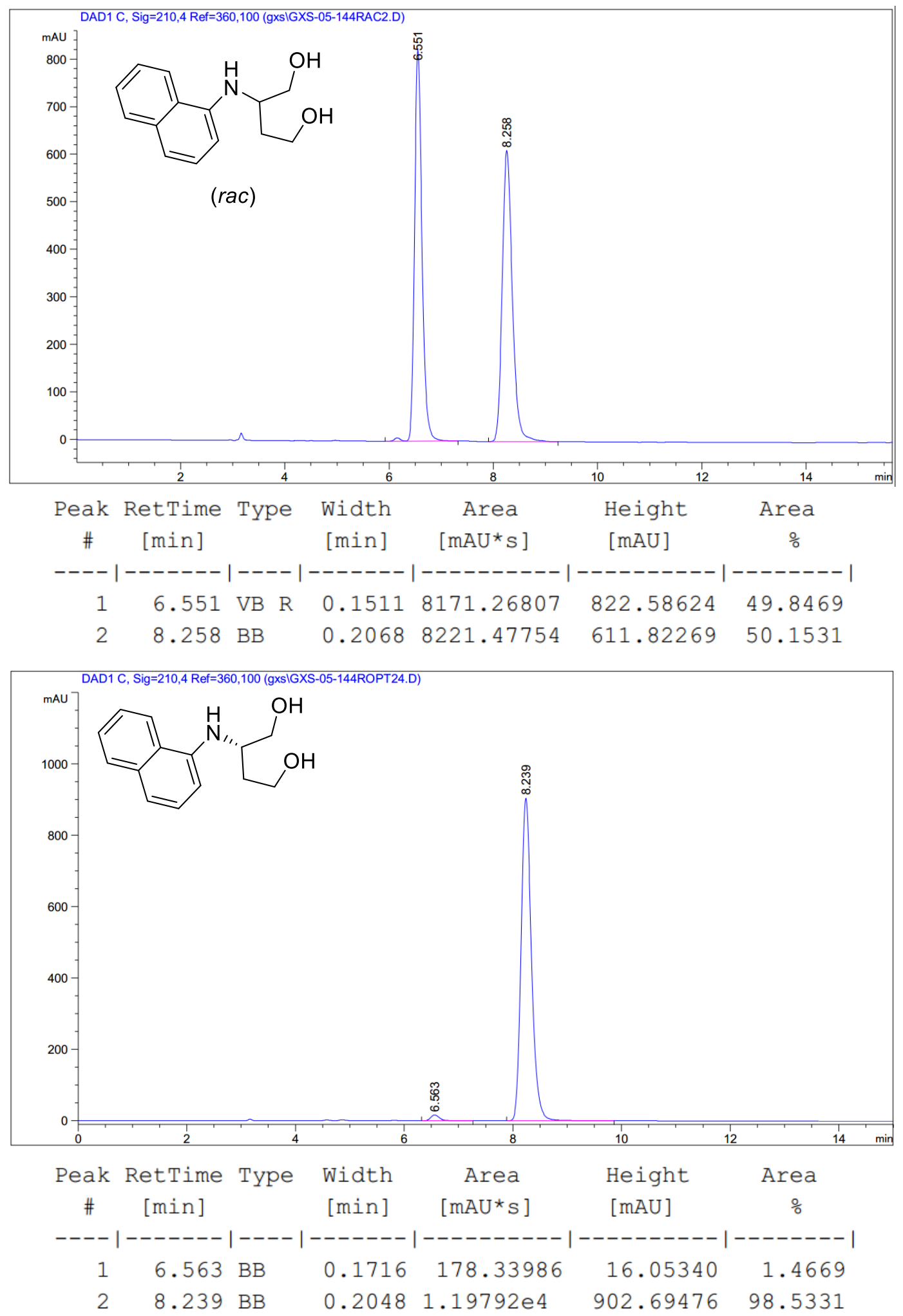


\section{(S)-2-(Benzo[d][1,3]dioxol-5-ylamino)butane-1,4-diol (7o)}
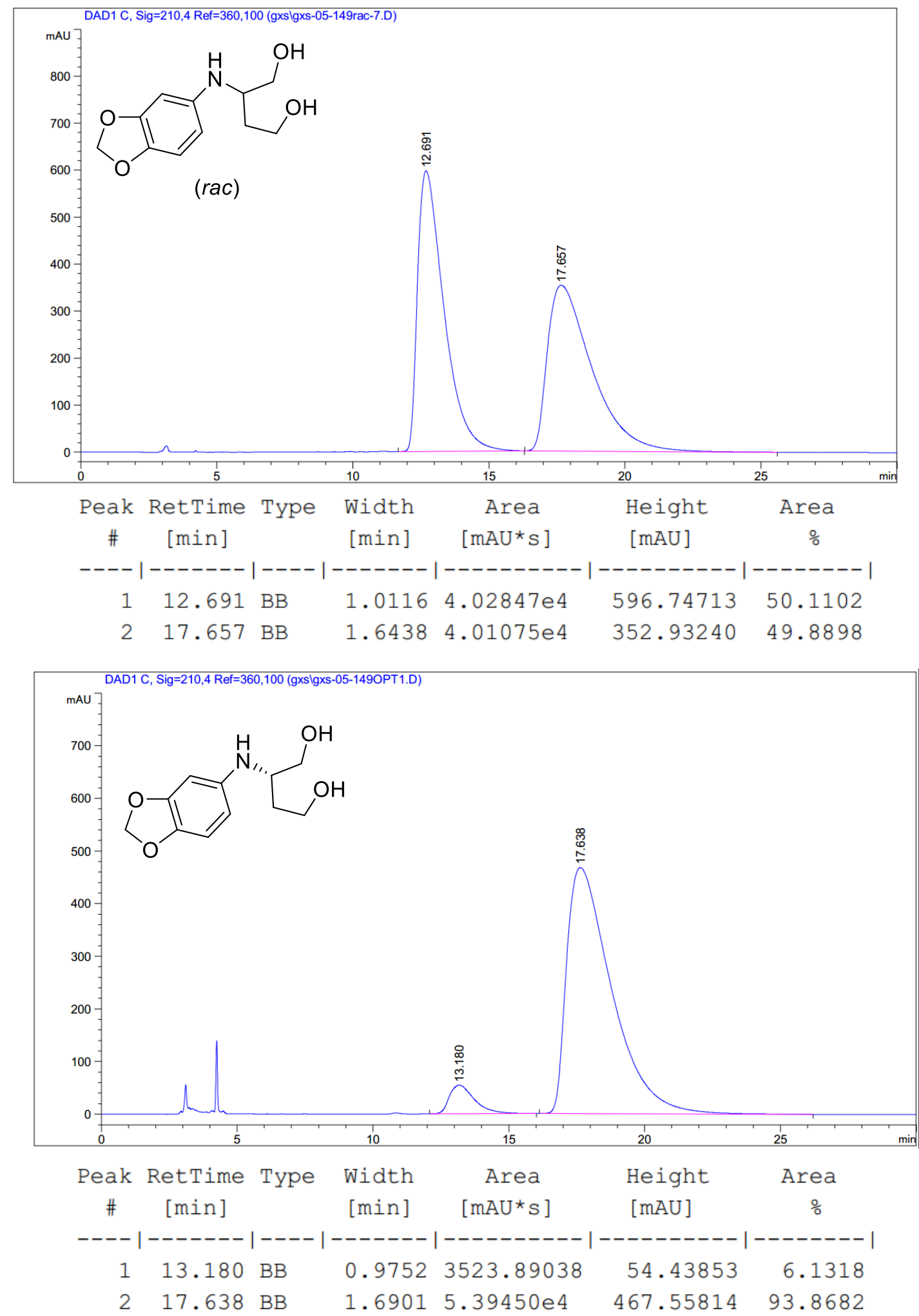
(S)-2-(6-Methylpyridin-2-ylamino)butane-1,4-diol (7p)
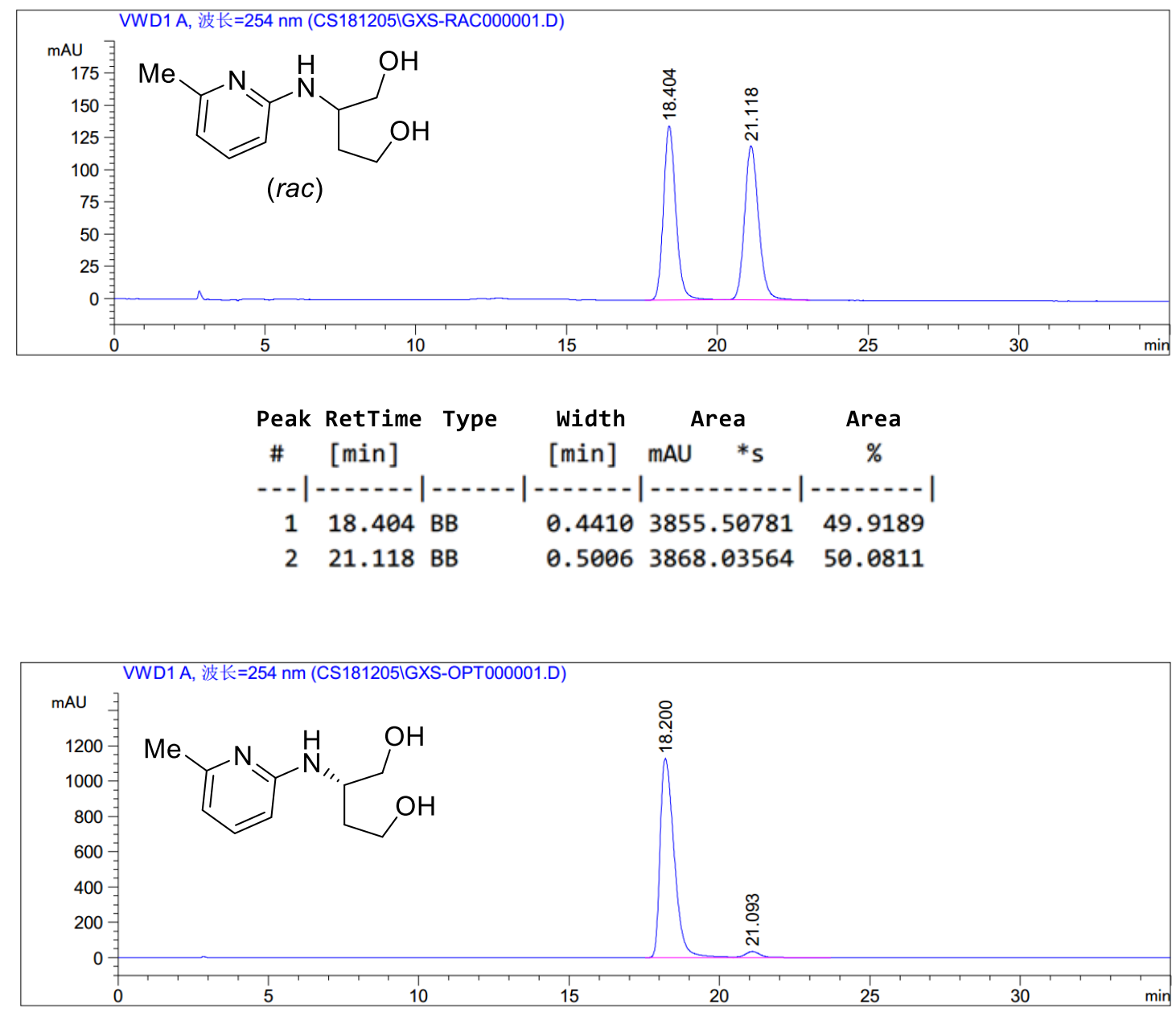

\begin{tabular}{|c|c|c|c|c|c|c|}
\hline $\begin{array}{c}\text { Peak } \\
\#\end{array}$ & $\begin{array}{c}\text { RetTime } \\
\text { [min] }\end{array}$ & Type & $\begin{array}{l}\text { Width } \\
\text { [min] }\end{array}$ & mAU & $\begin{array}{r}\text { Area } \\
* s\end{array}$ & $\begin{array}{c}\text { Area } \\
\%\end{array}$ \\
\hline & & & & & & \\
\hline & 200 & & 0.5057 & 3.67 & & $\begin{array}{r}96.6837 \\
2.2162\end{array}$ \\
\hline
\end{tabular}


(S)-1-(Hydroxymethyl)-3-hydroxypropyl) carbamic acid tert-butyl ester (7q)
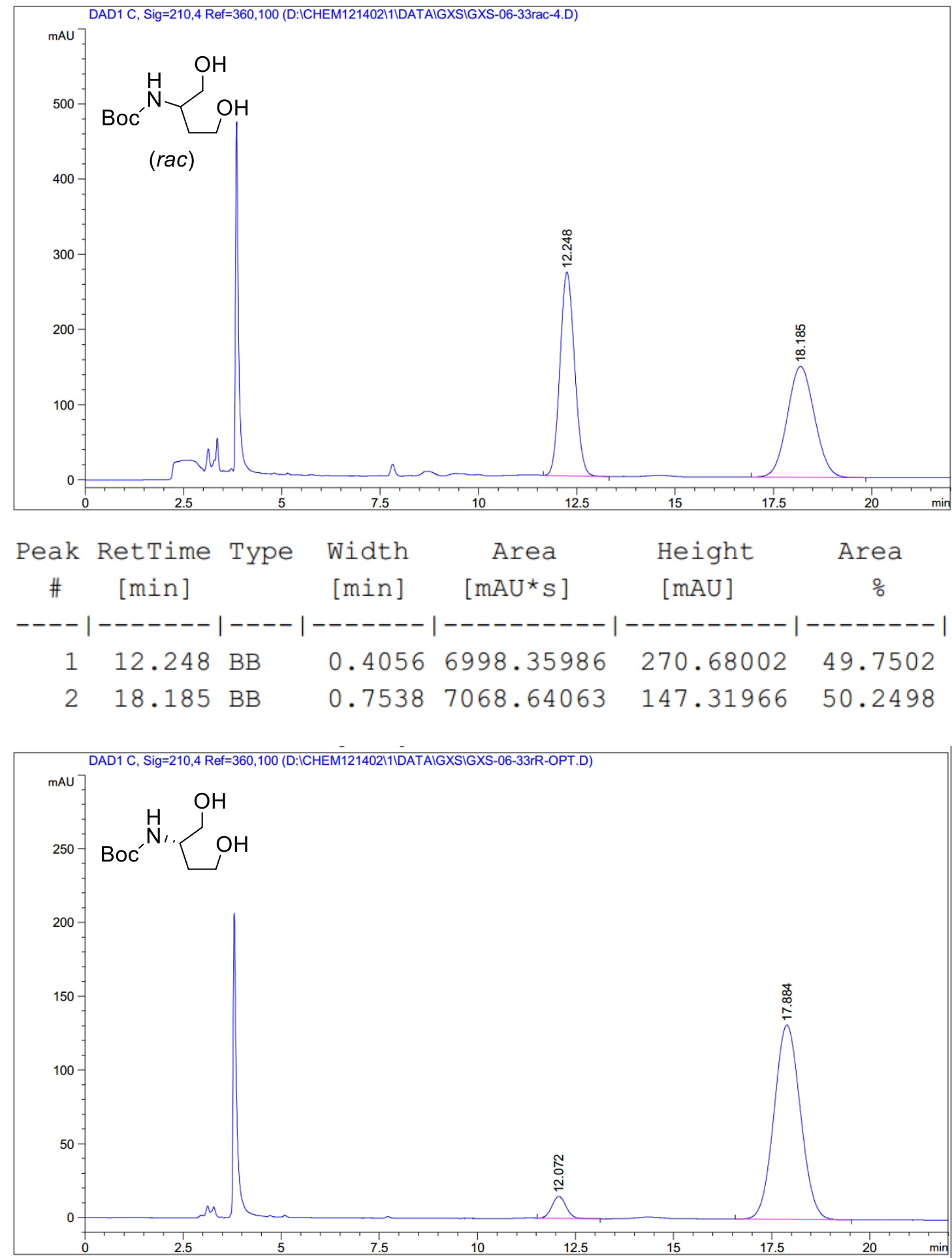

\begin{tabular}{|c|c|c|c|c|c|c|}
\hline $\begin{array}{c}\text { Peak } \\
\text { \# }\end{array}$ & $\begin{array}{c}\text { RetTime } \\
\text { [min] }\end{array}$ & Type & $\begin{array}{l}\text { Width } \\
\text { [min] }\end{array}$ & $\begin{array}{c}\text { Area } \\
{\left[\mathrm{mAU}{ }^{\star} \mathrm{S}\right]}\end{array}$ & $\begin{array}{l}\text { Height } \\
{[\mathrm{mAU}]}\end{array}$ & $\begin{array}{c}\text { Area } \\
\%\end{array}$ \\
\hline & & & & 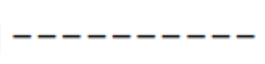 & ---------- & $---\mid$ \\
\hline 1 & & & & 374.43048 & .86681 & 340 \\
\hline 2 & 884 & $\mathrm{~B}$ & 247 & 6155.53369 & .83261 & 2660 \\
\hline
\end{tabular}




\section{$N$-(3-Hydroxy-1-hydroxymethyl-propyl)-4-methyl-benzenesulfonamide (7r)}
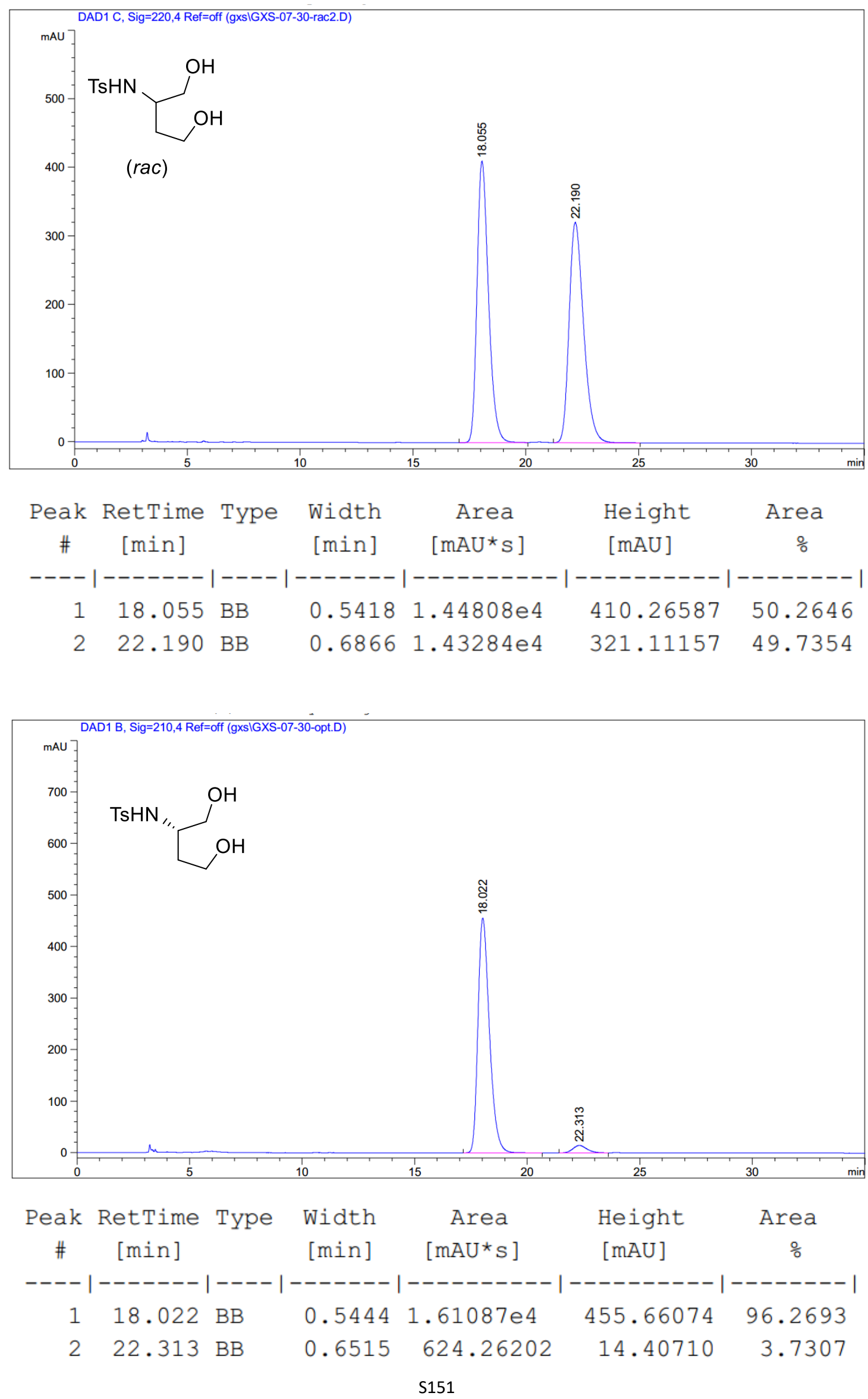


\section{(S)-2-(2-Phenylamino)pentane-1,5-diol (9a)}
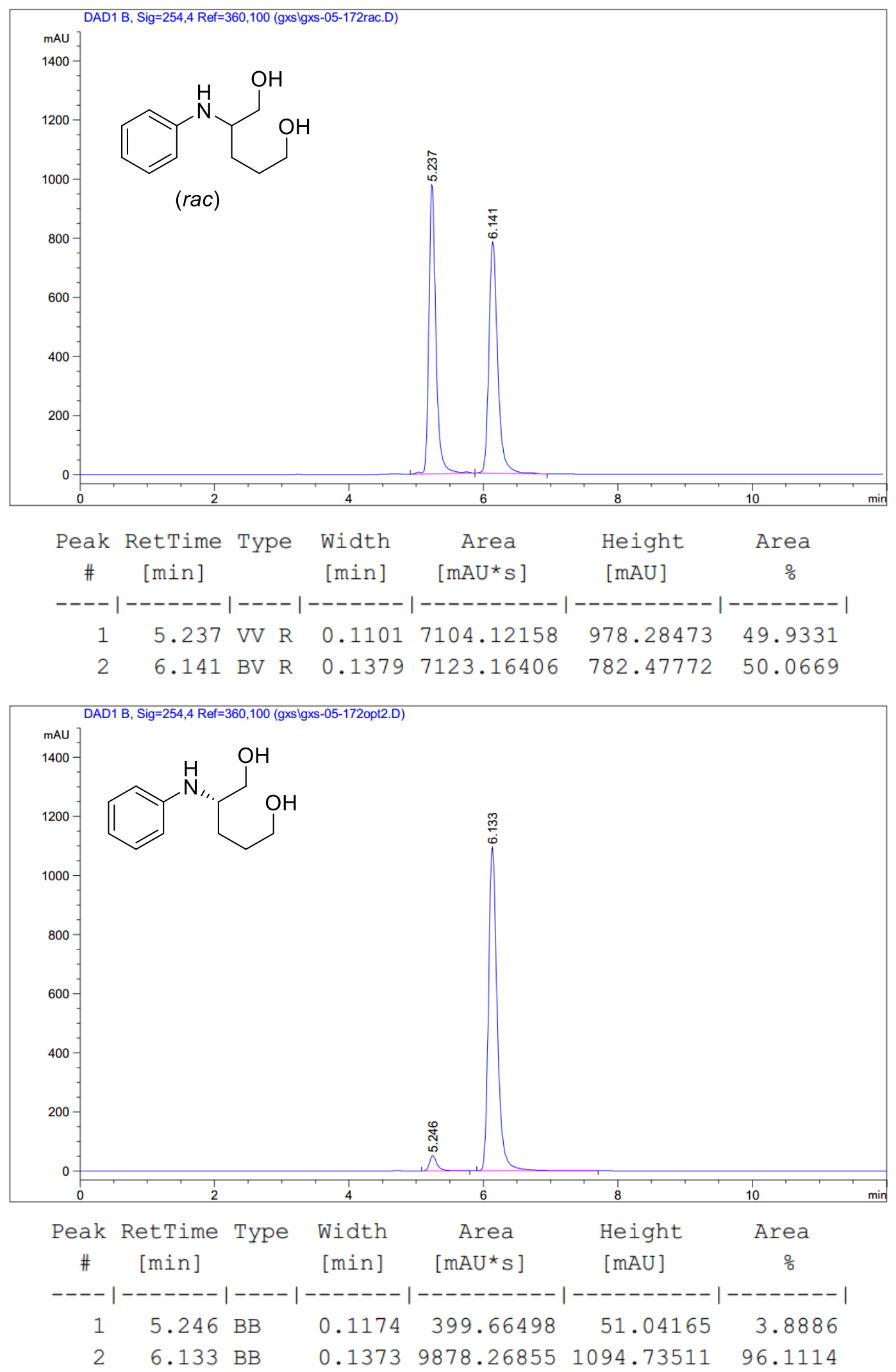


\section{(S)-2-(p-Tolylamino)pentane-1,5-diol (9b)}
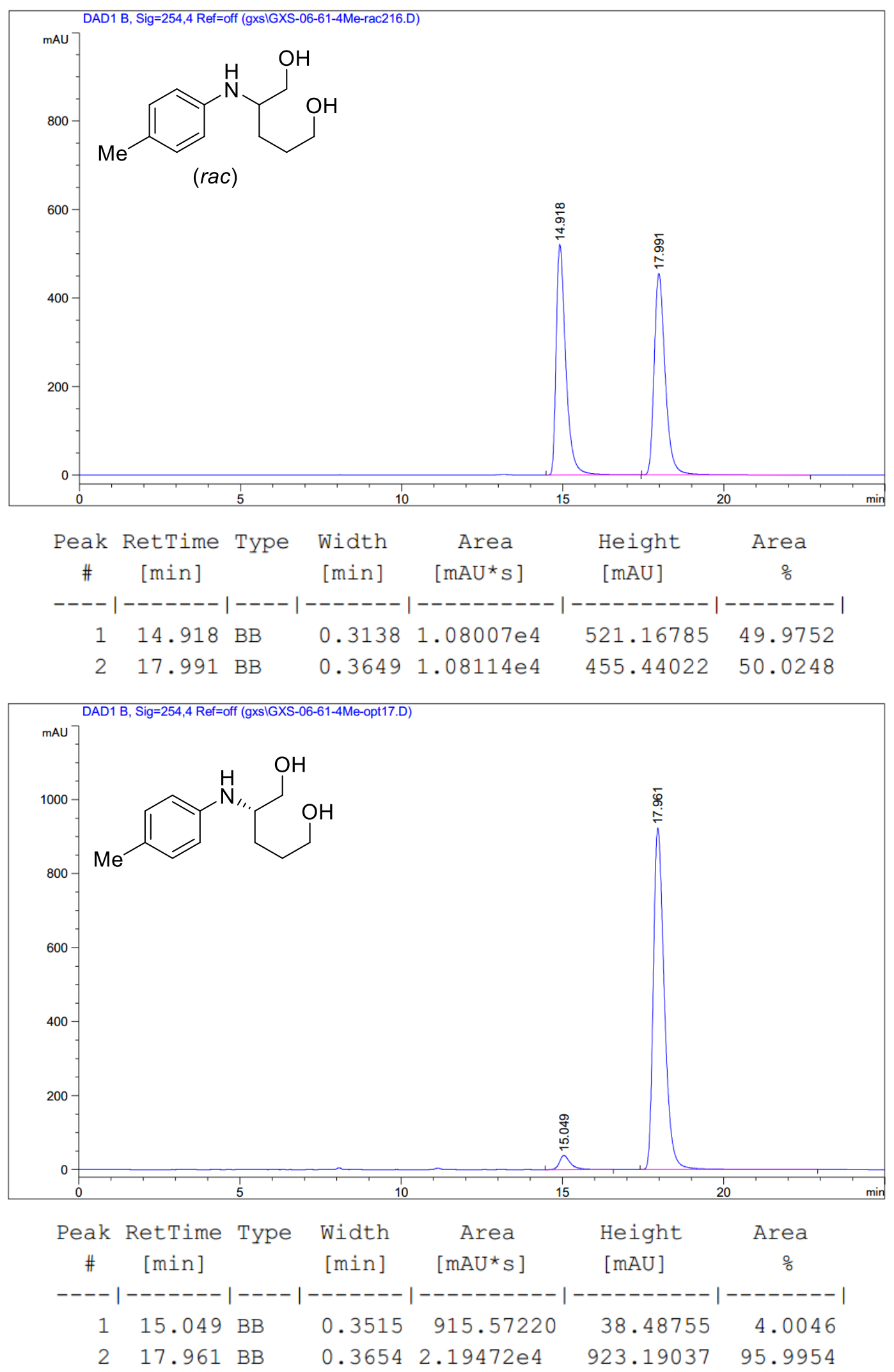
(S)-2-((4-Methoxyphenyl)amino)pentane-1,5-diol (9c)
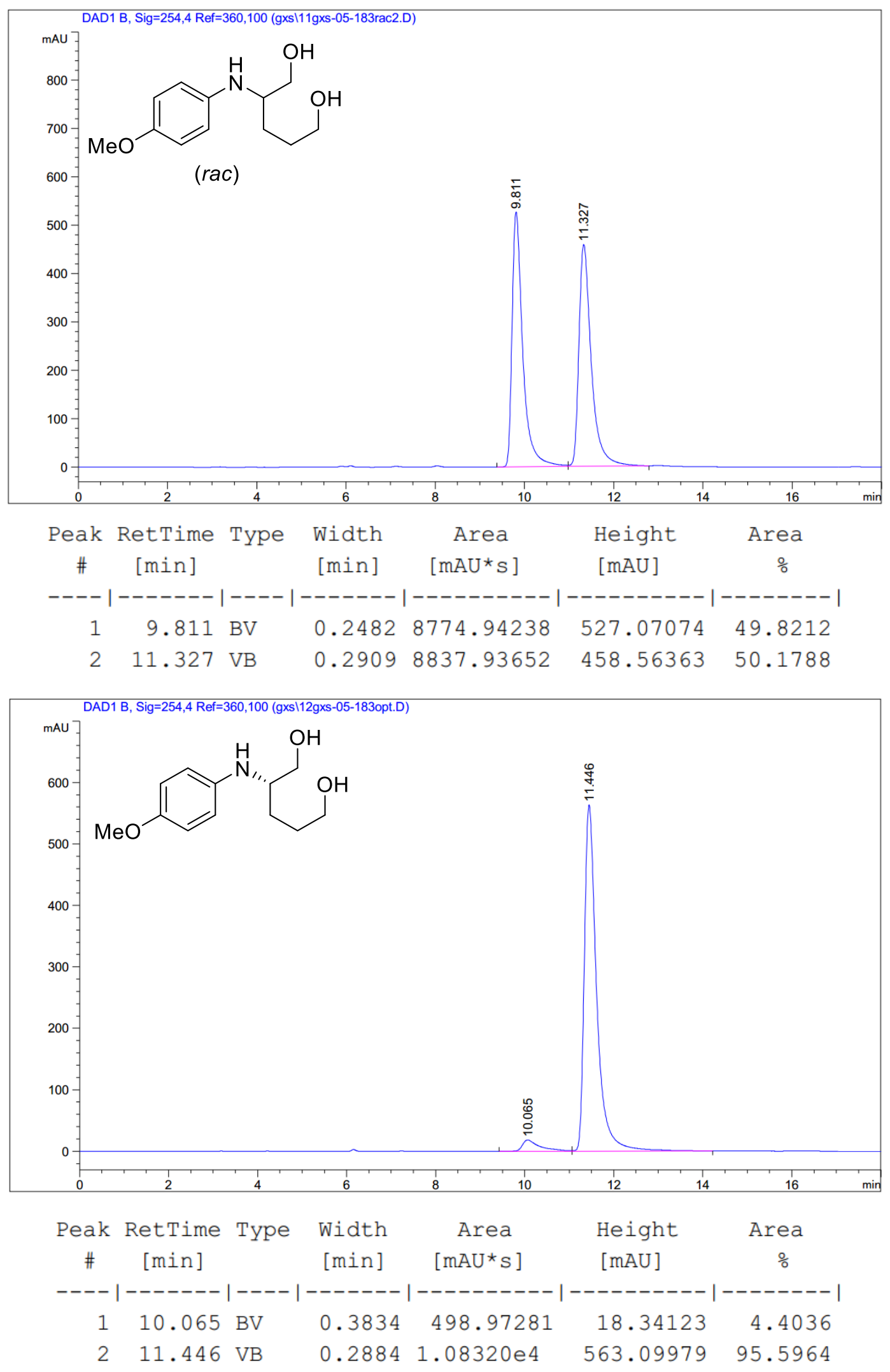


\section{(S)-2-((4-Fluorophenyl)amino)pentane-1,5-diol (9d)}
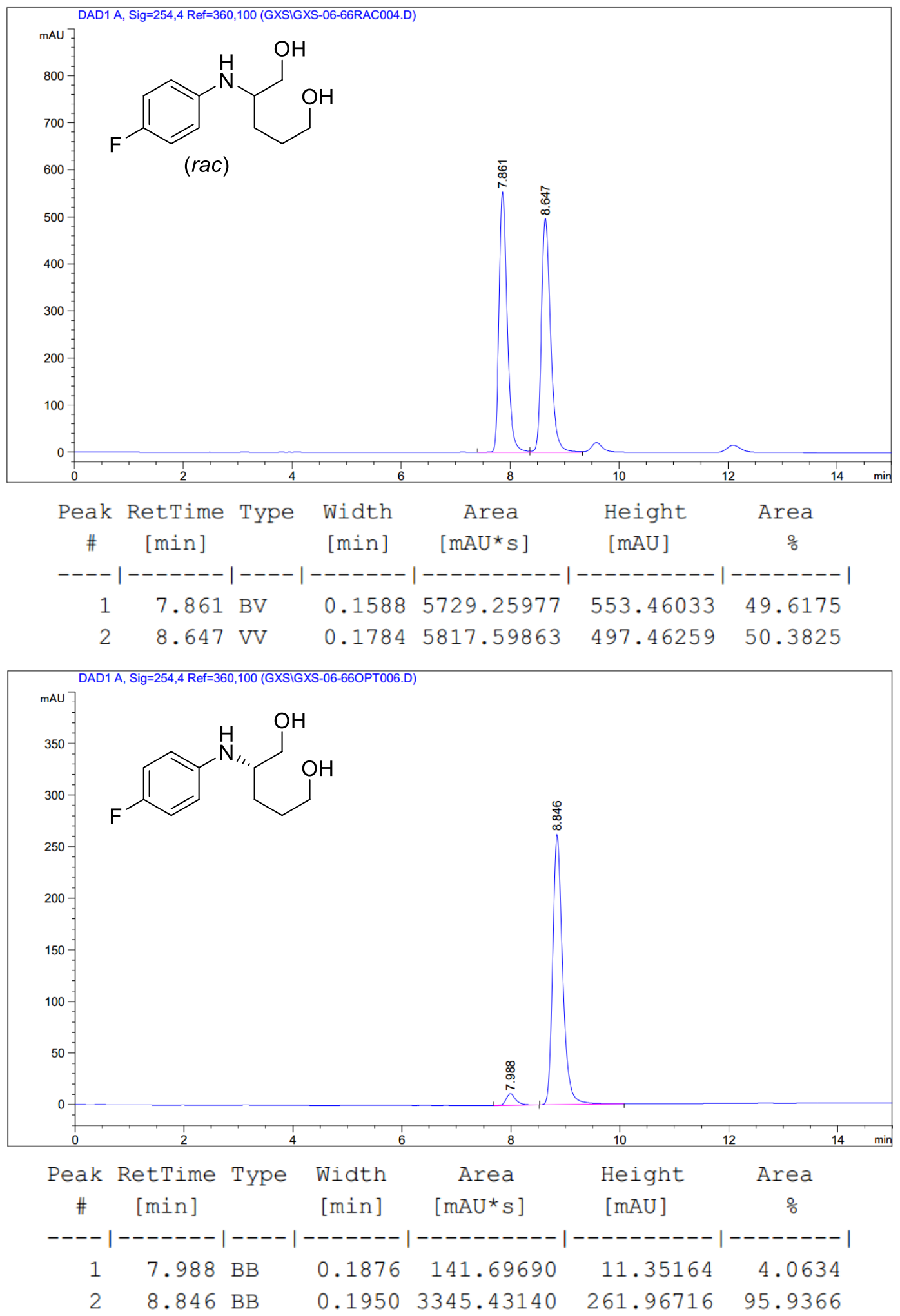


\section{(S)-2-((4-chlorophenyl)amino)pentane-1,5-diol (9e)}
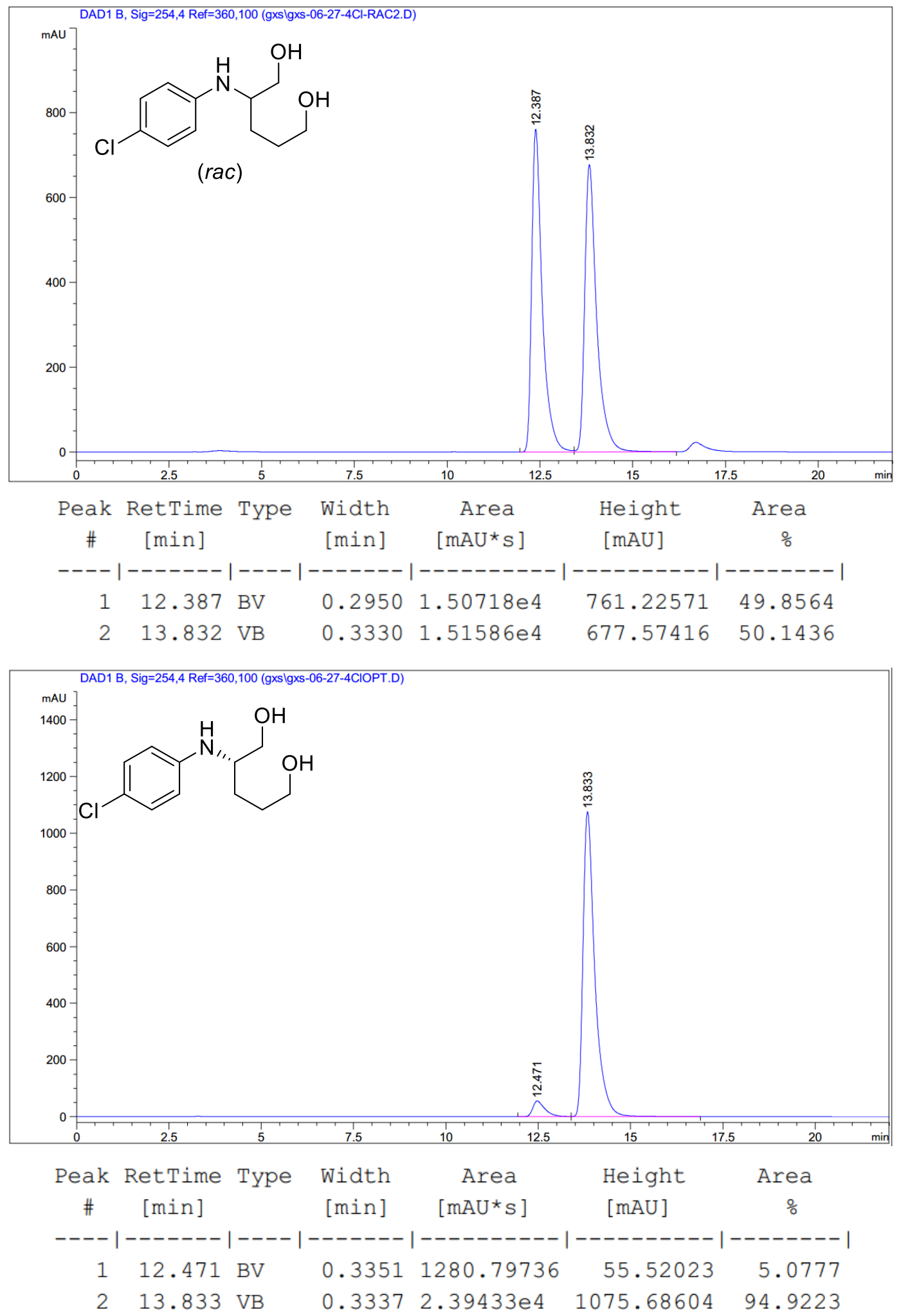
(S)-2-([1,1'-Biphenyl]-4-ylamino)pentane-1,5-diol (9f)
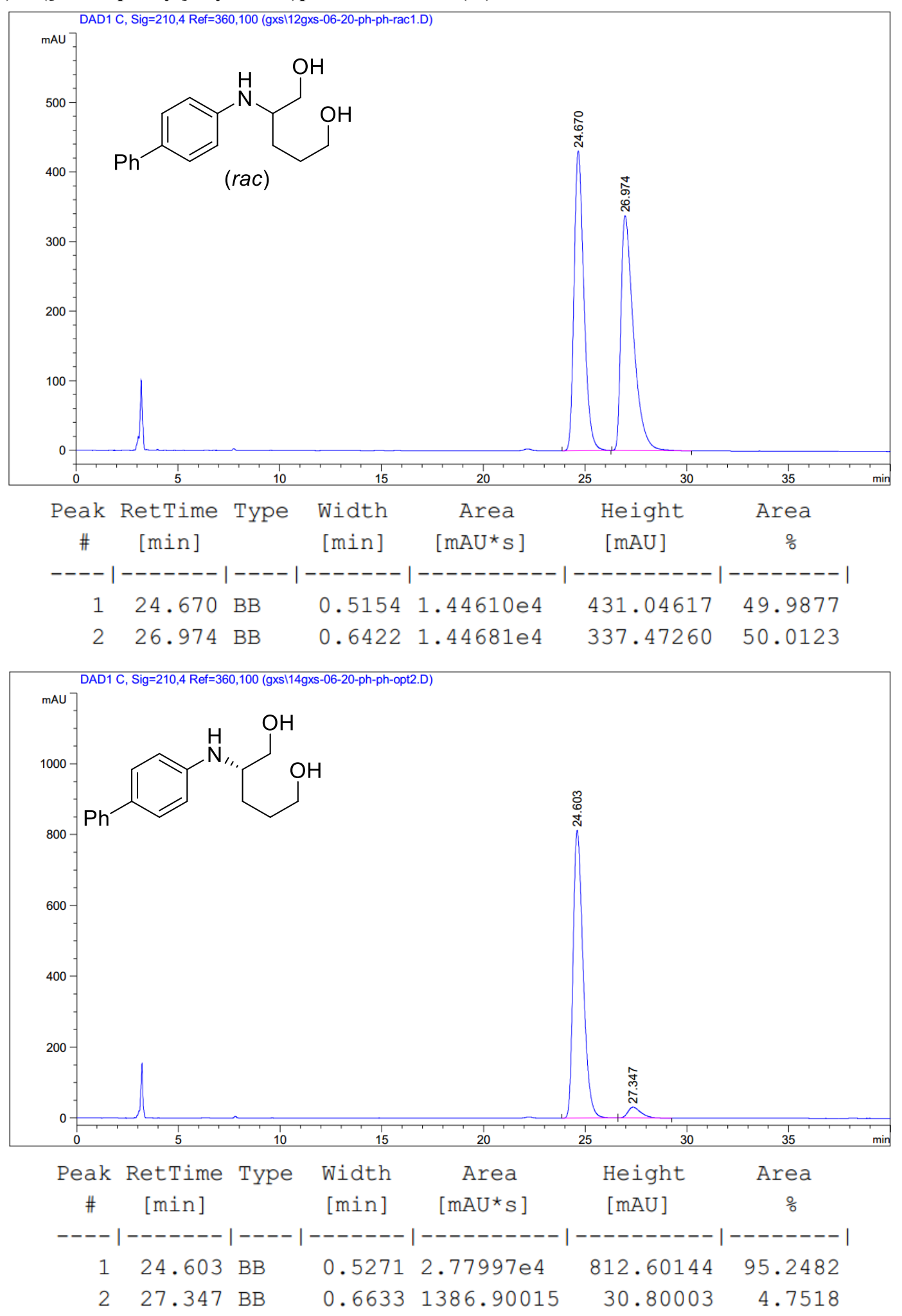


\section{(S)-2-(m-Tolylamino)pentane-1,5-diol (9g)}
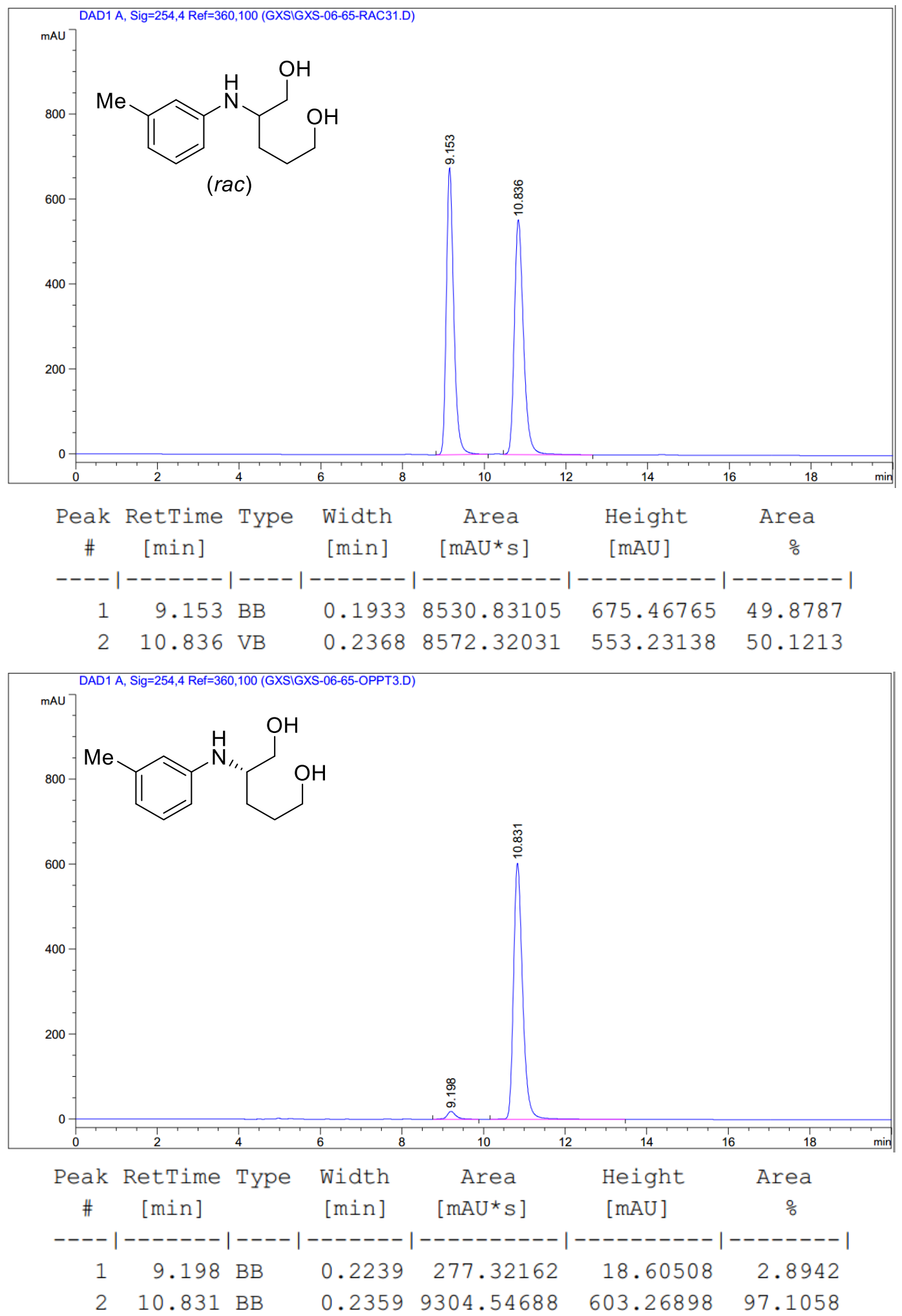
(S)-2-((3-methoxyphenyl)amino)pentane-1,5-diol (9h)
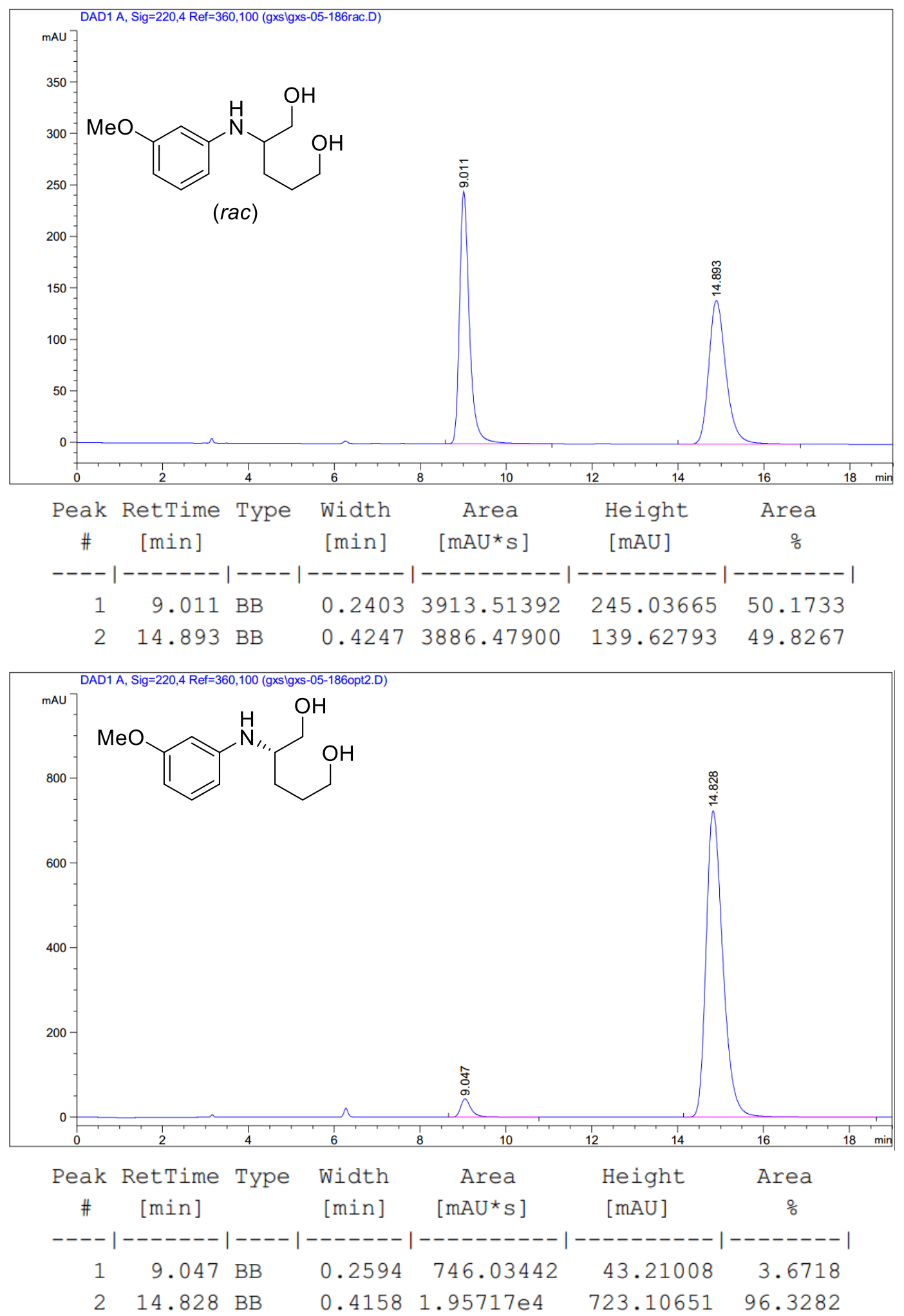
(S)-2-((3-Chlorophenyl)amino)pentane-1,5-diol (9i)
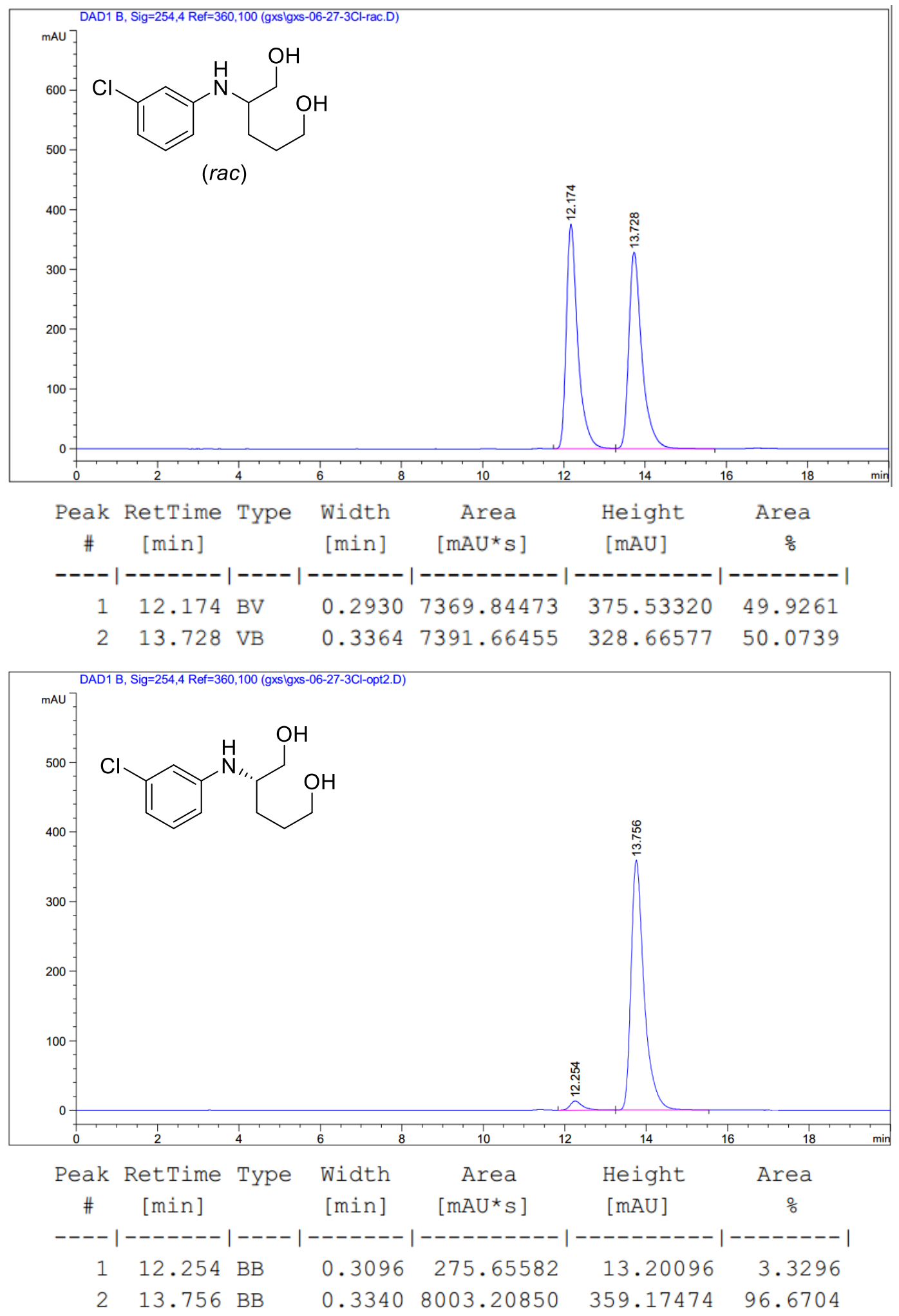


\section{(S)-2-(o-Tolylamino)pentane-1,5-diol (9j)}
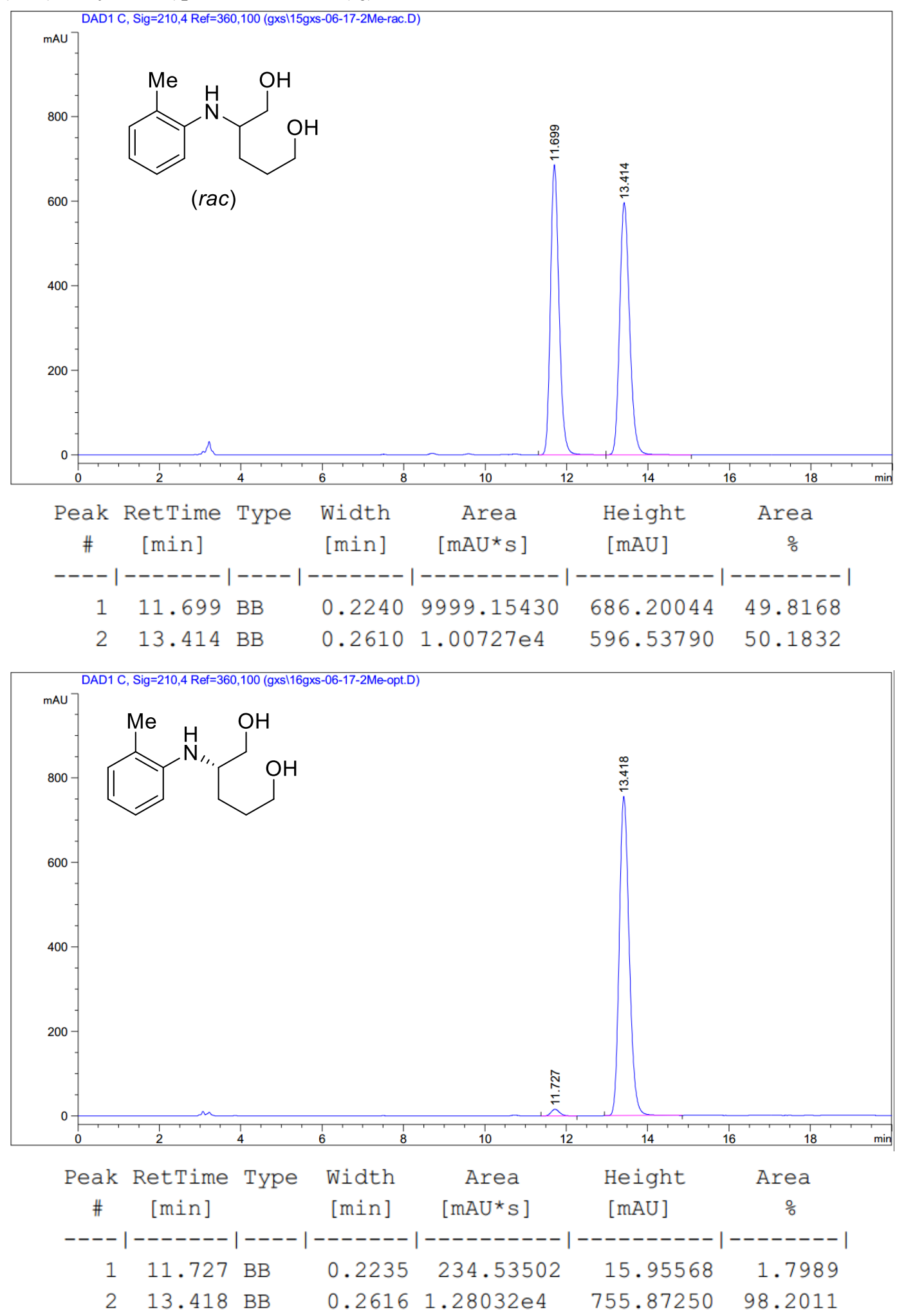


\section{(S)-2-((2-Ethylphenyl)amino)pentane-1,5-diol (9k)}
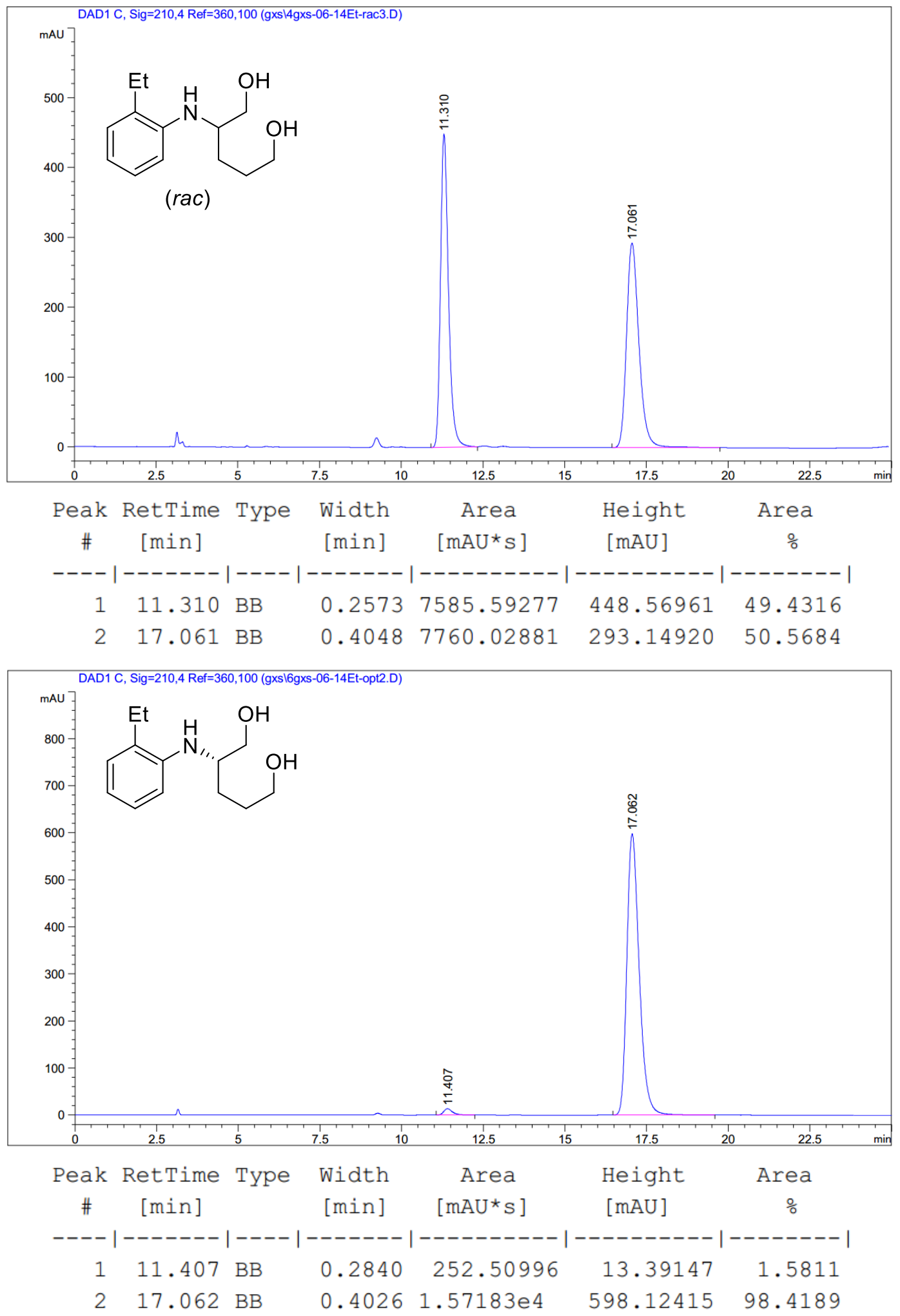


\section{(S)-2-((2-Isopropylphenyl)amino)pentane-1,5-diol (9l)}
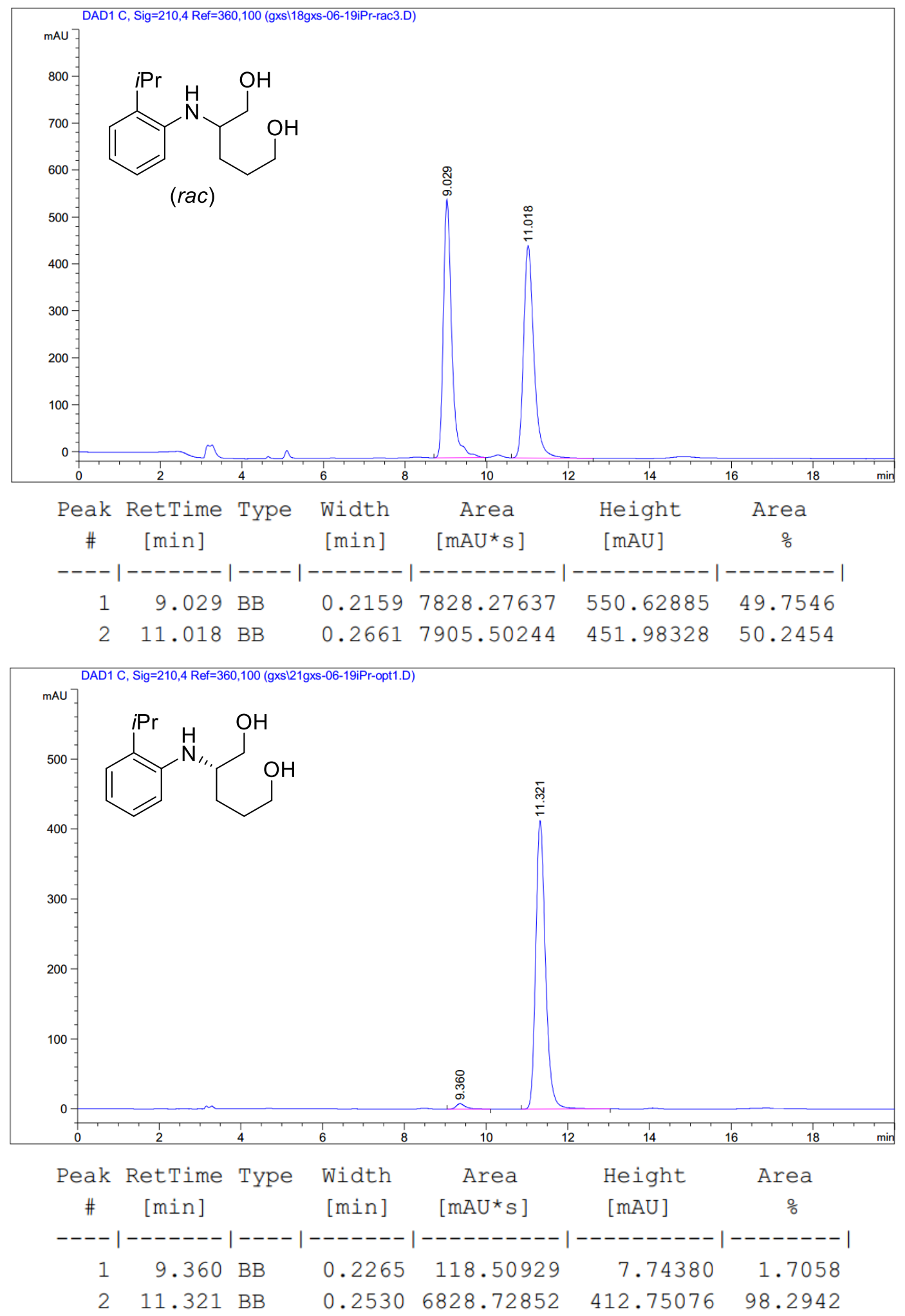


\section{(S)-2-((2-Methoxyphenyl)amino)pentane-1,5-diol (9m)}
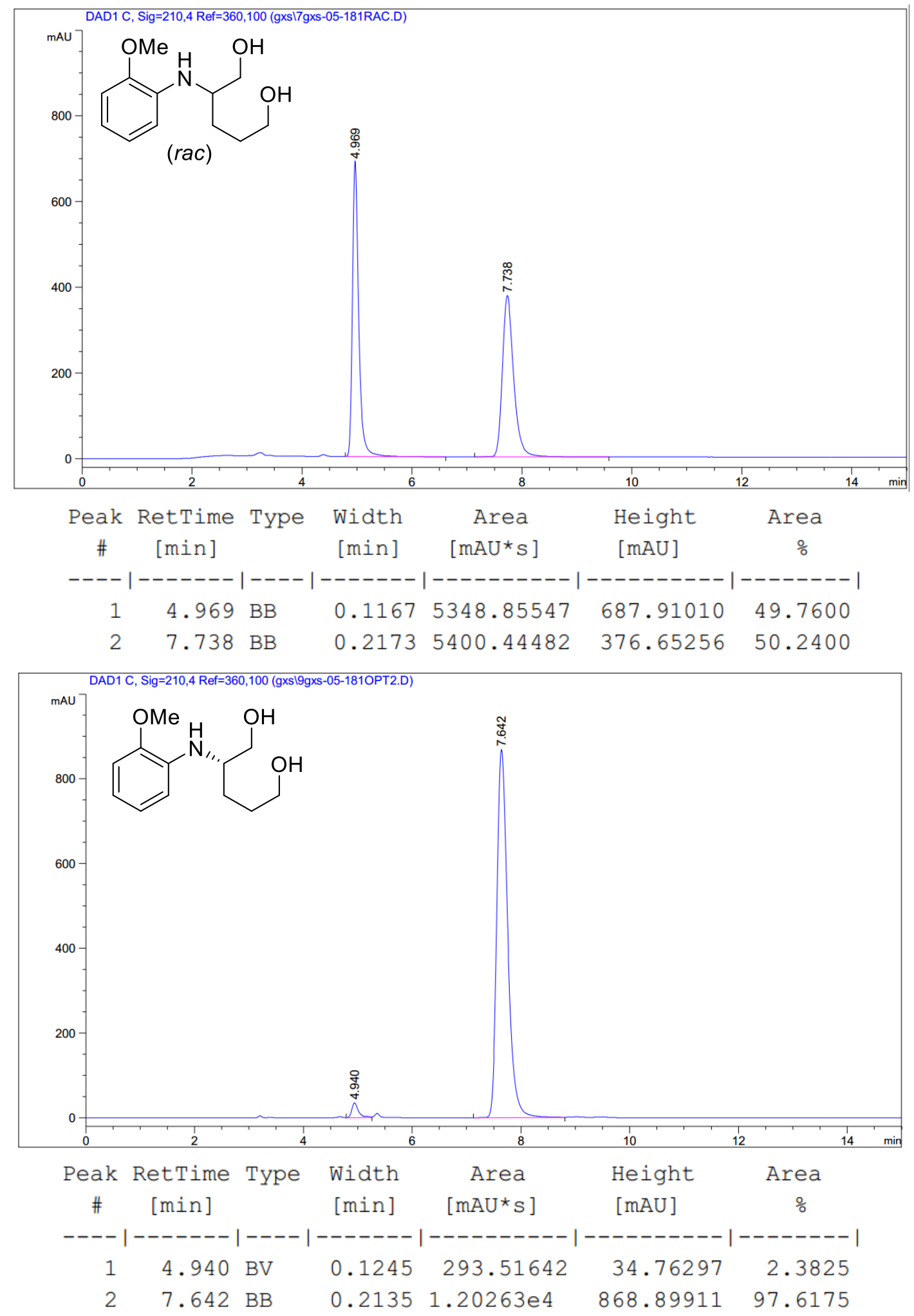
(S)-2-((2-Fluorophenyl)amino)pentane-1,5-diol (9n)
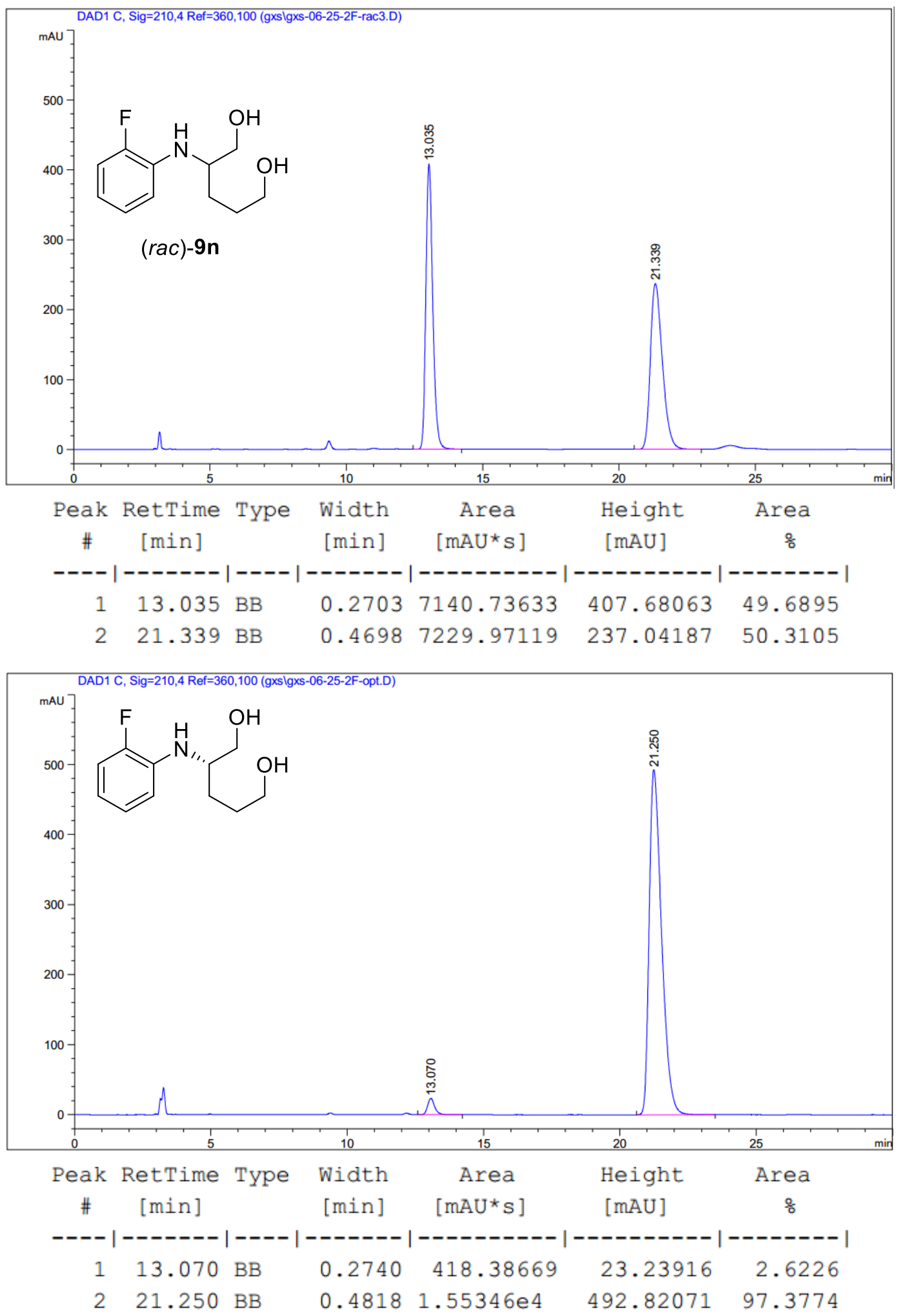
(S)-2-((2,4-Dimethoxyphenyl)amino)pentane-1,5-diol (9o)
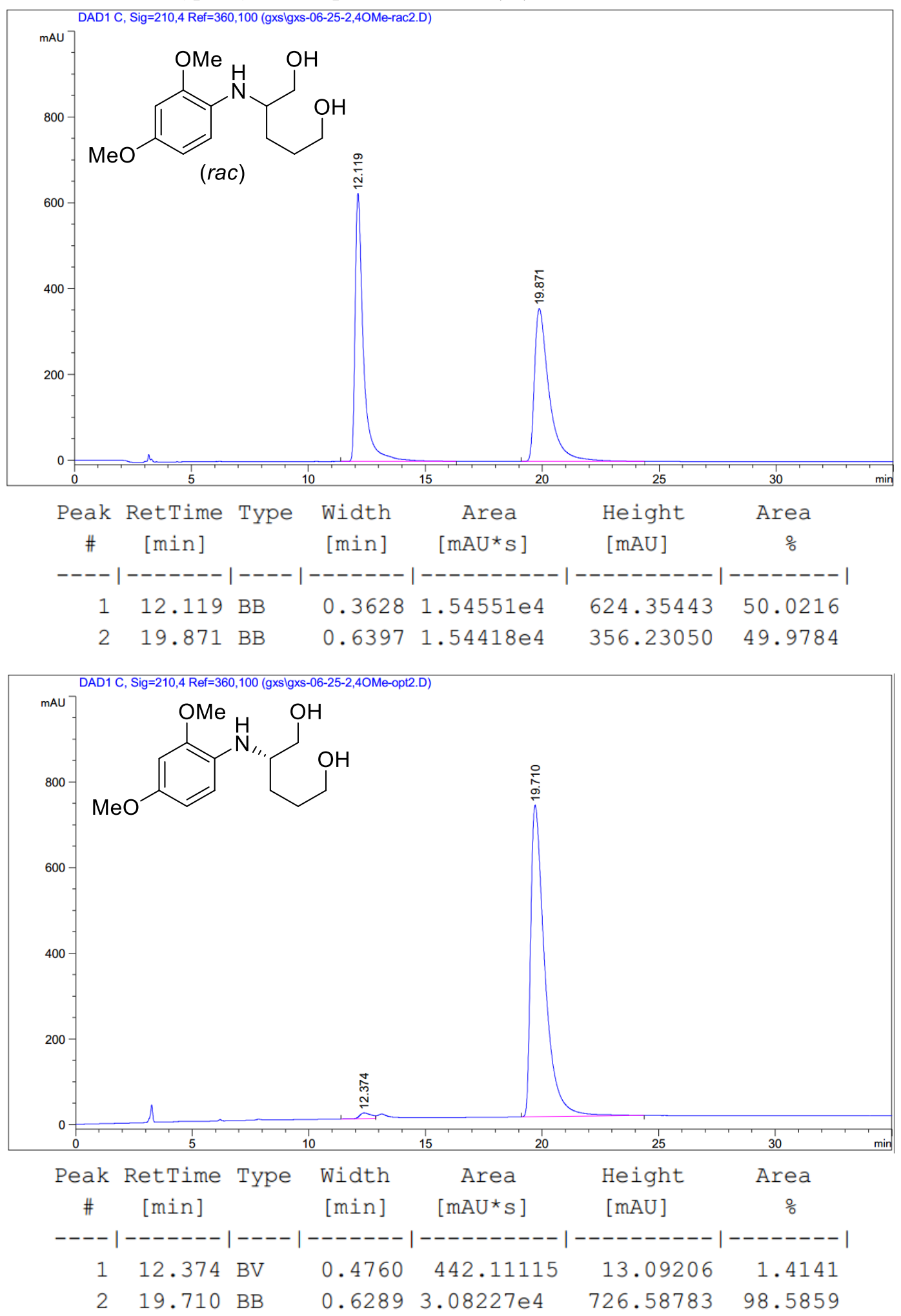
(S)-2-(Naphthalen-1-ylamino)pentane-1,5-diol (9p)
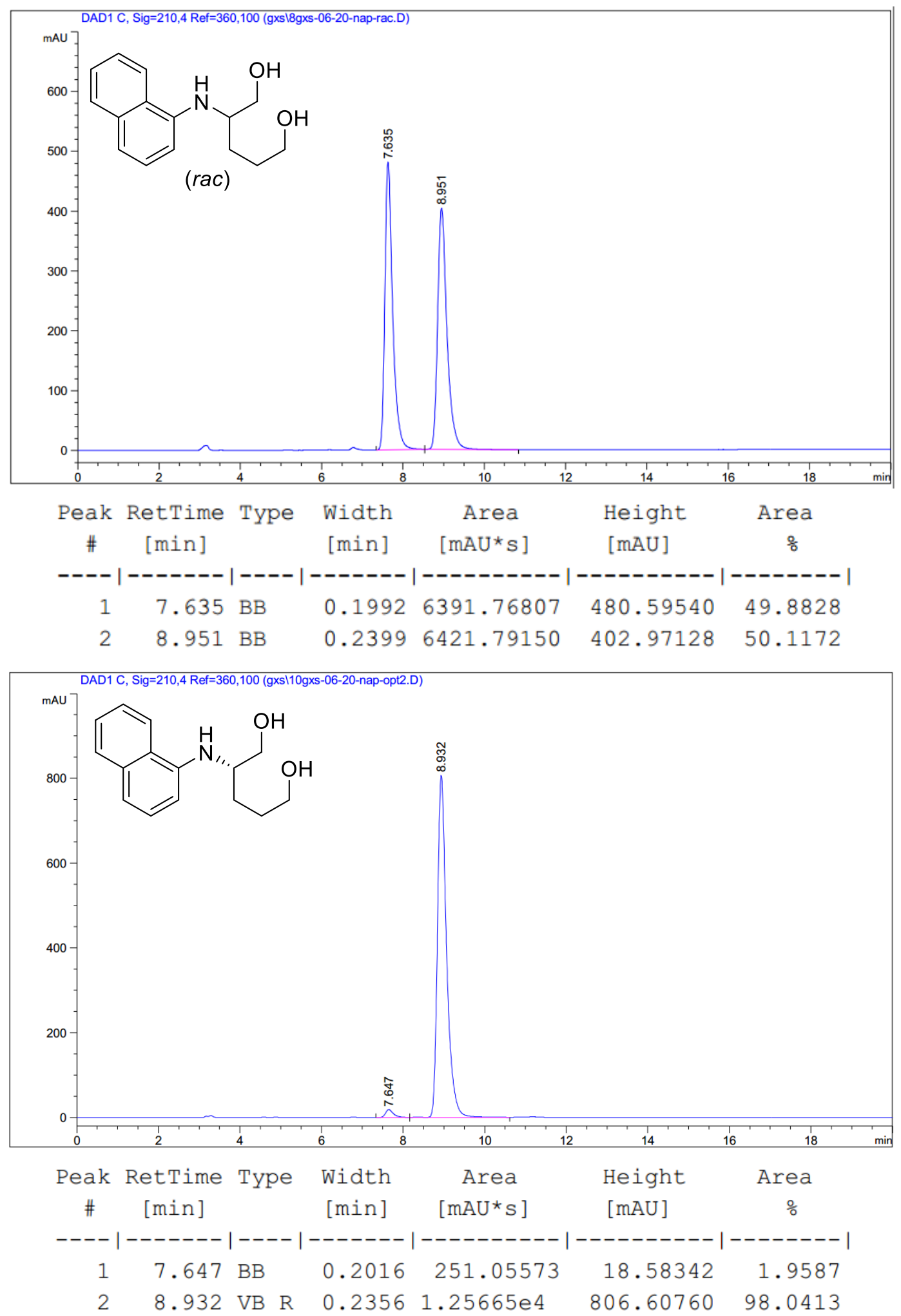